\title{
Cognitive Foundations for Visual Analytics
}

\author{
FL Greitzer \\ CF Noonan \\ LR Franklin
}

February 2011

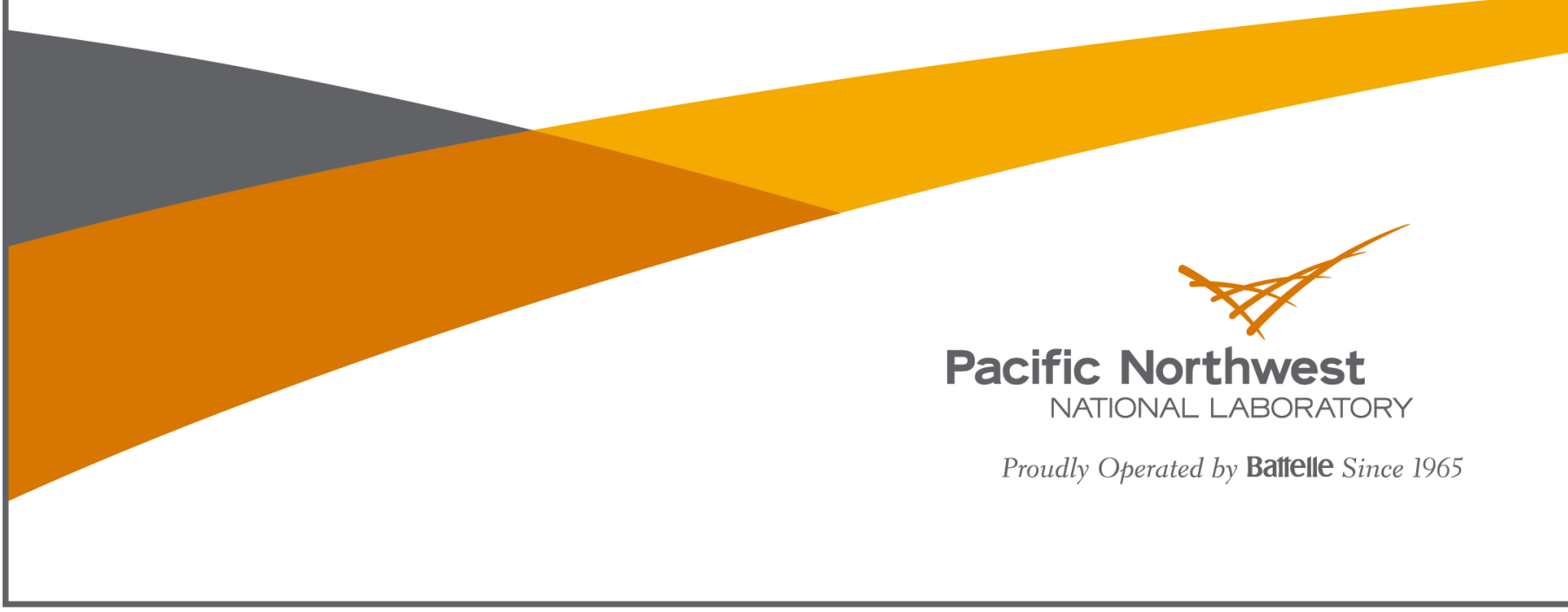




\title{
DISCLAIMER
}

This report was prepared as an account of work sponsored by an agency of the United States Government. Neither the United States Government nor any agency thereof, nor Battelle Memorial Institute, nor any of their employees, makes any warranty, express or implied, or assumes any legal liability or responsibility for the accuracy, completeness, or usefulness of any information, apparatus, product, or process disclosed, or represents that its use would not infringe privately owned rights. Reference herein to any specific commercial product, process, or service by trade name, trademark, manufacturer, or otherwise does not necessarily constitute or imply its endorsement, recommendation, or favoring by the United States Government or any agency thereof, or Battelle Memorial Institute. The views and opinions of authors expressed herein do not necessarily state or reflect those of the United States Government or any agency thereof.

\author{
PACIFIC NORTHWEST NATIONAL LABORATORY \\ operated by \\ BATTELLE \\ for the \\ UNITED STATES DEPARTMENT OF ENERGY \\ under Contract DE-AC05-76RL01830
}

\author{
Printed in the United States of America \\ Available to DOE and DOE contractors from the \\ Office of Scientific and Technical Information, \\ P.O. Box 62, Oak Ridge, TN 37831-0062; \\ ph: (865) 576-8401 \\ fax: (865) $576-5728$ \\ email: reports@adonis.osti.gov \\ Available to the public from the National Technical Information Service, \\ U.S. Department of Commerce, 5285 Port Royal Rd., Springfield, VA 22161 \\ ph: (800) 553-6847 \\ fax: (703) 605-6900 \\ email: orders@ntis.fedworld.gov \\ online ordering: http://www.ntis.gov/ordering.htm
}

This document was printed on recycled paper. $(9 / 2003)$ 


\section{Cognitive Foundations for Visual Analytics}

FL Greitzer

CF Noonan

LR Franklin

February 2011

Prepared for

the U.S. Department of Energy

under Contract DE-AC05-76RL01830

Pacific Northwest National Laboratory

Richland, Washington 99352 



\section{Summary}

Despite the growth of the visual analytics (VA) field, there has been limited systematic testing and evaluation to determine the effectiveness of VA solutions for improving knowledge discovery and decision making. The VA community acknowledges the need for a more scientific foundation to guide research on and evaluation of VA tools. A practical methodology and framework will not only inform the design of VA systems but also facilitate establishment of metrics to evaluate their effectiveness. This report describes the findings of a research project with the following scientific and operational objectives in support of the VA community: (a) Enhance understanding of the role of VA in knowledge discovery and insight; (b) Identify more rigorous scientific methods to evaluate effectiveness of VA tools; and (c) Inform design of deployable VA solutions based on this theoretical foundation.

U.S. Department of Homeland Security end users do not merely want more displays and tools; they need operational/deployable solutions that enhance information processing and decision making. There is also a need for user testing methodologies and metrics to assess performance effectiveness of VA tools in operationally relevant contexts. To this end, the present research examined scientific literature in cognitive science, human factors, and related fields to identify concepts and research results that inform the application of VA technologies to meet operational challenges. By updating previous taxonomies for VA approaches and applications, we hope to provide a more comprehensive framework and benchmarks for this expanding field.

In this report, we provide an overview of scientific/technical literature on information visualization and VA. Topics discussed include an update and overview of the extensive literature search conducted for this study, the nature and purpose of the field, major research thrusts, and scientific foundations. We review methodologies for evaluating and measuring the impact of VA technologies as well as taxonomies that have been proposed for various purposes to support the VA community. A cognitive science perspective underlies each of these discussions.

The following conclusions and recommendations are provided for advancing the VA field and for continuing and expanding this research program in cognitive foundations for VA.

Recommendations for future research:

- More research is needed on sensemaking/problem solving and the analytic process to help align visualization technologies and representation techniques to user's mental models and thought processes.

- Research is required to advance the science and engineering practices of VA tool evaluation.

- Research is needed to develop more effective means of communicating the results of analyses to stakeholders (intuitive and natural ways of conveying findings as well as providing rationale and background information supporting the decisions and recommendations).

- A more directed application of cognitive theories and results of empirical research on critical decision making and problem solving are needed to inform VA system design, development, and evaluation.

- Research is required to enhance methods for evaluating the effectiveness of VA tools. Evaluations are needed to determine if goals and requirements of a program or tool have been satisfied. 
- Researchers in the VA field have concluded that future research should always include evaluation studies and should seek to provide a scientific basis for their conclusions that transcends assessment of individual tools (moving beyond "point solutions").

- Further analysis is required to define requirements for the establishment of a test bed to support evaluation of VA tools.

- Stakeholders and thought leaders from the research community and end-user communities need to determine ways to foster more frequent and meaningful interactions and knowledge exchanges to provide an atmosphere and even formal processes that ensure the acceptance of VA tools by user communities. Two means of facilitating such exchanges are recommended:

- Conduct joint workshops involving researchers, practitioners, and operational users to survey the state of practice of VA tool development and identify operational needs and requirements that informs and provides directions for research.

- Establish an interactive forum at the VA Community Site to facilitate discussion and exchange of ideas.

This report represents an initial step toward meeting these research recommendations. In addition, we have established a Cognitive Foundations Forum at the VA Community Site, http://www.vacommunity.org, to facilitate interactions among the VA community to help advance research and deployment of successful products. We hope that this will lead the way toward increased information exchange between cognitive scientists and computer/information scientists engaged in visualization research and facilitate collaboration among the research community and stakeholders and users.

Appendix A of this report contains an exhaustive list of citations and available abstracts from most of the over 1700 research papers uncovered in the literature review. 


\section{Acknowledgments}

The authors gratefully acknowledge support from National Visualization and Analytics Center Director Richard May and Deputy Director Bill Pike, and from Joe Kielman at the U.S. Department of Homeland Security, for making this research possible. We thank Pacific Northwest National Laboratory scientists Andrew Cowell, Olga Kuchar, and Borik Zadeh for their helpful contributions and suggestions supporting this research and benefitting this report. We wish to thank our student intern, Eury Gallegos, for her contributions in supporting the literature search. Finally, we thank several visual analytics community collaborators who have provided helpful suggestions during an external review of the report. 



\section{Acronyms and Abbreviations}

MILCS

SA

SAGAT

VA
Multidimensional In-Depth Long-Term Case Studies situation awareness

Situational Awareness Global Assessment Technique visual analytics 



\section{Contents}

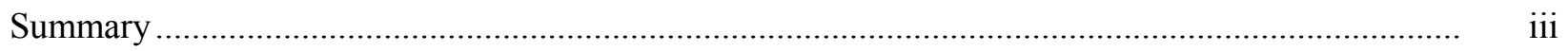

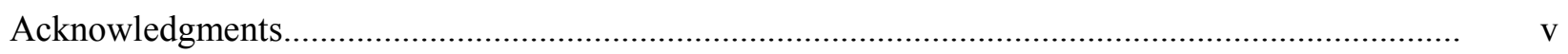

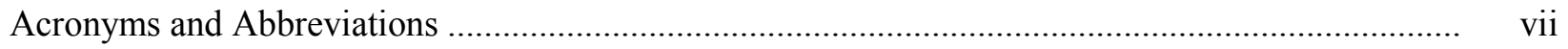

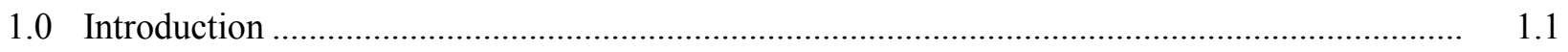

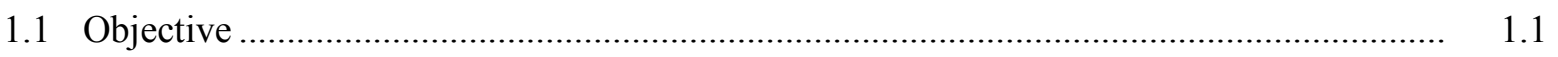

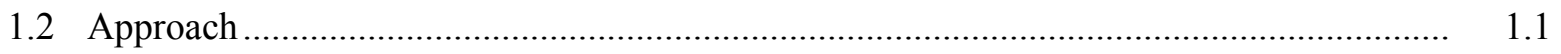

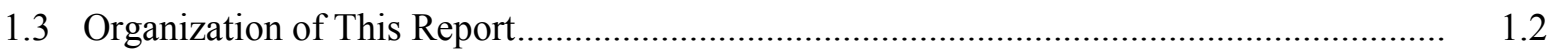

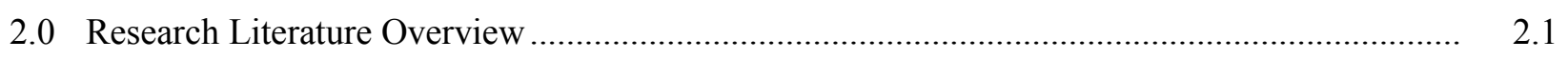

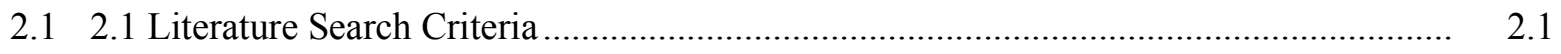

2.2 Literature Review ........................................................................................... 2.1

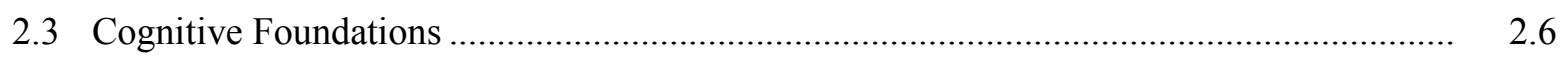

2.3.1 Situation Awareness Theories and Models ....................................................... 2.7

2.3.2 Cognitive/Sensemaking Perspective .............................................................. 2.8

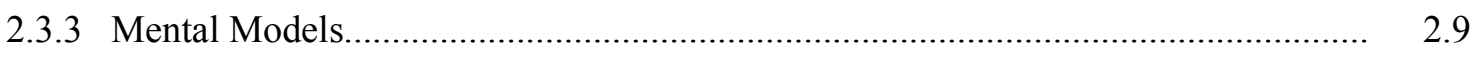

3.0 Methodologies and Metrics for Evaluation .................................................................... 3.1

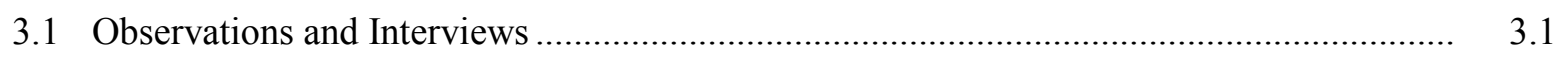

3.2 Questionnaires and Discussion Groups ................................................................ 3.2

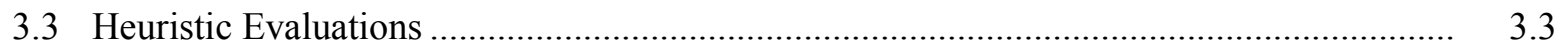

3.4 Longitudinal/Case Studies....................................................................................... 3.3

3.5 Controlled Experiments/Performance Testing ............................................................ 3.4

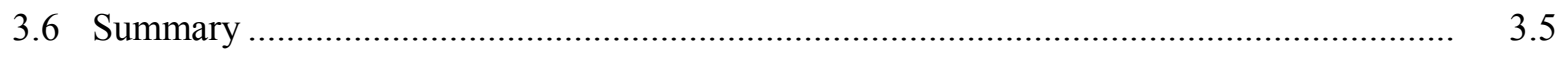

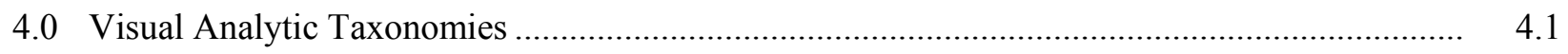

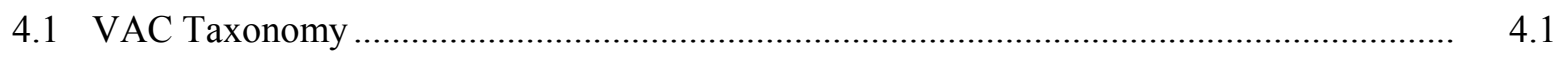

4.2 Review of Literature on VA Taxonomies ................................................................... 4.3

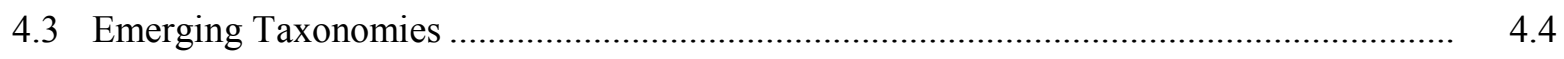

4.3.1 A Taxonomy of Problem Type/Purpose............................................................ 4.6

4.3.2 A Taxonomy of Visual Analytic Technologies..................................................... 4.8

4.3.3 A Taxonomy of Evaluation Methods \& Metrics.................................................... 4.9

4.4 A Work in Progress ................................................................................................ 4.10

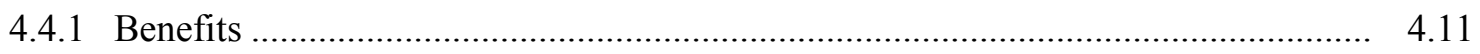

4.5 Status of Taxonomies for Visual Analytics............................................................... 4.12

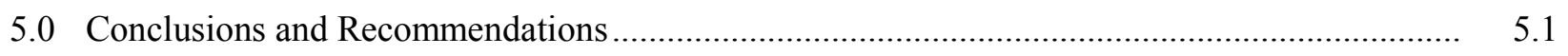

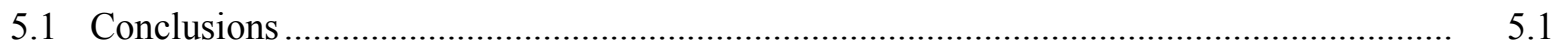

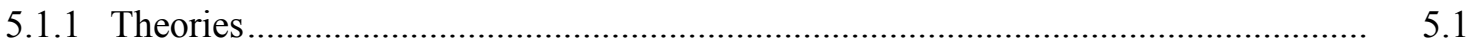

5.1.2 Cognitive/Sensemaking Research .......................................................... 5.2

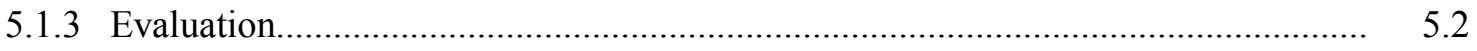




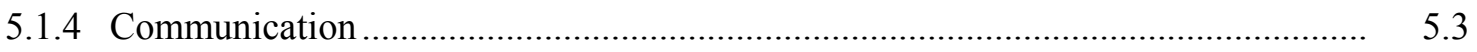

5.1.5 Bringing Communities Together.................................................................. 5.3

5.1 .6 VA Taxonomies …...................................................................................... 5.3

5.2 Summary of Recommendations for Research ............................................................. 5.4

5.3 Recommended Next Steps for Cognitive Foundations Project ........................................ 5.4

5.3.1 Set Up Experimental Test Bed ....................................................................... 5.4

5.3.2 Advance Community Involvement in Cognitive Foundations ............................... 5.5

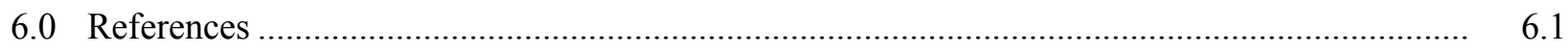

Appendix A - Annotated Bibliography …….............................................................................. A.1 


\section{Figures}

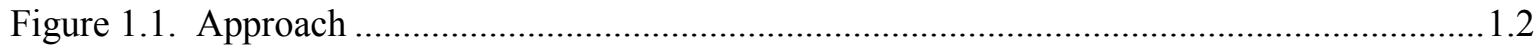

Figure 2.1. At a High Level, Visualization Can be Classified Based on Application Focus..........2.3

Figure 2.2. Classification of VA Field by Problem Type ..........................................................2.3

Figure 2.3. Classification of VA Solutions by Visualization Type.............................................2.4

Figure 2.4. Evaluation Approaches...............................................................................................2.5

Figure 2.5. Effectiveness of VA Tools Depends on Their Ability to Convey Optimal Representations (Mental Models) of the Problem ......................................................2.7

Figure 2.6. Situation Awareness According to Endsley .............................................................2.8

Figure 4.1. VA Taxonomy Developed in 2009 Workshop (Thomas, 2009).................................4.2

Figure 4.2. Process and Relationships Involved in Visualization Research, Development and

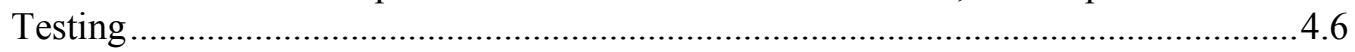

Figure 4.3. Problem Type Taxonomy showing examples of Evaluation Methods ........................4.6

Figure 4.4. Visualization Type Taxonomy ...............................................................................4.7

Figure 4.5. User Actions Organized According to Bloom's Taxonomy...................................... 4.8

Figure 4.6. Evaluation Method Taxonomy (Incomplete) ........................................................4.10

Figure 4.7. Cognitive Taxonomy............................................................................................ 4.11 


\section{Tables}

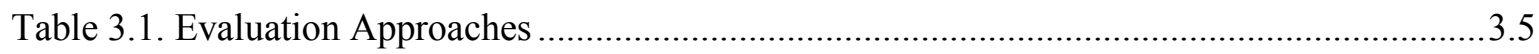

Table 4.1. Problem Type Taxonomy with Examples of Performance Metrics..............................4.7

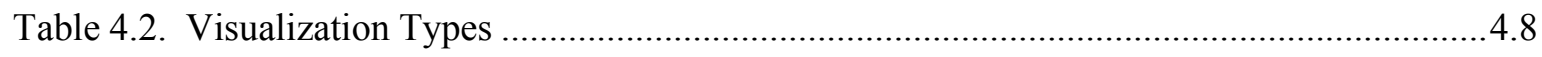

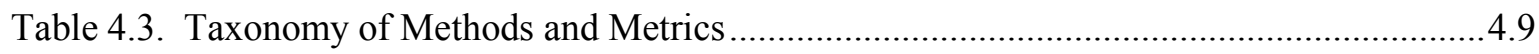




\subsection{Introduction}

Despite the growth of the visual analytics (VA) field, there has been limited systematic testing and evaluation to determine the effectiveness of VA solutions for improving knowledge discovery and decision making. Without a coherent, generalized theoretical framework, studies are unable to draw generalized conclusions; employment of visualizations tends to be based on ad hoc considerations or unduly influenced by aesthetics instead of a more balanced performance-based assessment. The VA community acknowledges the need for a more scientific foundation to guide research on and evaluation of VA tools (Liu, Nersessian and Stasko, 2008; Liu and Stasko, 2010). A practical methodology and framework will not only inform the design of VA systems but also facilitate establishment of metrics to evaluate their effectiveness.

\subsection{Objective}

This research project supports the VA community by pursuing the following scientific and operational objectives:

- Enhance understanding of the role of VA in knowledge discovery and insight

- Identify more rigorous scientific methods to evaluate effectiveness of VA tools

- Inform design of deployable VA solutions based on this theoretical foundation.

U.S. Department of Homeland Security end users do not merely want more displays and tools; they need operational/deployable solutions that enhance information processing and decision making performance. There is also a need for user testing methodologies and metrics to assess performance effectiveness of VA tools in operationally relevant contexts.

\subsection{Approach}

This research is examining scientific literature in cognitive science, human factors, and related fields to identify concepts and research results that inform the application of VA technologies to meet operational challenges. To provide a structure for the analysis, we are categorizing VA research to reveal where different cognitive science concepts and empirical results inform VA design and analysis. By updating previous taxonomies for VA approaches and applications, we hope to provide a more comprehensive framework and benchmarks for this expanding field. More specifically, as shown in Figure 1.1, the focus of the research is on: Scientific Foundations for visualization technology in problem solving, decision making, and insight research; Visualization Taxonomy to structure the research; and Experiment Designs and Scenarios for evaluating effects of visualization on decision making and insight. Follow-on research will define requirements for the establishment of a test bed laboratory for empirical research and validation of visualization tools. 


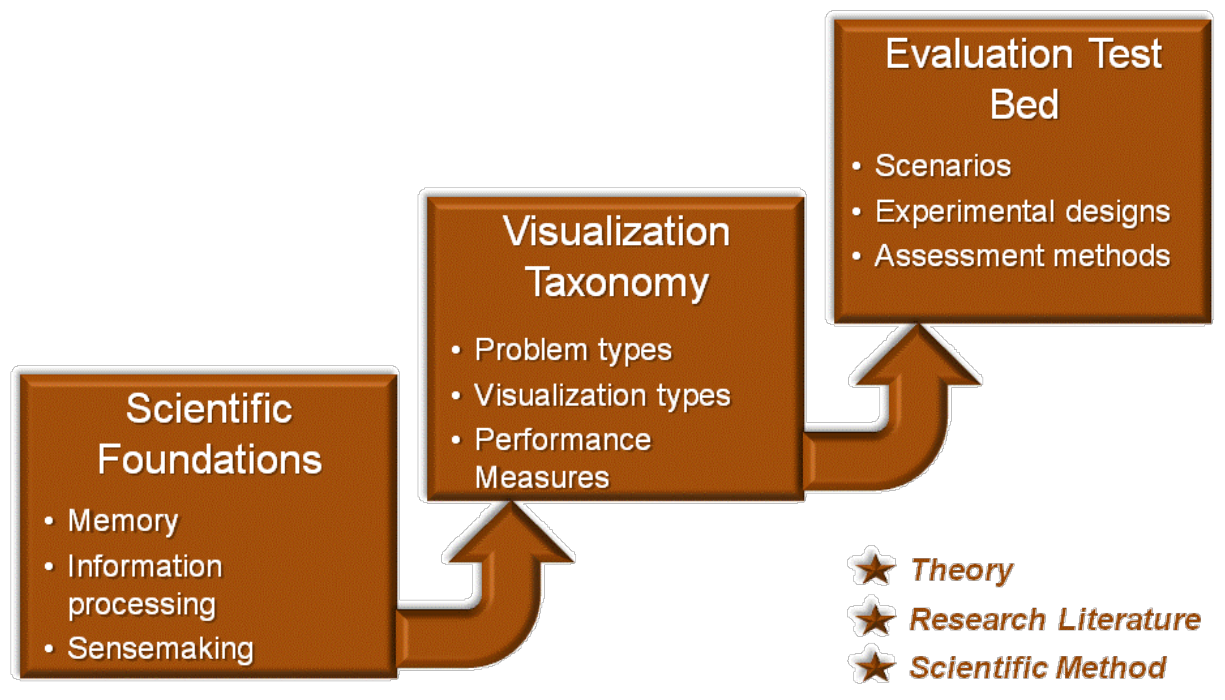

Figure 1.1. Approach

\subsection{Organization of This Report}

Section 2 of this report provides an overview of scientific/technical literature on information visualization and VA. Topics discussed include an update and overview of the extensive literature search conducted for this study, the nature and purpose of the field, major research thrusts, and scientific foundations. Section 3 reviews methodologies applied to evaluating and measuring the impact of VA technologies. Section 4 reviews selected literature that has attempted to produce taxonomies for various purposes to support the VA community. A cognitive science perspective underlies each of these discussions. Section 5 presents conclusions and recommendations for advancing the VA field and for continuing and expanding this research program in cognitive foundations for VA. References cited in the report are listed in Section 6. Following the report proper, Appendix A contains an exhaustive list of citations and available abstracts from most of the over 1700 research papers uncovered in the literature review. 


\subsection{Research Literature Overview}

\subsection{1 Literature Search Criteria}

We conducted an extensive literature search to update our understanding of the VA field from multiple perspectives. The focus of the review was to inform research and evaluation of VA technologies and products. A more complete description of search criteria and results is provided in Appendix A. ${ }^{1}$

Multiple searches were conducted in commercially available databases covering the broad areas of computer science, psychology, cognitive science, and human factors. No restrictions were placed on publication date or language of publication. The database queries identified over 1,900 citations. In addition, several large datasets were collected from VisWeek 2010 and National Visualization and Analytics Center archives. After removing redundancies and documents that were not research-oriented, the collection contains over 1700 research citations.

\subsection{Literature Review}

The study of scientific discovery has long been criticized by philosophers as irrelevant to analyzing the growth of scientific knowledge. In particular, little is known about how cognitive theories are discovered. Furthermore, neither the classical accounts of discovery as probabilistic induction nor the stock anecdotes about sudden "eureka" moments serve to deepen our insight into discovery (Gigerenzer, 1991). The ancient Greeks likened insight to a gift from the Muses, something that was believed to originate from the divine and/or the unconscious. The term insight is used to describe the "clear and sudden understanding of how to solve a problem" and is "thought to arise when a solver breaks free of unwarranted assumptions, or forms novel, task-related connections between existing concepts or skills" (Bowden, et al., 2005). It is natural then that the essence of insight has been deemed unpredictable and unexplainable through scientific measurement (Shanker, 1995). It has proven difficult to determine precisely the mechanisms underlying insight, primarily due to the limitations on insight research. This "problem" surrounding insight has been one of the leading conundrums in artificial intelligence, information visualization, and VA research.

At the core of this fundamental problem is the assumption that problem solving is a cognitive activity, and that the eureka moment, or moment of illumination, is caused by an unbroken chain of unconscious processes; i.e., it is processed in the background. This process of creative thinking is the starting point for Newell and Simon's $(1961,1963)$ theory of General Problem Solving, a theory designed to explain the nature of unconscious processes, the imagery employed in creative thinking and its significance to the thinking, and the phenomenon of "illumination," the flash of insight that reveals the solution of a problem being pursued. This theory is debated quite extensively by Shanker (1995) and will not be recapitulated here. In closing, Shanker observes that "what progress has been made in the psychology of creativity is largely the result of the increased attention that has been paid to the development of a repertoire of cognitive skills" (Shanker, 1995, p. 579) and he asserts that the greatest advances in our understanding of creative problem-solving have come from real-life and biographical studies (i.e.,

\footnotetext{
${ }^{1}$ Appendix A provides an alphabetic listing of the literature citations along with published abstracts, where available. Other, more usable forms of the repository, such as an organized list based on key words, will be made available.
} 
naturalistic/observational research as opposed to laboratory experiments and computational simulations). This argument has been reflected more recently in several papers advocating increased use of long-term case studies in VA research (e.g., Saraiya, North, and Duca, 2004; Shneiderman and Plaisant, 2006; Perer and Shneiderman, 2008; see also the discussion in Section 3.3).

Insight continues to be a core concept for many researchers in the field of visualization. Pike et al. $(2009$, p. 263) put it best: "A central precept of visual analytics is that the development of human insight is aided by interaction with a visual interface. As visual analytics is concerned with the relationship between visual displays and human cognition, merely developing novel visual metaphors is rarely sufficient to trigger this insight (where insight may be a new discovery or confirmation or negation of a prior belief). These visual displays must be embedded in an interactive framework that scaffolds the human knowledge construction process with the right tools and methods to support the accumulation of evidence and observations into theories and beliefs."

Visualization research has commonly focused on representing human mental processes (Zhu and Chen, 2005). This concept is now associated with the amplification of these mental processes and offers a link between the human eye and the computer to enable pattern recognition and to create insights from large quantities of data. According to Tufte (2006), science and art have "intense seeing" in common. He describes this union of data and its subsequent visualization as the "wide-eyed observing that generates empirical information." Empirical observations support explanations and presentations of evidence. Identification of patterns and analysis of data are important ingredients of scientific discovery as well as information analysis processes in identifying clues to help solve a crime, predicting terrorist threats, or forecasting the stock market. All of these activities have the potential to involve overwhelming and disparate quantities of data, whose value can be reduced because of the efforts involved in analysis and data exploration (Thomas and Cook, 2005). The frustration experienced by the end user can be alleviated with the use of visualization tools. Visualization strives to facilitate the analytical reasoning process by creating software that maximizes the human capacity to perceive, understand, and reason about complex, abstract, and dynamic data and situations (Thomas and Cook, 2005). The ultimate goal is to support rapid, high-quality decision-making to solve practical problems.

At a very high level, visualization can be classified based on application focus, as shown in Figure 2.1. These categories usually include scientific collaboration, software visualization, or information visualization (Zhu and Chen, 2005). The categories are not mutually exclusive and have fuzzy boundaries. Scientific visualization helps scientists and engineers more efficiently understand physical phenomena embedded in large volumes of data. The visualizations are typically 3-D renderings of volume, surface, etc. and may in addition contain a time component. Software visualization helps people understand and use computer software effectively (Stasko, et. al., 1998), while information visualization helps users identify patterns, correlations, or clusters of structured or unstructured data. Additionally, VA marries techniques from information visualization with techniques from computational transformation and data analysis (Thomas and Cook, 2005). 


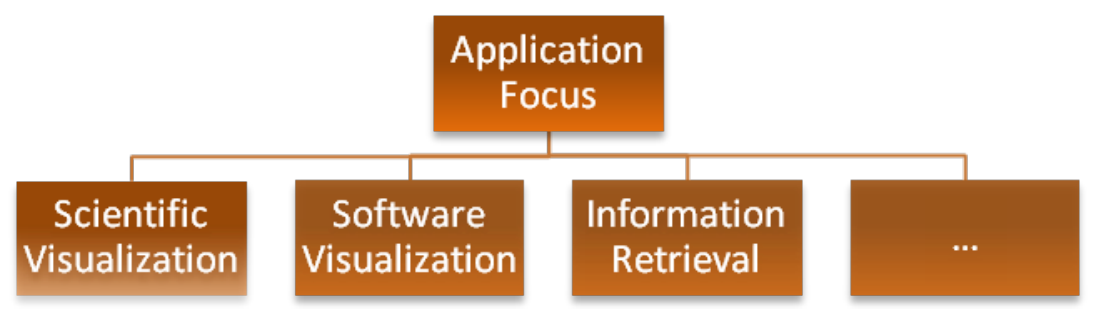

Figure 2.1. At a High Level, Visualization Can be Classified Based on Application Focus

Previous studies have constructed various taxonomies to categorize visualization research from a multitude of perspectives, many of which are discussed later in this report. Based on the type of problems addressed and/or objectives of the analysis, the visualization field may be further divided by problem type, such as visualizations to communicate an idea, support information retrieval, system monitoring, training, facilitate knowledge discovery, or enhance decision making (see Figure 2.2).

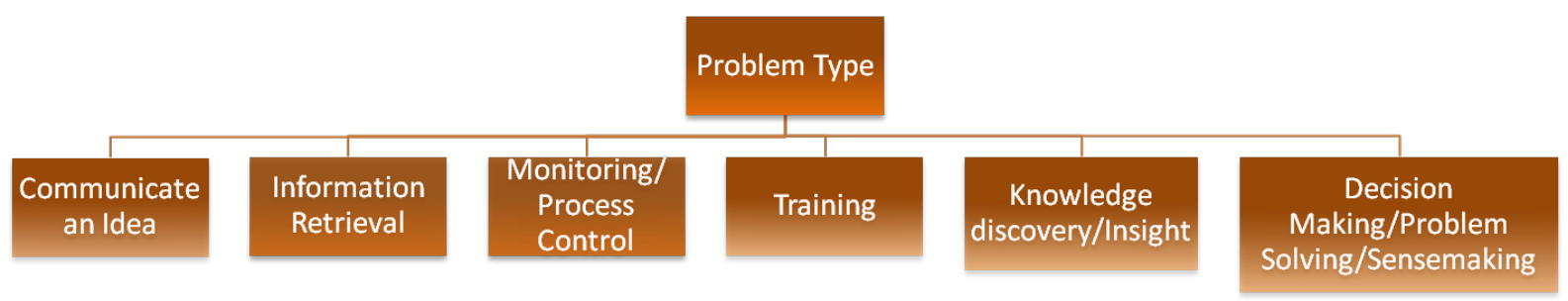

Figure 2.2. Classification of VA Field by Problem Type

Based on the features of VA techniques to meet these various objectives, VA solutions may be categorized according to visualization type on the basis of factors such as data types (including geographic information systems, multidimensional tables, nodes and links); information representation (1-D, 2-D, 3-D, temporal); user-interface interaction (zoom, filter, relate); and information analysis (index, extract, analyze); this classification is roughly conveyed in Figure 2.3. 


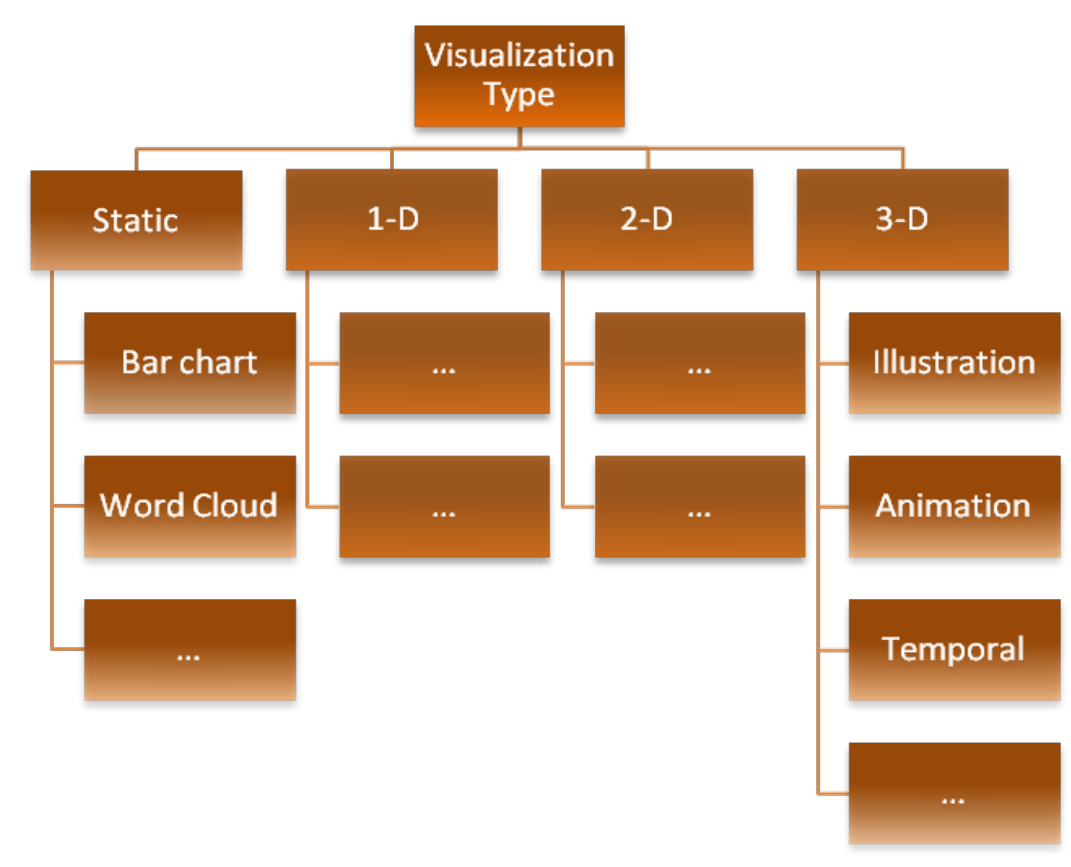

Figure 2.3. Classification of VA Solutions by Visualization Type

Because the development of a visualization system usually integrates several techniques, it is extremely helpful to provide a framework of visualization technologies based on their functionalities, analyst tasks, data types, and more. This same framework can be used to catalog and evaluate tools and to help with tool selection based on the tool's applicability to domain-specific tasks and/or activities. Indeed, information visualization can be applied to practically any domain where users need to extract insights from a vast amount of information including digital libraries and information repositories, patent analysis, and financial and market analysis. However, to maximize the utility of information visualization tools, evaluation metrics must consider the goals of such analysis. If indeed the goal is "to build knowledge, generate insight, and perform analysis" (Pike, et al., 2009), the discipline as a whole has fallen short when it comes to self-evaluation.

There are many types of visualization tools created for a variety of purposes. ${ }^{2}$ Due to the proliferation of tools available today, it is unfeasible and unwieldy to "try out" multiple tools to realize performance and suitability for a given task or activity. A clear need for the visualization field is support for choosing the right tool for a specific task, with the aim of enhancing decision making and reducing information overload. One type of support framework for selecting an appropriate VA tool is the use of taxonomies that organize tools using a standard set of criteria, allowing the end user to drill down into the taxonomy and choose important features or desired outcomes. Several proposed taxonomies for classifying visualization tools are discussed in Section 4 of this report.

\footnotetext{
${ }^{2}$ Because the focus of our study is on information analysis and knowledge discovery/problem solving, we shall not cover the interesting and popular topic of graphics design and use of graphical representation techniques for communicating concepts through data visualization. The IBM Many Eyes web site provides an interactive way to explore visualization techniques for information representation [http://services.alphaworks.ibm.com/manyeyes/home].
} 


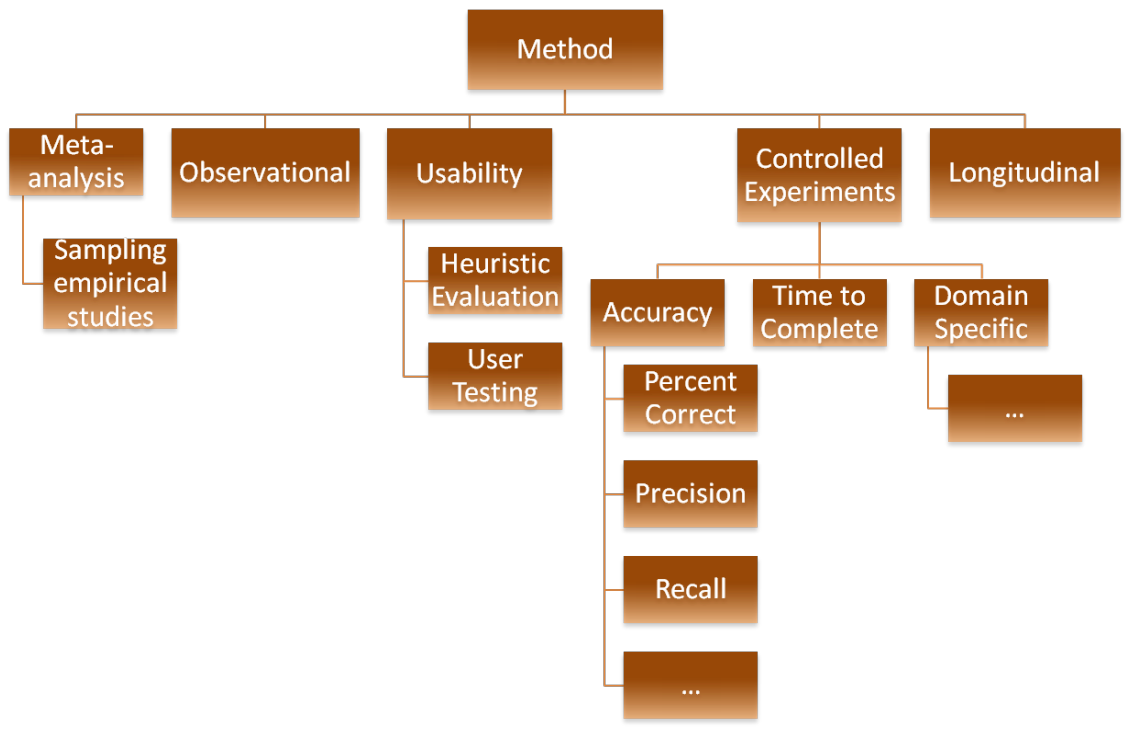

Figure 2.4. Evaluation Approaches

In addition to taxonomy, empirical evaluation is another technique in selecting a visualization tool. Many evaluation approaches employ controlled studies to validate the performance of visualization systems and designs. It is important to recognize that the entire evaluation process needs to be considered - from hypothesis forming to the presentation/communication of the results. However, because of the complexity of real-life system interface tasks, the validity of such studies and the applicability of research conclusions are sometimes questioned by practitioners. For example, low-level tasks such as spotting trends, clusters, and outliers are relatively easy to validate, whereas more complex tasks do not lend themselves well to standard lab experiments and validation. These complex, realistic, task-driven analyses are conducted frequently in visualization research and may take days or months to complete. A range of evaluation methods exist and include quantitative methods, qualitative methods, mixed method approaches, usability studies, and informal evaluation techniques (the classes not being mutually distinct) (Keim, et al., 2010). Accuracy of operation results and time to completion are two commonly used measures. For example, depending on the chosen method, one can examine very specific questions in a controlled laboratory environment in which a testable hypothesis can be formulated and which leads to conclusions with high confidence. Another type of evaluation looks at broader questions using qualitative methods with a focus on data acquisition through observation and interviewing. Mixed-method techniques combine aspects from both qualitative and quantitative evaluation. Usability evaluation is one such example that deals specifically with the ease of use of interactive tools. Finally, heuristic evaluations involve fewer people who give feedback on a visualization or the system used to create them, providing anecdotal evidence for its usefulness or effectiveness. Evaluation strives to be generalizable, precise, and realistic. As common principles, best practices, and theories are developed, they can be applied to visualization and problem-solving tools to validate performance. Figure 2.4 illustrates the range of evaluation approaches and identifies some associate performance measures. Evaluation approaches are described more fully in Section 3 of this report.

Many evaluation studies for VA tools are focused on specific functionalities that the visualization system aims to provide - we refer to these as transactions between the user and the system - and these studies do not necessarily consider the cognitive processes underlying these transactions that reflect 
problem solving and related mental processes (e.g., productive processes, Katona, 1940; Wertheimer, 1945/1959; Mayer,1996) that have historically been identified with knowledge discovery, complex decision making, and insight. A comprehensive, overarching taxonomy identifying not only tool traits but also design factors, cognitive foundations, and evaluation metrics can provide significant guidance in the design, selection, and utility of visualization tools and interfaces.

There are, of course, other factors to consider in developing, evaluating, or selecting VA tools. Two recent publications (Green \& Fisher, 2010; Green, et al., 2010) argue that the personality of the analyst has a direct impact on the efficacy of visualization and that personality factors influence the ability of an individual to carry-out a task, the individual's approach to solving a problem, and the conclusions that are drawn. Green and colleagues posit that behaviors such as identify-and-find (tasks that are more transactional, using our terminology) use a different combination of "tools in the cognitive toolbox" than do more cognitively complex, iterative reasoning behaviors that come into play during inferential tasks. If these differences are known, interaction performance can be predicted and used to develop design requirements and real-time interface interaction.

With the above overview of the VA field, we have attempted to provide both a historical perspective and a discussion of current thinking and research directions. ${ }^{3}$ A general message from this discussion is that advancement of the VA field will be facilitated by devoting more attention to cognitive foundations that will inform future research and evaluation approaches. Another observation is the conviction that successful deployment of VA technologies will require increased attention to practical considerations in facilitating selection of tools to match operational needs. The next section is devoted to further considerations of cognitive science foundations, and the remainder of the report addresses research directions and taxonomies for practical application and exploitation of VA technologies by the operational community.

\subsection{Cognitive Foundations}

As we have described, VA research seeks to enhance decision making, knowledge discovery, and insight in complex operational environments. With the expanding VA field, there is a need for a coherent theoretical framework to guide development of tools and evaluation of their effectiveness. An effective theoretical framework should build upon previous research in human factors including user interface design, information architecture, perceptual psychology, cognitive theory, and naturalistic decision making, as well as research and practice in fields of graphics design, communication theory, and statistics - with a goal of producing a coherent integrated framework. Cognitive theory suggests that the impact of VA on decision making is a function of the extent to which VA solutions enhance situation awareness (SA) and, by implication, facilitate or support optimal representations of the problem that contribute to correct decisions and increased understanding. In this view, the effectiveness of VA tools largely depends upon the consistency between the external representation they provide and the internal representations used by expert decision makers (see Figure 2.5). Internal representations and problemsolving strategies may be characterized using mental models. Mental models are well-defined, highly organized, and dynamic knowledge structures that are developed over time from experience. Appropriate and effective mental models help to integrate the large amount of information that is inherent in complex

\footnotetext{
${ }^{3}$ Please refer to the Appendix for a much broader collection of relevant research papers.
} 
operational environments; they enable experienced decision makers to correctly assess and interpret the current situation and to select an appropriate action.

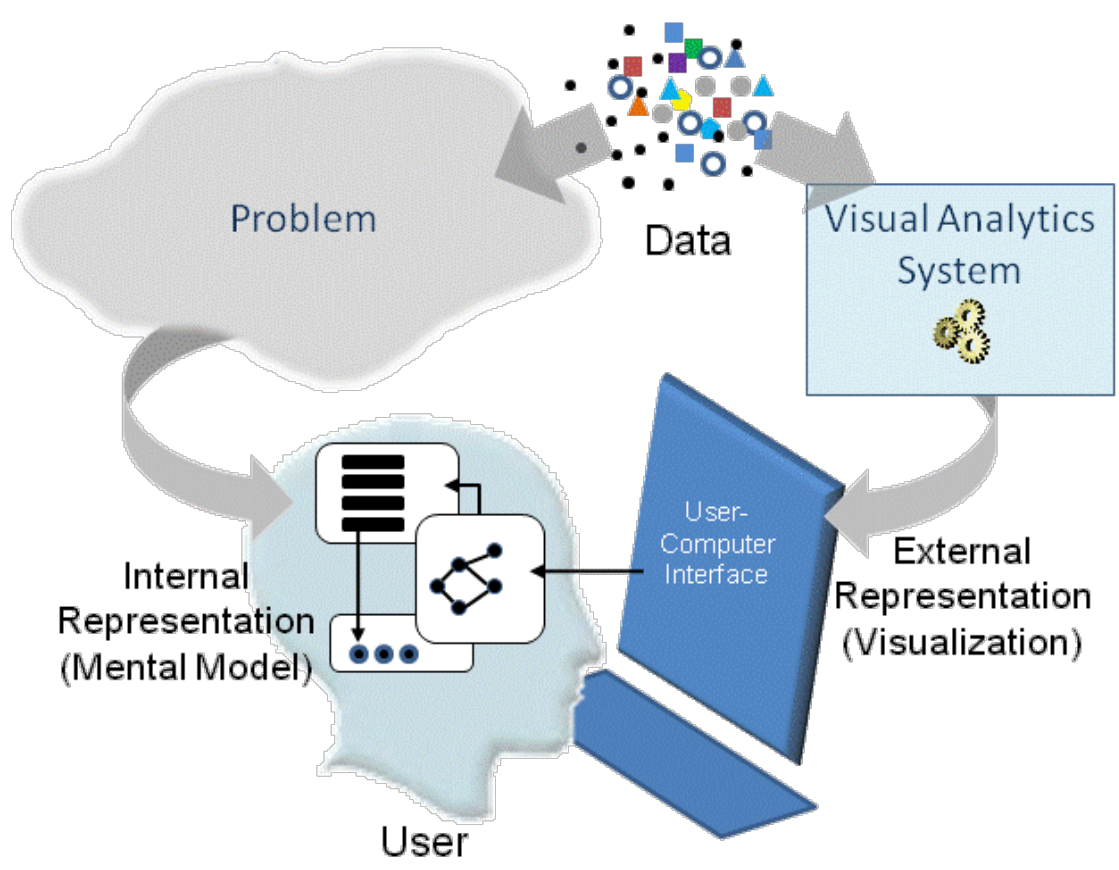

Figure 2.5. Effectiveness of VA Tools Depends on Their Ability to Convey Optimal Representations (Mental Models) of the Problem

\subsubsection{Situation Awareness Theories and Models}

Defining exactly what constitutes SA has been a challenging task because of the complexity in characterizing the construct in terms of a set of psychological processes. As Banbury and Tremblay (2004) have observed, "...the major impetus of research [on SA] has been on the development of techniques to measure SA, at the expense of a more rigorous understanding of why SA varies under certain psychological and environmental conditions." (p. xiv).

Micah Endsley (1988, 1995; Endsley et al. 2004) has provided the most highly recognized descriptive model of SA. She presents the model in two parts: a core SA model and a set of factors affecting SA. The core SA model, which is the basis for much of the current thinking about SA, is a three level system:

- Level 1, Perception of the elements in the environment

- Level 2, Comprehension of the current situation

- Level 3, Projection of future states.

As shown in Figure 2.6, The Perception level is the first step in achieving SA. In cognitive terms, this level involves interaction with long-term memory (comparing the information elements with what is already known); it is under attentional selection (modulated by the operator's selective attention processes as determined by task requirements); and the information content is held in active working memory. The Comprehension level provides an organized picture of the elements with a comprehension of the significance of objects and events. In cognitive terms, mental models stored in long-term memory 
provide a basis for Level 2 SA. The Projection level enables predictions about the states of the environment in the near future. This is achieved through knowledge of the status and dynamics of the elements and comprehension of the situation. In this view of SA, stimuli (information elements) are processed to yield meaning. It follows that a common "solution" to human information processing limitations is to design methods to facilitate processing of more information through limited processing channels.

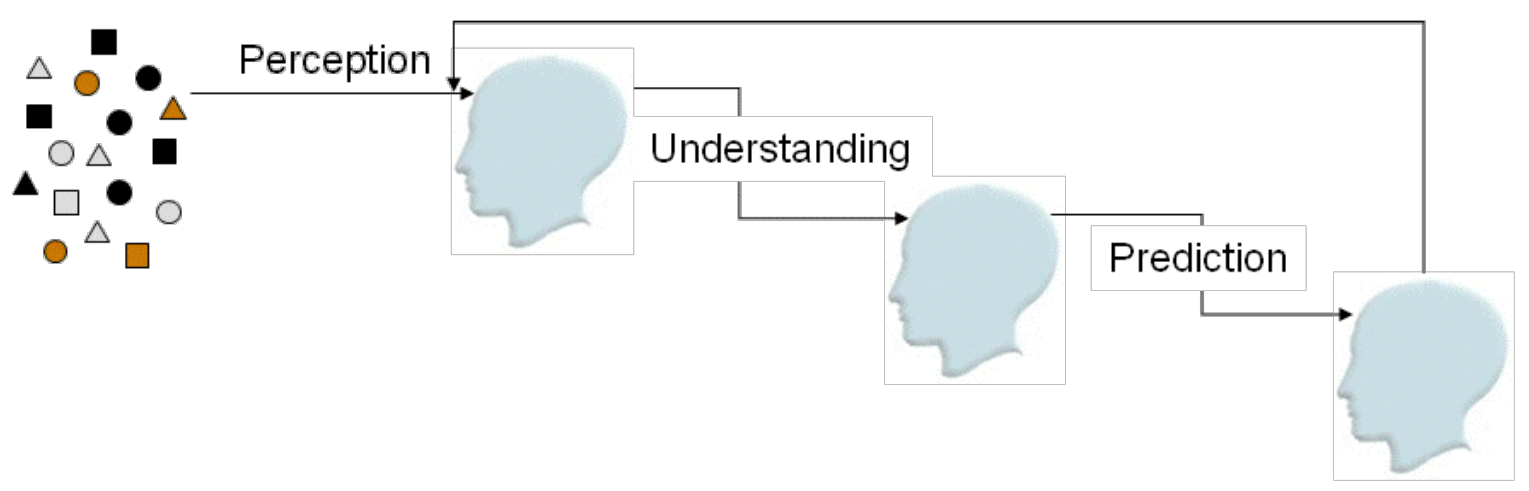

Figure 2.6. Situation Awareness According to Endsley ${ }^{4}$

An alternative view of SA as sensemaking is that it is not about providing more information (more data, more elements) but rather about providing priorities and clarity about preferences to help the decision maker understand what matters (Weick, 1995, p. 27). As Dekker and Lutzhoft (2004) argue, "SA is in part about constructing a plausible story of the process by which an outcome came about, and the reconstruction of immediate empirical history plays a dominant role in this. Few theories of SA acknowledge this, however, instead directing their attention to the creation of meaning from elements and the future projection of that meaning" (p. 29). Thus, in studying SA, rather than examining the (lack of) correspondence between actual and experienced worlds (a retrospective exercise), one should examine the decision makers' "unfolding experience of the situation in which they found themselves" (Dekker and Lutzhoft, 2004).

The traditional view of SA compels the investigator into a retrospective mode, to identify what the decision makers failed to notice, what they did not know, or what they should or should not have done. But from the point of view of the decision maker in the situation, such deficiencies do not exist - they may only be artifacts of retrospective hindsight. In the view of situated, or ecological, decision making, the focus of the investigation of SA is to understand why the decision makers experienced the situation the way they did or why what they saw made sense to them at the time.

\subsubsection{Cognitive/Sensemaking Perspective}

This cognitive/ecological approach to SA is a refreshing and potentially enlightening framework within which to study SA and explore more effective solutions to information visualization. Sensemaking is viewed as a cognitive process allowing individuals to interpret information in context so as to derive knowledge to initiate specific actions (Weick, 1995). Sensemaking is also referred to as knowledge construction that occurs at the individual as well as group level. Thus, sensemaking is both an individual and group cognitive process.

\footnotetext{
${ }^{4}$ This graphic taken from presentation by Greitzer et al. (2008) at IEEE Power \& Energy Society Annual Meeting.
} 
A sensemaking perspective leads us to ask different questions and to focus on understanding underlying cognitive processes of sensemaking - transactions between goals, observations, and actions rather than on more superficial questions about the extent to which certain human information processing limitations, display characteristics or visualization concepts might contribute to maintaining or losing SA. Consider, for example, the frequently cited human limitation described as Miller's (1956) magical number seven, plus or minus two: Miller's insightful observation that humans have a limited capacity in the number of items or "chunks" of information they can maintain in working memory tends to be interpreted as a hard limitation (information processing bottleneck). This factor is clearly situationdependent, and research has shown that experts have the ability to reduce complex stimuli into coherent chunks so that the $7 \pm 2$ constraint is rarely a limit on expert performance in natural environments.

Sensemaking can be applied to information visualization and/or to data mining activities with the intent to reduce complexity and to simplify the volume of data collected in order to create understanding. Ntuen, et al. (2010) summarize three major characteristics of sensemaking as: (1) an aspect of information foraging, which assumes that people modify strategies or the structure of their environment in order to maximize their rate of gaining valuable information; (2) as an information fusion tool, which can be viewed as a thinking process using new information to explain surprise and use new information to update prospective, predictive states of a situation; and (3) support for situation understanding in which a stimulus is placed into a framework to understand, explain, attribute, extrapolate, and predict, a process that leads to situation understanding. All three of these characteristics help to distinguish sensemaking from decision making. Decision making deals with making a choice from a given set of multiple alternatives. On the other hand, sensemaking deals with seeking, collating, and interpreting information to support decision making (Ntuen, et al., 2010). Visualization can serve to amplify (e.g., elaborate and strengthen) or to attenuate (weaken) cognitive ability. Visualization can thereby assist in a sensemaking task by providing some form of SA to enable the knowledge discovery process.

Flach, Mulder, and van Paasen (2004) observe that poor SA refers to the inability to see what matters. In this context, poor SA reflects the lack of a basis for decomposing complex data or information into coherent chunks. As they suggest, a useful model of SA should suggest how skilled decision makers might chunk information so they can navigate smoothly through the problem space, and it should suggest where they might make poor judgments and interpretations or how a novice's understanding of a situation would differ from that of an expert. It can be seen that this view of SA applies equally to VA. An implication is that mental models should be a key focus of study in developing a theoretical foundation for VA. Indeed, a prerequisite for SA is an accurate mental model (Sarter \& Woods, 1991).

Unfortunately, definitions and ideas about mental models vary widely, and some have argued that different uses of the term across varying disciplines have contributed to a decline in applying the concept to theoretical approaches in human-computer interaction research (Payne, 2003). Nevertheless, recent interest in mental models has brought a new appreciation for their potential contribution to VA research (Liu and Stasko, 2010).

\subsubsection{Mental Models}

A mental model is an internal representation of an external reality based on a person's perspective. This interpretation of mental models traces back to the Scottish psychologist Kenneth Craik (1943), who introduced the concept as a "small scale model of reality" that helps humans to reason and to anticipate 
events. Four decades later, Johnson-Laird's (1983) influential work provided a cognitive framework and theoretical base that describes mental models as psychological representations of real, hypothetical, or imaginary situations along with the relations among such entities. Norman (1983) extended the definition to focus on users' conceptions of how a system works.

Mental models can be constructed from perception, imagination and knowledge, or the comprehension of discourse. Gentner and Stevens (1983) emphasize that our views of the world, of ourselves, of our own capabilities, and of the tasks that we perform depend heavily on the conceptualizations that we bring to the task. In an interpretation that reflects the definitions of Craik, Johnson-Laird, and Norman, Senge (1990) describes mental models as deeply held internal images of how the world works. Senge observes that these images limit us to familiar ways of thinking and acting, and very often, we are not consciously aware of our mental models or their effects on our behavior.

Mental models have been described as well-defined, highly organized, and dynamic knowledge structures that are developed over time from experience (e.g., Kozlowski 1998). As representations of organized "chunks" of information in the environment, mental models serve to reduce the information load that would otherwise overwhelm the ability of novice decision makers to attend, process, and integrate the large amount of information that is inherent in complex operational environments. Thus, appropriate and effective mental models enable experienced decision makers to correctly assess and interpret the current situation (Level 1 and Level 2 SA) as well as to select an appropriate action based on patterns (mental models) stored in their long-term memory (Serfaty, MacMillan, Entin, \& Entin, 1997). Cues in the environment (including visualizations) activate these mental models, which in turn guide the decision-making process. Cognitive psychology research suggests that people who use visualizations have internal representations of the very visualizations they use (Larkin and Simon, 1987; Trafton et al., 2005), Because of cognitive limitations, including limits of working memory, use of visualizations supports decision making by providing an external "store" or what is referred to as external cognitioni.e., external visualizations can augment internal mental models. Consistent with the external cognition approach (Card, Mackinlay, and Shneiderman, 1999), this line of research has led researchers to design for elaborate visualizations. Liu and Stasko (2010) advise that approaches to design and development that focus only on external cognition (visualization) or only on internal cognition (mental models) tend to marginalize interaction (Yi et al., 2007). Thus, Liu and Stasko (2010) call for a unifying framework that integrates and articulates relationships among mental models, external visualizations, and interaction in the reasoning process. They advocate that the reasoning process can be explained in terms of the construction and simulation of mental models.

Thus, Liu and Stasko provide a definition of mental models for VA research that is largely concerned with using visualization in supporting sensemaking and reasoning:

A mental model is a functional analogue representation to an external interactive visualization system with the following characteristics:

- The structural and behavioral properties of external systems are preserved in mental models.

- A mental model can preserve schematic, semantic or item specific information about the underlying data.

- Given a problem, a mental model of an interactive visualization can be constructed and simulated in working memory for reasoning (Liu and Stasko, 2010, p. 1001). 
This definition emphasizes interactive aspects of information visualization. Most VA research that addresses interaction focuses on interaction techniques - actions initiated by users and features built into the visualization (e.g., Tweedie, 1997; Yi et al., 2007). Generally speaking, interaction techniques involve the user's physical motor actions and result in a change in the state of visualization (Liu and Stasko, 2010). A focus on surface-level interaction techniques (transactions between the user and the system, as we have called them) fails to capture the diversity of human actions in interacting with visualizations (Liu and Stasko, 2010) and the underlying cognitive activities in knowledge discovery and problem solving.

This new or updated perspective places a healthy emphasis on mental models, mental simulation, and model-based reasoning as a basis for a cognitive approach to VA research. This perspective leads us to ask different types of questions in conducting research in support of design, development, and evaluation of VA tools. In addition to addressing questions about transactional interaction techniques, we must seek to understand the mental models and mental simulations used in solving problems and the match between these internal representations and external ones. Liu and Stasko (2010) ask: "What specific simulative operations do people usually perform in their mental models? If we can specify and categorize examples of simulative transformations of InfoVis, such a taxonomy may serve as a basis for interaction design."

In summary, a cognitive approach to VA research produces a framework that acknowledges the role of sensemaking in information analysis/complex problem solving that leads to the study of mechanisms for mental models and mental simulations as a basis for understanding the role and effectiveness of visualizations and evaluating VA tool effectiveness. It is within this context and theoretical perspective that we have endeavored to review theories and experiments in VA to illuminate issues and gaps in this area of research. 



\subsection{Methodologies and Metrics for Evaluation}

In a review of 132 papers in the 2005-2007 IEEE Information Visualization Conferences and the 2006 and 2007 IEEE Symposium on Visual Analytics Science and Technology, Perer and Shneiderman (2009) found that only 39 papers had any type of user evaluation, and those that did tested users with no more than two hours of tool usage. Thus, the dominant approach has been to not perform user testing, and if such testing is used, then typically only novices serve as participants. Given a handful of calls and challenges over the last five years for the information visualization research community to adopt more effective and relevant evaluation strategies (e.g., Plaisant, 2004; Scholz, 2006; North, 2006; Perer and Shneiderman, 2008, 2009), perhaps this will change.

Our review of evaluation methods employed for VA technologies reveals the following dominant approaches (not counting the absence of evaluations, which is most frequent):

- Observations and interviews

- Questionnaires and discussion groups

- Heuristic evaluations

- Longitudinal/case studies

- Controlled experiments/performance testing.

Some studies use more than one of these methods in combination. There is some debate as to the best situations in which to make use of certain methods over others. Development of evaluation metrics generally yields measures that are particularly suited to the evaluation method employed. A useful overview of evaluation methods may be found in an article by Holzinger (2005), aimed at the humancomputer interaction community. Excellent reviews of evaluation methods and challenges for the VA field are provided by Shneiderman and Plaisant (2006) and Carpendale (2008). Carpendale's review describes varied methods and techniques, challenges and limitations, including discussion of qualitative and quantitative methods and concepts/risks to internal and external validity. Saraiya, North, Lam, and Duca (2006) provide a brief, but informative, review of VA tool evaluation methods.

\subsection{Observations and Interviews}

Observations and interviews are used most frequently. Perer and Shneiderman $(2008,2009)$ recognized limitations of controlled experiments for VA tool evaluation and described an evaluation methodology that includes longitudinal observation methods and in-depth interviews. Other notable observational studies include case studies conducted by Pirolli and Card (2005) and Chin, Kuchar, and Wolf (2009). Research exemplified by these studies typically focuses on gaining an understanding of the analytic process and/or examining the use of a proposed tool by experts, and the work tends not to involve comparison of two different tools or a single tool with current practice. A major contribution of these types of studies is to inform the design of future VA tools. A successful cognitive-based approach involving interviews that has been applied in this research is Cognitive Task Analysis (Gordon and Gill, 1997; Chipman, Schraagen, and Shalin, 2000). 
Interview-based methods have some possible risks in obtaining accurate information: as we noted earlier, frequently experts/users are not aware of (or they may not easily articulate) details about their knowledge discovery process and the strategies or methods that they employed-Ericsson and Simon (1993) demonstrated that retrospective verbalization about a task after it is completed is prone to forgetting and fabrication. ${ }^{5}$ A recent study by Lipford, Stukes, Dou, Hawkins, and Chang (2010) examined memories that intelligence analysts in the finance industry have about their strategies and methods, concluding that while their study participants exhibited good memory of the findings of their analyses, their memories about the strategy and methods employed were flawed. For example, they tended to overlook unfruitful steps that led to dead ends, they had difficulty remembering how their analytic processes evolved, and some even reported inaccurately on their processes during the analysis session. The researchers concluded that even if users were asked to document their reasoning along the way, they still might have difficulty creating an accurate record. An implication is that because both realtime and retrospective interviews of analysts may produce inaccurate characterizations of the analytic process, methods that employ user annotation or interviews should be augmented by other means of collecting information on the methods and steps that comprise the analysis process (e.g., logging of user transactions and interactions with a system and its visualizations).

Several metrics have been proposed for these qualitative evaluations. From their experience with observing the use of a visualization tool in a large corporate environment, Sedlmair et al. (2010) report on several recommendations to the practice of such a study. These recommendations cover all aspects of a study including its design, participant management, data collection, and the handling of results. In this case, observations were made during the course of their long-term study by researchers working with participating users. While not explicitly discussing them, Andrews (2006) covers observations in his discussion of testing methods as a part of formative testing (thinking aloud tests) and usage studies (including self-reporting and coding from log files). Observations as results of an evaluation are an indication that such a study is formative, even if originally designed as summative according to Ellis and Dix (2006). While they do not argue against the place of observations in visualization improvement, Ellis and Dix caution that as a method they are not suitable for reaching a summative conclusion such as determining if one visualization is more effective than another. Goodell et al. (2006) also treat observations as a basis for metrics used in later evaluations through other methods. However, as part of their visual analysis platform, they include a log of user activity and the ability to collect audio and text annotations that are indexed to system states. These annotations are participant-driven, rather than made by researchers, but they are also of the qualitative type of data that results from observation methods.

\subsection{Questionnaires and Discussion Groups}

Questionnaires and discussion groups are useful in assessing user satisfaction with proposed, prototype, or deployed tools. Questionnaires based on standard usability criteria (e.g., Nielsen, 1994b; Shneiderman, 1997) are often the preferred methodology for such evaluations. Interviews, both openended and structured, also rely on such standards, as well as combining in-depth interviews and observations using Cognitive Task Analysis methods. As we have indicated elsewhere in this report, because our focus is on identifying a path toward developing more rigorous methods and metrics for VA

\footnotetext{
${ }^{5}$ It has long been known that human memory is "reconstructive," as Bartlett (1932) described in his classic work on remembering, recounting how, with the passage of time (decades), individual memories become distorted, even fabricated by including details that were not originally present, and these distorted memories even grow in confidence.
} 
tool evaluation, we shall not describe or review this area of research but acknowledge that a large number of studies in information visualization field have employed these methodologies. A paper by Scholtz (2006) delivered to the VA Science and Technology community indicates the movement toward other, more rigorous evaluation approaches; she calls for the research community to go beyond usability evaluation and to develop methodologies and metrics to help researchers measure the progress of their work and assess the operational impact of VA tools.

\subsection{Heuristic Evaluations}

Developed by usability expert Jakob Nielsen (1994a), heuristic evaluation is a usability testing method designed to identify any problems associated with the design of a user interface. The method employs a set of usability design heuristics or rules of thumb that are used by a usability design expert during the usability inspection. This informal method is considered to save time and to quickly reveal design issues, although a constraint is that the results are influenced by the knowledge of the expert performing the evaluation. Typically, the heuristic evaluation is scoped to focus on a set of critical points and the targeted system is measured against predefined expectations. As Ardito et al. (2006) discuss, such heuristic inspection methods often involve expert evaluators only and are used as a cost-saving measure where a structured evaluation is needed but real users are difficult to access. To make heuristics more accessible to non-expert evaluators and allow for the reuse of heuristics across evaluations, they propose a standard method of defining heuristics that they call Abstract Tasks. Focusing on user tasks, they provide example definitions of several major tasks proposed by Shneiderman (1996), including overview, zoom, filter, details-on-demand, relate, history, and extract. Similar to Shneiderman, Lee et al. (2006) identify standard user tasks specifically scoped to assess graph visualizations that they organize into a taxonomy of five main task groups: topology-based tasks, attribute-based tasks, browsing tasks, and overview tasks. Lam (2008) focuses on the concept of cognitive costs or effort required by users to accomplish tasks. Adapted from Norman's Seven Stages of Action (Norman, 1988), there are seven costs to minimize: decision costs to form goals, system-power costs to form system operations, multiple input mode costs to form physical sequences, physical motion costs to execute sequences, visual-cluttering costs to perceive state, view-change costs to interpret perception, and state-change costs to evaluate interpretation. Another example of heuristic evaluation comes from the evaluation portion of the VA Science and Technology challenge (Scholtz, 2010) where analysts are given the opportunity to score participant entries based on a set of predefined criteria, including clarity of analysis process and the usefulness, efficiency, and intuitiveness of the analytical process, visualizations, and interactions.

\subsection{Longitudinal/Case Studies}

Catherine Plaisant (2004) challenged VA researchers to rethink their evaluation strategies and choose approaches that enable better metrics and long-term studies (benchmarks) to compare tools. She conducted a small literature survey of 50 user studies of information visualization systems and identified four thematic areas of evaluation: controlled experiments comparing design elements, usability evaluation of a tool, controlled experiments comparing two or more tools, and case studies of tools in realistic settings. Shneiderman and Plaisant (2006) proposed Multidimensional In-Depth Long-Term Case Studies (MILCS) in VA research. MILCS were originally developed to evaluate creativity support tools and can be adapted to evaluate VA tools. Their belief was that as information visualization research matures and tools developed in the laboratory are reaching users, there is a growing need for alternative methods of evaluation in order to present actionable evidence of measurable benefits that encourage more 
widespread use of VA solutions. They proposed that the efficacy of tools can be assessed by documenting (1) usage (observations, interviews, surveys, logging, etc.) and (2) expert users' success in achieving their goals. They also supported the use and adaptation of long-term ethnographic studies of large groups to better serve the goals of evaluation. In the article they also outline their proposal and guidelines for using MILCS in existing projects for better tool and use-case evaluation and to complement traditional evaluation methods.

Perer and Shneiderman (2008) noted that controlled laboratory-based experiments are less compelling for information visualization and VA research for the following reasons: (1) it is difficult to recruit sufficiently large numbers of analysts for a long time period, (2) exploratory tasks to be studied are poorly defined, (3) different backgrounds and experience of experiment participants leads to wide variation in performance, and (4) the research goals of the studies are often more than what quantitative analyses are able to provide. In their 2008 study, Perer and Shneiderman reported on two long-term case studies integrating visualizations and statistics. In a subsequent examination of four case studies, Perer and Shneiderman (2009) concluded that the combination of statistics and visualizations within an MILCS approach lead to new insights and discoveries. Thus, they contend that structured and replicated case study research methods offered by MILCS provide more useful assessments. Additional MILCS-based studies are described by Gonzalez and Kobsa (2003), Saraiya, North, and Duca (2004), and Seo and Shneiderman (2006).

\subsection{Controlled Experiments/Performance Testing}

Controlled experiments are designed to compare different conditions, such as examining the impact of a VA tool by comparing it with a baseline (current practice, for example), by examining the impact of the tool under varying operational conditions (such as difficulty of the problem), or by comparing the effectiveness of two or more alternative tools. Performance testing, as we are using the term, refers to the use of measures of performance (qualitative or quantitative) in a study that may or may not involve controlled experimentation. The use of controlled experimentation generally requires performance testing, but performance testing need not involve controlled experimentation. Controlled experiments are highly desired methods of evaluation, yet they also present some of the greatest challenges to VA researchers (as described in the above paragraph, Section 3.4). In addition to the limitations cited above, there is a challenge in determining appropriate data to use in the evaluation. As discussed by Plaisant (2004), two approaches are to use real data gathered from real-world instances or to use synthetically generated data. While use of real data may enhance external validity of the tool evaluation study, it may not be possible to determine ground truth and hence to generate useful performance metrics. Synthetic data affords an opportunity to insert known data that provides ground truth, but there is a risk to external validity to the extent that the simulated data do not reflect the operational environment. Greitzer (2005, 2008) discusses methodological issues in performing experiment-based evaluations of decision-support tools.

There have been several developments to create test environments in support of system effectiveness testing. An early effort in instrumenting an analytic environment is documented in reports and publications describing the Glass Box instrumentation test bed (Cowley, Nowell, and Scholz, 2005; Greitzer, 2005). The Glass Box instrumentation aimed to extract the analyst's reasoning processes through the examination of low-level user interaction, but it also implemented an optional annotation function that analysts could use to document their plans and ongoing thoughts. Several other research 
papers investigating VA tools have followed suit, with functions for in-depth data collection on user actions. For example, instrumentation by Goodell et al. (2006) automatically records user actions and collects audio and text annotations, which allows researchers to review user actions before and after critical points of interest in interacting with the tool. Gotz and Zhou (2008) reported an instrumentation approach that incorporates automatic tracking of semantic-level user interactions. Heer, Mackinlay, Stolte, and Agrawala (2008) described methods for logging user interactions and mechanisms to review, edit, and annotate the interactions. Other research efforts that include user annotations include Shrinivasan and van Wijk (2008) and the Scalable Reasoning System (Pike, May, and Turner, 2007). The Scalable Reasoning System facilitates collaboration among multiple analysts by allowing analysts to record their reasoning processes using node-link diagrams.

Performance measures used in traditional laboratory-based controlled experiments for VA tool evaluation include learning times, task performance times, and error rates. Perer and Shneiderman (2008) reviewed the use of laboratory experiments in the VA community and concluded that typical experiments have 20-60 participants who are given 10-30 minutes of training before the experimental manipulations are applied. In most cases, all participants perform the same experimental tasks during a 1-3 hour session, and performance is assessed using statistical hypothesis testing to show significant differences in mean values. These summary statistics are effective, especially if there is small variance across users. Obvious drawbacks of this approach include the fact that participants are non-experts with a very limited amount of experience; it is difficult to recruit a large number of experienced users to participate in these studies; and the nature of the tasks being studied (exploratory knowledge-discovery activities) is poorly defined, so applying experimental controls (e.g., asking users to perform specific tasks or specific instructions about how to perform tasks) is not compatible with discovery (Perer and Shneiderman, 2009).

\subsection{Summary}

Table 3.1 is a summary of advantages and disadvantages of different evaluation approaches:

Table 3.1. Evaluation Approaches

\begin{tabular}{lll}
\hline \multicolumn{1}{c}{ Method } & \multicolumn{1}{c}{ Most useful for... } & \multicolumn{1}{c}{ Limitations } \\
\hline Observations and Interviews & Revealing analytic process & Subjective \\
Questionnaires and Discussion Groups & Usability testing_-user satisfaction with & May not reflect true \\
& system & utility/effectiveness \\
Heuristic Evaluation & $\begin{array}{l}\text { Usability testing-focus on user } \\
\text { interactions/transactions with system }\end{array}$ & $\begin{array}{l}\text { May not reveal deeper } \\
\text { insights of cognitive process }\end{array}$ \\
Longitudinal Studies & $\begin{array}{l}\text { In-depth assessment of extent to which } \\
\text { tool aligns with process }\end{array}$ & $\begin{array}{l}\text { Tends to use a small sample } \\
\text { of participants }\end{array}$ \\
$\begin{array}{l}\text { Controlled Experiments/Performance } \\
\text { Testing }\end{array}$ & $\begin{array}{l}\text { Comparing alternative VA approaches } \\
\text { leading to enduring scientific conclusions }\end{array}$ & $\begin{array}{l}\text { Difficulty in obtaining } \\
\text { sufficient number of } \\
\text { participants }\end{array}$ \\
\hline
\end{tabular}





\subsection{Visual Analytic Taxonomies}

\subsection{Visual Analytics Taxonomy}

In a workshop on VA curricula hosted by Georgia Tech (Thomas, 2009), 20 participants discussed and brainstormed about developing a VA taxonomy. As Thomas stated, a taxonomy is the practice and science of classification: "The word has its roots in the Greek words $\tau a \xi_{1 \zeta}$ (taxis), meaning order, arrangement and vouos (nomos), meaning law or science. Taxonomy uses taxonomic units, known as taxa (which is the plural of taxon)" (Thomas, 2009, p. 6). As Thomas explains, the purpose for developing a taxonomy for VA is "... to provide an order and arrangement of topics - the taxa that are at the heart of studying visual analytics. The reason for such a "definition" is to more clearly describe the scope and intent of impact for the field of visual analytics."

As Thomas observed, the analysis revealed that usage of common terms was problematic: some terms appeared in multiple places with different meanings. For example "exploratory analytic" refers to both the name of a specific type of analytic method and a phrase that "... describes a very deep and powerful analytic science." The term "surveillance" is used both as a domain of application and an analytic goal with specific technologies (such as video motion analytics).

A higher-order classification that emerged from the VA curricula workshop, cited by Thomas (2009), is shown in Figure 4.1. The taxonomy comprises four main dimensions: Domain/Applications; Analytic Methods/Goals; Science and Technology; and Data Types/Structures. As stated, this taxonomy was oriented toward characterizing and informing educational strategies for academic programs. Elements of this framework are useful for some aspects of research and evaluation, but are perhaps less informative for guiding users and practitioners in selection of VA tools for particular purposes. Therefore, our study sought to review literature in the VA field to update VA taxonomies for varying aims in guiding research, evaluation, and practical applications. In so doing, we also seek to continue the specification of taxonomy (or taxonomies) by drilling down in providing additional detail at lower levels, as well as specifying applicable methods and metrics to guide evaluation research.

According to Chengzhi, et al. (2003), a taxonomy of visualization can help developers grasp the key techniques of visualization, assist domain users in understanding both how a particular visualization technique works and how it can facilitate selection of the right technique to use for analysis. Some research focuses on one or more factors, such as data type, display style, interactivity, etc. and many of these frameworks treat the developers of VA systems and the users of VA systems as the same. This confusion in perspective leads to ambiguous classifications and definitions for visualization techniques, systems, and ultimate utility. This can be seen in the number of taxonomies, classifications, and frameworks that have been published over the past decade. Our goal is to advance the field by addressing some of the published taxonomy shortcomings as well as their strengths, how taxonomies can be used to select the right tool for the job at hand, and how to better integrate perspectives from cognitive science. 


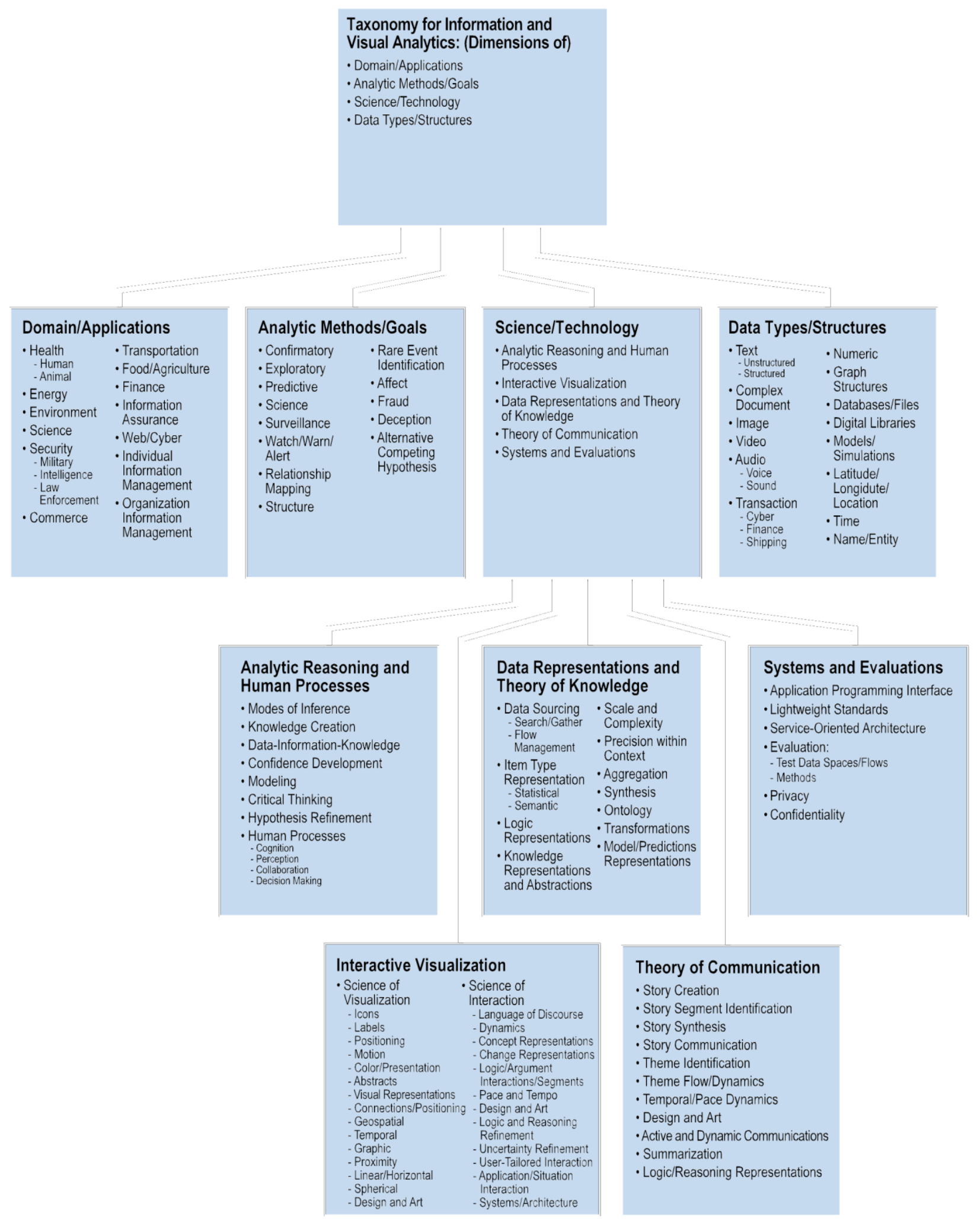

Figure 4.1. VA Taxonomy Developed in 2009 Workshop (Thomas, 2009) 


\subsection{Review of Literature on VA Taxonomies}

Our search and review of the relevant literature in VA revealed 228 unique citations of published works with a title that included either taxonomy or framework. Clearly, research efforts have heard the call for a VA taxonomy. A common approach is to focus on developing a taxonomy of user tasks, i.e., classifying visualization systems based on the types of basic user interaction tasks they support. Complex analytic tasks are often too nebulous to capture in easily codified terms, but as a way of approximation, each can be broken down into data and system interactions that are more universal and simpler to categorize.

Wehrend and Lewis (1990) provided an early and seminal classification of user tasks. They focused on domain-independent user actions, including locate data, identify objects, distinguish between values of variables, categorize objects, cluster data, describe distribution of data, rank data, compare within entities, compare between relations, associate entities, and correlate. While tasks such as clustering and ranking may imply certain types of VA systems, they may be more appropriate for describing the capabilities of many visualization systems operating on a variety of data types. In particular, clustering concerns techniques that allow users to identify associations among data, including finding gaps (absence of clusters). In this sense, a timeline visualization could reveal clusters - closely timed events - while other visualizations could show groups of related text documents based on keywords. The work by Wehrend and Lewis was later extended by Zhou and Feiner (1998), who added additional low-level tasks and regrouped tasks by three implications: organization, signaling, and transformation.

Shneiderman (1996) provided another task-based taxonomic framework that organizes evaluation of visual analytic systems using seven data types (one-, two-, three-dimensional, temporal, multidimensional, tree, and network) ${ }^{6}$ and seven user tasks (overview, zoom, filter, details-on-demand, relate, history, and extracts). Focus on data types is useful from a practitioner's viewpoint, especially if the taxonomy provides guidance on which types of visualizations are best suited for different types of data. Nevertheless, considerations that focus exclusively on visualization representation may miss critical thinking and problem-solving activity that underlies the interaction between the user and the visualization. Another limitation is that the inclusion of these user tasks into the taxonomic framework may tend to blur the distinction between interaction and task; for example, zoom reflects a transaction (action) performed by the user to meet a certain objective, but zooming per se isn't the objective that defines the task. Chi (2000) criticized use of classifications based on data types because of their limitations in providing guidance about selection of visualization techniques.

With his classification system, Shneiderman (1996) also delivers a "visual information seeking mantra" that advises: "overview first, zoom and filter, then details on demand strategy." That is, while tasks may be independently defined and perhaps iterative, there is still room to organize around user problem-solving strategies. Subsequent suggestions from other visualization researchers reflect this realization that taxonomies for VA should address not only aspects of the representation but also the analytic interactions supporting the problem-solving effort. For example, a framework by Pfitzner et al. (2001) includes interactivity type (static vs. dynamic), visualization type (textual vs. graphic, animated vs. images), and user skills level (as a continuum from novice to expert). Pfitzner, et al. (2001) suggest that their proposed framework addresses all the major factors involved in getting the user successfully from problem to solution, including bridging the divide between problem execution and data evaluation.

\footnotetext{
${ }^{6}$ A data type classification by Card, Mackinlay and Shneiderman (1999) is similar.
} 
Valiati et al. (2006) expanded the catalogue of low-level tasks and combined several similar higher-level tasks, acknowledging that the accomplishment of one task may require the execution of others. Thus, their high-level tasks can further be grouped into goals a user might have when using a visualization technique (identify, determine, compare, infer, locate) and more supportive tasks (visualize and configure). One aspect of note is that Valiati et al. have attempted to evaluate their taxonomy against users of a visualization system. Users were not told to use any specific strategy, and results indicate that the proposed taxonomy was able to capture the nature of user tasks.

More recent efforts by Gotz and Zhou (2008) also organize low-level user tasks into a taxonomy. Their taxonomy classifies tasks into three top-level semantic categories: exploration actions, insight actions, and meta actions. Meta actions include such data-managing tasks as delete and edit as well as undo and redo. Exploration actions include data exploration actions (filter, inspect, query, restore) and visual exploration actions (brush/highlight, change-metaphor, change-range, merge, sort, split). Insight actions are subtyped into visual insight actions (annotate, bookmark) and knowledge insight actions (create, modify, remove). Their classification of actions is based on understanding the intent of each lowlevel user action. For instance, annotation and bookmarking actions imply that a user is note taking. This classification recognizes the importance of visual information while filtering and inspecting, facilitating the process of constraining the visualization of the dataset.

To summarize, a common approach bases VA taxonomy on user tasks, and several low- to high-level tasks have been identified. While there is not always agreement on use of terms, there are commonalities across these task-oriented taxonomies. Few, however, have taken such task taxonomies beyond highlevel tasks and attempted to ground them in the cognitive domain.

A key exception comes from Xu et al. (2009) who borrow from Bloom's taxonomy of the cognitive domain (1956). While their motivation was largely to investigate and evaluate software visualization tools in regard to their support for learning a code base, $\mathrm{Xu}$ et al. provide a mapping of cognitive domain to sample tasks that support the domain. Several of these tasks have been identified by the previously discussed taxonomies. Bloom's taxonomy classifies stages of learning into ranked states, each of which subsumes the states that are lower in the hierarchy: knowledge, comprehension, application, analysis, synthesis, and evaluation. In order to have reached a state where one may engage in analysis, one must also comprehend and apply prior learning. User tasks identified elsewhere can be found as examples in $\mathrm{Xu}$ et al.'s mapping to supported domain (i.e., identify is supportive of the knowledge state while synthesis is supported by such tasks as relate and create).

This mapping of cognitive domain to user task is a critical step that until recently has been overlooked by the majority of the literature. However, in order to fully understand the usefulness of VA systems, it is important to understand the cognitive support they provide to users. Simply organizing the field by the tasks may not be enough to identify which visualizations are best suited to specific domain problems.

\subsection{Emerging Taxonomies}

Here we attempt to synthesize the above taxonomic frameworks by separating the different perspectives of researchers, developers, and users to help identify research needs, to help practitioners decide what approaches are best for particular types of problems, and to suggest evaluation methods that are most appropriate. In the following discussion, we describe taxonomic dimensions that emerge from 
this analysis and we attempt to convey them in different ways - tabular representations to provide some detail about their characteristics, representative research, etc. and graphic depictions that suggest ontological relationships that ultimately could be used to support decisions about choice of visualizations and evaluation approaches. We recognize the following categories:

- Application Focus. As described earlier, at a very high level, visualizations can be classified based on application focus such as information visualization, scientific visualization, software visualization, scientific collaboration, etc.

- Domain/Application. The Application Domain is important to include in the taxonomy to characterize the relationship between VA technology types and application domain - examining whether a particular type of technology is a more dominant choice of developers/users in a given domain of application. This dimension is the same as proposed by Thomas (2009) and therefore it is not depicted or described further here.

- Problem Type. The Problem Type is an important taxonomic dimension to support the need to identify the most appropriate VA technology to apply to a given type of problem. This taxonomic dimension corresponds to the dimensions of "analytic methods/goals" and "science/technology" offered in Thomas (2009).

- VA Technologies/Visualization Type. The VA Technology dimension incorporates some of the factors that Thomas (2009) included in the Science/Technology dimension.

- Evaluation Methods and Metrics. The Evaluation methods and metrics dimension corresponds roughly to portions of "Data Types/Structures" in Thomas (2009).

Figure 4.2 describes the process used and relationships involved in choosing a visualization approach for a given problem. The basic elements are shown in a circle, but the process is not simply a linear one, as there are times when the process jumps or iterates. Generally, the figure depicts how a given problem can be viewed as belonging to a certain application focus (and although not shown, the problem is associated with a given application domain). Given the problem type, one seeks to use existing theory and practice (ideally represented taxonomically) to choose a visualization approach or type. Given a visualization type, one selects a particular tool that is most appropriate for the objectives of the problem. The tool selection determines the types of user actions that are available, and these in turn help to define the methods used and performance metrics for evaluating the effectiveness of the visualization. 


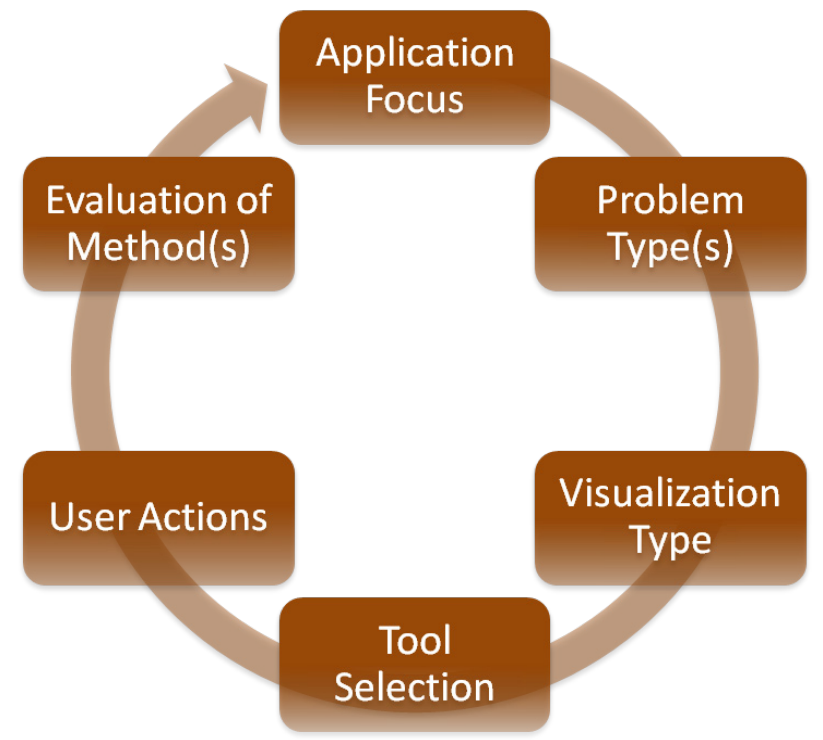

Figure 4.2. Process and Relationships Involved in Visualization Research, Development and Testing

\subsubsection{A Taxonomy of Problem Type/Purpose}

As we began to formulate a taxonomy similar to that proposed by Thomas (2009) and that follows concepts offered by other researchers, we realized that a deeper hierarchy would be useful and hence adopted a depiction that suggests this hierarchy for Problem Type (Figure 4.3 and Table 4.1), which also provides examples of methods used for evaluation.

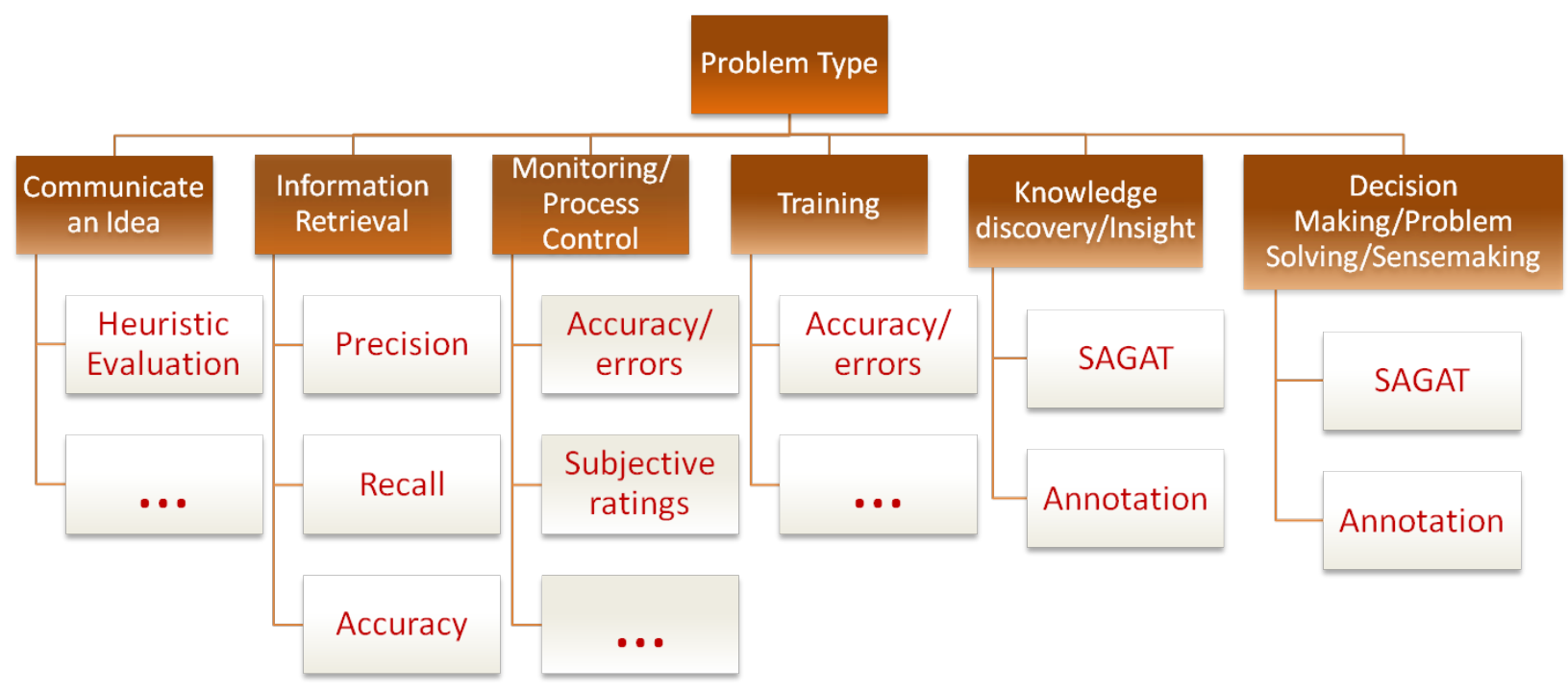

Figure 4.3. Problem Type Taxonomy showing examples of Evaluation Methods 
Table 4.1. Problem Type Taxonomy with Examples of Performance Metrics

\begin{tabular}{|c|c|c|}
\hline Problem Type & Data and Performance Metrics & Representative Studies \\
\hline Communicate an Idea & $\begin{array}{ll}\text { - } & \text { Cognitive Task Analysis } \\
\text { - Situational Awareness Global Assessment } \\
\text { Technique (SAGAT) (Endsley, 1988; 1995)) }\end{array}$ & $\begin{array}{ll}\text { - } & \text { Bertini and Santucci } 2006 \\
\text { - } & \text { Dasgupta and Kosara } 2010 \\
\text { - } & \text { Lam } 2008\end{array}$ \\
\hline Information Retrieval & $\begin{array}{l}\text { - Subjective ratings may be based on usability } \\
\text { factors (e.g., Nielsen, 1994a; 1994b) } \\
\text { - Accuracy/precision/recall }\end{array}$ & $\begin{array}{l}\text { - } \quad \text { Amar et al. } 2005 \\
\text { - } \quad \text { Ardito et al. } 2006 \\
\text { Matzen et al. } 2010\end{array}$ \\
\hline $\begin{array}{l}\text { Monitoring/Process } \\
\text { Control }\end{array}$ & $\begin{array}{l}\text { - Accuracy/errors } \\
\text { - Subjective ratings may be based on usability } \\
\text { factors (e.g., Nielsen, 1994a; 1994b) }\end{array}$ & $\begin{array}{ll}\text { - } & \text { Santos et al. } 2004 \\
\text { - } & \text { Ardito et al. } 2006 \\
\text { - } & \text { Lam } 2008 \\
\text { - } & \text { Liiv } 2010 \\
\text { - } & \text { Scholtz } 2010 \\
\text { Lee et al. } 2006\end{array}$ \\
\hline Training & $\begin{array}{l}\text { - User annotations (online or hand/journal input) } \\
\text { - Accuracy/percent correct/errors }\end{array}$ & - Chang et al. 2010 \\
\hline $\begin{array}{l}\text { Knowledge } \\
\text { discovery/Insight }\end{array}$ & $\begin{array}{l}\text { - } \quad \text { Accuracy/errors } \\
\text { - } \quad \text { Time to complete task/subtask } \\
\text { - } \quad \text { SAGAT (Endsley, 1988; 1995) } \\
\text { Domain-specific system metrics }\end{array}$ & $\begin{array}{ll}\text { - } & \text { Amar et al. } 2005 \\
\text { - } & \text { Ardito et al. } 2006 \\
\text { - } & \text { Matzen et al. } 2010\end{array}$ \\
\hline $\begin{array}{l}\text { Decision } \\
\text { Making/Problem } \\
\text { Solving/Sensemaking }\end{array}$ & $\begin{array}{l}\text { - } \quad \text { Accuracy/errors } \\
\text { - } \quad \text { Comparison with expert performance } \\
\text { - SAGAT (Endsley, 1988; 1995) }\end{array}$ & 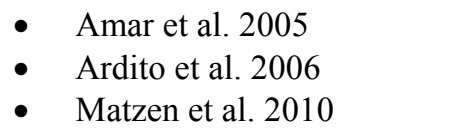 \\
\hline
\end{tabular}

Figure 4.4 illustrates the visualization type taxonomy (like other examples, we have not attempted a full specification; it is incomplete):

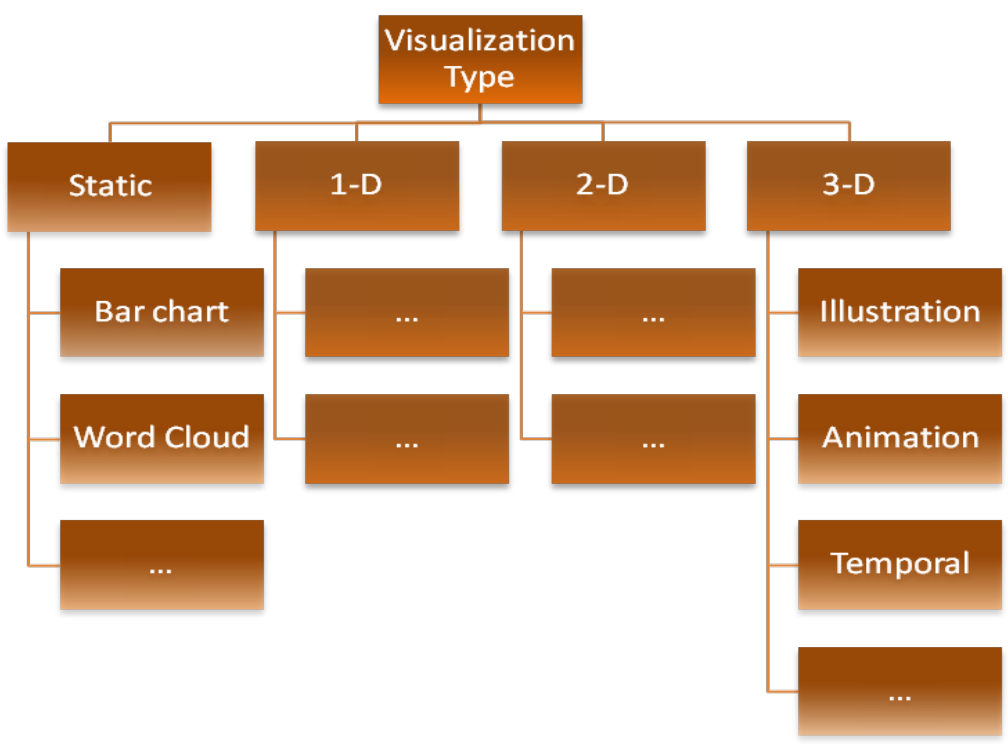

Figure 4.4. Visualization Type Taxonomy 


\subsubsection{A Taxonomy of Visual Analytic Technologies}

Table 4.2 illustrates the Visualization Type taxonomy. A great deal of research and practice characterizes this area, particular for static visualizations.

Table 4.2. Visualization Types

\begin{tabular}{|c|c|c|}
\hline Visualization Type & Examples & Purpose \\
\hline \multirow[t]{5}{*}{ Static Visualizations $^{7}$} & $\begin{array}{ll}\text { - } & \text { Network Diagram } \\
\text { - } & \text { Scatterplot } \\
\text { - } & \text { Matrix Chart }\end{array}$ & Demonstrate relationships \\
\hline & $\begin{array}{ll}\text { - } & \text { Bar Chart } \\
\text { - } & \text { Block Histogram } \\
\text { - } & \text { Bubble Chart }\end{array}$ & Compare a set of values \\
\hline & $\begin{array}{ll}\text { - } & \text { Line Graph } \\
\text { - } & \text { Stack Graph }\end{array}$ & Track trends \\
\hline & $\begin{array}{l}\text { - } \quad \text { Pie Chart } \\
\text { - Treemap }\end{array}$ & See parts of a whole \\
\hline & $\begin{array}{ll}\text { - } & \text { Word Tree } \\
\text { - } & \text { Word Cloud }\end{array}$ & Analyze text \\
\hline \multirow[t]{2}{*}{ 3-D Visualizations } & - Illustration & \\
\hline & - Animation & \\
\hline Heat Map & $\bullet$ & \\
\hline Interactive Visualizations & - & \\
\hline
\end{tabular}

As noted above, the selected VA technology or tool determines the type of user actions that will apply (subject to the functionality of the tool). We have begun to think of organizing user actions according to Bloom's taxonomy, as suggested by Xu et al. (2009). A depiction of this concept is shown in Figure 4.5.

\section{User Actions}

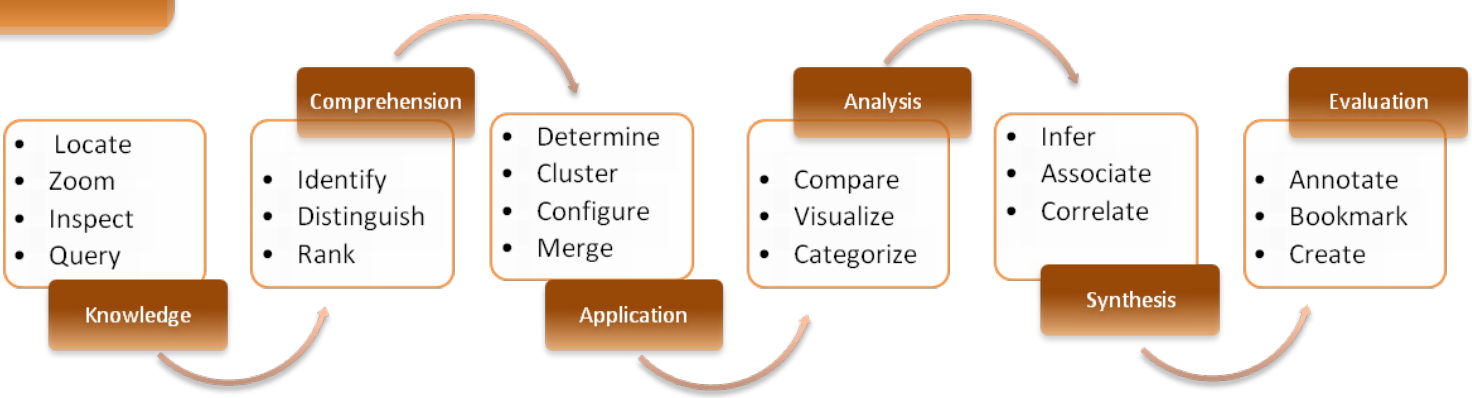

Figure 4.5. User Actions Organized According to Bloom's Taxonomy

\footnotetext{
${ }^{7}$ from Many Eyes web site, http://www-

958.ibm.com/software/data/cognos/manyeyes/page/Visualization_Options.html
} 


\subsubsection{A Taxonomy of Evaluation Methods \& Metrics}

Once choices have been made about the VA technology to apply to a given problem, decisions may be made about the method to be used in evaluating the impact of the technology. A partial depiction of relevant methods and relationships is shown in Table 4.3 and Figure 4.6.

Table 4.3. Taxonomy of Methods and Metrics

\begin{tabular}{|c|c|c|}
\hline Method & Data and Performance Metrics & Representative Studies \\
\hline $\begin{array}{l}\text { observations and } \\
\text { interviews }\end{array}$ & $\begin{array}{ll} & \text { Cognitive Task Analysis } \\
\text { - } & \text { SAGAT (Endsley,1988; 1995) }\end{array}$ & $\begin{array}{ll}\text { - } & \text { Sedlmair et al. } 2010 \\
\text { - } & \text { Andrews 2006 } \\
\text { - } & \text { Santos et al. } 2004 \\
\text { - } & \text { Ellis and Dix } 2006 \\
\text { - } & \text { Goodell et al. } 2006 \\
\text { - } & \text { Riche 2010 } \\
\text { - } & \text { Mayr et al. 2010 }\end{array}$ \\
\hline $\begin{array}{l}\text { questionnaires and } \\
\text { discussion groups }\end{array}$ & $\begin{array}{l}\text { - Subjective ratings may be based on usability } \\
\text { factors (e.g., Nielsen, 1994a; 1994b) }\end{array}$ & - $\quad$ Santos et al. 2004 \\
\hline $\begin{array}{l}\text { heuristic } \\
\text { evaluations }\end{array}$ & $\begin{array}{l}\text { - Subjective ratings may be based on usability } \\
\text { factors (e.g., Nielsen, 1994a; 1994b) }\end{array}$ & $\begin{array}{ll}\text { - } & \text { Santos et al. } 2004 \\
\text { - } & \text { Ardito et al. } 2006 \\
\text { - } & \text { Lam } 2008 \\
\text { - } & \text { Liiv } 2010 \\
\text { - } & \text { Scholtz } 2010 \\
\text { - } & \text { Lee et al. } 2006\end{array}$ \\
\hline $\begin{array}{l}\text { longitudinal/case } \\
\text { studies }\end{array}$ & - User annotations (online or hand/journal input) & - Shneiderman and Plaisant 2006 \\
\hline $\begin{array}{l}\text { controlled } \\
\text { experiments/ } \\
\text { performance testing }\end{array}$ & $\begin{array}{l}\text { - } \quad \text { Accuracy/errors } \\
\text { - Time to complete task/subtask } \\
\text { - Domain-specific system metrics }\end{array}$ & $\begin{array}{ll}\text { - } & \text { Santos et al. } 2004 \\
\text { - } & \text { Ellis and Dix } 2006 \\
\text { - } & \text { Goodell et al. } 2006 \\
\text { - } & \text { Richen et al. } 2010 \\
\text { - } & \text { Lee et al. } 2006\end{array}$ \\
\hline Meta-analysis & $\begin{array}{l}\text { - combines the results of several studies that } \\
\text { address a set of related research hypotheses }\end{array}$ & \\
\hline
\end{tabular}




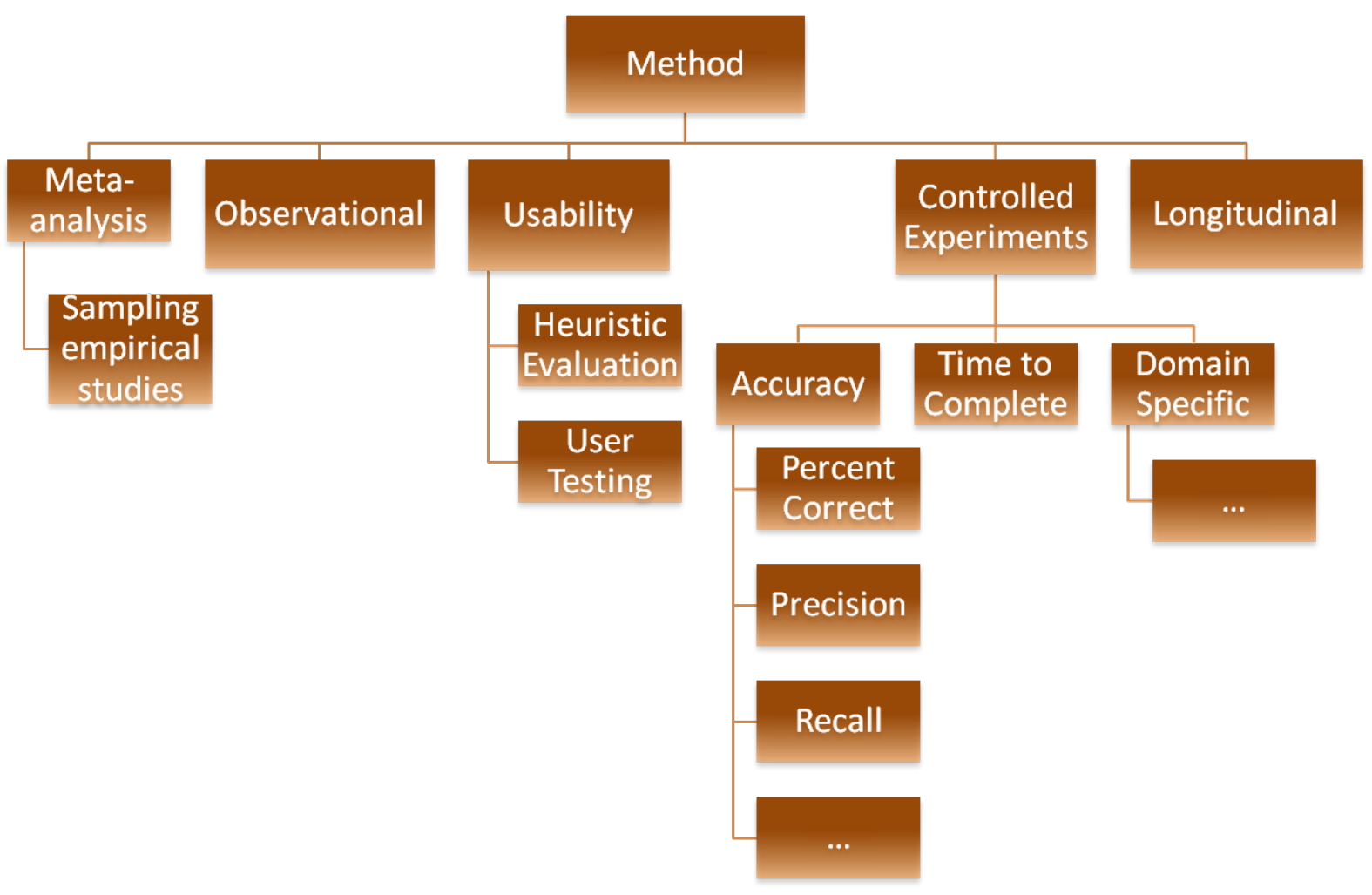

Figure 4.6. Evaluation Method Taxonomy (Incomplete)

\subsection{A Work in Progress}

As discussed throughout this report, there are numerous taxonomies published on a variety of facets of VA - collaboration, data type, interactivity type, actions, time dimensions, etc. It can be argued that there is a lack of agreement on how to organize the domain in a way that will (a) provide the right tool for the task at hand to solve practical problems and (b) generate valuable theoretical knowledge to advance the field as a whole. Taxonomies provide a mechanism to organize tools using a standard set of criteria, thereby making it easier to choose a tool for a specific purpose.

Figure 4.7 depicts, at a very high level, how a handful of the published taxonomies can be mapped to Bloom's taxonomy of cognitive domain (Bloom, 1956; Xu et al., 2009) with a focus on user actions. This unique application of a taxonomy from educational psychology assists in providing an overarching framework from which to derive a better understanding of the impact visual analysis in general, and information visualization in particular, have in sensemaking, problem-solving, and insight generation. The head with a question mark shown in the lower right of the figure conveys our assessment of the state of the description of taxonomies for information visualization at the level of problem solving and sensemaking; i.e., there is considerable more work to be done in specifying activities associated with deeper levels of cognition (e.g., more sophisticated activities associated with synthesis and evaluation in Bloom's taxonomy) that transcend user-system transactions falling into lower levels of Bloom's taxonomy (knowledge, comprehension, application). 


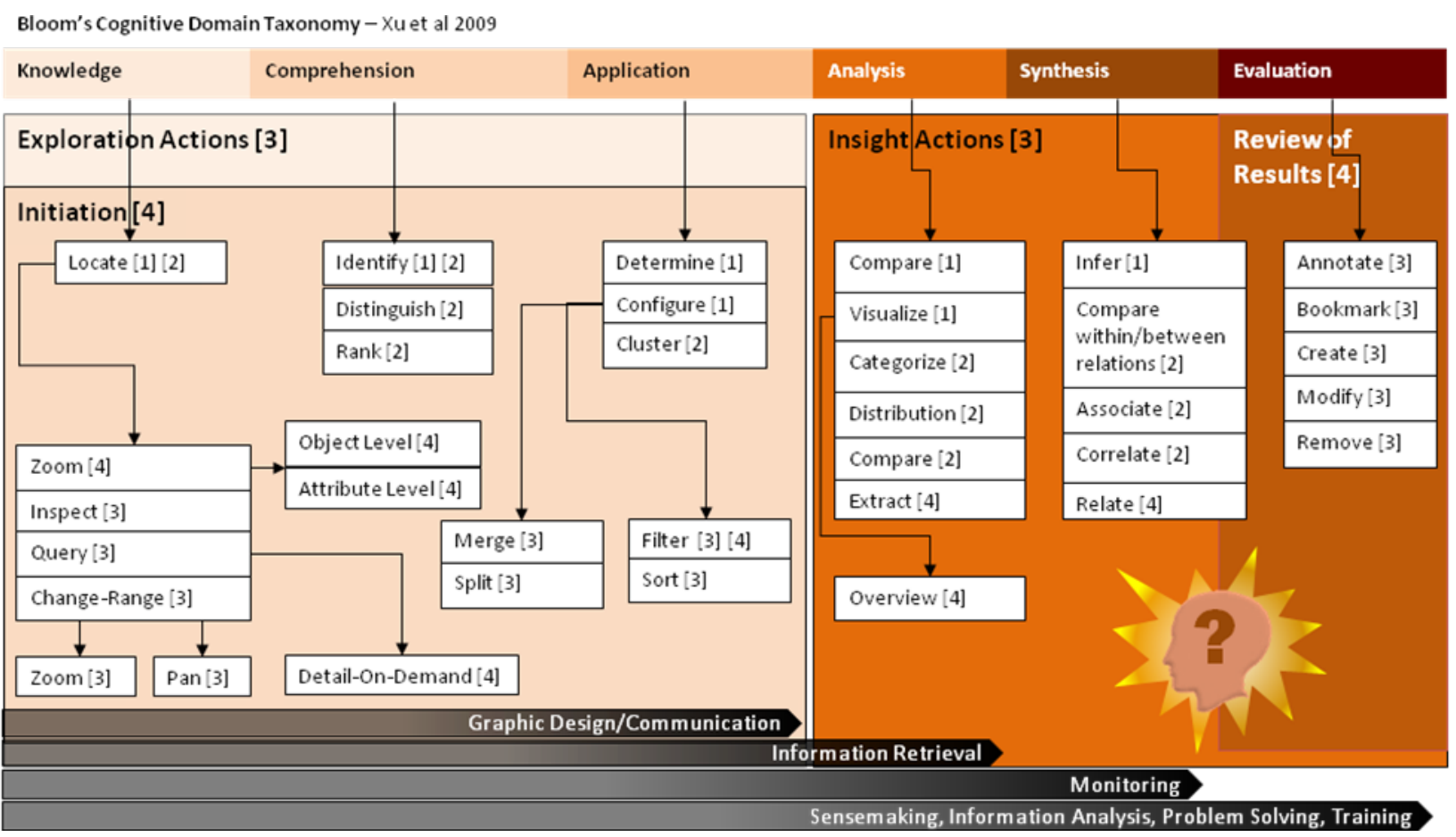

Figure 4.7. Cognitive Taxonomy. Sources: 1. Valiati et al., 2006, 2. Wehrend and Lewis, 1990, 3. Gotz and Zhou, 2008, 4. Pfitzner et al., 2001.

There is an extensive literature on high-level models of user strategies to identify information, solve problems, make decisions, etc. However, there is no consensus on the tasks required to get from point A to point $\mathrm{B}$ or about how to determine the best tool to facilitate human reasoning. Developing an understanding of the relationships among visual representations of data, complexity of task, and information retrieval will enable the community to build better tools. This can only be accomplished through robust, longitudinal, empirical studies. Identifying gaps in, among, and between taxonomies is a large task and requires input from experts in multiple domains salient to the National Visualization and Analytics Center agenda for VA.

With a lack of comparison studies and a rich theoretic history available, researchers look to other fields that may inform and advance the VA field. One recent article by Chen and Janicke (2010) examines the applicability of information theory taxonomy as a possible theoretic framework for visualization. Their quantitative evaluation yielded very positive results. Future work should explore application of existing theories and models from other disciplines.

\subsubsection{Benefits}

When the specification of taxonomies is completed to show best practices or most successful application of VA technologies to different types of problems, the resulting taxonomies will benefit the research community and the operational users alike by showing future research directions and informing developers and practitioners as to more optimal selection of tools and techniques. 


\subsubsection{Direct Future Research}

Once a large-scale taxonomy is developed, a web-based repository of visualization tools and resources can be developed. The repository is intended to be used by researchers to classify tools and determine if a particular visualization has been empirically validated to support decision-making in a particular domain or application. A byproduct of having this information in one place electronically is that it allows researchers and software developers to identify if there are gaps that can be filled, thus furthering the discipline.

The complexity involved in most analytic systems today is a major obstacle to usability as is the high cost of purchasing or developing a tool. According to Chen, "a prolonged lack of low-cost, ready-to-use, and reconfigurable information visualization systems will have an adverse impact on cultivating the critical user population" (2005, p. 12). To reach critical mass, the language of information visualization needs to be comprehensible to potential users. Generating a common ground through the development of theories and a common set of evaluation metrics is a start.

\subsubsection{Inform Selection of Tools}

An important function of VA taxonomies should be to help practitioners and stakeholders identify visualization techniques (and associated tools) that best address their problems, based on results from research and practical experience that ultimately would be represented in the collection of VA taxonomies that we seek.

\subsubsection{Inform Validation Efforts}

The purpose of empirical studies is to discover and explain facts and relationships. A study is only empirical if it explores and analyzes data associated with the use of information visualization functions and systems (Chen, 2004). Many are seduced by the eye-candy that can overwhelm the utility of visualization tools. However, empirical evidence is very important to fast-growing fields and remains an integral component of domain knowledge, e.g., what works, what failed, what is not known. Ideally, evaluative studies should be designed to capture the effectiveness of an information visualization design in terms of insights generated. However, additional measures of overall quality, uncertainty, novelty, usability, etc. are just as meaningful. One suggestion is that a large focus should be placed upon narrowing the gap between the "gulf of execution" and the "gulf of evaluation" so users can accomplish tasks smoothly and seamlessly and so they can judge their progress accurately (Chen, 2010: p. 395). Understanding how a tool works is just as important as why it works. A taxonomy capable of breaking down the constituent components of a visualization tool should, in theory, enable the researcher to better comprehend any shortcomings between tasks and visual cues as well as better comprehend the link between visual attributes and perceptual tasks.

\subsection{Status of Taxonomies for Visual Analytics}

In a special issue of the International Journal of Human-Computer Studies, Chen and Czerwinski (2000) acknowledge that the proliferation of information visualization tools and techniques has highlighted the need for principles and methodologies for empirical evaluation (2000: p. 631). Improved methods are needed in areas such as task analysis, usability evaluation, and usage analysis. The special 
issue highlights the growing interest in empirical evaluation of information visualization and signifies that the field has matured enough to start providing valuable contribution to stimulate further development of innovative techniques and a better understanding of the value of various visualization techniques. One approach used by Morse and Lewis (2000) evaluated visualization techniques using a task set based on a visual taxonomy. Their use of a taxonomy provided a way to deal with the complexity of visual tasks and concluded that, while successful in verifying the utility of visualization for supporting information retrieval activities, it could also be expanded and used to evaluate text-based tasks. They also established that it would be highly desirable to perform the evaluation as part of a study on the entire taxonomy, not just a portion. Another study combining evaluation with the application of taxonomies can be seen in Wohlfart, et al. (2008). Typically, empirical evaluations of visualization tools use predefined tasks to assess usability. However, when there is a lack of published studies on a particular data dimension, in this case temporal data, it is difficult to make a tool selection. Wohlfart et al. use a published categorization and a set of user tasks to perform a detailed comparison of several tools, focusing on temporal aspects of data. These studies demonstrate that use of taxonomies coupled with evaluation methods provides a valuable way to ascertain if the categorization is useful and enables easier comparisons between tools that take account of the type or types of data being analyzed.

Taxonomies for visual applications in particular can be positioned in a continuum between the user and the application. User-oriented categorizations take into account psychological aspects of the user, such as internal and external cognition, whereas categorizations oriented by the tool or application focus on features and types of interaction. On the other hand, empirical analyses of visualization applications use predefined tasks to evaluate their practical effect. Tasks are often selected according to their relevance to the task at hand. Users have to analyze one or more attributes of the data set to find correlations, make comparisons, or find meaningful clusters. Performance is typically measured by the time needed to complete each task and the correctness of their answers. Less frequently, evaluations include more subjective information like user preference and rating of the tested tools (Wohlfart, 2007).

No matter if you use the term categorization, classification, or taxonomy interchangeably (as have many of the authors cited in this document), they all enable the field to organize the various aspects of information visualization hierarchically. We propose that this organization is quite necessary to aid in the development of a more rigorous theoretical stance for the broad and multidisciplinary field of visualization. Much of the foundation of the field is based in other disciplines including psychology, statistics, and computer science. However, there is very little work purely motivated by visualization alone. In order to strengthen the field, more reliable and comparable empirical evidence of how information visualization is applied and how higher-level human capabilities, such as decision-making, contribute to our ability to elicit meaningful information from large volumes of data. Researchers will need this empirical data to identify patterns, propose hypotheses and theories, and test them. Some have claimed that the field of information visualization as a whole does not have an established scientific culture to collect comparable data and suggest that taxonomies for more precisely defined cognitive tasks will better enable researchers to describe their experiences and evaluation results more fully; that standardized measures enable verification of results and provide a mechanism to conduct meta-analysis; and finally that quantitative and physiological measures similar to those used in psychology will improve our understanding of information visualization (Ziemkiewicz, et al, 2010).

The tables and diagrams provided earlier in this section serve as an initial guide to future work on developing a high-level taxonomy and ontology for the discipline. A parallel activity to conduct as the taxonomy shapes up is a thorough review and analysis of published literature. 



\subsection{Conclusions and Recommendations}

\subsection{Conclusions}

The grand challenges described by Thomas and Cook (2005) apply as much today as they did six years ago at the beginning of the National Visualiztion and Analytics Center program. As they remarked, "Visual representations alone cannot satisfy analytical needs. Interaction techniques are needed to support the dialogue between the analyst and the data. While basic interactions such as search techniques are common in software today, more sophisticated interactions are also needed to support the analytical reasoning process." (p. 30). The reader should refer back to that work as well as other more recent work for a more complete discussion of research directions: For example, Keim et al. (2008) discuss technical challenges of scalability, quality of data and graphical representation, levels of detail of visual representation, novel interaction styles and metaphors to enhance user-system communication, research on a range of display devices from small portable assistants to large-scale power walls, development of a theoretically grounded evaluation framework, and a novel software infrastructure to support smooth interaction augmented by asynchronous processing. According to Keim et al. (2008), there is currently no technological approach or theoretical agreement among experts in the field of VA that claims to provide answers regarding the relevance of information, the adequacy of data processing methods and validity of results, and acceptability of the presentation of results for a given task.

Our discussion of research directions is focused on areas in which cognitive science research can help to advance VA research and practice. Major topics for research concern the consideration of how current theories can be applied to inform VA research; more focused cognitive research on sensemaking/problem solving and the analytic process to help align visualization technologies and representation techniques to user's mental models and thought processes; advancing the science and engineering practices of VA tool evaluation; research to develop more effective means of communicating the results of analyses to stakeholders (intuitive and natural ways of conveying findings as well as providing rationale and background information supporting the decisions and recommendations); and activities that foster a closer relationship between system developers and end users at all stages of the system design/development/deployment life cycle.

\subsubsection{Theories}

As we and others have argued, a more directed application of cognitive theories and results of empirical research on critical decision making and problem solving to VA system design, development, and evaluation. Research can inform future VA research. A wide array of theories and guidelines is likely to be appropriate for strengthening the theoretical foundations of VA research. A workshop titled "The Role of Theory in Information Visualization" at the recent VisWeek conference in Salt Lake City (organized by Robert Kosara, T.J. Jankun-Kelly, and Chris Weaver, Oct 25, 2010) ${ }^{8}$ included a productive discussion that identified theories from the following diverse fields that should be included in such a study:

- cognitive psychology/attention/perception/cognition

\footnotetext{
${ }^{8}$ Most of these suggestions came from a breakout session that FL Greitzer attended; we acknowledge the important contributions by Dr. Lucy Nowell, a participant in this session who provided many of the topics suggested below.
} 
- communication theory

- external cognition

- graphics design/layout

- human-computer interaction

- learning theory

- mapmaking

- sensemaking/decision making/problem solving

- statistical graph design

- studies of cognitive bias, change blindness.

\subsubsection{Cognitive/Sensemaking Research}

As we have discussed, VA technologies provide external memory to augment and accommodate human memory limitations, but when visualizations are difficult to interpret, a higher cognitive load results (Pfitzner, Hobbs, and Powers, 2001). If visualizations convey appropriate mental models, then they should enhance decision making, but if they are inconsistent with the mental models and analytic approaches used by the decision maker, then they will work against efficient sensemaking and problem solving. Very little research has been done to link observed performance to inferred mental models that represent the decision-making process. In addition, research on evaluation methods is required to help fill knowledge gaps in researcher- and practitioner-oriented taxonomies. Card, et al. (1999) suggest that many human cognition and performance studies need to be completely revisited with all of the research and theory development that has been accomplished within the field of information visualization. Undoubtedly, we can and have used them as a springboard to advance the field. But as the volume of data and data representations increase at a phenomenal rate, many of these studies that dealt with very small data sets and with limited technology may provide very limited application today.

\subsubsection{Evaluation}

Is visualization worthwhile - does it assist in reducing cognitive load, lead to insight, and facilitate problem-solving? How do we quantify the impact of VA tools or measure success? Research is required to enhance methods for evaluating the effectiveness of VA tools. Evaluations are needed to determine if goals and requirements of a program or tool have been satisfied. Certainly, this requires an appropriate amount of attention, up-front, to defining requirements for success that may be examined later in the evaluation phase of an effort. To do this effectively, developers need to have an evaluation plan in the beginning that specifies the types of evaluations that will be performed. Once again, research on taxonomies for evaluation is needed to help stakeholders and developers specify the most appropriate evaluation approaches that meet their operational objectives and to identify methods and performance metrics that are realistic and informative based on theory and research.

Researchers in the VA field have concluded that future research should always include evaluation studies and should seek to provide a scientific basis for their conclusions that transcends assessment of individual tools (moving beyond "point solutions"). Results of such research will help to complete the 
development of VA taxonomies that guide research, evaluation, and selection of VA tools based on benchmarks and rigorous theory/model-based and science-based principles. Thus, more evaluationscontrolled experiments and longitudinal studies - are needed that go beyond traditional user studies and that provide insights about why one type of visualization approach yields better results than another in a given problem domain.

\subsubsection{Communication}

Finally, we must not forget the importance of communicating the results of VA tasks in a way that effectively informs stakeholders about the conclusions reached as well as the rationale for the decisions. Results must be conveyed to a variety of potential users, from stakeholders to the general population. Human-computer interaction research is needed to devise intuitive methods of reviewing high-level analysis results/conclusions as well as examining evidence and relevant facts. Because most real-world problems are ill-defined and uncertain, the conclusions that derive from VA studies are necessarily uncertain; thus, it is important for VA displays and interfaces to portray concepts of uncertainty at an appropriate level of detail to be understood by the individual using the tool (this clearly varies depending on the user's role and intent).

\subsubsection{Bringing Communities Together}

Stakeholders and thought leaders from the research community and end-user communities need to determine ways to foster more frequent and meaningful interactions and knowledge exchanges to provide an atmosphere and even formal processes that ensure the acceptance of VA tools by user communities. It is widely acknowledged that systems must be designed with the users' tasks in mind, but current practice still fails to establish strong connections in developing system requirements that are tightly linked to realworld operational problems. While there has been movement toward more extended use of domain experts and users in the evaluation process (e.g., longitudinal case studies involving experts), the VA community needs to establish comparable associations during the concept development and requirements/design phases of work. A possible means of facilitating such exchanges is to conduct joint workshops involving researchers, practitioners, and operational users to survey the state of practice of VA tool development and identify operational needs and requirements that informs and provides directions for research.

\subsubsection{VA Taxonomies}

What can information visualization research achieve that other disciplines or fields can't? Several authors have documented milestones in the history of the field that should be useful in advancing our understanding of the needs and contributions of the VA field and derivation of a taxonomy or taxonomic framework that can be useful in guiding research and application (Card, MacKinley and Shneiderman, 1999; Chen, 2004; Spence, 2001; Thomas and Cook, 2005). However, very little work has been done in the area of evaluation and taxonomic development. Overall, paradigmatic analysis and characterization in the field of information visualization are lacking. The field of VA doesn't focus on specific methods to address these questions in one overarching "best practice." Each domain, of which there are many, contributes to the repertoire of approaches that contribute to the creation of solutions. Ultimately, it is up to the human user to utilize VA to complement the knowledge generation process in the context of their need. 


\subsection{Summary of Recommendations for Research}

Recommendations for future research based on the above discussion and conclusions are aggregated below:

- More research is needed on sensemaking/problem solving and the analytic process to help align visualization technologies and representation techniques to user's mental models and thought processes.

- Research is required to advance the science and engineering practices of VA tool evaluation.

- Research is needed to develop more effective means of communicating the results of analyses to stakeholders.

- A more directed application of cognitive theories and results of empirical research on critical decision making and problem solving are needed to inform VA system design, development, and evaluation.

- Research is required to enhance methods for evaluating the effectiveness of VA tools and determining if goals and requirements of a program or tool have been satisfied.

- In the future, VA research publications should include evaluations and should seek to provide a scientific basis for the conclusions that transcend assessment of individual tools (moving beyond "point solutions").

- Further analysis is required to define requirements for the establishment of a test bed to support evaluation of VA tools.

- Stakeholders and thought leaders from the research community and end-user communities need to determine ways to foster more frequent and meaningful interactions and knowledge exchanges. Two recommended ways to achieve this objective are to conduct joint workshops involving researchers, practitioners, and operational users and to establish an interactive forum at the VACommunity Site to facilitate discussion and exchange of ideas.

\subsection{Recommended Next Steps for Cognitive Foundations Project}

The following recommendations are offered specifically for following up on the research performed in this cognitive foundations project.

\subsubsection{Set Up Experimental Test Bed}

As Thomas and Cook (2005) advised, an evaluation infrastructure is needed to guide research in promising directions. Because no single visualization technique can be expected to be appropriate for all problems, combining a systematic exploration of the effectiveness of VA approaches within a taxonomic framework will help not only to guide research but also to inform practitioners in selecting or investing in VA approaches to meet operational challenges.

Further analysis is required to define requirements for the establishment of a test bed to support evaluation of VA tools. We have identified a number of drawbacks in current practice that should be addressed, including use of experienced participants instead of novices, use of extended tasks that exercise the cognitive/sensemaking processes, and development of methods and performance measures 
that help to reveal more cognitive activity (interactions involving human-information discourse) in addition to user-computer transactions to modify or search/retrieve data, navigate, or adjust visual mapping/representation. A test bed is needed that will support experiments and longitudinal studies involving experienced users. Two requirements for an evaluation infrastructure are:

- The test bed should support controlled experiments as well as more traditional usability studies.

- The test bed should include a portable system configuration that may be integrated into the user's environment.

\subsubsection{Advance Community Involvement in Cognitive Foundations}

In her keynote address at VisWeek 2010, Professor Mary Hegarty of the University of California, Santa Barbara, stated, “... design of effective visualizations is as much a challenge for cognitive science as for computer and information science, and ... these disciplines must collaborate closely on the development of new information technologies and visualization design" (2010: p. xxiv). To facilitate this collaboration, we have created a Cognitive Foundations Forum at the VA Community Site, http://vacommunity.org, to facilitate discussion and exchange of ideas on selected topics. We also intend to develop a blog where we can update this community about developments and discussion topics. We recommend continued maintenance of the forum, blog, and other social media methods for informing the research community and practitioners. Particular objectives are to encourage information exchange between cognitive scientists and computer/information scientists engaged in visualization research and to facilitate collaboration between the research community and stakeholders and users. Specific recommendations include:

- Maintain and update Cognitive Foundations Forum at the VA Community Site

- Use the site to disseminate information about events of interest and discussions, aimed at visualization designers/developers, cognitive scientists, and the user community

- Plan a workshop to bring together cognitive scientists and visualization researchers to continue to flesh out the research agenda. 



\subsection{References}

Amar, RA, and JT Stasko. 2005, "Knowledge Precepts for Design and Evaluation of Information Visualizations." Visualization and Computer Graphics, IEEE Transactions on 11(4):432-42.

Andrews, K. 2006, "Evaluating Information Visualisations." In AVI Workshop on BEyond Time and Errors: Novel Evaluation Methods for Information Visualization, pp. 1-5.

Ardito, C, MF Costabile, A De Angeli, and R Lanzilotti. 2006, "Systematic Evaluation of E-Learning Systems: An Experimental Validation." In Proceedings of the 4th Nordic Conference on HumanComputer Interaction: Changing Roles, pp. 195-202.

Banbury, S, and S Tremblay. 2004. A Cognitive Approach to Situation Awareness: Theory and Application. Ashgate Publishing Company, Burlington, VT.

Bartlett, FC. 1932. Remembering: A Study in Experimental and Social Psychology. Macmillan Co., New York, NY.

Bertini, E, and G Santucci. 2006, "Visual Quality Metrics." In Proceedings of the 2006 AVI Workshop on BEyond Time and Errors: Novel Evaluation Methods for Information Visualization, pp. 1-5.

Bloom, BS. 1956. Taxonomy of Educational Objectives: The Classificaiton of Educational Goals: Handbook I. Cognitive Domain. Longmans, Green, New York, NY.

Bowden, EM, M Jung-Beeman, J Fleck, and J Kounios. 2005, "New Approaches to Demystifying Insight." Trends in Cognitive Sciences 9(7):322-28.

Card, S, J Mackinlay, and B Shneiderman. 1999. Readings in Information Visualization: Using Vision to Think. Mogan Kaufmann Publishers, San Francisco, CA.

Carpendale, S. 2008, "Evaluating Information Visualizations.” In Information Visualization, Vol 4950, pp. 19-45. Springer-Verlag, Berlin.

Chen, C. 2004. Information Visualization: Beyond the Horizon, 2nd Ed. 2nd ed., Springer-Verlag, London.

Chen, C. 2005, “Top 10 Unsolved Information Visualization Problems.” Computer Graphics and Applications, IEEE 25(4):12-16.

Chen, C, and MP Czerwinski. 2000, "Empirical Evaluation of Information Visualizations: An Introduction.” International Journal of Human Computer Studies 53(5):631-36.

Chen, M, and H Janicke. 2010, “An Information Theoretic Framework for Visualization.” IEEE Transactions on Visualization and Computer Graphics 16(6):1206-15.

Chengzhi, Q, Z Chengu, and P Tao. 2003, “Taxonomy of Visualization Techniques and Systems Concerns between Users and Developers Are Different.” In Asia GIS Conference, pp. 1-14. 
Chi, EH. 2000, “A Taxonomy of Visualization Techniques Using the Data State Reference Model.” In IEEE Symposium on Information Visualization, pp. 69-75.

Chin, G, OA Kuchar, and KE Wolf. 2009, "Exploring the Analytical Processes of Intelligence Analysts." In Proceedings of the 27th International Conference on Human Factors in Computing Systems, pp. 11-20.

Chipman, SF, JM Schraagen, and VL Shalin. 2000, "Introduction to Cognitive Task Analysis." in Cognitive Task Analysis, eds. JM Schraagen, SF Chipman, and VL Shalin, pp. 3-23. Lawrence Erlbaoum Associates, Mahawah, NJ.

Cowley, P, L Nowell, and J Scholtz. 2005, "Glass Box: An Instrumented Infrastructure for Supporting Human Interaction with Information." In 38th Annual Hawaii International Conference on System Sciences, pp. 296c-96c.

Dasgupta, A, and R Kosara. 2010, "Pargnostics: Screen-Space Metrics for Parallel Coordinates." Visualization and Computer Graphics, IEEE Transactions on 16(6):1017-26.

Dekker, S, and M Lutzhoft. 2004, "Correspondence, Cognition and Sensemaking: A Radical Empiricist View of Situation Awareness: Theory and Application." pp. 22-41. In A Cognitive Approach to Situation Awareness: Theory and Application, eds. S Banbury and S Tremblays. Ashgate Publishing Company, Burlington, VT.

Ellis, G, and A Dix. 2007, "A Taxonomy of Clutter Reduction for Information Visualisation." Visualization and Computer Graphics, IEEE Transactions on 13(6):1216-23.

Endsley, MR. 1988, "Design and Evaluation for Situation Awareness Enhancement." In Human Factors Society 32nd Annual Meeting.

Endsley, MR. 1995, “Toward a Theory of Situation Awareness in Dynamic-Systems.” Human Factors 37(1):32-64.

Endsley, MR, B Bolte, and DG Jones. 2004. Designing for Situation Awareness. Taylor \& Francis, London, England.

Ericsson, AK, and HA Simon. 1993. Protocol Analysis: Verbal Reports as Data. MIT Press, Cambridge, MA.

Flach, J, M Mulder, and MM van Paasen. 2004, "The Concept of the Situation in Psychology." In $A$ Cognitive Approach to Situation Awareness, eds. S Banbury and S Tremblay, pp. 42-60. Ashgate Publishing Company, Burlington, VT.

Gentner, D, and AL Stevens. 1983. Mental Models. Lawrence Erlbaum Associates, Hillsdale, NJ.

Gigerenzer, G. 1991, "From Tools to Theories: A Heuristic of Discovery in Cognitive Psychology." Psychological Review 98(2):254-567.

Gonzalez, V, and A Kobsa. 2003, "Benefits of Information Visualization Systems for Administrative Data Analysts." In 7th International Conference on Information Visualization, pp. 331-36. 
Goodell, H, C Chiang, C Kelleher, A Bauman, and G Grinstein. 2006, "Collecting and Harnessing Rich Session Histories.” In 10th International Conference on Information Visualization, pp. 117-23.

Gordon, SE, and RT Gill. 1997, "Cognitive Task Analysis." in Naturalistic Decision Making, eds. C Zsombok and G Klein. Erlbaum, Mahwah, NJ.

Gotz, D, and MX Zhou. 2008, "Characterizing Users Visual Analytic Activity for Insight Provenance.” In IEEE Symposium on Visual Analytics Science \& Technology, pp. 123-30.

Green, TM, and B Fisher. 2010, "Towards the Personal Equation of Interaction : The Impact of Personality Factors on Visual Analytics Interface Interaction." In IEEE Symposium on Visual Analytics Science \& Technology, pp. 203-10.

Green, TM, DH Jeong, and B Fisher. 2010, "Using Personality Factors to Predict Interface Learning Performance." In 43rd Annual Hawaii International Conference on System Sciences.

Greitzer, FL. 2008, "Methodology, Methods and Metrics for Testing and Evaluating Augmented Cognition Systems." In Augmented Cognition: A Practitioner's Guide, eds. D Schmorrow and K Stanney, pp. 144-74. Human Factors Society, Santa Monica, CA.

Greitzer, FL. 2005, Methodology, Metrics and Measures for Testing and Evaluation of Intelligence Analysis. Technical Rpt. PNWD-3550, Battelle, Pacific Northwest Division, Richland, WA.

Greitzer, FL. 2005, “Toward the Development of a Cognitive Task Difficulty Metrics to Support Intelligence Anslysis Research.” In IEEE International Conference on Cognitive Informatics.

Greitzer, FL, A Schur, and RT Guttromson. 2008, "A Sensemaking Perspective on Situation Awareness in Power Grid Operations." In Power and Energy Society General Meeting - Conversion and Delivery of Electrical Energy in the 21st Century, 2008 IEEE, pp. 1-6.

Heer, J, J Mackinlay, C Stolte, and M Argawala. 2008, "Graphical Histories for Visualization: Supporting Analysis, Communication, and Evaluation." IEEE Transactions on Visualization and Computer Graphics 14(6):1189-96.

Hegarty, M. 2010, "Visweek Keynote Address." Visualization and Computer Graphics, IEEE Transactions on 16(6):xxiv-xxiv.

Holzinger, A. 2005, "Usability Engineering Methods for Software Developers.” Commun. ACM 48(1):7174.

Johnson-Laird, PN. 1983. Mental Models: Towards a Cognitive Science of Language, Inference, and Consciousness. Harvard University Press, Cambridge, MA.

Katona, G. 1940. Organizing and Memorizing: Studies in the Psychology of Learning and Teaching. Columbia University Press, New York, NY. 
Keim, D, G Andrienko, JD Fekete, C Görg, J Kohlhammer, and G Melancon. 2008, "Visual Analytics: Definition, Process, and Challenges." in Information Visualization, Human-Centered Issues and Perspectives, pp. 154-75. Springer-Verlag, Berlin.

Keim, D, J Kohlhammer, G Ellis, and F Mansmann, eds. 2010. Mastering the Information Age: Solving Problems with Visual Analytics. EuroGraphics, Goslar, Germany.

Kozlowski, SWJ. 1998, "Training and Developing Adaptive Teams: Theory, Principles, and Research." In Making Decisions under Stress: Implications for Individual and Team Training, eds. JA CannonBowers and E Salas, pp. 115-53. American Psychological Association, Washington, DC.

Lam, H. 2008, “A Framework of Interaction Costs in Information Visualization.” IEEE Transactions on Visualization and Computer Graphics 14(6):1149-56.

Larkin, JH, and HA Simon. 1987, "Why a Diagram Is (Sometimes) Worth Ten Thousand Words." Cognitive Science 11(1):65-100.

Lee, B, C Plasiant, CS Parr, J Fekete, and N Henry. 2006, "Task Taxonomy for Graph Visualization.” In Proceedings of the 2006 AVI Workshop on BEyond Time and Errors Novel Evaluation Methods for Information Visualization - BELIV'06, pp. 1-5.

Liiv, I. 2010, “Towards Information-Theoretic Visualization Evaluation Measure: A Practical Example for Bertin's Matrices." In BELIV'10: BEyond Time and Errors Novel Evaluation Methods for Information Visualization, April 10-11, 2010.

Lipford, HR, F Stukes, W Doub, ME Hawkins, and R Chang. 2010, "Helping Users Recall Their Reasoning Process.” In IEEE Symposium on Visual Analytics Science \& Technology, pp. 187-94.

Liu, Z, NJ Nersessian, and JT Stasko. 2008, "Distributed Cognition as a Theoretical Framework for Information Visualization." IEEE Transactions on Visualization and Computer Graphics 14(6):1173-80.

Liu, Z and J Stasko. 2010, "Mental Models, Visual Reasoning and Interaction in Information Visualization: A Top-Down Perspective." Visualization and Computer Graphics, IEEE Transactions on 16(6):999-1008.

Matzen, L, L McNamara, K Cole, A Bandlow, C Dornburg, and T Bauer. 2010, "Proposed Working Memory Measures for Evaluating Information Visualization Tools." In BELIV'10: BEyond Time and Errors: Novel Evaluation Methods for Information Visualization, April 10-11, 2010.

Mayer, RE. 1996, "The Search for Insight: Grappling with Gestalt Psychology's Unanswered Questions." In The Nature of Insight, eds. RJ Sternberg and JE Davidson, pp. 3-32. MIT Press.

Mayr, E, M Smuc, and H Risku. 2010, "Many Roads Lead to Rome. Mapping Users' Problem Solving Strategies." In BELIV'10: BEyond Time and Errors: Novel Evaluation Methods for Information Visualization, April 10-11, 2010.

Miller, GA. 1956, “The Magical Number Seven, Plus or Minus Two: Some Limits on Our Capacity to Process Information.” Psychological Review 63:81-96. 
Morse, E, M Lewis, and KA Olsen. 2000, “Evaluating Visualizations: Using a Taxonomic Guide.” Int. J. Hum.-Comput. Stud. 53(5):637-62.

Newell, A and HA Simon. 1963, "GPS: A Program That Simulates Human Thought.” In Computers and Thought, eds. EA Feigenbaum and J Feldman. MacGraw Hill, New York.

Nielsen, J. 1994, “Heuristic Evaluation.” In Usability Inspection Methods, eds. J Nielsen and RL Mack. John Wiley \& Sons, New York.

Nielsen, J. 1994. Usability Engineering. Morgan Kaufmann, San Francisco.

Norman, DA. 1988, "Psychology of Everyday Action.” In The Design of Everyday Things, pp. 45-46. Basic Books, New York.

Norman, DA. 1983, "Some Observations on Mental Models." In Mental Models, eds. D Dentner and AL Stevens, pp. 7-14. Lawrence Erlbaum Associates, Hillsdale, NJ.

North, C. 2006, “Toward Measuring Visualization Insight.” IEEE Computer Graphics and Applications 26(3):6-9.

Payne, SJ. 2003, "Users Mental Models: The Very Ideas." In HCI Models, Theories and Frameworks: Toward a Multidisciplinary Science, ed. JM Carroll, pp. 135-56. Morgan Kaufmann, San Francisco.

Perer, A and B Shneiderman. 2009, "Integrating Statistics and Visualization for Exploratory Power: From Long-Term Case Studies to Design Guidelines." IEEE Computer Graphics and Applications 29(3):39-51.

Perer, A and B Shneiderman. 2008, "Integrating Statistics and Visualization: Case Studies of Gaining Clarity During Exploratory Data Analysis.” In 26th Annual CHI Conference on Human Factors in Computing Systems, pp. 265-74.

Pfitzner, D, V Hobbs, and D Powers. 2001, “A Unified Taxonomic Framework for Information Visualization.” In 2nd Australian Institute of Computer Ethics Conference (AICE 2000), pp. 57-66.

Pike, W, R May, and A Turner. 2007, "Supporting Knowledge Transfer through Decomposable Reasoning Artifacts." In 40th Annual Hawaii International Conference on System Sciences, pp. 204c-04c.

Pike, WA, J Stasko, R Change, and TA O'Connell. 2009, “The Science of Interaction.” Information Visualization 8(4):263-74.

Pirolli, P and S Card. 2005, "The Sensemaking Process and Leverage Points for Analyst Technology as Identified through Cognitive Task Analysis.” In Conference on Intelligence Analysis, pp. 1-6.

Plaisant, C. 2004, "The Challenge of Information Visualization Evaluation." In Working Conference on Advanced Visual Interfaces (AVI'04), pp. 109-16.

Pohl, M, S Wiltner, and S Miksch. 2010, "Exploring Information Visualization - Describing Different Interaction Patterns." In BELIV'10: BEyond Time and Errors: Novel Evaluation Methods for Information Visualization, April 10-11, 2010. 
Riche, NH and T Dwyer. 2010, “Untangling Euler Diagrams." IEEE Transactions on Visualization and Computer Graphics 16(6):1090-9.

Santos, BS, F Zamfir, C Ferreira, O Mealha, and J Nunes. 2004, "Visual Application for the Analysis of Web-Based Information Systems Usage: A Preliminary Usability Evaluation." In 8th International Conference on Information Visualisation, pp. 812-18.

Saraiya, P, C North, and K Duca. 2004, "An Evaluation of Microarray Visualization Tools for Biological Insight.” In IEEE Symposium on Information Visualization, pp. 1-8.

Saraiya, P, C North, V Lam, and KA Duca. 2006, “An Insight-Based Longitudinal Study of Visual Analytics." IEEE Transactions on Visualization and Computer Graphics 12(6):1511-22.

Sarter, NB and DD Woods. 1991, "Situation Awareness: A Critical but Ill-Defined Phenomenon." International Journal of Aviation Psychology 1:45-57.

Scholtz, J. 2006, "Beyond Usability: Evaluation Aspects of Visual Analytic Environments." In IEEE Symposium on Visual Analytics Science \& Technology, pp. 145-50.

Scholtz, J. 2010, "Developing Qualitative Metrics for Visual Analytic Environments." In BELIV'10: Beyond Time and Errors: Novel Evaluation Methods for Information Visualization, April 10-11, 2010.

Sedlmair, M, P Isenberg, D Baur, and A Butz. 2010, "Evaluating Information Visualization in Large Companies: Challenges, Experiences, and Recommendations." In BELIV'10: BEyond Time and Errors: Novel Evaluation Methods for Information Visualization, April 10-11, 2010.

Senge, PM. 1990. The Fifth Discipline: The Art and Practice of the Learning Organization. Doubleday Currency, New York.

Seo, J and B Shneiderman. 2006, "Knowledge Discovery in High-Dimensional Data: Case Studies and a User Survey for the Rank-by-Feature Framework." IEEE Transactions on Visualization and Computer Graphics 12(3):311-22.

Serfaty, D, J MacMillan, EE Entin, and EB Entin. 1997, "The Decision-Making Expertise of Battle Commanders." in Naturalistic Decision Making, eds. CE Zsambok and G Klein, pp. 233-46. Lawrence Elrbaum Associates, Mahwah, NJ.

Shanker, SG. 1995, "The Nature of Insight." Minds and Machines 5(4):561-81.

Shneiderman, B. 1997. Designing the User Interface, 3rd Ed. Addison-Wesley, Reading, MA.

Shneiderman, B. 1996, "The Eyes Have It: A Task by Data Type Taxonomy for Information Visualizations.” In IEEE Symposium on Visual Languages, pp. 336-43.

Shneiderman, B and C Plaisant. 2006, "Strategies for Evaluating Information Visualization Tools: MultiDimensional In-Depth Long-Term Case Studies." In AVI Workshop on BEyond Time and Errors: Novel Evaluation Methods for Information Visualization (BELIV'06). 
Shrinivasan, YB and JJ van Wijk. 2008, "Supporting the Analytical Reasoning Process in Information Visualization." In 26th Annual SIGCHI Conference on Human Factors in Computing Systems, pp. 123746.

Smuc, M, E Mayr, and H Risku. 2010, "Is Your User Hunting or Gathering Insights? Identifying Insight Drivers across Domains." In BELIV'10: Beyond time and errors: novel evaluation methods for Information Visualization, April 10-11, 2010.

Spence, R. 2001. Information Visualization. Addison-Wesley Reading, MA.

Stasko, J, JB Domingue, MH Brown, and BA Price, eds. 1998. Software Visualization: Programming as a Multimedia Experience. The MIT Press, Cambridge, MA.

Thomas, JJ. 2009, “Taxonomy for Visual Analytics: Seeking Feedback.” VAC Views May:6-7.

Thomas, JJ, and KA Cook. 2005. Illuminating the Path: The Research and Development Agenda for Visual Analytics. IEEE.

Trafton, JG, SB Trickett, and FE Mintz. 2005, "Connecting Internal and External Representations: Spatial Transformations of Scientific Visualizations.” Foundations of Science 10(1):89-106.

Tufte, ER. 2006. Beautiful Evidence. Graphics Press, Cheshire, CT.

Tweedie, L. 1997, “Characterizing Interactive Externalizations.” In SIGCHI Conference on Human Factors in Computing Systems, pp. 375-75.

Valiati, ERA, MS Pimenta, and CMDS Freitas. 2006, "A Taxonomy of Tasks for Guiding the Evaluation of Multidimensional Visualizations." In AVI workshop on BEyond Time and Errors: Novel Evaluation Methods for Information Visualization (BELIV'06), pp. 1-6.

Wehrend, S and C Lewis. 1990, “A Problem-Oriented Classification of Visualization Techniques.” In $I^{\text {st }}$ IEEE Conference on Visualization, pp. 139-43.

Weick, KE. 1995. Sensemaking in Organizations. Sage, Thousand Oaks, CA.

Wetheimer, M. 1959. Productive Thinking. Harper, New York.

Wohlfart, E. 2007, A Detailed Comparison of Information Visualization Tools Using a Reference Data Set. Vol Master's Thesis. Technical University of Vienna.

Wohlfart, E, W Aigner, A Berone, and S Miksch. 2008, "Comparing Information Visualization Tools Focusing on the Temporal Dimensions." In 12th International Conference on Information Visualization, pp. 69-74.

$\mathrm{Xu}, \mathrm{S}, \mathrm{X}$ Chen, and D Liu. 2009, "Classifying Software Visualization Tools Using the Bloom's Taxonomy of Cognitive Domain." In 22nd Canadian Conference on Electrical and Computer Engineering, pp. 13-18. 
Yi, JS. 2010, "Implications of Individual Differences on Evaluating Information Visualization Techniques." In BELIV'10: BEyond Time and Errors: Novel Evaluation Methods for Information Visualization, April 10-11, 2010.

Yi, JS, Y ah Kang, J Stasko, and J Jacko. 2007, "Toward a Deeper Understanding of the Role of Interaction in Information Visualization." Visualization and Computer Graphics, IEEE Transactions on 13(6):1224-31.

Zhou, MX, and SK Feiner. 1998, "Visual Task Characterization for Automated Visual Discourse Synthesis." In SIGCHI Conference on Human Factors in Computing Systems - CHI '98, pp. 392-99.

Zhu, B, and HC Chen. 2005, "Information Visualization." Annual Review of Information Science and Technology 39:139-77.

Ziemkiewicz, C, P Kinnaird, R Kosara, J Mackinlay, B Rogowitz, and JS Yil. 2010, "Visualization Theory : Putting the Pieces Together." In IEEE Symposium on Information Visualization. 
Appendix A

Annotated Bibliography 



\section{Appendix A: Annotated Bibliography}

\section{A.1 Scope and Purpose}

This document is an Appendix to the report "Cognitive Foundations for Visual Analytics" [PNNL20207], which was produced through a research project with the same name, aiming to enhance understanding of the role of Visual Analytics (VA) in knowledge discovery and insight, identify more rigorous scientific methods to evaluate effectiveness of VA tools, and inform design of deployable VA solutions based on this theoretical foundation. In support of this project, the authors conducted an extensive literature search to update our understanding of the VA field from multiple perspectives.

This Appendix documents the method used in conducting the literature search and provides an alphabetical list of citations with abstracts for the research papers that were found. Ultimately, an interactive repository of the literature citations/abstracts is sought to provide researchers greater flexibility in searching for publications relevant to their fields, applications, and science approaches. Current status and plans for making an interactive repository available to the VA community are described below in Section A.3.

\section{A.2 Method}

A literature search is the active identification of existing information sources most relevant to the research question or need. For this project, subject matter experts were asked to supply keywords and phrases salient to the research topic and that would enable the information specialist to identify relevant literature. A sampling of keywords and phrases is in Table A.1 below:

Table A.1. Keywords and Phrases

\begin{tabular}{l}
\hline Information Analysis + \\
Discovery or "knowledge discovery" \\
Information visualization + \\
Insight \\
Intuition \\
Problem-solving, problem solving \\
Reasoning \\
Sensemaking, sense-making, sense making \\
Understanding \\
Wisdom \\
\hline
\end{tabular}

Multiple searches were conducted in commercially available databases covering the broad areas of computer science, psychology, cognitive science, and human factors. There were no restrictions placed on publication date or language of publication. The databases shown in Table A. 2 were queried, resulting in over 1,900 citations. In addition, several large datasets were collected from VisWeek 2010 and the National Visualization and Analytics Center. Collectively, we have gathered over 2,400 citations. 
Table A.2. Databases

\begin{tabular}{|c|c|}
\hline Database & Description of Coverage/Scope \\
\hline Scopus & $\begin{array}{l}\text { SciVerse Scopus is the world's largest abstract and citation database of peer- } \\
\text { reviewed literature and quality web sources. The database contains } \\
41 \text { million records, } 70 \% \text { with abstracts, and nearly } 18,000 \text { titles from } \\
5,000 \text { publishers worldwide. } 70 \% \text { of content is pulled from international } \\
\text { sources. Includes over } 3 \text { million conference papers. }\end{array}$ \\
\hline $\begin{array}{l}\text { Web of Science }+ \text { Conference } \\
\text { Proceedings Citation Index }\end{array}$ & $\begin{array}{l}\text { Science Citation Index Expanded: Over 7,100 major journals across } \\
150 \text { disciplines, from 1900-present. } \\
\text { Conference Proceedings Citation Index: Over 110,000 journals and book- } \\
\text { based proceedings in two editions: Science and Social Science and } \\
\text { Humanities, across } 256 \text { disciplines. }\end{array}$ \\
\hline $\begin{array}{l}\text { Compendex thru Engineering } \\
\text { Village }\end{array}$ & $\begin{array}{l}\text { Compendex is the most comprehensive bibliographic database of scientific } \\
\text { and technical engineering research available, covering all engineering } \\
\text { disciplines. It includes millions of bibliographic citations and abstracts from } \\
\text { thousands of engineering journals and conference proceedings. When } \\
\text { combined with the Engineering Index Backfile (1884-1969), Compendex } \\
\text { covers well over } 120 \text { years of core engineering literature. }\end{array}$ \\
\hline INSPEC thru Engineering Village & $\begin{array}{l}\text { Inspec includes bibliographic citations and indexed abstracts from } \\
\text { publications in the fields of physics, electrical and electronic engineering, } \\
\text { communications, computer science, control engineering, information } \\
\text { technology, manufacturing and mechanical engineering, operations research, } \\
\text { material science, oceanography, engineering mathematics, nuclear } \\
\text { engineering, environmental science, geophysics, nanotechnology, } \\
\text { biomedical technology, and biophysics. }\end{array}$ \\
\hline
\end{tabular}

\section{A.2.1 Citation Management}

Literature citations were downloaded into the EndNote X4 (Version 4) software. Thomson Reuters' EndNote is an industry standard software tool for publishing and managing bibliographies on the Windows and Macintosh desktop. Use of this tool enables the end user to manage reference document citations and their corresponding full-text, when available, among other capabilities.

Many abstracting and indexing databases have very broad coverage, which results in individual databases duplicating the content found in others. Duplicated bibliographic records were identified within the EndNote library and removed. To enable real-time collaboration and sharing of the literature, the EndNote library content was converted into an XML file and imported into a freely available citation management tool, Mendeley. Mendeley is a free desktop and web solution designed for managing and sharing research citations and papers and can be used for collaborating online. Mendeley enables the research team to share research citations through the internet using a private group that can be accessed using individual usernames/passwords from any computer, iPod touch, or iPad. 


\section{A.2.2 Organization of Results}

Our literature search resulted in the collection of over 2400 citations of published works on a variety of subtopics within cognitive science and visual analysis. To help organize the collected works, we performed an analysis of the collection with the assistance of IN-SPIRE ${ }^{\text {TM }}$. $^{9}$

As a first step in our analysis, we identified 36 papers that we felt were highly relevant to our task of understanding both the cognitive aspects of VA and the taxonomic organization of visual analytic tools. We processed these works as a dataset in IN-SPIRE and then used the Galaxy ${ }^{10}$ view to explore the clustering of the subset by topic. ${ }^{11}$ A visual inspection of the dataset (see Figure A.1) revealed several distinct groupings of documents, as shown below.

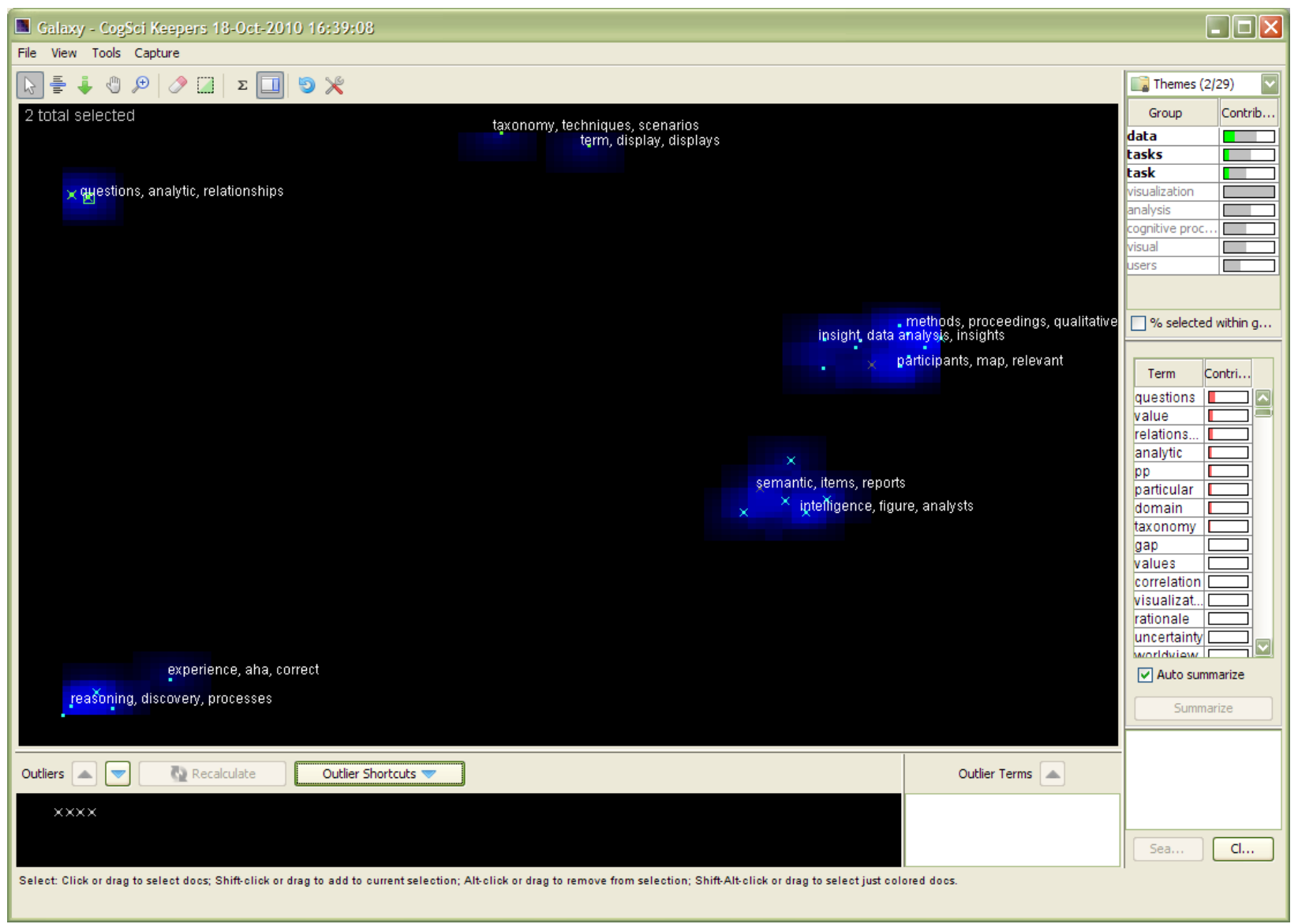

Figure A.1. IN-SPIRE Visualization of an Initial Set of 36 Papers

\footnotetext{
${ }^{9}$ IN-SPIRETM analyzes a multitude of text files and determines key topics or themes in each to create a signature for each document in the collection. More information at: http://in-spire.pnl.gov/index.stm.

${ }^{10}$ The Galaxy visualization uses the metaphor of the stars in the night sky with each star representing an individual document.

${ }^{11}$ Four documents were removed from the analysis because they were not parsed properly by IN-SPIRE.
} 
Using the extracted keywords, we developed the following labels for the clusters:

- reasoning processes and discovery

- sensemaking

- taxonomies and frameworks

- insight and actions

- insight and theory.

Our next step was to analyze in some detail as many of the collected citations as possible, given time and budget constraints. We had immediate access to 700 documents, including the 36 we identified earlier as highly relevant. These 36 were not treated in any special way when processed with the larger collection of documents; they were clustered normally with the others. Again, we removed a small number of documents due to poor parsing. The resulting clusters overlapped with the previously obtained clusters, particularly in clusters that we interpreted as reasoning, insight, and sensemaking. Clusters associated with other keywords became more prominent in the larger dataset, especially those associated with insight, creativity, and problem solving, cognition and insight, Information and Interaction Design, and Insight and Visualization. Absent from the featured clusters of the larger dataset was a cluster representing taxonomies and frameworks.

As a final step, we merged our two previous datasets to better understand where the original 36 highly relevant documents were clustered in the larger dataset. Several keywords were again prominent in this merged dataset, including reasoning and insight. Two separate clusters emerged to separate creativity from problem solving. The original 36 highly relevant documents were all located in clusters with similar topic keywords as their original clustering (see Figure A.2). Again, there were no clusters representing "taxonomies and frameworks." Instead, documents that we had earlier marked as "taxonomies and frameworks" were clustered with other documents under new labels such as "data visualization" and "visual analysis." "Participants" and "Subjects" appeared as a keyword in the merged dataset; however, as keywords they were included in several clusters and spread throughout the dataset, suggesting that while experimental evaluation is an emerging theme in the literature, efforts are still disjointed and lack unification or commonalities. 


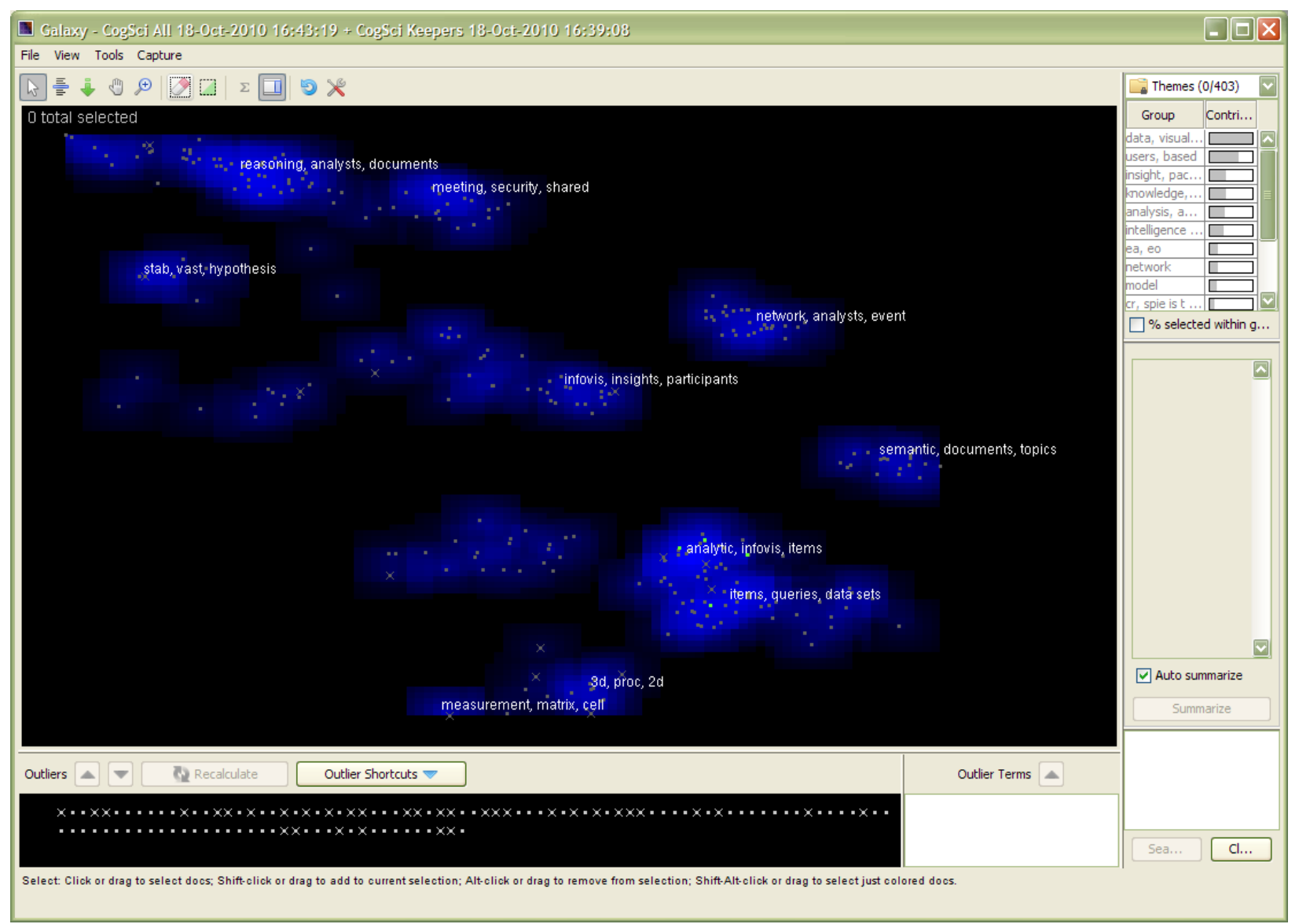

Figure A.2. IN-SPIRE Visualization of the More Complete Set of Papers

\section{A.3 Electronic Version of Sources}

Currently, our collection of relevant citations is available in an EndNote library. This library can be easily shared with others who have a recent copy of the software installed at their institution. However, a long-term goal is to create an online repository of the information. This collection of citations and abstracts could be organized with browse, search, and retrieval functionality and made available through the web. Ideally, the content will be organized using a structured taxonomy to enhance faceted navigation and retrieval. After addressing any copyright issues, this online repository would also be capable of including materials such as conference papers or journal articles, before (preprints) and after (postprints) undergoing peer review, and digital versions of grey literature, theses, and dissertations, all relevant to the cognitive foundations of VA and information visualization. The overall goal of such an endeavor is to continue the discussion on this topic with a wider audience and to make available source materials salient to VA research. 


\title{
A.4 Annotated Works: Alphabetical Listing of Annotated Bibliography
}

\author{
Abdalhakim, H, and M Abdelfattah. 2009, "An Intelligent Visualized Decision Toolbox Ivdt for Muzzy \\ Decision Maker." In 2nd ISECS International Colloquium on Computing, Communication, Control and \\ Management (CCCM 2009), pp. 115-18.
}

The importance of decision support systems is still increasing. Decision problems to be solved are often very complicated and require more powerful and often intelligent support tools. Sometimes, it is useful to build an expertise set in some decision steps. Such situation can be met when operating on very large databases and on solving complicated problems. However evolving a convenient intelligent components with visualization aspects in IDSS are big challenges to be developed; but it would provide a muzzy decision taker an insight, preference, and much capability during a decision choice. This paper opt the advanced information visualization schemes for both decision's fact discovery and supporting decision taking processes. It proposes a visualized toolbox for IDSS that integrates DSS and dynamic information visualization within enterprise functionality. Finally this work formulates a viability of implementing such toolbox and presents conclusions.

Abdalhakim, H, and M Abdelfattah. 2010, "A Visualization-Based Intelligent Decision Support System Conceptual Model." In International Joint Conference on Computer, Information, Systems Sciences and Engineering, pp. 353-58.

the development of Intelligent Decision Support Systems IDSS is till requiring much effort to cope with both: the complexity of today decisions and daily flood of risky decisions. However evolving a covenant intelligent components and visualization aspects in IDSS are big challenges to be developed; but it would provide a muzzy decision taker an insight, preference, and much capability during a decision choice. This paper opt the advanced information visualization schemes for both decision's fact finding and decision taking processes. It proposes a conceptual model for IDSS that integrates DSS and dynamic information visualization within enterprise functionality. And it has introduced a three-module IDSS conceptual model that assembles a model base subsystem, fact finding subsystem, and dynamic visualization subsystem as a practitioner solution. The paper will focus on information visualization as an emerging computing relevant to be incorporated in order to model hidden facts in data and to expose such patterns in a visual manner. Finally this work formulates a viability of implementing such architecture and presents the conclusions.

Adamoli, A, and M Hauswirth. 2010, "Trevis : A Context Tree Visualization \& Analysis Framework and Its Use for Classifying Performance Failure Reports." In 5th ACM Symposium on Software Visualization (SOFTVIS), pp. 73-82.

Adams, S, and AK Goel. 2007, "Making Sense of Vast Data." In Intelligence and Security Informatics Conference, pp. 270-73.

We view sensemaking in threat analysis as abducing a story whose plot explains the current data and makes predictions about the future. We have developed a preliminary computational system, called STAB, that abduces stories from data. STAB abduces multiple competing hypotheses by retrieving and instantiating story plots matching the current evidence. The story plots in STAB are represented as processes with goals and states, and organized in an abstraction hierarchy. STAB analyzes the VAST- 
2006 dataset. Given the VAST data incrementally, STAB generates multiple explanatory hypotheses, calculates their confidence values, and generates expectations about future data.

Adams, S, and AK Goel. 2007, "A Stab at Making Sense of Vast Data." In AAAI-2007 Workshop on Plan, Activity, and Intent Recognition, pp. 1-8.

We view sensemaking in threat analysis as abducing a story whose plot explains the current data and makes predictions about the future. We have developed a preliminary computational system, called STAB, that abduces stories from data. STAB abduces multiple competing hypotheses by retrieving and instantiating story plots matching the current evidence. The story plots in STAB are represented as processes with goals and states, and organized in an abstraction hierarchy. STAB analyzes the VAST2006 dataset. Given the VAST data incrementally, STAB generates multiple explanatory hypotheses, calculates their confidence values, and generates expectations about future data.

Addis, TR. 1990, "Knowledge and Machine Architectures." In IEE Colloquium on Symbols Versus Neurons, pp. 1-9.

A paradigm is proposed based upon a taxonomy of knowledge; a taxonomy that has been strongly influenced by the need to represent knowledge for machine processing. The importance of such a paradigm is to show an equivalence of activity in all spheres of system design from knowledge systems to machine architectures and thus open up the possibility of cross fertilization of techniques and a redistribution of tasks across fields. Both these possibilities will improve the design of knowledge systems within the framework of a rapidly evolving technology.

Adelson, B. 2010, In Press, "Task Analysis, Calculation and Approximation: The Work of Stuart K. Card, 2007 Bower Laureate in Computer \& Cognitive Science for Human-Centered Computing." Journal of the Franklin Institute.

In the early 1980s there was a large base of well-regarded cognitive theory--explaining human cognition. Additionally, personal technology was increasingly a factor in the workplace, but it was notoriously hard to use, leading to frustration rather than increased satisfaction and productivity [1], [2] and [3]. In 1974 Stuart Card accompanied Allen Newell from Carnegie Mellon University to Xerox's Palo Alto Research Center (PARC) to work on the problem of human-computer interaction. Newell returned to CMU, but Card remained to become the founding manager of the User Interface Research group. Card's first major work The Psychology of Human-Computer Interaction, was published in 1983. It was undertaken to address the problems of usability by closing the gap between theory and technology. It did this by providing a methodology for bringing theory to bear on application. The work served as a foundation for research and development in human-computer interaction for the following 15 years.

Adistambha, K, et al. 2010, "Efficient Multimedia Query-by-Content from Mobile Devices." Computers \& Electrical Engineering 36(4):626-42.

The phenomenal growth in multimedia content has lead to the development of a variety of multimedia description schemes, which can be used to facilitate querying of multimedia databases. In the increasingly mobile environment of today, multimedia query formats need to be applicable to mobile devices, which, compared to desktop PCs, have specific limitations such as small screen size, limited memory and processing power and high bandwidth cost. As a potential solution to multimedia querying in mobile 
environments, this paper introduces two concepts: query streaming and its application as targeted browsing. Targeted browsing is a technique for multimedia query-by-content designed especially for mobile devices while query streaming is a method for continually updating a query by sending additional terms to an existing query. This paper describes an implementation of query streaming that combines the Multimedia Query Format (MQF) (a standard communication language for querying multimedia databases) with Fragment Request Units (FRU) and Fragment Update Units (FUU) (which provide a standard way of randomly accessing fragments of XML documents). For efficient compression of the multimedia query XML files, the use of binary compression using MPEG BiM is proposed and a number of use case scenarios are examined. Results show that the proposed solution to provide a significant reduction in the file size required to perform multimedia querying.

Adnan, WAW, NGN Daud, and NLM Noor. 2008, "Expressive Information Visualization Taxonomy for Decision Support Environment." In 2008 Third International Conference on Convergence and Hybrid Information Technology, pp. 88-93.

Adnan, WW, N MdNoor, and R Aripin. 2007, "An Experimental Evaluation of Information Visualization Techniques and Decision Style." in Lecture Notes in Computer Science, ed. JA Jacko, Vol 4551, pp. 14350 .

This study aims to investigate the extent to which information visualization (IV) techniques and decision style affect decision performance and user preferences in a decision support environment. The study adopted an experimental method. Findings from this study provide theoretical, empirical and practical contributions. The results showed that there were significant differences in decision performance and user preference across IV techniques and decision style. The findings have important implications for the decision support system (DSS) designers, and provide important research issues for future work.

Aftandilian, EE, et al. 2010, "Heapviz : Interactive Heap Visualization for Program Understanding and Debugging." In 5th ACM Symposium on Software Visualization (SOFTVIS), pp. 53-62.

Aftandilian, EE, SL Su, and SZ Guyer. 2010, "Heapviz : A Programmer' S Tool for Data Structure Visualization." In VISWEEK (interactive demo), pp. 1-2.

Agrawala, M, C Stolte, and ACM Acm. 2001, "Rendering Effective Route Maps: Improving Usability through Generalization." In ACM SIGGRAPH Conference, pp. 241-50.

Route maps, which depict a path from one location to another, have emerged as one of the most popular applications on the Web. Current computer-generated route maps, however, are often very difficult to use. In this paper we present a set of cartographic generalization techniques specifically designed to improve the usability of route maps. Our generalization techniques are based both on cognitive psychology research studying how route maps are used and on an analysis of the generalizations commonly found in handdrawn route maps. We describe algorithmic implementations of these generalization techniques within LineDrive, a real-time system for automatically designing and rendering route maps. Feedback from over 2200 users indicates that almost all believe LineDrive maps are preferable to using standard computer-generated route maps alone. 
Ahlqvist, O. 2008, "Extending Post-Classification Change Detection Using Semantic Similarity Metrics to Overcome Class Heterogeneity: A Study of 1992 and 2001 U.S. National Land Cover Database Changes." Remote Sensing of Environment 112(3):1226-41.

The use of post-classification change methods for the analysis of land cover change provides intuitive and potentially reliable results. A recurring problem is the difference in land cover nomenclature that can occur over time or across space when multiple data sources are required. Building on work that uses category semantics as a foundation for reasoning with land cover classes, this paper uses a fuzzy sets based approach to develop attribute based prototype definitions of land cover classes. These formalized category descriptions are used to look at land cover changes as a semantic change evaluated through semantic similarity metrics. The methodology is illustrated on the U.S. National Land Cover Data from 1992 to 2001 over Chester County, PA. The results demonstrate that the proposed method is more versatile than the standard post-classification method in that it can both provide an overall, spatially explicit evaluation of land cover change, as well as nuanced assessments of graded changes for heterogeneous land cover types.

Ahlstrom, U, and FJ Friedman-Berg. 2006, "Using Eye Movement Activity as a Correlate of Cognitive Workload." International Journal of Industrial Ergonomics 36(7):623-36.

In the present study, we investigated the effect on air traffic controller operations and workload from the use of weather displays. Specifically, we assessed the impact on severe weather avoidance, controller efficiency, controller-pilot communications, and the safety of airspace operations when controllers managed traffic during adverse weather conditions. The results showed a significant impact on controller efficiency from the use of weather displays with an increase in sector throughput by up to $10 \%$. We found no significant effects of weather displays on severe weather avoidance, controller communications, and subjective workload ratings. However, using eye movement activity measures that correlate with cognitive workload, we found significantly shorter blink durations when controllers operated traffic in conditions lacking a weather display, indicating a higher workload level during these conditions. Also, the mean pupil diameter was significantly larger when controllers used a static storm forecast tool compared to when controllers used a dynamic forecast tool, indicating a higher workload level during the use of static tools. We conclude that eye movement activity measures can provide a more sensitive measure of controller workload, and that subjective ratings might not capture more transient fluctuations in workload levels during system or display interactions. Relevance to industry Using real-time eye movement activity measures, designers can better evaluate changes in operator workload during the design and evaluation of complex systems. If we can detect workload-inducing conditions early in the design process, we can improve the design, optimize operator workload, and reduce developmental costs.

Ahn, JW, and P Brusilovsky. 2009, "Adaptive Visualization of Search Results: Bringing User Models to Visual Analytics." Information Visualization 8(3):167-79.

Adaptive visualization is a new approach at the crossroads of user modeling and information visualization. Taking into account information about a user, adaptive visualization attempts to provide user-adapted visual presentation of information. This paper proposes Adaptive VIBE, an approach for adaptive visualization of search results in an intelligence analysis context. Adaptive VIBE extends the popular VIBE visualization framework by infusing user model terms as reference points for spatial document arrangement and manipulation. We explored the value of the proposed approach using data obtained from a user study. The result demonstrated that user modeling and spatial visualization 
technologies are able to reinforce each other, creating an enhanced level of user support. Spatial visualization amplifies the user model's ability to separate relevant and non-relevant documents, whereas user modeling adds valuable reference points to relevance-based spatial visualization. (C) 2009 Palgrave Macmillan.

Ahn, J-w, et al. 2010, "Semantic Annotation Based Exploratory Search for Information Analysts." Information Processing \& Management 46(4):383-402.

The system presented in this article aims to improve information access through the use of semantic annotation utilizing a non-traditional approach. Instead of applying semantic annotations to enhance the internal information access mechanisms, we use them to empower the user of an information access system through an innovative named entity-based user interface - NameSieve. NameSieve was built to support an intelligence analyst during the process of exploratory search, an advanced type of search requiring multiple iterations of retrieval interleaved with browsing and analyzing the retrieved information. The proposed approach was implemented in the NameSieve system so that the system can transparently present a summary of search results in the form of entity "clouds." Therefore, these clouds allow the analyst to further explore the results in a novel manner, acting together as a faceted browsing interface. We ran a user study (with ten subjects) to examine the effect of NameSieve, and the study results reported in the paper demonstrate that this new way of applying semantic annotation information was actively used and was evaluated positively by the subjects. It enabled the subjects to work more productively and bring back most relevant documents.

Ahonen-Rainio, P, and K Menno-Jan. 2005, "Towards Multi-Variate Visualization of Metadata Describing Geographic Information." in Exploring Geovisualization, eds. D Jason, MM Alan and K Menno-Jan, pp. 611-25. Elsevier, Oxford.

Summary A visual environment is proposed for the exploratory use of metadata relating to geographic datasets. Metadata has a prominent role when acquiring geographic data for solving spatial problems. Users need metadata only occasionally but when they do, they should be able to use metadata to decide how well the available datasets meet the needs of the intended use. This decision can be supported by interactive exploration of metadata in such a way that different characteristics of datasets can be studied simultaneously. Multi-variate visualization techniques such as the parallel coordinates plot, the scatter plot matrix, star plots and Chernoff faces convey different aspects of the metadata. A working prototype of a visualization environment for metadata is drafted. It combines the multi-variate visualization methods with a map of the region of interest, browse graphics and textual metadata in multiple linked views.

Aigner, W. 2009, "Assessing the Role and Value of Interactivity in Visual Business Intelligence - a User's Perspective." Vol MSc, pp. 1-103. Danube University, Krems.

The development and success of many organizations to a large extent depend on how well and how timely they manage to gain insight into collected data. Business Intelligence (BI) provides methods for analyzing business-critical information and supports decision-making processes. But the ever growing amounts of data and information clearly overwhelm traditional manual methods of data analysis such as spreadsheets, standard reporting or simple diagrams. One possibility to face this problem is Information Visualization (InfoVis). By utilizing the powerful human perceptual system that is extremely efficient in processing visual input, visualization, for example, can help to make sense of data, explore complex 
information spaces or spot patterns and relationships within the data. Interactivity or the possibility to engage in an active discourse with the representation lies at the core of Information Visualization. However, empirical evidence and detailed approaches to model the concept of interactivity are largely missing in research today. This thesis is a first step towards bridging this gap and approaching the concept of interactivity more in depth. In order to do so, a twofold approach was chosen. First, the concept of interactivity was investigated from a theoretical point of view. In particular, cognitive theories and models were analyzed in order to determine their ability to explain and predict the value and role of interactivity in visual methods. Second, a qualitative empirical study was conducted among six IT-managers working in the field of Business Intelligence to assess the current practice at work concerning visual methods and interactivity. The main results are that cognitive theories and models do not explicitly account for the role of interactivity but rather concentrate on modeling the elements of a cognitive system and explaining how these entities interact with each other to achieve a certain goal. How different types of interactivity influence these processes is usually not part of the theories, and the effect of interactivity on cognitive processes was hardly ever mentioned. Apart from that, the empirical study showed that interactive visual methods in the area of Business Intelligence aren't used very often. One of the main reasons reported is the fact that visualization is still two steps ahead and at the moment mostly more basic problems concerning data gathering, data modeling and data quality prevail. Moreover, most users are used to work with numbers and tables and are not aware of the possibilities in terms of visualization. However, the interviewed IT-managers acknowledged that using more interactive visual methods in Business Intelligence would be beneficial for users. vii

Aigner, W, A Bertone, and S Miksch. 2007, "Tutorial: Introduction to Visual Analytics." In $3^{\text {rd }}$ Symposium of the Workgroup Human-Computer Interaction and Usability Engineering of the Austrian Computer Society, pp. 453-56.

Visual Analytics is an emerging area of research and practice that aims for supporting analytical reasoning by interactive visual interfaces. The basic idea is the integration of the outstanding capabilities of humans in terms of visual information exploration and the enormous processing power of computers to forma powerful knowledge discovery environment. In the course of our half-day tutorial we will introduce this multi-disciplinary field by discussing its key issues of analytical reasoning, perception \& cognition, visualization interaction, computation mining, the visual analysis process, and show potential application areas.

Aigner, W, and S Miksch. 2006, "Carevis: Integrated Visualization of Computerized Protocols and Temporal Patient Data." Artificial Intelligence in Medicine 37(3):203-18.

SummaryObjective Currently, visualization support for patient data analysis is mostly limited to the representation of directly measured data. Contextual information on performed treatment steps is an important source to find reasons and explanations for certain phenomena in the measured patient data, but is mostly spared out in the analysis process. This work aims to fill this gap via integrating classical data visualization and visualization of treatment information.Methods and material We considered temporal as well as logical data aspects and applied a user-centered development approach that was guided by user input gathered via a user study, design reviews, and prototype evaluations. Furthermore, we investigated the novel PlanningLine glyph, that is used to represent plans in the temporal domain, via a comparative empirical user study.Results Our interactive visualization approach CareVis provides multiple simultaneous views to cover different aspects of the complex underlying data structure of treatment plans and patient data. The tightly coupled views use visualization methods well-known to domain experts and 
are designed to facilitate users' tasks. The views are based on the concepts of clinical algorithm maps and LifeLines which have been extended in order to cope with the powerful and expressive plan representation language Asbru. Initial feedback of physicians was encouraging and is accompanied by empirical evidence which verifies that PlanningLines are well suited to manage temporal uncertainty.Conclusion The interactive integration of different visualization methods forms a novel way of combining, relating, and analyzing different kinds of medical data and information that otherwise would be separated.

Aigner, W, and S Miksch. 2004, "Supporting Protocol-Based Care in Medicine Via Multiple Coordinated Views." In 2nd International Conference on Coordinated and Multiple Views in Exploratory Visualization, pp. 118-29.

Computer supported protocol-based care aims to aid physicians in the treatment process. The main focus of current research is directed towards the formal methods and representations used "behind the scenes" of such systems. This work on the contrary, is situated at the human end of the human-machine chain. We describe the development of interactive visualization methods to support protocol-based care. We provide multiple simultaneous views to cover different aspects of a complex underlying data structure of treatment plans and patient data. The tightly coupled views use visualization methods well-known to domain experts and are designed to facilitate users' tasks. The views are based on the concepts of clinical algorithm maps and Lifelines which have been extended in order to cope with the powerful and expressive plan representation language Asbru. The user-centered development approach applied for these interactive visualization methods has been guided by user input gathered via a user study, design reviews, and prototype evaluations.

Aigner, W, et al. 2007, "Visualizing Time-Oriented Data--a Systematic View." Computers \& Graphics 31(3):401-09.

The analysis of time-oriented data is an important task in many application scenarios. In recent years, a variety of techniques for visualizing such data have been published. This variety makes it difficult for prospective users to select methods or tools that are useful for their particular task at hand. In this article, we develop and discuss a systematic view on the diversity of methods for visualizing time-oriented data. With the proposed categorization we try to untangle the visualization of time-oriented data, which is such an important concern in Visual Analytics. The categorization is not only helpful for users, but also for researchers to identify future tasks in Visual Analytics.

Aktas, RN, and V Cortes. 2008, "Shell Nouns as Cohesive Devices in Published and Esl Student Writing." Journal of English for Academic Purposes 7(1):3-14.

This paper analyzes the use of a special type of unspecific noun, called shell nouns [Hunston, S., \& Francis, G. (1999). Pattern grammar. Amsterdam: Benjamins; Schmid, H. (2000). English abstract nouns as conceptual shells: From corpus to cognition. Berlin: Walter de Gruyter], which are frequently used as cohesive devices, in the written production of published scientists and international graduate students. These nouns act like "shells" because when they are used in this function, they can enclose or anticipate the meaning of the preceding or succeeding discourse. Using a corpus-based methodology and quantitative and qualitative procedures, our study analyzes the frequency of some of these shell nouns, the different lexico-grammatical patterns in which they occur, and the functions associated with these patterns. The result of this study indicates that students used some of these nouns more frequently than 
published authors and that the functional patterns in which these nouns were used varied between the two groups of writers. These results provide valuable information that can be directly applied to English for Academic Purposes (EAP) instruction.

Albers, D. 2010, "Poster: Perceptual Principles for Scalable Sequence Alignment Visualization." In $7^{\text {th }}$ Symposium on Applied Perception in Graphics and Visualization, pp. 1-2.

Sequence comparison is a fundamental task in the biological sciences. Scientists often need to understand the similarities and differences between genetic sequences to understand evolution, to infer common function, or identify differences. Because the sequences are too long for manual examination, scientists rely on alignment tools that automatically identify subsequences that match between the sequences being compared. Numerous approaches for displaying and exploring alignments exist, and have been incorporated into a wide variety of tools. See [Procter et al. 2010] for a survey of several existing approaches.

Albrecht, B, et al. 2010, "An Automatic Layout Algorithm for Bpel Processes." In 5th ACM Symposium on Software Visualization (SOFTVIS), pp. 173-82.

Albu, AB, et al. 2007, "Spatio-Temporal Modeling of Neural Source Activation from Eeg Data." In $20^{\text {th }}$ Annual Canadian Conference on Electrical and Computer Engineering, pp. 1014-17.

This paper proposes a new computer-vision based information visualization paradigm for the electrophysiological study of face recognition. The proposed approach first generates video sequences of voltage maps from EEG data. Next, projections of active sources are detected in each frame using colour information and spatiotemporal consistency. The evolution of source activation is thus translated into a deformable motion of 2D patterns. Hence, the last step of the proposed approach builds a new motion representation, called the Spatio-Temporal Activation Response (STAR), which extracts stimulus- and subject-specific information about neural source activations occurring during the experiment. It is shown that STAR is able to capture relevant information about differences in the cognitive representations elicited by two different visual stimuli.

Albuquerque, G, and DJ Lehmann. 2010, "Improving the Visual Analysis of High-Dimensional Datasets Using Quality Measures." In IEEE Symposium on Visual Analytics Science \& Technology, pp. 19-26.

Modern visualization methods are needed to cope with very high dimensional data. Efficient visual analytical techniques are required to extract the information content in these data. The large number of possible projections for each method, which usually grow quadratically or even exponentially with the number of dimensions, urges the necessity to employ automatic reduction techniques, automatic sorting or selecting the projections, based on their information bearing content. Different quality measures have been successfully applied for several specified user tasks and established visualization techniques, like Scatterplots, Scatterplot Matrices or Parallel Coordinates. Many other popular visualization techniques exist, but due to the structural differences, the measures are not directly applicable to them and new approaches are needed. In this paper we propose new quality measures for three popular visualization methods: Radviz, Pixel-Oriented Displays and Table Lenses. Our experiments show that these measures efficiently guide the visual analysis task. 
Alim, U, T Möller, and L Condat. 2010, "Gradient Estimation Revitalized." IEEE Transactions on Visualization and Computer Graphics 16(6):1495-504.

We investigate the use of a Fourier-domain derivative error kernel to quantify the error incurred while estimating the gradient of a function from scalar point samples on a regular lattice. We use the error kernel to show that gradient reconstruction quality is significantly enhanced merely by shifting the reconstruction kernel to the centers of the principal lattice directions. Additionally, we exploit the algebraic similarities between the scalar and derivative error kernels to design asymptotically optimal gradient estimation filters that can be factored into an infinite impulse response interpolation prefilter and a finite impulse response directional derivative filter. This leads to a significant performance gain both in terms of accuracy and computational efficiency. The interpolation prefilter provides an accurate scalar approximation and can be re-used to cheaply compute directional derivatives on-the-fly without the need to store gradients. We demonstrate the impact of our filters in the context of volume rendering of scalar data sampled on the Cartesian and Body-Centered Cubic lattices. Our results rival those obtained from other competitive gradient estimation methods while incurring no additional computational or storage overhead.

Allan, J, et al. 2001, "Evaluating Combinations of Ranked Lists and Visualizations of Inter-Document Similarity." Information Processing \& Management 37(3):435-58.

Allendoerfer, K, et al. 2005, "Adapting the Cognitive Walkthrough Method to Assess the Usability of a Knowledge Domain Visualization." In IEEE Symposium on Information Visualization, pp. 195-202.

The usability of knowledge domain visualization (KDViz) tools can be assessed at several levels. Cognitive Walkthrough (CW) is a well-known usability inspection method that focuses on how easily users can learn software through exploration. Typical applications of $\mathrm{CW}$ follow structured tasks where user goals and action sequences that lead to achievement of the goals are well-defined. KDViz and other information visualization tools, however, are typically designed for users to explore data and user goals and actions are less well understood. In this paper, we describe how the traditional CW method may be adapted for assessing the usability of these systems. We apply the adapted version of CW to CiteSpace, a KDViz tool that uses bibliometric analyses to create visualizations of scientific literatures. We describe usability issues identified by the adapted $\mathrm{CW}$ and discuss how CiteSpace supported the completion of tasks, such as identifying research fronts, and the achievement of goals. Finally, we discuss improvements to the adapted $\mathrm{CW}$ and issues to be addressed before applying it to a wider range of KDViz tools.

Alm, I, and I Johansson. 2001, "Cognitive Aspects in Visualisation of Complex Data." In International Conference on Imaging Science, Systems, and Technology (CISST 2001), pp. 633-38.

Information Visualization applications are dealing with fundamental difficulties related to overlap in cognitive models between designers and users, goal ambiguity, and accuracy in search strategies These difficulties are more obvious in applications aimed at reducing information overload by general users, than in applications aimed at visualising scientific data. General users have very likely quite different cognitive reference for approaching an abstract complex task, than designers. This can result in designs which can unintentionally increase information overload by users. In visualisation of scientific data the overlap of cognitive reference between specialists and designs is very likely much higher, but we need methods which can facilitate data exploration in real-time interaction. One possibility to facilitate 
exploration in a more or less systematic way is by means of metaphors which can support human perception in searching for patterns.

Almeida, MG. 1998, "Diversity of Edge Information Using Complementary Filter Banks Analysis." In IEEE Conference on Information Visualization, pp. 166-71.

We present the complementary filter (CF) banks, a signal processing technique for image processing. The two-dimensional (2D) multirate signal processing theory and complementary filter properties are the base of CF bank design. Procedures to design nonseparable complementary filters for an alias free decimation are presented. The conditions under which perfect reconstruction is achieved with 2-channel bank of filters, using complementary filters are given. Perfect reconstruction is guaranteed when a CF bank is implemented using a quincunx filter and sampling and although no perfect reconstruction for others methods, the analysis and synthesis are performed free of aliasing. We show that CF banks and Wavelet representation are related. The CF banks are implemented with images and their performance is analyzed. The CF banks were applied for edge detection and examples of diversity in edge information obtained for different types of filtering are shown

Almendros-Jiménez, JM, and L Iribarne. 2008, "An Extension of Uml for the Modeling of Wimp User Interfaces." Journal of Visual Languages \& Computing 19(6):695-720.

The Unified Modeling Language (UML) [OMG, Unified Modeling Language Specification, Version 2.0, Technical Report, Object Management Group $<$ http://www.omg.org/technology/documents/formal/uml.htm>, 2005] provides system architects working on analysis and design (A\&D) with one consistent language for specifying, visualizing, constructing, and documenting the artifacts of software systems, as well as for the business modeling. The user interface (UI), as a significant part of most applications, should be modeled using UML, and automatic CASE tools may help to generate UIs from UML designs. In this paper, we describe how to use and specialize UML diagrams in order to describe the UIs of a software system based on WIMP (Windows, Icons, Menus and Pointers). Use case diagrams are used for extracting the main UIs. Use cases are described by means of user-interaction diagrams, a special kind of activity diagrams in which states represent data output actions and transitions represent data input events. Input and output interactions in the user-interaction diagrams help the designer to extract the UI components used in each UI. We obtain a new and specialized version of the use case diagram for the UI modeling (called UI diagram) and a class diagram for UI components-called UI-class diagram. The user-interaction, UI and UI-class diagrams, can be seen as the UML-based UI models of the system. Finally, UI prototypes can be generated from UI-class diagrams with CASE tool support. As case study of our technique, we will describe an Internet book shopping system.

Alpert, SR. 2005, "Comprehensive Mapping of Knowledge and Information Resources: The Case of Webster." In International Workshop on Visual Artefacts for the Organization of Information and Knowledge, Searching for Synergies, pp. 220-37.

To maximize the representational and pedagogical effectiveness of computer-based concept maps, such maps should be able to incorporate any sort of media that can be represented in the computational environment. This chapter proposes cognitive and educational rationale for this thesis, and discusses an instantiation of these ideas in the form of a Web-based concept mapping tool named Webster. Webster permits broad flexibility in terms of the kinds of knowledge and information that may be represented and the structuring of their visual presentation. One result of this approach is the integration of knowledge 
visualization and information visualization in a single representational medium. These facilities also make Webster a convenient tool for personal knowledge management, facilitating individual organization of knowledge and external knowledge and information resources for reference and learning purposes.

Alsakran, J. 2010, "Poster : Visual Analysis of Stream Texts with Keywords Significance." In IEEE Symposium on Information Visualization, pp. 3-4.

Amar, R, J Eagan, and J Stasko. 2005, "Low-Level Components of Analytic Activity in Information Visualization." In IEEE Symposium on Information Visualization, pp. 111-17.

Existing system level taxonomies of visualization tasks are geared more towards the design of particular representations than the facilitation of user analytic activity. We present a set of ten low level analysis tasks that largely capture people's activities while employing information visualization tools for understanding data. To help develop these tasks, we collected nearly 200 sample questions from students about how they would analyze five particular data sets from different domains. The questions, while not being totally comprehensive, illustrated the sheer variety of analytic questions typically posed by users when employing information visualization systems. We hope that the presented set of tasks is useful for information visualization system designers as a kind of common substrate to discuss the relative analytic capabilities of the systems. Further, the tasks may provide a form of checklist for system designers.

Amar, R, and J Stasko. 2004, "Best Paper: A Knowledge Task-Based Framework for Design and Evaluation of Information Visualizations." In IEEE Symposium on Information Visualization, pp. 143-50.

The design and evaluation of most current information visualization systems descend from an emphasis on a user's ability to "unpack" the representations of data of interest and operate on them independently. Too often, successful decision-making and analysis are more a matter of serendipity and user experience than of intentional design and specific support for such tasks; although humans have considerable abilities in analyzing relationships from data, the utility of visualizations remains relatively variable across users, data sets, and domains. In this paper, we discuss the notion of analytic gaps, which represent obstacles faced by visualizations in facilitating higher-level analytic tasks, such as decisionmaking and learning. We discuss support for bridging the analytic gap, propose a framework for design and evaluation of information visualization systems, and demonstrate its use

Amar, RA, and JT Stasko. 2005, "Knowledge Precepts for Design and Evaluation of Information Visualizations." Visualization and Computer Graphics, IEEE Transactions on 11(4):432-42.

The design and evaluation of most current information visualization systems descend from an emphasis on a user's ability to "unpack" the representations of data of interest and operate on them independently. Too often, successful decision-making and analysis are more a matter of serendipity and user experience than of intentional design and specific support for such tasks; although humans have considerable abilities in analyzing relationships from data, the utility of visualizations remains relatively variable across users, data sets, and domains. In this paper, we discuss the notion of analytic gaps, which represent obstacles faced by visualizations in facilitating higher-level analytic tasks, such as decisionmaking and learning. We discuss support for bridging these gaps, propose a framework for the design and evaluation of information visualization systems, and demonstrate its use. 
Ament, M, D Weiskopf, and H Carr. 2010, "Direct Interval Volume Visualization." IEEE Transactions on Visualization and Computer Graphics 16(6):1505-14.

We extend direct volume rendering with a unified model for generalized isosurfaces, also called interval volumes, allowing a wider spectrum of visual classification. We generalize the concept of scaleinvariant opacity\&\#8212;typical for isosurface rendering\&\#8212; to semi-transparent interval volumes. Scale-invariant rendering is independent of physical space dimensions and therefore directly facilitates the analysis of data characteristics. Our model represents sharp isosurfaces as limits of interval volumes and combines them with features of direct volume rendering. Our objective is accurate rendering, guaranteeing that all isosurfaces and interval volumes are visualized in a crack-free way with correct spatial ordering. We achieve simultaneous direct and interval volume rendering by extending preintegration and explicit peak finding with data-driven splitting of ray integration and hybrid computation in physical and data domains. Our algorithm is suitable for efficient parallel processing for interactive applications as demonstrated by our CUDA implementation.

Amershi, S, et al. 2008, "Pedagogy and Usability in Interactive Algorithm Visualizations: Designing and Evaluating Cispace." Interacting with Computers 20(1):64-96.

Interactive algorithm visualizations (AVs) are powerful tools for teaching and learning concepts that are difficult to describe with static media alone. However, while countless AVs exist, their widespread adoption by the academic community has not occurred due to usability problems and mixed results of pedagogical effectiveness reported in the AV and education literature. This paper presents our experiences designing and evaluating CIspace, a set of interactive AVs for demonstrating fundamental Artificial Intelligence algorithms. In particular, we first review related work on AVs and theories of learning. Then, from this literature, we extract and compile a taxonomy of goals for designing interactive AVs that address key pedagogical and usability limitations of existing AVs. We advocate that differentiating between goals and design features that implement these goals will help designers of AVs make more informed choices, especially considering the abundance of often conflicting and inconsistent design recommendations in the AV literature. We also describe and present the results of a range of evaluations that we have conducted on CIspace that include semi-formal usability studies, usability surveys from actual students using CIspace as a course resource, and formal user studies designed to assess the pedagogical effectiveness of CIspace in terms of both knowledge gain and user preference. Our main results show that (i) studying with our interactive AVs is at least as effective at increasing student knowledge as studying with carefully designed paper-based materials; (ii) students like using our interactive AVs more than studying with the paper-based materials; (iii) students use both our interactive $\mathrm{AVs}$ and paper-based materials in practice although they are divided when forced to choose between them; (iv) students find our interactive AVs generally easy to use and useful. From these results, we conclude that while interactive AVs may not be universally preferred by students, it is beneficial to offer a variety of learning media to students to accommodate individual learning preferences. We hope that our experiences will be informative for other developers of interactive AVs, and encourage educators to exploit these potentially powerful resources in classrooms and other learning environments.

Amirkhanov, A, et al. 2010, "Visual Optimality and Stability Analysis of 3dct Scan Positions." IEEE Transactions on Visualization and Computer Graphics 16(6):1477-86.

Industrial cone-beam X-Ray computed tomography (CT) systems often face problems due to artifacts caused by a bad placement of the specimen on the rotary plate. This paper presents a visual-analysis tool 
for CT systems, which provides a simulation-based preview and estimates artifacts and deviations of a specimen's placement using the corresponding 3D geometrical surface model as input. The presented tool identifies potentially good or bad placements of a specimen and regions of a specimen, which cause the major portion of artefacts. The tool can be used for a preliminary analysis of the specimen before CT scanning, in order to determine the optimal way of placing the object. The analysis includes: penetration lengths, placement stability and an investigation in Radon space. Novel visualization techniques are applied to the simulation data. A stability widget is presented for determining the placement parameters' robustness. The performance and the comparison of results provided by the tool compared with real world data is demonstrated using two specimens.

Amitani, S, Z Bilda, and E Edmonds. 2008, "Our Content: Generative Montage Methods for Multimedia Data." Design Studies 29(6):572-86.

The concept underlying the research reported in this paper is that of a semi-automatic system with which groups of distributed users interact, primarily by exchanging multimedia data such as short sound recordings, photographs or video clips taken with mobile phones. The generative website is a place where the public can post their own contents such as articles and videos, and then the website generates possible and meaningful sequences of that data by combining the content provided in an interesting and stimulating way. The paper describes the interaction concept, the algorithms for the generative website and an implementation. An evaluation of user experience with the prototype system is also presented. The overall aim of this research is to design and develop methods for interactive generative systems as stimulants to human creativity and it is claimed that this aim is advanced by the research reported.

Ampatzoglou, A, and I Stamelos. 2010, "Software Engineering Research for Computer Games: A Systematic Review." Information and Software Technology 52(9):888-901.

Context Currently, computer game development is one of the fastest growing industries in the worldwide economy. In addition to that, computer games are rapidly evolving in the sense that newer game versions arrive in a very short interval. Thus, software engineering techniques are needed for game development in order to achieve greater flexibility and maintainability, less cost and effort, better design, etc. In addition, games present several characteristics that differentiate their development from classical software development. Objective This study aims to assess the state of the art on research concerning software engineering for computer games and discuss possible important areas for future research. Method We employed a standard methodology for systematic literature reviews using four well known digital libraries. Results Software engineering for computer games is a research domain that has doubled its research activity during the last 5 years. The dominant research topic has proven to be requirements engineering, while topics such as software verification and maintenance have been neglected up to now. Conclusion The results of the study suggest that software engineering for computer games is a field that embraces many techniques and methods from conventional software engineering and adapts them so as to fit the specific requirements of game development. In addition to that, the study proposes the employment of more elaborate empirical methods, i.e., controlled experiments and case studies, in game software engineering research, which, have not been extensively used up to now. 
Amrani, M, and B Shariat. 2000, "Deformable Organs Modeling with Multi Layer Particle Systems." In IEEE International Conference on Information Visualization, pp. 351-56.

We describe a general methodology for reconstruction and animation of volumetric deformable objects. In the scope of a medical application, we want to simulate the motion and the form alteration of the internal anatomical organs. Multi-layer particle systems are well adapted to represent the geometry. However, several problems occur related to the structure of our model and the nature of internal forces. For this, we have introduced the necessary tools to overcome these problems and to handle the dynamic and deformable behavior of cancerous tissues and organs. Our particle system seems to be suitable to be integrated in radiation dose evaluation software, developed within the European BIOMED2-ARROW project by North Western Medical Physics team, in Manchester

Anand, MK, S Bowers, and B Lud. 2010, "Provenance Browser : Displaying and Querying Scientific Workflow Provenance Graphs." In 26th International Conference on Data Engineering (ICDE), pp. 3-6.

This demonstration presents an interactive prove- nance browser for visualizing and querying data dependency (lineage) graphs produced by scientific workflow runs. The browser allows users to explore different views of provenance as well as to express complex and recursive graph queries through a highlevel query language (QLP). Answers to QLP queries are lineage preserving in that queries return sets of lineage dependencies (denoting provenance graphs), which can be further queried and visually displayed (as graphs) in the browser. By combining provenance visualization, navigation, and query, the provenance browser can enable scientists to more easily access and explore scientific workflow provenance information.

Anderson, DE, et al. 2007, "Stl and Local Regression for Modeling Disease Surveillance Counts." In $6^{\text {th }}$ Annual Conference, International Society for Disease Surveillance.

Andrews, K. 2006, "Evaluating Information Visualisations." In AVI workshop on BEyond time and errors: novel evaluation methods for information visualization, pp. 1-5.

Andrews, K, W Putz, and A Nussbaumer. 2007, "The Hierarchical Visualisation System (Hvs)." In $11^{\text {th }}$ International Conference Information Visualization, pp. 257-62.

Numerous techniques have been developed for visualising hierarchically structured information. This paper presents a new framework for the visualisation of hierarchies called the hierarchical visualisation system (HVS). HVS is a general framework implemented in Java. It provides a synchronised, multiple view environment for visualising, exploring and managing large hierarchies. HVS reads hierarchies either from the file system or from TreeML files. Eleven hierarchy browsers have so far been implemented within HVS, including: traditional tree views, the classic Walker tree layout, information pyramids, treemaps, a hyperbolic browser, sunburst, and cone trees. In addition to being a tool to explore and manage hierarchies, HVS was also designed to provide a platform for the empirical evaluation and comparison of different hierarchy browsers. The hierarchical visualisation testing environment (HVTE) is a semi-automated testing environment built on top of HVS. HVTE is being used for a series of comparative studies of hierarchy browsers. 
Andrienko, G, and N Andrienko. 2010, "Poster : Dynamic Time Transformation for Interpreting Clusters of Trajectories with Space-Time Cube." In IEEE Symposium on Information Visualization.

Andrienko, G, et al. 2005, "Creating Instruments for Ideation: Software Approaches to Geovisualization." in Exploring Geovisualization, eds. D Jason, MM Alan and K Menno-Jan, pp. 101-25. Elsevier, Oxford.

Summary New visualization techniques are frequently demonstrated and much academic effort goes into the production of software tools to support visualization. Here, the authors of subsequent chapters in this section identify reasons why they continue to enhance and develop the instruments that they design to support the process of geovisualization, justifying their ongoing work and in doing so offering some perspectives on and solutions to the issues that they address. A number of inter-related themes arise including: advances in technology that create opportunities and generate demands for new geovisualization solutions; increasingly rich data sets and sources that drive design due to the associated potential for revealing new structures and relationships; various and novel tasks to which geovisualization is being applied associated with debate and continuing research concerning the kinds of instrument that are required to best undertake particular tasks in particular conditions; an increasingly diverse set of users who require a variety of tools, environments and systems to support ideation in its numerous forms, including those who participate in simulations of visualization when learning; changes in the available expertise that prompt the development of ideas and instruments that borrow from advances and methods in cognate disciplines such as Cognitive science, Statistics, Information Visualization, Knowledge Discovery and Datamining (KDD), Human-Computer Interaction and Scientific Visualization.

Andrienko, G, et al. 2007, "Geovisual Analytics for Spatial Decision Support: Setting the Research Agenda." International Journal of Geographical Information Science 21(8):839-57.

This article summarizes the results of the workshop on Visualization, Analytics \& Spatial Decision Support, which took place at the GIScience conference in September 2006. The discussions at the workshop and analysis of the state of the art have revealed a need in concerted cross-disciplinary efforts to achieve substantial progress in supporting space-related decision making. The size and complexity of real-life problems together with their ill-defined nature call for a true synergy between the power of computational techniques and the human capabilities to analyze, envision, reason, and deliberate. Existing methods and tools are yet far from enabling this synergy. Appropriate methods can only appear as a result of a focused research based on the achievements in the fields of geovisualization and information visualization, human-computer interaction, geographic information science, operations research, data mining and machine learning, decision science, cognitive science, and other disciplines. The name 'Geovisual Analytics for Spatial Decision Support' suggested for this new research direction emphasizes the importance of visualization and interactive visual interfaces and the link with the emerging research discipline of Visual Analytics. This article, as well as the whole special issue, is meant to attract the attention of scientists with relevant expertise and interests to the major challenges requiring multidisciplinary efforts and to promote the establishment of a dedicated research community where an appropriate range of competences is combined with an appropriate breadth of thinking.

Andrienko, G, et al. 2010, "Discovering Bits of Place Histories from People ' S Activity Traces." In IEEE Symposium on Information Visualization, pp. 59-66.

Events that happened in the past are important for understanding the ongoing processes, predicting future developments, and making informed decisions. Significant and/or interesting events tend to attract 
many people. Some people leave traces of their attendance in the form of computer-processable data, such as records in the databases of mobile phone operators or photos on photo sharing web sites. We developed a suite of visual analytics methods for reconstructing past events from these activity traces. Our tools combine geocomputations, interactive geovisualizations and statistical methods to enable integrated analysis of the spatial, temporal, and thematic components of the data, including numeric attributes and texts. We demonstrate the utility of our approach on two large real data sets, mobile phone calls in Milano during 9 days and flickr photos made on British Isles during 5 years.

Andrienko, N, G Andrienko, and P Gatalsky. 2003, "Exploratory Spatio-Temporal Visualization: An Analytical Review." Journal of Visual Languages \& Computing 14(6):503-41.

Andrienko, N, G Andrienko, and P Gatalsky. 2005, "Impact of Data and Task Characteristics on Design of Spatio-Temporal Data Visualization Tools." in Exploring Geovisualization, eds. D Jason, MM Alan and K Menno-Jan, pp. 201-22. Elsevier, Oxford.

Summary It is widely recognized that data visualization may be a powerful methodology for exploratory analysis. In order to fulfill this claim, visualization software must be carefully designed taking into account two principal aspects: characteristics of the data to be visualized and the exploratory tasks to be supported. The tasks that may potentially arise in data exploration are, in their turn, dependent on the data. In the chapter, we present visualization software tools for three different types of spatio-temporal data developed using a task-driven approach to design. We demonstrate that different exploratory tasks may be anticipated in these three cases and that different techniques are required to properly support exploration of the data. Prior to the consideration of the examples, we briefly describe the typologies of data and tasks we use in our work.

Ankerst, M, S Berchtold, and DA Keim. 1998, "Similarity Clustering of Dimensions for an Enhanced Visualization of Multidimensional Data." In IEEE Symposium on Information Visualization, pp. 5260,153 .

The order and arrangement of dimensions (variates) is crucial for the effectiveness of a large number of visualization techniques such as parallel coordinates, scatterplots, recursive pattern, and many others. We describe a systematic approach to arrange the dimensions according to their similarity. The basic idea is to rearrange the data dimensions such that dimensions showing a similar behavior are positioned next to each other. For the similarity clustering of dimensions, we need to define similarity measures which determine the partial or global similarity of dimensions. We then consider the problem of finding an optimal one- or two-dimensional arrangement of the dimensions based on their similarity. Theoretical considerations show that both, the one- and the two-dimensional arrangement problem are surprisingly hard problems, i.e., they are NP complete. Our solution of the problem is therefore based on heuristic algorithms. An empirical evaluation using a number of different visualization techniques shows the high impact of our similarity clustering of dimensions on the visualization results

Anselma, L, et al. 2006, "Towards a Comprehensive Treatment of Repetitions, Periodicity and Temporal Constraints in Clinical Guidelines." Artificial Intelligence in Medicine 38(2):171-95.

SummaryObjective In this paper, we define a principled approach to represent temporal constraints in clinical guidelines and to reason (i.e., perform inferences in the form of constraint propagation) on them. We consider different types of constraints, including composite and repeated actions, and propose 
different types of temporal functionalities (e.g., temporal consistency checking).Background Constraints about actions, durations, delays and periodic repetitions of actions are an intrinsic part of most clinical guidelines. Although several approaches provide expressive temporal formalisms, only few of them deal with the related temporal reasoning issues.Methodology We first propose a temporal representation formalism and two temporal reasoning algorithms. Then, we consider the trade-off between the expressiveness of the formalism and the computational complexity of the algorithms, in order to devise a correct, complete and tractable approach. Finally, we show how the algorithms can be exploited to provide clinical guideline systems with different types of temporal facilities.Results Our approach offers several advantages. During the guideline acquisition phase, it enables to represent temporal constraints, and to check their consistency. In the execution phase, it checks the consistency between the execution times of the actions and the constraints in the guidelines, and provides query answering and simulation facilities.

Anslow, C, E Tempero, and R Biddle. 2010, "User Evaluation of Polymetric Views Using a Large Visualization Wall." In 5th ACM Symposium on Software Visualization (SOFTVIS), pp. 25-34.

Apted, T, et al. 2003, "Visualisation of Ontological Inferences for User Control of Personal Web Agents." In International Conference on Information Visualization, pp. 306-11.

We describe a visualisation tool, VlUM, designed to support users in scrutinising models of their interests, preferences and knowledge. We also describe MECUREO, a tool for building lightweight ontologies from online dictionaries. It enables a user to see a model describing both their interests and additional analogically inferred interests. We report a small qualitative evaluation of this combined system. This indicates that users were able to use the system to explore models of two hypothetical users, making use of varying levels of ontological inference.

Aragon, CR, et al. 2009, "Using Visual Analytics to Develop Situation Awareness in Astrophysics." Information Visualization 8(1):30-41.

We present a novel collaborative visual analytics application for cognitively overloaded users in the astrophysics domain. The system was developed for scientists who need to analyze heterogeneous, complex data under time pressure, and make predictions and time-critical decisions rapidly and correctly under a constant influx of changing data. The Sunfall Data Taking system utilizes several novel visualization and analysis techniques to enable a team of geographically distributed domain specialists to effectively and remotely maneuver a custom-built instrument under challenging operational conditions. Sunfall Data Taking has been in production use for 2 years by a major international astrophysics collaboration (the largest data volume supernova search currently in operation), and has substantially improved the operational efficiency of its users. We describe the system design process by an interdisciplinary team, the system architecture and the results of an informal usability evaluation of the production system by domain experts in the context of Endsley's three levels of situation awareness. Information Visualization (2009) 8, 30-41. doi: 10.1057/ivs.2008.30 
Ardito, C, et al. 2006, "Systematic Evaluation of E-Learning Systems: An Experimental Validation." In Proceedings of the 4th Nordic conference on Human-computer interaction: changing roles, pp. 195-202.

Arkoudas, K, and S Bringsjord. 2009, "Vivid: A Framework for Heterogeneous Problem Solving." Artificial Intelligence 173(15):1367-405.

We introduce Vivid, a domain-independent framework for mechanized heterogeneous reasoning that combines diagrammatic and symbolic representation and inference. The framework is presented in the form of a family of denotational proof languages (DPLs). We present novel formal structures, called named system states, that are specifically designed for modeling potentially underdetermined diagrams. These structures allow us to deal with incomplete information, a pervasive feature of heterogeneous problem solving. We introduce a notion of attribute interpretations that enables us to interpret first-order relational signatures into named system states, and develop a formal semantic framework based on 3valued logic. We extend the assumption-base semantics of DPLs to accommodate diagrammatic reasoning by introducing general inference mechanisms for the valid extraction of information from diagrams, and for the incorporation of sentential information into diagrams. A rigorous big-step operational semantics is given, on the basis of which we prove that the framework is sound. We present examples of particular instances of Vivid in order to solve a series of problems, and discuss related work.

Arning, K, and M Ziefle. 2007, "Understanding Age Differences in Pda Acceptance and Performance." Computers in Human Behavior 23(6):2904-27.

The present study addresses two basic determinants of technology utilization: the attitude towards a certain technology and the performance when using it. According to the technology acceptance model (TAM), perceived ease of use and usefulness are assumed to be strong determinants of the actual and successful utilization of technology. However, the relationship between the acceptance of technical devices and their successful utilization (i.e., performance) is not completely understood. In this study, users' attitudes towards technology and their performance when interacting with a computer simulated PDA device were examined. Moreover, the moderating role of individual variables like age, gender, subjective technical confidence, and computer expertise in the relationship between technical performance and acceptance was analyzed. The results showed significant associations between performance and TAM factors. However, this interrelation was much stronger for the older group, especially between performance and the ease of use. The factors computer expertise and technical selfconfidence played a minor role. Gender effects on technical self-confidence and TAM factors were identified, although they did not affect performance. Future research should focus on training formats for the older age group, which facilitate a successful interaction with technical devices.

Astefanoaie, A, et al. 2010, "Visual Analytics of a Pandemic Spread." In IEEE Symposium on Information Visualization, pp. 277-78.

Auer, T, et al. 2010, in press, "Herbariaviz: A Web-Based Client-Server Interface for Mapping and Exploring Flora Observation Data." Ecological Informatics.

The potential for physical flora collections to support scientific research is being enhanced by rapid development of digital databases that represent characteristics of the physical specimens held in those collections and make this information available remotely. One example is the unified database of California flora observations from the Consortium of California Herbaria that was developed to support 
the exploration of plant diversity patterns, distribution ranges of species, and vegetation associations for specimens held in physical collections. Many of the records in the herbaria database, and in complementary databases elsewhere, are geo-referenced; but, current web tools for accessing the data do not take advantage of that georeferencing. In this paper, we report on development and implementation of a web-based client-server map interface to facilitate open mapping and exploration of the dataset. Three research objectives were addressed: (1) develop a method for efficient web-map client-server interaction involving large volumes of spatiotemporal point data, (2) develop a symbology and symbol scaling method for representing those spatial-temporal data in the client, and (3) develop an interface for clientserver interactions and data exploration. With a focus on cartographically-sound visualization and userfriendly interaction, we introduce HerbariaViz, a web mapping application that provides space-timespecies data query responses efficiently. Following a discussion of relevant literature, we present opensource methods for aggregating point data spatially and temporally, outline our approach to sound cartographic representations of those data, and detail the design of a client interface for making requests and mapping responses. A focus group session involving domain experts was performed to provide user evaluation of the application. In our discussion, we present potential avenues of future work, including: facilitating query response comparisons, handling incomplete and inaccurate data, and generalizing the method presented here.

Augerat, P, C Martin, and B Stein. 2002, "Scalable Monitoring and Configuration Tools for Grids and Clusters." In 10th Euromicro Workshop on Parallet, Distributed and Network-based Processing, pp. 14753.

We present the Ka-admin project that addresses the problem of collecting, visualizing and feeding back any grid information, trace or snapshot, compliant to an XML-like model. Real use includes performance analysis of parallel applications and cluster administration. Ka-admin includes a generic "filter" module that processes monitored data independently of what they represent. Filters can remove, aggregate and transform data or pass them to external applications. The end user is responsible for activating the filters from within an interactive graphical interface. This allows him to focus on important information. We also present a "scatter/gather" module that allows efficient collection and distribution of data and commands in a large cluster. Early work on "MPI/threads" applications and system monitoring tools proved that the combination of both modules matches the objective of a scalable visualization of large data sets

Augusto, JC. 2005, "Temporal Reasoning for Decision Support in Medicine." Artificial Intelligence in Medicine 33(1):1-24.

SummaryObjective: Handling time-related concepts is essential in medicine. During diagnosis it can make a substantial difference to know the temporal order in which some symptoms occurred or for how long they lasted. During prognosis the potential evolutions of a disease are conceived as a description of events unfolding in time. In therapy planning the different steps of treatment must be applied in a precise order, with a given frequency and for a certain span of time in order to be effective. This article offers a survey on the use of temporal reasoning for decision support-related tasks in medicine.Material and methods: Key publications of the area, mainly circumscribed to the latest two decades, are reviewed and classified according to three important stages of patient treatment requiring decision support: diagnosis, prognosis and therapy planning/management. Other complementary publications, like those on timecentered information storage and retrieval, are also considered as they provide valuable support to the above mentioned three stages.Results: Key areas are highlighted and used to organize the latest 
contributions. The survey of previous research is followed by an analysis of what can still be improved and what is needed to make the next generation of decision support systems for medicine more effective.Conclusions: It can be observed that although the area has been considerably developed, there are still areas where more research is needed to make time-based systems of widespread use in decision support-related areas of medicine. Several suggestions for further exploration are proposed as a result of the survey.

Aurisicchio, M, et al. 2008, "How to Evaluate Reading and Interpretation of Differently Structured Engineering Design Rationales." Artificial Intelligence for Engineering Design, Analysis and Manufacturing: AIEDAM 22(4):345-58.

Documented engineering design rationale has the potential to become a key source of information about past designs. Ease of comprehension of design rationale might play a crucial role in ensuring that the full potential of documented information is realized and that the effort and time necessary to capture design rationale pay off. This research proposes an empirical methodology for evaluating how structuring design rationale and supplying it with visual nontextual cues influences reading and interpretation. The study compares reading and interpretation of technical documentation presented in different formats to engineering graduate trainees in the aerospace industry. Copyright (C) 2008 Cambridge University Press.

Azuma, S, I Fujishiro, and H Horii. 2006, "Hierarchical Causality Explorer: Making Complemental Use of $3 \mathrm{~d} / 2 \mathrm{~d}$ Visualizations - Art. No. 60600h." In Conference on Visualization and Data Analysis.

Hierarchical causality relationships reside ubiquitously in the reality. Since the relationships take intricate forms with two kinds of links - hierarchical abstraction and causal association. there exists no single visualization style that allows the user to comprehend them effectively. This paper introduces a novel information visualization framework which can change existing 3D and 2D display styles interactively according to the user's visual analysis demands. The two visualization styles play a complementary role, and the change in the style relies oil morphing so as to maintain the user's cognitive map. Based on this framework, we have developed a general-purpose prototype system, which provides the user with an enriched set of functions not only for supporting fundamental information seeking, but bridging analytic gaps to accomplishing high-level analytic tasks such as knowledge discovery and decision making. The effectiveness of the system is illustrated with an application to the analysis of a nuclear-hazard cover-up problem.

Baddeley, B, et al. 2008, "From Desktop to Field: Deploying Visual Incident Analysis for Law Enforcement." In IEEE Conference on Technologies for Homeland Security, pp. 209-14.

We present a prototype mobile application environment for law enforcement users to assist them in daily operations. This system supports the collection of real-time observations and allows users to quickly share their findings with team members. Mobile tools for law enforcement introduce safety and other operational constraints that must be considered in developing appropriate user interfaces. Our environment attempts to address these challenges and provide tools to increase information sharing among officers and, expedite form-filling and evidence collection. Moreover, real-time location tracking and mapping enables mobile users to view the locations of team members and to push data (such as fieldcollected images, video, or text) to them. 
Bailey, BP, CW Busbey, and ST Iqbal. 2007, "Taprav: An Interactive Analysis Tool for Exploring Workload Aligned to Models of Task Execution." Interacting with Computers 19(3):314-29.

Pupillary response is a valid indicator of mental workload and is being increasingly leveraged to identify lower cost moments for interruption, evaluate complex interfaces, and develop further understanding of psychological processes. Existing tools are not sufficient for analyzing this type of data, as it typically needs to be analyzed in relation to the corresponding task's execution. To address this emerging need, we have developed a new interactive analysis tool, TAPRAV. The primary components of the tool include; (i) a visualization of pupillary response aligned to the corresponding model of task execution, useful for exploring relationships between these two data sources; (ii) an interactive overview + detail metaphor, enabling rapid inspection of details while maintaining global context; (iii) synchronized playback of the video of the user's screen interaction, providing awareness of the state of the task; and (iv) interaction supporting discovery driven analysis. Results from a user study showed that users are able to efficiently interact with the tool to analyze relationships between pupillary response and task execution. The primary contribution of our tool is that it demonstrates an effective visualization and interaction design for rapidly exploring pupillary response in relation to models of task execution, thereby reducing the analysis effort.

Bailin, A, and A Peña. 2007, "Online Library Tutorials, Narratives, and Scripts." The Journal of Academic Librarianship 33(1):106-17.

In this article, we examine some issues that relate to effective presentation in online library tutorials. In so doing, we look at the differences between paper and online media in order to highlight distinctive properties of online presentations of information. We argue that one way to handle the particular challenges online tutorials pose is to use the concept of a script as developed by Schank and Abelson. We discuss how the concept of scripts has been incorporated in an online library tutorial developed at the Newman Library, Baruch College.

Baker, J, DR Jones, and J Burkman. 2009, "Using Visual Representations of Data to Enhance Sensemaking in Data Exploration Tasks." Journal of the Association of Information Systems 10(7):53359.

This paper explains how visual representations of data enable individual sensemaking in data exploration tasks. We build upon theories of human perception and cognition, including Cognitive Fit Theory, to explain what aspects of visual representations facilitate sensemaking for the viewer. We make three primary contributions. First, we give a general characterization of visual representations that would be used for data exploration tasks. These representations consist of a scene, objects within the scene, and the characteristics of those objects. Second, we extend Cognitive Fit Theory into the data exploration task domain. We explain that the data exploration task has a number of spatial subtasks including observing data points, looking for patterns or outliers, making inferences, comparing observed facts or patterns to one's own knowledge, generating hypotheses about the data, and drawing analogies from the context being observed to another context. Third, we offer a set of theoretical propositions about how visual representations of data can serve the sensemaking goal. Specifically, visual representations best facilitate sensemaking in data exploration tasks when they (1) support the four basic human visual perceptual approaches of association, differentiation, ordered perception, and quantitative perception, (2) have strong Gestalt properties, (3) are consistent with the viewer's stored knowledge, and (4) support analogical 
reasoning. We propose that visual representations should possess several of these four aspects to make them well-suited for the task of data exploration. (C) 2009, by the Association for Information Systems.

Baker, RSJd. 2010, "Data Mining." in International Encyclopedia of Education, eds. P Penelope, B Eva and M Barry, pp. 112-18. Elsevier, Oxford.

Educational data mining is the area of scientific inquiry centered around the development of methods for making discoveries within the unique kinds of data that come from educational settings, and using those methods to better understand students and the settings which they learn in. The recent advent of public educational data repositories has made it feasible for researchers to investigate a wide variety of scientific questions using data mining. In this article, five categories of educational data mining methods are discussed, as well as the key applications for which educational data mining methods have been used.

Balakrishnan, AD, SR Fussell, and S Kiesler. 2008, "Do Visualizations Improve Synchronous Remote Collaboration?" In 26th Annual CHI Conference on Human Factors in Computing Systems, pp. 1227-36.

Information visualizations can improve collaborative problem solving, but this improvement may depend on whether visualizations promote communication. In an experiment on the effect of network visualizations, remote pairs worked synchronously to identify a serial killer. They discussed disparate evidence distributed across the pair using IM. Four conditions, respectively, offered (a) spreadsheet only (controls), (b) individual unshared visualizations, (c) view-only shared visualizations, and (d) a fullaccess shared visualization of all evidence. We examined collaborative performance, use of the visualization tool, and communication as a function of condition. All visualization conditions improved remote collaborators' performance over the control condition. Full access to a shared visualization best facilitated remote collaboration by encouraging tool use and fostering discussion between the partners. Shared visualization without full access impaired performance somewhat and made communication even more vital to identifying the serial killer. This study provides direct evidence of visualization tool features and partner behavior that promote collaboration.

Balfanz, D, et al. 2003, "Mobile Situation-Awareness within the Project Map." Computers \& Graphics 27(6):893-98.

Mobile devices as PDAs evolve rapidly from digital calendars and address books to hosts of more complex functionality. Mobile access to business information such as customer, product or project databases is seen as one of the cutting edge IT solutions for improving productivity and customer satisfaction. However, mobility and scaled-down mobile technology lead to specific limitations in contrast to the usage of desktop computers. These restrictions are absolutely crucial to consider for the development of usable mobile applications. With reference to the project map--Multimedia Arbeitsplatz der Zukunft (Multimedia Workplace of the Future), this article outlines our main approach to situationaware support for mobile workers in response to mobile restrictions. We are pointing out focal components and sketch parts of the map architecture, in particular the prototypical application "BuddyAlert". 
Ball, R, and C North. 2007, "Realizing Embodied Interaction for Visual Analytics through Large Displays." Computers \& Graphics 31(3):380-400.

Visual analysts are engaged with the arduous task of scrutinizing increasingly larger data sets. Where conventional desktop displays are reaching their limits in terms of performance efficiency with large data sets, analysts can turn to larger displays. In a world of extensive multi-scale data sets, large highresolution displays have the potential to show both more overview and detail for a given data set than their smaller counterparts. In addition, people are able to use their embodied resources, such as spatial memory, proprioception, and optical flow to help them maintain orientation and improve performance times on analytic tasks when using larger displays. This paper looks at how physical navigation, physically interacting with large scale visualizations (e.g., walking, crouching, moving the head), affects user performance times on analytic tasks, such as finding patterns in geospatial data. The paper extends the space-scale diagram to take into account physical navigation and explains the theoretical repercussions. The paper then explains an empirical study performed for the purpose of further understanding how physical and virtual navigation affect performance times of tasks on varying size displays. In general, we found that large displays can decrease performance time of basic visualization tasks by more than ten times. In addition, we found overwhelming evidence from the empirical study that participants preferred physical navigation over virtual navigation (e.g., mouse interaction). Specifically, we found that for a number of tasks $100 \%$ of the participants chose to physically navigate--physically moving to different areas on the display instead of using virtual navigation to manipulate the view on the display.

Banbury, S, and S Tremblay. 2004. A Cognitive Approach to Situation Awareness: Theory and Application. Ashgate Publishing Company, Burlington, VT.

Bandte, O. 2004, "Visualizing Information in an Interactive Evolutionary Design Process." In Congress on Evolutionary Computation, pp. 691-98.

As interactive evolutionary computation is starting to penetrate scientific fields that otherwise rely heavily on numerical fitness evaluation, very little emphasis has been put on communicating the relevant information for decision making to the human during the interactive loop. This paper raises the issue of information presentation as a scientific question for interactive evolutionary applications that include automatic fitness evaluation and presents an example for how relevant information display can be for real world problems.

Bandura, A. 2001, "Social Cognitive Theory: An Agentic Perspective." Annual Review of Psychology 52:1-26.

The capacity to exercise control over the nature and quality of one's life is the essence of humanness. Human agency is characterized by a number of core features that operate through phenomenal and functional consciousness. These include the temporal extension of agency through intentionality and forethought, self-regulation by self-reactive influence, and self-reflectiveness about one's capabilities, quality of functioning, and the meaning and purpose of one's life pursuits. Personal agency operates within a broad network of sociostructural influences. In these agentic transactions, people are producers as well as products of social systems. Social cognitive theory distinguishes among three modes of agency: direct personal agency, proxy agency that relies on others to act on one's behest to secure desired outcomes, and collective agency exercised through socially coordinative and interdependent effort. 
Growing transnational embeddedness and interdependence are placing a premium on collective efficacy to exercise control over personal destinies and national life.

Barbosa, PT, and LZ Granville. 2010, "Interactive Snmp Traffic Analysis through Information Visualization." In IEEE Network Operations and Management Symposium, pp. 73-79.

The network management area deals with large amounts of data. Some of its protocols and techniques are not completely understood when it comes to usage patterns and most used features. The understanding of such characteristics is a challenging process, due to the massive data amount involved. This process can be supported by information visualization techniques. These consist in visual representations of data that make use of the unique properties of the human visual system to make insights about it in a more intuitive and effective way. In this context, interactivity has proved itself to be one of the main factors involved in providing such intuitiveness and effectiveness, specially in analysis of large datasets. Nevertheless, few interaction possibilities are available in current network management traffic visualization systems. In this paper we present a set of interactive information visualization techniques adapted to visualize SNMP trace files. We used an insight-based evaluation to show how the presented techniques can aid on the insight achievement process.

Bar-Ilan, J. 2008, "Informetrics at the Beginning of the 21st Century--a Review." Journal of Informetrics 2(1):1-52.

This paper reviews developments in informetrics between 2000 and 2006. At the beginning of the 21 st century we witness considerable growth in webometrics, mapping and visualization and open access. A new topic is comparison between citation databases, as a result of the introduction of two new citation databases Scopus and Google Scholar. There is renewed interest in indicators as a result of the introduction of the h-index. Traditional topics like citation analysis and informetric theory also continue to develop. The impact factor debate, especially outside the informetric literature continues to thrive. Ranked lists (of journal, highly cited papers or of educational institutions) are of great public interest.

Barlowe, S, et al. 2008, "Multivariate Visual Explanation for High Dimensional Datasets." In IEEE Symposium on Visual Analytics.

Understanding multivariate relationships is an important task in multivariate data analysis. Unfortunately, existing multivariate visualization systems lose effectiveness when analyzing relationships among variables that span more than a few dimensions. We present a novel multivariate visual explanation approach that helps users interactively discover multivariate relationships among a large number of dimensions by integrating automatic numerical differentiation techniques and multidimensional visualization techniques. The result is an efficient workflow for multivariate analysis model construction, interactive dimension reduction, and multivariate knowledge discovery leveraging both automatic multivariate analysis and interactive multivariate data visual exploration. Case studies and a formal user study with a real dataset illustrate the effectiveness of this approach.

Bar-On, D, and RE Oxman. 2002, "Context over Content: Icpd, a Conceptual Schema for the Building Technology Domain." Automation in Construction 11(4):467-93.

Computational systems in the field of building technology are, at present, mainly employed as electronic catalogs for the retrieval of technological information (content providers). However, the 
massive amount of information associated with building design demands explicit knowledge about the way various information pieces relate one to another in order to be able to achieve an intelligent use of those resources through the design process. This gives rise to a strong need for computational systems that help not only to retrieve information (design content) but also to explain the relationships between the elements of technological information (design context). The research presented in this paper has developed a conceptual model for the representation of knowledge in building technology based on specific context related to the field. The model employed an associative formalism reflecting the logical connections between pertinent information units used in the technological design process. The model is termed ICPD which stands for: Issue, Concept, Pattern and Detail. This model is to be employed in implementing a computational system to support design in building technology.

Barrera, D, and PC van Oorschot. 2009, "Security Visualization Tools and Ipv6 Addresses." In $6^{\text {th }}$ International Workshop on Visualization for Cyber Security, pp. 21-26.

Visualization is used by security analysts to help detect patterns and trends in large volumes of network traffic data. With IPv6 slowly being deployed around the world, network intruders are beginning to adapt their tools and techniques to work over IPv6 (vs. IPv4). Many tools for visualizing network activity, while useful for detecting large scale attacks and network behavior anomalies still only support IPv4. In this paper, we explore the current state of IPv6 support in some popular security visualization tools and identify the roadblocks preventing those tools from supporting the new protocol. We propose a filtering technique that helps reduce the occlusion of IPv6 sources on graphs. We also suggest using treemaps for visually representing the vast space of remote addresses in IPv6.

Bartlett, FC. 1932. Remembering: A Study in Experimental and Social Psychology. Macmillan Co., New York, NY.

Bartram, L. 1997, "Perceptual and Interpretative Properties of Motion for Information Visualization." In Workshop on New Paradigms in Information Visualization and Manipulation, pp. 3-7.

Bartram, L, C Ware, and T Calvert. 2003, "Moticons:: Detection, Distraction and Task." International Journal of Human-Computer Studies 58(5):515-45.

In this paper, we describe an empirical investigation of the utility of several perceptual properties of motion in information-dense displays applied to notification. Notification relates to awareness and how dynamic information is communicated from the system to the user. Key to a notification technique is how easily the notification is detected and identified. Our initial studies show that icons with simple motions, termed moticons, are effective coding techniques for notification and in fact are often better detected and identified than colour and shape codes, especially in the periphery. A subsequent experiment compared the detection and distraction effects of different motion types in several task conditions. Our results reveal how different attributes of motion contribute to detection, identification and distraction and provide initial guidelines on how motion codes can be designed to support awareness in information-rich interfaces while minimizing unwanted side effects of distraction and irritation. 
Basole, R, et al. 2010, "Poster : Visualizing Converging Business Ecosystems for Competitive Intelligence." In IEEE Symposium on Information Visualization, pp. 1-2.

Bass, WM, and RD Blanchard. 2011, "Examining Geographic Visualization as a Technique for Individual Risk Assessment." Applied Geography 31(1):53-63.

This research examined the extent to which geographic visualization might serve as a technique for assessing and understanding levels of personal risk. An exercise was created, consisting of a series of five animations, representing five historical flood events in flood-prone central Texas and displayed on an Internet site along with a survey questionnaire. Three questions guided this research: 1) To what extent can individuals correctly rank levels of intensities among five historical rainfall events, and, therefore levels of risk after viewing visualizations; 2) Does professional training and experience in a hazardsrelated field prove to be an advantage for correctly identifying and ranking levels of risk among the rainfall events after viewing visualizations; 3 ) Is prior experience with a flood, or any other hazard occurrence a factor in whether individuals can correctly assess levels of risk in visualizations depicting rainfall events? Our study demonstrated that computer-interested individuals are willing and able to access website information related to historical flood events, and interact with that website in viewing, interpreting and ranking computer animations of featured events; and, for the most part, regardless of prior experience, or workplace training, can, more or less, distinguish between levels of intensity of events. However, due to the fairly recent introduction of geographic visualization in hazards research, we call for more work in this area, and have offered an extensive list of research questions for assessing the viability of this technique for more accurate risk assessment and management at the individual level.

Bates-Brkljac, N. 2007, "Investigating Perceptual Responses and Shared Understanding of Architectural Design Ideas When Communicated through Different Forms of Visual Representations." In $11^{\text {th }}$ International Conference on Information Visualization, pp. 348-53.

Research to date has demonstrated the apparent differences how architects, as 'experts' and members of the public as 'non-experts' perceive and understand visual representations. However, the studies that compare architect' responses with other professionals in the field are rare. This paper reports the findings from an Economic and Social science Research Council (ESRC) funded study that investigated peoples' perceptual and cognitive responses to traditional and computer generated forms of architectural representations. The study and analysis provide an insight into factors that are relevant to the communication, shared understanding of design ideas and knowledge exchange amongst the participants in the process. Findings indicate strong resemblance between professionals' and 'lay' peoples' responses. Architects' responses vary depending on the age and length of work experience. The analysis of groups' accounts of how and why they prefer and value the forms they do shows the fracturing of communication and shared understanding amongst participants.

Baur, D, et al. 2010, "The Streams of Our Lives: Visualizing Listening Histories in Context." IEEE Transactions on Visualization and Computer Graphics 16(6):1119-28.

The choices we take when listening to music are expressions of our personal taste and character. Storing and accessing our listening histories is trivial due to services like Last.fm, but learning from them and understanding them is not. Existing solutions operate at a very abstract level and only produce statistics. By applying techniques from information visualization to this problem, we were able to provide average people with a detailed and powerful tool for accessing their own musical past. LastHistory is an 
interactive visualization for displaying music listening histories, along with contextual information from personal photos and calendar entries. Its two main user tasks are (1) analysis, with an emphasis on temporal patterns and hypotheses related to musical genre and sequences, and (2) reminiscing, where listening histories and context represent part of one's past. In this design study paper we give an overview of the field of music listening histories and explain their unique characteristics as a type of personal data. We then describe the design rationale, data and view transformations of LastHistory and present the results from both a lab- and a large-scale online study. We also put listening histories in contrast to other lifelogging data. The resonant and enthusiastic feedback that we received from average users shows a need for making their personal data accessible. We hope to stimulate such developments through this research.

Beale, R. 2007, "Supporting Serendipity: Using Ambient Intelligence to Augment User Exploration for Data Mining and Web Browsing." International Journal of Human-Computer Studies 65(5):421-33.

Serendipity is the making of fortunate discoveries by accident, and is one of the cornerstones of scientific progress. In today's world of digital data and media, there is now a vast quantity of material that we could potentially encounter, and so there is an increased opportunity of being able to discover interesting things. However, the availability of material does not imply that we will be able to actually find it; the sheer quantity of data mitigates against us being able to discover the interesting nuggets. This paper explores approaches we have taken to support users in their search for interesting and relevant information. The primary concept is the principle that it is more useful to augment user skills in information foraging than it is to try and replace them. We have taken a variety of artificial intelligence, statistical, and visualisation techniques, and combined them with careful design approaches to provide supportive systems that monitor user actions, garner additional information from their surrounding environment and use this enhanced understanding to offer supplemental information that aids the user in their interaction with the system. We present two different systems that have been designed and developed according to these principles. The first system is a data mining system that allows interactive exploration of the data, allowing the user to pose different questions and understand information at different levels of detail. The second supports information foraging of a different sort, aiming to augment users browsing habits in order to help them surf the internet more effectively. Both use ambient intelligence techniques to provide a richer context for the interaction and to help guide it in more effective ways: both have the user as the focal point of the interaction, in control of an iterative exploratory process, working in indirect collaboration with the artificial intelligence components. Each of these systems contains some important concepts of their own: the data mining system has a symbolic genetic algorithm which can be tuned in novel ways to aid knowledge discovery, and which reports results in a user-comprehensible format. The visualisation system supports high-dimensional data, dynamically organised in a three-dimensional space and grouped by similarity. The notions of similarity are further discussed in the internet browsing system, in which an approach to measuring similarity between web pages and a user's interests is presented. We present details of both systems and evaluate their effectiveness.

Beatriz. 2006, "An Introductory Course on Human-Computer Interaction: Programme, Bibliography, Practical Classes and Assignments." Computers \& Graphics 30(4):658-68.

As computing curricula have evolved, human-computer interaction has gradually become part of many of them, and the recent ACM/IEEE report on the core of computing science and engineering includes human-computer interaction as one of the fundamental sub-areas that should be addressed. 
However, both technology and human-computer interaction are developing rapidly, thus a continuous effort is needed to maintain a programme, bibliography, and a set of practical assignments up to date and adapted to the current technology. This paper briefly presents an introductory course on human-computer interaction offered to electrical and computer engineering students at the University of Aveiro.

Beck, F, and S Diehl. 2010, "Visual Comparison of Software Architectures." In 5th ACM Symposium on Software Visualization (SOFTVIS), pp. 183-92.

Beck, M. 2010, "Towards Automated Analysis and Visualization of Distributed Software Systems." In 5th ACM Symposium on Software Visualization (SOFTVIS), pp. 213-14.

Bederson, BB. 2003, "Fisheye Menus." in The Craft of Information Visualization, Readings and Reflections, eds. BB Benjamin and S Ben, pp. 299-307. Morgan Kaufmann, San Francisco.

Summary We introduce "fisheye menus" which apply traditional fisheye graphical visualization techniques to linear menus. This provides for an efficient mechanism to select items from long menus, which are becoming more common as menus are used to select data items in, for example, e-commerce applications. Fisheye menus dynamically change the size of menu items to provide a focus area around the mouse pointer. This makes it possible to present the entire menu on a single screen without requiring buttons, scrollbars, or hierarchies.

Bederson, BB, and A Boltman. 2003, "Does Animation Help Users Build Mental Maps of Spatial Information?" in The Craft of Information Visualization, Readings and Reflections, eds. BB Benjamin and S Ben, pp. 87-94. Morgan Kaufmann, San Francisco.

Summary We examine how animating a viewpoint change in a spatial information system affects a user's ability to build a mental map of the information in the space. We found that animation improves users' ability to reconstruct the information space, with no penalty on task performance time. We believe that this study provides strong evidence for adding animated transitions in many applications with fixed spatial data where the user navigates around the data space.

Bederson, BB, B Shneiderman, and M Wattenberg. 2003, "Ordered and Quantum Treemaps: Making Effective Use of 2d Space to Display Hierarchies." in The Craft of Information Visualization, Readings and Reflections, eds. BB Benjamin and S Ben, pp. 257-78. Morgan Kaufmann, San Francisco.

Bederson, BB, RS Wallace, and EL Schwartz. 1992, "A Miniaturized Active Vision System." In $11^{\text {th }}$ IAPR International Conference on Pattern Recognition, pp. 58-61.

The authors have developed a prototype miniaturized active vision system whose sensor architecture is based on a logarithmically structured space-variant pixel geometry. This system integrates a CCD sensor, miniature pan-tilt actuator, controller, general purpose processors and display. Due to the ability of space-variant sensors to cover large work-spaces yet provide high acuity with an extremely small number of pixels, space-variant active vision system architectures provide the potential for radical reductions in system size and cost. The authors describe a prototype space-variant active vision system which performs tasks such as moving object tracking and functions as a video telephone. The potential application domains for systems of this type include vision systems for mobile robots and robot manipulators, traffic monitoring systems, security and surveillance, and consumer video communications 
Bendich, P, H Edelsbrunner, and M Kerber. 2010, "Computing Robustness and Persistence for Images." IEEE Transactions on Visualization and Computer Graphics 16(6):1251-60.

We are interested in 3-dimensional images given as arrays of voxels with intensity values. Extending these values to acontinuous function, we study the robustness of homology classes in its level and interlevel sets, that is, the amount of perturbationneeded to destroy these classes. The structure of the homology classes and their robustness, over all level and interlevel sets, can bevisualized by a triangular diagram of dots obtained by computing the extended persistence of the function. We give a fast hierarchicalalgorithm using the dual complexes of oct-tree approximations of the function. In addition, we show that for balanced oct-trees, thedual complexes are geometrically realized in $\$ \mathrm{R}^{\wedge} 3 \$$ and can thus be used to construct level and interlevel sets. We apply these tools tostudy 3-dimensional images of plant root systems.

Benoît, G. 2005, "Application of Markov Chains in an Interactive Information Retrieval System." Information Processing \& Management 41(4):843-57.

Benson, JR, and F Wim. 2010, "Poster : Using Orthographic Projection and Animation to Convey Treemap Structure." In International Conference on Information Visualization (INFOVIS).

Berendt, B, B Krause, and S Kolbe-Nusser. 2010, "Intelligent Scientific Authoring Tools: Interactive Data Mining for Constructive Uses of Citation Networks." Information Processing \& Management 46(1):1-10.

Many powerful methods and tools exist for extracting meaning from scientific publications, their texts, and their citation links. However, existing proposals often neglect a fundamental aspect of learning: that understanding and learning require an active and constructive exploration of a domain. In this paper, we describe a new method and a tool that use data mining and interactivity to turn the typical search and retrieve dialogue, in which the user asks questions and a system gives answers, into a dialogue that also involves sense-making, in which the user has to become active by constructing a bibliography and a domain model of the search term(s). This model starts from an automatically generated and annotated clustering solution that is iteratively modified by users. The tool is part of an integrated authoring system covering all phases from search through reading and sense-making to writing. Two evaluation studies demonstrate the usability of this interactive and constructive approach, and they show that clusters and groups represent identifiable sub-topics.

Berg, AC, and SC Justo. 2003, "Aging of Orbicularis Muscle in Virtual Human Faces." In 7th International Conference on Information Visualization, pp. 164-68.

Given a facial image of a particular person, aging is to construct the facial image of the same person but at a different age. This assumption becomes an important limitation when modelling human aging from real data, especially in order to be used by plastic surgeons. We present a new approach to robustly analyze parts of face aging. Our analysis-synthesis cooperation, possible thanks to a highly realistic 3D head model and in comparison with a youthful age face of the same person. A prototype of aging a specific region of someone's face, which utilizes a framework for facial feature is presented. Via a number of experiments the validity of the rules that have been employed are demonstrated. The evaluation of the overall performance of the fully automated system indicates that facial aging is performed quite accurately by the system. 
Berg, M, I Kojo, and J Laarni. 2010, "Object Displays for Identifying Multidimensional Outliers within a Crowded Visual Periphery." Journal of Visual Communication and Image Representation 21(8):880-88.

This article discusses the human ability to detect, locate, or identify objects and their features using peripheral vision. The potential of peripheral vision is underused with user interfaces probably due to the limits of visual acuity. Peripheral preview can guide focused attention to informative locations, if the visual objects are large enough and otherwise within the limits of discrimination. Our experiments focused on the task of identifying an outlier and implicated another limiting factor, crowding, for integration of object features. The target object and the corresponding data dimension were located from an object display representation used for integrating multidimensional data. We measured performance on a peripheral vision task in terms of reaction times and eye movements. Subjects identified the target item from 480 alternatives within $100 \mathrm{~ms}$. Therefore, the identification process would not slow down the natural gaze sequence and focused attention during monitoring and data mining tasks.

Berger, W, and H Piringer. 2010, "Interactive Visual Analysis of Multiobjective Optimizations." In IEEE Symposium on Visual Analytics Science \& Technology, pp. 215-16.

Bernardi, ML, and G A. 2008, "A Taxonomy of Interactions Introduced by Aspects." In 32nd Annual IEEE International Computer Software and Applications Conference, pp. 726-31.

Aspects have a large impact on the static structure and dynamic behaviour of the system they belong. This is due to the intrinsic intrusive nature of aspects and the woven process allowing the alteration of the structure, the control and data flow of the components of the base system in aspect oriented (AO) systems. Several and different types of interactions among aspects and the other components can be introduced according to the different mechanisms provided by AO Programming. These interactions can make higher the complexity of the overall system affecting its comprehension. In this paper we propose a taxonomy to categorize these types of interactions among aspects and the components of the base system. The taxonomy can be used to understand how each type of interaction affects the complexity, and thus the comprehensibility, of the system.

Bertini, E, and G Santucci. 2006, "Visual Quality Metrics." In Proceedings of the 2006 AVI workshop on BEyond time and errors: novel evaluation methods for information visualization, pp. 1-5.

The definition and usage of quality metrics for Information Visualization techniques is still an immature field. Several proposals are available but a common view and understanding of this issue is still missing. This paper attempts a first step toward a visual quality metrics systematization, providing a general classification of both metrics and usage purposes. Moreover, the paper explores a quite neglected class of visual quality metrics, namely Feature Preservation Metrics, that allow for evaluating and improving in a novel way the effectiveness of basic Infovis techniques.

Best, DM, and RR Lewis. 2010, "Gwvis: A Tool for Comparative Ground-Water Data Visualization." Computers \& Geosciences 36(11):1436-42.

The Ground-Water Visualization application (GWVis) presents ground-water data visually in order to educate the public on ground-water issues. It is also intended for presentations to government and other funding agencies. GWVis works with ground-water level elevation data collected or modeled over a given time span, together with a matching fixed underlying terrain. GWVis was developed using the 
Python programming language in conjunction with associated extension packages and application program interfaces such as OpenGLTM to improve performance and allow us fine control of attributes of the model such as lighting, material properties, transformations, and interpolation. There are currently several systems available for visualizing ground-water data. We classify these into two categories: research-oriented models and static presentation-based models. While both of them have their strengths, we find the former overly complex and non-intuitive and the latter not engaging and presenting problems showing multiple data dimensions. GWVis bridges the gap between static and research based visualizations by providing an intuitive, interactive design that allows participants to view the model from different perspectives, infer information about simulations, and view a comparison of two datasets. By incorporating scientific data in an environment that can be easily understood, GWVis allows that information to be presented to a large audience base.

Bewley, WL. 2010, "Methods to Evaluate Technology." in International Encyclopedia of Education, eds. P Penelope, B Eva and M Barry, pp. 578-84. Elsevier, Oxford.

The goal of technology evaluation is to determine the effectiveness of technology for use in a particular context for a particular purpose in order to guide or justify decisions that lead to possibly large investments of scarce resources to implement technology-based educational interventions that may impact student learning, positively, negatively, or not at all, and with high or low cost-effectiveness. This article discusses the current state of technology evaluation methodology. It provides an overview of major methods, a discussion of issues to be addressed by any method, and a heuristic process for matching methods to evaluation requirements and situations.

Bezerianos, A, et al. 2010, "Geneaquilts: A System for Exploring Large Genealogies." IEEE Transactions on Visualization and Computer Graphics 16(6):1073-81.

GeneaQuilts is a new visualization technique for representing large genealogies of up to several thousand individuals. Thevisualization takes the form of a diagonally-filled matrix, where rows are individuals and columns are nuclear families. After identifyingthe major tasks performed in genealogical research and the limits of current software, we present an interactive genealogy explorationsystem based on GeneaQuilts. The system includes an overview, a timeline, search and filtering components, and a new interactiontechnique called Bring \&\#x0026; Slide that allows fluid navigation in very large genealogies. We report on preliminary feedback from domain experts and show how our system supports a number of their tasks.

Bhowmick, T, et al. 2008, "Informing Geospatial Toolset Design: Understanding the Process of Cancer Data Exploration and Analysis." Health \& Place 14(3):576-607.

There is an increasing need for new methods and tools that support knowledge construction from complex geospatial datasets related to public health. This study is part of a larger effort to develop, implement, and test such methods and tools. To be successful, the design of methods and tools must be grounded in a solid understanding of the work practices within the domain of use; the research reported here focuses on developing that understanding. We adopted a user-centered approach to toolset design where we investigated the work of cancer researchers and used the results of that investigation as inputs into the development of design guidelines for new geovisualization and spatial analysis tools. Specifically, we conducted key informant interviews focused on use, or potential use, of geographic information, methods, and tools and complemented this with a systematic analysis of published, peer- 
reviewed articles on geospatial cancer research. Results were used to characterize the typical process of analysis, to identify fundamental differences between intensive users of geospatial methods and infrequent users, and to outline key stages in analysis and tasks within the stages that methods and tools must support. Our findings inform design and implementation decisions for visual and analytic tools that support cancer prevention and control research and they provide insight into the processes used by cancer researchers for addressing the challenges of geographic factors in public health research and policy.

Billinghurst, M, R Grasset, and H Seichter. 2010, "Tangible Interfaces for Ambient Augmented Reality Applications." in Human-Centric Interfaces for Ambient Intelligence, eds. A Hamid, Delgado and Augusto, pp. 281-302. Academic Press, Oxford.

Summary Ambient intelligence has the goal of embedding context-sensitive technology into the user's surroundings. In many ways augmented reality (AR) is complementary to this in that AR interfaces seamlessly enhance the user's real environment with a virtual information overlay. The two merge in context-aware ambient AR applications, allowing users to easily perceive and interact with the interface using the AR overlay of the real world. In this chapter we describe how tangible interaction techniques can be used to design human-centric interfaces for ambient AR applications. Examples will be drawn from current and previous research in augmented reality and ambient interfaces, and design guidelines will be given to show how human-centric tangible AR interfaces can be developed.

Bin, C, HM Tse, and W Wenping. 1998, "Design of a Walkthrough System for Indoor Environments from Floor Plans." In IEEE Conference on Information Visualization, pp. 50-57.

Shares some experience in designing a practical walkthrough system for indoor environments. We discuss issues from the design of efficient utility programs for building 3D models to the design of a walkthrough engine. Our utility programs allow the creation of the interior of a several-storied building in a few man-days. We also propose several rendering speedup techniques implemented in our walkthrough system. Tests have been carried out to benchmark the amount of speedup brought about by different techniques. One of these techniques, called dynamic visibility, proves to be more efficient than existing standard visibility preprocessing methods

Bin, W, and L Suo. 2009, "Literminer: A Literature Visual Analytic System." In 1st International Conference on Information Science and Engineering (ICISE), pp. 891-94.

The data set of scientific literature contains abundant knowledge. Currently, most bibliography search engines provide only keyword search or similarity search functions for retrieving information directly stored in the database but neglect mining hidden information. In this article, we design a visual analytic tool called LiterMiner to extract entities such as article, author, affiliation, and keyword from these literature records, establish the relationships among them and display them to help analyst understand them effectively. Especially, LiterMiner uses community detection algorithm to explore academic teams. Our tool has three principle features. First, a sophisticated data clean process is designed for integration of many different formatted literature records. Second, LiterMiner can find academic teams and analyze the resume of an academic team. Third, an interactive and cooperate visualization solution is used to display the analysis result, which help user understand the result better. 
Bin, Z, G Shankar, and Y Cai. 2007, "Integrating Data Quality Data into Decision-Making Process: An Information Visualization Approach." in Lecture Notes in Computer Science, Vol 4557, pp. 366-69. Springer-Verlag, Berlin.

Poor data quality (DQ) has been a troubling issue within many organizations. Thus it is important for a decision-maker to be aware of the data quality of the information based on which he/she makes a decision. However, the mental integration of DQ metadata in to a decision process poses a significant cognitive challenge. A decision-maker could be overloaded and may not effectively take advantage of the DQ metadata [2]. This paper thus proposes a visualization approach to reduce the amount of cognitive resource required for such mental integration.

Bitterman, N, E Lerner, and H Bitterman. 2010, "Evaluation of Data Display for Patient-Oriented Electronic Record of Anticoagulant Therapy." Telemedicine Journal and E-Health 16(7):799-806.

Objective: Our aim was to evaluate visualization methods for specific tasks performed with personal healthcare e-record systems for lay adults and older patients. We investigated common visualization methods for data entry and follow-up of personal and clinical information for self-control of blood coagulation functions. Methods: Twenty-five old ( $72.2+/-5.5$ years) and 25 young ( $30.4+/-4.9$ years) participants completed tasks based on common scenarios, on experimental Web sites with hidden tracking programs. Functional parameters (time, accuracy), subjective parameters (preference, satisfaction), and physiological parameters (heart rate, skin temperature, sweat, respiratory rate, and muscle tension) monitored with miniature sensors were used. Results: Total time for data entry and information follow-up were significantly longer for older compared with younger participants, with no significant differences in accuracy (errors), in stress-related physiological parameters, in preferences, or in satisfaction between age group. The Menu display was the significantly preferred configuration for data entry in both age groups, based on functional, physiological, and subjective criteria $(\mathrm{p}<0.05$, Duncan test). The Calendar configuration was significantly preferred for mixed tasks of follow-up and information retrieval, in both age groups, based on functional, physiological, and subjective criteria ( $\mathrm{p}<0.05$, Duncan test).

Conclusions: Our study supports equal capabilities of old and young people to use interactive healthcare systems for management of chronic diseases and further encourages using physiological, functional, and subjective methods for evaluating personal healthcare records.

Bittmann, RM, and R Gelbard. 2009, "Visualization of Multi-Algorithm Clustering for Better Economic Decisions -- the Case of Car Pricing." Decision Support Systems 47(1):42-50.

Clustering decisions frequently arise in business applications such as recommendations concerning products, markets, human resources, etc. Currently, decision makers must analyze diverse algorithms and parameters on an individual basis in order to establish preferences on the decision-making issues they face; because there is no supportive model or tool which enables comparing different result-clusters generated by these algorithms and parameters combinations. The Multi-Algorithm-Voting (MAV) methodology enables not only visualization of results produced by diverse clustering algorithms, but also provides quantitative analysis of the results. The current research applies MAV methodology to the case of recommending new-car pricing. The findings illustrate the impact and the benefits of such decision support system. 
Bjork, S, et al. 2000, "Powerview - Using Information Links and Information Views to Navigate and Visualize Information on Small Displays." in Lecture Notes in Computer Science, eds. P Thomas and HW Gellersen, Vol 1927, pp. 46-62. Springer-Verlag, Berlin.

PowerView is a PDA application designed to support people with situational information, primarily during conversations and meetings with other people. PowerView was designed to address a number of issues in interface design concerning both information visualization and interaction on small, mobile devices. In terms of information visualization, the system was required to provide the user With a single integrated information System that enabled quick access to related information once an object of interest had been selected. In terms of interaction, the system was required to enable easy and efficient information retrieval, including single-handed use of the device. These problems were addressed by introducing Information Links and Information Views. An evaluation of the application against the standard application suite bundle of die PDA, a Casio Cassiopeia E-11, proved the interfaces equivalent in usability even though the PowerView application uses a novel interface paradigm and the test subjects were given no training time with the system.

Blaas, J, CP Botha, and FH Post. 2008, "Extensions of Parallel Coordinates for Interactive Exploration of Large Multi-Timepoint Data Sets." Visualization and Computer Graphics, IEEE Transactions on 14(6):1436-51.

Parallel coordinate plots (PCPs) are commonly used in information visualization to provide insight into multi-variate data. These plots help to spot correlations between variables. PCPs have been successfully applied to unstructured datasets up to a few millions of points. In this paper, we present techniques to enhance the usability of PCPs for the exploration of large, multi-timepoint volumetric data sets, containing tens of millions of points per timestep. The main difficulties that arise when applying PCPs to large numbers of data points are visual clutter and slow performance, making interactive exploration infeasible. Moreover, the spatial context of the volumetric data is usually lost. We describe techniques for preprocessing using data quantization and compression, and for fast GPU-based rendering of PCPs using joint density distributions for each pair of consecutive variables, resulting in a smooth, continuous visualization. Also, fast brushing techniques are proposed for interactive data selection in multiple linked views, including a 3D spatial volume view. These techniques have been successfully applied to three large data sets: Hurricane Isabel (Vis'04 contest), the ionization front instability data set (Vis'08 design contest), and data from a large-eddy simulation of cumulus clouds. With these data, we show how PCPs can be extended to successfully visualize and interactively explore multi-timepoint volumetric datasets with an order of magnitude more data points.

Black, JB. 1995, "Using a Knowledge Representaitons Approach to Cognitive Task Analysis." In Proceedings of the 1995 Annual National Convention of the Association for Educational Communications and Technology, pp. 10-10.

Blanchard, J, et al. 2007, "A 2d-3d Visualization Support for Human-Centered Rule Mining." Computers \& Graphics 31(3):350-60.

On account of the enormous amounts of rules that can be produced by data mining algorithms, knowledge post-processing is a difficult stage in an association rule discovery process. In order to find relevant knowledge, the user needs to rummage through the rules. To make this task easier, we propose a new interactive mining methodology based on well adapted dynamic visual representations. It allows the 
user to drive the discovery process by focusing his/her attention on limited subsets of rules. We have implemented our methodology with two complementary 2D and 3D visualization supports. These implementations exploit the user's focus to guide the generation of the rules by means of a specific constraint-based rule-mining algorithm.

Blasio, AJ, and AM Bisantz. 2002, "A Comparison of the Effects of Data-Ink Ratio on Performance with Dynamic Displays in a Monitoring Task." International Journal of Industrial Ergonomics 30(2):89-101.

This study investigated the applicability of recommendations for the design of graphs to the design of dynamic graphic displays. Specifically, recommendations regarding the level of data-ink ratio (the ratio of ink showing data to the total ink) were tested. Participants monitored dynamic process control variables in one of three display conditions corresponding to high, medium, and low data-ink ratios. Speed of response to out-of-range variables and level of situation awareness were measured, and aspects of subjective mental workload were assessed. Results indicated some support for principles of non-data-ink reduction: reaction times to out-of-range events were fastest for the highest data-ink ratio condition. Reaction times were similar for the low and medium data-ink conditions, perhaps because these conditions relied on the same graphical specifier. Level of situation awareness showed no effect of dataink ratio; however, participants appeared to have better awareness of variables, which changed more frequently.Relevance to industry Increased capabilities and decreased costs of computer displays have increased the likelihood that such technology will be applied in an industrial context. This study suggests that while there may be some applicability of available design guidelines for static graphics to dynamic displays, a simple translation of the guidelines from a static to dynamic environment is not a guarantee for improved performance.

Blazy, S, and P Facon. 1997, "Application of Formal Methods to the Development of a Software Maintenance Tool." In 12th International Conference on Automated Software Engineering, pp. 162-71.

Partial evaluation is an optimization technique traditionally used in compilation. We have adapted this technique to the understanding of scientific application programs during their maintenance, and we have implemented a tool that analyzes Fortran 90 application programs and performs an interprocedural pointer analysis. This paper presents how we have specified this analysis with different formalisms (inference rules with global definitions and set and relational operators). Then we present the tool implementing these specifications. It has been implemented in a generic programming environment and a graphical interface has been developed to visualize the information computed during the partial evaluation (values of variables, already-analyzed procedures, scope of variables, removed statements, etc.)

Blomé, M, CR Johansson, and P Odenrick. 2006, "Visualization of Ergonomic Guidelines -- a Comparison of Two Computer Aided Systems to Support Vehicle Design." International Journal of Industrial Ergonomics 36(6):571-80.

Guidelines are used in information and quality management systems to ensure high quality by supporting design, production and communication processes. These guidelines can be presented as a conventional system on an intranet suitable for printouts, visualized as a traditional text-based report with a list of headings accompanied by tables and pictures or presented as paper documents in manuals or binders. Such approaches do not always correspond to some companys' requirements for usability. However, multimedia techniques can be used to visualize and let users interact with the information 
through hyperlinked text, pictures, and animations. The aim of this study was to design a computer aided system of ergonomic guidelines visualized by means of interactive multimedia technology based on cognitive theories and practical examples. Furthermore, the efficiency in and attitudes towards using the interactive multimedia system were evaluated and compared with a conventional system which visualized ergonomic guidelines in the form of a traditional report on scrollable web pages. The interactive multimedia system was the result of a participatory design process with experts and potential users in collaboration with Saab Automobile. The system was, in general, faster to use with lower dispersion in performance speed and number of incorrect answers compared to the conventional system. No statistically significant differences were found between males and females or between the five groups of potential users. In addition, the interactive multimedia system was experienced as more enjoyable to use, which is likely to promote interest and learning about ergonomic issues.Relevance to industry The results from this study can be used to design and improve visualization of ergonomic guidelines on an intranet, increase efficiency, and engender a positive attitude towards using ergonomic information.

Bloom, BS. 1956. Taxonomy of Educational Objectives: The Classificaiton of Educaitonal Goals: Handbook I. Cognitive Domain. Longmans, Green, New York, NY.

Boccuzzo, S, and HC Gall. 2008, "Software Visualization with Audio Supported Cognitive Glyphs." In IEEE International Conference on Software Maintenance, pp. 366-75.

There exist numerous software visualization techniques that aim to facilitate program comprehension. One of the main concerns in every such software visualization is to identify relevant aspects fast and provide information in an effective way. In previous work, we developed a cognitive visualization technique and tool called CocoViz that uses common place metaphors for an intuitive understanding of software structures and evolution. In this paper, we address software comprehension by a combination of visualization and audio. Evolution and structural aspects are annotated with different audio to represent concepts such as design erosion, code smells or evolution metrics. We use audio concepts such as loudness, sharpness, tone pitch, roughness or oscillation and map those to properties of classes and packages. As such we provide an audio annotation of software entities along their version history for software analysis and software browsing. Our first results with the prototype and a small user study show that with this combination of visual and aural means we can facilitate program comprehension and provide additional information that usually is not provided by current visualization approaches.

Boff, KR. 2006, "Revolutions and Shifting Paradigms in Human Factors \& Ergonomics." Applied Ergonomics 37(4):391-99.

The "Revolution in Information Technology" has spawned a series of transformational revolutions in the nature and practice of human factors and ergonomics (HFE). "Generation 1" HFE evolved with a focus on adapting equipment, workplace and tasks to human capabilities and limitations. Generation 2, focused on cognitive systems integration, arose in response to the need to manage automation and dynamic function allocation. Generation 3 is focused on symbiotic technologies that can amplify human physical and cognitive capabilities. Generation 4 is emergent and is focused on biological enhancement of physical or cognitive capabilities. The shift from HFE Generations 1 and 2 to Generations 3 and 4 profoundly alters accepted boundary constraints on the adaptability of humans in complex systems design. Furthermore, it has opened an ethical divide between those that see cognitive and physical enhancement as a great benefit to society and those who perceive this as tampering with the fundamentals of human nature. 
Böhlen, M, et al. 2003, "3d Visual Data Mining--Goals and Experiences." Computational Statistics \& Data Analysis 43(4):445-69.

The visual exploration of large databases raises a number of unresolved inference problems and calls for new interaction patterns between multiple disciplines--both at the conceptual and technical level. We present an approach that is based on the interaction of four disciplines: database systems, statistical analyses, perceptual and cognitive psychology, and scientific visualization. At the conceptual level we offer perceptual and cognitive insights to guide the information visualization process. We then choose cluster surfaces to exemplify the data mining process, to discuss the tasks involved, and to work out the interaction patterns.

Bohner, SA, et al. 2007, "Evolutional Insights from Uml and Source Code Versions Using Information Visualization and Visual Analysis." In 4th IEEE International Workshop on Visualizing Software for Understanding and Analysis, pp. 145-48.

Risks associated with producing today's software applications are increasingly linked with size and complexity - just too many aspects of software to fit in the head of even the most talented software engineer. Understanding software entails more than browsing the source code or reviewing the models in the other software artifacts. We use information visualization and visual analysis techniques to parse data sets generated from UML and Java SDK source code versions to examine patterns. This visual perspective provides relevant insights and additional navigation opportunities for software engineers during development and maintenance activities.

Bolger, AF. 2006, "Do You See What I See?: Agreeing to Disagree." Journal of the American College of Cardiology 47(1):129-30.

Bonsignore, EM, et al. 2009, "First Steps to Netviz Nirvana: Evaluating Social Network Analysis with Nodexl." In International Conference on Computational Science and Engineering, pp. 332-39.

Social Network Analysis (SNA) has evolved as a popular, standard method for modeling meaningful, often hidden structural relationships in communities. Existing SNA tools often involve extensive preprocessing or intensive programming skills that can challenge practitioners and students alike. NodeXL, an open-source template for Microsoft Excel, integrates a library of common network metrics and graph layout algorithms within the familiar spreadsheet format, offering a potentially low-barrier-to-entry framework for teaching and learning SNA. We present the preliminary findings of 2 user studies of 21 graduate students who engaged in SNA using NodeXL. The majority of students, while information professionals, had little technical background or experience with SNA techniques. Six of the participants had more technical backgrounds and were chosen specifically for their experience with graph drawing and information visualization. Our primary objectives were (1) to evaluate NodeXL as an SNA tool for a broad base of users and (2) to explore methods for teaching SNA. Our complementary dual case-study format demonstrates the usability of NodeXL for a diverse set of users, and significantly, the power of a tightly integrated metrics/visualization tool to spark insight and facilitate sense-making for students of SNA. 
Booch, G. 2010, "Capstone Presentation Why Don' T Developers Draw Diagrams ?" In 5th International Symposium on Software Visualization, pp. 4503-03.

Common sense (and common practice in other engineering disciplines) tells us that modeling is a Good Thing. There is undeniable value in having and using simple, standard, and expressive graphical notations to help one reason about complex artifacts. However, while software-intensive systems are among the most complex of artifacts, reality is that the vast majority of developers live fully in the textual dimension and, like Flatlanders, have no understanding of or desire for the visual dimension save for a few diagrams with dubious semantics that they may hastily and ethereally sketch on a whiteboard. This community (of SoftVis researchers) have produced some compelling ideas - but none of them are what one can claim to be fully mainstream. This keynote will discuss why this is so and what we might do to narrow this gap to attend to the pain points of developers.

Borchers, J, et al. 1996, "Layout Rules for Graphical Web Documents." Computers \& Graphics 20(3):415-26.

The number of companies, institutions, and individuals competing for attention in the World Wide Web is growing exponentially. This makes designing informative, easy-to-grasp, and visually appealing documents not only important for user-friendly information presentation, but also the key to success for any information provider. In this paper, we present layout guidelines for textual and graphical, static and dynamic, 2-D and 3-D Web documents which are drawn from fields as diverse as typography, Gestalt psychology, architecture, hypertext authoring, and human-computer interaction. Web documents are classified into five basic types, and our layout rules are applied to each of these. Finally, we show how currently evolving standards (HTML 3.0 for text and still graphics, Java for 2-D animation, and VRML for 3-D worlds) support applying those rules.

Borgo, R, et al. 2010, "Evaluating the Impact of Task Demands and Block Resolution on the Effectiveness of Pixel-Based Visualization." IEEE Transactions on Visualization and Computer Graphics 16(6):963-72.

Pixel-based visualization is a popular method of conveying large amounts of numerical data graphically. Application scenarios include business and finance, bioinformatics and remote sensing. In this work, we examined how the usability of such visual representations varied across different tasks and block resolutions. The main stimuli consisted of temporal pixel-based visualization with a white-red color map, simulating monthly temperature variation over a six-year period. In the first study, we included 5 separate tasks to exert different perceptual loads. We found that performance varied considerably as a function of task, ranging from $75 \%$ correct in low-load tasks to below $40 \%$ in high-load tasks. There was a small but consistent effect of resolution, with the uniform patch improving performance by around $6 \%$ relative to higher block resolution. In the second user study, we focused on a high-load task for evaluating month-to-month changes across different regions of the temperature range. We tested both CIE $\mathrm{L}^{*} \mathrm{u}^{*} \mathrm{v}^{*}$ and RGB color spaces. We found that the nature of the change-evaluation errors related directly to the distance between the compared regions in the mapped color space. We were able to reduce such errors by using multiple color bands for the same data range. In a final study, we examined more fully the influence of block resolution on performance, and found block resolution had a limited impact on the effectiveness of pixel-based visualization. 
Born, S, et al. 2010, "Illustrative Stream Surfaces." IEEE Transactions on Visualization and Computer Graphics 16(6):1329-38.

Stream surfaces are an intuitive approach to represent 3D vector fields. In many cases, however, they are challenging objects to visualize and to understand, due to a high degree of self-occlusion. Despite the need for adequate rendering methods, little work has been done so far in this important research area. In this paper, we present an illustrative rendering strategy for stream surfaces. In our approach, we apply various rendering techniques, which are inspired by the traditional flow illustrations drawn by Dallmann and Abraham $\backslash \& \# x$ 0026; Shaw in the early 1980s. Among these techniques are contour lines and halftoning to show the overall surface shape. Flow direction as well as singularities on the stream surface are depicted by illustrative surface streamlines. ;To go beyond reproducing static text book images, we provide several interaction features, such as movable cuts and slabs allowing an interactive exploration of the flow and insights into subjacent structures, e.g., the inner windings of vortex breakdown bubbles. These methods take only the parameterized stream surface as input, require no further preprocessing, and can be freely combined by the user. We explain the design, GPU-implementation, and combination of the different illustrative rendering and interaction methods and demonstrate the potential of our approach by applying it to stream surfaces from various flow simulations. ;

Borner, K, WR Hazlewood, and SM Lin. 2002, "Visualizing the Spatial and Temporal Distribution of User Interaction Data Collected in Three-Dimensional Virtual Worlds." In 6th International Conference on Information Visualization, pp. 25-31.

This paper reports work in progress on the analysis and visualization of the spatial and temporal distribution of user interaction data collected in three-dimensional (3-D) virtual worlds. Two tools are introduced. The "WorldMapper" reads in a so-called propdump file and creates a 2-D clickable map showing the layout of the world as well as interaction possibilities such as teleports and clickable web links. The second tool visualizes user interaction data such as navigation, chatting, and Web access activity overlaid on the world neap. Resulting visualizations are meant to support social navigation, design evaluation and optimization, and the study of virtual communities. Both tools are demonstrated on a 19-person information treasure hunt for information in a 3-D virtual world. The paper concludes with a discussion and an outlook.

Borner, K, et al. 2004, "Visualizing the Vlearn3d 2002 Conference in Space and Time." In Conference on Visualization and Data Analysis, pp. 24-32.

This paper introduces a set of visualization tools that aim to support social navigation, the evaluation and optimization of three-dimensional virtual worlds and the study of their evolving communities. Previous work (Bomer Lin, 2001; Bomer et al., 2002) demonstrated how this toolset can be used to analyze data acquired during a information treasure hunt. This paper extends and applies this tool set to visualize an entire virtual Conference on the topic Virtual Learning in Three Dimensions (VLearn3D)) that was held in December 2002.

Börner, K, and A Scharnhorst. 2009, "Visual Conceptualizations and Models of Science." Journal of Informetrics 3(3):161-72.

This is the Guest Editor's introduction to the Special Issue on "Science of Science: Conceptualizations and Models of Science", Journal of Informetrics. The introduction discusses challenges towards a 
theoretically grounded and practically useful science of science. It provides a brief chronological review of relevant work and argues for (1) the development of common frameworks for the comparison and combination of existing approaches, theories, laws, and measurements, (2) the combination of quantitative and qualitative studies of science, and (3) the operationalization of theoretical concepts in terms of measurement and empirical evidence. Next, three visual conceptualizations of science are discussed and compared. Each of them provides a framework for the comparison and combination of existing works, means to combine quantitative and qualitative data, and helps to operationalize and communicate theoretical concepts using empirical data. Last but not least, the contributions of and interlinkages among the papers included in this issue are discussed.

Böttger, S, and H Barthel. 2010, "Fault Forest Visualization." In 5th International Symposium on Software Visualization, pp. 219-20.

Bouchard, A, et al. 2008, "Evaluation of Information Visualization Approaches for an Enhanced Recognized Maritime Picture - Art. No. 694510." In Conference on Optics and Photonics in Global Homeland Security IV, pp. 94510-10.

This paper presents a MISR Visualization Experimental Environment which provides support to the development, evaluation, experimentation, and transitioning of information visualization approaches to emulate the essential elements of a future RMP. The environment provides a means of integrating and sharing the output of visualization tools; storing, accessing and managing showcase examples of visual representations via an underlying visualization reference model; and providing access to underlying data sources supplied through simulation, representative data, and operational data. Visualization design and experimentation activities are also briefly introduced. The MISR experimental environment may be used to characterize the various techniques evaluated and the results of experiments will be introduced as inputs of the environment knowledge base. It is expected that the experimentation undertaken, supported by a MISR experimental environment, will identify novel visualization approaches to be integrated in the future RMP and have the potential to enhance maritime domain awareness.

Boukhelifa, N, and F Chevalier. 2010, "Real-Time Aggregation of Wikipedia Data for Visual Analytics." In IEEE Symposium on Visual Analytics Science \& Technology, pp. 147-54.

Bourbeillon, J, C Garbay, and F Giroud. 2009, "Mass Data Exploration in Oncology: An Information Synthesis Approach." Journal of Biomedical Informatics 42(4):612-23.

New technologies and equipment allow for mass treatment of samples and research teams share acquired data on an always larger scale. In this context scientists are facing a major data exploitation problem. More precisely, using these data sets through data mining tools or introducing them in a classical experimental approach require a preliminary understanding of the information space, in order to direct the process. But acquiring this grasp on the data is a complex activity, which is seldom supported by current software tools. The goal of this paper is to introduce a solution to this scientific data grasp problem. Illustrated in the Tissue MicroArrays application domain, the proposal is based on the synthesis notion, which is inspired by Information Retrieval paradigms. The envisioned synthesis model gives a central role to the study the researcher wants to conduct, through the task notion. It allows for the implementation of a task-oriented Information Retrieval prototype system. Cases studies and user studies were used to validate this prototype system. It opens interesting prospects for the extension of the model or extensions towards other application domains. 
Bowden, EM, et al. 2005, "New Approaches to Demystifying Insight." Trends in Cognitive Sciences 9(7):322-28.

After a person has become stuck on a problem, they sometimes achieve a clear and sudden solution through insight - the so-called Aha! experience. Because of its distinctive experience, the origins and characteristics of insight have received considerable attention historically in psychological research. However, despite considerable progress in characterizing insight, the underlying mechanisms remain mysterious. We argue that research on insight could be greatly advanced by supplementing traditional insight research, which depends on a few complex problems, with paradigms common in other domains of cognitive science. We describe a large set of mini-insight problems to which multiple methods can be applied, together with subjective reports to identify insight problem-solving. Behavioral priming and neuroimaging methods are providing evidence about what, where, and how neural activity occurs during insight. Such evidence constrains theories of component processes, and will help to demystify insight.

Bowman, I, and JDV Horn. 2010, "Large-Scale Neuroanatomical Visualization Using a Manifold Embedding Approach." In IEEE Symposium on Visual Analytics Science \& Technology, pp. 237-38.

Boyack, KW, and N Rahal. 2005, "Evaluation of Laboratory Directed Research and Development Investment Areas at Sandia." Technological Forecasting and Social Change 72(9):1122-36.

Sandia National Laboratories conducts a variety of research projects each year under its LaboratoryDirected Research and Development (LDRD) program. Recently, information visualization techniques have been used with corporate data to map several LDRD investment areas for the purpose of understanding strategic overlaps and identifying potential opportunities for future development outside of our current technologies. Tools, techniques, and specific analyses are presented here. We find that these tools and techniques hold great promise for aiding the future direction of the science and technology enterprise.

Boyack, KW, BN Wylie, and GS Davidson. 2002, "Information Visualization, Human-Computer Interaction, and Cognitive Psychology: Domain Visualizations." in Lecture Notes in Computer Science, eds. K Borner and C Chen, Vol 2539, pp. 145-58. Springer-Verlag, Berlin.

Digital libraries stand to benefit from technology insertions from the fields of information visualization, human-computer interaction, and cognitive psychology, among others. However, the current state of interaction between these fields is not well understood. We use our knowledge visualization tool, VxInsight, to provide several domain visualizations of the overlap between these fields. Relevant articles were extracted from the Science Citation Indexes (SCI and Social SCI) using keyword searches. An article map, a semantic (co-term) map, and a co-author network have been generated from the data. Analysis reveals that while there are overlaps between fields, they are not substantial. However, the most recent work suggests areas where future collaboration could have a great impact on digital libraries of the future.

Bradley, E, D Roberts, and C Still. 2010, "Design of an Image Analysis Website for Phenological and Meteorological Monitoring." Environmental Modelling \& Software 25(1):107-16.

Web camera image databases and web-based services can be valuable components for a variety of modelling applications, but are still areas of relatively new exploration. Investigating design and 
information flow for an online image archive and analysis site for plant phenology and meteorological research has broader relevance to considerations of interoperability and website features. Currently, numerous online weather cameras provide images, but have no or limited-utility archives and do not support quantitative image analysis. We describe the design and implementation of a website (http://zulu.geog.ucsb.edu/Data/camera.html) that both provides different display options for archived image review, as well as the ability to chart time-series values extracted for user-specified regions of interest. This interface is distinguished by content-enabled charts with the ability to click on data points and directly access the corresponding image for reference purposes. A linked website to the meteorological data from the camera station further extends the potential for exploratory analysis and pedagogical utility. Online quantification of the color change related to plant senescence and insolation impacts due to cloud cover are demonstrated. We conclude that dynamic web pages are a powerful and useful tool for adding educational and scientific value to repeat digital photography systems.

Brandes, U, and J Lerner. 2008, "Visual Analysis of Controversy in User-Generated Encyclopedias." Information Visualization 7(1):34-48.

Wikipedia is a large and rapidly growing Web-based collaborative authoring environment, where anyone on the Internet can create, modify, and delete pages about encyclopedic topics. A remarkable property of some Wikipedia pages is that they are written by up to thousands of authors who may have contradicting opinions. In this paper, we show that a visual analysis of the 'who revises whom'-network gives deep insight into controversies. We propose a set of analysis and visualization techniques that reveal the dominant authors of a page, the roles they play, and the alters they confront. Thereby we provide tools to understand how Wikipedia authors collaborate in the presence of controversy. Information Visualization (2008) 7, 34-48. doi: 10.1057/palgrave.ivs.9500171

Brasch, S, L Linsen, and G Fuellen. 2009, "Vanlo - Interactive Visual Exploration of Aligned Biological Networks." Bmc Bioinformatics 10:327.

Background: Protein-protein interaction (PPI) is fundamental to many biological processes. In the course of evolution, biological networks such as protein-protein interaction networks have developed. Biological networks of different species can be aligned by finding instances (e. g. proteins) with the same common ancestor in the evolutionary process, so-called orthologs. For a better understanding of the evolution of biological networks, such aligned networks have to be explored. Visualization can play a key role in making the various relationships transparent. Results: We present a novel visualization system for aligned biological networks in 3D space that naturally embeds existing 2D layouts. In addition to displaying the intra-network connectivities, we also provide insight into how the individual networks relate to each other by placing aligned entities on top of each other in separate layers. We optimize the layout of the entire alignment graph in a global fashion that takes into account inter-as well as intranetwork relationships. The layout algorithm includes a step of merging aligned networks into one graph, laying out the graph with respect to application-specific requirements, splitting the merged graph again into individual networks, and displaying the network alignment in layers. In addition to representing the data in a static way, we also provide different interaction techniques to explore the data with respect to application-specific tasks. Conclusion: Our system provides an intuitive global understanding of aligned PPI networks and it allows the investigation of key biological questions. We evaluate our system by applying it to real-world examples documenting how our system can be used to investigate the data with respect to these key questions. Our tool VANLO (Visualization of Aligned Networks with Layout Optimization) can be accessed at http://www.math-inf.uni-greifswald.de/VANLO. 
Brath, R. 1997, "Concept Demonstration - Metrics for Effective Information Visualization." In IEEE Symposium on Information Visualization, pp. 108-11.

Metrics for information visualization will help designers create and evaluate 3D information visualizations. Based on experience from $60+3 \mathrm{D}$ information visualizations, the metrics we propose are: number of data points and data density; number of dimensions and cognitive overhead; occlusion percentage; and reference context and percentage of identifiable points.

Brath, R. 1997, "Metrics for Effective Information Visualization." In IEEE Symposium on Information Visualization, pp. 108-11.

Metrics for information visualization will help designers create and evaluate 3D information visualizations. Based on experience from $60+3 \mathrm{D}$ information visualizations, the metrics we propose are: number of data points and data density; number of dimensions and cognitive overhead; occlusion percentage; and reference context and percentage of identifiable points.

Bremm, S, and T Schreck. 2010, "Cluster Correspondence Views for Enhanced Analysis of Som Displays." In IEEE Symposium on Visual Analytics Science \& Technology, pp. 217-18.

Bresciani, S, and MJ Eppler. 2009, "The Benefits of Synchronous Collaborative Information Visualization: Evidence from an Experimental Evaluation." Visualization and Computer Graphics, IEEE Transactions on 15(6):1073-80.

A great corpus of studies reports empirical evidence of how information visualization supports comprehension and analysis of data. The benefits of visualization for synchronous group knowledge work, however, have not been addressed extensively. Anecdotal evidence and use cases illustrate the benefits of synchronous collaborative information visualization, but very few empirical studies have rigorously examined the impact of visualization on group knowledge work. We have consequently designed and conducted an experiment in which we have analyzed the impact of visualization on knowledge sharing in situated work groups. Our experimental study consists of evaluating the performance of 131 subjects (all experienced managers) in groups of 5 (for a total of 26 groups), working together on a real-life knowledge sharing task. We compare (1) the control condition (no visualization provided), with two visualization supports: (2) optimal and (3) suboptimal visualization (based on a previous survey). The facilitator of each group was asked to populate the provided interactive visual template with insights from the group, and to organize the contributions according to the group consensus. We have evaluated the results through both objective and subjective measures. Our statistical analysis clearly shows that interactive visualization has a statistically significant, objective and positive impact on the outcomes of knowledge sharing, but that the subjects seem not to be aware of this. In particular, groups supported by visualization achieved higher productivity, higher quality of outcome and greater knowledge gains. No statistically significant results could be found between an optimal and a suboptimal visualization though (as classified by the pre-experiment survey). Subjects also did not seem to be aware of the benefits that the visualizations provided as no difference between the visualization and the control conditions was found for the self-reported measures of satisfaction a- - nd participation. An implication of our study for information visualization applications is to extend them by using real-time group annotation functionalities that aid in the group sense making process of the represented data. 
Bresciani, S, and MJ Eppler. 2009, "Beyond Knowledge Visualization Usability: Toward a Better Understanding of Business Diagram Adoption." In 13th International Conference on Information Visualisation, pp. 474-79.

Information visualization research typically focuses on the formal aspects of specific graphic solutions in terms of their thinking and/or communication support. This type of analysis often focuses on quantitative diagrams and emphasizes their attributes for specific tasks. Our contribution, by contrast, focuses on qualitative visual solutions and the factors that contribute to their wider diffusion. As many knowledge visualization solutions have not achieved wider use, the question of which factors foster diagram adoption seems to be a particularly relevant but under- researched topic. In this paper we develop and discuss three dimensions that foster knowledge visualization adoption: perceived ease of use, perceived usefulness and perceived authority. We base our analysis on the Technology Adoption Model by Davis and the Perceived Characteristics of Innovations by Rogers. We illustrate these dimensions and their items through examples of business diagrams that have received wide recognition and we contrast them with negative examples.

Brewer, I, et al. 2000, "Collaborative Geographic Visualization: Enabling Shared Understanding of Environmental Processes." In IEEE Symposium on Information Vizualization, pp. 137-41.

We describe a prototype same-time/different-place collaborative geovisualization environment. We outline an approach to understanding use and usability and present results of interviews with domain experts about the ways in which collaborative visualization might enable groups to work at a distance. One goal for our research is to design an effective and flexible system that can support group work on environmental science research mediated through dynamic geovisualization displays. We are addressing this goal using a four-step human-centered system design process, modeled on that proposed by (Gabbard et al., 1999) for development and evaluation of virtual environments. The steps they delineate are: user task analysis; expert guideline-based evaluation; formative user-centered evaluation; and summative comparative evaluation

Brivio, P, M Tarini, and P Cignoni. 2010, "Browsing Large Image Datasets through Voronoi Diagrams." IEEE Transactions on Visualization and Computer Graphics 16(6):1261-70.

Conventional browsing of image collections use mechanisms such as thumbnails arranged on a regular grid or on a line,often mounted over a scrollable panel. However, this approach does not scale well with the size of the datasets (number of images).In this paper, we propose a new thumbnail-based interface to browse large collections of images. Our approach is based on weightedcentroidal anisotropic Voronoi diagrams. A dynamically changing subset of images is represented by thumbnails and shown on the screen. Thumbnails are shaped like general polygons, to better cover screen space, while still reflecting the original aspect ratios or orientation of the represented images. During the browsing process, thumbnails are dynamically rearranged, reshaped and rescaled. The objective is to devote more screen space (more numerous and larger thumbnails) to the parts of the dataset closer to the current region of interest, and progressively lesser away from it, while still making the dataset visible as a whole. During the entire process, temporal coherence is always maintained. GPU implementation easily guarantees the frame rates needed for fully smooth interactivity. 
Brodlie, K. 2005, "Models of Collaborative Visualization." in Exploring Geovisualization, eds. D Jason, MM Alan and K Menno-Jan, pp. 463-75. Elsevier, Oxford.

As more and more research is tackled by teams, often geographically distributed, it becomes increasingly important to find good approaches to "collaborative geovisualization". In this chapter, we develop three models for collaborative visualization: in the first, there is a single application, but its interface is shared amongst several researchers; in the second, each researcher runs their own instance of a single application, keeping their input parameters synchronized; and in the third, each researcher runs their own distinct application, independently of the others, but data may be passed between them. We then discuss tools that support these three models of working, and report on practical experiences of using these tools over a number of years, most recently in a collaborative geovisualization experiment with colleagues in New Zealand.

Brown, KS. 1996, "Excitement and Emotion Mark Scientists' Fleeting Moments of Discovery." The Scientist 10(23):1-5.

Bruckner, S, and T Möller. 2010, "Result-Driven Exploration of Simulation Parameter Spaces for Visual Effects Design." IEEE Transactions on Visualization and Computer Graphics 16(6):1468-76.

Graphics artists commonly employ physically-based simulation for the generation of effects such as smoke, explosions, and similar phenomena. The task of finding the correct parameters for a desired result, however, is difficult and time-consuming as current tools provide little to no guidance. In this paper, we present a new approach for the visual exploration of such parameter spaces. Given a three-dimensional scene description, we utilize sampling and spatio-temporal clustering techniques to generate a concise overview of the achievable variations and their temporal evolution. Our visualization system then allows the user to explore the simulation space in a goal-oriented manner. Animation sequences with a set of desired characteristics can be composed using a novel search-by-example approach and interactive direct volume rendering is employed to provide instant visual feedback.

Bryden, A. 2010, "Collaborative Visualization of Structural Biology." In IEEE International Conference on Information Visualization, pp. 1-2.

Buckeridge, DL, et al. 2002, "Making Health Data Maps: A Case Study of a Community/University Research Collaboration." Social Science \& Medicine 55(7):1189-206.

This paper presents the main findings from a collaborative community/university research project in Canada. The goal of the project was to improve access to community health information, and in so doing, enhance our knowledge of the development of community health information resources and community/university collaboration. The project built on a rich history of community/university collaboration in Southeast Toronto (SETO), and employed an interdisciplinary applied research and action design. Specific project objectives were to: (1) develop via active community/university collaboration a geographic information system (GIS) for ready access to routinely collected health data, and to study logistical, conceptual and technical problems encountered during system development; and (2) to document and analyze issues that can emerge in the process of community/university research collaboration. System development involved iteration through community user assessment of need, development or refinement of the GIS, and assessment of the GIS by community users. Collaborative process assessment entailed analysis of archival material, interviews with investigators and participant 
observation. Over the course of the project, a system was successfully developed, and favorably assessed by users. System development problems fell into four main areas: maintaining user involvement in system development, understanding and integrating data, bringing disparate data sources together, and making use of assembled data. Major themes emerging from the community/university collaborative research process included separate community and university cultures, time as an important issue for all involved, and the impact of uncertainty and ambiguity on the collaborative process.

Buddelmeijer, H, and S Trager. 2010, "Finding and Visualizing Relevant Subspaces for Clustering HighDimensional Astronomical Data Using Connected Morphological Operators." In IEEE Symposium on Visual Analytics Science \& Technology, pp. 35-42.

Buering, T, J Gerken, and H Reiterer. 2006, "User Interaction with Scatterplots on Small Screens - a Comparative Evaluation of Geometric-Semantic Zoom and Fisheye Distortion." Visualization and Computer Graphics, IEEE Transactions on 12(5):829-36.

Larger, higher resolution displays can be used to increase the scalability of information visualizations. But just how much can scalability increase using larger displays before hitting human perceptual or cognitive limits? Are the same visualization techniques that are good on a single monitor also the techniques that are best when they are scaled up using large, high-resolution displays? To answer these questions we performed a controlled experiment on user performance time, accuracy, and subjective workload when scaling up data quantity with different space-time-attribute visualizations using a large, tiled display. Twelve college students used small multiples, embedded bar matrices, and embedded timeseries graphs either on a 2 megapixel (Mp) display or with data scaled up using a $32 \mathrm{Mp}$ tiled display. Participants performed various overview and detail tasks on geospatially-referenced multidimensional time-series data. Results showed that current designs are perceptually scalable because they result in a decrease in task completion time when normalized per number of data attributes along with no decrease in accuracy. It appears that, for the visualizations selected for this study, the relative comparison between designs is generally consistent between display sizes. However, results also suggest that encoding is more important on a smaller display while spatial grouping is more important on a larger display. Some suggestions for designers are provided based on our experience designing visualizations for large displays

Bugajska, M. 2005, "Framework for Spatial Visual Design of Abstract Information." In 9th International Conference on Information Visualization, pp. 713-23.

Spatially-organized information can be accessed and operated on rapidly and effortlessly, especially when a spatial arrangement reveals the conceptual organization of information. Therefore, spatial perception plays an important role for cognitive processing when interacting with abstract information. The process of spatial information visualization is shaped by various factors including interactive, perceptual, navigational as well as organizational and metaphorical aspects and as such requires an interdisciplinary approach. Consequently, bringing the knowledge from different disciplines requires the development of a framework which can host and classify the interdisciplinary features important in designing effective spatial visualizations. In this paper we present a framework which manifests a holistic approach in designing spatial visualization of abstract information. 
Bullinger, H-J, et al. 2010, "Towards User Centred Design (Ucd) in Architecture Based on Immersive Virtual Environments." Computers in Industry 61(4):372-79.

This paper describes a generic concept of how to combine the experience of user centred design (UCD) in the field of Human Computer Interaction (HCI) with the traditional approach of participatory design (PD) in an architectural design process. Even if some basic requirements of this generic method are not available yet, this paper will also describe an approach, which enables planners even now to involve end users by using virtual environments (VE) as immersive and spatial prototype. It will be described and illustrated by the way of example using the building project Centre of Virtual Engineering of the Fraunhofer Institute for Industrial Engineering (IAO) in Stuttgart. It demonstrates that the transfer of the UCD approach to architectural planning combined with the provision of an adequate prototype can make a significant contribution towards an increase in quality and performance in building and construction projects.

Bunte, K, et al. 2010, "Adaptive Local Dissimilarity Measures for Discriminative Dimension Reduction of Labeled Data." Neurocomputing 73(7-9):1074-92.

Due to the tremendous increase of electronic information with respect to the size of data sets as well as their dimension, dimension reduction and visualization of high-dimensional data has become one of the key problems of data mining. Since embedding in lower dimensions necessarily includes a loss of information, methods to explicitly control the information kept by a specific dimension reduction technique are highly desirable. The incorporation of supervised class information constitutes an important specific case. The aim is to preserve and potentially enhance the discrimination of classes in lower dimensions. In this contribution we use an extension of prototype-based local distance learning, which results in a nonlinear discriminative dissimilarity measure for a given labeled data manifold. The learned local distance measure can be used as basis for other unsupervised dimension reduction techniques, which take into account neighborhood information. We show the combination of different dimension reduction techniques with a discriminative similarity measure learned by an extension of learning vector quantization (LVQ) and their behavior with different parameter settings. The methods are introduced and discussed in terms of artificial and real world data sets.

Burch, M, et al. 2008, "Cartesian Vs. Radial - a Comparative Evaluation of Two Visualization Tools." in Lecture Notes in Computer Science, ed. G Bebis, Vol 5358, pp. 151-60. Springer-Verlag, Berlin.

Many recently developed information visualization techniques are radial variants of originally Cartesian visualizations. Almost none of these radial variants have been evaluated with respect to their benefits over their original visualizations. In this work we compare a radial and a Cartesian variant of a visualization tool for sequences of transactions in information hierarchies. The Timeline Trees (TLT) approach uses a Cartesian coordinate system to represent both the hierarchy and the sequence of transactions whereas the The Timeline Trees (TRT) technique is the radial counterpart which makes use of a radial tree, as well as circle slices and sectors to show the sequence of transactions. For the evaluation we use both quantitative as well as qualitative evaluation methods including eye tracking. 
Burigat, S, and L Chittaro. 2008, "Interactive Visual Analysis of Geographic Data on Mobile Devices Based on Dynamic Queries." Journal of Visual Languages \& Computing 19(1):99-122.

The capabilities of current mobile devices, especially PDAs, are making it possible to design and develop mobile applications that employ visual techniques for using geographic data in the field. These applications can be extremely useful in areas as diverse as tourism, business, natural resources management and homeland security. In this paper, we present a system aimed at supporting users in the exploratory analysis of geographic data on PDAs through a highly interactive interface based on visual dynamic queries. We propose alternative visualizations to display query results and present an experimental evaluation aimed at comparing their effectiveness on a PDA in a tourist scenario. Our findings provide an experimental confirmation of the unsuitability of the typical visualization, employed by classic dynamic query systems, which displays only those results that fully satisfy a query, in those cases where only sub-optimal results are obtainable. For such cases, the results of our study highlight the usefulness of visualizations that display all results and their degree of satisfaction of the query.

Burigat, S, L Chittaro, and S Gabrielli. 2008, "Navigation Techniques for Small-Screen Devices: An Evaluation on Maps and Web Pages." International Journal of Human-Computer Studies 66(2):78-97.

Several techniques have been proposed to support user navigation of large information spaces (e.g., maps or web pages) on small-screen devices such as PDAs and Smartphones. In this paper, we present the results of an evaluation that compared three of these techniques to determine how they might affect performance and satisfaction of users. Two of the techniques are quite common on mobile devices: the first one (DoubleScrollbar) is the standard combination of two scrollbars for separate horizontal and vertical scrolling with zoom buttons to change the scale of the information space, the second one (Grab\&Drag) enables users to navigate the information space by directly dragging its currently displayed portion, while zooming is handled through a slider control. The last technique (Zoom-Enhanced Navigator or ZEN) is an extension and adaptation to mobile screens of Overview\&Detail approaches, which are based on displaying an overview of the information space together with a detail view of a portion of that space. In these approaches, navigation is usually supported by (i) highlighting in the overview which portion of space is displayed in the detail view, and (ii) allowing users to move the highlight within the overview area to change the corresponding portion of space in the detail area. Our experimental evaluation concerned tasks involving maps as well as web page navigation. The paper analyzes in detail the obtained results in terms of task completion times, number and duration of user interface actions, accuracy of the gained spatial knowledge, and subjective preferences.

Buring, T, J Gerken, and H Reiterer. 2007, "Dynamic Text Filtering for Improving the Usability of Alphasliders on Small Screens." In 11th International Conference Information Visualization, pp. 145-49.

Previous research has shown that alphasliders are an effective tool for searching an alphabetically sorted list when only limited screen space is available for the graphical user interface. To improve user satisfaction, we propose equipping the widget with a novel text filter to dynamically limit the slider range. In this way, users are supported in locating target items and in identifying records that are missing. The results of a comparative user evaluation run on a personal digital assistant showed that 8 out of 12 participants preferred the filter widget to the classic interface. We further suggest an enhanced alphaslider design to speed up user interaction. 
Buring, T, J Gerken, and H Reiterer. 2006, "User Interaction with Scatterplots on Small Screens - a Comparative Evaluation of Geometric-Semantic Zoom and Fisheye Distortion." IEEE Transactions on Visualization and Computer Graphics 12(5):829-36.

Existing information-visualization techniques that target small screens are usually limited to exploring a few hundred items. In this article we present a scatterplot tool for Personal Digital Assistants that allows the handling of many thousands of items. The application's scalability is achieved by incorporating two alternative interaction techniques: a geometric-semantic zoom that provides smooth transition between overview and detail, and a fisheye distortion that displays the focus and context regions of the scatterplot in a single view. A user study with 24 participants was conducted to compare the usability and efficiency of both techniques when searching a book database containing 7500 items. The study was run on a pen-driven Wacom board simulating a PDA interface. While the results showed no significant difference in task-completion times, a clear majority of 20 users preferred the fisheye view over the zoom interaction. In addition, other dependent variables such as user satisfaction and subjective rating of orientation and navigation support revealed a preference for the fisheye distortion. These findings partly contradict related research and indicate that, when using a small screen, users place higher value on the ability to preserve navigational context than they do on the ease of use of a simplistic, metaphor-based interaction style.

Burkhard, RA. 2004, "Learning from Architects: The Difference between Knowledge Visualization and Information Visualization." In 8th International Conference on Information Visualisation, pp. 519-24.

This paper focuses on an aspect which has been neglected, but which is decisive: The transfer of knowledge to different stakeholders; especially the transfer of insights derived from information visualization tools. In knowledge management the transfer of knowledge is a core process, which can be improved by using our innate abilities to process visual representations. The potential of visualizations are manifold. But business managers miss a holistic framework on the use of visualization methods for information exploration and communication tasks. This paper analyzes how architects use visualizations to amplify cognition and to transfer knowledge. It introduces a mediating framework that brings together isolated research directions and defines the new research focus knowledge visualization. Knowledge visualization examines the use of visualizations for the transfer of knowledge between at least two peoples. We found that the new focus is decisive and has implications for researchers in information visualization and knowledge management.

Burkhard, RA. 2005, "Towards a Framework and a Model for Knowledge Visualization: Synergies between Information and Knowledge Visualization." in Knowledge and Information Visualization, pp. 238-55. Springer-Verlag, Berlin.

Burkhard, RA, and M Meier. 2005, "Tube Map Visualization: Evaluation of a Novel Knowledge Visualization Application for the Transfer of Knowledge in Long-Term Projects." Journal of Universal Computer Science 11(4):473-94.

This article introduces two theoretical concepts for the emerging field Knowledge Visualization and discusses a new visualization application that was used to communicate a long-term project to various stakeholders in an organization. First, we introduce a theoretical framework and a model for Knowledge Visualization. The framework and the model identify and relate the key-aspects for successful Knowledge Visualization applications. Next, we present an evaluation of an implemented Knowledge Visualization 
application: The Tube Map Visualization. A quality development process had to be established in an education centre for health care professions. Traditional project plans, flyers, and mails did not manage to get the attention, did present overview and detail insufficiently, and did not motivate the employees for actions. Because Visual Metaphors are effective for Knowledge Communication we developed a customized Knowledge Map based on the tube system metaphor. The Tube Map Visualization illustrates the whole project, where each tube line represents a target group and each station a milestone. The visualization was printed as a poster and displayed at prominent locations in the organization. The findings of an evaluation indicate that the Tube Map Visualization is a powerful metaphor to communicate a complex project to different target groups and to build up a mutual story. The employees considered it useful because it provides overview and detailed information in one image and because it initiates discussion. The Tube Map Visualization is therefore helpful to complement traditional project plans of ( 1) long-term projects where (2) different stakeholders are involved. The theoretical framework, the model, and the findings have implications for researchers in the fields of Knowledge Management, Knowledge Visualization, Information Visualization, and Communication Sciences.

\section{Burmeister, D. 2005, "The Exchangeability of Speech by Cognitive Metaphors." In 9th International} Conference on Information Visualisation, pp. 89-96.

This article proceeds the reports regarding the development of learning techniques for deaf people based on information visualization. It connects Piagets research about the use of symbol systems with contributions taken from cognitive linguistics concerning cognitive metaphors. On the one hand it is shown, that audible speech is only one of many ways to access abstract content. It is possible to exchange it by visualizations of cognitive metaphors which are a proper visual aid used in special languages. On the other hand a demonstrator program for a knowledge editor based on cognitive metaphors is introduced. This editor may be a useful tool even for analyzing metaphors.

Burnett, M, et al. 2002, "End-User Programming of Time as an [']Ordinary' Dimension in Grid-Oriented Visual Programming Languages." Journal of Visual Languages \& Computing 13(4):421-47.

Specifying varying speeds and temporal relationships is necessary when programming graphical animations, but support for temporal programming has usually been done by adding new language features to a VPL, and these features must be mastered over and above the other aspects of the VPL. However, some researchers have believed that time should be able to be treated like just another dimension. In this paper, we explore whether programming of temporal relationships can be supported usingexactly the same devices as in spatial programming in grid-oriented VPLs. Toward this end, we provide a continuum of models aimed at this goal, with their advantages and disadvantages, and identify core issues that help illuminate the essence of the problem. We also describe an empirical study comparing one of these models against a widely used traditional approach. The results were that end users were significantly more successful using the one model tested than with the traditional approach.

Butavicius, MA, and MD Lee. 2007, "An Empirical Evaluation of Four Data Visualization Techniques for Displaying Short News Text Similarities." International Journal of Human-Computer Studies 65(11):931-44.

An experiment was conducted comparing user performance on four data visualization techniques--an unstructured display condition consisting of a random one-dimensional (1D) list and three proximitybased representations including a 1D list ranked by a greedy nearest-neighbor algorithm and two 2D 
spatial visualizations using the ISOMAP layout algorithm and multidimensional scaling (MDS). Eighty-one participants completed an information retrieval task where the visualization techniques were used to display a corpus consisting of 50 short news texts. Human pairwise similarity judgments for this corpus were used to create the three proximity-based displays. Results demonstrated an advantage in accuracy, the number of documents accessed, and, to a lesser extent, subjective confidence in these displays over the Random List condition and in the 2D over the 1D displays. Similar, but smaller, advantages were observed in the MDS display over ISOMAP however none of these pairwise comparisons were statistically significant. A sequential analysis of participant actions in terms of the proximity of document representations accessed provided some explanation for variations in performance between the displays as well as indicating strategic differences in interactions particularly between visualizations of different dimensionality.

Butkiewicz, T, R Chang, and Z Wartell. 2008, "Visual Analysis for Live Lidar Battlefield Change Detection." In SPIE Defense and Security.

Butkiewicz, T, et al. 2007, "Analyzing Sampled Terrain Volumetrically with Regard to Error and Geologic Variation." In SPIE Conference on Visualization and Data Analysis, pp. 64950O-50O-10.

Butkiewicz, T, et al. 2008, "Multi-Focused Geospatial Analysis Using Probes." IEEE transactions on visualization and computer graphics 14(6):1165-72.

Traditional geospatial information visualizations often present views that restrict the user to a single perspective. When zoomed out, local trends and anomalies become suppressed and lost; when zoomed in for local inspection, spatial awareness and comparison between regions become limited. In our model, coordinated visualizations are integrated within individual probe interfaces, which depict the local data in user-defined regions-of-interest. Our probe concept can be incorporated into a variety of geospatial visualizations to empower users with the ability to observe, coordinate, and compare data across multiple local regions. It is especially useful when dealing with complex simulations or analyses where behavior in various localities differs from other localities and from the system as a whole. We illustrate the effectiveness of our technique over traditional interfaces by incorporating it within three existing geospatial visualization systems: an agent-based social simulation, a census data exploration tool, and an 3D GIS environment for analyzing urban change over time. In each case, the probe-based interaction enhances spatial awareness, improves inspection and comparison capabilities, expands the range of scopes, and facilitates collaboration among multiple users.

Buxton, B. 2007, "References and Bibliography." in Sketching User Experiences: Getting the Design Right and the Right Decision, pp. 421-35. Morgan Kaufmann, Burlington.

Byelas, H, and A Telea. 2009, "Towards Realism in Drawing Areas of Interest on Architecture Diagrams." Journal of Visual Languages and Computing 20(2):110-28.

Areas of interest (AOIs) are defined as groups of elements of system architecture diagrams that share some common property. Visualizing AOIs is a useful addition to plain diagrams, such as UML diagrams. Some methods have been proposed to automatically draw AOIs on UML diagrams. However, it is not clear whether actual users perceive the results of such methods to be better or worse as compared to human-drawn AOI, and what needs to be improved. We present here a process of studying and improving the perceived quality of computer-drawn AOI. For this, we conducted a qualitative evaluation that 
delivered insight in how users perceive the quality of computer-drawn AOIs as compared to hand-drawn diagrams. Following these results, we derived and implemented several improvements to an existing algorithm for computer-drawn AOIs. Next, we designed a distance metric to quantitatively compare different AOI drawings, and used this metric to show that our improved rendering algorithm creates drawings which are closer to (good) human drawings than the original rendering algorithm. We present here the results of the user evaluation, our improved algorithm for drawing AOIs, and the quantitative analysis performed to compare different drawings. The combined user evaluation, algorithmic improvements, and quantitative comparison method support our claim of having improved the perceived quality and understandability of AOI rendered on architecture diagrams. (C) 2008 Elsevier Ltd. All rights reserved.

Byrne, MD, R Catrambone, and JT Stasko. 1999, "Evaluating Animations as Student Aids in Learning Computer Algorithms." Computers \& Education 33(4):253-78.

We conducted two experiments designed to examine whether animations of algorithms would help students learn the algorithms more effectively. Across the two studies we used two different algorithms -depth-first search and binomial heaps -- and used two different subject populations -- students with little or no computer science background and students who were computer science majors -- and examined whether animations helped students acquire procedural and conceptual knowledge about the algorithms. The results suggest that one way animations may aid learning of procedural knowledge is by encouraging learners to predict the algorithm's behavior. However, such a learning improvement was also found when learners made predictions of an algorithm's behavior from static diagrams. This suggests that prediction, rather than animation per se, may have been the key factor in aiding learning in the present studies. These initial experiments served to highlight a number of methodological issues that need to be systematically addressed in future experiments in order to fully test the relationship between animation and prediction as well as to examine other possible benefits of animations on learning.

Byung-Geuk, K, et al. 1997, "An Integrated Visualization Framework for Interprocessor Communication Using 3-D Virtual Space." In High-Performance Computing on the Information Superhighway, pp. 38388.

As a parallel system employs more processors to become more complex, it is very hard to understand, evaluate, and tune the system without the aid of the performance information visualization. Many researchers have found that the criteria of good visualization usually include scalability, multiple views, context and user interaction. In spite of much research in performance visualization of a parallel system, only a few results are available which meet those criteria in an integrated fashion. The paper presents an integrated visualization framework satisfying those criteria for interprocessor communication using 3-D virtual space. The proposed framework is currently applied to a parallel system SPAX (Scalable Parallel Architecture computer based on Xcent-net) being developed by the ETRI (Electronics and Telecommunications Research Institute)

Caat, M, NM Maurits, and JBTM Roerdink. 2007, "Design and Evaluation of Tiled Parallel Coordinate Visualization of Multichannel Eeg Data." Visualization and Computer Graphics, IEEE Transactions on 13(1):70-79.

The field of visualization assists data interpretation in many areas, but does not manage all types of data equally well. This holds, in particular, for time-varying multichannel EEG data. No existing method 
can successfully visualize simultaneous information from all channels in use at all time steps. To address this problem, a new visualization method is presented based on the parallel coordinate method and making use of a tiled organization. This tiled organization employs a two-dimensional row-column representation, rather than a one-dimensional arrangement in columns as used for classical parallel coordinates. The usefulness of the new method, referred to as tiled parallel coordinates (TPC), is demonstrated by a particular type of EEG data. It can be applied to an arbitrary number of time steps, handling the maximum number of channels currently in use. An extensive user evaluation shows that, for a typical EEG assessment task, data evaluation by the TPC method is faster than by an existing clinical EEG visualization method, without loss of information. The generality of the TPC method makes it widely applicable to other time-varying multivariate data types

Cadik, M, and P Slavik. 2004, "Evaluation of Two Principal Approaches to Objective Image Quality Assessment." In 8th International Conference on Information Visualization, pp. 513-18.

Nowadays, it is evident that we must consider human perceptual properties to visualize information clearly and efficiently. We may utilize computational models of human visual systems to consider human perception well. Image quality assessment is a challenging task that is traditionally approached by such computational models. Recently, a new assessment methodology based on structural similarity has been proposed. We select two representative models of each group, the visible differences predictor and the structural similarity index, for evaluation. We begin with the description of these two approaches and models. We then depict the subjective tests that we have conducted to obtain mean opinion scores. Inputs to these tests included uniformly compressed images and images compressed non-uniformly with regions of interest. Then, we discuss the performance of the two models, and the similarities and differences between the two models. We end with a summary of the important advantages of each approach.

Cai, G, and B Tomaszewski. 2006, "Understanding Government Contexts in Geocollaborative Crisis Management." In 11th International Conference on Intelligent User Interfaces, pp. 441-42.

This panel presentation serves as a forum for sharing perspectives, methodologies, experiences, and lessons on how geocollaborative crisis management (GCCM) is facilitated and supported by advanced information technologies in a variety of government contexts. The goal is to generate intellectual synergy towards understanding the scientific and practical issues for effective facilitation of GCCM.

Caldwell, BS. 2008, "Knowledge Sharing and Expertise Coordination of Event Response in Organizations." Applied Ergonomics 39(4):427-38.

This paper provides an overview of opportunities and challenges for expert coordination, knowledge sharing, and task performance using advanced information and communication technologies. Evolving in part from [Hendrick, H., 1991. Ergonomics in organizational design and management. Ergonomics 34(6), 743-756] discussion of macroergonomics, this paper describes the author's framework for systems engineering analysis of information flow and performance at team and organizational units of analysis. Work in the author's research lab has focused on several aspects of information technology use and team interactions to support shared understandings, task demands, and effective responses in responses to events. Multiple empirical studies are summarized describing evaluations of technology use, task cycles and expert knowledge coordination in several settings, including aerospace, healthcare, and project management. 
Callahan, SP, et al. 2006, "Vistrails: Visualization Meets Data Management." In SIGMOD International Conference on Management of Data, pp. 745-47.

Scientists are now faced with an incredible volume of data to analyze. To successfully analyze and validate various hypothesis, it is necessary to pose several queries, correlate disparate data, and create insightful visualizations of both the simulated processes and observed phenomena. Often, insight comes from comparing the results of multiple visualizations. Unfortunately, today this process is far from interactive and contains many error-prone and time-consuming tasks. As a result, the generation and maintenance of visualizations is a major bottleneck in the scientific process, hindering both the ability to mine scientific data and the actual use of the data. The VisTrails system represents our initial attempt to improve the scientific discovery process and reduce the time to insight. In VisTrails, we address the problem of visualization from a data management perspective: VisTrails manages the data and metadata of a visualization product. In this demonstration, we show the power and flexibility of our system by presenting actual scenarios in which scientific visualization is used and showing how our system improves usability, enables reproducibility, and greatly reduces the time required to create scientific visualizations.

Cammarano, M, et al. 2007, "Visualization of Heterogeneous Data." Computer 13(6):1200-07.

Both the resource description framework (RDF), used in the semantic web, and Maya Viz u-forms represent data as a graph of objects connected by labeled edges. Existing systems for flexible visualization of this kind of data require manual specification of the possible visualization roles for each data attribute. When the schema is large and unfamiliar, this requirement inhibits exploratory visualization by requiring a costly up-front data integration step. To eliminate this step, we propose an automatic technique for mapping data attributes to visualization attributes. We formulate this as a schema matching problem, finding appropriate paths in the data model for each required visualization attribute in a visualization template.

Campbell, BD, et al. 2008, "Emergency Response Planning and Training through Interactive Simulation and Visualization with Decision Support." Architecture:176-80.

Teams of first responders work together to effectively manage a community-wide crisis. Traditionally, key groups such as police, fire, and medical are each trained for specific emergency procedures. Emergency response teams design training exercises to augment an individual's cognition associated with performing time critical roles. The aggregation of all individual cognition, distributed through communications, suggests a situation awareness that an incident commander requires to perform optimal decision-making. We are developing a computer-supported simulation environment with a decision support tool called the RimSim, to facilitate emergency response planning and training of first responders. We modularize RimSim for synchronous multi-player use or asynchronous individual use with simulated participants. Through interactive computer- supported role-play with shared visualizations, we are able to study distributed cognition with a long-term goal of identifying opportunities for improving information management during emergency response. We aim to improve mindful distributed cognition for first responders during emergency response to natural and man-made crises. 
Campbell, C, et al. 2010, "Multi-Viz Data Fusion Vast 2010 Grand Challenge Award : Outstanding Debrief." In IEEE Symposium on Visual Analytics Science \& Technology, pp. 265-66.

Campbell, C, et al. 2010, "Protoviz and Processing : Tracing the Mutations of Disease Vast 2010 Mini Challenge 3." In IEEE Symposium on Visual Analytics Science \& Technology.

Campbell, CS, and PP Maglio. 1999, "Facilitating Navigation in Information Spaces: Road-Signs on the World Wide Web." International Journal of Human-Computer Studies 50(4):309-27.

A series of experiments was conducted to evaluate whether simple hyperlink annotations--traffic lights that represent Internet connection speeds--can facilitate web navigation. Traffic lights are small red, yellow or green images added around the anchor text of each link indicating its connection speed, red for slow, yellow for somewhat fast and green for fastest. The first two experiments showed that traffic lights do not facilitate perceptual processes involved in web navigation (i.e., link localization and visual search). However, traffic lights also do not distract from the process of finding links in hypertext documents and, thus have no perceptual performance cost. The third experiment showed that traffic lights facilitate web navigation performance by improving link evaluation and decision processes. This improvement is particularly marked when link relevance is low or undifferentiated. It was concluded that supplying users with information about Internet connection speeds improves web navigation performance. Thus, traffic lights provide functional cues for efficiently navigating the web.

Cañas, AJ, et al. 2005, "Concept Maps: Integrating Knowledge and Information Visualization." in Knowledge and Information Visualization, pp. 205-19. Springer-Verlag, Berlin.

Cao, N, et al. 2010, "Facetatlas: Multifaceted Visualization for Rich Text Corpora." IEEE Transactions on Visualization and Computer Graphics 16(6):1172-81.

Documents in rich text corpora usually contain multiple facets of information. For example, an article about a specific disease often consists of different facets such as symptom, treatment, cause, diagnosis, prognosis, and prevention. Thus, documents may have different relations based on different facets. Powerful search tools have been developed to help users locate lists of individual documents that are most related to specific keywords. However, there is a lack of effective analysis tools that reveal the multifaceted relations of documents within or cross the document clusters. In this paper, we present FacetAtlas, a multifaceted visualization technique for visually analyzing rich text corpora. FacetAtlas combines search technology with advanced visual analytical tools to convey both global and local patterns simultaneously. We describe several unique aspects of FacetAtlas, including (1) node cliques and multifaceted edges, (2) an optimized density map, and (3) automated opacity pattern enhancement for highlighting visual patterns, (4) interactive context switch between facets. In addition, we demonstrate the power of FacetAtlas through a case study that targets patient education in the health care domain. Our evaluation shows the benefits of this work, especially in support of complex multifaceted data analysis.

Cao, YW, et al. 2007, "A Toolkit to Support Dynamic Social Network Visualization." eds. GP Qiu, et al., Vol 4781, pp. 512-23. Springer-Verlag, Berlin.

In this paper we introduce the design, implementation and evaluation of the Dynamic Visualization Toolkit (DyVT) to support complex dynamic social network visualization. Dynamic aspects of social networks such as spatiotemporal as well as personalized information can be visualized in a common 
toolkit. To that end, an XML-based target language DyVTML is an extension of existing schemata enabling expression, storage and interchange of rich animated social network data. With the language and the available tool support, even less-experienced users can visualize temporal data in animations and spatial data in maps and personalize it with icons and colors. The prototype is evaluated by the visualization of large mailing list data sets.

Cappetti, N, and E Santoro. 1998, "An Application of Computer Visualisation for Solving a Mechanical Design by Fuzzy Set." In 2nd International Conference on Information Visualization, pp. 69-75.

A computer visualisation application has been presented for the fuzzy evaluation of windscreen wiper systems which must satisfy several requirements. Some requirements are modelled by fuzzy sets, and a compensating arithmetic mean is utilised for computing the overall performance. A computer animation of the wiper mechanism motion and rainfall is shown, which enables experts, drivers and passengers to evaluate their degree of satisfaction and the correctness of their judgements. This fuzzy evaluation approach is illustrated by considering five \&ldquo; subjective\&rdquo; requirements (driver visibility, passenger visibility, wiped surface, visibility sensation and trouble sensation), and three design alternatives (traditional single- and double-wiped systems and a variable course system). The results are shown

Caquard, S, and DRF Taylor. 2005, "Chapter 12 Art, Maps and Cybercartography: Stimulating Reflexivity among Map-Users." in Modern Cartography Series: Cybercartography, Theory and Practice, ed. DRF Taylor, Vol Volume 4, pp. 285-307. Academic Press.

In this chapter the case is made for encouraging reflexivity by making map-users aware that any map is a "construction of the image of space" and is thus inherently subjective. Informing map-users of the constructed dimension of maps is particularly vital given the exponential production of maps via the Internet. This growth expands the presence of potentially dogmatic and misleading messages. To address these issues, the interrelation between aesthetics, science and technology, and its impact on the perception of maps by most users is discussed. On the one hand cartographers have widely used the aesthetic dimension of art to increase the impression of objectivity associated with maps and on the other hand, developments in the humanities provide grounds to challenge this concept of map objectivity. In this context the potential of cybercartography to combine multiple media, art, technologies, and perspectives into maps is highlighted. This chapter concludes by arguing for stronger presence of artistic creativity and social criticism in maps on the Internet to stimulate map reflexivity.

Card, S. 2004, "Foreword." in Information Visualization: Perception for Design, pp. xvii-xviii. Academic Press, San Diego.

Card, S. 2004, "From Information Visualization to Sensemaking: Connecting the Mind's Eye to the Mind's Muscle." In IEEE Symposium on Information Visualization.

Looking back on the tenth anniversary of the first Symposium on Information Visualization, much has been accomplished. The computer's power has been exploited to give quick visual form to abstract data, to interact, and to warp detail to follow the user's changing interest. Moreover, the design space of visualizations has been systematized with reference models, taxonomies, and monographs relating visualization to perceptual and graphical constraints. Looking ahead, however, I will argue that the era of pure information visualization is over. The path ahead depends on giving much more attention to the 
purposes of visualization and its use. Leaving aside communication, the purpose of information visualization is insight, or more particularly, a larger process that might be called sensemaking. I will sketch out the nature of sensemaking, exemplify it empirically in a practical, urgent setting, and suggest how theories of sensemaking could be developed. I will then describe systems that subsume information visualization as part of an emerging class of sensemaking systems combining visualization (the mind's eye) with semantic content analysis and sensemaking operations (the mind's muscle). Not surprisingly, a focus on sensemaking is a good generator of new visualizations. But these developments also suggest that it may be time for the information visualization field to alter its boundaries to go beyond the merely visual.

Card, S, J Mackinlay, and B Shneiderman. 1999. Readings in Information Visualization: Using Vision to Think. Mogan Kaufmann Publishers, San Francisco, CA.

Card, SK, and J Mackinlay. 1997, "The Structure of the Information Visualization Design Space." In IEEE Symposium on Information Visualization.

Research on information visualization has reached the point where a number of successful point designs have been proposed and a variety of techniques have been discovered. It is now appropriate to describe and analyze portions of the design space so as to understand the differences among designs and to suggest new possibilities. This paper proposes an organization of the information visualization literature and illustrates it with a series of examples. The result is a framework for designing new visualizations and augmenting existing designs.

Card, SK, P Pirolli, and JD Mackinlay. 1994, "The Cost-of-Knowledge Characteristic Function: Display Evaluation for Direct-Walk Dynamic Information Visualizations." In Human Factors in Computing Systems: Celebrating Interdependence, pp. 238-44.

Card, SK, GG Robertson, and JD Mackinlay. 1991, "The Information Visualizer, an Information Workspace." In SIGCHI conference on Human factors in computing systems: Reaching through technology pp. 181-86.

Cardinaels, E, and PMG van Veen-Dirks. 2010, "Financial Versus Non-Financial Information: The Impact of Information Organization and Presentation in a Balanced Scorecard." Accounting, Organizations and Society 35(6):565-78.

This paper investigates how the organization and presentation of performance measures affect how evaluators weight financial and non-financial measures when evaluating performance. We conduct two experiments, in which participants act as senior executives charged with evaluating two business-unit managers. Performance differences between business units are contained in either a financial or one of the three non-financial categories. Specifically, the first experiment studies how organizing measures in a Balanced Scorecard (BSC) format affects performance evaluations. Our results show that when the performance differences are contained in the financial category, evaluators that use a BSC-format place more weight on financial category measures than evaluators using an unformatted scorecard. Conversely, when performance differences are contained in the non-financial categories, whether measures are organized into a BSC-format or into an unformatted scorecard has no impact on the evaluation. The second experiment shows that when performance markers are added to the scorecards (i.e.,,,+- and $=$ signs for above-target, below-target, and on-target performance), evaluators that use a BSC-format weight 
measures in any category containing a performance difference more heavily than evaluators using an unformatted scorecard. Our findings suggest that firms should carefully consider how to present and organize measures to get the intended effect on performance evaluations.

Carenini, G, and JD Moore. 2006, "Generating and Evaluating Evaluative Arguments." Artificial Intelligence 170(11):925-52.

Evaluative arguments are pervasive in natural human communication. In countless situations people attempt to advise or persuade their interlocutors that something is desirable (vs. undesirable) or right (vs. wrong). With the proliferation of on-line systems serving as personal advisors and assistants, there is a pressing need to develop general and testable computational models for generating and presenting evaluative arguments. Previous research on generating evaluative arguments has been characterized by two major limitations. First, researchers have tended to focus only on specific aspects of the generation process. Second, the proposed approaches were not empirically tested. The research presented in this paper addresses both limitations. We have designed and implemented a complete computational model for generating evaluative arguments. For content selection and organization, we devised an argumentation strategy based on guidelines from argumentation theory. For expressing the content in natural language, we extended and integrated previous work in computational linguistics on generating evaluative arguments. The key knowledge source for both tasks is a quantitative model of user preferences. To empirically test critical aspects of our generation model, we have devised and implemented an evaluation framework in which the effectiveness of evaluative arguments can be measured with real users. Within the framework, we have performed an experiment to test two basic hypotheses on which the design of the computational model is based; namely, that our proposal for tailoring an evaluative argument to the addressee's preferences increases its effectiveness, and that differences in conciseness significantly influence argument effectiveness. The second hypothesis was confirmed in the experiment. In contrast, the first hypothesis was only marginally confirmed. However, independent testing by other researchers has recently provided further support for this hypothesis.

Carpendale, S. 2008, "Evaluating Information Visualizations." in Information Visualization, Vol 4950, pp. 19-45. Springer-Verlag, Berlin.

Information visualization research is becoming more established, and as a result, it is becoming increasingly important that research in this field is validated. With the general increase in information visualization research there has also been an increase, albeit disproportionately small, in the amount of empirical work directly focused on information visualization. The purpose of this chapter is to increase awareness of empirical research in general, of its relationship to information visualization in particular; to emphasize its importance; and to encourage thoughtful application of a greater variety of evaluative research methodologies in information visualization. (C) 2008 Springer-Verlag Berlin Heidelberg. 
Carr, DB. 2001, "Graphics in the Physical Sciences." in Encyclopedia of Physical Science and Technology, ed. AM Robert, pp. 1-14. Academic Press, New York.

Carr, DB. 2005, "Some Recent Graphics Templates and Software for Showing Statistical Summaries." in Handbook of Statistics, eds. EJ Wegman and JL Solka, Vol 24, pp. 415-36. Elsevier.

Carriere, J, and R Kazman. 1995, "Research Report. Interacting with Huge Hierarchies: Beyond Cone Trees." In IEEE Symposium on Information Visualization, pp. 74-81.

The paper describes an implementation of a tool for visualizing and interacting with huge information hierarchies, and some preliminary empirical evaluation of the tool's efficacy. Existing systems for visualizing huge hierarchies using cone trees "break down" once the hierarchy to be displayed exceeds roughly 1000 nodes, due to increasing visual clutter. The paper describes a system called fsviz which visualizes arbitrarily large hierarchies while retaining user control. This is accomplished by augmenting cone trees with several graphical and interaction techniques: usage-based filtering, animated zooming, hand-coupled rotation, fish-eye zooming, coalescing of distant nodes, texturing, effective use of colour for depth cueing, and the applications of dynamic queries. The fsviz system also improves upon earlier cone tree visualization systems through a more elaborate node layout algorithm. This algorithm enhances the usefulness of cone tree visualization for large hierarchies by all but eliminating clutter.

Carroll, JM. 2010, "Conceptualizing a Possible Discipline of Human-Computer Interaction." Interacting with Computers 22(1):3-12.

This essay is a personal reflection on John Long's keynote address at the BCS People and Computers meeting in Nottingham in the summer of 1989. I try to locate the paper's purpose and significance within the history of human-computer interaction (HCI), both prior to 1989 and subsequently, and particularly with respect to the abiding questions of what sort of enterprise HCI is, and of what sorts of knowledge it uses and produces.

Carroll, JM. 2003, "References." in Hci Models, Theories, and Frameworks, ed. MC John, pp. 475-519. Morgan Kaufmann, San Francisco.

Carroll, JM, and MB Rosson. 2008, "Theorizing Mobility in Community Networks." International Journal of Human-Computer Studies 66(12):944-62.

Community networks emerged in North America during the late 1970s and early 1980s. During the past three decades, paradigms for networked information, services, and collaboration as resources for community development have evolved in many respects. In this paper we revisit a theoretical analysis of broadband Internet community networks [Carroll, J.M., Rosson, M.B., 2003a. A trajectory for community networks. The Information Society 19(5), 381-393], and extend that analysis to mobile/wireless community networks. This analysis is part of the planning for a specific municipal wireless project in the town of State College, Pennsylvania. But more broadly, it is intended to engage and to help focus humancomputer interaction (HCI) design perspectives in the development of wireless community networks throughout North America and elsewhere. 
Cartwright, W. 2003, "Chapter 3 - Maps on the Web." in Maps and the Internet, ed. P Michael, pp. 35-56. Elsevier Science, Oxford.

Summary Providing maps on the Web and the different strategies required for Web Cartography has changed the genre of the communication of geographical information. Web cartography, in its portrayal methods and viewing conditions, differs from paper maps, including these produced by CAD or DeskTop Publishing (DTP) packages.

Cartwright, WE. 2005, "Chapter 14 Linking Geographical Facts with Cartographic Artifacts." in Modern Cartography Series: Cybercartography - Theory and Practice, ed. DRF Taylor, Vol Volume 4, pp. 33147. Academic Press.

The design of a paper atlas took into account how the various elements -- maps, diagrams, photographs, etc. -- could be "fused" to provide a harmonious and complete product. Paper atlases allowed users to explore geography through the use of cartographic artifacts alongside presentations of geographical facts. Now, we are presented with a myriad of Rich Media alternatives and interactive and online enhancements with atlases delivered through the Internet, and particularly the World Wide Web (WWW). It could be argued that cartographic publishers of online products may be guilty of planning to assault their potential users with a bombardment of geographical resources and tools. The combination of an efficient delivery mechanism, the Web; engaging information displays, New Media products; powerful tools for exploring geography, maps; online access to current geographical information, data resources available via Web-enabled repositories; and good interface and interaction practice, innovative maps and maprelated products; can provide a wonderful mechanism for data prospecting and information mining. Consequently, there is now much interest in ensuring that such packages are designed and built in a way that best exploits existing and evolving media, both delivery medium and access/usage medium. This chapter explores the elements of the design of contemporary New Media atlas products, and how designers need to focus on how best to deliver both geographical facts and cartographic artifacts so as to ensure the provision of an "exploitable" information resource.

Carvalho, E, et al. 2008, "A Prototype for Cartographic Human Body Analysis." Computer Graphics and Applications, IEEE 28(5):16-21.

A cartographic-oriented model uses algebraic map operations to perform spatial analysis of medical data relative to the human body. A prototype system uses 3D visualization techniques to deliver analysis results. A prototype implementation suggests the model might provide the basis for a medical application tool that introduces new information insight.

Carvalho, ES. 1998, "An Application Model for Scientific Visualization in Hydrologic Basins Management." In IEEE International Conference on Information Visualization, pp. 93-98.

Scientific visualization is a science of great importance nowadays. There are already several visual techniques, but in the field of hydrological basins there are still many questions to be answered. There is no computer application model that uses scientific visualization techniques as a key for information transmission in this scientific area. Another important aspect is that hydrological basins imply geographical data too. This means that GIS techniques and potentialities should be also considered. As a matter of fact, there is a lack of proper computer models in this area. Such a model should include some of the emerging hybrid aspects that are appearing in scientific visualization and in general systems design. 
It should combine GIS and scientific visualization techniques properly. This paper introduces a computer application model that can be used to develop an application for hydrological basin management, defining its general architectural model, processes, software and hardware supports, and visual and cognitive models

Castellani, U, et al. 2008, "Visual Mri: Merging Information Visualization and Non-Parametric Clustering Techniques for Mri Dataset Analysis." Artificial Intelligence in Medicine 44(3):183-99.

SummaryObjective This paper presents Visual MRI, an innovative tool for the magnetic resonance imaging (MRI) analysis of tumoral tissues. The main goal of the analysis is to separate each magnetic resonance image in meaningful clusters, highlighting zones which are more probably related with the cancer evolution. Such non-invasive analysis serves to address novel cancer treatments, resulting in a less destabilizing and more effective type of therapy than the chemotherapy-based ones. The advancements brought by Visual MRI are two: first, it is an integration of effective information visualization (IV) techniques into a clustering framework, which separates each MRI image in a set of informative clusters; the second improvement relies in the clustering framework itself, which is derived from a recently rediscovered non-parametric grouping strategy, i.e., the mean shift.Methodology The proposed methodology merges visualization methods and data mining techniques, providing a computational framework that allows the physician to move effectively from the MRI image to the images displaying the derived parameter space. An unsupervised non-parametric clustering algorithm, derived from the mean shift paradigm, and called MRI-mean shift, is the novel data mining technique proposed here. The main underlying idea of such approach is that the parameter space is regarded as an empirical probability density function to estimate: the possible separate modes and their attraction basins represent separated clusters. The mean shift algorithm needs sensibility threshold values to be set, which could lead to highly different segmentation results. Usually, these values are set by hands. Here, with the MRI-mean shift algorithm, we propose a strategy based on a structured optimality criterion which faces effectively this issue, resulting in a completely unsupervised clustering framework. A linked brushing visualization technique is then used for representing clusters on the parameter space and on the MRI image, where physicians can observe further anatomical details. In order to allow the physician to easily use all the analysis and visualization tools, a visual interface has been designed and implemented, resulting in a computational framework susceptible of evaluation and testing by physicians.Results Visual MRI has been adopted by physicians in a real clinical research setting. To describe the main features of the system, some examples of usage on real cases are shown, following step by step all the actions scientists can do on an MRI image. To assess the contribution of Visual MRI given to the research setting, a validation of the clustering results in a medical sense has been carried out.Conclusions From a general point of view, the two main objectives reached in this paper are: (1) merging information visualization and data mining approaches to support clinical research and (2) proposing an effective and fully automated clustering technique. More particularly, a new application for MRI data analysis, named Visual MRI, is proposed, aiming at improving the support of medical researchers in the context of cancer therapy; moreover, a nonparametric technique for cluster analysis, named MRI-mean shift, has been drawn. The results show the effectiveness and the efficacy of the proposed application.

Catarci, T. 2000, "What Happened When Database Researchers Met Usability." Information Systems 25(3):177-212.

This paper is about database researchers building interactive information systems. It tells the reader of their initial enthusiasm and user-induced frustration, the consequent discovery of both human-computer 
interaction (hci) world and the concept of system "usability", and finally their quite successful coupling of hci and databases.

Cawthon, N, and AV Moere. 2006, "A Conceptual Model for Evaluating Aesthetic Effect within the User Experience of Information Visualization." In 10th International Conference on Information Visualization, pp. 374-79.

This paper proposes a conceptual model for which one might begin to assess aesthetic effect within the user experience of information visualization. Through first defining, then illustrating new dimensions of aesthetic that exist within the context of information visualization this paper goes on to suggest that a user centered evaluation method not solely centered around task efficiency metrics is now imperative.

Cawthon, N, and AV Moere. 2007, "The Effect of Aesthetic on the Usability of Data Visualization." In 11th International Conference on Information Visualization, pp. 637-48.

Aesthetic seems currently under represented in most current data visualization evaluation methodologies. This paper investigates the results of an online survey of 285 participants, measuring both perceived aesthetic as well as the efficiency and effectiveness of retrieval tasks across a set of 11 different data visualization techniques. The data visualizations represent an identical hierarchical dataset, which has been normalized in terms of color, typography and layout balance. This study measured parameters such as speed of completion, accuracy rate, task abandonment and latency of erroneous response. Our findings demonstrate a correlation between latency in task abandonment and erroneous response time in relation to visualization's perceived aesthetic. These results support the need for an increased recognition for aesthetic in the typical evaluation process of data visualization techniques.

Cedilnik, A, et al. 2008, "Integration of Information and Volume Visualization for Analysis of Cell Lineage and Gene Expression During Embryogenesis." In SPIE Conference on Visualization and Data Analysis.

Dramatic technological advances in the field of genomics have made it possible to sequence the complete genomes of many different organisms. With this overwhelming amount of data at hand, biologists are now confronted with the challenge of understanding the function of the many different elements of the genome. One of the best places to start gaining insight on the mechanisms by which the genome controls an organism is the study of embryogenesis. There are multiple and inter-related layers of information that must be established in order to understand how the genome controls the formation of an organism. One is cell lineage which describes how patterns of cell division give rise to different parts of an organism. Another is gene expression which describes when and where different genes are turned on. Both of these data types can now be acquired using fluorescent laser-scanning (confocal or 2-photon) microscopy of embryos tagged with fluorescent proteins to generate 3D movies of developing embryos. However, analyzing the wealth of resulting images requires tools capable of interactively visualizing several different types of information as well as being scalable to terabytes of data. This paper describes how the combination of existing large data volume visualization and the new Titan information visualization framework of the Visualization Toolkit (VTK) can be applied to the problem of studying the cell lineage of an organism. In particular, by linking the visualization of spatial and temporal gene expression data with novel ways of visualizing cell lineage data, users can study how the genome regulates different aspects of embryonic development. 
Chakrabarty, R. 2010, "A Visual Analytics System to Study the Spread of Pandemics." In IEEE Symposium on Visual Analytics Science \& Technology, pp. 1-2.

Chakravarty, S, and Y Shahar. 2001, "Acquisition and Analysis of Repeating Patterns in Time-Oriented Clinical Data." Methods of Information in Medicine 40(5):410-20.

Objectives: (1) Creation of an expressive language for specification of temporal patterns in clinical domains, (2) Development of a graphical knowledge-aquisition tool allowing expert physicians to define meaningful domain-specific patterns, (3) Implementation of on interpreter capable of detecting such patterns in clinical databases, and (4) Evaluation of the tools in the domains of diabetes and oncology. Methods. We describe a constraint-based language, named CAPSUL, for specification of temporal patterns. We implemented a knowledge-acquisition tool and a temporal-pattern interpreter within Resume, a larger temporal-abstraction architecture. We evaluated the knowledge-acquisition process with the help of domain experts. In collaboration with the Rush Presbyterain/St. Luke's Medical Center, we analyzed data of bone-marrow transplantation patients. The expert compared the detected patterns to a manual inspection of the data, with the help of on experimental information-visualization tool we are developing in a related project. Results: The CAPSUL language was expressive enough during the knowledge-acquisition process to capture almost all of the patterns that-the experts found useful. The patterns detected in the data by the pattern interpreter were all verified as correct. Completeness (whether all correct patterns were found) was difficult to assess, due to the size of the database. Conclusions: The CAPSUL language enables medical experts to express temporal patterns involving multiple-levels of abstraction of clinical data. The ability to reuse both domain-patterns and abstract constraints seems highly useful. The Resume interpreter, augmented by the CAPSUL semantics, finds the complex patterns within a clinical time-oriented database in a sound fashion.

Chan, B, et al. 2008, "Vispedia * : Interactive Visual Exploration of Wikipedia Data Via Search-Based Integration." Visualization and Computer Graphics 14(6):1213-20.

Wikipedia is an example of the collaborative, semi-structured data sets emerging on the Web. These data sets have large, non-uniform schema that require costly data integration into structured tables before visualization can begin. We present Vispedia, a Web-based visualization system that reduces the cost of this data integration. Users can browse Wikipedia, select an interesting data table, then use a search interface to discover, integrate, and visualize additional columns of data drawn from multiple Wikipedia articles. This interaction is supported by a fast path search algorithm over DBpedia, a semantic graph extracted from Wikipedia's hyperlink structure. Vispedia can also export the augmented data tables produced for use in traditional visualization systems. We believe that these techniques begin to address the "long tail" of visualization by allowing a wider audience to visualize a broader class of data. We evaluated this system in a first-use formative lab study. Study participants were able to quickly create effective visualizations for a diverse set of domains, performing data integration as needed. 
Chan, Y-h, and CD Correa. 2010, "Flow-Based Scatterplots for Sensitivity Analysis." In IEEE Symposium on Visual Analytics Science \& Technology, pp. 43-50.

Chang, H-S, S-C Kang, and P-H Chen. 2009, "Systematic Procedure of Determining an Ideal Color Scheme on 4d Models." Advanced Engineering Informatics 23(4):463-73.

Four-dimensional models, which are 3D models with an added dimension to represent schedule information, have become an important tool in representing construction processes. These models usually rely on colors to represent the different construction states, such that when an ideal color scheme is used, engineers are able to understand the model and identify the potential problems more easily. However, up to this point, little research has been conducted in this area. This paper presents the selection, examination, and user test (SEUT) procedure, a systematic procedure to determine the ideal color scheme for a 4D model. This procedure can be performed iteratively to obtain the ideal color scheme, which would fit a 4D model according to its construction purposes. After conducting an example case following the proposed procedure, we determined an ideal color scheme for six construction states of a 4D model for plant construction. In total ten color schemes were examined and testing was conducted by 58 users over two iterations. The results show that the SEUT procedure is an effective method for determining color schemes to present 4D models and an ideal color scheme was validated and recommended in this research.

Chang, R, T Butkiewicz, and C Ziemkiewicz. 2006, "Hierarchical Simplification of City Models to Maintain Urban Legibility." In ACM SIGGRAPH.

Mesh simplification and discrete levels of detail (LOD) are wellstudied areas of research in computer graphics. However, until recently, most of the developed algorithms have focused on simplification and viewing of a single object with a large number of polygons. When these algorithms are used on a large collection of simple models, many objects may be completely erased, leading to results that are misleading to the viewer. In this paper, we present a novel approach to simplifying city-sized collections of 2.5D buildings based on the principles of "urban legibility" as defined by architects and city planners. Our main contributions include a clustering algorithm tailored towards forming logical groups while respecting roads, a polyline simplification algorithm that maintains boundary facades, and a LOD process that preserves landmarks and skylines. The advantage of our approach is that the legibility and understandability of a complex urban space is preserved at all levels of simplification.

Chang, R, et al. 2008, "Legible Simplification of Textured Urban Models." IEEE Computer Graphics and Applications 28(3):27-36.

Chang, R, et al. 2007, "Wirevis: Visualization of Categorical, Time-Varying Data from Financial Transactions." In IEEE Symposium on Visual Analytics Science and Technology.

Large financial institutions such as Bank of America handle hundreds of thousands of wire transactions per day. Although most transactions are legitimate, these institutions have legal and financial obligations in discovering those that are suspicious. With the methods of fraudulent activities ever changing, searching on predefined patterns is often insufficient in detecting previously undiscovered methods. In this paper, we present a set of coordinated visualizations based on identifying specific keywords within the wire transactions. The different views used in our system depict relationships among keywords and accounts over time. Furthermore, we introduce a search-by-example technique which 
extracts accounts that show similar transaction patterns. In collaboration with the Anti-Money Laundering division at Bank of America, we demonstrate that using our tool, investigators are able to detect accounts and transactions that exhibit suspicious behaviors.

Chang, R, et al. 2008, "Scalable and Interactive Visual Analysis of Financial Wire Transactions for Fraud Detection." Information Visualization 7(1):63-76.

Chang, R, et al. 2007, "Legible Cities: Focus-Dependent Multi-Resolution Visualization of Urban Relationships." IEEE Transactions on Visualization and Computer Graphics 13(6):1169-75.

Numerous systems have been developed to display large collections of data for urban contexts; however, most have focused on layering of single dimensions of data and manual calculations to understand relationships within the urban environment. Furthermore, these systems often limit the user's perspectives on the data, thereby diminishing the user's spatial understanding of the viewing region. In this paper, we introduce a highly interactive urban visualization tool that provides intuitive understanding of the urban data. Our system utilizes an aggregation method that combines buildings and city blocks into legible clusters, thus providing continuous levels of abstraction while preserving the user's mental model of the city. In conjunction with a 3D view of the urban model, a separate but integrated information visualization view displays multiple disparate dimensions of the urban data, allowing the user to understand the urban environment both spatially and cognitively in one glance. For our evaluation, expert users from various backgrounds viewed a real city model with census data and confirmed that our system allowed them to gain more intuitive and deeper understanding of the urban model from different perspectives and levels of abstraction than existing commercial urban visualization systems.

Chang, R, et al. 2009, "Defining Insight for Visual Analytics." IEEE Computer Graphics and Applications 29(2):14-17.

Many have proposed that the goal of visualization is insight. However, few rigorous definitions of insight exist in the visualization community, and none have been commonly accepted. We propose that two types of insight are relevant to the visualization community. We examine the definition of insight cognitive scientists use (spontaneous insight) and compare it with the definitions the visualization community uses (knowledge-building insight). The two, although distinct, are related and contribute toward each other. Only by understanding how the two types differ and interact with each other can the visualization community accurately measure insight and determine the effectiveness of visualizations. (C) 2009 IEEE.

Chang, R, et al. 2010, "Learning-Based Evaluation of Visual Analytic Systems." In BELIV'10: Beyond time and errors: novel evaluation methods for Information Visualization, April 10-11, 2010.

Evaluation in visualization remains a difficult problem because of the unique constraints and opportunities inherent to visualization use. While many potentially useful methodologies have been proposed, there remain significant gaps in assessing the value of the open-ended exploration and complex task-solving that the visualization community holds up as an ideal. In this paper, we propose a methodology to quantitatively evaluate a visual analytics (VA) system based on measuring what is learned by its users as the users reapply the knowledge to a different problem or domain. The motivation for this methodology is based on the observation that the ultimate goal of a user of a VA system is to gain knowledge of and expertise with the dataset, task, or tool itself. We propose a framework for describing 
and measuring knowledge gain in the analytical process based on these three types of knowledge and discuss considerations for evaluating each. We propose that through careful design of tests that examine how well participants can reapply knowledge learned from using a VA system, the utility of the visualization can be more directly assessed.

Chang, S-K, and E Jungert. 1996, "Visual Queries." in Symbolic Projection for Image Information Retrieval and Spatial Reasoning, pp. 227-45. Academic Press, San Diego.

Chao, WO. 2010, "Poster : Rapid Pen-Centric Authoring of Improvisational Visualizations with Napkinvis." In IEEE International Conference on Information Visualization.

Chapman, A, HV Jagadish, and A Arbor. 2009, "Why Not?" In SIGMOD Conference, pp. 523-34.

As humans, we have expectations for the results of any action, e.g., we expect at least one student to be returned when we query a uni- versity database for student records. When these expectations are not met, traditional database users often explore datasets via a se- ries of slightly altered SQL queries. Yet most database access is via limited interfaces that deprive end users of the ability to alter their query in any way to garner better understanding of the dataset and result set. Users are unable to question why a particular data item is Not in the result set of a given query. In this work, we develop a model for answers toWHY NOT? queries. We show through a user study the usefulness of our answers, and describe two algorithms for finding themanipulation that discarded the data itemof interest. Moreover, we work through two different methods for tracing the discarded data item that can be used with either algorithm. Using our algorithms, it is feasible for users to find the manipulation that excluded the data item of interest, and can eliminate the need for exhausting debugging.

Chávez, E, R Ide, and T Kirste. 1999, "Interactive Applications of Personal Situation-Aware Assistants." Computers \& Graphics 23(6):903-15.

Ultraportable mobile computers provide electronic assistance for environments and usage situations, where computer support up to now has not been feasible. For the first time, a true physical and cognitive integration of computer support into the everyday business of the real-world becomes possible, as envisioned in Mark Weiser's concept of "ubiquitous computing" (Weiser, Communication of the ICM 1993, 36 (12): 75-85). However, although Handheld-PC, etc. today; already support a good deal of personal information management and basic access to distributed multimedia information services such as the World-Wide Web, they are still surprisingly difficult to use in "full action". Specifically, lengthy interaction sequences and the inability to find quickly that important piece of information which is embedded somewhere in the machine, makes using those devices sometimes a very disappointing experience. In this, paper, we outline a new approach to realizing an easy-to-use personal digital assistant systems, based on the concept of Situation Awareness. Using knowledge about task structures, situation dependencies, and task contexts, our concept allows a mobile assistant to proactively provide the right information at the right time and the right place, without intruding upon the users primary task: interacting with reality. 
Chen, C. 2010, "Information Visualization." Wiley Interdisciplinary Reviews: Computational Statistics 2(4):387-403.

Abstract This overview introduces the key structure of the field of information visualization, a number of influential exemplars in the field, and challenging as well as promising directions of future developments. The focus is on explaining some of the most fundamental concepts, prominent approaches, and commonly held criteria. The overview also aims to point out theoretical and practical challenges that the community as a whole has been addressing. Copyright (C) 2010 John Wiley \& Sons, Inc. For further resources related to this article, please visit the WIREs website

Chen, C. 2004. Information Visualization: Beyond the Horizon, 2nd Ed. 2nd ed., Springer-Verlag, London.

Chen, C. 2008, "An Information-Theoretic View of Visual Analytics." IEEE Computer Graphics and Applications 28(1):18-23.

Visual analytics is an emerging discipline that helps connect dots. It facilitates analytical reasoning and decision making through integrated and highly interactive visualization of complex and dynamic data and situations. Solving mysteries is only part of the game. Visual analytics must augment analyst and decision-maker capabilities to assimilate complex situations and reach informed decisions. In information theory, the information value carried by a message is the difference in information entropy before and after receipt of the message. Information entropy is a macroscopic measure of uncertainty defined on a frequency or probability distribution. The information-theoretical approach attempts to quantify discrepancies of the information content of distributions

Chen, C. 2005, "Top 10 Unsolved Information Visualization Problems." Computer Graphics and Applications, IEEE 25(4):12-16.

The top 10 unsolved problems list described in this article is a revised and extended version of information visualization problems. These problems are not necessarily imposed by technical barriers, rather, they are problems that might hinder the growth of information visualization as a field. The first three problems highlight issues from a user-centered perspective. The fifth, sixth, and seventh problems are technical challenges in nature. The last three are the ones that need tackling at the disciplinary level. The author broadly defines information visualization as visual representations of the semantics, or meaning, of information. In contrast to scientific visualization, information visualization typically deals with nonnumeric, nonspatial, and high-dimensional data.

Chen, C, and L Carr. 1999, "Visualizing the Evolution of a Subject Domain: A Case Study." In Visualization' '99, pp. 449-561.

We explore the potential of information visualization techniques in enhancing existing methodologies for domain analysis and modeling. In this case study, we particularly focus on visualizing the evolution of the hypertext field based on author co-citation patterns, including the use of a sliding-window scheme to generate a series of annual snapshots of the domain structure, and a factor-referenced color-coding scheme to highlight predominant specialties in the field. 
Chen, C, et al. 2009, "Towards an Explanatory and Computational Theory of Scientific Discovery." Journal of Informetrics 3(3):191-209.

We propose an explanatory and computational theory of transformative discoveries in science. The theory is derived from a recurring theme found in a diverse range of scientific change, scientific discovery, and knowledge diffusion theories in philosophy of science, sociology of science, social network analysis, and information science. The theory extends the concept of structural holes from social networks to a broader range of associative networks found in science studies, especially including networks that reflect underlying intellectual structures such as co-citation networks and collaboration networks. The central premise is that connecting otherwise disparate patches of knowledge is a valuable mechanism of creative thinking in general and transformative scientific discovery in particular. In addition, the premise consistently explains the value of connecting people from different disciplinary specialties. The theory not only explains the nature of transformative discoveries in terms of the brokerage mechanism but also characterizes the subsequent diffusion process as optimal information foraging in a problem space. Complementary to epidemiological models of diffusion, foraging-based conceptualizations offer a unified framework for arriving at insightful discoveries and optimizing subsequent pathways of search in a problem space. Structural and temporal properties of potentially highimpact scientific discoveries are derived from the theory to characterize the emergence and evolution of intellectual networks of a field. Two Nobel Prize winning discoveries, the discovery of Helicobacter pylori and gene targeting techniques, and a discovery in string theory demonstrated such properties. Connections to and differences from existing approaches are discussed. The primary value of the theory is that it provides not only a computational model of intellectual growth, but also concrete and constructive explanations of where one may find insightful inspirations for transformative scientific discoveries.

Chen, C, et al. 2002, "Footprints of Information Foragers: Behaviour Semantics of Visual Exploration." International Journal of Human-Computer Studies 57(2):139-63.

Social navigation exploits the knowledge and experience of peer users of information resources. A wide variety of visual-spatial approaches become increasingly popular as a means to optimize information access as well as to foster and sustain a virtual community among geographically distributed users. An information landscape is among the most appealing design options of representing and communicating the essence of distributed information resources to users. A fundamental and challenging issue is how an information landscape can be designed such that it will not only preserve the essence of the underlying information structure, but also accommodate the diversity of individual users. The majority of research in social navigation has been focusing on how to extract useful information from what is in common between users' profiles, their interests and preferences. In this article, we explore the role of modelling sequential behaviour patterns of users in augmenting social navigation in thematic landscapes. In particular, we compare and analyse the trails of individual users in thematic spaces along with their cognitive ability measures. We are interested in whether such trails can provide useful guidance for social navigation if they are embedded in a visual-spatial environment. Furthermore, we are interested in whether such information can help users to learn from each other, for example, from the ones who have been successful in retrieving documents. In this article, we first describe how users' trails in sessions of an experimental study of visual information retrieval can be characterized by Hidden Markov Models. Trails of users with the most successful retrieval performance are used to estimate parameters of such models. Optimal virtual trails generated from the models are visualized and animated as if they were actual trails of individual users in order to highlight behavioural patterns that may foster social navigation. The findings of the research will provide direct input to the design of social navigation systems as well as to 
enrich theories of social navigation in a wider context. These findings will lead to the further development and consolidation of a tightly coupled paradigm of spatial, semantic and social navigation.

Chen, C, and MP Czerwinski. 2000, "Empirical Evaluation of Information Visualizations: An Introduction." International Journal of Human Computer Studies 53(5):631-36.

Chen, C, et al. 2008, "The Thematic and Citation Landscape of Data and Knowledge Engineering (19852007)." Data \& Knowledge Engineering 67(2):234-59.

The thematic and citation structures of Data and Knowledge Engineering (DKE) (1985-2007) are identified based on text analysis and citation analysis of the bibliographic records of full papers published in the journal. Temporal patterns are identified by detecting abrupt increases of frequencies of noun phrases extracted from titles and abstracts of DKE papers over time. Conceptual structures of the subject domain are identified by clustering analysis. Concept maps and network visualizations are presented to illustrate salient patterns and emerging thematic trends. A variety of statistics are reported to highlight key contributors and DKE papers that have made profound impacts.

Chen, C, and YUE Yu. 2000, "Empirical Studies of Information Visualization: A Meta-Analysis." International Journal of Human-Computer Studies 53(5):851-66.

A meta-analysis is conducted on a set of empirical studies of information visualization. To be included in the meta-analysis, a study must meet a set of selection criteria. The meta-analysis synthesizes significant levels and effect sizes, tests the heterogeneity of findings from individual studies included and tests the linear trends over a range of information visualization features with ascending visual-spatial complexity. Recommendations for future experimental studies of information visualizations are included.

Chen, C, et al. 2007, "Tracing Conceptual and Geospatial Diffusion of Knowledge." in Online Communities and social computing, Vol 4564, pp. 265-74. Springer-Verlag, Berlin.

Chen, CD, A Woodcock, and SAR Scrivener. 2003, "The Development of a Method to Inform Cmc Design for Visual Communication." In 7th International Conference on Information Visualization, pp. 421-26.

An essential characteristic of design communication is the need to talk about things (objects and concepts). We concern the development of a practical method to assist the iterative development of computer-mediated communication (CMC) systems that support talk by designers working co-operatively over distance. Previous approaches to the study of CMC systems limit the extent to which the value of new media and mechanisms can be explored. We have developed an alternative method to exploring CMCs based on shifts between communication resources during design activity, based on Scrivener's (2000) postulate that users in a communication environment satisfy their communication purposes by selecting from the available resources those most appropriate for their communication purpose. Such shifts reveal insights into the relative strengths and weaknesses of the communication channels. We outline the rationale behind shift analysis, provide examples of the analysis and conclude by reviewing the steps needed to use this method in iterative CMC development. 
Chen, CM, et al. 2002, "Footprints of Information Foragers: Behaviour Semantics of Visual Exploration." International Journal of Human-Computer Studies 57(2):139-63.

Social navigation exploits the knowledge and experience of peer users of information resources. A wide variety of visual-spatial approaches become increasingly popular as a means to optimize information access as well as to foster and sustain a virtual community among geographically distributed users. An information landscape is among the most appealing design options of representing and communicating the essence of distributed information resources to users. A fundamental and challenging issue is how an information landscape can be designed such that it will not only preserve the essence of the underlying information structure, but also accommodate the diversity of individual users. The majority of research in social navigation has been focusing on how to extract useful information from what is in common between users' profiles, their interests and preferences. In this article, we explore the role of modelling sequential behaviour patterns of users in augmenting social navigation in thematic landscapes. In particular, we compare and analyse the trails of individual users in thematic spaces along with their cognitive ability measures. We are interested in whether such trails can provide useful guidance for social navigation if they are embedded in a visual-spatial environment. Furthermore, we are interested in whether such information can help users to learn from each other, for example, from the ones who have been successful in retrieving documents. In this article, we first describe how users' trails in sessions of an experimental study of visual information retrieval can be characterized by Hidden Markov Models. Trails of users with the most successful retrieval performance are used to estimate parameters of such models. Optimal virtual trails generated from the models are visualized and animated as if they were actual trails of individual users in order to highlight behavioural patterns that may foster social navigation. The findings of the research will provide direct input to the design of social navigation systems as well as to enrich theories of social navigation in a wider context. These findings will lead to the further development and consolidation of a tightly coupled paradigm of spatial, semantic and social navigation. (C) 2002 Elsevier Science Ltd. All rights reserved.

Chen, F, Y Zhao, and Z Yuan. 2010, "Spectral Modeling of Divergence-Free Vector Fields." In IEEE International Visualization Conference, pp. 1-2.

Chen, H, and AL Houston. 1999, "Digital Libraries: Social Issues and Technological Advances." Advances in Computers 48:257-314.

Abstract The location and provision of information services has dramatically changed over the last ten years. There is no need to leave the home or office to locate and access information now readily available on-line via digital gateways furnished by a wide variety of information providers, (e.g., libraries, electronic publishers, businesses, organizations, individuals). Information access is no longer restricted to what is physically available in the nearest library. It is electronically accessible from a wide variety of globally distributed information repositories--"digital libraries". In this chapter we will focus on digital libraries, starting with a discussion of the historical visionaries, definitions, driving forces and enabling technologies and some key research issues. We will discuss some of the US and international digital library projects and research initiatives. We will then describe some of the emerging techniques for building large-scale digital libraries, including a discussion of semantic interoperability, the "Grand Challenge" of digital library research. Finally, we offer our conclusions and a discussion of some future directions for digital libraries. 
Chen, IX, CZ Yang, and TL Hsu. 2006, "Design and Evaluation of a Panoramic Visualization Environment on Semantic Web." Information and Software Technology 48(6):402-09.

Information visualization has been popularly applied to the Semantic Web to facilitate the presentation of data semantics. Since, the past visualization interfaces, based either on RDF or on Topic Maps, only present a partial view of the Semantic Web space, an integrated view would be needed to demonstrate the panorama and help users access the Semantic Web resources. We have presented an Integrated Semantic Web interactive visualization environment (ISWIVE) to incorporate topic features from Topic Maps into RDF, and showed that both the detailed resource descriptions and the overall topic relationships can be clearly visualized. In this paper, we present an improved ISWIVE interface that provides a mixed-model visualization in a clearer layout and supports a concurrent display of both RDF and Topic Maps views. Quantitative analyses were conducted to show the enhancement of the improved ISWIVE interface and the differences between the textual interface and the original ISWIVE prototype. (C) 2006 Elsevier B.V. All fights reserved.

Chen, J. 2006, "Visual Inquiry of Spatio-Temporal Multivariate Patterns." In IEEE Symposium on Visual Analytics Science and Technology, pp. 1-2.

Chen, J, et al. 2007, "Getting Human-Centered Computing and Scientific Visualization Married : The Myth and Critical Issues." In 2007 IEEE Visualization Conference.

Chen, J, A Maceachren, and E Lengerich. 2008, "Visual Analytics of Spatial Scan Statistic Results." In Geospatial Visual Analytics Workshop, pp. 2-5.

Chen, J, and AM MacEachren. 2008, "Resolution Control for Balancing Overview and Detail in Multivariate Spatial Analysis." Cartographic Journal, The 45(4):261-73.

Chen, J, AM Maceachren, and D Guo. 2008, "Supporting the Process of Exploring and Interpreting Space-Time Multivariate Patterns: The Visual Inquiry Toolkit." Cartography 35(1):33-50.

While many data sets carry geographic and temporal references, our ability to analyze these datasets lags behind our ability to collect them because of the challenges posed by both data complexity and tool scalability issues. This study develops a visual analytics approach that leverages human expertise with visual, computational, and cartographic methods to support the application of visual analytics to relatively large spatio-temporal, multivariate data sets. We develop and apply a variety of methods for data clustering, pattern searching, information visualization, and synthesis. By combining both human and machine strengths, this approach has a better chance to discover novel, relevant, and potentially useful information that is difficult to detect by any of the methods used in isolation. We demonstrate the effectiveness of the approach by applying the Visual Inquiry Toolkit we developed to analyze a data set containing geographically referenced, time-varying and multivariate data for U.S. technology industries.

Chen, J, AM MacEachren, and DJ Peuquet. 2009, "Constructing Overview Plus Detail DendrogramMatrix Views." IEEE Transactions on Visualization and Computer Graphics 15(6):889-96.

A dendrogram that visualizes a clustering hierarchy is often integrated with a reorderable matrix for pattern identification. The method is widely used in many research fields including biology, geography, statistics, and data mining. However, most dendrograms do not scale up well, particularly with respect to 
problems of graphical and cognitive information overload. This research proposes a strategy that links an overview dendrogram and a detail-view dendrogram, each integrated with a re-orderable matrix. The overview displays only a user-controlled, limited number of nodes that represent the "skeleton" of a hierarchy. The detail view displays the sub-tree represented by a selected meta-node in the overview. The research presented here focuses on constructing a concise overview dendrogram and its coordination with a detail view. The proposed method has the following benefits: dramatic alleviation of information overload, enhanced scalability and data abstraction quality on the dendrogram, and the support of data exploration at arbitrary levels of detail. The contribution of the paper includes a new metric to measure the "importance" of nodes in a dendrogram; the method to construct the concise overview dendrogram from the dynamically-identified, important nodes; and measure for evaluating the data abstraction quality for dendrograms. We evaluate and compare the proposed method to some related existing methods, and demonstrating how the proposed method can help users find interesting patterns through a case study on county-level U.S. cervical cancer mortality and demographic data.

Chen, KK, and L Liu. 2003, "A Visual Framework Invites Human into the Clustering Process." In $15^{\text {th }}$ International Conference on Scientific and Statistical Database Management, pp. 97-106.

Clustering is a technique commonly used in scientific research. The task of clustering inevitably involves human participation - The clustering is not finished when the computer/algorithm finishes but the user has evaluated, understood and accepted the patterns. This defines a human involved "clusteringanalysis/evaluation" iteration. Instead of neglecting this human involvement, we provide a visual framework (VISTA) with all power of algorithmic approaches (since their result can be visualized), and in addition we allow the user to steer/monitor/refine the clustering process with domain knowledge. The visual-rendering result also provides a precise pattern for fast post-processing.

Chen, M, et al. 2008, "Data, Information, and Knowledge in Visualization." Computer Graphics and Applications, IEEE 29(1):12-19.

Chen, M, and H Janicke. 2010, "An Information Theoretic Framework for Visualization." IEEE Transactions on Visualization and Computer Graphics 16(6):1206-15.

In this paper, we examine whether or not information theory can be one of the theoretic frameworks for visualization. We formulate concepts and measurements for qualifying visual information. We illustrate these concepts with examples that manifest the intrinsic and implicit use of information theory in many existing visualization techniques. We outline the broad correlation between visualization and the major applications of information theory, while pointing out the difference in emphasis and some technical gaps. Our study provides compelling evidence that information theory can explain a significant number of phenomena or events in visualization, while no example has been found which is fundamentally in conflict with information theory. We also notice that the emphasis of some traditional applications of information theory, such as data compression or data communication, may not always suit visualization, as the former typically focuses on the efficient throughput of a communication channel, whilst the latter focuses on the effectiveness in aiding the perceptual and cognitive process for data understanding and knowledge discovery. These findings suggest that further theoretic developments are necessary for adopting and adapting information theory for visualization. 
Chen, TT, and LC Hsieh. 2006, "Uncovering the Latent Underlying Domains of a Research Field: Knowledge Visualization Revealed." In 10th International Conference on Information Visualization, pp. 252-56.

This paper illustrates how to clarify a new research topic - knowledge visualization - in terms of analyzing a vast amount of citations related to this field. The study presents a method that may help scholars who are interested in an inter-disciplinary and not-well-understood research area, such as knowledge visualization by revealing for them the underlying disciplines that a particular research is based upon. Key papers relevant to the research in question are also identified that may help researchers gain an overview of the field. The method discussed in this paper is generic in nature and generally applicable to other research topics

Chen, V, et al. 2010, "Model Based Interactive Analysis of Interwoven , Imprecise Narratives." In IEEE Symposium on Visual Analytics Science \& Technology, pp. 275-76.

Chen, V, et al. 2010, "Interactive Demo : Using Czsaw to Analyze Entities in Collections." In IEEE International Conference on Information Visualization, pp. 1-2.

Chen, VY, CZ Qian, and RF Woodbury. 2007, "Visualizing Collaborative Filtering in Digital Collections." In 11th International Conference on Information Visualization, pp. 203-10.

The NEAR (navigating exhibitions, annotations and resources) panel is a method of managing digital collections and user preferences through collaborative filtering and graphically revealing implicit data relations such as sharing, reference and similarity. It is implemented on AldrVIldrRE, an online multimedia repository. AldrVIldrRE supports semi-structured collections (exhibitions) which containing various resources and annotations. Its users are encouraged to contribute, share, annotate and interpret resources. Similar to the act of adding items into shopping carts in the e-commence applications, a user's activities of searching, organizing and interpreting data in AldrVIldrRE are considered as evidence of user's preferences. The design process of NEAR was guided by several principles from the visualization literature. It implements new navigation and communication approaches that support discovery of relations. Having tested NEAR with several users, we further analyze the design, report the evaluation and consider its use in other applications.

Chen, W, A Lu, and DS Ebert. 2007, "Shape-Aware Volume Illustration." Computer Graphics Forum 26(3):705-14.

Chen, W, et al. 2008, "Abstractive Representation and Exploration of Hierarchically Clustered Diffusion Tensor Fiber Tracts." Computer Graphics Forum 27(3):1071-78.

Chen, Y, and S Barlowe. 2010, "Click2annotate : Automated Insight Externalization with Rich Semantics." In IEEE Symposium on Visual Analytics Science \& Technology, pp. 155-62.

Chen, Y, GL Tan, and LH Hou. 2008. Research on Visual Analysis Technique of Multi-Dmensional Health Data Based on Parallel Coordinates.

Health data set usually is a huge, complex and multivariate data set. It is difficult for doctors to understand and analyse these large amount of data and the relationships between them without visual techniques. Parallel coordinate is an effective technique to visualize complex multivariate data set. In this 
paper, we present a visual analysis method on multidimensional health data. With this method, the target properties are first selected from data set. Then the selected data are visualized with parallel coordinate, using coloured subsection property axes and property value marker to solve the disorder problem when there are large amount of data on the screen. And last, the cluster method based on parallel coordinate is provided to help user analysing the data. The method was used in HIMES (Health Information Management and Evaluation System) that can provide relation analysis, trend analysis and cluster analysis etc. The application result of HIMES proved that this method is effect in helping doctor to analyse the complex health data set. The method presented in this paper can also be used in data analysis in other field such as population statistics, market researching and so on.

Chen, Y, J Yang, and W Ribarsky. 2009, "Toward Effective Insight Management in Visual Analytics Systems." In IEEE Pacific Visualization Symposium, pp. 49-56.

Although significant progress has been made toward effective insight discovery in visual sense making approaches, there is a lack of effective and efficient approaches to manage the large amounts of insights discovered. In this paper, we propose a systematic approach to leverage this problem around the concept of facts. Facts refer to patterns, relationships, or anomalies extracted from data under analysis. They are the direct products of visual exploration and permit construction of insights together with user's mental model and evaluation. Different from the mental model, the type of facts that can be discovered from data is predictable and applicationindependent. Thus it is possible to develop a general Fact Management Framework (FMF) to allow visualization users to effectively and efficiently annotate, browse, retrieve, associate, and exchange facts. Since facts are essential components of insights, it will be feasible to extend FMF to effective insight management in a variety of visual analytics approaches. Toward this goal, we first construct a fact taxonomy that categorizes various facts in multidimensional data and captures their essential attributes through extensive literature survey and user studies. We then propose a conceptual framework of fact management based upon this fact taxonomy. A concrete scenario of visual sense making on real data sets illustrates how this FMF will work. C2009 IEEE.

Cheng, H. 1994, "Towards the Next Generation User Interface: The Viu Model Using a Universe Paradigm and the Globe Metaphor for Enterprise Information Visualization." In IEEE International Conference on Systems, Man and Cybernetics, pp. 1838-42.

The next generation user interface for information systems, and indeed for all significant computer environments, will feature model-assisted visualization as its definitive element. This visualization goes beyond the straightforward drawing and rendering of imagery for physically visual objects or phenomena and extends to creating visual interpretation of logical subjects, such as enterprise information, using information and decision models and other profound contextual knowledge of the application. Dynamic animation in continual and interactive environments based on application models is the hallmark of this visualization. A universe paradigm is conceptualized for visualizing the enterprise information and is referred to as the visual information universe (VIU) model. The globe model is developed as the universe paradigm's basic metaphor, which features both the interior and the surface in an integrated cognitive design. The metadatabase model for enterprise information integration is exploited to afford an experimental environment 
Cheng, Y-p, et al. 2010, "Xdiva : Automatic Animation between Debugging Break." In 5th ACM Symposium on Software Visualization (SOFTVIS), pp. 221-22.

Chengzhi, Q, Z Chengu, and P Tao. 2003, "Taxonomy of Visualizationtechniques and Systems Concerns between Users and Developers Are Different." In Asia GIS Conference, pp. 1-14.

Cheung, W, EY Li, and LW Yee. 2003, "Multimedia Learning System and Its Effect on Self-Efficacy in Database Modeling and Design: An Exploratory Study." Computers \& Education 41(3):249-70.

Metadatabase modeling and design integrate process modeling and data modeling methodologies. Both are core topics in the information technology (IT) curriculum. Learning these topics has been an important pedagogical issue to the core studies for management information systems (MIS) and computer science (CSc) students. Unfortunately, the learning curve for the associated metadatabase modeling methodologies and their corresponding computer aided software engineering (CASE) tools have made it difficult for students to grasp. Addressing this learning issue, this study developed a multimedia learning system (MLS) and examines the effect of this MLS on the self-efficacy of learning these topics. It confirms that the MLS has significant impact on the self-efficacy in learning and that the software usefulness and software ease-of-use indeed affect the learning self-efficacy of MIS students. Furthermore, longer usage of the MLS can improve the self-efficacy in learning, but cannot make the improvement faster. Finally, the MLS can help the MIS students improve their self-efficacy in learning more than helping the CSc students.

Chevalier, F, and S Diamond. 2010, "The Use of Real Data in Fine Arts for Insight and Discovery : Case Studies in Text Analysis." In IEEE International Conference on Information Visualization, pp. 6-9.

Chhatwal, SD, and SJ Rose. 2008, "Visually Exploring Worldwide Incidents Tracking System Data." Proceedings of SPIE 6809:1-7.

Chi, EH. 2000, "A Taxonomy of Visualization Techniques Using the Data State Reference Model." In IEEE Symposium on Information Visualization, pp. 69-75.

In previous work, researchers have attempted to construct taxonomies of information visualization techniques by examining the data domains that are compatible with these techniques. This is useful because implementers can quickly identify various techniques that can be applied to their domain of interest. However, these taxonomies do not help the implementers understand how to apply and implement these techniques. The author extends and proposes a new way to taxonomize information visualization techniques by using the Data State Model (E.H. Chi and J.T. Reidl, 1998). In fact, as the taxonomic analysis in the paper will show, many of the techniques share similar operating steps that can easily be reused. The paper shows that the Data State Model not only helps researchers understand the space of design, but also helps implementers understand how information visualization techniques can be applied more broadly

Chi, EH, and SK Card. 1999, "Sensemaking of Evolving Web Sites Using Visualization Spreadsheets." In IEEE Symposium on Information Visualization, pp. 18-25,142.

In the process of knowledge discovery, workers examine available information in order to make sense of it. By sensemaking, we mean interacting with and operating on the information with a variety of 
information processing mechanisms. Previously, we introduced a concept that uses the spreadsheet metaphor with cells containing visualizations of complex data. We extend and apply a cognitive model called \&ldquo; visual sensemaking\&rdquo; to the visualization spreadsheet. We use the task of making sense of a large Web site as a concrete example throughout the paper for demonstration. Using a variety of visualization techniques, such as the Disk Tree and Cone Tree, we show that the interactions of the visualization spreadsheet help users draw conclusions from the overall relationships of the entire information set

Chi, EH-h, and JT Riedl. 1998, "An Operator Interaction Framework for Visualization Systems." In IEEE Symposium on Information Visualization, pp. 63-70.

Information visualization encounters a wide variety of different data domains. The visualization community has developed representation methods and interactive techniques. As a community, we have realized that the requirements in each domain are often dramatically different. In order to easily apply existing methods, researchers have developed a semiology of graphic representations. We have extended this research into a framework that includes operators and interactions in visualization systems, such as a visualization spreadsheet. We discuss properties of this framework and use it to characterize operations spanning a variety of different visualization techniques. The framework developed in this paper enables a new way of exploring and evaluating the design space of visualization operators, and helps end--users in their analysis tasks.

Chiew, V, and Y Wang. 2004, "Formal Description of the Cognitive Process of Decision Making." In $3^{\text {rd }}$ International Conference on Cognitive Informatics, pp. 124-30.

One of the fundamental human cognitive processes is problem solving. Most of the decisions we make relate to some kind of problems we try to solve no matter how trivial and critical the problem may be. The problem solving process entails performing in a new situation with information acquired and knowledge learned from past situations. As a higher level cognitive process, problem solving involves the correlation process effort to connect newly encounter problem object(s) with the object-attribute-relation (OAR) model representation of knowledge in the brain. The goal of problem solving is to search along various solution paths within the problem solver's knowledge base in the memory. When a problem object is identified, problem solving can be perceived as a search process in the memory space for finding a relationship between a set of problem-solving goals and a set of alternative paths. This paper presents a mathematical and cognitive model that describes problem solving as a cognitive process. The cognitive structures of the brain and the mechanisms of internal knowledge representation behind the cognitive process of problem solving are explained. The cognitive process is then formally and rigorously described using real-time process algebra (RTPA) base on the aforementioned models. Extended discussions are presented on applications of the cognitive process model of problem solving in software engineering and psychology.

Chignell, M, PA Hancock, and H Takeshita. 1999, "Chapter 11 - Human--Computer Interaction: The Psychology of Augmented Human Behavior." in Human Performance and Ergonomics, ed. PA Hancock, pp. 291-328. Academic Press, San Diego.

Summary This chapter examines the evolution of human--computer interfaces and the implications of technological augmentation for the study of human psychology. Human capability and behavior is changing as more artifacts become part of the standard equipment that people carry around and use. This 
leads to a question: Who or what is the subject of analysis in the experimental psychology of the future? A redefinition of the experimental unit in psychology is proposed both as a means of more realistic assessment of human behavior and as a basis for design. One of the critical limiting factors in technological augmentation of people is the human-computer interface. Augmenting technology has to be designed appropriately so that it assists in the performance of meaningful tasks without overloading the perceptual, motor, and cognitive capacities of people. This chapter reviews selected topics in human-computer interaction and shows how the constructive nature of perception and cognition may facilitate the implementation of augmenting technologies.

Chih, CH, and DS Parker. 2008, "The Persuasive Phase of Visualization." In 14th ACM SIGKDD International Conference on Knowledge Discovery and Data Mining, pp. 884-92.

Research in visualization often revolves around visualizing information. However, visualization is a process that extends over time from initial exploration to hypothesis confirmation, and even to result presentation. It is rare that the final phases of visualization are solely about information. In this paper we present a more biased kind of visualization, in which there is a message or set of assumptions behind the presentation that is of interest to both the presenter and the viewer, and emphasizes points that the presenter wants to convey to the viewer. This kind of persuasive visualization -- presenting data in a way that emphasizes a point or message -- is not only common in visualization, but also often expected by the viewer. Persuasive visualization is implicit in the deliberate emphasis on interestingness and also in the deliberate use of graphical elements that are processed preattentively by the human visual system, which automatically groups these elements and guiding attention so that they "stand out". We discuss how these ideas have been implemented in the Morpherspective system for automated generation of information graphics.

Chin, G, OA Kuchar, and KE Wolf. 2009, "Exploring the Analytical Processes of Intelligence Analysts." In Proceedings of the 27th international conference on Human factors in computing systems, pp. 11-20.

Chin, G, et al. 2009, "Visual Analysis of Dynamic Data Streams." Information Visualization 8(3):212-29.

For scientific data visualizations, real-time data streams present many interesting challenges when compared to static data. Real-time data are dynamic, transient, high-volume and temporal. Effective visualizations need to be able to accommodate dynamic data behavior as well as abstract and present the data in ways that make sense to and are usable by humans. The Visual Content Analysis of Real-Time Data Streams project at the Pacific Northwest National Laboratory is researching and prototyping dynamic visualization techniques and tools to help facilitate human understanding and comprehension of high-volume, real-time data. The general strategy of the project is to develop and evolve visual contexts that will organize and orient high-volume dynamic data in conceptual and perceptive views. The goal is to allow users to quickly grasp dynamic data in forms that are intuitive and natural without requiring intensive training in the use of specific visualization or analysis tools and methods. Thus far, the project has prototyped five different visualization prototypes that represent and convey dynamic data through human-recognizable contexts and paradigms such as hierarchies, relationships, time and geography. We describe the design considerations and unique features of these dynamic visualization prototypes as well as our findings in the exploration and evaluation of their use. Information Visualization (2009) 8, 212 229. doi: $10.1057 /$ ivs. 2009.18 
Chipman, SF, JM Schraagen, and VL Shalin. 2000, "Introduction to Cognitive Task Analysis." in Cognitive Task Analysis, eds. JM Schraagen, SF Chipman and VL Shalin, pp. 3-23. Lawrence Erlbaoum Associates, Mahawah, NJ.

Chittaro, L. 2001, "Information Visualization and Its Application to Medicine." Artificial Intelligence in Medicine 22(2):81-88.

This paper provides an introduction to the field of information visualization (IV) and a discussion of its application to medical systems. More specifically, it aims at: (i) defining what IV is and what are its goals (ii) highlighting the similarities and differences between IV and traditional medical imaging (iii) illustrating the potential of IV for medical applications by examining several examples of implemented systems and (iv) giving some general indications about the purposes and the effective exploitation of an IV component into a medical system.

Chittaro, L, and C Combi. 2001, "Representation of Temporal Intervals and Relations: Information Visualization Aspects and Their Evaluation." In 8th International Symposium on Temporal Representation and Reasoning, pp. 13-20.

A crucial component for turning any temporal reasoning system into a real-world application that can be adopted by a wide base of users is given by its user interface. After analyzing and discussing the state of the art for the visualization of temporal intervals and relations, this paper proposes three new solutions, also evaluating them with a proper user study

Chittaro, L, and C Combi. 2003, "Visualizing Queries on Databases of Temporal Histories: New Metaphors and Their Evaluation." Data \& Knowledge Engineering 44(2):239-64.

Chittaro, L, C Combi, and IC Society. 2001, "Representation of Temporal Intervals and Relations: Information Visualization Aspects and Their Evaluation." In 8th International Symposium on Temporal Representation and Reasoning (TIME 2001), pp. 13-20.

A crucial component for turning any temporal reasoning system into a real-world application that can he adopted by a wide base of users is given by its user interface. After analyzing and discussing the state of the art for the visualization of temporal intervals and relations, this paper proposes three new solutions, also evaluating them with a proper user study.

Chittaro, L, C Combi, and G Trapasso. 2003, "Data Mining on Temporal Data: A Visual Approach and Its Clinical Application to Hemodialysis." Journal of Visual Languages and Computing 14(6):591-620.

The quantity and complexity of data acquired, time-stamped and stored in clinical databases by automated medical devices is rapidly and continuously increasing. As a result, it becomes more and more important to provide clinicians with easy-to-use interactive tools to analyze huge amounts of this data. This paper proposes an approach for visual data mining on temporal data and applies it to a real medical problem, i.e., the management of hemodialysis. The approach is based on the integration of 3D and 2D information visualization techniques and offers a set of interactive functionalities that will be described in detail in the paper. We will also discuss how the system has been evaluated with end users and how the evaluation led to changes in system design. (C) 2003 Elsevier Ltd. All rights reserved. 
Chittaro, L, R Ranon, and L Ieronutti. 2006, "Vu-Flow: A Visualization Tool for Analyzing Navigation in Virtual Environments." IEEE Transactions on Visualization and Computer Graphics 12(6):1475-85.

This paper presents a tool for the visual analysis of navigation patterns of moving entities, such as users, virtual characters, or vehicles in 3D Virtual Environments (VEs). The tool, called VU-Flow, provides a set of interactive visualizations that highlight interesting navigation behaviors of single or groups of moving entities that were the VE together or separately. The visualizations help to improve the design of VEs and to study the navigation behavior of users, e. g., during controlled experiments. Besides VEs, the proposed techniques could also be applied to visualize real-world data recorded by positioning systems, allowing one to employ VU-Flow in domains such as urban planning, transportation, and emergency response.

Choi, H, H Lee, and H Kim. 2009, "Fast Detection and Visualization of Network Attacks on Parallel Coordinates." Computers \& Security 28(5):276-88.

This article presents what we call the parallel coordinate attack visualization (PCAV) for detecting unknown large-scale Internet attacks including Internet worms, DDoS attacks and network scanning activities. PCAV displays network traffic on the plane of parallel coordinates using the flow information such as the source IP address, destination IP address, destination port and the average packet length in a flow. The parameters are used to draw each flow as a connected line on the plane, where a group of polygonal lines form a particular shape in case of attack. From the observation that each attack type of significance forms a unique pattern, we develop nine signatures and their detection mechanism based on an efficient hashing algorithm. Using the graphical signatures, PCAV can quickly detect new attacks and enable network administrators to intuitively recognize and respond to the attacks. Compared with existing visualization works, PCAV can handle hyper-dimensions, i.e., can visualize more than 3 parameters if necessary, which significantly reduces false positives. As a consequence, Internet worms are more precisely detectable by machine and more easily recognizable by human. Another strength of PCAV is handling flows instead of packets. Per-flow visualization greatly reduces the processing time and further provides compatibility with legacy routers which export flow information, e.g., as NetFlow does in Cisco routers. We demonstrate the effectiveness of PCAV using real-life Internet traffic traces. The PCAV program is publicly available.

Cholewiak, Sa, HZ Tan, and DS Ebert. 2008, "Haptic Identification of Stiffness and Force Magnitude." In Symposium on Haptic Interfaces for Virtual Environment and Teleoperator Systems, pp. 87-91.

As haptics becomes an integral component of scientific data visualization systems, there is a growing need to study "haptic glyphs" (building blocks for displaying information through the sense of touch) and quantify their information transmission capability. The present study investigated the channel capacity for transmitting information through stiffness or force magnitude. Specifically, we measured the number of stiffness or force- magnitude levels that can be reliably identified in an absolute identification paradigm. The range of stiffness and force magnitude used in the present study, $0.2-3.0 \mathrm{~N} / \mathrm{mm}$ and $0.1-5.0 \mathrm{~N}$, respectively, was typical of the parameter values encountered in most virtual reality or data visualization applications. Ten individuals participated in a stiffness identification experiment, each completing 250 trials. Subsequently, four of these individuals and six additional participants completed 250 trials in a force-magnitude identification experiment. A custom-designed 3 degrees-of-freedom force-feedback device, the ministick, was used for stimulus delivery. The results showed an average information transfer of 1.46 bits for stiffness identification, or equivalently, 2.8 correctly-identifiable stiffness levels. The 
average information transfer for force magnitude was 1.54 bits, or equivalently, 2.9 correctly-identifiable force magnitudes. Therefore, on average, the participants could only reliably identify 2-3 stiffness levels in the range of 0.2-3.0 N/mm, and 2-3 force- magnitude levels in the range of 0.1-5.0 N. Individual performance varied from 1 to 4 correctly-identifiable stiffness levels and 2 to 4 correctly-identifiable force-magnitude levels. Our results are consistent with reported information transfers for haptic stimuli. Based on the present study, it is recommended that 2 stiffness or force-magnitude levels (i.e., high and low) be used with haptic glyphs in a data visualization system, with an additional third level (medium) for mo- - re experienced users.

Choo, J, H Lee, and H Park. 2010, "Ivisclassifier : An Interactive Visual Analytics System for Classification Based on Supervised Dimension Reduction." In IEEE International Symposium on Information Visualization, pp. 27-34.

Chou, C, C-C Tsai, and H-F Tsai. 2001, "Developing a Networked Vrml Learning System for Health Science Education in Taiwan." International Journal of Educational Development 21(4):293-303.

This study discusses applying virtual reality (VR) and Virtual Reality Modeling Language (VRML) to promote health science education in Taiwan. It first describes the needs of health science education in Taiwan, and the advantages of using computer technology in health science teaching and learning. A networked desktop VR-based system and courseware entitled "Travelling with Our Food" were developed for health science learning. The design of the course, the development of the system (platform and software), and expert-based and user-based evaluations are reported. Evaluation results, research issues, and possible future work are also discussed.

Chou, P-H, et al. 2010, "Integrating Web Mining and Neural Network for Personalized E-Commerce Automatic Service." Expert Systems with Applications 37(4):2898-910.

Electronic commerce (EC) has become a trend in the world nowadays. However, most researches neglect a fundamental issue - the user's product-specific knowledge on which the useful intelligent systems are based. This research employs the user's product-specific knowledge and mine his/her interior desire on appropriate target products as a part of personalization process to construct the overall EC strategy for businesses. This paper illustrates a novel web usage mining approach, based on the sequence mining technique applied to user's navigation behaviour, to discover patterns in the navigation of websites. Three critical contributions are made in this paper: (1) using the footstep graph to visualize the user's click-stream data and any interesting pattern can be detected more easily and quickly; (2) illustrating a novel sequence mining approach to identify pre-designated user navigation patterns automatically and integrates back-propagation network (BPN) model smoothly; and (3) applying the empirical research to indicate that the proposed approach can predict and categorize the users' navigation behaviour with high accuracy.

Chourasia, A, et al. 2007, "Visual Insights into High-Resolution Earthquake Simulations." IEEE Computer Graphics and Applications 27(5):28-34.

This study focuses on the visualization of a series of large earthquake simulations, collectively called TeraShake. The simulation series aims to assess the impact of Southern San Andreas Fault earthquake scenarios on Southern California. The authors discuss the role of visualization in gaining scientific insight and aiding unexpected discovery. Examples include significant differences in the ground motion pattern 
for different rupture directions; wave-guide effects leading to strong, localized amplification; variation in rupture speed for a spontaneous rupture model; and a star-burst pattern, indicating an unusual radiation of energy. The authors also cite instances of how visualization helped in finding numerical instabilities, leading to the use alternate absorbing boundary conditions for the wave propagation code. Without the sophisticated level of visualization carried out for the TeraShake, important scientific results would have remained undiscovered or less clearly understood. Moreover, some of the visualizations provide invaluable instructional material on earthquake phenomena to the public. C 2007 IEEE.

Christel, MG. 2006, "Evaluation and User Studies with Respect to Video Summarization and Browsing Art. No. 60730m." In Conference on Multimedia Content Analysis, Management and Retrieval, pp. M730-M30.

The Informedia group at Carnegie Mellon University has since 1994 been developing and evaluating surrogates, summary interfaces, and visualizations for accessing digital video collections containing thousands of documents, millions of shots, and terabytes of data. This paper surveys the Informedia user studies that have taken place through the years, reporting on how these studies can provide a user pull complementing the technology push as automated video processing advances. The merits of discount usability techniques for iterative improvement and evaluation are presented, as well as the structure of formal empirical investigations with end users that have ecological validity while addressing the human computer interaction metrics of efficiency, effectiveness, and satisfaction. The difficulties in evaluating video summarization and browsing interfaces are discussed. Lessons learned from Informedia user studies are reported with respect to video summarization and browsing, ranging from the simplest portrayal of a single thumbnail to represent video stories, to collections of thumbnails in storyboards, to playable video skims, to video collages with multiple synchronized information perspectives.

Christel, MG. 2007, "Examining User Interactions with Video Retrieval Systems - Art. No. 650606." In Conference on Multimedia Content Access - Algorithms and Systems, pp. 50606-06.

The Informedia group at Carnegie Mellon University has since 1994 been developing and evaluating surrogates, summary interfaces, and visualizations for accessing digital video collections containing thousands of documents, millions of shots, and terabytes of data. This paper reports on TRECVID 2005 and 2006 interactive search tasks conducted with the Informedia system by users having no knowledge of Informedia or other video retrieval interfaces, but being experts in analyst activities. Think-aloud protocols, questionnaires, and interviews were also conducted with this user group to assess the contributions of various video summarization and browsing techniques with respect to broadcast news test corpora. Lessons learned from these user interactions are reported, with recommendations on both interface improvements for video retrieval systems and enhancing the ecological validity of video retrieval interface evaluations.

Christmann, O, N Carbonell, and S Richir. 2010, "Visual Search in Dynamic 3d Visualisations of Unstructured Picture Collections." Interacting with Computers 22(5):399-416.

We present two empirical studies of visual search in dynamic 3D visualisations of large, randomly ordered, photo collections. The aim is to assess the possible effects of geometrical distortions on visual search effectiveness, efficiency and comfort, by comparing the influence of two perspective representations of photo collections on participants' performance results and subjective judgments. Thumbnails of the 1000 or so photographs in each collection are plastered on the lateral surface of a 
vertical cylinder, either on the inside (inner view, IV) or on the outside (outer view, OV). IV and OV suggest two different interaction metaphors: locomotion in a virtual space (IV) versus manipulation of a virtual object (OV). They also implement different perspective distortions: enlargement and distortion of lateral columns (IV) versus enlargement of central columns and dwindling plus distortion of lateral columns (OV). Presentation of results focus on the second study, S2, which involved 20 participants and offered them strictly identical interaction facilities with the two views, unlike the initial pilot study, S1 (8 participants and slightly different interaction facilities between the two views). Participants in both studies were experienced computer users (average age: 25.15 years, SD: 3.13). They performed two types of basic visual tasks that are carried out repeatedly while navigating photo collections: (i) searching for a photo meeting specific, visual and thematic, criteria, the photo and its location in the collection being unknown to participants (ST1) and (ii) looking for a visually familiar photo, the location of the photo being familiar to participants (ST2). According to post-experiment questionnaires and debriefings, all participants in S2 save one judged both 3D views positively in reference to standard 2D visualisations. Half of them preferred IV over OV, four appreciated OV better, and six expressed no clear opinion. Preferences were mainly motivated by the effects of perspective distortions on thumbnail visibility. They were barely influenced by interaction metaphors (e.g., the feeling of immersion induced by IV). Despite large inter-individual differences in performance, a majority of participants carried out ST1 tasks more effectively and efficiently with IV than with OV, as regards error rates (statistically significant difference) and search times (tendency). Performance results for ST2 tasks were similar with the two views, due, probably, to the simplicity and brevity of ST2 tasks. Perspective distortions seem to have exerted less influence on participants' visual strategies than horizontal scrolling, a dynamic feature common to both views. Qualitative analyses of participants' behaviours suggest that IV has the potential to support spatial memory better than OV, presumably thanks to the locomotion metaphor. These results indicate that perspective views have the potential to facilitate and improve visual search in unstructured picture collections provided that distortions are adapted to users' individual visual capabilities. Further research is needed to better understand: (i) the actual relations between visual exploration strategies and geometrical properties of perspective visualisations and (ii) the influence of the manipulation and locomotion metaphors on spatial memory. This knowledge is necessary to further improve the comfort and effectiveness of visual search in large unstructured picture collections, using 3D visualisations.

Chuah, MC, and SF Roth. 1996, "On the Semantics of Interactive Visualizations." In Information Visualization '96, Proceedings IEEE Symposium on, pp. 29-36.

Interactive techniques are powerful tools for manipulating visualizations to analyze, communicate and acquire information. This is especially true for large data sets or complex 3D visualizations. Although many new types of interaction have been introduced recently, very little work has been done on understanding what their components are, how they are related and how they can be combined. This paper begins to address these issues with a framework for classifying interactive visualizations. Our goal is a framework that will enable us to develop toolkits for assembling visualization interfaces both interactively and automatically 
Chuang, J, and P Hanrahan. 2009, "Statistically Identifying Basic Color Terms." In 31st Annual Meeting of the Cognitive Science Society, pp. 1-6.

Chuang, J, et al. 2009, "A Probabilistic Model of the Categorical Association between Colors." In $16^{\text {th }}$ Color Imaging Conference.

Chung, H, et al. 2010, "Vizcept : Supporting Synchronous Collaboration for Constructing Visualizations in Intelligence Analysis." In IEEE Symposium on Information Visualization, pp. 107-14.

Chung, W. 2006, "Studying Information Seeking on the Non-English Web: An Experiment on a Spanish Business Web Portal." International Journal of Human-Computer Studies 64(9):811-29.

The Internet is estimated to grow significantly as access to Web content in some non-English languages continues to increase. However, prior research in human-computer interaction (HCI) has implicitly assumed the primary language used on the Web to be English. This assumption is not true for many non-English-speaking regions where rapidly growing on-line populations access the Web in their native languages. For example, Latin America, where the majority of people speak Spanish, will have the fastest growing population in coming decades. However, existing Spanish search engines lack search, browse, and analysis capabilities. The research reported here studied human information seeking on the non-English Web. In it we developed a Spanish business Web portal that supports searching, browsing, summarization, categorization, and visualization of Spanish business Web pages. Using 42 Spanish speakers as subjects we conducted a two-phase experiment to evaluate this portal and found that, compared with a Spanish search engine and a Spanish Web directory, it achieved significantly better user ratings on information quality, cross-regional search capability, system performance attributes, and overall satisfaction. Subjects' verbal comments strongly favored the search and browse functionality and user interface of our portal. As the Web becomes more international, this research makes three contributions: (1) an empirical evaluation of the performance level of a Spanish search portal; (2) an examination of the information quality, cross-regional search capability and usability of search engines for the non-English Web; and (3) a better understanding of non-English Web searching.

Chung, W. 2010, "Web Searching and Browsing: A Multilingual Perspective." in Advances in Computers: Improving the Web, ed. VZ Marvin, Vol 78, pp. 41-69. Elsevier.

Since the publication of "The World Wide Web" in a 1999 volume of Advances in Computers, worldwide Internet usage has grown tremendously, with the most rapid growth in some non-Englishspeaking regions. A widening gap exists between the surging demand for non-English Web content and the availability of non-English resources. This chapter reviews previous works on computer-mediated information seeking on the Web, computing technologies that support Web searching and browsing, and Web portals in several major regions and languages of the world. We introduce a general framework for supporting Web searching and browsing in a multilingual world. Three Web portals were developed to support searching, browsing, and postretrieval analysis of Chinese business intelligence, Spanish business intelligence, and Arabic medical intelligence. Results of experiments involving 67 native speakers of the three languages confirm the usability and benefits of the portals and support the applicability of the framework. The review, framework, and findings presented in this chapter contribute to the fields of Web analysis, text mining, business and medical informatics, and human-computer interaction. 
Chung, W, et al. 2006, "Supporting Non-English Web Searching: An Experiment on the Spanish Business and the Arabic Medical Intelligence Portals." Decision Support Systems 42(3):1697-714.

Although non-English-speaking online populations are growing rapidly, support for searching nonEnglish Web content is much weaker than for English content. Prior research has implicitly assumed English to be the primary language used on the Web, but this is not the case for many non-Englishspeaking regions. This research proposes a language-independent approach that uses meta-searching, statistical language processing, summarization, categorization, and visualization techniques to build highquality domain-specific collections and to support searching and browsing of non-English information. Based on this approach, we developed SBizPort and AMedPort for the Spanish business and Arabic medical domains respectively. Experimental results showed that the portals achieved significantly better search accuracy, information quality, and overall satisfaction than benchmark search engines. Subjects strongly favored the portals' search and browse functionality and user interface. This research thus contributes to developing and validating a useful approach to non-English Web searching and providing an example of supporting decision-making in non-English Web domains.

Chung, W, et al. 2005, "Evaluating Event Visualization: A Usability Study of Coplink Spatio-Temporal Visualizer." International Journal of Human-Computer Studies 62(1):127-57.

Event visualization holds the promise of alleviating information overload in human analysis and numerous tools and techniques have been developed and evaluated. However, previous work does not specifically address either the coordination of event dimensions with the types of tasks involved or the way that visualizing different event dimensions can benefit human analysis. In this paper, we propose a taxonomy of event visualization and present a methodology for evaluating a coordinated event visualization tool called COPLINK Spatio-Temporal Visualizer (STV). The taxonomy encompasses various event dimensions, application domains, visualization metaphors, evaluation methods and performance measures. The evaluation methodology examines different event dimensions and different task types, thus juxtaposing two important elements of evaluating a tool. To achieve both internal and external validity, a laboratory experiment with students and a field study with crime analysis experts were conducted. Findings of our usability study show that STV could support crime analysis involving multiple, coordinated event dimensions as effectively as it could analyze individual, uncoordinated event dimensions. STV was significantly more effective and efficient than Microsoft Excel in performing coordinated tasks and was significantly more efficient in doing uncoordinated tasks related to temporal, spatial and aggregated information. Also, STV had compared favorably with Excel in completing uncoordinated tasks related to temporal and spatial information, respectively. Subjects' comments showed STV to be intuitive, useful and preferable to existing crime analysis methods.

Chung, W, and A Leung. 2007, "Supporting Web Searching of Business Intelligence with Information Visualization." In IEEE/WIC/ACM International Conference on Web Intelligence, pp. 807-11.

In this research, we proposed and validated an approach to using information visualization to augment search engines in supporting the analysis of business stakeholder information on the Web. We report in this paper findings from a preliminary evaluation comparing a visualization prototype with a traditional method of stakeholder analysis (Web browsing and searching). We found that the prototype achieved a higher perceived usefulness and perceived analysis effectiveness and was perceived favorably in expediting the subjects' decision making and in helping them understand the analysis results. Overall, the 
proposed approach was found to augment traditional methods of analyzing business stakeholders. We discuss implications to researchers and practitioners and future directions.

Chung, WY, et al. 2005, "Evaluating Event Visualization: A Usability Study of Coplink Spatio-Temporal Visualizer." International Journal of Human-Computer Studies 62(1):127-57.

Event visualization holds the promise of alleviating information overload in human analysis and numerous tools and techniques have been developed and evaluated. However, previous work does not specifically address either the coordination of event dimensions with the types of tasks involved or the way that visualizing different event dimensions can benefit human analysis. In this paper, we propose a taxonomy of event visualization and present a methodology for evaluating a coordinated event visualization tool called COPLINK Spatio-Temporal Visualizer (STV). The taxonomy encompasses various event dimensions, application domains, visualization metaphors, evaluation methods and performance measures. The evaluation methodology examines different event dimensions and different task types, thus juxtaposing two important elements of evaluating a tool. To achieve both internal and external validity, a laboratory experiment with students and a field study with crime analysis experts were conducted. Findings of our usability study show that STV could support crime analysis involving multiple, coordinated event dimensions as effectively as it could analyze individual, uncoordinated event dimensions. STV was significantly more effective and efficient than Microsoft Excel in performing coordinated tasks and was significantly more efficient in doing uncoordinated tasks related to temporal, spatial and aggregated information. Also, STV had compared favorably with Excel in completing uncoordinated tasks related to temporal and spatial information, respectively. Subjects' comments showed STV to be intuitive, useful and preferable to existing crime analysis methods. (C) 2004 Elsevier Ltd. All rights reserved.

Clarkson, E, J Day, and J Foley. 2005, The Development of an Educational Digital Library for HumanCentered Computing. Technical Rpt. GIT-GVU-05-33, Georgia Tech.

Clarkson, E, JA Day, and JD Foley. 2006, "An Educational Digital Library for Human-Centered Computing." In CHI'06 extended abstracts on Human factors in computing systems pp. 646-51.

Digital libraries have great potential to improve the educational experience. As a result, there are a wide variety of such repositories, especially those that focus specifically on education. But relatively few focus on topics as specific as Human-Computer Interaction (HCI) or Human-Centered Computing (HCC). In addition, support for browsing behavior, with a few exceptions, is both weak and not suitable for user needs. This paper presents our work to create a repository of educational materials for a relatively narrowly-targeted field $(\mathrm{HCC} / \mathrm{HCI})$, including our requirements gathering methods and results. Finally, we discuss the HCC Education Digital Library (HCC EDL) as a platform for investigating broader digital library research questions, such as exploring alternative designs for content browsing mechanisms. 
Clarkson, E, and JD Foley. 2007, "Augmenting Digital Library Search Interfaces with Visual Analysis Tools." In IEEE Information Visualization Conference, pp. 2-3.

Clarkson, E, and JD Foley. 2006, "Browsing Affordance Designs for the Human-Centered Computing Education Digital Library." In 6th ACM/IEEE-CS joint conference on Digital libraries, pp. 361-61.

Browsing is a widespread user behavior in the digital library (DL) environment; there are an array of existing techniques that afford browsing and are readily applicable to digital libraries. We outline the designs of two such methods based on well-known techniques: treemaps and ScentTrails.

Clarkson, EC, and JD Foley. 2009, "Augmenting Faceted Exploration with Resultmaps." In IEEE Conference on Information Visualization, pp. 1-4.

ResultMaps are a treemap-based [5] search visualization concept; for online digital repositories; designed to leverages hierarchical; metadata often already present in digital libraries. We have; previously reported our application of ResultMaps to a digital; library's keyword search engine result pages (SERPs) [3]. Here; we discuss the use of ResultMaps in the more interesting context; of faceted metadata and plans for their formal evaluation.; ResultMaps perform several useful functions in that environment:; placing items within a consistent visual context; provide a; mechanism for outlier; cluster and correlation detection; and act; as a visual medium for previewing prospective selections.

Clarkson, EC, and JD Foley. 2007, "Resultmaps : Search Result Visualization for Hierarchical Information Spaces." In ACM CHI '07 Workshop on Exploratory Search and HCI.

Cockburn, A, and B McKenzie. 2004, "Evaluating Spatial Memory in Two and Three Dimensions." International Journal of Human-Computer Studies 61(3):359-73.

Prior research has shown that the efficient use of graphical user interfaces is strongly dependent on human capabilities for spatial cognition. One facet of spatial cognition is the ability to quickly and accurately recall and access the location of objects in a spatial arrangement. This paper describes a series of experiments aimed at determining whether three-dimensional user interfaces better support spatial memory than their more traditional two-dimensional counterparts. The experiments are conducted using both computer-supported systems and physical models that vary the depth and perspective cues in spatial arrangements of interface items. The physical models were used to escape some of the dimensional ambiguities that are hard to control using computer displays. Results strongly suggest that adding a third dimension to computer displays does not aid users' spatial memory. Although there were no significant differences between the effectiveness of spatial memory when using two- and three-dimensional computer interfaces, participants' memory for the location of cards representing web-pages was reliably better when using a two-dimensional physical model than when using an equivalent three-dimensional physical model.

Cohen, M, and K Brodlie. 2007, "Focus and Context for Volume Visualization." In Theory and Practice of Computer Graphics, pp. 32-39.

Scientific investigation and simulation have been producing increasingly large datasets. Usually that kind of data is visualized employing some scientific visualization technique. Another related field, information visualization, deals with nonscientific large scale data, using different approaches to achieve 
an effective understanding. Our aim is to bring these two fields together, proposing a taxonomy for volume visualization, employing information visualization techniques, particularly the focus and context idea. A prototype is being developed, which aims to implement and evaluate a number of solutions for this problem, identifying through demonstration which applications would benefit most from focus + context approaches. We describe work in progress

Cohen, S, et al. 2010, "Panel : Using Visualization for Storytelling on the Web." In IEEE Conference on Information Visualization.

Cohen, T, and D Widdows. 2009, "Empirical Distributional Semantics: Methods and Biomedical Applications." Journal of Biomedical Informatics 42(2):390-405.

Over the past 15 years, a range of methods have been developed that are able to learn human-like estimates of the semantic relatedness between terms from the way in which these terms are distributed in a corpus of unannotated natural language text. These methods have also been evaluated in a number of applications in the cognitive science, computational linguistics and the information retrieval literatures. In this paper, we review the available methodologies for derivation of semantic relatedness from free text, as well as their evaluation in a variety of biomedical and other applications. Recent methodological developments, and their applicability to several existing applications are also discussed.

Cokrojoyo, H. 2005, "Multidimensional Multivariate Information Visualization Using Dynamic Iconography." Vol M.S., pp. 150. University of Missouri - Columbia.

Large multidimensional multivariate (mdmv) datasets are now common in many fields. The data in various digital archives exist in a multitude of formats and are too voluminous to inspect or explore manually using numeric or tabular formats. This thesis proposes a new informational visualization approach for exploration of mdmv datasets to enhance data mining and knowledge discovery. The proposed technique for qualitative exploratory data analysis is to use dynamic iconography and tree icons. Visual iconography relies on the use of icons to visually map the multidimensional feature vectors of the dataset to three-dimensional representation to enable observers to effectively obtain qualitative understanding and insight. Conventional iconography such as stick figure visualization, however, is limited to two dimensions for graphical display and static icons are of limited use for mdmv datasets. We propose two enhancements through a repertoire of animations and through the use of tree icons that are more flexible and scalable three-dimensional icon maps. Three different types of icons were explored including propeller type motions and stylized humanoid gait, along with branching structures for tree icons, each with their own unique features, properties and capabilities to accommodate the various types of mdmv data. Three different datasets were collected and analyzed including multi-spectral Landsat data for landcover classification, handwritten digit recognition for machine learning and an e-commerce financial survey data for web assurance. The results using the StickViz software developed in this thesis using dynamic iconography demonstrates the utility, potential and promise of a new approach for understanding mdmv datasets. 
Cole, C, B Mandelblatt, and J Stevenson. 2002, "Visualizing a High Recall Search Strategy Output for Undergraduates in an Exploration Stage of Researching a Term Paper." Information Processing \& Management 38(1):37-54.

Collins, TD. 2003, "Applying Software Visualization Technology to Support the Use of Evolutionary Algorithms." Journal of Visual Languages \& Computing 14(2):123-50.

This paper describes an approach to supporting the use of evolutionary algorithms through the integration of software visualization into the working practices of evolutionary algorithm users. A case is made for the provision of principled visualization support at an accessible level of abstraction for the user. Henson, a visualization framework for genetic algorithms is introduced that provides multiple levels of abstraction for the development of interactive visualizations. A genetic algorithm visualization tool called Gonzo is then described to illustrate the application of the framework approach and the integration of principled visualization design in an easily adopted visualization environment. This paper concludes with an overview of the approach described and the benefits revealed through the integration of visualization within the software design and development process.

Compieta, P, et al. 2007, "Exploratory Spatio-Temporal Data Mining and Visualization." Journal of Visual Languages \& Computing 18(3):255-79.

Spatio-temporal data sets are often very large and difficult to analyze and display. Since they are fundamental for decision support in many application contexts, recently a lot of interest has arisen toward data-mining techniques to filter out relevant subsets of very large data repositories as well as visualization tools to effectively display the results. In this paper we propose a data-mining system to deal with very large spatio-temporal data sets. Within this system, new techniques have been developed to efficiently support the data-mining process, address the spatial and temporal dimensions of the data set, and visualize and interpret results. In particular, two complementary 3D visualization environments have been implemented. One exploits Google Earth to display the mining outcomes combined with a map and other geographical layers, while the other is a Java3D-based tool for providing advanced interactions with the data set in a non-geo-referenced space, such as displaying association rules and variable distributions.

Conti, G, M Ahamad, and J Stasko. 2005, "Attacking Information Visualization System Usability Overloading and Deceiving the Human." In Symposium on Usable Privacy and Security, pp. 89-100.

Information visualization is an effective way to easily comprehend large amounts of data. For such systems to be truly effective, the information visualization designer must be aware of the ways in which their system may be manipulated and protect their users from attack. In addition, users should be aware of potential attacks in order to minimize or negate their effect. These attacks target the information visualization system as well as the perceptual, cognitive and motor capabilities of human end users. To identify and help counter these attacks we present a framework for information visualization system security analysis, a taxonomy of visualization attacks and technology independent principles for countering malicious visualizations. These themes are illustrated with case studies and working examples from the network security visualization domain, but are widely applicable to virtually any information visualization system. 
Convertino, G, et al. 2008, "The Cache Study: Group Effects in Computer-Supported Collaborative Analysis." Computer Supported Cooperative Work 17(4):353-93.

The present study evaluates some effects of group composition in distributed intelligence analysis. Prior work suggests that bias makes this task difficult. We experimentally investigated the impact of heterogeneity of view point and prior knowledge on bias and information coverage, specifically on anchoring and confirmation bias. Bias was substantially reduced whether individuals worked alone or in heterogeneous groups yet, importantly, net process costs were not increased for collaborating individuals. Also, despite similar initial bias, individuals in homogeneous groups showed increasing bias and greater final bias than in the other conditions suggesting that ill-formed groups exacerbate bias. All participants used CACHE, a web-based environment that supports a collaborative version of Analysis of Competing Hypotheses $(\mathrm{ACH})$-a method employed by professional intelligence analysts. We suggest that CACHE may be effective in reducing both bias and process costs in collaborative analysis.

Cook, K, R Earnshaw, and J Stasko. 2007, "Discovering the Unexpected." IEEE Computer Graphics and Applications 27(5):15-9.

The marriage of computation, visual representation, and interactive thinking supports intensive analysis. The goal is not only to permit users to detect expected events, such as might be predicted by models, but also to help users discover the unexpected - the surprising anomalies, changes, patterns, and relationships that are then examined and assessed to develop new insight. The Guest Editors discuss the key issues and challenges associated with discovering the unexpected, as well as introduce the articles that make up this Special Issue.

Cook, K, R Earnshaw, and J Stasko. 2007, "Guest Editors' Introduction: Discovering the Unexpected." Computer Graphics and Applications, IEEE 27(5):15-19.

The marriage of computation, visual representation, and interactive thinking supports intensive analysis. The goal is not only to permit users to detect expected events, such as might be predicted by models, but also to help users discover the unexpected\&\#x2014; the surprising anomalies, changes, patterns, and relationships that are then examined and assessed to develop new insight. The Guest Editors discuss the key issues and challenges associated with discovering the unexpected, as well as introduce the articles that make up this Special Issue.

Cook, MB, and HS Smallman. 2008, "Human Factors of the Confirmaiton Bias in Inteligence Analysis: Decision Support from Graphical Evidence Landscapes." Human Factors 50(5):745-54.

OBJECTIVE: This study addresses the human factors challenge of designing and validating decision support to promote less biased intelligence analysis. BACKGROUND: The confirmation bias can compromise objectivity in ambiguous medical and military decision making through neglect of conflicting evidence and judgments not reflective of the entire evidence spectrum. Previous debiasing approaches have had mixed success and have tended to place additional demands on users' decision making. METHOD: Two new debiasing interventions that help analysts picture the full spectrum of evidence, the relation of evidence to a hypothesis, and other analysts' evidence assessments were manipulated in a repeated-measures design: (a) an integrated graphical evidence layout, compared with a text baseline; and (b) evidence tagged with other analysts' assessments, compared with participants' own assessments. Twenty-seven naval trainee analysts and reservists assessed, selected, and prioritized 
evidence in analysis vignettes carefully constructed to have balanced supporting and conflicting evidence sets. Bias was measured for all three evidence analysis steps. RESULTS: A bias to select a skewed distribution of confirming evidence occurred across conditions. However, graphical evidence layout, but not other analysts' assessments, significantly reduced this selection bias, resulting in more balanced evidence selection. Participants systematically prioritized the most supportive evidence as most important. CONCLUSION: Domain experts exhibited confirmation bias in a realistic intelligence analysis task and apparently conflated evidence supportiveness with importance. Graphical evidence layout promoted more balanced and less biased evidence selection. APPLICATION: Results have application to real-world decision making, implications for basic decision theory, and lessons for how shrewd visualization can help reduce bias.

Cooper, W, and C Jarvis. 2004, "A Java-Based Intelligent Advisor for Selecting a Context-Appropriate Spatial Interpolation Algorithm." Computers \& Geosciences 30(9-10):1003-18.

The choice of an appropriate interpolation technique is an imposing task. There are many techniques to choose from depending on the characteristics of the phenomenon, the purpose of the study and also the expertise of the user. This paper describes the implementation and architecture of a Java-based intelligent advisor to assist a generic user, ranging from the casual to the specialist, in selecting the interpolator most appropriate for a given task and data set. The software integrates procedural knowledge from disparate sources through an expert system shell, and uses multiple sensory channels to present and abstract contextual knowledge from the user regarding both the data and the task at hand. The system has been tested for use in a meteorological domain in the first instance. The interpolation methods currently assessed by the system are multiple forms of kriging, thin plate smoothing splines, inverse-distance weighting and trend surface/polynomial analysis.

Cornelissen, B, et al. 2008, "Execution Trace Analysis through Massive Sequence and Circular Bundle Views." Journal of Systems and Software 81(12):2252-68.

An important part of many software maintenance tasks is to gain a sufficient level of understanding of the system at hand. The use of dynamic information to aid in this software understanding process is a common practice nowadays. A major issue in this context is scalability: due to the vast amounts of information, it is a very difficult task to successfully navigate through the dynamic data contained in execution traces without getting lost. In this paper, we propose the use of two novel trace visualization techniques based on the massive sequence and circular bundle view, which both reflect a strong emphasis on scalability. These techniques have been implemented in a tool called Extravis. By means of distinct usage scenarios that were conducted on three different software systems, we show how our approach is applicable in three typical program comprehension tasks: trace exploration, feature location, and topdown analysis with domain knowledge.

Corral, K, D Schuff, and R D. 2006, "The Impact of Alternative Diagrams on the Accuracy of Recall: A Comparison of Star-Schema Diagrams and Entity-Relationship Diagrams." Decision Support Systems 42(1):450-68.

Data warehouses can be constructed using either a relational or a dimensional model. This research compares the diagrammatic representations of these two models. Semantic network theory suggests that, because of their structure, star-schema diagrams (SSDs) are more understandable than entity-relationship diagrams (ERDs). Through two experiments that test both the accuracy and pattern of users' recall, we 
find strong evidence that SSDs are easier to understand than ERDs. Our findings imply that the use of the dimensional model should help decision makers query the warehouse directly, thereby reducing costs and the need for intervention and support by IT professionals.

\section{Correll, MA. 2010, "Poster : Understanding Tagged Text." In IEEE Conference on Information Visualization.}

Cosmides, L, and J Tooby. 1996, "Are Humans Good Intuitive Statisticians after All? Rethinking Some Conclusions from the Literature on Judgment under Uncertainty." Cognition 58(1):1-73.

Professional probabilists have long argued over what probability means, with, for example, Bayesians arguing that probabilities refer to subjective degrees of confidence and frequentists arguing that probabilities refer to the frequencies of events in the world. Recently, Gigerenzer and his colleagues have argued that these same distinctions are made by untutored subjects, and that, for many domains, the human mind represents probabilistic information as frequencies. We analyze several reasons why, from an ecological and evolutionary perspective, certain classes of problem-solving mechanisms in the human mind should be expected to represent probabilistic information as frequencies. Then, using a problem famous in the "heuristics and biases" literature for eliciting base rate neglect, we show that correct Bayesian reasoning can be elicited in $76 \%$ of subjects - indeed, $92 \%$ in the most ecologically valid condition - simply by expressing the problem in frequentist terms. This result adds to the growing body of literature showing that frequentist representations cause various cognitive biases to disappear, including overconfidence, the conjunction fallacy, and base-rate neglect. Taken together, these new findings indicate that the conclusion most common in the literature on judgment under uncertainty - that our inductive reasoning mechanisms do not embody a calculus of probability - will have to be re-examined. From an ecological and evolutionary perspective, humans may turn out to be good intuitive statisticians after all.

Cosmides, L, and J Tooby. 1994, "Beyond Intuition and Instinct Blindness: Toward an Evolutionarily Rigorous Cognitive Science." Cognition 50(1-3):41-77.

Cognitive psychology has an opportunity to turn itself into a theoretically rigorous discipline in which a powerful set of theories organize observations and suggest focused new hypotheses. This cannot happen, however, as long as intuition and folk psychology continue to set our research agenda. This is because intuition systematically blinds us to the full universe of problems our minds spontaneously solve, restricting our attention instead to a minute class of unrepresentative "high-level" problems. In contrast, evolutionarily rigorous theories of adaptive function are the logical foundation on which to build cognitive theories, because the architecture of the human mind acquired its functional organization through the evolutionary process. Theories of adaptive function specify what problems our cognitive mechanisms were designed by evolution to solve, thereby supplying critical information about what their design features are likely to be. This information can free cognitive scientists from the blinders of intuition and folk psychology, allowing them to construct experiments capable of detecting complex mechanisms they otherwise would not have thought to test for. The choice is not between no-nonsense empiricism and evolutionary theory; it is between folk theory and evolutionary theory. 
Costello, L, et al. 2009, "Advancing User-Centered Evaluation of Visual Analytic Environments through Contests." Information Visualization 8(3):230-38.

In this paper, the authors describe the Visual Analytics Science and Technology (VAST) Symposium contests run in 2006 and 2007 and the VAST 2008 and 2009 challenges. These contests were designed to provide researchers with a better understanding of the tasks and data that face potential end users. Access to these end users is limited because of time constraints and the classified nature of the tasks and data. In that respect, the contests serve as an intermediary, with the metrics and feedback serving as measures of utility to the end users. The authors summarize the lessons learned and the future directions for VAST Challenges. Information Visualization (2009) 8, 230 - 238. doi: 10.1057/ivs.2009.16

Couclelis, H. 1998, "Worlds of Information: The Geographic Metaphor in the Visualization of Complex Information." Cartography and Geographic Information Science 25(4):209-20.

Cowley, P, L Nowell, and J Scholtz. 2005, "Glass Box: An Instrumented Infrastructure for Supporting Human Interaction with Information." In 38th Annual Hawaii International Conference on System Sciences, pp. 296c-96c.

In this paper, we discuss the challenges involved in developing an infrastructure to support a new generation of analytic tools for information analysts. The infra- structure provides data for establishing context about what the analyst is doing with the analytic tools, supports an integration environment to allow suites of tools to work together, and supports evaluation of the analytic tools. We discuss the functionality of the Glass Box, the challenges of evaluating adaptive systems including the capture of data for evaluation metrics, and lessons learned from our experiences to date.

Craft, B, and P Cairns. 2008, "Directions for Methodological Research in Information Visualization." In 12th International Conference Information Visualisation, pp. 44-50.

People within and outside the information visualization community are motivated to create new tools to address their own unique problems of understanding data. However, the techniques which visualizations use to enhance cognition of data are not widely known outside the field. Also, there are currently few resources which comprehensively describe methods for designing novel visualizations. Consequently, people who seek to build new information visualization tools are left to consult design examples, guidelines, and reference models, which do not adequately describe the visualization design process or suggest ways to undertake the process. We identify some of these shortcomings, provide an overview Of methodological knowledge in Information Visualization design to date, and suggest that methods from other design-oriented disciplines can bridge these shortcomings.

Craik, K. 1943. The Nature of Explanation. Cambridge University Press, Cambridge.

Crapo, AW, et al. 2000, "Visualization and the Process of Modeling: A Cognitive-Theoretic View." In $6^{\text {th }}$ ACM SIGKDD international conference on Knowledge discovery and data mining pp. 218-26.

Crescenzi, P, and G Innocenti. 2002, "Towards a Taxonomy of Network Protocol Visualization Tools." in Software Visualization, ed. S Diehl, Vol 2269, pp. 538-42. Springer Berlin Heidelberg, Berlin.

Due to the rising importance of visualization for teaching purposes, several network protocol visualization applications and applets are flowing on the Internet. So far, no taxonomy has been 
developed to classify this rather broad material. In this paper, we propose a taxonomy of network protocol visualization resources, to be used either by software visualization researchers to classify their tools or by educators to easily determine if a particular visualization actually satisfies their teaching needs. The taxonomy (which has been obtained by analyzing stand-alone applications and applets available on the Internet) is mainly intended to integrate previously existing taxonomies of algorithm visualization tools by mostly considering some characteristic features which are specific of the considered subject of network protocols.

Crestani, F, J Vegas, and P de la Fuente. 2004, "A Graphical User Interface for the Retrieval of Hierarchically Structured Documents." Information Processing \& Management 40(2):269-89.

Past research has proved that graphical user interfaces (GUIs) can significantly improve the effectiveness of the information access task. Our work is based on the consideration that structured document retrieval requires different user graphical interfaces from standard information retrieval. In structured document retrieval a GUI has to enable a user to query, browse retrieved documents, provide query refinement and relevance feedback based not only on full documents, but also on specific document parts in relation to the document structure. In this paper, we present a new GUI for structured document retrieval specifically designed for hierarchically structured documents. A user task-oriented evaluation has shown that the proposed interface provides the user with an intuitive and powerful set of tools for structured document searching, retrieved list navigation, and search refinement.

Cribbin, T, and CM Chen. 2001, "Visual-Spatial Exploration of Thematic Spaces: A Comparative Study of Three Visualisation Models." In 8th Meeting of the Visual Data Exploration and Analysis Conference, pp. 199-209.

Scatter graphs (e.g., SPIRE Galaxies, Bead, VR-VIBE) are a popular medium for visualising spatialsemantic structures derived from abstract information spaces. For small spaces (i.e., less than one hundred nodes), such graphs can be an effective means of reducing high-dimensional information into two or three spatial dimensions. As dimensionality increases, representing the thematic diversity of documents using spatial proximity alone becomes less and less effective. This paper reports an experiment designed to determine whether, for larger spaces, benefits are to be gained from adding visual links between document nodes as an additional means of representing the most important semantic relationships. Two well known algorithms, minimum spanning trees (MST) and pathfinder associative networks (PFNET), were tested against both a scatter graph visualisation, derived from factor analysis, and a traditional listbased hypertext interface. It was hypothesised that visual links would facilitate users' comprehension of the information space with corresponding gains in information seeking performance. Navigation performance and user impressions were analysed across a range of different search tasks. Results indicate both significant performance gains and more positive user feedback for MST and PFNET visualisations over scatter graphs. Performance on all visualisations was generally poorer and never better than that achieved on the text list interface, although the magnitude of these differences was found to be highly task dependent.

Crippa, A, et al. 2011, "Heuristics for Connectivity-Based Brain Parcellation of Sma/Pre-Sma through Force-Directed Graph Layout." NeuroImage 54(3):2176-84.

We propose the use of force-directed graph layout as an explorative tool for connectivity-based brain parcellation studies. The method can be used as a heuristic to find the number of clusters intrinsically 
present in the data (if any) and to investigate their organisation. It provides an intuitive representation of the structure of the data and facilitates interactive exploration of properties of single seed voxels as well as relations among (groups of) voxels. We validate the method on synthetic data sets and we investigate the changes in connectivity in the supplementary motor cortex, a brain region whose parcellation has been previously investigated via connectivity studies. This region is supposed to present two easily distinguishable connectivity patterns, putatively denoted by SMA (supplementary motor area) and preSMA. Our method provides insights with respect to the connectivity patterns of the premotor cortex. These present a substantial variation among subjects, and their subdivision into two well-separated clusters is not always straightforward.

Crnovrsanin, T, et al. 2009, "Proximity-Based Visualization of Movement Trace Data." In IEEE Conference on Information Visualization, pp. 11-18.

The increasing availability of motion sensors and video cameras in living spaces has made possible the analysis of motion patterns and collective behavior in a number of situations. The visualization of this movement data, however, remains a challenge. Although maintaining the actual layout of the data space is often desirable, direct visualization of movement traces becomes cluttered and confusing as the spatial distribution of traces may be disparate and uneven. We present proximity-based visualization as a novel approach to the visualization of movement traces in an abstract space rather than the given spatial layout. This abstract space is obtained by considering proximity data, which is computed as the distance between entities and some number of important locations. These important locations can range from a single fixed point, to a moving point, several points, or even the proximities between the entities themselves. This creates a continuum of proximity spaces, ranging from the fixed absolute reference frame to completely relative reference frames. By combining these abstracted views with the concrete spatial views, we provide a way to mentally map the abstract spaces back to the real space. We demonstrate the effectiveness of this approach, and its applicability to visual analytics problems such as hazard prevention, migration patterns, and behavioral studies.

Crosby, ME, J Scholtz, and P Ward. 2006, "Special Issue of Interacting with Computers: Symbiotic Performance between Humans and Intelligent Systems." Interacting with Computers 18(6):1165-69.

Crowley, RS, and O Medvedeva. 2006, "An Intelligent Tutoring System for Visual Classification Problem Solving." Artificial Intelligence in Medicine 36(1):85-117.

SummaryObjective This manuscript describes the development of a general intelligent tutoring system for teaching visual classification problem solving.Materials and methods The approach is informed by cognitive theory, previous empirical work on expertise in diagnostic problem-solving, and our own prior work describing the development of expertise in pathology. The architecture incorporates aspects of cognitive tutoring system and knowledge-based system design within the framework of the unified problem-solving method description language component model. Based on the domain ontology, domain task ontology and case data, the abstract problem-solving methods of the expert model create a dynamic solution graph. Student interaction with the solution graph is filtered through an instructional layer, which is created by a second set of abstract problem-solving methods and pedagogic ontologies, in response to the current state of the student model.Results In this paper, we outline the empirically derived requirements and design principles, describe the knowledge representation and dynamic solution graph, detail the functioning of the instructional layer, and demonstrate two implemented interfaces to the 
system.Conclusion Using the general visual classification tutor, we have created SlideTutor, a tutoring system for microscopic diagnosis of inflammatory diseases of skin.

Csikszentmihalyi, M, and K Sawyer. 1996, "Chapter 10 - Creative Insight: The Social Dimension of a Solitary Moment." in The Nature of Insight, eds. RJ Sternberg and JE Davidson, pp. 329-61. MIT Press.

Cugini, J, S Laskowski, and M Sebrechts. 2000, "Design of 3-D Visualization of Search Results: Evolution and Evaluation." In 7th Meeting on Visual Data Exploration and Analysis, pp. 198-210.

We discuss the evolution of the NIST Information Retrieval Visualization Engine (NIRVE), This prototype employs modern interactive visualization techniques to provide easier access to a set of documents resulting from a query to a search engine. The motivation and evaluation of several design features, such as keyword to concept mapping, explicit clustering, the use of 3-D vs. 2-D, and the relationship of visualization to logical structure are described. in particular, the results of an extensive usability experiment show how visualization may lead to either increased or decreased cognitive load.

Cui, J, et al. 2010, "A Curved Ray Camera for Handling Occlusions through Continuous Multiperspective Visualization." IEEE Transactions on Visualization and Computer Graphics 16(6):1235-42.

Most images used in visualization are computed with the planar pinhole camera. This classic camera model has important advantages such as simplicity, which enables efficient software and hardware implementations, and similarity to the human eye, which yields images familiar to the user. However, the planar pinhole camera has only a single viewpoint, which limits images to parts of the scene to which there is direct line of sight. In this paper we introduce the curved ray camera to address the single viewpoint limitation. Rays are C1-continuous curves that bend to circumvent occluders. Our camera is designed to provide a fast 3-D point projection operation, which enables interactive visualization. The camera supports both 3-D surface and volume datasets. The camera is a powerful tool that enables seamless integration of multiple perspectives for overcoming occlusions in visualization while minimizing distortions.

Cui, Y, and J Widom. 2003, "Lineage Tracing for General Data Warehouse Transformations." The VLDB Journal The International Journal on Very Large Data Bases 12(1):41-58.

Data warehousing systems integrate information from operational data sources into a central repository to en- able analysis and mining of the integrated information. Dur- ing the integration process, source data typically undergoes a series of transformations, which may vary from simple alge- braic operations or aggregations to complex "data cleansing" procedures. In a warehousing environment, the data lineage problem is that of tracing warehouse data items back to the original source items from which they were derived.We for- mally define the lineage tracing problem in the presence of general data warehouse transformations, and we present al- gorithms for lineage tracing in this environment. Our tracing procedures take advantage of known structure or properties of transformations when present, but alsowork in the absence of such information. Our results can be used as the basis for a lineage tracing tool in a generalwarehousing setting, and also can guide the design of data warehouses that enable efficient lineage tracing. 
Cvek, U, et al. 2008, "From Microarrays to Promoters: The Visual Story of Stat3." In 5th International Conference on BioMedical Visualization, pp. 15-20.

High-throughput technologies have established themselves as indispensable for the study of biological systems, from gene expression level changes, protein concentrations. to their modifications and interactions in complex diseases and systems. This kind of data analysis is not well served by the biostatistical techniques traditionally applied to biomedical and clinical data sets. Non-trivial patterns are most often discovered using visual and other computational tools applied to this data. We derive patterns and information from a series of differentially expressed genes on eight microarrays combined with analysis of promoter regions using regular expression-driven examination of short representative sequences called motifs. These non-trivial patterns are used to aid the discovery process through the use of information visualization and by harnessing user's perceptual and cognitive capabilities.

Cyr, D, M Head, and A Ivanov. 2006, "Design Aesthetics Leading to M-Loyalty in Mobile Commerce." Information \& Management 43(8):950-63.

Researchers have previously examined the technology acceptance model (TAM) in many contexts, including the Internet. More recently TAM has been enhanced to include a hedonic component of enjoyment but the effect has rarely been investigated in a mobile commerce context. In addition, specific antecedents of TAM related to design aesthetics have not been examined within the mobile domain. Our research filled these gaps, and discovered that visual design aesthetics did significantly impact perceived usefulness, ease of use, and enjoyment, all of which ultimately influenced users' loyalty intentions towards a mobile service.

Cyr, D, M Head, and A Ivanov. 2009, "Perceived Interactivity Leading to E-Loyalty: Development of a Model for Cognitive-Affective User Responses." International Journal of Human-Computer Studies 67(10):850-69.

Novel applications of website interactivity are important to attract and retain online users. In this empirical study five designs for interactivity are examined using different web-poll interfaces. The goal of the investigation is to examine perceived interactivity in a model which includes most commonly tested cognitive elements such as efficiency and effectiveness, but augments this model with the inclusion of a cognitive-affective element for trust, and an affective element of enjoyment. More specifically, a model is created to validate the relationship of perceived interactivity (comprised of user control, user connectedness, and responsiveness of the web-poll application) to efficiency, effectiveness, trust and enjoyment, of the website. In turn, efficiency, effectiveness, trust, and enjoyment are tested for their influence on user behavioral intentions for e-loyalty. All relationships in the model are supported. In addition, exploratory evaluation of qualitative comments is conducted to investigate additional insights between the five web-poll treatments in this investigation. The research confirms the complexity of a model in which cognitive, cognitive-affective and affective elements are present, and advances knowledge on the consequences of perceived interactivity. In additional to theoretical advancements, the research has merit for web designers and online marketers regarding how to enhance interactive online web applications. 
Czerwinski, MP, et al. 1999, "The Contribution of Thumbnail Image, Mouse-over Text and Spatial Location Memory to Web Page Retrieval in 3d." In International Conference on Human-Computer Interaction (INTERACT 99), pp. 163-70.

We present an empirical evaluation of the contribution of pictorial image and spatial location information on the retrieval of previously stored Web pages. Subjects were given 100 snapshots of Web pages that they stored in spatial locations on an inclined plane in a desktop 3D environment (Data Mountain). We had them return and try to retrieve their pages again, using a variety of retrieval cues. Even though users had not seen their Web page layout for several months, their retrieval times were not significantly slower. In addition, on half of the trials, stored pages were not presented as thumbnail images of the Web pages but as blank icons. Taking the pictorial thumbnail images away initially led to a significant drop in subjects' ability to find the pages, although within a short period of time subjects were able to find the pages equally fast without the thumbnail information. These results indicate that the use of $3 \mathrm{D}$ visualization techniques such as those described in this paper can lead to improved user memory for where favourite or frequently used information is stored in an electronic environment.

Dabdoub, SM, S Member, and WC Ray. 2010, "Aesthetics and Understanding in Molecular Motion." In IEEE Conference on Information Visualization, pp. 1-2.

Dachselt, R, and A Hübner. 2007, "Three-Dimensional Menus: A Survey and Taxonomy." Computers \& Graphics 31(1):53-65.

Various interaction techniques have been developed in the field of virtual and augmented reality. Whereas techniques for object selection, manipulation, travel, and wayfinding have already been covered in existing taxonomies in some detail, application control techniques have not yet been sufficiently considered. However, they are needed by almost every mixed reality application, e.g., for choosing from alternative objects or options. For this purpose a great variety of distinct three-dimensional (3D) menu selection techniques is available. This paper surveys existing 3D menus from the corpus of literature and classifies them according to various criteria. The taxonomy introduced here assists developers of interactive 3D applications to better evaluate their options when choosing, optimizing, and implementing a 3D menu technique. Since the taxonomy spans the design space for 3D menu solutions, it also aids researchers in identifying opportunities to improve or create novel virtual menu techniques.

Dadzie, AS, V Lanfranchi, and D Petrelli. 2009, "Seeing Is Believing: Linking Data with Knowledge." Information Visualization 8(3):197-211.

The analysis of data using a visual tool is rarely a task done in isolation, it tends to be part of a wider goal: that of making sense of the current situation, often to support decision-making. A user-centred approach is needed in order to properly design interaction that supports sense-making incorporating visual data analysis. This paper reports the experience gained in X-Media, a project that aims to support knowledge management (KM), sharing and reuse across different media in large enterprises. We report the user-centred design approach adopted and the design phases that led to the first prototype. A user evaluation was conducted to assess the design and how different levels of data, information and knowledge were mapped using alternative visual tools. The results show that a clear separation of the visual data analysis from other sense-making sub-tasks helps users in focussing their attention. Users particularly appreciated the data analysis across different media and formats, as well as the support for contextualising information within the broader perspective of KM. Further work is needed to develop 
more fully intuitive visualisations that exploit the richer information in multimedia documents and make the multiple connections between data more easily accessible. Information Visualization (2009) 8, 197 211. doi: 10.1057/ivs.2009.11;

Dahlberg, TA, and KR Subramanian. 2001, "Visualization of Mobile Network Simulations." Simulation 77(3-4):128-40.

The use of adaptive techniques in mobile networks permits scalable resource allocation policies to meet varying demand as well as Quality of Service (QoS) performance objectives. As these algorithms operate at multiple layers of communications architecture, evaluation of such techniques must take into account a variety of scenarios, which are in turn parameterized by a large number of variables. The need to monitor algorithm behavior in real time results in a data explosion. In this work, we propose new realtime metrics to characterize an identify the critical states of a mobile network in the wake of channel failures, congestion, signal degradation, etc. We use these metrics to define a survivability index, a measure of mobile network performance in the wake of failures. We demonstrate the effectiveness of information visualization techniques in understanding the complex spatial and temporal relationships between performance and cost metrics that influence adaptive algorithms. Our visualization system is highly scalable and interactive, permitting multiple algorithms to be simultaneously evaluated. We demonstrate applications to network monitoring and to the design and evaluation of adaptive admission control algorithms.

D'Amico, A, and M Kocka. 2005, "Information Assurance Visualizations for Specific Stages of Situational Awareness and Intended Uses: Lessons Learned." In IEEE Workshop on Visualization for Computer Security, pp. 107-12.

Information visualization has proven to be a valuable tool for working more effectively with complex data and maintaining situational awareness in demanding operational domains. Unfortunately, many applications of visualization technology fall short of expectations because the technology is used inappropriately: the wrong tool applied in the wrong way. A study of visualization techniques as applied to one particularly demanding area - information assurance - leads to the conclusion that there is a proper and formal way to approach designing visualization techniques for maintaining situational awareness in complex domains. Visualization techniques should be specifically designed or selected to align with one of the three identified stages of situational awareness - perception, comprehension, or projection - and with one of five standard uses of visualization: monitoring, inspecting, exploring, forecasting, or communicating. Greater value can be realized by selecting the right visualization technique to focus on each operational task, rather than searching for a single allencompassing solution to fit every need. Examples of how visualizations can be used to support specific tasks of IA analysis are presented, with examples based on a review of available literature, a formal cognitive task analysis performed by the authors, and lessons learned from direct experience with developing IA visualizations and training analysts in their use.

D'Amico, AD, et al. 2007, "Visual Discovery in Computer Network Defense." Computer Graphics and Applications, IEEE 27(5):20-27.

Computer network defense (CND) requires analysts to detect both known and novel forms of attacks in massive volumes of network data. It's through discovering the unexpected that CND analysts detect new versions of mal ware (such as viruses and Trojan horses) that have passed through their antivirus 
products, new methods of intrusion that have breached their firewalls and intrusion detection systems (IDSs), and new groups of cyber-criminals pressing the attack. This paper presents visual assistant for information assurance analysis. VIAssist is a visualization framework based on a comprehensive cognitive task analysis of CND analysts, and so fits their work practices and operational environment.

Dang, TN, L Wilkinson, and A Anand. 2010, "Stacking Graphic Elements to Avoid over-Plotting." IEEE Transactions on Visualization and Computer Graphics 16(6):1044-52.

An ongoing challenge for information visualization is how to deal with over-plotting forced by ties or the relatively limitedvisual field of display devices. A popular solution is to represent local data density with area (bubble plots, treemaps), color(heatmaps), or aggregation (histograms, kernel densities, pixel displays). All of these methods have at least one of three deficiencies:1) magnitude judgments are biased because area and color have convex downward perceptual functions, 2) area, hue, and brightnesshave relatively restricted ranges of perceptual intensity compared to length representations, and/or 3) it is difficult to brush or link toindividual cases when viewing aggregations. In this paper, we introduce a new technique for visualizing and interacting with datasetsthat preserves density information by stacking overlapping cases. The overlapping data can be points or lines or other geometricelements, depending on the type of plot. We show real-dataset applications of this stacking paradigm and compare them to othertechniques that deal with over-plotting in high-dimensional displays.

Dansereau, DF, and DD Simpson. 2009, "A Picture Is Worth a Thousand Words: The Case for Graphic Representations." Professional Psychology: Research and Practice 40(1):104-10.

Research in psychology and communication shows a strong advantage for visual displays in comparison with typical language, and technological innovations in computer graphics and printing capabilities now make them cost-effective as well. It can be argued that the greater use of evidence-based visualization strategies can enhance communication occurring in the interactions among and between health service delivery counselors, clients, evaluators, administrators, and clinical supervisors. In this article we present conceptual, empirical, and practical reasons for increasing the use of node-link (boxline) graphic representations in psychological treatment systems. These general-purpose displays offer clinical advantages by clearly representing interrelationships among ideas, emotions, and actions that are often lost in verbal discourse.

Danziger, M. 2008, "Information Visualization for the People." in Dept. of Comparative Media Studies, Vol M.S. MIT.

Includes bibliographical references (p. 85-89). The design of information visualization, defined as the interactive, graphical presentation of data, is on the verge of a significant paradigm shift brought on by the continued maturation of the Information Age. Its traditional role as a scientific tool deployed by rigorous data analysts is in the process of expanding to include more mainstream uses and users, reflecting fundamental changes to the role of information and data in our increasingly digital society. However, visualization design theory remains rooted in earlier conceptions of its use, largely ignoring the needs of this new, non-expert audience. Accordingly, this thesis attempts to re-contextualize information visualization as a public-facing practice, and explores ways in which its design can shift from being described as "by experts, for experts" to a new characterization as "for the people." by Michael Danziger. S.M. 
Darling, E, et al. 2004, "An Experimental Investigation of Magnification Lens Offset and Its Impact on Imagery Analysis." In IEEE Symposium on Information Visualization, pp. p5-p5.

A digital lens is a user interface mechanism that is a potential solution to information mangement problems. We investigated the use of digital lensing applied to imagery analysis. Participants completed three different types of tasks (locate, follow, and compare) using a magnification lens with three different degrees of offset (aligned, adjacent, and docked) over a high-resolution aerial photo. Although no lens offset mode was significantly better than another, most participants preferred the adjacent mode for the locate and compare tasks, and the docked mode for the follow tasks. This paper describes the results of a user study of magnification lenses and provides new insights into preferences of and interactions with digital lensing.

Daschiel, H, and M Datcu. 2005, "Design and Evaluation of Human-Machine Communication for Image Information Mining." Multimedia, IEEE Transactions on 7(6):1036-46.

Very large volumes of heterogenous data, like multimedia, Earth observation images, scientific and engineering measurements, for instance, are continuously generated and stored. A typical case is the field of Earth observation. The widespread availability of high resolution images does not only explore the volumes of data, but also brings order at magnitude in the image detail, thus enormously increasing the information content. However, today's concepts and technologies are still limited in communicating the information content to people for use in real life applications. In this paper, we overview a new concept for knowledge-driven image information mining (KIM) and both analyze and evaluate it from the perspective of human-machine communication. The KIM concept enables the information communication from a very large image repository to users via the Internet. The communication is at a semantic level of representation and is adapted to the user's conjecture.

Dasgupta, A, and R Kosara. 2010, "Pargnostics: Screen-Space Metrics for Parallel Coordinates." Visualization and Computer Graphics, IEEE Transactions on 16(6):1017-26.

Interactive visualization requires the translation of data into a screen space of limited resolution. While currently ignored by most visualization models, this translation entails a loss of information and the introduction of a number of artifacts that can be useful, (e.g., aggregation, structures) or distracting (e.g., over-plotting, clutter) for the analysis. This phenomenon is observed in parallel coordinates, where overlapping lines between adjacent axes form distinct patterns, representing the relation between variables they connect. However, even for a small number of dimensions, the challenge is to effectively convey the relationships for all combinations of dimensions. The size of the dataset and a large number of dimensions only add to the complexity of this problem. To address these issues, we propose Pargnostics, parallel coordinates diagnostics, a model based on screen-space metrics that quantify the different visual structures. Pargnostics metrics are calculated for pairs of axes and take into account the resolution of the display as well as potential axis inversions. Metrics include the number of line crossings, crossing angles, convergence, overplotting, etc. To construct a visualization view, the user can pick from a ranked display showing pairs of coordinate axes and the structures between them, or examine all possible combinations of axes at once in a matrix display. Picking the best axes layout is an NP-complete problem in general, but we provide a way of automatically optimizing the display according to the user's preferences based on our metrics and model. 
Dastbaz, M. 2000, "Hypermedia Aided Learning (Hal): A Viewpoint on Delivering Education in the New Millennium." In IEEE International Conference on Information Visualization, pp. 44-48.

The paper discusses the emergence of multimedia systems and with it the emergence of hypermedia as a new and powerful learning medium. The effects of this new technology on computer aided learning (CAL) as well as problems associated with CAL are discussed. The paper argues that hypermedia aided learning (HAL) could remedy some of the problems associated with CAL and pave the way for a new generation of powerful learning tools to emerge. Evaluation of HAL as a learning resource is also presented. The problems associated with HAL and the prospect ahead are also discussed

Davidson, JE. 1996, "Chapter 4 - the Suddenness of Insight." in The Nature of Insight, eds. RJ Sternberg and JE Davidson, pp. 125-55. MIT Press.

Davidson, S, et al. 2007, "Provenance in Scientific Workflow Systems." Bulletin of the IEEE Computer Society Technical Committee on Data Engineering 30(4):44-50.

The automated tracking and storage of provenance information promises to be a major advantage of scientific workflow systems. We discuss issues related to data and workflow provenance, and present techniques for focusing user attention on meaningful provenance through "user views," for managing the provenance of nested scientific data, and for using information about the evolution of a workflow specification to understand the difference in the provenance of similar data products.

Debije-Meessen, AEJ, and JAH Jansen. 2006, "The Balance between Aesthetics, Usability and Corporate Identity: Graphic User Interface Design within a Commercial Company." In 10th International Conference on Information Visualization, pp. 357-61.

This paper describes the search for a balance between aesthetics, usability and corporate identity in product development. This case study shows that usability has been integrated in the process for some time now. Aesthetics and corporate identity is relatively new and not yet incorporated at the same level. Over the past three years we developed a new software style called Ocean. For the first time, as a starting point of the design process, the design team created an aesthetic concept. During the following design process, small, splintered checks are carried out on usability and on perception. However, no overall test is carried out to evaluate the perception of the product as a whole. Do our users experience the software product as professional, independent and human? Do they recognise the Oce brand they know from earlier marketing campaigns? Looking back on the Ocean design process, aesthetics proved itself to be a valid requirement. Expanding our tests with professional checks on perception would complete the embedding of aesthetics in our design process. This is a bridge that still needs to be crossed. To be able to cross this bridge we, as graphic user interface designers, need proper evaluation methods, better information and more case studies. In other words, we need backup from the information visualisation field in order to achieve this acceptance 
Decker, JW. 2010, "An Interactive, Visual Composite Tuner for Multi-Layer Spatial Data Sets." In IEEE Conference on Information Visualization.

Defanti, TA, and MD Brown. 1991, "Visualization in Scientific Computing." in Advances in Computers, ed. CY Marshall, Vol 33, pp. 247-307. Elsevier.

Dekker, S, and M Lutzhoft. 2004, "Correspondence, Cognition and Sensemaking: A Radical Empiricist View of Situation Awareness: Theory and Application." pp. 22-41. Ashgate Publishing Company, Burlington, VT.

Del Nero, HS. 1994, "Cognitive Systems and Cognitive Science." In IEEE International Conference on Systems, Man, and Cybernetics, pp. 1962-67.

There are two main strategies in cognitive science that pursue the very nature of mental powers. One is seeking the origins of the processes that can be called intelligent. The other is seeking the nature of what one might call mental objects. This article attempts to establish why mental objects are manipulated in the central nervous system, trying to understand one of the most intriguing features of this manipulation, i.e., switching from automatic to conscious modes of operation. In my view cognitive science has a broader view than cognitive systems, while setting the question about the nature of conscious control as opposed to automatic control. A tentative model of automatic to conscious manipulation is presented using the phase-locked-loop approach. Conscious control would be in this way the resulting process that emerges every time an object, here defined as an oscillation of a group of neurons, displays structural instability, i.e., there is a bifurcation point in the parameters space

Demsar, U. 2007, "Investigating Visual Exploration of Geospatial Data: An Exploratory Usability Experiment for Visual Data Mining." Computers, Environment and Urban Systems 31(5):551-71.

This study presents a small exploratory usability experiment with the goal to observe how people visually explore geospatial data. The well-known iris dataset from pattern recognition was put into geographical context for this experiment, in order to provide the participants with a dataset with easily observable spatial and other relationships. The participants were given free hand to explore this dataset with a visual data mining system in any way they liked. The protocols collected during the experiment with the thinking-aloud method were analysed with the aim to understand what types of hypotheses the participants formed, which visualisations they used to either derive, confirm or reject their hypotheses and what exploration strategies they adopted.

Derthick, M, and SF Roith. 2001, "Enhancing Data Exploration with a Branching History of User Operations." Knowledge-Based Systems 14(1-2):65-74.

Backtracking and investigating alternative scenarios are integral parts of exploratory data analysis. Yet today's interfaces cannot represent alternative exploration paths as a branching history, forcing the user to recognize conceptual branch points in a linear history. Further, the interface can only show information from one state at a time, forcing users to rely on memory to compare scenarios. Our system includes a tree-structured visualization for navigating across time and scenarios. The visualization also allows browsing the history and selectively undoing/redoing events within a scenario or across scenarios. It uses the AI formalism of contexts to maintain multiple, possibly mutually inconsistent, knowledge base 
states. Cross-context formulas can be written for explicit scenario comparison, including visualizations of scenario differences.

Deshpande, G, P Santhanam, and X Hu. 2011, "Instantaneous and Causal Connectivity in Resting State Brain Networks Derived from Functional Mri Data." NeuroImage 54(2):1043-52.

Background Most neuroimaging studies of resting state networks have concentrated on functional connectivity (FC) based on instantaneous correlation in a single network. In this study we investigated both FC and effective connectivity (EC) based on Granger causality of four important networks at resting state derived from functional magnetic resonance imaging data -- default mode network (DMN), hippocampal cortical memory network (HCMN), dorsal attention network (DAN) and fronto-parietal control network (FPCN).Methodology/principle findings A method called correlation-purged Granger causality analysis was used, not only enabling the simultaneous evaluation of FC and EC of all networks using a single multivariate model, but also accounting for the interaction between them resulting from the smoothing of neuronal activity by hemodynamics. FC was visualized using a force-directed layout upon which causal interactions were overlaid. FC results revealed that DAN is very tightly coupled compared to the other networks while the DMN forms the backbone around which the other networks amalgamate. The pattern of bidirectional causal interactions indicates that posterior cingulate and posterior inferior parietal lobule of DMN act as major hubs. The pattern of unidirectional causal paths revealed that hippocampus and anterior prefrontal cortex (aPFC) receive major inputs, likely reflecting memory encoding/retrieval and cognitive integration, respectively. Major outputs emanating from anterior insula and middle temporal area, which are directed at aPFC, may carry information about interoceptive awareness and external environment, respectively, into aPFC for integration, supporting the hypothesis that aPFC-seeded FPCN acts as a control network.Conclusions/significance Our findings indicate the following. First, regions whose activities are not synchronized interact via time-delayed causal influences. Second, the causal interactions are organized such that cingulo-parietal regions act as hubs. Finally, segregation of different resting state networks is not clear cut but only by soft boundaries.

Deursen, AV. 2010, "A Pragmatic Perspective on Software Visualization." In 5th ACM Symposium on Software Visualization (SOFTVIS), pp. 4503-03.

Devai, F. 2002, "An Analysis Technique and an Algorithm for Line Clipping." In IEEE Conference on Information Visualization, pp. 157-65.

A mathematical model for the expected-time analysis of line-clipping algorithms is proposed. Assuming that all clipping windows are equally likely, we demonstrate that the probability of a line segment being totally outside the window approaches the value of $8 / 9$, provided that the line segments tend to be short and evenly distributed within a rectangle. Acceptance-rejection matrices are introduced for a machine-independent comparison of algorithms. A new line-clipping method, called QuickClip, is also proposed. QuickClip is not only simpler and more concise than the Cohen-Sutherland algorithm, the most widely used method in computer graphics, but also faster both in terms of machine-independent analysis and timing results on random line segments. Timing results also indicate that QuickClip is significantly faster than the Nicholl-Lee-Nicholl (1987) algorithm. The theoretical results generalise to $3 \mathrm{D}$, with the important consequence that the average clipping volume is $1 / 27$ of the volume of the model 
Dhillon, IS, DS Modha, and WS Spangler. 2002, "Class Visualization of High-Dimensional Data with Applications." Computational Statistics \& Data Analysis 41(1):59-90.

The problem of visualizing high-dimensional data that has been categorized into various classes is considered. The goal in visualizing is to quickly absorb inter-class and intra-class relationships. Towards this end, class-preserving projections of the multidimensional data onto two-dimensional planes, which can be displayed on a computer screen, are introduced. These class-preserving projections maintain the high-dimensional class structure, and are closely related to Fisher's linear discriminants. By displaying sequences of such two-dimensional projections and by moving continuously from one projection to the next, an illusion of smooth motion through a multidimensional display can be created. Such sequences are called class tours. Furthermore, class-similarity graphs are overlaid on the two-dimensional projections to capture the distance relationships in the original high-dimensional space. The above visualization tools are illustrated on the classical Iris plant data, the ISOLET spoken letter data, and the PENDIGITS on-line handwriting data set. It is shown how the visual examination of the data can uncover latent class relationships.

Diakopoulos, N, M Naaman, and F Kivran-swaine. 2010, "Diamonds in the Rough : Social Media Visual Analytics for Journalistic Inquiry." In IEEE Symposium on Visual Analytics Science \& Technology, pp. 115-22.

Diehl, S, and Acm. 2005, "Software Visualization." In 27th International Conference on Software Engineering (ICSE 2005), pp. 718-19.

This half-day tutorial gives an overview of the current state-of-the-art in software visualization. Software visualization encompasses the development and evaluation of methods for graphically representing different aspects of software, including its structure, its execution, and its evolution. In contrast to visual programming and diagramming for software design, software visualization is not so much concerned with the construction, but with the analysis of programs and their development process. Software visualization combines techniques from areas like software engineering, programming languages, data mining, computer graphics, information visualization and human-computer interaction. Topics covered in this tutorial include static program visualization, algorithm animation, visual debugging, as well as the visualization of the evolution of software. In particular we identify common principles illustrated by many examples and give pointers to tools available today.

Diehl, S, F Beck, and M Burch. 2010, "Uncovering Strengths and Weaknesses of Radial Visualizations--an Empirical Approach." IEEE Transactions on Visualization and Computer Graphics 16(6):935-42.

Radial visualizations play an important role in the information visualization community. But the decision to choose a radial coordinate system is rather based on intuition than on scientific foundations. The empirical approach presented in this paper aims at uncovering strengths and weaknesses of radial visualizations by comparing them to equivalent ones in Cartesian coordinate systems. We identified memorizing positions of visual elements as a generic task when working with visualizations. A first study with 674 participants provides a broad data spectrum for exploring differences between the two visualization types. A second, complementing study with fewer participants focuses on further questions raised by the first study. Our findings document that Cartesian visualizations tend to outperform their radial counterparts especially with respect to answer times. Nonetheless, radial visualization seem to be more appropriate for focusing on a particular data dimension. 
Dillman, DA, et al. 2009, "Response Rate and Measurement Differences in Mixed-Mode Surveys Using Mail, Telephone, Interactive Voice Response (Ivr) and the Internet." Social Science Research 38(1):1-18.

The potential for improving response rates by changing from one mode of data collection to another mode and the consequences for measurement and nonresponse errors are examined. Data collection from 8999 households was done in two phases. Phase 1 data collection was conducted by telephone interview, mail, interactive voice response, or the Internet, while Phase 2 focused on nonrespondents to Phase 1, and was conducted by a different mode, either telephone or mail. Results from our study suggest that switching to a second mode is an effective means of improving response. We also find that for the satisfaction-dissatisfaction questions asked in this survey, respondents to the aural modes (telephone and IVR) are significantly more likely than are respondents to the visual modes (mail and web) to give extreme positive responses, a difference that cannot be accounted for by a tendency towards recency effects with telephone. In general, switching to a second mode of data collection was not an effective means of reducing nonresponse error based on demographics.

Ding, Y, GG Chowdhury, and S Foo. 2001, "Bibliometric Cartography of Information Retrieval Research by Using Co-Word Analysis." Information Processing \& Management 37(6):817-42.

Ding, Y, et al. 2000, "Bibliometric Information Retrieval System (Birs): A Web Search Interface Utilizing Bibliometric Research Results." Journal of the American Society for Information Science 51(13):1190-204.

The aim of this article is to test whether the results obtained from a specific bibliographic research can be applied to a real search environment and enhance the level of utility of an information retrieval session for all levels of end users. In this respect, a Web-based Bibliometric Information Retrieval System (BIRS) has been designed and created, with facilities to assist the end users to get better understanding of their search domain, formulate and expand their search queries, and visualize the bibliographic research results. There are three specific features in the system design of the BIRS: the information visualization feature of the BIRS (cocitation maps) to guide the end users to identify the important research groups and capture the detailed information about the intellectual structure of the search domain; the multilevel browsing feature to allow the end users to go to different levels of interesting topics; and the common user interface feature to enable the end users to search all kinds of databases regardless of different searching systems, different working platforms, different database producer and supplier, such as different Web search engines, different library OPACs, or different on-line databases, A preliminary user evaluation study of BIRS revealed that users generally found it easy to form and expand their queries, and that BIRS helped them acquire useful background information about the search domain. They also pointed out aspects of information visualization, multilevel browsing, and common user interface as novel characteristics exhibited by BIRS.

Dittenbach, M, A Rauber, and D Merkl. 2002, "Uncovering Hierarchical Structure in Data Using the Growing Hierarchical Self-Organizing Map." Neurocomputing 48(1-4):199-216.

Discovering the inherent structure in data has become one of the major challenges in data mining applications. It requires stable and adaptive models that are capable of handling the typically very highdimensional feature spaces. In particular, the representation of hierarchical relations and intuitively visible cluster boundaries are essential for a wide range of data mining applications. Current approaches based on neural networks hardly fulfill these requirements within a single model. In this paper we present the 
growing hierarchical self-organizing map (), a neural network model based on the self-organizing map. The main feature of this novel architecture is its capability of growing both in terms of map size as well as in a three-dimensional tree-structure in order to represent the hierarchical structure present in a data collection during an unsupervised training process. This capability, combined with the stability of the self-organizing map for high-dimensional feature space representation, makes it an ideal tool for data analysis and exploration. We demonstrate the potential of the with an application from the information retrieval domain, which is prototypical both of the high-dimensional feature spaces frequently encountered in today's applications as well as of the hierarchical nature of data.

Dix, A. 2010, "Human-Computer Interaction: A Stable Discipline, a Nascent Science, and the Growth of the Long Tail." Interacting with Computers 22(1):13-27.

This paper represents a personal view of the state of HCI as a design discipline and as a scientific discipline, and how this is changing in the face of new technological and social situations. Going back 20 years a frequent topic of discussion was whether HCI was a [']discipline'. It is unclear whether this was ever a fruitful topic, but academic disciplines are effectively about academic communities and there is ample evidence of the long-term stability of the international HCI/CHI community. However, as in computer [']science', the central scientific core of HCI is perhaps still unclear; for example, a strength of $\mathrm{HCI}$ is the closeness between theory and practice, but the corresponding danger is that the two are often confused. The paper focuses particularly on the challenge of methodological thinking in HCI, especially as the technological and social context of HCI rapidly changes. This is set alongside two other challenges: the development of reliable knowledge in HCI and the clear understanding of interlinked human roles within the discipline. As a case study of the need for methodological thinking, the paper considers the use of single person studies in research and design. These are likely to be particularly valuable as we move from a small number of applications used by many people to a [']long tail' where large numbers of applications are used by small numbers of people. This change calls for different practical design strategies; focusing on the peak experience of a few rather than acceptable performance for many. Moving back to the broader picture, as we see more diversity both in terms of types of systems and kinds of concerns, this may also be an opportunity to reflect on what is core across these; potential fragmentation becoming a locus to understand more clearly what defines HCI, not just for the things we see now, but for the future that we cannot see.

do Nascimento, HAD, and P Eades. 2005, "User Hints: A Framework for Interactive Optimization." Future Generation Computer Systems 21(7):1177-91.

Innovative improvements in the area of human-computer interaction and user interfaces have enabled intuitive and effective applications for a variety of problems. On the other hand, there has also been the realization that several real-world optimization problems still cannot be totally automated. Very often, user interaction is necessary for refining the optimization problem, managing the computational resources available, or validating or adjusting a computer-generated solution. This paper presents an interactive framework called user hints for having humans help optimization methods to solve difficult problems. In the framework users play a dynamic and important role by providing hints. Hints are actions that help to insert domain knowledge, to escape from local minima, to reduce the space of solutions to be explored, or to avoid ambiguity when there is more than one optimal solution. User hints are given in an intuitive way through a graphical interface. Visualization tools are also included in order to inform the user about the state of the optimization process. We discuss applications of the user hints framework to the graph 
drawing and the map labeling problems. An evaluation of some user hints systems indicates that optimization processes can benefit from human interaction. (c) 2004 Elsevier B.V. All rights reserved.

Dobson, A. 1997, "Architectural Composition in the Electronic Design Studio: Conceptual Design Using Cad Visualisation and Virtual Reality Modelling." In IEEE Conference on Information Visualization, pp. 179-85.

The paper evaluates the possibilities for the use of computer aided design and desktop virtual reality technologies as tools for architectural composition. An experimental teaching programme involving undergraduate architectural students at the University of Luton, in which aspects of compositional theory are explored through the direct creation of architectural form and space in digital formats is described. In the programme principles of architectural composition, based upon the ordering and organisation of typological architectural elements according to established rules of composition are introduced to the students through the study of recognised works of design theory. CAD and desktop virtual reality are then used to define and manipulate architectural elements, and to make formal and spatial evaluations of the environments created. The paper describes the theoretical context of the work, assesses the suitability of the software used for performing compositional manipulations, and evaluates the qualities of immersion and intuitive feedback which virtual reality based modelling can offer in the design visualisation process. The teaching programme utilises standard software packages, including AutoCAD, and 3D Studio, as well as Superscape VRT, a PC based desktop VR package

Dodge, S, R Weibel, and A-K Lautenschütz. 2008, "Towards a Taxonomy of Movement Patterns." Information Visualization 7(3-4):240-52.

Dollner, J, and M Walther. 2003, "Real-Time Expressive Rendering of City Models." In 7th International Conference on Information Visualization, pp. 245-50.

City models have become central elements for visually communicating spatial information related to urban areas and have manifold applications. Our real-time nonphotorealistic rendering technique aims at abstract, comprehensible, and vivid drawings of assemblies of polygonal 3D urban objects. It takes into account related principles in cartography, cognition, and nonphotorealism. Technically, the geometry of a building is rendered using expressive line drawings to enhance the edges, two-tone or three-tone shading to draw the faces, and simulated shadows. The edge enhancement offers several degrees of freedom, such as interactively changing the style, width, tilt, color, transparency, and length of the strokes. Traditional drawings of cities and panoramas inspired the tone shading that achieves a pleasing visual color effect. The rendering technique can be applied not only to city models but to polygonal shapes in general.

Dominowski, RL, and P Dallob. 1996, "Insight and Problem Solving." in The Nature of Insight, eds. RJ Sternberg and JE Davidson, pp. 33-62. MIT Press.

Donato, JM, et al. 1999, "Mining Multi-Dimensional Data for Decision Support." Future Generation Computer Systems 15(3):433-41.

Personal bankruptcy is an increasingly common yet little understood phenomenon. Attempts to predict bankruptcy have involved the application of data mining techniques to credit card data. This is a difficult problem, since credit card data is multi-dimensional, consisting of monthly account records and 
daily transaction records. In this paper, we describe a two-stage approach that combines decision trees and neural networks to predict personal bankruptcy using credit card data.

Dörk, M, et al. 2010, "A Visual Backchannel for Large-Scale Events." IEEE Transactions on Visualization and Computer Graphics 16(6):1129-38.

We introduce the concept of a Visual Backchannel as a novel way of following and exploring online conversations aboutlarge-scale events. Microblogging communities, such as Twitter, are increasingly used as digital backchannels for timely exchange ofbrief comments and impressions during political speeches, sport competitions, natural disasters, and other large events. Currently,shared updates are typically displayed in the form of a simple list, making it difficult to get an overview of the fast-paced discussions asit happens in the moment and how it evolves over time. In contrast, our Visual Backchannel design provides an evolving, interactive,and multi-faceted visual overview of large-scale ongoing conversations on Twitter. To visualize a continuously updating informationstream, we include visual saliency for what is happening now and what has just happened, set in the context of the evolving conversation.As part of a fully web-based coordinated-view system we introduce Topic Streams, a temporally adjustable stacked graphvisualizing topics over time, a People Spiral representing participants and their activity, and an Image Cloud encoding the popularityof event photos by size. Together with a post listing, these mutually linked views support cross-filtering along topics, participants, andtime ranges. We discuss our design considerations, in particular with respect to evolving visualizations of dynamically changing data.Initial feedback indicates significant interest and suggests several unanticipated uses.

Dornburg, CC, et al. 2009, "Working Memory Load as a Novel Tool for Evaluating Visual Analytics." In IEEE Symposium on Visual Analytics Science and Technology, pp. 217-18.

The current visual analytics literature highlights design and evaluation processes that are highly variable and situation dependent, which raises at least two broad challenges. First, lack of a standardized evaluation criterion leads to costly re-designs for each task and specific user community. Second, this inadequacy in criterion validation raises significant uncertainty regarding visualization outputs and their related decisions, which may be especially troubling in high consequence environments like those of the Intelligence Community. As an attempt to standardize the "apples and oranges" of the extant situation, we propose the creation of standardized evaluation tools using general principles of human cognition. Theoretically, visual analytics enables the user to see information in a way that should attenuate the user's memory load and increase the user's task-available cognitive resources. By using general cognitive abilities like available working memory resources as our dependent measures, we propose to develop standardized evaluative capabilities that can be generalized across contexts, tasks, and user communities. (C)2009 IEEE.

Dörner, R, and C Ware. 2004, "Visual Interactive Stimuli Techniques: Interactive Tools for Exploring Data Using Behavioral Animation." Journal of Visual Languages \& Computing 15(2):161-81.

Data driven behavioral animation is a new visual computing technique that involves mapping data attributes to the behavioral functions of a set of graphical data avatars. When the avatars are allowed to interact, their individual and group behavior reveals qualitative structures in the data as well as relationships. In this paper we present a set of novel techniques for interacting with systems of data avatars. These are especially intended to support the user in exploring the data visually in the early stages of data analysis. We show that the methodology supports a comprehensive set of interactive tasks 
including the abstracting of meaning, comparing groups, identifying relationships, imposing structures and hypothesis testing. The techniques are implemented in a system called AnimVis that supports behavioral data exploration in a two-dimensional arena.

Dou, W, et al. 2009, "Recovering Reasoning Processes from User Interactions." IEEE Computer Graphics and Applications 29(3):52-61.

Understanding how analysts use visual-analytics (VA) tools can help reveal their reasoning processes when using these tools. By examining analysts' interaction logs, the authors identified the analysts' strategies, methods, and findings when using a financial VA tool.

Dou, W, et al. 2010, "Comparing Different Levels of Interaction Constraints for Deriving Visual Problem Isomorphs." In IEEE Conference on Information Visualization, pp. 195-202.

Downing, RE, JL Moore, and SW Brown. 2005, "The Effects and Interaction of Spatial Visualization and Domain Expertise on Information Seeking." Computers in Human Behavior 21(2):195-209.

Information seeking skills are becoming increasingly important as rapid and widespread developments in technology have made information available in more formats and from more sources than ever before. Research in human computer interaction (HCI) has demonstrated that primary cognitive abilities represent a powerful predictor of information-seeking success in electronic information systems. Specifically, spatial visualization ability (SVA) seems to be particularly related to hierarchical menus systems navigation within databases, online learning environments, information archival systems, and virtually all internet web sites. Research indicates that individuals with low SVA take longer to complete tasks and experience more errors on first attempts to find information in hierarchical databases compared to those with high SVA. Understanding the influences of SVA as well as its interaction with other aspects of individual differences, such as domain expertise, is critical to the design of systems intended to accommodate individual differences in users. Thirty-five college students (23 males and 12 females) were selected from the general student body of two universities and assigned to groups based upon their selfreported membership in one of two specific disciplines: business $(n=26)$ or biology $(n=9)$. Participants were then assigned to groups based upon scores on tests of SVA using a median-split. Each participant conducted five searches: one neutral search, two searches for business related information, and two searches for biology related information using the FirstSearch archival search tool. A $2 \times 2$ factorial Analysis of Variance with one between-groups variable (high vs. low SVA) and one within-group variable (high vs. low domain expertise) indicated a significant main effect of SVA as well as a significant main effect of Domain Expertise on the time required to find their first relevant article on the search topic. The analysis also revealed that there was no main effect for SVA on the total number of relevant articles found during the search period but there was a significant main effect of Domain Expertise on the total total-number-of-relevant-articles found. There was no interaction between SVA and Domain Expertise on either time to first article or total number of articles found. Results of the study extend existing knowledge regarding the effects of SVA and domain expertise on information seeking by demonstrating a strong effect of SVA and domain expertise on information seeking skills. The results of this study also provide evidence in support of interface designs that are friendlier to information seekers who have low SVA. Related findings and suggestions for further research are discussed. 
Driessen, S, J Jacobs, and WO Huijsen. 2007, "Combining Query and Visual Search for Knowledge Mapping." In 10th International Conference on Information Visualization, pp. 216-24.

Searching through information can be done in two ways: by querying and browsing. In the literature these approaches are treated separately. However every search usually consists of a combination of browsing and querying. Therefore this paper is about combining querying and browsing in one search interface. Our hypothesis is that both strategies can be combined and users will be better supported in their search for information. A model is presented how to combine these approaches and this model is evaluated by applying it in the knowledge-mapping domain. We will show that combining both strategies in one model is possible. Based on our evaluation of the application of this model we conclude that the users understand this combination and find it to be helpful. However several technical issues need to be addressed in our future research to confirm the usefulness of this combination

Droste, P, et al. 2010, "Customizable Visualization on Demand for Hierarchically Organized Information in Biochemical Networks." In 2nd International Symposium on Computational Modeling of Objects Represented in Images, pp. 163-74.

Systems biology is concerned with systemic and integrative studies of complex interactions between molecular components within microorganisms and higher cells, and aims at generating holistic understanding of biological processes. The interactions between genes, proteins and metabolites are naturally represented by hierarchically interconnected biochemical networks on different levels. Moreover, the network topology is typically supplemented by qualitative information and quantitative data on individual mechanisms and system states, for example enzyme kinetics and flow rates. Measurement data are often combined with simulation results and knowledge from databases. Due to constantly improving high throughput techniques we increasingly face the challenge of visualizing manifold and extensive data within large biochemical network diagrams. This issue is effectively addressed by the interactive Visualization on Demand (VoD) approach for hierarchically structured network diagrams presented in this contribution.

Druin, A, et al. 2003, "Designing a Digital Library for Young Children: An Intergenerational Partnership." in The Craft of Information Visualization, Readings and Reflections, eds. BB Benjamin and S Ben, pp. 178-85. Morgan Kaufmann, San Francisco.

Summary As more information resources become accessible using computers, our digital interfaces to those resources need to be appropriate for all people. However when it comes to digital libraries, the interfaces have typically been designed for older children or adults. Therefore, we have begun to develop a digital library interface developmentally appropriate for young children (ages 5-10 years old). Our prototype system we now call "SearchKids" offers a graphical interface for querying, browsing and reviewing search results. This paper describes our motivation for the research, the design partnership we established between children and adults, our design process, the technology outcomes of our current work, and the lessons we have learned. 
Dubchak, PI, et al. 2010, "Panel : Challenges in Visualizing Biological Data." In IEEE Conference on Information Visualization.

Dudzic, S, and JA Godwin. 2010, "Visualization of Temporal Relationships within Coordinated Views." In IEEE Symposium on Visual Analytics Science \& Technology, pp. 219-20.

Duez, P, and KJ Vicente. 2005, "Ecological Interface Design and Computer Network Management: The Effects of Network Size and Fault Frequency." International Journal of Human-Computer Studies 63(6):565-86.

This article describes an experiment investigating the impact of ecological interface design (EID) on human performance in computer network management. This work domain is more dynamic than those previously studied under EID because there is a constant potential for the addition and removal of devices, as well as changing configurations, making it important to study the generalizability of the framework. Two interfaces were created for the University of Toronto campus network consisting of 220 nodes: a P interface based on existing design practices which presented primarily physical information and a $\mathrm{P}+\mathrm{F}$ interface based on EID which presented both physical and functional information identified by an abstraction hierarchy analysis. Participants used one of the two interfaces to detect and diagnose faults or disturbances in the simulated network in real-time. Network size and fault frequency were both manipulated as within-participants variables. The $\mathrm{P}+\mathrm{F}$ interface led to faster detection times overall, as well as improved fault detection rate and more accurate fault diagnosis under higher fault loads. These results suggest that the EID framework may lead to more robust monitoring performance in computer network management compared to existing interfaces.

Duke, DJ, KW Brodlie, and Da Duce. 2004, "Building an Ontology of Visualization." In IEEE Visualization 2004, pp. 7p-7p.

Recent activity within the UK National e-Science Programme has identified a need to establish an ontology for visualization. Motivation for this includes defining web and grid services for visualization (the 'semantic grid'), supporting collaborative work, curation, and underpinning visualization research and education. At a preliminary meeting, members of the UK visualization community identified a skeleton for the ontology. We have started to build on this by identifying how existing work might be related and utilized. We believe that the greatest challenge is reaching a consensus within the visualization community itself. This poster is intended as one step in this process, setting out the perceived needs for the ontology, and sketching initial directions. It is hoped that this will lead to debate, feedback and involvement across the community.

Duke, DJ, K W, and I Herman. 2005, "Do You See What I Mean ?" IEEE Computer Graphics and Applications 25(3):6-9.

Visualizers have to think about how people extract meaning from pictures (psychophysics), what people understand from a picture (cognition), how pictures are imbued with meaning (semiotics), and how in some cases that meaning arises within a social and/or cultural context. 
Duke, J, A Faiola, and H Kharrazi. 2009, "A Novel Visualization Tool for Evaluating Medication SideEffects in Multi-Drug Regimens." In 13th International Conference on Human-Computer Interaction, pp. 478-87.

The evaluation and management of medication side-effects is a common and complex task for physicians. Information visualization has the potential to increase the efficiency and reduce the cognitive load involved in this process. We describe the design and development of Rxplore, a novel tool for assessing medication side-effects. Rxplore supports simultaneous lookup of multiple medications and an intuitive visual representation of query results. In a pilot study of Rxplore's usability and utility, physicians rated the system highly for efficiency, intuitiveness, and clinical value.

Duke, JD, X Li, and SJ Grannis. 2010, "Data Visualization Speeds Review of Potential Adverse Drug Events in Patients on Multiple Medications." Journal of Biomedical Informatics 43(2):326-31.

Patients on multiple medications are at increased risk for adverse drug events. While physicians can reduce this risk by regularly reviewing the side-effect profiles of their patients' medications, this process can be time-consuming. We created a decision support system designed to expedite reviewing potential adverse reactions through information visualization. The system includes a database containing 16,340 unique drug and side-effect pairs, representing 250 common medications. A numeric score is assigned to each pair reflecting the strength of association between drug and effect. Based on these scores, the system generates graphical adverse reaction maps for any user-selected combination of drugs. A study comparing speed and accuracy of retrieving side-effect data using this tool versus UpToDate ${ }^{\circledR}$ demonstrated a $60 \%$ reduction in time to complete a query (61 s vs. $155 \mathrm{~s}, \mathrm{p}<0.0001)$ with no decrease in accuracy. These findings suggest that information visualization can significantly expedite review of potential adverse drug events.

Dumontier, M, L Ferres, and N Villanueva-Rosales. 2010, "Modeling and Querying Graphical Representations of Statistical Data." Web Semantics: Science, Services and Agents on the World Wide Web 8(2-3):241-54.

Although pictorial renditions of statistical data are ubiquitous, few techniques and standards exist to exchange, search and query these graphical representations. We present several improvements to humangraph interaction including (i) a new approach to manage statistical graph knowledge by semantic annotation of graphs that bridges the gap between Web 2.0 social tagging and formal, logic-based approaches, (ii) knowledge management and discovery across a non-trivial graph knowledge base and (iii) sophisticated question answering that requires background knowledge. 
Dumoulin, J, et al. 2010, "Poster : Multimedia Information Browsing and Visualization." In IEEE Conference on Information Visualization.

Dunbar, K. 1996, "How Scientists Really Reason : Scientific Reasoning in Real -World Laboratories." in Mechanisms of Insight, eds. RJ Sternberg and JE Davidson, pp. 365-95. MIT Press.

Dunsmore, A, M Roper, and M Wood. 2000, "The Role of Comprehension in Software Inspection." Journal of Systems and Software 52(2-3):121-29.

In spite of code inspections having been demonstrated as an effective defect detection process, little work has been done to determine how this process best supports the object-oriented paradigm. In contrast, this paradigm (or at least its questionable manifestation in $\mathrm{C}++$ ) is well supported by tools that purport to aid comprehension. These tools typically take the form of visualisation tools designed to assist in the maintenance process, and it is natural to consider that these tools (or adaptations thereof) might also support inspection. However, since these tools claim to aid comprehension, it is important to consider the role of comprehension in inspection. Or put simply, does comprehension matter, or are there simple techniques in existence which are similarly effective in detecting defects? This paper presents the issues associated with inspections (and the complications presented by the object-oriented paradigm) and comprehension, and presents the results of two experiments which considered the relationship between comprehension and inspection. The results indicate a relationship, but further work is needed to determine the precise nature of this relationship and how inspections might best be supported in the future.

Dunsmuir, D, et al. 2010, "Czsaw , Imas \& Tableau : Collaboration among Teams." In IEEE Symposium on Visual Analytics Science \& Technology, pp. 267-68.

Durbha, SS, et al. 2009, "A Framework for Semantic Reconciliation of Disparate Earth Observation Thematic Data." Computers \& Geosciences 35(4):761-73.

There is a growing demand for digital databases of topographic and thematic information for a multitude of applications in environmental management, and also in data integration and efficient updating of other spatially oriented data. These thematic data sets are highly heterogeneous in syntax, structure and semantics as they are produced and provided by a variety of agencies having different definitions, standards and applications of the data. In this paper, we focus on the semantic heterogeneity in thematic information sources, as it has been widely recognized that the semantic conflicts are responsible for the most serious data heterogeneity problems hindering the efficient interoperability between heterogeneous information sources. In particular, we focus on the semantic heterogeneities present in the land cover classification schemes corresponding to the global land cover characterization data. We propose a framework (semantics enabled thematic data Integration (SETI)) that describes in depth the methodology involved in the reconciliation of such semantic conflicts by adopting the emerging semantic web technologies. Ontologies were developed for the classification schemes and a sharedontology approach for integrating the application level ontologies as described. We employ description logics (DL)-based reasoning on the terminological knowledge base developed for the land cover characterization which enables querying and retrieval that goes beyond keyword-based searches. 
Durso, FT, BR Johnson, and JM Crutchfield. 2010, "Dimensions of Air Traffic Control Tower Information Needs: From Information Requests to Display Design." Journal of Experimental Psychology: Applied 16(3):219-37.

In an effort to determine the information needs of tower air traffic controllers, instructors from the Federal Aviation Administration's Academy in Oklahoma City were asked to control traffic in a highfidelity tower cab simulator. Information requests were made apparent by eliminating access to standard tower information sources. Instead, controllers were required to ask for precisely the information they needed during the scenarios. The information requests were classified using an elaboration of Zwaan and Radvansky's (1998) dimensions of situation models. The vast majority of requests were about three of the dimensions originally developed for reading comprehension: the protagonist, intentionality, and space. The information requests were also classified into 28 operational categories (e.g., aircraft identification, destination). From these results, the data were summarized, not just statistically, but by the creation of display-hypotheses. The display-hypotheses were organized according to the situation-model dimensions. Controllers preferred data blocks organized by the situation-model principle over those that violated this organization. The summary display-hypotheses were quite simple and accounted for the vast majority of the information requests controllers made. The display-hypotheses accounted for the information needs of controllers during routine as well as off-nominal events.

Dykes, J. 2000, "An Approach to Virtual Environments for Visualization Using Linked Geo-Referenced Panoramic Imagery." Computers, Environment and Urban Systems 24(2):127-52.

The relentless pace at which technological advances are being made is resulting in a whole series of new digital media, data and data collection devices. The ways in which digital spatial information can be represented are changing and so are the types of use to which digital map products are put. Maps are increasingly used in the analytical stages of the research process for visualization. Cartographers are currently undertaking research into visualization with foci on: the potential for extending the forms of representation and the types of phenomena that are represented; providing mechanisms for interaction with these representations; managing the volumes of data involved; and developing a more complete understanding of their use. Here 360-degree panoramic imagery is used as a means of representing geographic information in a visualization context. Techniques are outlined for linking a number of panoramic images to a geographic base. These methods have been used to create navigable virtual environments in which qualitative and quantitative spatial information is embedded and analytical tools are provided. Such an environment has utility in a number of scenarios and applications. A software implementation that has been designed specifically for use in supporting student fieldwork is introduced and practical experience of using the software provided. It is extremely flexible, taking advantage of Internet protocols to communicate with remote databases that can be searched for spatial information and providing a virtual environment for visualization that acts as a geo-graphic user interface to multimedia information.

Dykes, J. 2005, "Chapter 13 - Facilitating Interaction for Geovisualization." in Exploring Geovisualization, eds. D Jason, MM Alan and K Menno-Jan, pp. 265-91. Elsevier, Oxford.

Summary Drawing upon the experience gained from developing geovisualization applications software, a cartographer's perspective is offered on using and creating instruments that support the process of visualization. In line with a belief that high levels of interaction are increasingly dominant as the defining requirement of geovisualization tools, a number of methods of achieving and supporting this 
are explored. Three highly interactive applications are introduced that demonstrate opportunities afforded by technological advances, propose particular designs and permit geovisualization. Parallels are drawn between interactivityand flexibilityand a range of methods of increasing the levels and flexibility of interaction between scientist and data are discussed, beyond the options available in standard applications software. Efficient combination of these various ways of instructing computers is identified as a key objective to overcoming impediments to the process of geovisualization, and the concept of the "visualization effort" required to chase ideas and support the thought process is emphasized. A number of means of increasing efficiencies, sharing software components and reusing resources to facilitate interaction are discussed. Scripting is identified as an approach that offers much to fields where application design involves combining existing software functionality in new and unpredictable ways in an iterative process of continual change. Geovisualization is one such application area and flexible highlevel environments for instructing computers that offer rapid results, efficiencies and flexibility by drawing upon existing functionality and the opportunity to augment this through integration with lower level languages possess considerable scope for use as instruments for ideation.

\section{Dykes, J, and DF Keefe. 2010, "Perspectives on Teaching Data Visualization." In IEEE Conference on} Information Visualization.

Dykes, J, AM MacEachren, and M-J Kraak. 2005, "Chapter 36 - Advancing Geovisualization." in Exploring Geovisualization, eds. D Jason, MM Alan and K Menno-Jan, pp. 691-703. Elsevier, Oxford.

Summary This short concluding chapter identifies a number of themes, issues and tensions that permeate through the contributions presented in this collected volume. These include: a focus on the use of geovisualization tools to support a wide range of users in tasks involving the acquisition, processing, and sharing of information; the need to support collaborative work in geovisualization; the various relationships between Information Visualization and geovisualization and ways in which synergies can be developed; the many issues surrounding the advantages and disadvantages of using $2 \mathrm{D}$ and $3 \mathrm{D}$ representations; the increasing demand for supporting geovisualization through mobile devices and the specific differences and constraints associated with static and mobile tools; the various drivers for developing geovisualization applications and the relationships between them. An argument for grounding efforts to support geovisualization in a deep understanding of the broader scientific process is drawn upon. It is suggested that doing so enables us to link the issues of instrument design, graphical representation, connecting distributed resources and the effective use of technological developments that structure the contributions to this book. Such a perspective provides a framework upon which the work reported here, emanating from a series of related disciplines, can draw. Doing so can help us develop the functionality and tools required to deploy and operationalize geovisualization through effective usable solutions that better employ the knowledge and visual functionality we have separately developed. The concept of "distributed cognition" is drawn from cognitive science as a means of informing such efforts to develop "the map" as a tool for presenting, using, interpreting and understanding information about geographic phenomena. 
Dykes, J, AM MacEachren, and M-J Kraak. 2005, "Introduction - Exploring Geovisualization." in Exploring Geovisualization, eds. D Jason, MM Alan and K Menno-Jan, pp. 1-19. Elsevier, Oxford.

Dykes, J, J Wood, and A Slingsby. 2010, "Rethinking Map Legends with Visualization." Visualization and Computer Graphics, IEEE Transactions on 16(6):890-99.

This design paper presents new guidance for creating map legends in a dynamic environment. Our contribution is a set ofguidelines for legend design in a visualization context and a series of illustrative themes through which they may be expressed. Theseare demonstrated in an applications context through interactive software prototypes. The guidelines are derived from cartographicliterature and in liaison with EDINA who provide digital mapping services for UK tertiary education. They enhance approaches tolegend design that have evolved for static media with visualization by considering: selection, layout, symbols, position, dynamismand design and process. Broad visualization legend themes include: The Ground Truth Legend, The Legend as Statistical Graphicand The Map is the Legend. Together, these concepts enable us to augment legends with dynamic properties that address specificneeds, rethink their nature and role and contribute to a wider re-evaluation of maps as artifacts of usage rather than statements offact. EDINA has acquired funding to enhance their clients with visualization legends that use these concepts as a consequence ofthis work. The guidance applies to the design of a wide range of legends and keys used in cartography and information visualization.

E., M Bertolotto, and DC Wilson. 2008, "Understanding Geospatial Interests by Visualizing Map Interaction Behavior." Information Visualization 7(3-4):275-86.

Much information can be derived about users' geospatial information requirements based on how they interact with a geospatial system. Our research focuses on the analysis of mouse movements and map navigation operations as a proxy to implicitly determine users' interests. Visualization provides an effective way of investigating how these interactions can provide an insight into users' preferences and task at hand. This article describes GIViz (Geospatial Interactions Visualizer), a visualization tool that enables system designers to analyze user interface behavior with a geospatial data set. Behavior traits identified can be exploited to improve map personalization engines. In particular, this article discusses the visualization of user interface behavior to gain a better understanding of the correlation between users' actions, the interaction strategy employed for approaching a particular type of task and users' interests. Information Visualization (2008) 7, 275-286. doi: 10.1057/ivs.2008.24

Easter, JR, and WC Elm. 1988, "Human Engineering in the Design of Expert Systems for Microcomputers System Troubleshooting." In 4th Conference on Human Factors and Power Plants, pp. 359-59.

Summary form only given. The development of a computer-based expert system to be used as a trouble-shooting aid for microcomputer systems is described. A knowledge structure for an expert system that describes the purposes or design objectives of the faulted microcomputer system has been created. The knowledge structure, along with appropriate design data about the faulted system has been computerized in a rapid prototyping AI (artificial intelligence) environment which permits potential customers to see the development results as they evolve and to inject their comments and ideas. The internal problem solving by the expert system can be initiated by the user at any point in the knowledge structure depending on the viewpoint of the user. The expert system is assumed to completely 'touch' the faulted system and is intended to initiate tests that will exercise both the hardware and the software of the 
faulted system. This approach is being tailored to microprocessor-based process control systems installed in process plants

Eastman, MC, DD Woods, and WC Elm. 1986, "Specifying and Communicating Data Structure in Computer-Based Graphic Displays." In Human Factors and Ergonomics Society Annual Meeting, pp. 1034-37.

Presents a set of techniques that were developed to respond to an important need in the design of computer-based graphic displays: how to specify requirements for communicating data structure to the user. These techniques were developed in the context of designing a display system to aid nuclear power plant operators in supervising the automatic controller of a feedwater system

Ebert, A, et al. 2010, "Tiled Plus Plus : An Enhanced Tiled Hi-Res Display Wall." IEEE Transactions on Visualization and Computer Graphics 16(1):120-32.

In recent years, high-resolutiondisplays have become increasingly important to decision makers and scientists because large screens combined with a high pixel count facilitate content rich, simultaneous display of computer-generated imagery and high-definition video data from multiple sources. Tiled displays are attractive due to their extended screen real estate, scalability, and low cost. LCD panels are usually preferred over projectors because of their superior resolution. One of the drawbacks of LCDbased tiled displays is the fact that users sometimes get distracted by the screens' bezels, which cause discontinuities in rendered images, animations, or videos. Most conventional solutions either ignore the bezels and display all pixels, causing objects to become distorted, or eliminate the pixels that would normally fall under the bezels, causing pixels to be missing in the display of static images. In animations, the missing pixels will eventually reappear when the object moves, providing an experience that is similar to looking through a French window. In this paper, we present a new scalable approach that leads neither to discontinuities nor to significant loss of information. By projecting onto the bezels, we demonstrate that a combination of LCD-based tiled displays and projection significantly reduces the bezel problem. Our technique eliminates ambiguities that commonly occur on tiled displays in the fields of information visualization, visual data analysis, human-computer interaction, and scientific data display. It improves the usability of multimonitor systems by virtually eliminating the bezels. We describe a setup and provide results from an evaluation experiment conducted on a $3 \times 3$ and on a $10 \times 5$ tiled display wall.

---. 2010, "Tiled++: An Enhanced Tiled Hi-Res Display Wall." Visualization and Computer Graphics, IEEE Transactions on 16(1):120-32.

In recent years, high-resolution displays have become increasingly important to decision makers and scientists because large screens combined with a high pixel count facilitate content rich, simultaneous display of computer-generated imagery and high-definition video data from multiple sources. Tiled displays are attractive due to their extended screen real estate, scalability, and low cost. LCD panels are usually preferred over projectors because of their superior resolution. One of the drawbacks of LCDbased tiled displays is the fact that users sometimes get distracted by the screens' bezels, which cause discontinuities in rendered images, animations, or videos. Most conventional solutions either ignore the bezels and display all pixels, causing objects to become distorted, or eliminate the pixels that would normally fall under the bezels, causing pixels to be missing in the display of static images. In animations, the missing pixels will eventually reappear when the object moves, providing an experience that is similar to looking through a French window. In this paper, we present a new scalable approach that leads neither 
to discontinuities nor to significant loss of information. By projecting onto the bezels, we demonstrate that a combination of LCD-based tiled displays and projection significantly reduces the bezel problem. Our technique eliminates ambiguities that commonly occur on tiled displays in the fields of information visualization, visual data analysis, human-computer interaction, and scientific data display. It improves the usability of multimonitor systems by virtually eliminating the bezels. We describe a setup and provide results from an evaluation experiment conducted on a 3 times 3 and on a 10 times 5 tiled display wall.

Ebert, DS. 2005, "Chapter 39 - Extending Visualization to Perceptualization: The Importance of Perception in Effective Communication of Information." in Visualization Handbook, eds. DH Charles and RJ Chris, pp. 771-80. Butterworth-Heinemann, Burlington.

Summary The essence of any human-computer interface is to convey information. Visualization has been a good information communication tool for more than two decades. While many visualization systems provide users with a better understanding of their data, they are often difficult to use and are not a reliable, accurate tool for conveying information, which limits their acceptance and use. As scientists, medical researchers, and information analysts face drastic growth in the size of their datasets, the efficient, accurate, and reproducible communication of information becomes essential. These problems become even worse when the datasets under investigation are multivariate and/or vector datasets.

Ebert, DS, et al. 2006, "Resource- and Task-Driven Visualization Adaptation." In CHI'06 Workshop: Information Visualization and Interaction Techniques for Collaboration across Multiple Displays.

We believe that for the most effective information communication to the user, the content and visual representation of the content needs to be adapted based on the task the user is performing and the resource capabilities of their display, including battery life, memory, display resolution, and communication bandwidth. By adapting the content that is presented and the visual representation (rendering) of the content, the user can more effectively complete tasks and make decisions.

Eberts, RE. 1988, "Development of Mental Models by Display Augmentation." IEEE Transactions on Systems, Man, and Cybernetics 18(4):506-13.

Eccles, R, et al. 2008, "Stories in Geotime." Information Visualization 7(1):3-17.

Astory is a powerful abstraction used by intelligence analysts to conceptualize threats and understand patterns as part of the analytical process. This paper demonstrates a system that detects geo-temporal patterns and integrates story narration to increase analytic sense-making cohesion in GeoTime. The GeoTime geo-temporal event visualization tool was augmented with a story system that uses narratives, hypertext-linked visualizations, visual annotations, and pattern detection to create an environment for analytic exploration and communication, thereby assisting the analyst in identifying, extracting, arranging, and presenting stories within the data. The story system lets analysts operate at the story level with higher level abstractions of data, such as behaviors and events, while staying connected to the evidence. The story system was developed in collaboration with analysts. A formal evaluation was completed that showed high utility and usability. Information Visualization (2008) 7, 3-17. doi:

10.1057/palgrave.ivs.9500173 
Eckert, CM, et al. 2006, "Supporting Change Processes in Design: Complexity, Prediction and Reliability." Reliability Engineering \& System Safety 91(12):1521-34.

Change to existing products is fundamental to design processes. New products are often designed through change or modification to existing products. Specific parts or subsystems are changed to similar ones whilst others are directly reused. Design by modification applies particularly to safety critical products where the reuse of existing working parts and subsystems can reduce cost and risk. However change is rarely a matter of just reusing or modifying parts. Changing one part can propagate through the entire design leading to costly rework or jeopardising the integrity of the whole product. This paper characterises product change based on studies in the aerospace and automotive industry and introduces tools to aid designers in understanding the potential effects of change. Two ways of supporting designers are described: probabilistic prediction of the effects of change and visualisation of change propagation through product connectivities. Change propagation has uncertainties which are amplified by the choices designers make in practice as they implement change. Change prediction and visualisation is discussed with reference to complexity in three areas of product development: the structural backcloth of connectivities in the existing product (and its processes), the descriptions of the product used in design and the actions taken to carry out changes.

Edmonds, E, et al. 2003, "Macaroni Synthesis: A Creative Multimedia Collaboration." In $7^{\text {th }}$ International Conference on Information Visualization, pp. 646-51.

We describe a collaborative project between an HCI team and an internationally known Japanese artist, based in New York, who was artist-in-residence with the group in the UK. The collaboration resulted in a new performance art work and a new interactive instrument. The research included a full study of the process of collaboration and innovation. We describe the work that was created, the interactive instrument developed and illustrates its use in a performance.

Edsall, R. 2009, "Map Interactivity." in International Encyclopedia of Human Geography, eds. K Rob and T Nigel, pp. 323-28. Elsevier, Oxford.

Map interaction represents an important development in the functionality and power of geographic representations. The addition of interactivity has the potential to circumvent many of the frustrating limitations of cartography, primarily the necessarily limited perspective of the cartographer. Static maps are the result of a long series of subjective choices of the cartographer, but with the addition of interactivity, many of those choices are offloaded onto the map user, who can customize the map in order to better suit his or her needs. Cartographers, whose role has traditionally been the optimal representation of geographic data, now face an additional role of providing the means for responsible and advantageous interaction. This article summarizes the many different ways that interaction on maps can facilitate geographic understanding and increase the functionality of maps. It presents a systematic approach to thinking about interaction that can inform future designs, reviews existing approaches to interacting with modern computer maps (e.g., GIS and Google Earth), and speculates about technological advances of the future that may require a significant rethinking of the nature and uses of interaction in cartography. 
Edsall, RM, M Harrower, and JL Mennis. 2000, "Tools for Visualizing Properties of Spatial and Temporal Periodicity in Geographic Data." Computers \& Geosciences 26(1):109-18.

Exploration of complex spatio-temporal environmental data demands creative methods of analysis. We present a suite of interactive visualization techniques to explore a three-dimensional Fourier transformation of a geographic time series. Such a transformation decomposes geographic time series data into coherent spatial and temporal periodicities that may be otherwise difficult to observe. The tools reported herein facilitate the exploration of three-dimensional representations of both physical and spectral space. These visualization techniques are demonstrated using both idealized and real-world environmental space-time data. Using the tools described, a user may explore existing hypotheses as well as generate new insights into environmental process. Because the spatial and temporal parameters of the phenomena under investigation are not necessarily predetermined, exploration of the data itself using the techniques described in this paper can drive the phenomena definition. Ultimately, this technique may lead to insight concerning the behavior of a given environmental phenomenon over a variety of scales, or indicate appropriate spatial and temporal scales for further analysis.

Edsall, RM, and LR Sidney. 2005, "Chapter 30 - Applications of a Cognitively Informed Framework for the Design of Interactive Spatio-Temporal Representations." in Exploring Geovisualization, eds. D Jason, MM Alan and K Menno-Jan, pp. 577-89. Elsevier, Oxford.

Summary In dynamic geographic information and visualization systems, the ways in which a user is allowed to manipulate the map and the data represented (through various interaction capabilities) are just as important as the ways the data are presented (as marks on the screen). This chapter describes strategies that will help cartographers design geovisualization interfaces and environments. We present a framework, informed by cognitive science, for designing and developing modes of interaction for use in geovisualization environments. We also review some applications of that framework in the context of representing the temporal component of geographic data. Consideration of the factors that guided us in these applications may assist both geovisualization users in understanding the limitations and opportunities presented by the tools they are using and geovisualization developers in the creation of such tools.

Effken, JA. 2002, "Different Lenses, Improved Outcomes: A New Approach to the Analysis and Design of Healthcare Information Systems." International Journal of Medical Informatics 65(1):59-74.

Healthcare systems are complex sociotechnical systems in which many information system innovations fail because of problems in planning or design. One of the reasons for this is that traditional analysis methods were designed for stable, relatively simple systems and single users. New analytical approaches are needed that can encompass the complexity of changing systems and multiple, interacting users. The author suggests that integrating two seemingly disparate approaches may be helpful in achieving successful designs for the complexities of healthcare systems. The first is derived from Carper's four ways of knowing; the second is Cognitive Work Analysis (CWA). The utility of the approach is demonstrated via a case study. 
Effken, JA, et al. 2008, "Clinical Information Displays to Improve Icu Outcomes." International Journal of Medical Informatics 77(11):765-77.

Purpose In a previous study, we compared a prototype ecological display (ED) that represented physiological data in a structured pictorial format with two bar graph displays [J.A. Effken, Improving clinical decision making through ecological interfaces, Ecol. Psych. 18 (2006) 283-318]. In ED and the first bar graph display, data were grouped hierarchically based on a cognitive work analysis (CWA); in the second bar graph display they were grouped as usually collected. Treatment efficiency (i.e., percentage of time seven variables in the CWA model were in target range) improved similarly with the two displays incorporating the CWA order for intensive care unit (ICU) residents, but not for novice ICU nurses. Hypothesized reasons for this result included: insufficient practice with novel displays; use of identical histories across displays; insufficient clinical knowledge; and the variables used in the efficiency analysis, which included only one of ED's four integrated design elements. In the current study we tested these hypotheses.Methods We asked ICU nurses assigned to three knowledge groups based on intensive care and hemodynamic monitoring pretests to identify and treat oxygenation problems presented via ED and the first bar graph display (BGD) in an experimental laboratory simulation. We measured the impact of display, clinical scenario, data level, knowledge, presentation order, and practice extent on event recognition, treatment efficiency, cognitive workload, and user satisfaction.Results The two displays produced little difference in recognition speed or overall cognitive workload, but user satisfaction was greater with ED. When 12 variables were included in the analysis, treatment efficiency improved with ED; when only 7 were measured, BGD prevailed. The results suggest benefits for the kind of synthesis provided in ED, but also a potential limitation. If too many different pictorial formats are used in a display, detecting critical events may be more difficult.

Efremov, R, DR Insua, and A Lotov. 2009, "A Framework for Participatory Decision Support Using Pareto Frontier Visualization, Goal Identification and Arbitration." European Journal of Operational Research 199(2):459-67.

There is a growing interest in promoting participation of lay stakeholders in public decision-making processes, possibly with the aid of Internet-based systems. This implies supporting non-sophisticated users and, consequently, developing user-friendly, yet rigorous, participatory decision support methods. We outline a framework to develop such methods based on interactive Pareto frontier visualization combined with expression of preferences in terms of feasible goals and using feasible goal-based arbitration.

Eick, SG. 2000, "Visual Discovery and Analysis." Visualization and Computer Graphics, IEEE Transactions on 6(1):44-58.

We have developed a flexible software environment called ADVIZOR for visual information discovery. ADVIZOR complements existing assumptive-based analyses by providing a discovery-based approach. ADVIZOR consists of five parts: a rich set of flexible visual components, strategies for arranging the components for particular analyses, an in-memory data pool, data manipulation components, and container applications. Working together, ADVIZOR's architecture provides a powerful production platform for creating innovative visual query and analysis applications 
Eidenberger, H, and C Breiteneder. 2003, "Vizir--a Framework for Visual Information Retrieval." Journal of Visual Languages \& Computing 14(5):443-69.

In this paper the visual information retrieval project VizIR is presented. The goal of the project is the implementation of an open visual information retrieval (VIR) prototype as basis for further research on major problems of VIR. The motivation behind VizIR is the implementation of an open platform for supporting and facilitating research, teaching, the exchange of research results and research cooperation in the field in general. The availability of this platform could make cooperation and such research (especially for smaller institutions) easier. The intention of this paper is to inform interested researchers about the VizIR project and its design and to invite people to participate in the design and implementation process. We describe the goals of the VizIR project, the intended design of the querying framework, the user interface design and major implementation issues. The querying framework consists of classes for feature extraction, similarity measurement, media handling and database access. User interface design includes a description of visual components and their class structure, the communication between panels and the communication between visual components and query engines. The latter is based on the multimedia retrieval markup language (MRML, Website. http://www.mrml.net (last visited: 2003-0320)). To be compatible with our querying paradigm, we extend MRML with additional elements. Implementation issues include a sketch on advantages and drawbacks of existing cross-platform media processing frameworks: Java Media Framework, OpenML and DirectX/DirectShow and details on the Java components used for user interface implementation, 3D graphics with Java and Java XML parsing.

Eilouti, BH. 2009, "Design Knowledge Recycling Using Precedent-Based Analysis and Synthesis Models." Design Studies 30(4):340-68.

New models of abstracted typological classes that encode forms, functions, processes, concepts, scenarios, principles and components of previous design products are introduced. They are presented as seven precedent-based knowledge management constructs that depart from the dissection of existing design compositions into simple explicit forms in order to facilitate classifying, analyzing and reusing their information to derive new designs. They aim to formulate reference prototypes that may help guide pre-design reasoning, inspire design processing, and direct post-design evaluation. The research design consists of theoretical, action and survey researches. These are applied to devise the models, involve participants in improving their designing techniques, and test participants' feedback, respectively. Examples and results of the action and survey researches are illustrated and discussed.

Eklund, P, J Ducrou, and P Brawn. 2004, "Concept Lattices for Information Visualization: Can Novices Read Line-Diagrams?" In 2nd International Conference on Formal Concept Analysis (ICFCA 2004), pp. 57-73.

MAIL-SLEUTH is a personal productivity tool that allows individuals to manage email and visualize its contents using line diagrams. Based on earlier work on the Conceptual Email Manager (CEM), a major hypothesis of MAIL-SLEUTH is that novices to Formal Concept Analysis can read a lattice diagram. Since there is no empirical evidence for this in the Formal Concept Analysis literature this paper is a first attempt to test this hypothesis by following a user-centred design and evaluation process. Our results suggest that, with some adjustments, novice users can read line diagrams without specialized background in mathematics or computer science. This paper describes the process and outcomes based on usability testing and explains the evolution of the MAIL-SLEUTH design responding to the evaluation at the Access Testing Centre. 
Ellis, G, and A Dix. 2007, "A Taxonomy of Clutter Reduction for Information Visualisation." Visualization and Computer Graphics, IEEE Transactions on 13(6):1216-23.

Information visualisation is about gaining insight into data through a visual representation. This data is often multivariate and increasingly, the datasets are very large. To help us explore all this data, numerous visualisation applications, both commercial and research prototypes, have been designed using a variety of techniques and algorithms. Whether they are dedicated to geo-spatial data or skewed hierarchical data, most of the visualisations need to adopt strategies for dealing with overcrowded displays, brought about by too much data to fit in too small a display space. This paper analyses a large number of these clutter reduction methods, classifying them both in terms of how they deal with clutter reduction and more importantly, in terms of the benefits and losses. The aim of the resulting taxonomy is to act as a guide to match techniques to problems where different criteria may have different importance, and more importantly as a means to critique and hence develop existing and new techniques.

Ellis, JB, et al. 2007, "Task and Social Visualization in Software Development: Evaluation of a Prototype." In Conference on Human Factors in Computing Systems, pp. 577-86.

As open source development has evolved, differentiation of roles and increased sophistication of collaborative processes has occurred. Recently, we described coordination issues in software development and an interactive Visualization tool called the Social Health Overview (SHO) developed to address them [12]. This paper presents an empirical evaluation of SHO intended to identify its strengths and weaknesses. Eleven informants in various open source roles were interviewed about their work practices. Eight of these participated in an evaluation comparing three change management tasks in SHO and Bugzilla. Results are discussed with respect to task strategy with each tool and participants' roles.

Elm, W, et al. 2005, "Finding Decision Support Requirements for Effective Intelligence Analysis Tools." In 49th Annual Meeting of the Human Factors and Ergonomics Society, pp. 297-301.

Within ARDA's GI2Vis program, we developed a unique framework for the definition of decision support requirements for intelligence analysis tools. This framework, based on a first-of-a-kind integration of a model of inferential analysis and principles for designing effective human-computer teams from Cognitive Systems Engineering, has defined the essential support functions to be provided to the intelligence analyst(s). This model has proven to be extremely useful in assessing the support provided by a large set of visualization tools. This assessment has identified clusters of support functions that are addressed by many tools as well as key missing support functions. In this way, the Support Function Model has been used to identify gaps in the support function coverage of existing tools. This can serve as a valuable focusing mechanism for future design and development efforts. In addition, we believe this would be a useful mechanism to enhance cross-discussions among research teams involved in Cognitive Task Analysis efforts within the Intelligence Community. Having others integrate their analytic results with this framework would provide the mechanism for expansion of this model to become a more robust tool and have an even greater impact on the Intelligence Community.

Elm, WC. 1988, "How Artificial Intelligence Can Help." Nuclear Engineering International 33(Copyright 1988, IEE):36-40.

The operator is ultimately responsible for the safe and economical operation of the plant, and must evaluate the accuracy of any system-recommended action or other output. Decision support systems offer 
a means to improve the man-machine interface by explicitly supporting operator problem solving, rather than complicating decision-making by the need to request an explanation of the rationale behind an expert system's advice during a high stress situation

Elm, WC, et al. 2004, "Designing Support for Intelligence Analysts." Human Factors and Ergonomics Society Annual Meeting Proceedings 48:406-10.

Elm, WC, et al. 2008, "Integrating Cognitive Systems Engineering Throughout the Systems Engineering Process." Journal of Cognitive Engineering and Decision Making 2:249-73.

Elm, WC, et al. 2003, "Chapter 16 - Applied Cognitive Work Analysis: A Pragmatic Methodology for Designing Revolutionary Cognitive Affordances." in Handbook of Cognitive Task Design, ed. E Hollnagel, pp. 357--57-. Lawrence Erlbaum Associates, Inc., Publishers, Mahwah, NJ.

Elm, WC, and DD Woods. 1985, "Getting Lost: A Case Study in Interface Design." In Human Factors and Ergonomics Society Annual Meeting, pp. 927-31.

Elm, WC, et al. 2002, "Behind the Curtain: The Cognitive Tasks Behind the Visualizations." In Human Factors and Ergonomics Society Annual Meeting, pp. 285-88.

This panel includes participants from academia and industry who have each made significant contributions to the design of effective visualizations to support decision making in a variety of domains. Panel members will offer an example of an innovative decision support visualization concept and discuss the underlying cognitive demands it is meant to support as well as any artifacts they used in its development. Both the nature of the visualization itself, and the linkage to the processes 'behind the curtain' will be presented. Panelists will discuss the various techniques used and the pro's and con's of each

Elmagarmid, AK, and S Member. 2007, "Duplicate Record Detection : A Survey." Knowledge Creation Diffusion Utilization 19(1):1-16.

Often, in the real world, entities have two or more representations in databases. Duplicate records do not share a common key and/or they contain errors that make duplicate matching a difficult task. Errors are introduced as the result of transcription errors, incomplete information, lack of standard formats, or any combination of these factors. In this paper, we present a thorough analysis of the literature on duplicate record detection. We cover similarity metrics that are commonly used to detect similar field entries, and we present an extensive set of duplicate detection algorithms that can detect approximately duplicate records in a database. We also cover multiple techniques for improving the efficiency and scalability of approximate duplicate detection algorithms. We conclude with coverage of existing tools and with a brief discussion of the big open problems in the area

Elmagarmid, AK, A Samuel, and M Ouzzani. 2008, "Community-Cyberinfrastructure- Enabled Discovery in Science and Engineering." Genomics 10(5):46-53.

A community cyberinfrastructure would enable a new era of multidisciplinary research and collaboration in science and engineering. With such an infrastructure, researchers could share knowledge and results along with computing cycles, storage, and bandwidth. A generic, transparent 
cyberinfrastructure would also foster more meaningful analyses of data and visualization, modeling, and simulation of real-world phenomena.

Elmeleegy, H, M Ouzzani, and A Elmagarmid. 2008, "Usage-Based Schema Matching." In $24^{\text {th }}$ International Conference on Data Engineering, pp. 20-29.

Existing techniques for schema matching are classified as either schema-based, instance-based, or a combination of both. In this paper, we define a new class of techniques, called usage-based schema matching. The idea is to exploit information extracted from the query logs to find correspondences between attributes in the schemas to be matched. We propose methods to identify co-occurrence patterns between attributes in addition to other features such as their use in joins and with aggregate functions. Several scoring functions are considered to measure the similarity of the extracted features, and a genetic algorithm is employed to find the highest- score mappings between the two schemas. Our technique is suitable for matching schemas even when their attribute names are opaque. It can further be combined with existing techniques to obtain more accurate results. Our experimental study demonstrates the effectiveness of the proposed approach and the benefit of combining it with other existing approaches.

Elmqvist, N, and P Tsigas. 2007, "View-Projection Animation for 3d Occlusion Management." Computers \& Graphics 31(6):864-76.

Inter-object occlusion is inherent to 3D environments and is one of the challenges of using 3D instead of 2D computer graphics for visualization. Based on an analysis of this effect, we present an interaction technique for view-projection animation that reduces inter-object occlusion in $3 \mathrm{D}$ environments without modifying the geometrical properties of the objects themselves. The technique allows for smooth ondemand animation between parallel and perspective projection modes as well as online manipulation of view parameters, enabling the user to quickly and easily adapt the view to reduce occlusion. A user study indicates that the technique provides many of the occlusion reduction benefits of traditional camera movement, but without the need to actually change the viewpoint. We have also implemented a prototype of the technique in the Blender 3D modeler.

Eltabakh, MY, M Ouzzani, and WG Aref. 2007, "Duplicate Elimination in Space-Partitioning Tree Indexes." In 19th International Conference on Scientific and Statistical Database Management, pp. 1818.

Space-partitioning trees, like the disk-based trie, quadtree, kd-tree and their variants, are a family of access methods that index multi-dimensional objects. In the case of indexing non-zero extent objects, e.g., line segments and rectangles, space-partitioning trees may replicate objects over multiple space partitions, e.g., PMR quadtree, expanded MX-CIF quadtree, and extended kd-tree. As a result, the answer to a query over these indexes may include duplicates that need to be eliminated, i.e., the same object may be reported more than once. In this paper, we propose generic duplicate elimination techniques for the class of space-partitioning trees in the context of SP-GiST; an extensible indexing framework for realizing space-partitioning trees. The proposed techniques are embedded inside the INDEX-SCAN operator. Therefore, duplicate copies of the same object do not propagate in the query plan, and the elimination process is transparent to the end-users. Two cases for the index structures are considered based on whether or not the objects' coordinates are stored inside the index tree. The theoretical and experimental analysis illustrate that the proposed techniques achieve savings in the storage requirements, 
I/O operations, and processing time when compared to adding a separate duplicate elimination operator in the query plan.

Endsley, MR. 1988, "Design and Evaluation for Situation Awareness Enhancement." In Human Factors Society 32nd Annual Meeting.

Endsley, MR. 1995, "Toward a Theory of Situation Awareness in Dynamic-Systems." Human Factors 37(1):32-64.

This paper presents a theoretical model of situation awareness based on its role in dynamic human decision making in a variety of domains. Situation awareness is presented as a predominant concern in system operation, based on a descriptive view of decision making. The relationship between situation awareness and numerous individual and environmental factors is explored. Among these factors, attention and working memory are presented as critical factors limiting operators from acquiring and interpreting information from the environment to form situation awareness, and mental models and goal-directed behavior are hypothesized as important mechanisms for overcoming these limits. The impact of design features, workload, stress, system complexity, and automation on operator situation awareness is addressed, and a taxonomy of errors in situation awareness is introduced, based on the model presented. The model is used to generate design implications for enhancing operator situation awareness and future directions for situation awareness research.

Endsley, MR, B Bolte, and DG Jones. 2004. Designing for Situation Awareness. Taylor \& Francis, London, England.

Engelmann, T, et al. 2009, "Knowledge Awareness in Cscl: A Psychological Perspective." Computers in Human Behavior 25(4):949-60.

In this paper, a specific group awareness approach for CSCL settings, namely knowledge awareness, is presented. We classify knowledge awareness in relation to already well-established concepts like shared mental models, common ground, and transactive memory system in order to provide a comprehensive definition of this approach. The functionality of knowledge awareness tools aiming at fostering knowledge awareness is conceptualized in a cyclical model. Three application scenarios are presented to illustrate how knowledge awareness tools work in three different tasks. All these knowledge awareness tools have been found to be effective, that is all tools could foster knowledge awareness and led to increased learning performance.

Eppler, MJ. 2006, "Toward a Pragmatic Taxonomy of Knowledge Maps: Classification Principles, Sample Typologies, and Application Examples." In Tenth International Conference on Information Visualisation (IV'06), pp. 195-204.

This paper discusses pragmatic ways of classifying knowledge maps to give an overview of their application formats and contexts. We show where and how the term knowledge map has been previously used and what criteria must be met in a sound and useful knowledge map classification. Various possible classification principles are presented. A table matches map formats to purposes and contents to serve as a selection framework in knowledge management processes. Examples of some of the main types of knowledge maps are presented to illustrate the varieties of knowledge mapping present in the classifications. The article concludes by discussing its limitations and future research questions. 
Eppler, MJ, and K Platts. 2007, "An Empirical Classification of Visual Methods for Management: Results of Picture Sorting Experiments with Managers and Students." In 11th International Conference on Information Visualization, pp. 335-41.

This paper presents an empirically derived taxonomy of managerial visualization methods by reporting on the results of picture sorting experiments with a total of 65 participants ( 37 students and 15 experienced and 13 young managers). Participants were asked to group 30 thumb nailed images of visualization- based methods based on their spontaneous judgments of similarity. By conducting an average cluster analysis on the resulting similarity matrix (based on a pair-wise analysis of group comembership), an overall classification system emerged from the participants' groupings that revealed salient classification attributes as well as insights into the grouping rationale of students and (junior and senior) managers. As a main result, we present a dendrogram of thirty visual methods and its limitations due to biases in the perception of the participants, diagram novelty, and due to ambiguous conventions employed in some diagrams. In addition, we present a list of salient grouping attributes used by the participants. These attributes, as well as the derived guidelines, can be used for future classification systems in the area of managerial visualization methods.

Eppler, MJ, and KW Platts. 2009, "Visual Strategizing: The Systematic Use of Visualization in the Strategic-Planning Process." Long Range Planning 42(1):42-74.

This article shows how visualization can be used in the strategic-planning process, by exaining the use of real-time, interactive visual representations in the business strategy process. Starting with a concise review of literature, we postulate that visualization can improve the quality of the strategic planning process by addressing many of its cognitive, social, and emotional challenges. We develop a conceptual framework for strategy visualization, and use this structure to group and position interactive visual representations of information along the strategic-planning process. We highlight the benefits of visual methods for strategizing, and illustrate them with five case studies covering the entire strategizing process from analysis to implementation. The cases also highlight the use of visualization at different organizational levels, and we consider some of the challenges involved in employing graphic means in strategy work, and how to address them. We highlight resulting risks and practices for visual strategizing and articulate a research agenda for this emergent domain. The key lesson for executives is that visualization should not just be seen as an attractive way to communicate strategic planning process outcomes and monitor its progress, but as a powerful process enabler that can enable strategizing as a joint managerial practice - if facilitated properly. Visualization is, however, a double edged sword and we present several caveats that need to be considered in its application in the strategy context.

Erbacher, RF. 2007, "Exemplifying the Inter-Disciplinary Nature of Visualization Research." In $11^{\text {th }}$ International Conference on Information Visualization, pp. 623-30.

This paper proposes an interdisciplinary design process for the development of novel visualization techniques and discusses requirements for ensuring that such a process results in effective techniques which meet the demands of a the target user base. In essence, we are attempting to make explicit the process by which many researchers implicitly design visualizations and urge for greater explicit collaborations between fields to improve the effectiveness of visualization design. This process is dependent on the application of cognitive psychology, art and architecture, domain experts, and target users. We discuss how such a wide and disparate group must work together in an integrated fashion within the design process. We lay out the process itself as well as typical design goals. Given the 
importance of perception, we highlight the impact of perception, and thus cognitive psychology, on the visual interpretation process.

Erbacher, RF. 2001, "Visual Traffic Monitoring and Evaluation." In Conference on Internet Performance and Control of Network Systems II, pp. 153-60.

As computer networks and associated infrastructures become ever more important to the nation's commerce and communication, it is becoming exceedingly critical that these networks be managed effectively. Current techniques, which rely on manual or log based analysis, are too slow and ineffective to handle the explosive growth of network infrastructures. We have developed visualization techniques geared towards aiding the analysis of network based infrastructures such that network managers can quickly identify usage characteristics of the network and reallocate bandwidth or restructure portions of the network to better improve connectivity. In this fashion, bottlenecks can be quickly identified along with their cause so the issues can be remedied expeditiously. The techniques can also be used for long range infrastructure planning and network misuse detection.

Erbacher, RF, et al. 2010, "A Multi-Phase Network Situational Awareness Cognitive Task Analysis." Information Visualization 9(3):204-19.

The goal of our project is to create a set of next-generation cyber situational-awareness capabilities with applications to other domains in the long term. The objective is to improve the decision-making process to enable decision makers to choose better actions. To this end, we put extensive effort into making certain that we had feedback from network analysts and managers and understand what their genuine needs are. This article discusses the cognitive task-analysis methodology that we followed to acquire feedback from the analysts. This article also provides the details we acquired from the analysts on their processes, goals, concerns, the data and metadata that they analyze. Finally, we describe the generation of a novel task-flow diagram representing the activities of the target user base. Information Visualization (2010) 9, 204-219. doi:10.1057/ivs.2010.5

Erhardt, RAA, R Schneider, and C Blaschke. 2006, "Status of Text-Mining Techniques Applied to Biomedical Text." Drug Discovery Today 11(7-8):315-25.

Scientific progress is increasingly based on knowledge and information. Knowledge is now recognized as the driver of productivity and economic growth, leading to a new focus on the role of information in the decision-making process. Most scientific knowledge is registered in publications and other unstructured representations that make it difficult to use and to integrate the information with other sources (e.g., biological databases). Making a computer understand human language has proven to be a complex achievement, but there are techniques capable of detecting, distinguishing and extracting a limited number of different classes of facts. In the biomedical field, extracting information has specific problems: complex and ever-changing nomenclature (especially genes and proteins) and the limited representation of domain knowledge. 
Ericsson, AK, and HA Simon. 1993. Protocol Analysis: Verbal Reports as Data. MIT Press, Cambridge, MA.

Ernst, NA, MA Storey, and P Allen. 2005, "Cognitive Support for Ontology Modeling." International Journal of Human-Computer Studies 62(5):553-77.

Knowledge engineering tools are becoming ever more complex, and therefore increased cognitive support will be necessary to leverage the potential of those tools. Our paper motivates this claim by examining some previous work in this domain and explaining the nature of cognitive support. We discuss some of the problem areas we have encountered in our research. Through user questionnaires and observations carried out at the National Cancer Institute (NCI) and the University of Washington Foundational Model of Anatomy (FMA) Project, we have begun to gain an understanding of the cognitive barriers experienced by the users of knowledge engineering tools. We present some proposed solutions that could address the problems we identified, and in addition, discuss how our own tool, called Jambalaya, could be applied to provide cognitive support. We analyse the support Jambalaya provides using some non-functional design criteria and illustrate some trade-offs inherent in tool design. We suggest that the need for cognitive support in knowledge engineering is immediate and essential. (c) 2005 Elsevier Ltd. All rights reserved.

Eslambolchilar, P, and R Murray-Smith. 2008, "Control Centric Approach in Designing Scrolling and Zooming User Interfaces." International Journal of Human-Computer Studies 66(12):838-56.

The dynamic systems approach to the design of continuous interaction interfaces allows the designer to use simulations, and analytical tools to analyse the behaviour and stability of the controlled system alone and when it is coupled with a manual control model of user behaviour. This approach also helps designers to calibrate and tune the parameters of the system before the actual implementation, and in response to user feedback. In this work we provide a dynamic systems interpretation of the coupling of internal states involved in speed-dependent automatic zooming, and test our implementation on a text browser on a Pocket PC instrumented with a tilt sensor. We illustrate simulated and experimental results of the use of the proposed coupled navigation and zooming interface using tilt and touch screen input.

Fabrikant, SI, and DR Montello. 2008, "The Effect of Instructions on Distance and Similarity Judgements in Information Spatializations." International Journal of Geographical Information Science 22(4):463-78.

We investigate the relationship of perceived distances to judged similarities between document points in various types of spatialized displays. Our findings suggest that the distance-similarity relationship is not as self-evident to viewers as is commonly assumed in the information visualization literature. We further investigate how participants interpret instructions to judge distances when those instructions do or do not specify the type of distance. We find that in all types of spatialization displays, there is no significant difference between default and direct judgements of distance; people clearly interpret default distance instructions to refer to direct (straight-line) distance. These findings provide direct evidence on the conditions under which people employ distance when assessing similarity between data objects in various types of spatialized views and, when they do, which type of distance. They also give insight into how people explore the similarity of geographic features depicted in cartographic maps or GIS displays. 
Fabrikant, SI, and A Skupin. 2005, "Chapter 35 - Cognitively Plausible Information Visualization." in Exploring Geovisualization, eds. D Jason, MM Alan and K Menno-Jan, pp. 667-90. Elsevier, Oxford.

Summary Information Visualization is concerned with the art and technology of designing and implementing highly interactive, computer supported tools for knowledge discovery in large non-spatial databases. Information Visualization displays, also known as information spaces or graphic spatializations, differ from ordinary data visualization and geovisualization in that they may be explored as if they represented spatial information. Information spaces are very often based on spatial metaphors such as location, distance, region, scale, etc., thus potentially affording spatial analysis techniques and geovisualization approaches for data exploration and knowledge discovery. Two major concerns in spatialization can be identified from a GIScience/ geovisualization perspective: the use of space as a data generalization strategy, and the use of spatial representations or maps to depict these data abstractions. A range of theoretical and technical research questions needs to be addressed to assure the construction of cognitively adequate spatializations. In the first part of this chapter we propose a framework for the construction of cognitively plausible semantic information spaces. This theoretical scaffold is based on geographic information theory and includes principles of ontological modeling such as semantic generalization (spatial primitives), geometric generalization (visual variables), association (source-target domain mapping through spatial metaphors), and aggregation (hierarchical organization). In the remainder of the chapter we discuss ways in which the framework may be applied towards the design of cognitively adequate spatializations.

Faisal, S, P Cairns, and A Blandford. 2007, "Building for Users Not for Experts: Designing a Visualization of the Literature Domain." In 11th International Conference on Information Visualization, pp. 707-12.

As researchers we are constantly working with academic literature. Literature data is growing exponentially. Interacting with this huge amount of information has been a challenge for the field of HCI for years. The goal is to assist users in making sense of this information by producing usable designs. Information Visualization (InfoVis) augments users' cognition when interacting with complex data structures. Although the use of InfoVis as a tool for representing literature data is nor new, we have found that most of the existing Literature Knowledge Domain Visualizations (LKDViz) target specialists known as domain analysts who study publication patterns. Our goal is to design a LKDViz tool for academic users. Due to the diversity of academic literature users we captured their experiences and used it as the main source for our design. Interestingly, this method generated design criteria that have nor been applied in most of the academic literature visualizations.

Faisal, S, et al. 2008, "Internalization, Qualitative Methods, and Evaluation." In Conference on BEyond Time and Errors: Novel EvaLuation Methods for Information Visualization, BELIV'08.

Information Visualization (InfoVis) is at least in part defined by a process that occurs within the subjective internal experience of the users of visualization tools. Hence, users' interaction with these tools is seen as an 'experience'. Relying on standard quantitative usability measures evaluates the interface. Yet, there is more to users' interaction with InfoVis tools than merely the interface. Qualitative methods targets users' subjective experiences. In this paper we demonstrate the potential benefits of qualitative methods, more specifically Grounded Theory, for generating a theoretical understanding of users' InfoVis experiences through discussing the results of a qualitative study we conducted. The study was conducted in order to evaluate a visualization of the academic literature domain, which we have designed and built 
using a user-centered design approach. The study resulted in us identifying categories that are essential to the InfoVis experience. This paper argues that these categories can be used as a foundation for building an InfoVis theory of interaction. Copyright 2008 ACM.

Falconer, SM, and MA Storey. 2007, "A Cognitive Support Framework for Ontology Mapping." In $6^{\text {th }}$ international The semantic web and 2 nd Asian conference on Asian semantic web conference, pp. 11427.

Ontology mapping is the key to data interoperability in the semantic web. This problem has received a lot of research attention, however, the research emphasis has been mostly devoted to automating the mapping process, even though the creation of mappings often involve the user. As industry interest in semantic web technologies grows and the number of widely adopted semantic web applications increases, we must begin to support the user. In this paper, we combine data gathered from background literature, theories of cognitive support and decision making, and an observational case study to propose a theoretical framework for cognitive support in ontology mapping tools. We also describe a tool called COGZ that is based on this framework.

Falkman, G. 2001, "Information Visualisation in Clinical Odontology: Multidimensional Analysis and Interactive Data Exploration." Artificial Intelligence in Medicine 22(2):133-58.

In 1995, the MedView project, based on a co-operation between computing science and clinical medicine was initiated. The overall goal of the project was to develop models, methods and tools to support clinicians in their daily diagnostic work. As part of MedView, two information visualisation tools were developed and tested as solutions to the problem of visualising clinical experience derived from large amounts of clinical data. The first tool (The Cube) was based on the idea of dynamic threedimensional (3D) parallel diagrams, an idea similar to the notion of 3D parallel co-ordinates. The Cube was developed to enhance the clinician's ability to intelligibly analyse existing patient material and to allow for pattern recognition and statistical analysis. The second tool (SimVis) was based on a similarity assessment-based interaction model for exploring data, and was designed to help clinicians to classify and cluster clinical examination data. User interaction was supported by 3D visualisation of clusters and similarity measures. Both tools were tested on a knowledge base containing about 1500 examinations obtained from different clinics. Clinical practice indicated that the basic ideas are conceptually appealing to the involved clinicians as the tools can be used for generating and testing of hypotheses.

Fan, J, Y Gao, and H Luo. 2008, "Integrating Concept Ontology and Multitask Learning to Achieve More Effective Classifier Training for Multilevel Image Annotation." IEEE transactions on image processing : a publication of the IEEE Signal Processing Society 17(3):407-26.

In this paper, we have developed a new scheme for achieving multilevel annotations of large-scale images automatically. To achieve more sufficient representation of various visual properties of the images, both the global visual features and the local visual features are extracted for image content representation. To tackle the problem of huge intraconcept visual diversity, multiple types of kernels are integrated to characterize the diverse visual similarity relationships between the images more precisely, and a multiple kernel learning algorithm is developed for SVM image classifier training. To address the problem of huge interconcept visual similarity, a novel multitask learning algorithm is developed to learn the correlated classifiers for the sibling image concepts under the same parent concept and enhance their discrimination and adaptation power significantly. To tackle the problem of huge intraconcept visual 
diversity for the image concepts at the higher levels of the concept ontology, a novel hierarchical boosting algorithm is developed to learn their ensemble classifiers hierarchically. In order to assist users on selecting more effective hypotheses for image classifier training, we have developed a novel hyperbolic framework for large-scale image visualization and interactive hypotheses assessment. Our experiments on large-scale image collections have also obtained very positive results.

Fan, J, et al. 2008, "A Novel Approach to Enable Semantic and Visual Image Summarization for Exploratory Image Search." Proceeding of the 1st ACM international conference on Multimedia information retrieval - MIR '08:358-58.

Fan, J, and DA Keim. 2008, "Justclick : Personalized Image Recommendation Via Exploratory Search from Large-Scale Flickr Image Collections." IEEE Transactions on Circuits and Systems 18(8):1-20.

In this paper, we have developed a novel framework called JustClick to enable personalized image recommendation via exploratory search from large-scale collections of Flickr images. First, a topic network is automatically generated to summarize large-scale collections of Flickr images at a semantic level. Hyperbolic visualization is further used to enable interactive navigation and exploration of the topic network, so that users can gain insights of large-scale image collections at the first glance, build up their mental query models interactively and specify their queries (i.e., image needs) more precisely by selecting the image topics on the topic network directly. Thus, our personalized query recommendation framework can effectively address both the problem of query formulation and the problem of vocabulary discrepancy and null returns. Second, a small set of most representative images are recommended for the given image topic according to their representativeness scores. Kernel principal component analysis and hyperbolic visualization are seamlessly integrated to organize and layout the recommended images (i.e., most representative images) according to their nonlinear visual similarity contexts, so that users can assess the relevance between the recommended images and their real query intentions interactively. An interactive interface is implemented to allow users to express their time-varying query intentions precisely and to direct our JustClick system to more relevant images according to their personal preferences. Our experiments on large-scale collections of Flickr images show very positive results.

Farrimond, B, and R Hetherington. 2004, "Using 3d to Visualise Temporal Data." In 8th International Conference on Information Visualization, pp. 108-17.

Dynamic 3D models generated from historical, time stamped data provide opportunities for insights and understanding that would otherwise be difficult or impossible to gain in applications as diverse as the teaching and learning of history and the analysis of aerospace experiments. This paper describes how historical data in a temporal database can be used to generate dynamic 3D models expressed in VRML. The models include facilities to move back and forth in time, to hide or reveal groups of objects and their labels and to show the paths traced by individual objects as they move through space and time. The change of shape of objects over time can also be modelled. The tools described in this paper enable the user to create these 3D worlds automatically without needing to know anything about the underlying 3D modelling language employed. 
Fekete, J-D. 2009, "Visualizing Networks Using Adjacency Matrices: Progresses and Challenges." In 11th International Conference on Computer-Aided Design and Computer Graphics, pp. 32-32.

Visualizing networks has become a very important research and application topic in the recent years, due to the availability of network data through the web, but also to the need of analyzing several types of networks such as computer networks, social networks, biological networks (e.g., gene similarities or biological pathways). Until 2000, the node-link diagram was the only representation used. However, this representation suffers from many readability issues when the network becomes dense. In 2003, we showed that the adjacency matrix representation was more effective to visualize networks when they were dense. In this talk, we will present new methods designed by our group and others to make adjacency matrices usable for network analysis and exploration. We will explore various alternatives around the matrix representations (combined views, augmented matrices, hybrid node-link and matrix views), interaction and navigation methods for very large networks and algorithmic methods to reorder the matrices in order to show high-level structures. We will conclude with some research challenges ahead in matrix-based visualization of networks.

Fekete, JD. 2010, "Advanced Interaction for Information Visualization." In Pacific Visualization Symposium, pp. 1-1.

Information Visualization (InfoVis) is a research field dedicated to the design and evaluation of visual representations and interactions to explore and understand large data set. Until recently, the focus of InfoVis has been more on the graphical representation and less on the interaction. However, several new interactive techniques have been published in the past few years, opening perspectives for InfoVis and HCI practitioners. I describe the work conducted at our group, AVIZ, linking traditional HCI interaction research with InfoVis interaction research. I present our latest techniques for navigating large information spaces with varying dimensionalities and topologies. I outline their applicability in HCI problems that are not traditionally viewed as InfoVis in the hope that they will be experimented and adopted in standard interfaces and, more importantly, to emphasize the fact that interaction design for large information spaces is required for our complex specialized applications as well as our everyday working environment.

Fekete, JD, et al. 2008, "The Value of Information Visualization." Information Visualization:1-18.

Feldt, N, et al. 2005, "Tailor-Made Exploratory Visualization for Statistics Sweden." In

Third International Conference on Coordinated \& Multiple Views in Exploratory Visualization, pp. 13342.

Sweden's statistical databases are maintained by Statistics Sweden and can be accessed free of charge via the Web. We provide an easy-to-use, exploratory visualization application, called "GeoWizard" that lets users of these databases explore data, construct hypotheses, discover, refine, test knowledge and evaluate results. Our target user group is not restricted to experts, but we want a broader group of analysts to feel comfortable with our human interaction tools. The voluminous high-dimensional nature of the statistical databases calls for high interactive performance and creative integrated information visualization and geovisualization methods. Tailor-made and web-enabled applications based on layered component thinking are the foundation for our research. We present a development platform approach that, instead of Java, uses Microsoft's .NET framework, which can integrate a wide range of problemsolving components, both computationally and visually. The approach facilitates NET hierarchical layout management for implementation of dynamic and resizeable views in a single coherent GUI window and 
the OpenViz data model optimized for efficiency and interactivity in handling large multivariate data sets. We introduce a parallel coordinates browser (PCB) that serves as the control panel for easier identification of multivariate relationships across spatial domains in the choropleth map. The PCB integrates range sliders for both dynamic queries and conditioning that constrains the data displayed to those meeting specified parameters on all attributes in the PCB. Finally, we present a client-side, plug-in architecture that enables a light-weight GeoWizard application to be distributed across the Web to the users of the statistical databases.

Feng, D, et al. 2010, "Matching Visual Saliency to Confidence in Plots of Uncertain Data." IEEE Transactions on Visualization and Computer Graphics 16(6):980-9.

Conveying data uncertainty in visualizations is crucial for preventing viewers from drawing conclusions based on untrustworthy data points. This paper proposes a methodology for efficiently generating density plots of uncertain multivariate data sets that draws viewers to preattentively identify values of high certainty while not calling attention to uncertain values. We demonstrate how to augment scatter plots and parallel coordinates plots to incorporate statistically modeled uncertainty and show how to integrate them with existing multivariate analysis techniques, including outlier detection and interactive brushing. Computing high quality density plots can be expensive for large data sets, so we also describe a probabilistic plotting technique that summarizes the data without requiring explicit density plot computation. These techniques have been useful for identifying brain tumors in multivariate magnetic resonance spectroscopy data and we describe how to extend them to visualize ensemble data sets.

Fernandes, KJ, VH Raja, and J Eyre. 2003, "Immersive Learning System for Manufacturing Industries." Computers in Industry 51(1):31-40.

Virtual reality-based training systems are advanced computer-assisted training systems using virtual reality (VR) technology. To have better structure and easier implementation, a virtual training system can be modeled as an integrated system consisting of a training visualization suite, an interface module and instruction module. This paper discusses how a fully immersive VR visualization suite, called "Cybersphere", can be used in conjunction with a collaborative product suite to achieve an ideal training environment for manufacturing industries. The design and development of the system, and expert- and user-based evaluations are reported.

Ferstl, F, et al. 2010, "Interactive Separating Streak Surfaces." IEEE Transactions on Visualization and Computer Graphics 16(6):1569-77.

Streak surfaces are among the most important features to support 3D unsteady flow exploration, but they are also among the computationally most demanding. Furthermore, to enable a feature driven analysis of the flow, one is mainly interested in streak surfaces that show separation profiles and thus detect unstable manifolds in the flow. The computation of such separation surfaces requires to place seeding structures at the separation locations and to let the structures move correspondingly to these locations in the unsteady flow. Since only little knowledge exists about the time evolution of separating streak surfaces, at this time, an automated exploration of 3D unsteady flows using such surfaces is not feasible. Therefore, in this paper we present an interactive approach for the visual analysis of separating streak surfaces. Our method draws upon recent work on the extraction of Lagrangian coherent structures (LCS) and the real-time visualization of streak surfaces on the GPU. We propose an interactive technique for computing ridges in the finite time Lyapunov exponent (FTLE) field at each time step, and we use 
these ridges as seeding structures to track streak surfaces in the time-varying flow. By showing separation surfaces in combination with particle trajectories, and by letting the user interactively change seeding parameters such as particle density and position, visually guided exploration of separation profiles in 3D is provided. To the best of our knowledge, this is the first time that the reconstruction and display of semantic separable surfaces in 3D unsteady flows can be performed interactively, giving rise to new possibilities for gaining insight into complex flow phenomena.

Finke, RA. 1996, "Creative Insight and Preinventive Forms." in The Nature of Insight, eds. RJ Sternberg and JE Davidson, pp. 276-78. MIT Press.

Fisher, D, et al. 2010, "Visualizations Everywhere : A Multiplatform Infrastructure for Linked Visualizations." IEEE Transactions on Visualization and Computer Graphics 16(6):1157-63.

Fisher, D, et al. 2008, "Using Visualization to Support Network and Application Management in a Data Center." In Internet Network Management Workshop, pp. 1-6.

We argue that there is a continuum between completely manual and completely automated management of networks and distributed applications. The ability to visualize the status of the network and applications inside a data center allows human users to rapidly asses the health of the system quickly identifying problems that span across components and solving problems that challenge fully autonomic and machine-learning based management systems. We present a case study that highlights the requirements on visualization systems designed for the management of networks and applications inside large data centers. We explain the design of the Visual-I system that meets these requirements and the experience of an operations team using Visual-I.

Fishwick, P. 2005, "Enhancing Experiential and Subjective Qualities of Discrete Structure Representations with Aesthetic Computing." Journal of Visual Languages \& Computing 16(5):406-27.

The task of visualization, as it applies to computing, includes by default the notion of pluralism and perspectivism since there is an explicit attempt at representing one, often textual, interface in terms of a more graphical one. This desire for alternate, subjective perspectives is consistent with art theory and practice, and even though rigor and formalism generally mean different things to artists and computer scientists, there is room for collaboration and connection by applying artistic aesthetics to computing, while maintaining that which makes computing a viable, usable field. This new area is called aesthetic computing. Within this area, there is an attempt to balance qualitative with quantitative representational aspects of visual computing, recognizing that aesthetics creates a dimension that is consistent with supporting numerous visual perspectives. We introduce one aspect of aesthetic computing, with specific examples from our research and teaching to illustrate the potential and possibilities associated with alternate representations of discrete structures such as finite state automata and a data flow network. We limit ourselves, and our methodology, to model notations with components that bear a largely symbolic connection to what they represent, thus providing greater degrees of representational freedom. We show that by exploring aesthetics, we surface some important philosophical and cultural questions regarding notation, which turn out to be at least as important as the algorithmic and procedural means of achieving customized model component representations. 
Fivelsdal, J. 2005, "Using Information Visualization to Give Rapid Insight into Information-Intensive Problems." J. Comput. Small Coll. 20(5):128-29.

Because most interesting tasks are too difficult to be dealt with purely mentally, information visualization can aid in the completion of useful tasks, thus increasing user efficiency, by providing a way for the computer to act as a co-participant in such tasks. It accomplishes this by allowing mental operations to rapidly access large amounts of data outside the mind.

Flach, J, M Mulder, and MM van Paasen. 2004, "The Concept of the Situation in Psychology." in $A$ Cognitive Approach to Situation Awareness, eds. S Banbury and S Tremblay, pp. 42-60. Ashgate Publishing Company, Burlington, VT.

Florentz, B, and T Muecke. 2006, "Unification and Evaluation of Graph Drawing Algorithms for Different Application Domains." In 10th International Conference on Information Visualization, pp. 47582.

Today formalisms with graphical representations are used to model structure, behavior, and other views on software and systems. The UML is just one example for modeling languages with graphical representation of its models. Although most models are executable without explicit graphical information of the model's elements, the appearance of a model is of particular importance for its readability. To improve the so called embedding of a graph representing a model or to create a initial embedding, an algorithmic support for arranging the elements is useful. We provide a software environment in which layout algorithms can be developed and evaluated. By defining proper interfaces for graph elements, we provide easy access to our library of layout algorithms for external use

Fluit, C, and J Wester. 2002, "Using Visualization for Information Management Tasks." In $6^{\text {th }}$ International Conference on Information Visualization, pp. 447-54.

Taxonomies are a powerful modelling tool when building interfaces for disclosing large information repositories. However, their actual use is far from trivial; tasks such as creation, instantiation and maintenance of taxonomies are often difficult and time-consuming. We present a number of ways in which the cluster map, a component for the visualization of instantiated taxonomies, can help in these tasks. Using the proposed visualizations, a user gains insight in the information, detects anomalies, monitors the information as it evolves over time and assesses the quality of the output of automatic classification tools. The proposed visualizations are presented in the context of one of our customers, for which we create Web portals based on taxonomies, providing access to a large document collection.

Foehrenbach, S, et al. 2009, "Tactile Feedback Enhanced Hand Gesture Interaction at Large, HighResolution Displays." Journal of Visual Languages \& Computing 20(5):341-51.

Human beings perceive their surroundings based on sensory information from diverse channels. However, for human-computer interaction we mostly restrict the user on visual perception. In this paper, we contribute to the investigation of tactile feedback as an additional perception modality. Therefore, we will first discuss existing user studies and provide a classification scheme for tactile feedback techniques. We will then present and discuss a comparative evaluation study based on the ISO 9241-9 [Ergonomic requirements for office work with visual display terminals (VDTs) - Part 9: requirements for nonkeyboard input devices, 2000]. The 20 participants performed horizontal and vertical one-directional 
tapping tasks with hand gesture input with and without tactile feedback in front of a large, high-resolution display. In contrast to previous research, we cannot confirm a benefit of tactile feedback on user performance. Our results show no significant effect in terms of throughput (effective index of performance (IPe)) and even a significant higher error rate for horizontal target alignment when using tactile feedback. Based on these results, we suggest that tactile feedback can interfere with other senses in a negative way, resulting in the observed higher error rate for horizontal targets. Therefore, more systematic research is needed to clarify the influencing factors on the usefulness of tactile feedback. Besides these results, we found a significant difference in favor of the horizontal target alignment compared with the vertical one in terms of the effective index of performance (IPe), confirming the work by Dennerlein et al. [Force feedback improves performance for steering and combined steering-targeting tasks, in: CHI '00: Proceedings of the SIGCHI Conference on Human Factors in Computing Systems, ACM, New York, NY, USA, 2000, pp. 423-429].

Foley, J, et al. 2006, "Visual Analytics Education." In IEEE Symposium on Visual Analytics Science \& Technology, pp. 209-11.

Visual analytics is a newly evolving field that spans across several more established disciplines. This panel discusses how VA system developers and researchers are best educated at the MS and PhD levels. This paper describes several ways in which VA can be characterized - with the goal of using these characterizations to identify knowledge domains that can be used to define VA curricula. Also, a digital library of VA educational resources is described

Follett, M, and O Hoeber. 2010, "Impactviz : Visualizing Class Dependencies and the Impact of Changes in Software Revisions Categories and Subject Descriptors." In 5th ACM Symposium on Software Visualization (SOFTVIS), pp. 209-10.

Følstad, A, and K Hornbæk. 2010, "Work-Domain Knowledge in Usability Evaluation: Experiences with Cooperative Usability Testing." Journal of Systems and Software 83(11):2019-30.

Usability evaluation helps to determine whether interactive systems support users in their work tasks. However, knowledge about those tasks and, more generally, about the work-domain is difficult to bring to bear on the processes and outcome of usability evaluation. One way to include such work-domain knowledge might be Cooperative Usability Testing, an evaluation method that consists of (a) interaction phases, similar to classic usability testing, and (b) interpretation phases, where the test participant and the moderator discuss incidents and experiences from the interaction phases. We have studied whether such interpretation phases improve the relevance of usability evaluations in the development of work-domain specific systems. The study included two development cases. We conclude that the interpretation phases generate additional insight and redesign suggestions related to observed usability problems. Also, the interpretation phases generate a substantial proportion of new usability issues, thereby providing a richer evaluation output. Feedback from the developers of the evaluated systems indicates that the usability issues that are generated in the interpretation phases have substantial impact on the software development process. The benefits of the interpretation phases may be explained by the access these provide both to the test participants' work-domain knowledge and to their experiences as users. 
Forbes, AG, T H, and G Legrady. 2010, "Behaviorism : A Framework for Dynamic Data Visualization." IEEE Transactions on Visualization and Computer Graphics 16(6):1164-71.

Forsell, C. 2010, "A Guide to Scientific Evaluation in Information Visualization." In 14th International Conference on Information Visualization, pp. 162-69.

This paper addresses some fundamental and practical issues that should be considered when pursuing evaluation studies in Information Visualization. The main focus is on quantitative experimental research but the general information applies to all kinds of studies. The purpose is to increase awareness of what constitutes a sound scientific approach to evaluation and to point out common pitfalls and mistakes during the phases of such study. These phases cover how to plan, design, conduct and analyse the outcome of an evaluation and finally how to report in a way that enhances readability, provides details relevant to the outcome and that allows replication. The paper could be used as a guide when conducting evaluation and it could also be helpful when reviewing publications since the same rules apply.

Fowler, S, and V Stanwick. 2004, "Resources." in Web Application Design Handbook, Best Practices for Web-Based Software, pp. 611-32. Morgan Kaufmann, San Francisco.

Fraedrich, R, S Auer, and R Westermann. 2010, "Efficient High-Quality Volume Rendering of Sph Data." IEEE Transactions on Visualization and Computer Graphics 16(6):1533-40.

High quality volume rendering of SPH data requires a complex order-dependent resampling of particle quantities along the view rays. In this paper we present an efficient approach to perform this task using a novel view-space discretization of the simulation domain. Our method draws upon recent work on GPU-based particle voxelization for the efficient resampling of particles into uniform grids. We propose a new technique that leverages a perspective grid to adaptively discretize the view-volume, giving rise to a continuous level-of-detail sampling structure and reducing memory requirements compared to a uniform grid. In combination with a level-of-detail representation of the particle set, the perspective grid allows effectively reducing the amount of primitives to be processed at run-time. We demonstrate the quality and performance of our method for the rendering of fluid and gas dynamics SPH simulations consisting of many millions of particles.

Franken, N. 2009, "Visual Exploration of Algorithm Parameter Space." In IEEE Congress on Evolutionary Computation, pp. 389-98.

In this article we apply information visualization techniques to the domain of swarm intelligence. We describe an intuitive approach that enables researchers and designers of stochastic optimization algorithms to efficiently determine trends and identify optimal regions in an algorithm's parameter search space. The parameter space is evenly sampled using low-discrepancy sequences, and visualized using parallel coordinates. Various techniques are applied to iteratively highlight areas that influence the optimization algorithm's performance on a particular problem. By analyzing experimental data with this technique, we were able to gain important insight into the complexity of the target problem domain. For example, we were able to confirm some underlying theoretical assumptions of an important class of population-based stochastic algorithms. Most importantly, the technique improves the efficiency of finding good parameter settings by orders of magnitude. 
Fraser Taylor, DR. 2005, "Chapter 1 - the Theory and Practice of Cybercartography: An Introduction." in Modern Cartography Series, ed. DRF Taylor, Vol 4, pp. 1-13. Academic Press.

The concept of cybercartography was introduced in 1997 in the keynote address entitled Maps and Mapping in the Information Era, presented to the International Cartographic Conference in Sweden. The central argument made was that if cartography was to play a more important role in the information era, then a new paradigm was required. An initial version of cybercartography was introduced as that paradigm. This chapter describes developments in cybercartography since that time and, in particular, the developments emerging from the Cybercartography and the New Economy research project, funded for a 4 year period, by the Social Sciences and Humanities Research Council of Canada. Many of the chapters in this book grew out of this research project. Parallel developments in the theory and practice of cybercartography are taking place in a very different cultural context at Mexico by CentroGeo, a new and dynamic research agency, which is part of the Consejo Nacional de Ciencia y Technologica (CONACYT) network. The significant differences between cybercartography and Geographic Information Systems (GIS) and the related concept of Geographical Information Science will be discussed, and this chapter will establish the context for the rest of this book.

Frauenberger, C, and T Stockman. 2009, "Auditory Display Design--an Investigation of a Design Pattern Approach." International Journal of Human-Computer Studies 67(11):907-22.

We present the evaluation of a methodological design framework that supports expert and novice designers in creating auditory artefacts in human-technology interaction. We first motivate the development of our framework by analysing available guidance and the current practice in the field. Subsequently, we recapitulate on the design of the framework--paco, pattern design in the context space-and present its key concepts and methods. The evaluation of paco aimed to investigate how useful this framework is in a real-world environment. It was conducted in two phases: experts in auditory display design first captured successful designs through paco and created a body of design patterns. These patterns were subsequently used in a controlled experiment with novice designers who were given a design task that forced them to use audio. The results demonstrate that paco has facilitated the transfer of design knowledge and good practice from experts to novices through design patterns. The context space, a key concept in paco, improves the contextual awareness of designers and provides an organising principle for problems, patterns and artefacts. We close by reflecting on the results and discussing future lines of research.

Freire, M. 2010, "Gene Similarity Uncovers Mutation Path Vast 2010 Mini Challenge 3 Award : Innovative Tool Adaptation." In IEEE Symposium on Visual Analytics Science \& Technology, pp. 287-88.

Freitas, C, et al. 2002, "On Evaluating Information Visualization Techniques." In Working Conference on Advanced Visual Interfaces pp. 373-74.

Freitas, C, et al. 2010, "Evaluating Usability of Information Visualization Techniques." In International Conference on Advanced Visual Interfaces, pp. 373-74.

Evaluation is a key research challenge within the international Information Visualization (InfoVis) community, and Heuristic Evaluation is one recognized method. Various sets of heuristics have been proposed but there remains no consensus as to which heuristics are most useful for addressing aspects specific to the complex interactive visual displays used in modern InfoVis systems. This paper presents a 
first effort to empirically determine a new set of such general heuristics tailored for Heuristic Evaluation of common and important usability problems in InfoVis techniques. Participants in the study rated how well a total of 63 heuristics from 6 earlier published heuristic sets could explain a collection of 74 usability problems derived from earlier InfoVis evaluations. The results were used to synthesize 10 heuristics that, as a set, provided the highest explanatory coverage. The paper also stresses the challenges for future research to validate and further improve upon this set.

Friedler, SA, et al. 2008, "Enabling Teachers to Explore Grade Patterns to Identify Individual Needs and Promote Fairer Student Assessment." Computers \& Education 51(4):1467-85.

Exploring student test, homework, and other assessment scores is a challenge for most teachers, especially when attempting to identify cross-assessment weaknesses and produce final course grades. During the course, teachers need to identify subject weaknesses in order to help students who are struggling with a particular topic. This identification often needs to happen across multiple assessment data points and should be considered in comparison to the class's progress as a whole. When determining grades, fairness to all is essential, but there are special needs for students who did poorly on one exam or had a steadily increasing grasp of the subject. We present eduViz, a visualization tool designed to help teachers explore and assign grades. Teachers can see the trajectory of student scores, the relationship of a particular student to the class, and use categories they have defined in order to filter their assessment information. Query response is immediate and all logical comparisons are possible. Teachers can easily compare their query to the class or per student average as well as view scores by raw point total or percentage. Additionally, eduViz provides a grade assignment interface which allows teachers to view sorted student scores in a scatterplot. This scatterplot is coupled with a unique partition slider which allows users to move color coordinated bands on the scatterplot to indicate grade ranges. As these grade ranges are set, a histogram is updated to show the number of students assigned to each grade range. These features give teachers new and powerful ways to explore and assign grades so that they can better understand student strengths and weaknesses and make the most of the time they have available. Interviews with 16 expert teachers indicate that eduViz is a success across fields, provides teachers with a useful tool to understand and help their classes, and encourages reflective practice. 
Friedman, E. 2010, "Epidemic Outbreak Visualizer." In IEEE Symposium on Visual Analytics Science \& Technology.

Friedman, E. 2010, "Ner Visualiser and Pinwallvis." In IEEE Symposium on Visual Analytics Science \& Technology, pp. 1-2.

Friedman, E. 2010, "Phylogenetic Tree Visualizer for Analogous Genetic Sequences." In IEEE Symposium on Visual Analytics Science \& Technology, pp. 3-4.

Friendly, M. 2007, "A Brief History of Data Visualization." in Handbook of Data Visualization, Vol 2, pp. 15-56. Springer-Verlag, Berlin.

1. Michael Friendly, "A Brief History of Data Visualization", pp 15-56 2. Antony Unwin, "Good Graphics?", pp 57-78 3. Paul Murrell, "Static Graphics", pp 79-101 4. George Michailidis, "Data Visualization Through Their Graph Rep.", pp 103-120 5. Lelend Wilkinson, "Graph-theoretic Graphics", pp 121-150 6. Martin Theus, "High-dimensional Data Visualization", pp 151-178 7. Matthew O. Ward, "Multivariate Data Glyphs: Principles and Practice", pp 179-198 8. Adalbert Wilhelm, "Linked Views for Visual Exploration", pp 199-215 9. Graham Wills, "Linked Data Views", pp 217-241 10. Simon Urbanek, "Visualizing Trees and Forests", pp 243-264

Friendly, M, and E Kwan. 2003, "Effect Ordering for Data Displays." Computational Statistics \& Data Analysis 43(4):509-39.

This paper outlines a general framework for ordering information in visual displays (tables and graphs) according to the effects or trends which we desire to see. This idea, termed effect-ordered data displays, applies principally to the arrangement of unordered factors for quantitative data and frequency data, and to the arrangement of variables and observations in multivariate displays (star plots, parallel coordinate plots, and so forth). As examples of this principle, we present several techniques for ordering items, levels or variables "optimally", based on some desired criterion. All of these may be based on eigenvalue or singular-value decompositions. Along the way, we tell some stories about data display, illustrated by graphs--some surprisingly bad, and some surprisingly good--for showing patterns, trends, and anomalies in data. We hope to raise more questions than we can provide answers for.

Frigioni, D, and L Tarantino. 2003, "Multiple Zooming in Geographic Maps." Data \& Knowledge Engineering 47(2):207-36.

We present a zooming model, based on a level-of-detail (LOD) approach, aimed at visualizing sequences of gradually simplified representations of a given geographic map. We first describe a set of basic zooming primitives as transitions between maps at different LOD. We then define a new multiple zooming primitive on top of the basic ones, and show theoretical results concerning this new primitive with practical implications. Finally, we put the proposed theoretical model into practice, and suggest an implementation of maps and zooming primitives in terms of PLA-structures, a topological invariant suitable for the inclusion in a visual interaction environment based on a focus + context approach. 
Frisch, M, and R Dachselt. 2010, "Off-Screen Visualization Techniques for Class Diagrams." In 5th ACM Symposium on Software Visualization (SOFTVIS), pp. 163-72.

Fuchs, R, J Waser, and ME Groller. 2009, "Visual Human Plus Machine Learning." IEEE Transactions on Visualization and Computer Graphics 15(6):1327-34.

In this paper we describe a novel method to integrate interactive visual analysis and machine learning to support the insight generation of the user. The suggested approach combines the vast search and processing power of the computer with the superior reasoning and pattern recognition capabilities of the human user. An evolutionary search algorithm has been adapted to assist in the fuzzy logic formalization of hypotheses that aim at explaining features inside multivariate, volumetric data. Up to now, users solely rely on their knowledge and expertise when looking for explanatory theories. However, it often remains unclear whether the selected attribute ranges represent the real explanation for the feature of interest. Other selections hidden in the large number of data variables could potentially lead to similar features. Moreover, as simulation complexity grows, users are confronted with huge multidimensional data sets making it almost impossible to find meaningful hypotheses at all. We propose an interactive cycle of knowledge-based analysis and automatic hypothesis generation. Starting from initial hypotheses, created with linking and brushing, the user steers a heuristic search algorithm to look for alternative or related hypotheses. The results are analyzed in information visualization views that are linked to the volume rendering. Individual properties as well as global aggregates are visually presented to provide insight into the most relevant aspects of the generated hypotheses. This novel approach becomes computationally feasible due to a GPU implementation of the time-critical parts in the algorithm. A thorough evaluation of search times and noise sensitivity as well as a case study on data from the automotive domain substantiate the usefulness of the suggested approach.

Fuchs, R, J Waser, and ME Groller. 2009, "Visual Human+Machine Learning." Visualization and Computer Graphics, IEEE Transactions on 15(6):1327-34.

In this paper we describe a novel method to integrate interactive visual analysis and machine learning to support the insight generation of the user. The suggested approach combines the vast search and processing power of the computer with the superior reasoning and pattern recognition capabilities of the human user. An evolutionary search algorithm has been adapted to assist in the fuzzy logic formalization of hypotheses that aim at explaining features inside multivariate, volumetric data. Up to now, users solely rely on their knowledge and expertise when looking for explanatory theories. However, it often remains unclear whether the selected attribute ranges represent the real explanation for the feature of interest. Other selections hidden in the large number of data variables could potentially lead to similar features. Moreover, as simulation complexity grows, users are confronted with huge multidimensional data sets making it almost impossible to find meaningful hypotheses at all. We propose an interactive cycle of knowledge-based analysis and automatic hypothesis generation. Starting from initial hypotheses, created with linking and brushing, the user steers a heuristic search algorithm to look for alternative or related hypotheses. The results are analyzed in information visualization views that are linked to the volume rendering. Individual properties as well as global aggregates are visually presented to provide insight into the most relevant aspects of the generated hypotheses. This novel approach becomes computationally feasible due to a GPU implementation of the time-critical parts in the algorithm. A thorough evaluation of search times and noise sensitivity as well as a case study on data from the automotive domain substantiate the usefulness of the suggested approach. 
Fuhrmann, S. 2003, "Chapter 16 - Supporting Wayfinding in Desktop Geovirtual Environments." in Maps and the Internet, ed. P Michael, pp. 271-87. Elsevier Science, Oxford.

Summary Advances in the technology for both dynamic visual representation and dissemination of those representations using the Internet are changing the way geoscientists access and apply geospatial and related data. Desktop geovirtual environments (GeoVEs) offer new ways to interactively display geospatial structures and phenomena for geovisualization. However, users have aggravating difficulties in utilizing the potentials of desktop GeoVEs because of navigation and wayfinding constraints. This chapter is concerned with the design of graphical user interface methods that support navigation and orientation within desktop GeoVEs and facilitate user's wayfinding tasks.

Fuhrmann, S, et al. 2005, "Making Useful and Useable Geovisualization: Design and Evaluation Issues." eds. D Jason, MM Alan and K Menno-Jan, pp. 551-66. Elsevier, Oxford.

Summary The design of geovisualization tools is not only a technical research question. For many years geovisualization tool design was largely technology driven, where system designers and final users were mostly one and the same. Nowadays geovisualization tools are applied in and developed for a broader geosoftware market with the goal of providing useful and usable geovisualization. Sometimes this goal is not reached for many reasons, resulting in frustrated users and unsolved tasks. The aim of this overarching chapter is to give an introduction into methods and research questions on user-centered geovisualization tool design, bridging the gap between developers and users. In order to stimulate the development of geovisualization theory, the authors of this chapter contribute their views and discuss issues from Computer Science, Information Visualization, Geoinformation Science, Geography and Cartography.

Furmanski, C, R Azuma, and M Daily. 2002, "Augmented-Reality Visualizations Guided by Cognition: Perceptual Heuristics for Combining Visible and Obscured Information." In International Symposium on Mixed and Augmented Reality, pp. 215-+.

One of the unique applications of Mixed and Augmented Reality (MR/AR) systems is that hidden and occluded objects can be readily visualized. We call this specialized use of MR/AR Obscured Information Visualization (OIV). In this paper, we describe the beginning of a research program designed to develop such visualizations through the use of principles derived from perceptual psychology and cognitive science. In this paper we surveyed the cognitive science literature as it applies to such visualization tasks, described experimental questions derived from these cognitive principles, and generated general guidelines that can be used in designing future OIV systems (as well improving AR displays more generally). Here we also report the results from an experiment that utilized a functioning AR-OIV system: we found that in a relative depth judgment, subjects reported rendered objects as being in front of realworld objects, except when additional occlusion and motion cues were presented together.

G. 1993, "Visualization Education in the USA." Education and Computing 8(4):339-45.

The use of advanced computer graphics techniques to help visualize large volumes of multivariate information has become increasingly important. Most of the research in this area has been in the area of scientific visualization, and visualization has become one of the most important tools of modern computational science. It should be noted that computational science has become the third supporting methodology for the physical and biological sciences, alongside the more traditional theoretical and 
laboratory science areas. It is receiving considerable emphasis from the National Science Foundation in the United States. This development has raised the issue of providing visualization education for both computer science students and students in the physical and biological sciences. The computer graphics community has started to examine the question of incorporating visualization concepts and techniques into undergraduate and graduate computer science curricula. The author co-chaired, with Steve Cunningham, an Educators' Seminar on "Education for Visualization" at SIGGRAPH '90 and the ACM SIGGRAPH Education Committee has recently formed a subcommitte (currently consisting of the author, Steve Cunningham, and Norman Soong of Villanova University) to address these educational issues. Steve Cunningham has just been elected to the Board of Directors of ACM SIGCSE (Special Interest Group on Computer Science Education) and intends to work to support the development of computational science studies in computer science programs. There are many issues in visualization education. Students need to be familiar with a wide range of tools, because visualization environments typically include many networked hardware and software tools that support particular aspects of visualization. Equipment in a visualization center typically includes high-performance computing and specialized codes for numerical experiments, specialized rendering machines with accelerated graphics, individual workstations for scientists' viewing, and specialized devices for making video or film images for study and publication. Many commercial visualization tools, such as the Silicon Graphics, Wavefront and Alias software systems, are now available for different computer platforms. In addition, a substantial amount of public domain visualization software is available, such as the set of image tools from the National Center for Supercomputer Applications (NCSA) at the University of Illinois and the Khoros system from the Vision Lab at the University of New Mexico. Finally, some visualization software is developed, especially for special projects. Some of the educational questions to be considered are as follows: - Should all computer science students learn some visualization concepts? - What computational environment should be offered to visualization students? - Should visualization techniques be a part of an introductory computer graphics course, part of an advanced computer graphics course, or should there be a separate course in the area? Just what visualization techniques should be taught? - What are the appropriate underlying principles? What textbooks and other course materials are available or need to be developed? This paper will report on the current status of education for visualization in the United States, attempt to answer some of the above questions, and make some preliminary recommendations for future curriculum development.

Gahegan, M, et al. 2007, "Measures of Similarity for Integrating Conceptual Geographical Knowledge: Some Ideas and Some Questions." In International Conference on Spatial Information Theory (COSIT), pp. 1-6.

Gahegan, M, et al. 2009, "Connecting Geon: Making Sense of the Myriad Resources, Researchers and Concepts That Comprise a Geoscience Cyberinfrastructure." Computers \& Geosciences 35(4):836-54.

Simply placing electronic geoscience resources such as datasets, methods, ontologies, workflows and articles in a digital library or cyberinfrastructure does not mean that they will be used successfully by other researchers or educators. It is also necessary to provide the means to locate potentially useful content, and to understand it. Without suitable provision for these needs, many useful resources will go undiscovered, or else will be found but used inappropriately. In this article, we describe an approach to discovering, describing and understanding e-resources based on the notion that meaning is carried in the interconnections between resources and the actors in the cyberinfrastructure (including individuals, groups, organizations), as well as by ontologies and conventional metadata. Navigation around this universe is achieved by implementing the idea of perspectives as dynamic, conceptual views (defined by SPARQL-like queries against an OWL schema) that not only act as filters, but also dynamically promote 
and demote concepts, relationships and properties according to their immediate relevance. We describe a means to represent a wide variety of interactions between resources using the notion of a knowledge nexus, and we illustrate its use with resources and actors from the Geosciences Network (GEON) cyberinfrastructure community. We also closely link browsing and visualizing strategies to our nexus, drawing on ideas from semiotics to move resources and connections not currently of interest from the foreground to the background, and vice versa, using a new form of adaptive perspective. We illustrate our ideas via ConceptVista, an open-source concept mapping application that provides rich, visual depictions of the resources, cyber-community and myriad connections between them. Examples are presented that show how geoscientific knowledge can be explored not only via ontological structure, but also by use cases, social networks, citation graphs and organization charts; all of which may carry some aspects of meaning for the user.

Gaio, M, et al. 2008, "A Global Process to Access Documents' Contents from a Geographical Point of View." Journal of Visual Languages \& Computing 19(1):3-23.

Local cultural heritage document repositories are characterized by contents strongly attached to a territory (i.e., geographical references). The user must be able to consider such repositories according to a focus, which takes into account his/her geographical interests, and which allows one to access the relevant document's contents from a geographical point of view. This paper presents the Virtual Itineraries in the Pyrenees (PIV) project. Spatial and temporal core models are proposed to give a formal representation of geographical information. The models take into account the characteristics of heterogeneous human modes of expression: written language and captures of drawings, maps, pictures, etc. Semantic processes have been built to automatically manage the spatial and temporal information from non-structured data. A "back office" prototype, which adds these processes to classic information extraction (IE) approaches, while associating a geographical information retrieval (GIR) service is proposed. This service searches for any links between formal representations of geographic information in document collections, and similar representations in a user's information query. Finally, the paper presents the design work, giving the details of the principles of result visualization and navigation, while proposing a "front office" first implementation of the system.

Gallagher, K, A Hatch, and M Munro. 2008, "Software Architecture Visualization: An Evaluation Framework and Its Application." IEEE Transactions on Software Engineering 34(2):260-70.

Garcia, CA, et al. 2009, "Evaluation of Clustered Molecules in the Process of New Drugs Design." In 10th International Symposium on Smart Graphics, pp. 3-14.

Drug design is very complex and expensive. Finding new active chemical structures is a very important goal. Both experimental and virtual (in silico) screenings call be used to explore chemical space [11][12]. With virtual screening it is possible to reduce the amount of compounds for experimental evaluations. Moreover, when the 3D structure of the target is known, candidate molecules can be put, to fit in the target hole in different positions and later cluster these positions in order to find the best to fit. Therefore, we propose a visual tool that couples with Jmol[21] viewer, provides together with a means of visually exploring clustered molecules, all overview of the majority of the data, supporting thus the decision making in the process of new drugs design. 
Garcia-Molina, H. 2008, "Photospread: A Spreadsheet for Managing Photos." In 24th International Conference on Data Engineering, pp. 5-6.

The goal of the BioACT Project at Stanford is to help biodiversity researchers Acquire digital materials in the field, manage these online holdings (Curate), and Transfer the knowledge (or disseminate) to other researchers, museums, and the public. We are developing three sets of tools: (i) tools for speedy data entry and small-group collaboration in the field, (ii) tools for large scale collaboration in distributed collection curation, and (iii) tools for semidirected search and browsing of digital biodiversity materials.Currently, the biologists use spreadsheets to analyze the photos. Each photograph is represented by a row in the spreadsheet. One column gives the file name where the photo is stored, and the other columns give the metadata (date, species, etc.). Once the data is in the spreadsheet, they can select and group photos of interest and compute relevant statistics. In this paper, we describe Photospread in more detail. We discuss the supported formula language, and we describe how drag-anddrop actions work. We also compare Photospread to other photo browsing and analysis tools.

Garg, S, IV Ramakrishnan, and K Mueller. 2010, "A Visual Analytics Approach to Model Learning." In IEEE Symposium on Visual Analytics Science \& Technology, pp. 67-74.

Garlandini, S, and SI Fabrikant. 2009, "Evaluating the Effectiveness and Efficiency of Visual Variables for Geographic Information Visualization." In 9th International Conference on Spatial Information Theory, pp. 195-211.

We propose an empirical, perception-based evaluation approach for assessing the effectiveness and efficiency of longstanding cartographic design principles applied to 2D map displays. The approach includes bottom-up visual saliency models that are compared with eye-movement data collected in human-subject experiments on map stimuli embedded in the so-called flicker paradigm. The proposed methods are applied to the assessment of four commonly used visual variables for designing 2D maps: size, color value, color hue, and orientation. The empirical results suggest that the visual variable size is the most efficient (fastest) and most effective (accurate) visual variable to detect change under flicker conditions. The visual variable orientation proved to be the least efficient and effective of the tested visual variables. These empirical results shed new light on the implied ranking of the visual variables that have been proposed over 40 years ago. With the presented approach we hope to provide cartographers, GIScientists and visualization designers a systematic assessment method to develop effective and efficient geovisualization displays.

Garrett, JH. 2000, "Evaluating Visualizations Based on the Performed Task." In IEEE International Conference on Information Visualization, pp. 135-42.

Describes an ongoing project with the goal of designing and implementing a method to evaluate visualizations based on the tasks supported. The method evaluates time to perform the task and the quality of the user solution. This method is described in detail in this paper. A software tool which uses visualization to support users performing environmental analysis was used to test the first version of this method. The software tool evaluated helps users to perform computations of the environmental impacts of two products. Two versions of the same software tool were used. The first version uses interactive tables and the second version displays the information using different types of interactive visualizations. The software tool was evaluated with users solving a task which consists of selecting a product from two alternatives based on environmental parameters. The users were divided into two groups. Each group 
of users solved the same task but they used different software tools. A summary of the findings and possible future research is presented at the end of this paper

Garth, C, and KI Joy. 2010, "Fast, Memory-Efficient Cell Location in Unstructured Grids for Visualization." IEEE Transactions on Visualization and Computer Graphics 16(6):1541-50.

Applying certain visualization techniques to datasets described on unstructured grids requires the interpolation of variables of interest at arbitrary locations within the dataset's domain of definition. Typical solutions to the problem of finding the grid element enclosing a given interpolation point make use of a variety of spatial subdivision schemes. However, existing solutions are memory- intensive, do not scale well to large grids, or do not work reliably on grids describing complex geometries. In this paper, we propose a data structure and associated construction algorithm for fast cell location in unstructured grids, and apply it to the interpolation problem. Based on the concept of bounding interval hierarchies, the proposed approach is memory-efficient, fast and numerically robust. We examine the performance characteristics of the proposed approach and compare it to existing approaches using a number of benchmark problems related to vector field visualization. Furthermore, we demonstrate that our approach can successfully accommodate large datasets, and discuss application to visualization on both CPUs and GPUs.

Gedeck, P, and P Willett. 2001, "Visual and Computational Analysis of Structure-Activity Relationships in High-Throughput Screening Data." Current Opinion in Chemical Biology 5(4):389-95.

Novel analytic methods are required to assimilate the large volumes of structural and bioassay data generated by combinatorial chemistry and high-throughput screening programmes in the pharmaceutical and agrochemical industries. Recent work in visualisation and data mining has been used to develop structure-activity relationships from such chemical-biological datasets.

Gedik, AC, and B Bozkurt. 2010, "Pitch-Frequency Histogram-Based Music Information Retrieval for Turkish Music." Signal Processing 90(4):1049-63.

This study reviews the use of pitch histograms in music information retrieval studies for western and non-western music. The problems in applying the pitch-class histogram-based methods developed for western music to non-western music and specifically to Turkish music are discussed in detail. The main problems are the assumptions used to reduce the dimension of the pitch histogram space, such as, mapping to a low and fixed dimensional pitch-class space, the hard-coded use of western music theory, the use of the standard diapason (A4=440 Hz), analysis based on tonality and tempered tuning. We argue that it is more appropriate to use higher dimensional pitch-frequency histograms without such assumptions for Turkish music. We show in two applications, automatic tonic detection and makam recognition, that high dimensional pitch-frequency histogram representations can be successfully used in Music Information Retrieval (MIR) applications without such pre-assumptions, using the data-driven models.

Gel, J, et al. 2003, "The Dynamic Response of Stereoscopic Depth Cognition." In 25th Annual International Conference of the IEEE Engineering in Medicine and Biology Society, pp. 2913-16.

Owing to high complexity of life activity, the dynamic complex response of objective visual channel had never been studied by human being but it is very important to understand life activity actually. Along 
with the measure sequences of human genome has successfully been carried out. Proteome is investigating its functions and structures. As a matter of fact, all these are not dynamic to get the hereditary information of organism based on off body condition, the organisms only have activity but they are dead already. We hope to research micro-dynamics of life activity in the physiological system based on physiology step by step and to developmentally investigate at cell level or even at gene level for understanding life activity and modulating mechanism simultaneously in order to make important progress little by little. In the article, relatively comparative independent visual system resolved from sophisticated human body and connected cognition studies the dynamic complex response of cognitive progresses with visual stimuli and uses to understand the function of information processing system in brain. As a result of cognitive psychology related to cognitive process, on one hand, researchers can directly take part in studies that investigated how to get perception from recognizable objects and surroundings by subject and worked on the cognitive information. On other hand they can learn from experience about the sense of characteristics of dynamic response at the same time. Due to our researches directly participates in experiments by themselves, they much better know the response progresses which what have happened directly perceived through the senses. It much helps to theoretical analysis. Using a multi-channel of visual cortex evoked potential (VCEP) collecting system for this research; we studied the mechanisms of the brain function and stereoscopic information processing, and identified a new N2 wave of VCEP as the characteristic response to the disparity depth stimuli. The VCEP of vary visual functional areas evoked by the specially designed RDS uncovered that the information processing of the disparity depth perception starts at the striate cortex but ends at the advanced cortical areas. Besides, we employed several independent methods to have- proved the new N2 wave existence. The result also convincingly proved the supposition, which proposed that the depth cognition of stereoscopic vision is a dynamic multi-factor synchronizes cooperated processing and the consequence of depth perception in advanced cortical areas may get through biological feedback to the whole process of visual signal processing. The delays and peak to peak in time series of dynamic response happened in advanced visual cortex are about $340 \mathrm{~ms}$ and $5.0 \mathrm{uv}$ in lead P4, $339 \mathrm{~ms}$ and $3.7 \mathrm{uv}$ in P3, $339 \mathrm{~ms}$ and $4.0 \mathrm{uv}$ in T1. Both experimental and complex dynamic computing results are given in the text.

Gentner, D, and AL Stevens. 1983. Mental Models. Lawrence Erlbaum Associates, Hillsdale, NJ.

Gerber, S, et al. 2010, "Visual Exploration of High Dimensional Scalar Functions." IEEE Transactions on Visualization and Computer Graphics 16(6):1271-80.

An important goal of scientific data analysis is to understand the behavior of a system or process based on a sample of the system. In many instances it is possible to observe both input parameters and system outputs, and characterize the system as a high-dimensional function. Such data sets arise, for instance, in large numerical simulations, as energy landscapes in optimization problems, or in the analysis of image data relating to biological or medical parameters. This paper proposes an approach to analyze and visualizing such data sets. The proposed method combines topological and geometric techniques to provide interactive visualizations of discretely sampled high-dimensional scalar fields. The method relies on a segmentation of the parameter space using an approximate Morse-Smale complex on the cloud of point samples. For each crystal of the Morse-Smale complex, a regression of the system parameters with respect to the output yields a curve in the parameter space. The result is a simplified geometric representation of the Morse-Smale complex in the high dimensional input domain. Finally, the geometric representation is embedded in $2 \mathrm{D}$, using dimension reduction, to provide a visualization platform. The geometric properties of the regression curves enable the visualization of additional information about each crystal such as local and global shape, width, length, and sampling densities. The method is 
illustrated on several synthetic examples of two dimensional functions. Two use cases, using data sets from the UCI machine learning repository, demonstrate the utility of the proposed approach on real data. Finally, in collaboration with domain experts the proposed method is applied to two scientific challenges. The analysis of parameters of climate simulations and their relationship to predicted global energy flux and the concentrations of chemical species in a combustion simulation and their integration with temperature.

Geroimenko, V, and L Geroimenko. 2000, "Visualising Human Consciousness Content Using Java3d/X3d and Psychological Techniques." In IEEE International Conference on Information Visualization, pp. 529-32.

Describes a new approach to visualising the contents of human consciousness that combines the Twin VR (virtual reality) technology with relevant psychological methods. The Twin VR technology is based on the use of two dynamically connected virtual environments: the first one serves for the elicitation of required information and the second for its visualisation. A prototype VRGrid system is presented as a practical implementation of this approach. The system allows eliciting and visualising some personal conceptual models in the context of personal construct psychology (PCP). Several aspects of the development of the prototype using emerging technologies such as Java 3D and X3D (Extensible 3D) are discussed

Gerth, J, et al. 2008, "Maintaining Interactivity While Exploring Massive Time Series." In IEEE Symposium on Visual Analytics Science \& Technology, pp. 59-66.

The speed of data retrieval qualitatively affects how analysts visually explore and analyze their data. To ensure smooth interactions in massive time series datasets, one needs to address the challenges of computing ad hoc queries, distributing query load, and hiding system latency. In this paper, we present ATLAS, a visualization tool for temporal data that addresses these issues using a combination of high performance database technology, predictive caching, and level of detail management. We demonstrate ATLAS using commodity hardware on a network traffic dataset of more than a billion records.

Gestwicki, P. 2010, "Poster : Design and Evaluation of an Interactive Curriculum Visualization System." In IEEE Conference on Information Visualization.

Ghanbari, M. 2007, "Purpose of Data Visualization."133-37.

Ghanbari, M. 2008, "Scalability of Visualization's Evaluation." In Southeastcon, pp. 318-22.

Information visualization has progressed and taken big steps in previous decade, despite challenging complexities of presenting and transforming the data. Visualization binds the perceptual capabilities of the human visual system. In the data, Human being looks for structure, pattern, features, anomalies, and relationship. Visualization, support this by preparing the data in a way to drive particular sense that differentiate various interactions and understanding. How human being receives and interacts with a visualization tools, can strongly influences his understanding of the data as well as the system's usefulness. Therefore, understanding the tools, relationships, and how well be able to depict the blue print of the model in mind, is not an easy task. Too often, successful decision-making and analysis are more a matter of serendipity and user experience than of intentional design and specific support for such a task [2]. We need better metrics and benchmark repositories to compare tools, and we should also seek reports 
of successful adoption and demonstrated utility. Moreover, there is a large range of target audience with different background and therefore, examining the concept, data, and analytic methodologies for these class of audience also is a big step in the right way. Furthermore, we also should consider how tools -for transformation and presentation - can improve mental activities of developer. This mental support has been defined as "; cognitive support"; [3]. So, are we able to explicitly state and compare claims about how particular tool support cognition? Are there capable theories for backdrop, onto which suitable theories and claims can be painted? Unfortunately, there are too many factors and relations which we should consider in order to be able to have a clear cut of measuring the relationships and their boundaries. In this paper, I'll try to open the question and shed on some important and very difficult aspect of visualization evaluation.

Ghanbari, M. 2008, "Scalability of Visualization's Evaluation." In IEEE Southeastcon 2008, pp. 318-22.

Information visualization has progressed and taken big steps in previous decade, despite challenging complexities of presenting and transforming the data. Visualization binds the perceptual capabilities of the human visual system. In the data, Human being looks for structure, pattern, features, anomalies, and relationship. Visualization, support this by preparing the data in a way to drive particular sense that differentiate various interactions and understanding. How human being receives and interacts with a visualization tools, can strongly influences his understanding of the data as well as the system's usefulness. Therefore, understanding the tools, relationships, and how well be able to depict the blue print of the model in mind, is not an easy task. Too often, successful decision-making and analysis are more a matter of serendipity and user experience than of intentional design and specific support for such a task [2]. We need better metrics and benchmark repositories to compare tools, and we should also seek reports of successful adoption and demonstrated utility. Moreover, there is a large range of target audience with different background and therefore, examining the concept, data, and analytic methodologies for these class of audience also is a big step in the right way. Furthermore, we also should consider how tools -for transformation and presentation - can improve mental activities of developer. This mental support has been defined as "; cognitive support"; [3]. So, are we able to explicitly state and compare claims about how particular tool support cognition? Are there capable theories for backdrop, onto which suitable theories and claims can be painted? Unfortunately, there are too many factors and relations which we should consider in order to be able to have a clear cut of measuring the relationships and their boundaries. In this paper, I'll try to open the question and shed on some important and very difficult aspect of visualization evaluation.

Ghoniem, M, JD Fekete, and P Castagliola. 2004, "A Comparison of the Readability of Graphs Using Node-Link and Matrix-Based Representations." In IEEE Symposium on Information Visualization, pp. 17-24.

In this paper, we describe a taxonomy of generic graph related tasks and an evaluation aiming at assessing the readability of two representations of graphs: matrix-based representations and node-link diagrams. This evaluation bears on seven generic tasks and leads to important recommendations with regard to the representation of graphs according to their size and density. For instance, we show that when graphs are bigger than twenty vertices, the matrix-based visualization performs better than node-link diagrams on most tasks. Only path finding is consistently in favor of node-link diagrams throughout the evaluation 
Ghoniem, M, et al. 2007, "Newslab: Exploratory Broadcast News Video Analysis." In IEEE Symposium on Visual Analytics Science \& Technology, pp. 123-30.

In this paper, we introduce NewsLab, an exploratory visualization approach for the analysis of large scale broadcast news video collections containing many thousands of news stories over extended periods of time. A river metaphor is used to depict the thematic changes of the news over time. An interactive lens metaphor allows the playback of fine-grained video segments selected through the river overview. Multi-resolution navigation is supported via a hierarchical time structure as well as a hierarchical theme structure. Themes can be explored hierarchically according to their thematic structure, or in an unstructured fashion using various ranking criteria. A rich set of interactions such as filtering, drilldown/roll-up navigation, history animation, and keyword based search are also provided. Our case studies show how this set of tools can be used to find emerging topics in the news, compare different broadcasters, or mine the news for topics of interest.

Giannopoulou, EG, et al. 2009, "Proteomic Feature Maps: A New Visualization Approach in Proteomics Analysis." Journal of Biomedical Informatics 42(4):644-53.

The different steps of a proteomics analysis workflow generate a plethora of features for each extracted proteomic object (a protein spot in 2D gel electrophoresis (2-DE), or a peptide peak in liquid chromatography-mass spectrometry (LC-MS) analysis). Yet, the joint visualization of multiple object features on 2D gel-like maps is rather limited in currently available proteomics software packages. We introduce a new, simple, and intuitive visualization method that utilizes spheres to represent proteomic objects on proteomic feature maps, and exploits the spheres size and color to provide simultaneous visualization of user-selected feature pairs. Our contribution, a unified and flexible visualization mechanism that can be easily applied at any stage of a 2-DE or a LC-MS based differential proteomics study, is demonstrated and discussed using five representative scenarios. The joint visualization of proteomic object features and their spatial distribution is a powerful tool for inspecting and comparing the proteomics analysis results, attracting the users attention to useful information, such as differential expression trends and patterns, and even assisting in the evaluation and refinement of a proteomics experiment.

Gick, ML, and RS Lockhart. 1996, "Cognitive and Affective Components of Insight." in The Nature of Insight, eds. RJ Sternberg and JE Davidson, pp. 197-228. MIT Press.

Giereth, M, and T Ertl. 2008, "Visualization Enhanced Semantic Wikis for Patent Information." In $12^{\text {th }}$ International Conference on Information Visualization, pp. 185-90.

In this paper we present a new approach for using semantic wikis for collaborative patent search and annotation. We describe an extension that allows integrating interactive visualizations into semantic wikis for getting deeper insights into the classificatory, geographical, and temporal distribution of large patent sets. This approach differs from typical wiki usage scenarios in the sense that it combines automatic content generation based on patent search activities of the users with user driven semantic annotation of patent information, e.g., patent rating, linking with prior art, reviews, translations, discussions, etc. The content generation involves a semantic model that is described in terms of different ontologies for patent information. A modern wiki system is used for semantic annotation, comments, discussions, versioning, notification, and full-text search. Our approach is motivated by using available functionalities of a modern 
wiki system in combination with visualization techniques to directly implement major user requirements for supporting the knowledge-intensive tasks of patent search and understanding.

Gigerenzer, G. 1991, "From Tools to Theories: A Heuristic of Discovery in Cognitive Psychology." Psychological Review 98(2):254-567.

The study of scientific discovery-where do new ideas come from?- has long been denigrated by philosophers as irrelevant to analyzing the growth of scientific knowledge. In particular, little is known about how cognitive theories are discovered, and neither the classical accounts of discovery as either probabilistic induction (e.g., Reichenbach, 1938) or lucky guesses (e.g., Popper, 1959), nor the stock anecdotes about sudden "eureka" moments deepen the insight into discovery. A heuristics approach is taken in this review, where heuristics are understood as strategies of discovery less general than a supposed unique logic of discovery but more general than lucky guesses. This article deals with how scientists' tools shape theories of mind, in particular with how methods of statistical inference have turned into metaphors of mind. The tools-to-theories heuristic explains the emergence of a broad range of cognitive theories, from the cognitive revolution of the 1960s up to the present, and it can be used to detect both limitations and new lines of development in current cognitive theories that investigate the mind as an "intuitive statistician."

Gilger, M. 2006, "Addressing Information Display Weaknesses for Situational Awareness." In IEEE Military Communications Conference, pp. 2785-91.

Situational awareness-monitoring, understanding, and responding to a situation-is critical for effective operations within industries such as air traffic control, aviation, disaster response, maintenance, medical, military, power systems, and transportation. The key to successful situational awareness is capturing data from a large number of distributed, heterogeneous information-sources and presenting that data in a manner that facilitates the operator's understanding of evolving events in complex and dynamic situations-including flight control, supply logistics, or battlefield management. Supporting situational awareness is a complex problem. How do we fuse a varied mixture of raw data from disparate sources into a higher order-into meaningful information that maximizes human understanding and comprehension without increasing operator stress? Current data display technologies fall short in the area of information visualization due to display-size constraints and differences in the types and sources of the data. Many display technologies approach the problem by "rolling-up" (aggregating) the data, and then allowing the operator to "drill-down"for details. The result is a time-consuming and cognitively expensive process. This paper introduces an advanced, visual display language that enables far richer data display (more data onscreen) while significantly reducing the operator's cognitive loading to process that data, thus allowing the operator to apply more cognitive abilities to decision-making and problemsolving.

Girot, C, and F Truniger. 2006, "The Walker's Perspective: Strategies for Conveying Landscape Perception Using Audiovisual Media." In 10th International Conference on Information Visualization, pp. 225-31.

This paper presents a method and three case studies for the integration of video into the practice of landscape architecture. As a design practice, landscape architecture is heavily dependent on visualisations. Traditionally maps, perspectives and photographic stills were used, but today these techniques are clearly no longer up to the task: they convey little information about the way we perceive landscape. Landscape perception has changed dramatically since the introduction of the moving image 
and highspeed travel. Contemporary visualisations of landscapes must therefore include these dynamic parameters in order to meet the profession's needs. We propose video as a new tool for visualizing the perception of and communicating about, landscape. In this paper we first introduce an analytical grid for the evaluation of video as a means of visualising landscape perception, from the vantage point of the slowest traveller, the pedestrian. Using three case studies, we then describe how video may be used to understand the specific situation of a walking perceiver. The findings are important both for practitioners and researchers in landscape architecture and for researchers of all disciplines interested in human perception

Glander, T, and J Döllner. 2009, "Abstract Representations for Interactive Visualization of Virtual 3d City Models." Computers, Environment and Urban Systems 33(5):375-87.

Virtual 3D city models increasingly cover whole city areas; hence, the perception of complex urban structures becomes increasingly difficult. Using abstract visualization, complexity of these models can be hidden where its visibility is unnecessary, while important features are maintained and highlighted for better comprehension and communication. We present a technique to automatically generalize a given virtual 3D city model consisting of building models, an infrastructure network and optional land coverage data; this technique creates several representations of increasing levels of abstraction. Using the infrastructure network, our technique groups building models and replaces them with cell blocks, while preserving local landmarks. By computing a landmark hierarchy, we reduce the set of initial landmarks in a spatially balanced manner for use in higher levels of abstraction. In four application examples, we demonstrate smooth visualization of transitions between precomputed representations; dynamic landmark highlighting according to virtual camera distance; an implementation of a cognitively enhanced route representation, and generalization lenses to combine precomputed representations in focus + context visualization.

Glanfield, J, et al. 2009, "Overflow : An Overview Visualization for Network Analysis." In 6th International Workshop on Visualization for Cyber Security, pp. 11-19.

Many network visualizations make the assumption that an administrator has previously determined the subset of data that should be visualized. Yet the problem remains that if the visualization provides no insight into the network events that warrant further consideration, then the administrator must go back to the data to determine what should be visualized next. This is a critical issue given the amount of network data under consideration, only a small portion of which can be examined at any one time. In this paper we present a visualization that provides context for network visualizations by providing a high-level view of network events. Our visualization not only provides a starting point for network visualization, but also reduces the cognitive burden of the analyst by providing a visual paradigm for both the filtering of network data and the selection of network data to drill into and visualize with alternative representations. We demonstrate, through the use of a case study, that our visualization can provide motivation for further investigation into anomalous network activity.

Gloor, PA, and Z Yan. 2006, "Analyzing Actors and Their Discussion Topics by Semantic Social Network Analysis." In IEEE Conference on Information Visualization, pp. 130-35.

iQuest is a novel software system to improve understanding of organizational phenomena with greater precision, clarity, and granularity than has previously been possible. It permits to gain new insights into organizational behavior, addressing issues such as tracking information while respecting privacy, 
comparing different interaction channels, network membership, and correlating organizational performance and creativity. It extends automatic visualization of social networks by mining communication archives such as e-mail and blogs through including analysis of the contents of those archives

Gnambs, T, M Appel, and B Batinic. 2010, "Color Red in Web-Based Knowledge Testing." Computers in Human Behavior 26(6):1625-31.

Computer- and web-based testing procedures are increasingly popular for the assessment of cognitive abilities and knowledge. This paper identified color red as a critical context factor that may influence the results. Two studies showed that color red may harm the performance in web-based tests of general knowledge. In Study $1(\mathrm{~N}=131)$ a red (vs. green) progress bar impeded the performance in a knowledge test, but only for the male participants. In Study $2(\mathrm{~N}=190)$ the color of the survey's forward-button was manipulated (red vs. blue vs. mixed color) which led to a replication of the gender-dependent color effect. Evolutionary psychology and stereotype threat research explain why red impedes the activation of knowledge among men, but not among women.

Godwin, A, R Chang, and R Kosara. 2008, "Visual Analysis of Entity Relationships in Global Terrorism Database." SPIE Defense and.

With the increase of terrorist activity around the world, it has become more important than ever to analyze and understand these activities over time. Although the data on terrorist activities are detailed and relevant, the complexity of the data has rendered the understanding and analysis difficult. We present a visual analytical approach to effectively identify related entities such as terrorist groups, events, locations, etc. based on a 2D layout. Our methods are based on sequence comparison from bioinformatics, modified to incorporate the element of time. By allowing the user the freedom to link entities by their activities over time, we provide a new framework for comparison of event sequences. Our scoring mechanism is robust and flexible, giving the user the flexibility to define the extent to which time is considered in aligning entities. Incorporated with high interactivity, the user can efficiently navigate through tens of thousands of records recorded in over a hundred dimensions of data by choosing combinations of categories to examine. Exploration of the terrorist activities in our system reveals relationships between entities that are not easily detectable using traditional methods.

Godwin, JA, and RM Kilgore. 2010, "Conveying Network Features in Geospatial Battlespace Displays." In IEEE Symposium on Visual Analytics Science \& Technology, pp. 221-22.

Goebel, PM, and M Vincze. 2007, "Vision for Cognitive Systems: A New Compound Concept Connecting Natural Scenes with Cognitive Models." In 5th International Conference on Industrial Informatics, pp. 705-10.

Vision, as a key perceptional capability for cognitive systems relates to rather difficult problems -such as visual object recognition, representation, categorization, and scene understanding. State-of-the-art solutions, using object appearance based models, already reached certain maturity. They achieve excellent recognition performance and provide learning structures that are subsequently utilized for object recognition and tracking. However, in context of object topology understanding for cognitive tasks, these methods cannot be directly compared with human performance, because it is obvious that appearance based methods do not contribute to understanding of structures in 3D. Research findings from infant 
psychology and animal investigation give evidence for using hierarchical models of object representation, based on image primitives e.g., such as edges, corners, shading or homogeneity of object colors. It is the objective of this paper to present an approach based on both, findings from biological studies and cognitive science, as enablers for autonomous cognitive investigation of natural scenes and their understanding. We present the architecture of a compound cognitive framework and its first behavioral level with the implementation of a vision model of the mammalian striate visual cortex in five layers. The proposed implementation is exemplified with an object similar to the Necker cube.

Goel, A, et al. 1999, Playing Detective: Using Ai for Sensemaking in Investigative Analysis. Technical Rpt. GIT-GVU-09-03, Georgia Tech.

---. 2009, Using Ai for Sensemaking in Investigative Analysis. Technical Rpt. GIT-GVU-09-09-03, Georgia Tech.

Goguen, J, and F Harrell. 2003, "Information Visualization and Semiotic Morphisms." In $2^{\text {nd }}$ International Conference on Visual Representations and Interpretations, pp. 9-12.

Golovchinsky, G, and MH Chignell. 1997, "The Newspaper as an Information Exploration Metaphor." Information Processing \& Management 33(5):663-83.

The newspaper represents a mature information presentation medium that is well-suited to the display of relatively short, loosely related pieces of text. This work examines the implementation of the newspaper metaphor in an information exploration interface. Based on an analysis of differences between electronic books and electronic newspapers, we submit that the newspaper metaphor is an appropriate interface paradigm for large-scale full-text databases. Similarities between newspapers and hypertext databases lead us to suggest that this metaphor is appropriate for large automatically generated hypertexts, independent of the nature of their content. We describe VOIR, a software prototype that we have used as an electronic newspaper workbench. The program constructs newspaper pages interactively, and allows users to specify their information-seeking intent in a variety of ways, including graphical Boolean queries, hypertext links, and typed-in queries. We report some empirical evidence that indicates that users perform better when they are shown more articles simultaneously. These results suggest that the newspaper metaphor may be an effective organizing principle for a class of information exploration tasks. Finally, we discuss some implications that this work has for hypertext and information retrieval in general.

Gomez, GM, et al. 2000, Io/Ia Visualization Technologies, State-of-the-Art Report, May 11, 2000 [Limited Distribution]. Technical, Information Assurance Technology Analysis Center.

Gonzalez, V, and A Kobsa. 2003, "Benefits of Information Visualization Systems for Administrative Data Analysts." In 7th International Conference on Information Visualization, pp. 331-36.

We report results from a study on the adoption of an information visualization system by administrative data analysts. Despite the fact that the system was neither fully integrated with their current software tools nor with their existing data analysis practices, analysts identified a number of key benefits that visualization systems provide to their work. These benefits for the most part occurred when analysts went beyond their habitual and well-mastered data analysis routines and engaged in creative discovery processes. We analyze the conditions under which these benefits arose, to inform the design of visualization systems that can better assist the work of administrative data analysts. 
Good, L, and BB Bederson. 2003, "Zoomable User Interfaces as a Medium for Slide Show Presentations." in The Craft of Information Visualization, Readings and Reflections, eds. BB Bederson and B Shneiderman, pp. 105-19. Morgan Kaufmann, San Francisco.

Summary In this paper, the authors propose Zoomable User Interfaces as an alternative presentation medium to address several common presentation problems. Zoomable User Interfaces offer new techniques for managing multiple versions of a presentation, providing interactive presentation navigation, and distinguishing levels of detail. These zoomable presentations may also offer several cognitive benefits over their commercial slide show counterparts. The authors also introduce CounterPoint, a tool to simplify the creation and delivery of zoomable presentations, discuss the techniques they have used to make authoring and navigation manageable in the multidimensional space. Lastly, some of the visualization principles compiled by the authors for designing these types of presentations are presented.

Goodall, JR. 2008, "Introduction to Visualization for Computer Security." In 4th International Workshop on Computer Security, pp. 1-17.

Networked computers are ubiquitous, and are subject to attack, misuse, and abuse. Automated systems to combat this threat are one potential solution, but most automated systems require vigilant human oversight. This automated approach under-values the strong analytic capabilities of humans. While automation affords opportunities for increased scalability, humans provide the ability to handle exceptions and novel patterns. One method to counteracting the ever increasing cyber threat is to provide the human security analysts with better tools to discover pattems, detect anomalies, identify correlations, and communicate their findings. This is what visualization for computer security (VizSec) researchers and developers are doing. VizSec is about putting robust information visualization tools into the hands of humans to take advantage of the power of the human perceptual and cognitive processes in solving computer security problems. This chapter is an introduction to the VizSec research community and the papers in this volume.

Goodell, H, et al. 2006, "Collecting and Harnessing Rich Session Histories." In 10th International Conference on Information Visualization, pp. 117-23.

To be most useful, evaluation requires detailed observation and effective analysis of a full spectrum of system use. We have developed an approach and architecture for in-depth data collection and analysis of all use of a visualization system. User interface components in a large visualization and analysis platform automatically record user actions, and can restore previous system states on demand. Audio and text annotations are collected and indexed to states, allowing users to find a comment and restore the system state in which they made it; then explore actions before and after. History is visible as data; so a variety of visual displays and analysis techniques may be used to develop insights about the user's experience. States of any part of the interface may be analyzed separately. Actions are categorized in a taxonomy as the user interface is built, allowing comparison of similar patterns in all tools. History data can co-exist with other data during data exploration, supporting further individual or group data exploration 
Gordon, SE, and RT Gill. 1997, "Cognitive Task Analysis." in Naturalistic Decision Making, eds. C Zsombok and G Klein. Erlbaum, Mahwah, NJ.

Gorg, C, et al. 2007, "Jigsaw Meets Blue Iguanodon - the Vast 2007 Contest." In IEEE Symposium on Visual Analytics Science \& Technology, pp. 235-36.

This article describes our use of the Jigsaw system in working on the VAST 2007 contest. Jigsaw provides multiple views of a document collection and the individual entities within those documents, with a particular focus on exposing connections between entities. We describe how we refined the identified entities in order to better facilitate Jigsaw's use and how the different views helped us to uncover key parts of the underlying plot.

Gorg, C, et al. 2007, "Visual Analytics with Jigsaw." In IEEE Symposium on Visual Analytics Science and Technology, pp. 201-02.

This article briefly introduces the Jigsaw system and describes how we used it in analysis activities for the VAST '07 Contest. Jigsaw is a visual analytic system that provides multiple coordinated views to show connections between entities that are extracted from a collection of documents.

Gotz, D, M Zhou, and V Aggarwal. 2006, "Interactive Visual Synthesis of Analytic Knowledge." In IEEE Symposium On Visual Analytics And Technology, pp. 51-58.

A visual investigation involves both the examination of existing in- formation and the synthesis of new analytic knowledge. This is a progressive process in which newly synthesized knowledge be- comes the foundation for future discovery. In this paper, we present a novel system supporting interactive, progressive synthesis of ana- lytic knowledge. Here we use the term "analytic knowledge" to re- fer to concepts that a user derives from existing data along with the evidence supporting such concepts. Unlike existing visual analytic tools, which typically support only exploration of existing informa- tion, our system offers two unique features. First, we support user- system cooperative visual synthesis of analytic knowledge from ex- isting data. Specifically, users can visually define new concepts by annotating existing information, and refine partially formed con- cepts by linking additional evidence or manipulating related con- cepts. In response to user actions, our system can automatically manage the evolving corpus of synthesized knowledge and its cor- responding evidence. Second, we support progressive visual analy- sis of synthesized knowledge. This feature allows analysts to visu- ally explore both existing knowledge and synthesized knowledge, dynamically incorporating earlier analytic conclusions into the en- suing discovery process. We have applied our system to two com- plex but very different analytic applications. Our preliminary eval- uation shows the promise of our work.

Gotz, D, and MX Zhou. 2008, "Characterizing Users Visual Analytic Activity for Insight Provenance." In IEEE Symposium on Visual Analytics Science \& Technology, pp. 123-30.

Insight provenance - a historical record of the process and rationale by which an insight is derived - is an essential requirement in many visual analytics applications. While work in this area has relied on either manually recorded provenance (e.g., user notes) or automatically recorded event-based insight provenance (e.g., clicks, drags, and key-presses), both approaches have fundamental limitations. Our aim is to develop a new approach that combines the benefits of both approaches while avoiding their deficiencies. Toward this goal, we characterize users visual analytic activity at multiple levels of 
granularity. Moreover, we identify a critical level of abstraction, Actions, that can be used to represent visual analytic activity with a set of general but semantically meaningful behavior types. In turn, the action types can be used as the semantic building blocks for insight provenance. We present a catalog of common actions identified through observations of several different visual analytic systems. In addition, we define a taxonomy to categorize actions into three major classes based on their semantic intent. The concept of actions has been integrated into our labs prototype visual analytic system, HARVEST, as the basis for its insight provenance capabilities.

Gotz, D, and MX Zhou. 2009, "Characterizing Users' Visual Analytic Activity for Insight Provenance." Information Visualization 8(1):42-55.

Insight provenance-a historical record of the process and rationale by which an insight is derived-is an essential requirement in many visual analytics applications. Although work in this area has relied on either manually recorded provenance (for example, user notes) or automatically recorded event-based insight provenance (for example, clicks, drags and key-presses), both approaches have fundamental limitations. Our aim is to develop a new approach that combines the benefits of both approaches while avoiding their deficiencies. Toward this goal, we characterize users' visual analytic activity at multiple levels of granularity. Moreover, we identify a critical level of abstraction, Actions, that can be used to represent visual analytic activity with a set of general but semantically meaningful behavior types. In turn, the action types can be used as the semantic building blocks for insight provenance. We present a catalog of common actions identified through observations of several different visual analytic systems. In addition, we define a taxonomy to categorize actions into three major classes based on their semantic intent. The concept of actions has been integrated into our lab's prototype visual analytic system, HARVEST, as the basis for its insight provenance capabilities. Information Visualization (2009) 8, 42-55. doi: $10.1057 /$ ivs.2008.31

Gotz, D, MX Zhou, and V Aggarwal. 2006, "Interactive Visual Synthesis of Analytic Knowledge." In IEEE Symposium on Visual Analytics Science \& Technology, pp. 51-58.

A visual investigation involves both the examination of existing information and the synthesis of new analytic knowledge. This is a progressive process in which newly synthesized knowledge becomes the foundation for future discovery. In this paper, we present a novel system supporting interactive, progressive synthesis of analytic knowledge. Here we use the term "analytic knowledge" to refer to concepts that a user derives from existing data along with the evidence supporting such concepts. Unlike existing visual analytic-tools, which typically support only exploration of existing information, our system offers two unique features. First, we support user-system cooperative visual synthesis of analytic knowledge from existing data. Specifically, users can visually define new concepts by annotating existing information, and refine partially formed concepts by linking additional evidence or manipulating related concepts. In response to user actions, our system can automatically manage the evolving corpus of synthesized knowledge and its corresponding evidence. Second, we support progressive visual analysis of synthesized knowledge. This feature allows analysts to visually explore both existing knowledge and synthesized knowledge, dynamically incorporating earlier analytic conclusions into the ensuing discovery process. We have applied our system to two complex but very different analytic applications. Our preliminary evaluation shows the promise of our work 
Govindaraju, RS, et al. 2009, "Vision of Cyberinfrastructure for End-to-End Environmental Explorations (C4e4)." Journal of Hydrologic 14(1):53-64.

Holistic approaches are needed for understanding and addressing a wide range of environmental issues that require multidisciplinary studies of complex and interlocking systems. The writers' vision of a cyberinfrastructure for end-to-end environmental exploration (C4E4) that combines data and modeling tools in an integrated environment across different spatial and temporal scales is presented. The overall goal behind C4E4 is to enable a broad environmental research and remediation community to address the challenges of environmental data management and integration in real-world settings. The St. Joseph Watershed in northern Indiana is chosen as a test bed in this effort. The C4E4 framework will allow researchers to combine heterogeneous data resources with state-of-the-art modeling and visualization tools through a user-friendly web portal. By engaging TeraGrid resources, C4E4 will have the computational resources to store, manipulate, and query large data sets, thereby facilitating new science. C4E4 will serve as a prototype, and provide valuable experience for scaling up to larger observatories at the national level. This paper presents the writers' vision and goals, initial efforts, and briefly describes how C4E4 can benefit the environmental community.

Graham, D, I Benest, and P Nicholl. 2007, "Cognitive Issues in Information Visualisation Design for Interaction for the Visually Impaired." In 11 th International Conference Information Visualization, pp. 917-20.

This paper reports on the findings of a study on improving interaction design for visually impaired students, focusing upon the cognitive criteria for information visualisation.

Graham, M, J Kennedy, and D Benyon. 2000, "Towards a Methodology for Developing Visualizations." International Journal of Human-Computer Studies 53(5):789-807.

Grammel, L. 2010, "Poster : Choosel - Web-Based Visualization Construction and Coordination for Information Visualization Novices." IEEE transactions on visualization and computer graphics:2-3.

Grammel, L, M Tory, and M-A Storey. 2010, "How Information Visualization Novices Construct Visualizations." IEEE Transactions on Visualization and Computer Graphics 16(6):943-52.

Granitzer, M, et al. 2005, "Evaluating a System for Interactive Exploration of Large, Hierarchically Structured Document Repositories." In IEEE Symposium on Information Visualization, pp. 127-34.

The InfoSky visual explorer is a system enabling users to interactively explore large, hierarchically structured document collections. Similar to a real-world telescope, InfoSky employs a planar graphical representation with variable magnification. Documents of similar content are placed close to each other and displayed as stars, while collections of documents at a particular level in the hierarchy are visualised as bounding polygons. Usability testing of an early prototype implementation of InfoSky revealed several design issues which prevented users from fully exploiting the power of the visual metaphor. Evaluation results have been incorporated into an advanced prototype, and another usability test has been conducted. A comparison of test results demonstrates enhanced system performance and points out promising directions for further work 
Green, TM. 2010, "Alida : Using Machine Learning for Intent Discernment in Visual Analytics Interfaces." In IEEE Symposium on Visual Analytics Science \& Technology, pp. 223-24.

Green, TM, and B Fisher. 2010, "Towards the Personal Equation of Interaction : The Impact of Personality Factors on Visual Analytics Interface Interaction." In IEEE Symposium on Visual Analytics Science \& Technology, pp. 203-10.

Green, TM, DH Jeong, and B Fisher. 2010, "Using Personality Factors to Predict Interface Learning Performance." In 43rd Annual Hawaii International Conference on System Sciences.

This current study explored the impact of individual differences in personality factors on interface interaction and learning performance in both an interactive visualization and a menu-driven web application. Participants were administered 6 psychometric measures designed to assess trait anxiety, locus of control, and other personality traits. Participants were then asked to complete 3 procedural tasks and 3 inferential tasks in each interface. Results demonstrated that participants with an external locus of control completed inferential tasks more quickly than those with an internal locus. Factor analysis of items from the 6 psychometric scales isolated a 9-item short measure, which showed trending with procedural scores. Additionally, data demonstrated that the visualization interface was more effective and efficient for the completion of the inferential tasks. Participants also preferred the visualization to the web interface for both types of task. Implications and future directions of this research are also discussed. (C) 2010 IEEE.

Green, TM, and W Ribarsky. 2008, Using a Human Cognition Model in the Creation of Collaborative Knowledge Visualizations. Technical Rpt. CVC-UNCC-09-17, UNCC.

This paper explores the basis and usefulness of a predictive model for the architecture of data and knowledge visualizations based on human higher-cognition, including human tendencies in reasoning heuristics and cognitive biases. The strengths and weakness of would-be human and computer collaborators are explored, and a model framework is outlined and discussed.

Green, TM, W Ribarsky, and B Fisher. 2009, "Building and Applying a Human Cognition Model for Visual Analytics." Information Visualization 8(1):1-13.

It is well known that visual analytics addresses the difficulty of evaluating and processing large quantities of information. Less often discussed are the increasingly complex analytic and reasoning processes that must be applied in order to accomplish that goal. Success of the visual analytics approach will require us to develop new visualization models that predict how computational processes might facilitate human insight and guide the flow of human reasoning. In this paper, we seek to advance visualization methods by proposing a framework for human higher cognition that extends more familiar perceptual models. Based on this approach, we suggest guidelines for the development of visual interfaces that better integrate complementary capabilities of humans and computers. Although many of these recommendations are novel, some can be found in existing visual analytics applications. In the latter case, much of the value of our contribution lies in the deeper rationale that the model provides for those principles. Lastly, we assess these visual analytics guidelines through the evaluation of several visualization examples. 2009 Palgrave Macmillan. 
Green, TM, W Ribarsky, and B Fisher. 2008, "Visual Analytics for Complex Concepts Using a Human Cognition Model." In IEEE Symposium on Visual Analytics Science \& Technology, pp. 91-98.

As the information being visualized and the process of understanding that information both become increasingly complex, it is necessary to develop new visualization approaches that facilitate the flow of human reasoning. In this paper, we endeavor to push visualization design a step beyond current user models by discussing a modeling framework of human ldquohigher cognition.rdquo Based on this cognition model, we present design guidelines for the development of visual interfaces designed to maximize the complementary cognitive strengths of both human and computer. Some of these principles are already being reflected in the better visual analytics designs, while others have not yet been applied or fully applied. But none of the guidelines have explained the deeper rationale that the model provides. Lastly, we discuss and assess these visual analytics guidelines through the evaluation of several visualization examples.

Green, TRG, et al. 2006, "Cognitive Dimensions: Achievements, New Directions, and Open Questions." Journal of Visual Languages \& Computing 17(4):328-65.

The cognitive dimensions framework has inspired research both more and less varied than expected. In this paper, we revisit the original aims and briefly describe some subsequent research, to consider whether the original aims were too austere in rejecting knowledge-based dimensions; whether the dimensions can be shown to have real-world relevance; and whether their definitions can be improved, either piecemeal or by refactoring the entire set. We mention some issues that remain unexplored, and conclude by describing two different ventures into defining clear procedures for real-life application, operating in very different milieux but both accepting that the framework should be developed from its original formulation.

Green, TRG, SP Davies, and DJ Gilmore. 1996, "Delivering Cognitive Psychology to Hci: The Problems of Common Language and of Knowledge Transfer." Interacting with Computers 8(1):89-111.

Although cognitive psychology showed much initial promise, it has failed to make significant contributions to the study of human-computer interaction, which has led to a rejection of cognitivism in favour of situated action theory. The authors accept that the critique has much to offer, but reject the outright abandoning of cognitivism. Cognitive psychology needs a common language in which to describe interaction between people and artifacts: two examples of research in progress are described, one focused on events, the other on representations and the relationship between the information display and the conceptual model. Cognitive psychology also needs a better delivery method than the traditional research paper, and the idea is proposed of a vocabulary of [']cognitive dimensions', terms which can be meaningfully used by non-specialists (who will recognise familiar but uncrystallised concepts) and which can be used as indexes to the professional literature. These two components form a proposal for improving the effectiveness of cognitive psychology. The paper ends with the hope that mainstream cognitive psychology will broaden its area of enquiry. 
Greene, R, et al. 2010, "An Approach to Gis-Based Multiple Criteria Decision Analysis That Integrates Exploration and Evaluation Phases: Case Study in a Forest-Dominated Landscape." Forest Ecology and Management 260(12):2102-14.

The increasing importance and complexity of land and natural resource management are creating a need for ecosystem-based management (EBM). Multiple criteria decision analysis (MCDA) combined with geographic information systems (GIS) can integrate factors related to the triple bottom line of ecological, economic, and social perspectives required by EBM. However, GIS-based MCDA is limited in this role because (i) it rarely integrates or encourages an exploration phase in preparation for structured evaluation and (ii) inexperienced users may find MCDA methods and GIS software difficult to use. This paper presents a novel approach for (i) supporting an exploration phase to help structure a problem and (ii) integrating the exploration and evaluation phases in an easy-to-use software system. The approach was validated through a land-management case study in a forest-dominated landscape with a variety of stakeholders. Case-study participants used the approach to rate areas within a timber harvest plan based on their potential for conflict with conservation values. The case-study decision analysis determined that between $1.3 \%$ and $6.6 \%$ of the harvest plan area had a conservation rating of 0.30 or higher on a scale of $0-1$. The system was made available to the forest industry and other stakeholders to support harvest plan adjustments, demonstrating how such tools can be used to improve and integrate our knowledge of forest ecology and management. Assessment of participant feedback reveals that an exploration phase is effective in helping understand a problem and prepare for multiple criteria evaluation (MCE).

Greenspan, B. 2005, "Chapter 13 Mapping Play: What Cybercartographers Can Learn from Popular Culture." in Modern Cartography Series, Cybercartography - Theory and Practive, ed. DRF Taylor, Vol 4, pp. 309-29. Academic Press.

Although cybercartography is a new field of study, its arrival has long been rehearsed in popular culture and entertainment, including novels, films, Web sites and digital games. The widespread representation of cartographic, navigational and location technologies in popular texts have created cultural expectations that far exceed the current technical limits of mapping tools. At the same time, these popular narratives enable a growing familiarity with the concept of cybercartography that cannot but shape the way users approach the cybermaps of the future. This chapter will explore cybermaps as a popular concept, examining some of the cybercartographic interfaces (both real and fictional) that have been imagined and implemented in printed fiction, hyperfiction, Web sites, films and digital games. Through various forms of cultural analysis, I will show how the stories we tell about cybermaps today can inform future cybercartographic theory and practice. Note: View the SIM City Screen Capture Images on the Accompanying CD-Rom. 
Greitzer, FL. 2008, "Methodology, Methods and Metrics for Testing and Evaluating Augmented Cognition Systems." in Augmented Cognition: A Practitioner's Guide, eds. D Schmorrow and K Stanney, pp. 144-74. Human Factors Society, Santa Monica, CA.

Greitzer, FL. 2005, Methodology, Metrics and Measures for Testing and Evaluation of Intelligence Analysis. Technical Rpt. PNWD-3550, Battelle, Pacific Northwest Division, Richland, WA.

Greitzer, FL. 2005, "Toward the Development of a Cognitive Task Difficulty Metrics to Support Intelligence Anslysis Research." In IEEE International Conference on Cognitive Informatics.

Intelligence analysis is a cognitively complex task that is the subject of considerable research aimed at developing methods and tools to aid the analysis process. To support such research, it is necessary to characterize the difficulty or complexity of intelligence analysis tasks in order to facilitate assessments of the impact or effectiveness of tools that are being considered for deployment. A number of informal accounts of "What makes intelligence analysis hard" are available, but there has been no attempt to establish a more rigorous characterization with well-defined difficulty factors or dimensions. This paper takes an initial step in this direction by describing a set of proposed difficulty metrics based on cognitive principles.

Greitzer, FL, et al. 2005, "Metrics and Measures for Intelligence Analysis Task Difficulty." In First Annual Conference on Intelligence Analysis Methods and Tools.

Greitzer, FL, et al. 2008, "A Sensemaking Perspective on Situation Awareness in Power Grid Operations." In Power and Energy Society General Meeting - Conversion and Delivery of Electrical Energy in the 21st Century, 2008 IEEE, pp. 1-6.

With increasing complexity and interconnectivity of the electric power grid, the scope and complexity of grid operations continues to grow. New paradigms are needed to guide research to improve operations by enhancing situation awareness of operators. Research on human factors/situation awareness is described within a taxonomy of tools and approaches that address different levels of cognitive processing. While user interface features and visualization approaches represent the predominant focus of human factors studies of situation awareness, this paper argues that a complementary level, sensemaking, deserves further consideration by designers of decision support systems for power grid operations. A sensemaking perspective on situation awareness may reveal new insights that complement ongoing human factors research, where the focus of the investigation of errors is to understand why the decision makers experienced the situation the way they did, or why what they saw made sense to them at the time.

Griffin, AL. 2009, "Information Graphics." in International Encyclopedia of Human Geography, eds. K Rob and T Nigel, pp. 459-68. Elsevier, Oxford.

Information graphics include a wide variety of static and dynamic visual representations of information. These displays take different forms depending on the purpose for which the graphic is being constructed (e.g., for thinking or communication), characteristics of the data, potential visual display forms, and whether or not the information graphic is interactive. Choosing an appropriate method for representing data requires a basic understanding of the perceptual and cognitive processing that occurs in the human visual system. 
Grimstead, IJ, DW Walker, and NJ Avis. 2005, "Collaborative Visualization: A Review and Taxonomy." In 9th International Symposium on Distributed Simulation and Real-time Applications, pp. 61-69.

We present a brief review of 42 collaborative visualization systems, grouped into four application areas: collaborative problem-solving environments, virtual reality environments, multi-player online games and multi-user enabling of single user applications. The systems are then compared by five attributes: number of simultaneous users, user access control, communication architecture, type of transmitted data and user synchronization. We review the characteristic properties of each application area, overall trends in characteristics and recommend improvements for future systems. The taxonomy of visualization and accompanying bibliography are available on-line via the RAVE project pages by Grimstead, I.J., (2005), with on-line references hyper-linked where available.

Grinstein, G, et al. 2003, "Which Comes First, Usability or Utility?" In IEEE Conference on Informaiton Visualization, pp. 605-06.

Grinstein, G, et al. 2010, "Vast 2010 Challenge : Arms Dealings and Pandemics." In IEEE Conference on Information Visualization, pp. 263-64.

Grinstein, G, et al. 2007, "Vast 2007 Contest - Blue Iguanodon." In IEEE Conference on Information Visualization, pp. 231-32.

Visual analytics experts realize that one effective way to push the field forward and to develop metrics for measuring the performance of various visual analytics components is to hold an annual competition. The second visual analytics science and technology (VAST) contest was held in conjunction with the 2007 IEEE VAST symposium. In this contest participants were to use visual analytic tools to explore a large heterogeneous data collection to construct a scenario and find evidence buried in the data of illegal and terrorist activities that were occurring. A synthetic data set was made available as well as tasks. In this paper we describe some of the advances we have made from the first competition held in 2006.

---. 2008, "Vast 2008 Challenge : Introducing Mini-Challenges." In IEEE Conference on Information Visualization, pp. 195-96.

Visual analytics experts realize that one effective way to push the field forward and to develop metrics for measuring the performance of various visual analytics components is to hold an annual competition. The VAST 2008 Challenge is the third year that such a competition was held in conjunction with the IEEE Visual Analytics Science and Technology (VAST) symposium. The authors restructured the contest format used in 2006 and 2007 to reduce the barriers to participation and offered four minichallenges and a Grand Challenge. Mini Challenge participants were to use visual analytic tools to explore one of four heterogeneous data collections to analyze specific activities of a fictitious, controversial movement. Questions asked in the Grand Challenge required the participants to synthesize data from all four data sets. In this paper we give a brief overview of the data sets, the tasks, the participation, the judging, and the results. 
Grinstein, G, et al. 2006, "Vast 2006 Contest a Tale of Alderwood." In IEEE Conference on Information Visualization, pp. 215-16.

Visual analytics experts realize that one effective way to push the field forward and to develop metrics for measuring the performance of various visual analytics components is to hold an annual competition. The first visual analytics science and technology (VAST) contest was held in conjunction with the 2006 IEEE VAST Symposium. The competition entailed the identification of possible political shenanigans in the fictitious town of Alderwood. A synthetic data set was made available as well as tasks. We summarize how we prepared and advertised the contest, developed some initial metrics for evaluation, and selected the winners. The winners were invited to participate at an additional live competition at the symposium to provide them with feedback from senior analysts

Groller, E. 2002, "Insight into Data through Visualization." In 9th International Symposium on Graph Drawing (GD 2001), pp. 352-66.

Computer graphics, scientific visualization, information visualization, and graph drawing are areas which deal with visual information layout. They all use the remarkable properties of the human visual perception to rapidly absorb and analyse visual information. The paper discusses important visualization aspects and gives examples of how visualization techniques facilitate insight into data characteristics. The connection between visualization and graph drawing is shortly discussed.

Gross, M, and C Voegeli. 2007, "A Multimedia Framework for Effective Language Training." Computers \& Graphics 31(5):761-77.

We present a novel framework for the multimodal display of words using topological, appearance, and auditory representations. The methods are designed for effective language training and serve as a learning aid for individuals with dyslexia. Our topological code decomposes the word into its syllables and displays it graphically as a tree structure. The appearance code assigns color attributes and shape primitives to each letter and takes into account conditional symbol probabilities, code ambiguities, and phonologically confusable letter combinations. An additional auditory code assigns midi events to each symbol and thus generates a melody for each input string. The entire framework is based on information theory and utilizes a Markovian language model derived from linguistic analysis of language corpora for English, French, and German. For effective word repetition a selection controller adapts to the user's state and optimizes the learning process by minimizing error entropy. The performance of the method was evaluated in a large scale experimental study involving 80 dyslexic and non-dyslexic children. The results show significant improvements in writing skills in both groups after small amounts of daily training. Our approach combines findings from 3D computer graphics, visualization, linguistics, perception, psychology, and information theory.

Groth, DP. 2004, "Information Provenance and the Knowledge Rediscovery Problem." In $8^{\text {th }}$ International Conference on Information Visualisation, pp. 345-51.

Visualizations leverage innate human capabilities for rec- ognizing interesting aspects of data. Even if users might agree on what is interesting about a visualization, the steps that they use in the knowledge discovery process may be significantly different. This results in an inabil- ity to effectively recreate the exact conditions of the dis- covery process, which we call the knowledge rediscovery problem. Because we cannot expect a user to fully doc- ument each of their interactions, there is a need for vi- sualization 
systems to maintain user trace data in a way that enhances a user's ability to communicate what they found to be interesting, as well as how they found it. We present a model for representing user interactions that ar- ticulates with a corresponding set of annotations, or ob- servations that are made during the exploration. Such an ability is critical to addressing the knowledge rediscovery problem, and is a fundamental component for systems that must provide information provenance

Groth, DP. 2006, "Visualizing Distributions and Classification Accuracy." In 10th International Conference on Information Visualization, pp. 389-94.

Data mining is the search for novel, actionable information within data. It is important to note that the number of records in the data being analyzed is only one (and perhaps a small) factor in determining the complexity of a given data mining technique. Most complexity in data mining arises from the distribution of values contained in the data - not the number of records. In this paper, we utilize straightforward histogram-based visualizations to gain insight into how the performance of a well-studied data mining technique, the naive-Bayes classifier, performs under various discretization schemes for both continuous and discrete values. The resulting visualization system provides users with a tool that describes the underlying model of the data used by the classifier. Exploratory visualizations of the distributions of training data can be selected based on expert domain knowledge and then combined to apply to the test data

Groth, DP, and K Streefkerk. 2006, "Provenance and Annotation for Visual Exploration Systems." IEEE Transactions on Visualization and Computer Graphics 12(6):1500-10.

Exploring data using visualization systems has been shown to be an extremely powerful technique. However, one of the challenges with such systems is an inability to completely support the knowledge discovery process. More than simply looking at data, users will make a semipermanent record of their visualizations by printing out a hard copy. Subsequently, users will mark and annotate these static representations, either for dissemination purposes or to augment their personal memory of what was witnessed. In this paper, we present a model for recording the history of user explorations in visualization environments, augmented with the capability for users to annotate their explorations. A prototype system is used to demonstrate how this provenance information can be recalled and shared. The prototype system generates interactive visualizations of the provenance data using a spatio-temporal technique. Beyond the technical details of our model and prototype, results from a controlled experiment that explores how different history mechanisms impact problem solving in visualization environments are presented.

Groth, P. 2009, "A Model of Process Documentation to Determine Provenance in Mash-Ups University of Southern California." ACM Transactions on Internet Technology 9(1):1-31.

Through technologies such as RSS (Really Simple Syndication), Web Services, and AJAX (Asynchronous JavaScript and XML), the Internet has facilitated the emergence of applications that are composed from a variety of services and data sources. Through tools such as Yahoo Pipes, these "mashups" can be composed in a dynamic, just-in-time manner from components provided by mul- tiple institutions (i.e., Google, Amazon, your neighbor). However, when using these applications, it is not apparent where data comes from or how it is processed. Thus, to inspire trust and confi- dence in mashups, it is critical to be able to analyze their processes after the fact. These trailing analyses, in particular the determination of the provenance of a result (i.e., the process that led to it), are enabled by process documentation, which is documentation of an application's past process created by the components of 
that application at execution time. In this article, we define a generic conceptual data model that supports the autonomous creation of attributable, factual process doc- umentation for dynamic multi-institutional applications. The data model is instantiated using two Internet formats, OWLand XML, and is evaluated with respect to questions about the provenance of results generated by a complex bioinformatics mash-up.

Gruber, HE. 1996, "Chapter 12 - Insight and Affect in the History of Science." in The Nature of Insight, eds. RJ Sternberg and JE Davidson, pp. 397-431. MIT Press.

Gschwandtner, T, et al. 2010, "Easing Semantically Enriched Information Retrieval--an Interactive SemiAutomatic Annotation System for Medical Documents." International Journal of Human-Computer Studies 68(6):370-85.

Mapping medical concepts from a terminology system to the concepts in the narrative text of a medical document is necessary to provide semantically accurate information for further processing steps. The MetaMap Transfer (MMTx) program is a semantic annotation system that generates a rough mapping of concepts from the Unified Medical Language System (UMLS) Metathesaurus to free medical text, but this mapping still contains erroneous and ambiguous bits of information. Since manually correcting the mapping is an extremely cumbersome and time-consuming task, we have developed the MapFace editor. The editor provides a convenient way of navigating the annotated information gained from the MMTx output, and enables users to correct this information on both a conceptual and a syntactical level, and thus it greatly facilitates the handling of the MMTx program. Additionally, the editor provides enhanced visualization features to support the correct interpretation of medical concepts within the text. We paid special attention to ensure that the MapFace editor is an intuitive and convenient tool to work with. Therefore, we recently conducted a usability study in order to create a well founded background serving as a starting point for further improvement of the editor's usability.

Gu, YX, et al. 2010, "Facilitating Learning Interests through Mobile Information Visualization." In $10^{\text {th }}$ IEEE International Conference on Advanced Learning Technologies, pp. 323-27.

In this paper, a mobile approach for ambient learning is presented. As an example of the concept, modeling is done to deliver educational content on weather phenomenon to schoolchildren. Information visualization techniques are utilized to present an alternative approach of knowledge delivery that could be considered to be beyond conventional classroom based teachings. The concept is demonstrated with the 'Interactive Weather Information System' (IWIS) platform. During the user tests, the effectiveness of the proposed method on knowledge conveyance was evaluated. Usability tests showed that IWIS serves as a good stimulus to captivate the learning interests of the target user group (children from 10 to 12 years old). In addition, the new issues that surfaced could be used as design considerations or factors in subsequent research work in this area.

Gualtieri, JW, and WC Elm. 2002, "Power Tool for Countering Cyberwar: Visualizations for Information Assurance and Computer Network Defense." In Human Factors and Ergonomics Society Annual Meeting, pp. 463-67.

There has been a growing need for military decision-makers to maintain the integrity of the information contained within their computer network. Tools to support Information Assurance and Computer Network Defense (IA-CND) are needed to defend their information infrastructure and conduct computer network operations with a new level of insight and understanding. This paper describes 
one effort to develop visualizations to aid these decision-makers in the highly abstract, complex and dynamic mission of IA-CND. This paper describes the development of a IA-CND communications display. Using a cognitive systems engineering methodology, this project transitioned from a broad description of a work domain, to the development of decision aiding concepts for a particular portion of that domain. This methodology also provided a means to develop breakthrough support for a decision difficult domain

Gualtieri, JW, et al. 2001, "Analysis with a Purpose: Narrowing the Gap with a Pragmatic Approach." In Human Factors and Ergonomics Society Annual Meeting, pp. 444-48.

Gualtieri, JW, et al. 2005, Cognitive Analysis of Air Operations Center Strategy Division's Work Domain. Technical Rpt. AFRL-HE-WP-TR-2006-0016.

Gualtieri, JW, et al. 2003, Aie Cseds: Initial Cognitive Systems Engineering Design Specification (Cseds) for the Acwa(Trademark) Integrated Environment (Aie). Technical Rpt. AFRL-HE-WP-TR-2004-0072.

---. 2003, Aie: Acwa (Trademark) Integrated Environment Technical Survey. Technical Rpt. AFRL-HEWP-TR-2005-0177.

Gualtieri, JW, S Szymczak, and WC Elm. 2005, "Cognitive System Engineering - Based Design: Alchemy or Engineering." In Human Factors and Ergonomics Society Annual Meeting, pp. 254-58.

Guerrero-Bote, VP, et al. 2006, "Binary Pathfinder: An Improvement to the Pathfinder Algorithm." Information Processing \& Management 42(6):1484-90.

The Pathfinder algorithm is widely used to prune social networks. The pruning maintains the geodesic distances between nodes. It has shown itself to be very useful in the analysis of, amongst others, citations in BIS (bibliometrics, informetrics, and scientometrics). It has even been proposed for the online display of the search results in an information retrieval system. However, its great time and space complexity limits its use in real-time applications and in networks of any considerable size. The present work describes an improved algorithm with considerably reduced time and space complexity. Its lower execution costs thus increase its applicability both in real time and to large networks.

Guetat, A, et al. 2010, "Pre-Integrated Volume Rendering with Non-Linear Gradient Interpolation." IEEE Transactions on Visualization and Computer Graphics 16(6):1487-94.

Shading is an important feature for the comprehension of volume datasets, but is difficult to implement accurately. Current techniques based on pre-integrated direct volume rendering approximate the volume rendering integral by ignoring non-linear gradient variations between front and back samples, which might result in cumulated shading errors when gradient variations are important and / or when the illumination function features high frequencies. In this paper, we explore a simple approach for preintegrated volume rendering with non-linear gradient interpolation between front and back samples. We consider that the gradient smoothly varies along a quadratic curve instead of a segment in-between consecutive samples. This not only allows us to compute more accurate shaded pre-integrated look-up tables, but also allows us to more efficiently process shading amplifying effects, based on gradient filtering. An interesting property is that the pre-integration tables we use remain two-dimensional as for 
usual pre-integrated classification. We conduct experiments using a full hardware approach with the Blinn-Phong illumination model as well as with a non-photorealistic illumination model.

Guimarães, R, A Meiguins, and B Meiguins. 2010, "A Pragmatic Approach to Solve the Vast 2010 Mini Challenge 3." In IEEE Symposium on Visual Analytics Science \& Technology, pp. 2-3.

Guo, D, and M Gahengan. 2004, "Spatial Ordering and Encoding for Geographic Data Mining and Visualization." Journal of Intelligent Information Systems 27(3):243-66.

Geographic information (e.g., locations, networks, and nearest neighbors) are unique and different from other aspatial attributes (e.g., population, sales, or income). It is a challenging problem in spatial data mining and visualization to take into account both the geographic information and multiple aspatial variables in the detection of patterns. To tackle this problem, we present and evaluate a variety of spatial ordering methods that can transform spatial relations into a one-dimensional ordering and encoding which preserves spatial locality as much possible. The ordering can then be used to spatially sort temporal or multivariate data series and thus help reveal patterns across different spaces. The encoding, as a materialization of spatial clusters and neighboring relations, is also amenable for processing together with aspatial variables by any existing (non-spatial) data mining methods. We design a set of measures to evaluate nine different ordering/encoding methods, including two space-filling curves, six hierarchical clustering based methods, and a one-dimensional Sammon mapping (a multidimensional scaling approach). Evaluation results with various data distributions show that the optimal ordering/encoding with the complete-linkage clustering consistently gives the best overall performance, surpassing wellknown space-filling curves in preserving spatial locality. Moreover, clustering-based methods can encode not only simple geographic locations, e.g., $\mathrm{x}$ and $\mathrm{y}$ coordinates, but also a wide range of other spatial relations, e.g., network distances or arbitrarily weighted graphs.

Guo, H, et al. 2010, "Multi-Dimensional Transfer Function Design Based on Combined Interface of Parallel Coordinates and Dimension Projection." In IEEE Conference on Information Visualization.

Guoray, C. 2007, "Formalizing Analytical Discourse in Visual Analytics." In IEEE Syposium on Visual Analytics Science \& Technology, pp. 217-18.

This paper presents a theory of analytical discourse and a formal model of the intentional structure of visual analytic reasoning process. Our model rests on the theory of collaborative discourse, and allows for cooperative human-machine communication in visual interactive dialogues. Using a sample discourse from a crisis management scenario, we demonstrated the utility of our theory in characterizing the discourse context and collaboration. In particular, we view analytical discourse as plans consisting of complex mental attitude towards analytical tasks and issues. Under this view, human reasoning and computational analysis become integral part of the collaborative plan that evolves through discourse.

Gürkan, A, et al. 2010, "Mediating Debate through on-Line Large-Scale Argumentation: Evidence from the Field." Information Sciences 180(19):3686-702.

Web 2.0 technologies, such as forums and wikis, are enabling an explosion of global knowledge sharing through distributed large-scale conversations, but they seem to be less successful at supporting collaborative deliberation around complex and controversial questions. In order to cope with this limitation, many scholars have proposed to adopt on-line argumentation platforms to improve information 
visualization, organization and reuse. However, such research has mostly focused on the design of adequate argument-based knowledge formalisms. Less attention has been paid to the empirical analysis of actual interactions mediated by argumentation technology with reasonably large user communities. In this paper, we present an in-depth analysis of the data obtained in the empirical test of an argumentation platform where a 160-member community created, in 3 weeks, what is to our knowledge the largest single online argument map ever built (around 5000 posts). Our results show that (i) users were able to quickly and comprehensively explore and map the debate on the selected discussion topic; (ii) substantial moderation was needed to ensure that the argument map was well-organized and users were confident with the argumentation formalism; (iii) considerable out-of-the map communication occurred, possibly as a way to allow for conversational flows inhibited by the argumentation formalism, (iv) formal rating of contributions favored exploration of the map, understanding the debate structure, and improving the quality of content.

Gurses, AP, Y Xiao, and P Hu. 2009, "User-Designed Information Tools to Support Communication and Care Coordination in a Trauma Hospital." Journal of Biomedical Informatics 42(4):667-77.

Background In response to inherent inadequacies in health information technologies, clinicians create their own tools for managing their information needs. Little is known about these clinician-designed information tools. With greater appreciation for why clinicians resort to these tools, health information technology designers can develop systems that better meet clinicians' needs and that can also support clinicians in design and use of their own information tools. Objective To describe the design characteristics and use of a clinician-designed information tool in supporting information transfer and care coordinationDesign Observations, semi-structured interviews, and photographing were used to collect data. Participants were six nurse coordinators in a high-volume trauma hospital. Content analysis was carried out and interactions with information tools were analyzed.Results Nurse coordinators used a paper-based information tool (a nurse coordinator's clipboard) that consisted of the compilation of essential data from disparate information sources. The tool was assembled twice daily through (1) selecting and formatting key data from multiple information systems (such as the unit census and the EHR), (2) data reduction (e.g., by cutting and whitening out non-essential items from the print-outs of computerized information systems), (3) bundling (e.g., organizing pieces of information and taping them to each other), and (4) annotating (e.g., through the use of colored highlighters and shorthand symbols). It took nurse coordinators an average of $41 \mathrm{~min}$ to assemble the clipboard. The design goals articulated by nurse coordinators to fit the tool into their tasks included (1) making information compatible with the mobile nature of their work, (2) enabling rapid information access and note-taking under time pressure, and (3) supporting rapid information processing and attention management through the effective use of layout design, shorthand symbols, and color-coding.Conclusions Clinicians design their own information tools based on the existing health information technologies to meet their information needs. The characteristics of these clinician-designed tools provide insights into the "realities" of how clinicians work with health information technologies. The findings suggest an often overlooked role for health information technologies: facilitating user creation of information tools that will best meet their needs. 
Gutman, M, et al. 2010, "Enhancing Text-Based Chat with Visuals for Hazardous Weather Decision Making." In IEEE Symposium on Visual Analytics Science \& Technology, pp. 225-26.

Gwizdka, J, and M Chignell. 2004, "Individual Differences and Task-Based User Interface Evaluation: A Case Study of Pending Tasks in Email." Interacting with Computers 16(4):769-97.

This paper addresses issues raised by the ever-expanding role of email as a multi-faceted application that combines communication, collaboration, and task management. Individual differences analysis was used to contrast two email user interfaces in terms of their demands on users. The results of this analysis were then interpreted in terms of their implications for designing more inclusive interfaces that meet the needs of users with widely ranging abilities. The specific target of this research is the development of a new type of email message representation that makes pending tasks more visible. We describe a study that compared a new way of representing tasks in an email inbox, with a more standard representation (the Microsoft Outlook inbox). The study consisted of an experiment that examined how people with different levels of three specific cognitive capabilities (flexibility of closure, visual memory, and working memory) perform when using these representations. We then identified combinations of representation and task that are disadvantageous for people with low levels of the measured capabilities.

Haber, J, and S Carpendale. 2010, "Poster : Qr Vis : Turning Printed Infographics into Interactive Visualizations." In IEEE Conference on Information Visualization, pp. 2-3.

Hall, CM, SAH McMullen, and DL Hall. 2006, Cognitive Engineering Research Methodology : A Proposed Study of Visualization Analysis Techniques. Technical Rpt. RTO-MP-IST-063, Tech Reach, Inc., State College, PA.

The rapid development of new sensors and wide-band communications provides the capability to collect enormous amounts of data. An increasing challenge involves how to understand and interpret the data to yield knowledge about evolving situations or threats (e.g., of military situations, state of complex systems, etc.). New visualization tools and techniques are becoming available to support advanced visualization including three-dimensional, full immersion display environments and tools to support novel visualizations. Examples include network system display tools and evolving multi-sensory situation environments. Despite the emergence of such tools, there has been limited systematic test and evaluations to determine the efficacy of such tools for knowledge understanding and decision making. This paper provides an overview of this problem and argues for the need to conduct controlled experiments. A sample experiment is suggested.

Hall, LE, and X Bescos. 1995, "Menu -- What Menu?" Interacting with Computers 7(4):383-94.

In recent years information system interface design has become increasingly dominated by the use of menus, with the majority of systems relying on static menus as their main dialogue structure. Alternatives to this interface style are explored, and through the discussion of an application developed in banking a number of alternative styles which can be used are detailed. The usability and utility of this application is evaluated with positive results. It is suggested that while menus are a viable and useful interface design design technique, the use of other techniques should also be encouraged, and that small-scale innovations in interface design will improve the variety, usability and acceptability of information systems. 
Ham, D-H, WC Yoon, and B-T Han. 2008, "Experimental Study on the Effects of Visualized Functionally Abstracted Information on Process Control Tasks." Reliability Engineering \& System Safety 93(2):254-70.

Two distinct design problems of information display for process control are information content representation and visual form design. Regarding information content, we experimentally showed the effectiveness of functionally abstracted information without the benefits of sophisticated graphical presentation in various task situations. However, since it is obvious that the effects of the information display are also influenced by display formats (i.e., visual forms) as well as the information content, further research was required to investigate the effectiveness of visualized functionally abstracted information. For this purpose, this study conducted an experiment in complex process control tasks (operation and fault diagnosis). The experimental purposes were to confirm the effectiveness of the functionally abstracted information visualized with emergent features or peculiar geometric forms and to examine the additional effects of the visualization on task performance. The results showed that functionally abstracted information presented with sophisticated visual forms helped operators perform process control tasks in more efficient and safe way. The results also indicated the importance of explicit visualization of goal-means relation between higher and lower abstraction levels. Lastly, this study proposed a framework for designing visual forms for process control display.

Hamdi, MS. 2011, "Somse: A Semantic Map Based Meta-Search Engine for the Purpose of Web Information Customization." Applied Soft Computing 11(1):1310-21.

To combat information overload, systems that are often referred to as information customization systems are needed. Such systems act on the user's behalf and can rely on existing information services like search engines that do the resource-intensive part of the work. These systems will be sufficiently lightweight to run on an average PC and serve as personal assistants. Since such an assistant has relatively modest resource requirements it can reside on an individual user's machine. If the assistant resides on the user's machine, there is no need to turn down intelligence. The system can have substantial local intelligence. In this paper, we propose an information customization system that combines meta-search and unsupervised learning. A meta-search engine simultaneously searches multiple search engines and returns a single list of results. The results retrieved by this engine can be highly relevant, since it is usually grabbing the first items from the relevancy-ranked list of hits returned by the individual search engines. The Kohonen Feature Map is then used to construct a self-organizing semantic map such that documents of similar contents are placed close to one another.

Haniff, DJ, and C Baber. 2003, "User Evaluation of Augmented Reality Systems." In 7th International Conference on Information Visualization, pp. 505-11.

Augmented reality (AR) systems need to be evaluated for their appropriateness for a given task. Three approaches are used to evaluate the waterpump augmented reality tool (WART). The system is assessed using verbal protocol, performance time and a questionnaire. The WART system is compared with a paper version of the assembly instructions. The verbal protocol revealed that there was more cognitive processing for the paper version of task than WART, it took longer to complete the task with WART than the paper version and the questionnaire revealed the effect on performance of problems associated with AR. All of the participants in the evaluation, however, were positive about WART, and appreciated its usefulness. 
Hannafin, M. 2006, "Disciplined Inquiry and Research in Computer-Supported Learning." Computers in Human Behavior 22(1):149-53.

Hanrahan, P, D Keim, and S Card. 2010, "The State of Visual Analytics : Views on What Visual Analytics Is and Where It Is Going." In IEEE Conference on Information Visualization, pp. 257-59.

Hansen, DL, B Shneiderman, and MA Smith. 2011, "Getting Started with Nodexl, Layout, Visual Design, and Labeling." in Analyzing Social Media Networks with Nodexl, pp. 53-67. Morgan Kaufmann, Boston.

Hao, MC, et al. 2010, "Visual Analysis of Frequent Patterns in Large Time Series." In IEEE Symposium on Visual Analytics Science \& Technology, pp. 227-28.

Hao, MC, et al. 2005, "Importance-Driven Visualization Layouts for Large Time Series Data." In IEEE Conference on Information Visualization, pp. 203-10.

Time series are an important type of data with applications in virtually every aspect of the real world. Often a large number of time series have to be monitored and analyzed in parallel. Sets of time series may show intrinsic hierarchical relationships and varying degrees of importance among the individual time series. Effective techniques for visually analyzing large sets of time series should encode the relative importance and hierarchical ordering of the time series data by size and position, and should also provide a high degree of regularity in order to support comparability by the analyst. In this paper, we present a framework for visualizing large sets of time series. Based on the notion of inter time series importance relationships, we define a set of objective functions that space-filling layout schemes for time series data should obey. We develop an efficient algorithm addressing the identified problems by generating layouts that reflect hierarchy and importance based relationships in a regular layout with favorable aspect ratios. We apply our technique to a number of real world data sets including sales and stock data, and we compare our technique with an aspect ratio aware variant of the well known TreeMap algorithm. The examples show the advantages and practical usefulness of our layout algorithm.

Haque, A. 2010, "Tensor Visualization in Computational Turbulent Combustion : A Case Study." In IEEE Conference on Information Visualization.

Hardisty, F, and G Center. 2008, "Geojabber: Finding Significant Analytic Events in Collaborative Visual Analysis Sessions." In GIScience 2008, pp. 1-4.

The integration of a peer-to-peer instant messaging system into visual analytic software allows automatic extraction of significant analytic events, such as inference drawing, causality determination, or hypothesis generation, during the course of an analysis. It does so by examining the textual communications between collaborators and marking those analytic events which are determined to be significant using term extraction and term matching. These events can be used as entry points into the analysis session, as a way to better understand both the subject of analysis (such as a possible Sarin gas attack), the collaborative behavior of the analysts, and patterns of tool use. This approach can potentially make visual analytics more productive through support for sharing fragments of reasoning among analysts. The GeoViz Toolkit introduced here is an open source software project that enables multivariate visual analysis of geospatial data. The open XMPP communication protocol (also known as Jabber) was used in the GeoViz Toolkit software to create a working prototype of a geocollaboration system, by creating extensions to Jabber to support tool state sharing, including geospatial aspects of tool state. 
Advantages and disadvantages of using XMPP vs. other implementation methods are detailed through a set of examples and discussion of those examples.

Hartson, RH. 1998, "Human-Computer Interaction: Interdisciplinary Roots and Trends." Journal of Systems and Software 43(2):103-18.

Methodology, theory, and practice in the field of Human-Computer Interaction (HCI) all share the goal of producing interactive software that can be used efficiently, effectively, safely, and with satisfaction. HCI is cross-disciplinary in its conduct and multidisciplinary in its roots. The central concept of HCI is usability, ease of use plus usefulness. Achieving good usability requires attention to both product and development process, particularly for the user interaction design, which should serve as requirements for the user interface software component. This paper reviews some of the theory and modeling supporting the practice of HCI, development life cycles and activities, and much of the practice that constitutes "usability engineering". Future application areas of interest in HCI include new interaction styles, virtual environments, the World Wide Web, information visualization, and wearable computing.

Harutyunyan, HA, and W Shengjian. 2006, "Efficient Multicast Algorithms for Mesh-Connected Multicomputers." In IEEE Conference on Information Visualization, pp. 504-10.

Performance of multicomputers largely depends on that of the underlying network communications such as multicast. Two major parameters used to evaluate multicast routing are the time it takes to deliver the message to all destinations and the traffic which refers to the total number of links involved. Mesh is a network topology widely used in multicomputers. It has been proved that, in mesh network, it is NP-hard to find the multicast routing which is optimal on both time and traffic. In this paper, we proposed two efficient multicast algorithms designed for store-and-forward switched mesh-connected multicomputers: DIAG and DDS. They are both tree-based shortest path multicast algorithms whose complexity is $\mathrm{O}(\mathrm{KN})$ or less. Performance evaluations of these algorithms resulted from simulations are given at the end

He, Q, X Zhao, and Z Shi. 2008, "A Cognitive Data Visualization Method Based on Hyper Surface." In 6th IEEE International Conference on Cognitive Informatics, pp. 85-91.

The understanding of data is highly relevant to how one senses and perceives them. The existing approaches for classification have been developed mainly based on exploring the intrinsic structure of dataset itself less or no emphasis paid on simulating human visual cognition. A new hyper surface classification method (HSC) has been studied since 2002. HSC is a universal classification method, in which a model of hyper surface is obtained by adoptively dividing the sample space and then the hyper surface is directly used to classify large database based on Jordan curve theorem in topology. In this paper we point out that HSC is a cognitive data visualization method. Simulation results show the effectiveness of the proposed method on large test data with complex distribution and high density. In particular, we show that HSC can very often bring a significant reduction of computation effort without loss of prediction capability. 
Heer, J, and M Agrawala. 2007, "Design Considerations for Collaborative Visual Analytics." In IEEE Symposium on Visual Analytics Science and Technology, pp. 171-78.

Heer, J, and M Bostock. 2010, "Crowdsourcing Graphical Perception: Using Mechanical Turk to Assess Visualization Design." In 28th Annual CHI Conference on Human Factors in Computing Systems, pp. 203-12.

Understanding perception is critical to effective visualization design. With its low cost and scalability; crowdsourcing presents an attractive option for evaluating the large design space of visualizations; however, it first requires validation. In this paper, we assess the viability of Amazon's Mechanical Turk as a platform for graphical perception experiments. We replicate previous studies of spatial encoding and luminance contrast and compare our results. We also conduct new experiments on rectangular area perception (as in treemaps or cartograms) and on chart size and gridline spacing. Our results demonstrate that crowdsourced perception experiments are viable and contribute new insights for visualization design. Lastly, we report cost and performance data from our experiments and distill recommendations for the design of crowdsourced studies.

Heer, J, and M Bostock. 2010, "Declarative Language Design for Interactive Visualization." IEEE Transactions on Visualization and Computer Graphics 16(6):1149-56.

We investigate the design of declarative, domain-specific languages for constructing interactive visualizations. By separatingspecification from execution, declarative languages can simplify development, enable unobtrusive optimization, and supportretargeting across platforms. We describe the design of the Protovis specification language and its implementation within anobject-oriented, staticallytyped programming language (Java). We demonstrate how to support rich visualizations without requiring atoolkit-specific data model and extend Protovis to enable declarative specification of animated transitions. To support cross-platformdeployment, we introduce rendering and event-handling infrastructures decoupled from the runtime platform, letting designers retargetvisualization specifications (e.g., from desktop to mobile phone) with reduced effort. We also explore optimizations such as runtimecompilation of visualization specifications, parallelized execution, and hardware-accelerated rendering. We present benchmark studiesmeasuring the performance gains provided by these optimizations and compare performance to existing Java-based visualizationtools, demonstrating scalability improvements exceeding an order of magnitude.

Heer, J, SK Card, and JA Landay. 2005, "Prefuse: A Toolkit for Interactive Information Visualization." In SIGCHI conference on Human factors in computing systems pp. 421-30.

Heer, J, et al. 2008, "Graphical Histories for Visualization: Supporting Analysis, Communication, and Evaluation." IEEE Transactions on Visualization and Computer Graphics 14(6):1189-96.

Interactive history tools, ranging from basic undo and redo to branching timelines of user actions, facilitate iterative forms of interaction. In this paper, we investigate the design of history mechanisms for information visualization. We present a design space analysis of both architectural and interface issues, identifying design decisions and associated trade-offs. Based on this analysis, we contribute a design study of graphical history tools for Tableau, a database visualization system. These tools record and visualize interaction histories, support data analysis and communication of findings, and contribute novel mechanisms for presenting, managing, and exporting histories. Furthermore, we have analyzed 
aggregated collections of history sessions to evaluate Tableau usage. We describe additional tools for analyzing users' history logs and how they have been applied to study usage patterns in Tableau.

Heer, J, and A Maneesh. 2006, "Software Design Patterns for Information Visualization." Visualization and Computer Graphics, IEEE Transactions on 12(5):853-60.

Despite a diversity of software architectures supporting information visualization, it is often difficult to identify, evaluate, and re-apply the design solutions implemented within such frameworks. One popular and effective approach for addressing such difficulties is to capture successful solutions in design patterns, abstract descriptions of interacting software components that can be customized to solve design problems within a particular context. Based upon a review of existing frameworks and our own experiences building visualization software, we present a series of design patterns for the domain of information visualization. We discuss the structure, context of use, and interrelations of patterns spanning data representation, graphics, and interaction. By representing design knowledge in a reusable form, these patterns can be used to facilitate software design, implementation, and evaluation, and improve developer education and communication

Hegarty, M. 2010, "Components of Spatial Intelligence." in Psychology of Learning and Motivation, ed. HR Brian, Vol Volume 52, pp. 265-97. Academic Press.

This chapter identifies two basic components of spatial intelligence, based on analyses of performance on tests of spatial ability and on complex spatial thinking tasks in domains such as mechanics, chemistry, medicine, and meteorology. The first component is flexible strategy choice between mental imagery (or mental simulation more generally) and more analytic forms of thinking. Research reviewed here suggests that mental simulation is an important strategy in spatial thinking, but that it is augmented by more analytic strategies such as task decomposition and rule-based reasoning. The second is meta-representational competence [diSessa, A. A. (2004). Metarepresentation: Native competence and targets for instruction. Cognition and Instruction, 22, 293-331], which encompasses ability to choose the optimal external representation for a task and to use novel external representations productively. Research on this aspect of spatial intelligence reveals large individual differences in ability to adaptively choose and use external visual-spatial representations for a task. This research suggests that we should not just think of interactive external visualizations as ways of augmenting spatial intelligence, but also consider the types of intelligence that are required for their use.

Hegarty, M. 2004, "Dynamic Visualizations and Learning: Getting to the Difficult Questions." Learning and Instruction 14(3):343-51.

Hegarty, M. 2010, "Visweek Keynote Address." Visualization and Computer Graphics, IEEE Transactions on 16(6):xxiv-xxiv.

Hegarty, M, MS Canham, and SI Fabrikant. 2010, "Thinking About the Weather: How Display Salience and Knowledge Affect Performance in a Graphic Inference Task." Journal of Experimental Psychology: Learning, Memory, and Cognition 36(1):37-53.

Three experiments examined how bottom-up and top-down processes interact when people view and make inferences from complex visual displays (weather maps). Bottom-up effects of display design were investigated by manipulating the relative visual salience of task-relevant and task-irrelevant information 
across different maps. Top-down effects of domain knowledge were investigated by examining performance and eye fixations before and after participants learned relevant meteorological principles. Map design and knowledge interacted such that salience had no effect on performance before participants learned the meteorological principles; however, after learning, participants were more accurate if they viewed maps that made task-relevant information more visually salient. Effects of display design on task performance were somewhat dissociated from effects of display design on eye fixations. The results support a model in which eye fixations are directed primarily by top-down factors (task and domain knowledge). They suggest that good display design facilitates performance not just by guiding where viewers look in a complex display but also by facilitating processing of the visual features that represent task-relevant information at a given display location.

Heidemann, G. 2005, "Unsupervised Image Categorization." Image and Vision Computing 23(10):86176.

Large image collections require efficient organization and visualization. This paper describes an approach to establish image categories automatically by unsupervised learning. The method works free of context and previous knowledge: in a first stage, features are formed automatically, then images are clustered to form categories. The human database designer has to decide only whether a category is useful or too inhomogeneous from a high level point of view. To collect images that cannot be categorized automatically, an additional [']miscellaneous' category exists. Categories are visualized by displaying the most typical image(s) of the categories as thumbnails. The main benefit of the approach is that it deals with color and shape in a unified way on a local scale, combined with the advantages of histogram techniques on the global scale. To judge results, an evaluation scheme which is adequate for the task of categorization is proposed.

Heinrich, J, et al. 2010, "Geneiusvis - Visual Analytics for Genetic Sequences." In IEEE Symposium on Visual Analytics Science \& Technology, pp. 3-4.

Heinrichs, JH, LJ Hudspeth, and JS Lim. 2003, "Knowledge Management." in Encyclopedia of Information Systems, ed. B Hossein, pp. 13-31. Elsevier, New York.

Heiser, J, and B Tversky. 2006, "Arrows in Comprehending and Producing Mechanical Diagrams." Cognitive Science 30(3):581-92.

Mechanical systems have structural organizations - parts, and their relations - and functional organizations - temporal, dynamic, and causal processes - which can be explained using text or diagrams. Two experiments illustrate the role of arrows in diagrams of mechanical systems. In Experiment 1, people described diagrams with or without arrows, interpreting diagrams without arrows as conveying structural information and diagrams with arrows as conveying functional information. In Experiment 2, people produced sketches of mechanical systems from structural or functional descriptions. People spontaneously used arrows to indicate functional processes in diagrams. Arrows can play a powerful role in augmenting structural diagrams to convey dynamic, causal, or functional information. 
Helminen, J, and L Malmi. 2010, "Jype - a Program Visualization and Programming Exercise Tool for Python Categories and Subject Descriptors." In 5th ACM Symposium on Software Visualization (SOFTVIS), pp. 153-62.

Hendley, RJ, et al. 1995, "Case Study. Narcissus: Visualising Information." In IEEE Conference on Information Visualization, pp. 90-96.

It is becoming increasingly important that support is provided for users who are dealing with complex information spaces. The need is driven by the growing number of domains where there is a requirement for users to understand, navigate and manipulate large sets of computer based data; by the increasing size and complexity of this information and by the pressures to use this information efficiently. The paradigmatic example is the World Wide Web, but other domains include software systems, information systems and concurrent engineering. One approach to providing this support is to provide sophisticated visualisation tools which lead the users to form an intuitive understanding of the structure and behaviour of their domain and which provide mechanisms which allow them to manipulate objects within their system. The paper describes such a tool and a number of visualisation techniques that it implements.

Henley, M, M Hagen, and RD Bergeron. 2007, "Evaluating Two Visualization Techniques for Genome Comparison." In 11th International Conference Information Visualization, pp. 551-58.

Genomic study is fairly novel. Typical research processes are not established yet. Many new discoveries are happening in this area all the time. Are current methods of visualizations effective? What works well? What could be improved? These are some of the questions we are interested in evaluating for two graphical tools used to compare nucleotide sequences. Scatter plots and parallel coordinate-like visuals have been used in genomics for identifying similarities in genetic code. Our preliminary evaluation focuses on determining the aspects of the two visualizations that are successful and those that need enhancements.

Henry, JAG, and NF Polys. 2010, "The Effects of Immersion and Navigation on the Acquisition of Spatial Knowledge of Abstract Data Networks." Procedia Computer Science 1(1):1737-46.

With increasing frequency immersive virtual environments (IVEs) are being used to present multidimensional information visualizations. Networks and graphs are a common type of abstract data; in order to understand the varied relationships between entities in a network, it is crucial to acquire some spatial knowledge about the layout and connectivity of its components. While there is a good body of evidence for the benefits of IVE displays, most work on the effects of immersion and of navigation on the acquisition of spatial knowledge has been concerned with wayfinding in realistic environments; much less is known about how to leverage IVE technology to benefit a user'ss spatial understanding of (abstract) data networks. In this paper we present an empirical study designed to determine what effect level of immersion and navigation technique can have on a user's acquisition of spatial knowledge of network data, specifically cell signaling pathways. For this CAVE study (CAVE Automatic Virtual Environment), the level of immersion is controlled by changing the Field-Of-Regard, while we also vary navigation between one egocentric and one exocentric technique. The results show that both immersion and navigation technique can affect the acquisition of spatial knowledge regarding abstract networks in an immersive virtual environment. 
Hepting, DH. 2007, "Decision Support for Local Environmental Impact Assessment." Environmental Modelling \& Software 22(4):436-41.

Sustainability has become a key concern for consumers and industry. However, coming to understand its implications in the context of daily life can be difficult. It may be hard to come to terms with the idea that traditional activities present environmental risks and may lead to real emergencies, manifested differently depending on local conditions. Everyone will be asked to do more but the move towards sustainability may require the total transformation of current attitudes and actions. This paper considers the role of informatics in responding to, and possibly averting, environmental emergencies by making information available to decision makers, whether they are individual consumers, institutional buyers, or emergency response personnel. If all choices can be presented within a spectrum of best and worst possible impacts for sustainability, decision makers can meaningfully weigh their options. Any evaluation on this basis is presently very difficult because one needs to independently assess and integrate possibly incomplete and conflicting information from a wide variety of sources. This paper describes the use of the cogito software, which embodies an approach for data access and presentation that is expected to provide significant benefits over existing web-based tools. The paper also considers the issues related to the collection and synthesis of disparate data.

Hepting, DH. 2002, "Towards a Visual Interface for Information Visualization." In 6th International Conference on Information Visualisation, pp. 295-302.

Information visualization, aided by ever more accessible computational resources, continues to grow in popularity and significance. The capability to generate complex imagery by computer is often necessary but not always sufficient to gain the desired insight. The success of a visual representation in a given context may be affected by many variables, not the least of which is the individual user's experience. Even if a precise relationship could be found between context and "best" visual representation, the complete articulation of a context is practically impossible. In other fields, this is known as sensitive dependence to initial conditions. A more feasible alternative is to begin with an incomplete articulation of a context and allow the user to interactively develop and refine it. Although most computer interfaces for information visualization tools are predominantly verbal, a predominantly visual interface can have significant advantages. Such an interface allows users to avoid the usual translations between visual and verbal modes and it removes users' need for a specialized visualization vocabulary. A visual interface can also shift the focus of the visualization process from the data towards the user. These ideas are discussed in the context of a prototype tool, the design of which is illustrated with an example, and the evaluation of which has provided many positive results.

Herman, I, G Melancon, and MS Marshall. 2000, "Graph Visualization and Navigation in Information Visualization: A Survey." IEEE Transactions on Visualization and Computer Graphics 6(1):24-43.

This is a survey on graph visualization and navigation techniques, as used in information visualization. Graphs appear in numerous applications such as web browsing, state-transition diagrams, and data structures. The ability to visualize and to navigate in these potentially large, abstract graphs is often a crucial part of an application. Information visualization has specific requirements, which means that this survey approaches the results of traditional graph drawing from a different perspective. 
Herman, I, G Melançon, and MS Marshall. 2000, "Graph Visualization and Navigation in Information Visualization: A Survey." IEEE Transactions on Visualization and Computer Graphics 6(1):24-43.

Hernandez, T. 2007, "Enhancing Retail Location Decision Support: The Development and Application of Geovisualization." Journal of Retailing and Consumer Services 14(4):249-58.

Geovisualization refers to the visual exploration, analysis, synthesis and presentation of geospatial data. This paper presents findings from research that has focused on developing and applying geovisualization techniques and technologies for use within retail location decision support. To date, retailers represent a major user group of geographic information system (GIS) -based decision support technologies, with applications ranging from trade area mapping to store portfolio planning. However, the ability to handle spatial-temporal data, visualize change, and explore the temporal dimension of spatial data is limited within conventional GIS. The paper details the development of a prototype geovisualization system that has been designed to enable visualization of spatial-temporal change of retail-related data. From this explicitly visual paradigm, a number of examples of potential analysis are examined at four different scales of analysis: national, regional, market and micro-level. The paper highlights both the challenges and potential to enhance retail decision support by integrating geovisualization techniques and technology within decision support activities.

Herold, J, et al. 2010, "Integrating Semantic Annotation and Information Visualization for the Analysis of Multichannel Fluorescence Micrographs from Pancreatic Tissue." Computerized Medical Imaging and Graphics 34(6):446-52.

The challenging problem of computational bioimage analysis receives growing attention from life sciences. Fluorescence microscopy is capable of simultaneously visualizing multiple molecules by staining with different fluorescent dyes. In the analysis of the result multichannel images, segmentation of ROIs resembles only a first step which must be followed by a second step towards the analysis of the ROI's signals in the different channels. In this paper we present a system that combines image segmentation and information visualization principles for an integrated analysis of fluorescence micrographs of tissue samples. The analysis aims at the detection and annotation of cells of the Islets of Langerhans and the whole pancreas, which is of great importance in diabetes studies and in the search for new anti-diabetes treatments. The system operates with two modules. The automatic annotation module applies supervised machine learning for cell detection and segmentation. The second information visualization module can be used for an interactive classification and visualization of cell types following the link-and-brush principle for filtering. We can compare the results obtained with our system with results obtained manually by an expert, who evaluated a set of example images three times to account for his intra-observer variance. The comparison shows that using our system the images can be evaluated with high accuracy which allows a considerable speed up of the time-consuming evaluation process. (C) 2009 Elsevier Ltd. All rights reserved.

Herr, BW, et al. 2009, "The Nih Visual Browser: An Interactive Visualization of Biomedical Research." In 13th International Conference Information Visualisation, pp. 505-09.

This paper presents a technical description of the methods used to generate an interactive, twodimensional visualization of 60,568 grants funded by the National Institutes of Health in 2007. The visualization is made intelligible by providing interactive features for assessing the data in a web-based visual browser, see http://www.nihmaps.org. The key features include deep zooming, selection, full-text 
querying, overlays, color-coding schemes, and multi-level labeling. Major insights, broader applicability, and future directions are discussed.

Herr, BW, et al. 2007, "Movies and Actors: Mapping the Internet Movie Database." In 11th International Conference on Information Visualization, pp. 465-69.

This paper presents the results of an analysis and visualization of 428,440 movies from the Internet Movie Database (IMDb) provided for the Graph Drawing 2005 contest. Simple statistics are presented as well as a tapestry of all movies with an overlay of the giant component of the co-actor network. Academy award winners are highlighted. Major insights are discussed.

Hervás, R, and J Bravo. 2011, "Towards the Ubiquitous Visualization: Adaptive User-Interfaces Based on the Semantic Web." Interacting with Computers 23(1):40-56.

This manuscript presents an infrastructure that contributes to ubiquitous information. Advances in Ambient Intelligence may help to provide us with the right information at the right time, in an appropriate manner and through the most suitable device for each situation. It is therefore crucial for such devices to have contextual information; that is, to know the person or persons in need of information, the environment, and the available devices and services. All of this information, in appropriate models, can provide a simplified view of the real world and let the system act more like a human and, consequently, more intelligently. A suitable context model is not enough; proactive user interface adaptation is necessary to offer personalized information to the user. In this paper, we present mechanisms for the management of contextual information, reasoning techniques and adaptable user interfaces to support visualization services, providing functionality to make decisions about what and how available information can be offered. Additionally, we present the ViMos framework, an infrastructure to generate context-powered information visualization services dynamically.

Hesse, BW. 2008, "Of Mice and Mentors: Developing Cyber-Infrastructure to Support Transdisciplinary Scientific Collaboration." American Journal of Preventive Medicine 35(2, Supplement 1):S235-S39.

Hesse, BW, and B Shneiderman. 2007, "Ehealth Research from the User's Perspective." American Journal of Preventive Medicine 32(5, Supplement 1):S97-S103.

Abstract The application of information technology (IT) to issues of healthcare delivery has had a long and tortuous history in the United States. Within the field of eHealth, vanguard applications of advanced computing techniques, such as applications in artificial intelligence or expert systems, have languished in spite of a track record of scholarly publication and decisional accuracy. The problem is one of purpose, of asking the right questions for the science to solve. Historically, many computer science pioneers have been tempted to ask "what can the computer do?" New advances in eHealth are prompting developers to ask "what can people do?" How can eHealth take part in national goals for healthcare reform to empower relationships between healthcare professionals and patients, healthcare teams and families, and hospitals and communities to improve health equitably throughout the population? To do this, eHealth researchers must combine best evidence from the user sciences (human factors engineering, human-computer interaction, psychology, and usability) with best evidence in medicine to create transformational improvements in the quality of care that medicine offers. These improvements should follow recommendations from the Institute of Medicine to create a healthcare system that is (1) safe, (2) effective (evidence based), (3) patient centered, and (4) timely. Relying on the eHealth researcher's 
intuitive grasp of systems issues, improvements should be made with considerations of users and beneficiaries at the individual (patient-physician), group (family-staff), community, and broad environmental levels.

Hessinger, M, et al. 2008, "Hemodynamic Models for Education in Physiology." Mathematics and Computers in Simulation 79(4):1039-47.

By application of case-based learning (CBL) various effects can be analyzed and demonstrated more easily. In the area of medicine one rapidly reaches boundaries in the visualization of complex information [J.L.M. Poiseuille, Recherches experimentales sur le mouvement des liquids dans les tubes de tres petits diametres, Memoires Savant des Etrangers 9 (1846) 433-544]. Learning and teaching without recourse to patients is difficult. Consequently the use of models and simulations are useful. In this paper the authors report about experiences gained with HAEMOSIM, a web-based project in medical education. The goal of this project is the design and development of interactive simulations in local hemodynamics by the application of mathematical-physiological models. These include the modelling of arterial blood flow dependent on the pressure gradient, radius and bifurcations, as well as blood flow profiles in dependency of viscosity, density and radius and finally pulse-wave dynamics with regard to local and global compliance.

Hetzler, B, et al. 2004, "In-Spire Infovis 2004 Contest Entry." In IEEE Conference on Information Visualization, pp. r2-r2.

This is the first part (summary) of a three-part contest entry submitted to IEEE InfoVis 2004. The contest topic is visualizing InfoVis symposium papers from 1995 to 2002 and their references. The paper introduces the visualization tool IN-SPIRE, the visualization process and results, and presents lessons learned.

Hetzler, B, et al. 1998, "Multi-Faceted Insight through Interoperable Visual Information Analysis Paradigms." In IEEE Symposium on Information Visualization, pp. 137-+.

To gain insight and understanding of complex information collections, users must be able to visualize and explore many facets of the information. This paper presents several novel visual methods from an information analyst's perspective. We present a sample scenario, using the various methods to gain a variety of insights from a large information collection. We conclude that no single paradigm or visual method is sufficient for many analytical tasks. Often a suite of integrated methods offers a better analytic environment in today's emerging culture of information overload and rapidly changing issues. We also conclude that the interactions among these visual paradigms are equally as important as, if not more important than, the paradigms themselves.

Hetzler, E, and A Turner. 2005, "Analysis Experiences Using Information Visualization." Computer Graphics and Applications, IEEE 24(5):22-26.

Heylighen, A, and IM Verstijnen. 2003, "Close Encounters of the Architectural Kind." Design Studies 24(4):313-26.

Throughout their career architects collect an extensive record of architectural cases, which they use as a source of inspiration and knowledge during design. Being novices, student architects do not yet have 
such a record. In order to compensate for this lack of knowledge, teachers in architecture engage their students into realistic yet simulated projects and introduce them to relevant architectural precedents for these projects. Within the realm of AI, case-based reasoning (CBR) stresses the importance of cases too. So far, however, applications that flow from CBR research have rarely found their way into architecture. The experiment that is reported in this article examines the conditions under which CBR technology can be useful in architectural education. The results show that in order for students to benefit from this technology, it should supply cases that are closely related to the project at hand. These results are consistent with psychological theories of knowledge representation in novices.

Hibbs, M, et al. 2007, "Viewing the Larger Context of Genomic Data through Horizontal Integration." In 11th International Conference on Information Visualization, pp. 326-34.

Genomics is an important emerging scientific field that relies on meaningful data visualization as a key step in analysis. Specifically, most investigation of gene expression microarray data is performed using visualization techniques. However, as microarrays become more ubiquitous, researchers must analyze their own data within the context of previously published work in order to gain a more complete understanding. No current method for microarray visualization and analysis enables biology researchers to observe the greater context of data that surrounds their own results, which severely limits the ability of researchers draw novel conclusions. Here we present a system, called HIDRA, that visually integrates the simultaneous display of multiple microarray datasets to identify important parallels and dissimilarities. We demonstrate the power of our approach through examples of real-world biological insights that can be observed using HIDRA that are not apparent using other techniques.

Hightower, RR, et al. 2003, "Graphical Multiscale Web Histories: A Study of Padprints." in The Craft of Information Visualization, Readings and Reflections, eds. BB Benjamin and S Ben, pp. 220-27. Morgan Kaufmann, San Francisco.

Summary We have implemented a browser companion called PadPrints that dynamically builds a graphical history-map of visited web pages. PadPrints relies on Pad++, a zooming user interface (ZUI) development substrate, to display the history-map using minimal screen space. PadPrints functions in conjunction with a traditional web browser but without requiring any browser modifications.

Hochheiser, H. 2003, "Interactive Graphical Querying of Time Series and Linear Sequence Data Sets." Vol Ph.D., pp. 284-84. University of Maryland, College Park.

Numerous analytic domains involve the study of measurable quantities that change over time. This widespread interest in time series data sets has led to substantial work in algorithmic strategies for querying and indexing data. Much less work has been done in the development of interactive tools for identifying patterns in these data sets. This dissertation uses a graphical mechanism for specifying queries on time series data to provide the basis for an exploration of the algorithmic and semantic issues surrounding interactive querying of time series data. Contributions of this dissertation include: (1) The definition of timeboxes--rectangular widgets that can be used in direct-manipulation Graphical User Interfaces (GUIs) to specify query constraints on time series data sets. Timeboxes are used to simultaneously specify two sets of constraints: given a set of $\mathrm{N}$ time series profiles, a timebox covering time periods x $1 \ldots \mathrm{x} 2(\mathrm{x} 1 \mathrm{~d}$ x 2$)$ and values y $1 \ldots \mathrm{y} 2(\mathrm{y} 1 \mathrm{~d}$ y 2$)$ will retrieve only those $\mathrm{n}$ that have values y $1 \mathrm{~d}$ y d y 2 during all times $\mathrm{x} 1 \mathrm{~d} \times \mathrm{d} \times 2$. (2) The TimeSearcher information visualization tool, which is based on the time-box query model. TimeSearcher's object-oriented architecture can easily be 
extended to support variants of the timebox model that provide additional expressive power. (3) The design and implementation of query models and widgets that extend the timebox model, including variable-time timeboxes (VTTs), angular queries, leaders \& laggards queries, multiple search attributes, and query inversion. (4) Analysis of algorithmic issues: A comparison of multiple alternative search algorithms found that simple sequential scans outperformed geometric indices for processing timebox queries. (5) Empirical evaluation of timeboxes: Two empirical studies, each with 12 subjects, provided preliminary insight into the utility of timeboxes and led to design improvements for input and display. (6) Validation through case studies: TimeSearcher has been used by molecular biologists to explore gene expression data and nucleotide frequencies. This work has validated the utility of the tool and identified design suggestions and opportunities. (7) A framework for extending the timebox model, including the description of numerous possible extensions.

Hodgkinson, GP, J Langan-Fox, and E Sadler-Smith. 2008, "Intuition: A Fundamental Bridging Construct in the Behavioural Sciences." British Journal of Psychology 99(1):1-27.

The concept of intuition has, until recently, received scant scholarly attention within and beyond the psychological sciences, despite its potential to unify a number of lines of inquiry. Presently, the literature on intuition is conceptually underdeveloped and dispersed across a range of domains of application, from education, to management, to health. In this article, we clarify and distinguish intuition from related constructs, such as insight, and review a number of theoretical models that attempt to unify cognition and affect. Intuition's place within a broader conceptual framework that distinguishes between two fundamental types of human information processing is explored. We examine recent evidence from the field of social cognitive neuroscience that identifies the potential neural correlates of these separate systems and conclude by identifying a number of theoretical and methodological challenges associated with the valid and reliable assessment of intuition as a basis for future research in this burgeoning field of inquiry.

Hoeber, O. 2009, "User Evaluation Methods for Visualweb Search Interfaces." In 13th International Conference on Information Visualisation, pp. 139-45.

In recent years, numerous visual Web search interfaces have been developed in the research community. However, the user evaluations of these interfaces have been performed using a wide range of methods, making it difficult to compare and verify the relative value of the proposed advancements. In this paper, we survey these evaluation methods, and propose a stepped evaluation and refinement model for the systematic study and enhancement of visual Web search interfaces. We suggest that this stepped model can be generalized to support the evaluation of other information visualization systems that target exploratory or knowledge-centric domains. (C) 2009 IEEE.

Hoeber, O. 2010, "Visually Representing Geo-Temporal Differences." In IEEE Conference on Information Visualization, pp. 229-30.

Hoeber, O, D Schroeder, and M Brooks. 2009, "Real-World User Evaluations of a Visual and Interactive Web Search Interface." In 4th Information Visualization Conference, pp. 119-26.

Although laboratory user studies are the most common method for validating the utility of information visualization systems, it may be difficult to determine if such studies accurately reflect the tasks of real users. In this paper, we describe a longitudinal real-world user evaluation of a visual and 
interactive Web search interface designed to support exploratory searching: theHotMap.com. Although we experienced some attrition during the course of the four-week study, the results indicate that many of the features of the system are beneficial and useful in real-world use. Although exploratory search comprised a small portion of the total search activities undertaken, the features of theHotMap.com which support query refinement and search results exploration did not interfere with the more common targeted searching tasks. As a result, most participants indicated that they found the system useful and easy to use.

Hoeber, O, and XD Yang. 2008, "Evaluating Wordbars in Exploratory Web Search Scenarios." Information Processing \& Management 44(2):485-510.

Web searchers commonly have difficulties crafting queries to fulfill their information needs; even after they are able to craft a query, they often find it challenging to evaluate the results of their Web searches. Sources of these problems include the lack of support for constructing and refining queries, and the static nature of the list-based representations of Web search results. WordBars has been developed to assist users in their Web search and exploration tasks. This system provides a visual representation of the frequencies of the terms found in the first 100 document surrogates returned from an initial query, in the form of a histogram. Exploration of the search results is supported through term selection in the histogram, resulting in a re-sorting of the search results based on the use of the selected terms in the document surrogates. Terms from the histogram can be easily added or removed from the query, generating a new set of search results. Examples illustrate how WordBars can provide valuable support for query refinement and search results exploration, both when vague and specific initial queries are provided. User evaluations with both expert and intermediate Web searchers illustrate the benefits of the interactive exploration features of WordBars in terms of effectiveness as well as subjective measures. Although differences were found in the demographics of these two user groups, both were able to benefit from the features of WordBars.

Hoffman, RR, and WC Elm. 2006, "Hcc Implications for the Procurement Process." Ieee Intelligent Systems 21(1):74-81.

The view of author for the procurement of information processing and intelligent technology for complex sociotechnical domains are discussed. Various case studies including the US National Weather Service's Advanced Weather Information Processing System (AWIPS) and Department of Defense (DoD) are also presented. Human-centered computational tools need to support active organization, active search, active exploration of information as well as reflect and evaluate the meaning of information. It is suggested that designers must understand users' need and the goals for the system being created.

Hoffman, RR, and DD Woods. 2005, "Toward a Theory of Complex and Cognitive Systems." Intelligent Systems, IEEE 20(1):76-79.

We present nine propositions that we've referred to us principles of human-centered computing. We discussed the reductive tendency, which is a necessary consequence of learning. We pointed out that this tendency also applies to those who are creating new information technologies, especially complex and cognitive systems. Indeed, the people who try to create new complex and cognitive systems are themselves prone to generate reductive understandings, in which complexities are simplified. 
Hollan, JD, BB Bederson, and J Helfman. 1997, "Information Visualization." Handbook of humancomputer interaction:33-48.

Holzinger, A. 2005, "Usability Engineering Methods for Software Developers." Commun. ACM 48(1):7174.

The human-computer interaction community aims to increase the awareness and acceptance of established methods among software practitioners. Indeed, awareness of the basic usability methods will drive an Information Society for all.

Holzinger, A, et al. 2009, "Learning Performance with Interactive Simulations in Medical Education: Lessons Learned from Results of Learning Complex Physiological Models with the Haemodynamics Simulator." Computers \& Education 52(2):292-301.

Objective Since simulations are often accepted uncritically, with excessive emphasis being placed on technological sophistication at the expense of underlying psychological and educational theories, we evaluated the learning performance of simulation software, in order to gain insight into the proper use of simulations for application in medical education.Design The authors designed and evaluated a software packet, following of user-centered development, which they call Haemodynamics Simulator (HAEMOSIM), for the simulation of complex physiological models, e.g., the modeling of arterial blood flow dependent on the pressure gradient, radius and bifurcations; shear-stress and blood flow profiles depending on viscosity and radius.Measurements In a quasi-experimental real-life setup, the authors compared the learning performance of 96 medical students for three conditions: (1) conventional textbased lesson; (2) HAEMOSIM alone and (3) HAEMOSIM with a combination of additional material and support, found necessary during user-centered development. The individual student's learning time was unvarying in all three conditions. Results While the first two settings produced equivalent results, the combination of additional support and HAEMOSIM yielded a significantly higher learning performance. These results are discussed regarding Mayer's multimedia learning theory, Sweller's cognitive load theory, and claims of prior research on utilizing interactive simulations for learning.Conclusion The results showed that simulations can be beneficial for learning complex concepts, however, interacting with sophisticated simulations strain the limitation of cognitive processes; therefore successful application of simulations require careful additional guidance from medical professionals and a certain amount of previous knowledge on the part of the learners. The inclusion of pedagogical and psychological expertise into the design and development of educational software is essential.

Hopfer, S, and aM MacEachren. 2007, "Leveraging the Potential of Geospatial Annotations for Collaboration: A Communication Theory Perspective." International Journal of Geographical Information Science 21(8):921-34.

Hoppe, B, and C Reinelt. 2010, "Social Network Analysis and the Evaluation of Leadership Networks." The Leadership Quarterly 21(4):600-19.

Leadership development practitioners are increasingly interested in social networks as a way to strengthen relationships among leaders in fields, communities, and organizations. This paper offers a framework for conceptualizing different types of leadership networks and uses case examples to identify outcomes typically associated with each type of network. Evaluating leadership networks is a challenge for the field of leadership development. Social network analysis (SNA) is an evaluation approach that 
uses mathematics and visualization to represent the structure of relationships between people, organizations, goals, interests, and other entities within a larger system. In this article we describe core social network concepts and the application of them to illuminate the value of SNA as an evaluation tool.

Hosny, HM. 1999, "An Interactive Lab Environment for Computing Concepts and Courseware." Computers \& Industrial Engineering 37(1-2):313-17.

This paper describes a new system which is being designed and implemented by senior and graduate Computer Science students, under faculty supervision, to help younger CS students understand and visualize the new computing concepts with which they are in constant struggle. Broadly speaking, the system is to achieve two major goals at the same time : first to provide senior CS students and fresh graduates with the opportunity to develop educational software for junior fellow students in their major using new technology; and second to make the junior ones (freshmen and sophomore levels) experience the new revolution in education of which they will soon become a part while they learn new concepts in their discipline.

Hosssain, Z, and T Möller. 2010, "Edge Aware Anisotropic Diffusion for 3d Scalar Data." IEEE Transactions on Visualization and Computer Graphics 16(6):1376-85.

In this paper we present a novel anisotropic diffusion model targeted for 3D scalar field data. Our model preserves material boundaries as well as fine tubular structures while noise is smoothed out. One of the major novelties is the use of the directional second derivative to define material boundaries instead of the gradient magnitude for thresholding. This results in a diffusion model that has much lower sensitivity to the diffusion parameter and smoothes material boundaries consistently compared to gradient magnitude based techniques. We empirically analyze the stability and convergence of the proposed diffusion and demonstrate its de-noising capabilities for both analytic and real data. We also discuss applications in the context of volume rendering.

Hostler, RE, VY Yoon, and T Guimaraes. 2005, "Assessing the Impact of Internet Agent on End Users' Performance." Decision Support Systems 41(1):313-23.

Intelligent software agents that can perform tasks on the user's behalf independently of direct control of the user themselves, promise to evolutionize the way in which we use the Internet to conduct business. Research on how these agents will change the nature of Internet-based e-commerce and what its impact will be on consumers and businesses is only just beginning. To assess the impact of agent usage in a retail online shopping environment, an empirical study was conducted to determine what impact, if any, the use of Shopbots, a form of Internet agent, had on consumers looking to purchase a DVD player online via the World Wide Web [WWW]. Of particular interest was the Internet agent's impact on the user's task performance and task outcomes. These included the time spent on shopping activities, the shopper's confidence in their purchase decision, the quality of the purchase decision made by the shopper and the amount of cognitive effort required to select a product for purchase. 
Hourcade, JP, et al. 2003, "The International Children's Digital Library: Viewing Digital Books Online." in The Craft of Information Visualization, Readings and Reflections, eds. BB Benjamin and S Ben, pp. 186-95. Morgan Kaufmann, San Francisco.

Summary Reading books plays an important role in children's cognitive and social development. However, many children do not have access to diverse collections of books due to the limited resources of their community libraries. We have begun to address this issue by creating a large-scale digital archive of children's books, the International Children's Digital Library (ICDL). In this paper we discuss our initial efforts in building the ICDL, concentrating on an informal evaluation of innovative digital book readers.

Hourizi, R, and P Johnson. 2003, "Towards an Explanatory, Predictive Account of Awareness." Computers \& Graphics 27(6):859-72.

In this paper we investigate the utility of an explanatory and predictive account of awareness in the (re)design of complex human-computer interaction. We start by describing both the wider field of human computer awareness and the specific sub-field of situation awareness in the aviation domain. We also discuss the wide range of observations and descriptions, which detail both success and failure in the acquisition, maintenance and repair of such awareness across a range of domains. Next, we assert the need for an explanatory and predictive model of the phenomenon in the search for a reduction in the breakdowns reported and propose such a model. We then go on to investigate the utility of our model as a guide for design through the discussion of a recent experiment involving manipulations of an animated warning signal on a simulated cockpit control panel. Our results show initial support both for the model and for our earlier assertion of its utility. We conclude that our composite view of awareness yields practical benefit in the design of human computer awareness support and provides a basis for future research.

Howell, M, S Love, and M Turner. 2006, "Visualisation Improves the Usability of Voice-Operated Mobile Phone Services." International Journal of Human-Computer Studies 64(8):754-69.

Three different versions of a voice-operated mobile city guide service with a hierarchically structured dialogue were evaluated in a mobile setting. One numbered menu style (standard) service, and two services which contained terms derived from underlying real-world referents, were implemented. The real-world referents (metaphors) used were: an office filing system and a computer desktop. It was hypothesized that the use of interface metaphors would allow more participants to visualize the service structure, leading to an improvement in performance relative to the standard service. Forty-two phone users undertook three different tasks with one of the three phone services. User performance and attitudes to the services were recorded, and post-task interviews were conducted. Results showed that significantly more participants using the metaphor-based services visualized the services. Visualizers performed significantly better than non-visualizers, with visualization emerging as a significant predictor of both attitude and performance. We argue that designing speech-based mobile phone services using an appropriate spatial metaphor leads to high levels of visualisation, which allows participants to orient themselves and to navigate more effectively within the hierarchical service architecture. The usability benefits afforded by visualization may become especially important when using phone services in cognitively demanding mobile settings. 
Hu, PJ-H, P-C Ma, and PYK Chau. 1999, "Evaluation of User Interface Designs for Information Retrieval Systems: A Computer-Based Experiment." Decision Support Systems 27(1-2):125-43.

In this study, we conducted a computer-based experiment to evaluate and compare the effectiveness of six different interface designs, graphical or list-based, in supporting communication of an object's "relevance" from an information retrieval (IR) system to its users. We adopted the Model Human Processor to provide a necessary framework to incorporate relevant cognitive psychology theories and user-centered design principles in the development of different interfaces. The study had a wellresearched theoretical foundation, complied with relevant design principles, and included a large-scale empirical evaluation. Our results suggest that interface design may have a significant effect on systemuser concept communication, regardless of users' familiarity with the search task, and that a graphical user interface may be more effective in supporting such communication than a list-based design. Furthermore, we also examined the cognitive load and user satisfaction resulting from each investigated interface design. Findings of the study have important implications for the design of IR systems (including online library systems and Internet-based search systems) as well as for the information representation and visualization of knowledge management systems, which ordinarily depend on text-based display methods to support system-user concept communication.

Huang, L Jie, and Nguyen. 2008, "A Usability Study on the Use of Multi-Context Visualization." In $5^{\text {th }}$ International Conference on Computer Graphics, Imaging and Visualization, pp. 311-16.

Graph visualization has been widely used in realworld applications, as it provides better presentation of overall data structure. However, there are navigation problems existing in deep and large relational datasets. To address these challenges, a new technique called multi-context visualization, which provides users with rich contextual information, has been proposed as the solution to the navigation in large scale datasets. This paper evaluates the multi-context visualization by conducting an experiment-based user study. To answer whether the more contextual information positively assist in making more accurate and easier decisions, it aims to evaluate the effectiveness and efficiency of the multicontext visualization, by measuring the user performance. Specifically, this usability test was designed to test if the use of multiple context views can improve navigation problems for deep and large relational data sets.

Huang, H-M. 2006, "Do Print and Web Surveys Provide the Same Results?" Computers in Human Behavior 22(3):334-50.

With an increased percentage of the population using the Internet, the Web is the most popular medium to collect and disseminate information. Web surveys are widely used instead of paper-and-pencil (print) surveys to measure employee motivation, program effectiveness and staff performance. However, few researchers worry that the same questions posed on the Web and in print can yield very different answers. The purpose of this study was to determine if participants would respond differently to a gradient of questions, when the survey method was modified and complete anonymity was available for each group. Finally, implications and possible issues of Web surveys were also discussed.

Huang, L, M Hemmje, and EJ Neuhold. 2000, "Admire: An Adaptive Data Model for Meta Search Engines." Computer Networks 33(1-6):431-48.

Considering the diversity among search engines, efficient integration of them is an important but difficult job. It is essential to provide a data model that can provide a detailed description of the query 
capabilities of heterogeneous search engines. By means of this model, the meta-searcher can map users' queries into specific sources more accurately, and it can achieve good precision and recall. Moreover, it will benefit the selection of target source and computing priority. Because new search engines emerge frequently and old ones are updated when their function and content change, the data model needs good adaptivity and scalability to keep in step with the rapidly developing World Wide Web. This paper gives a formal description of the query capabilities of heterogeneous search engines and an algorithm for mapping a query from a general mediator format into the specific wrapper format of a specific search engine. Compared with related work, the special features of our work are that we focus more on the constraint of/between the terms, attribute order, and the impact of logical operator restraints. The contribution of our work is that we offer a data model that is both expressive enough to meticulously describe the query capabilities of current World Wide Web search engines and flexible enough to integrate them efficiently.

Huang, L, and H Pashler. 2007, "A Boolean Map Theory of Visual Attention." Psychological Review 114(3):599-631.

A theory is presented that attempts to answer two questions. What visual contents can an observer consciously access at one moment? Answer: only one feature value (e.g., green) per dimension, but those feature values can be associated (as a group) with multiple spatially precise locations (comprising a single labeled Boolean map). How can an observer voluntarily select what to access? Answer: in one of two ways: (a) by selecting one feature value in one dimension (e.g., selecting the color red) or (b) by iteratively combining the output of (a) with a preexisting Boolean map via the Boolean operations of intersection and union. Boolean map theory offers a unified interpretation of a wide variety of visual attention phenomena usually treated in separate literatures. In so doing, it also illuminates the neglected phenomena of attention to structure.

Huang, ML, P Eades, and RF Cohen. 1998, "Webofdav -- Navigating and Visualizing the Web on-Line with Animated Context Swapping." Computer Networks and ISDN Systems 30(1-7):638-42.

This paper presents a novel navigation approach that helps the user, not only by providing a visual aid to guide the Web journey, but also by preserving the user's mental map [2] of the view while the user interactively navigates the Web by swapping of views. This approach does not predefine the geometry of whole visualization at once; instead it incrementally calculates and maintains a small local visualization on-line corresponding to the change of the user's focus. This feature enables the user to explore the Webspace without requiring the whole Web graph to be known.

Huang, ML, J Liang, and QV Nguyen. 2008, "A Usability Study on the Use of Multi-Context Visualization." In 5th International Conference on Computer Graphics, Imaging and Visualization, pp. 311-16.

Graph visualization has been widely used in real-world applications, as it provides better presentation Of overall data structure. However, there are navigation problems existing in deep and large relational datasets. To address these challenges, a new technique called multi-context visualization, which provides users with rich contextual information, has been proposed as the solution to the navigation in large scale datasets. This paper evaluates the multi-context visualization by conducting an experiment-based user study. To answer whether the more contextual information positively assist in making more accurate and easier decisions. it aims to evaluate the effectiveness and efficiency of the multi-context visualization, by 
measuring the user performance. Specifically, this usability test was designed to test if the use of multiple context views can improve navigation problems for deep and large relational data sets.

Huang, ML, and W Quan. 2005, "Exploring Spatially Referenced Information through 2d Marching Graph." In 5th IASTED International Conference on Visualization, Imaging, and Image Processing, pp. 703-08.

In this paper, we proposed a new visualization framework called Marching Graph that integrates the graph metaphor and the spatial metaphor into a single visualization. Marching Graph allows users to navigate the spatially referenced relational data across two different visual metaphors. We use a forcedirected layout algorithm to draw a sequence of progressive graphs, G(1), G(2) ... G(n) in a 2D geometric space that present the spatially referenced relational data. Each graph G(i) is associated with a particular geographic region R-i presented by the spatial metaphor. We allow the user to "march" through the thematic map by altering the focus region Ri and the display of its corresponding graph G(i) -> R-i. The use of 2D visual metaphors facilitates the navigation activities and human cognition process significantly.

Huang, SH, et al. 2008, "Knowledge-Based Evaluation of Nursing Care Practice." In IEEE International Conference on Information Reuse and Integration, pp. 171-74.

Providing people with a complete and responsive healthcare solution requires a multi-tiered health service delivery system. One aspect in the healthcare hierarchy is the need for care provided by nurses. Nursing care and observation provide the basis for nurses to communicate their practice with others in the healthcare system. It is necessary to capture and manage knowledge Of nursing care to improve the quality nursing practice. This paper proposes a novel knowledge-based decision support system for nurses to capture and manage nursing practice, and further, to monitor nursing care quality, as well as to test aspects of an electronic health record for recording and reporting nursing practice. As part of ongoing collaborative research of the nursing school and the department of computer science, a prototype toolset was developed to capture and manage nursing practice in order to improve the quality of care. A case study is presented to demonstrate the toolset used by nurses in a local hospital environment.

Huang, W, P Eades, and SH Hong. 2008, "Beyond Time and Error: A Cognitive Approach to the Evaluation of Graph Drawings." In Conference on BEyond Time and Errors: Novel EvaLuation Methods for Information Visualization, BELIV'08.

Time and error are commonly used to measure the effectiveness of graph drawings. However, such measures are limited in providing more fundamental knowledge that is useful for general visualization design. We therefore apply a cognitive approach in evaluations. This approach evaluates graph drawings from a cognitive perspective, measuring more than just time and error. Three user studies are conducted to demonstrate the usefulness of this approach. Copyright 2008 ACM.

Huang, W, P Eades, and SH Hong. 2009, "Measuring Effectiveness of Graph Visualizations: A Cognitive Load Perspective." Information Visualization 8(3):139-52.

Graph visualizations are typically evaluated by comparing their differences in effectiveness, measured by task performance such as response time and accuracy. Such performance-based measures have proved to be useful in their own right. There are some situations, however, where the performance measures alone may not be sensitive enough to detect differences. This limitation can be seen from the 
fact that the graph viewer may achieve the same level of performance by devoting different amounts of cognitive effort. In addition, it is not often that individual performance measures are consistently in favor of a particular visualization. This makes design and evaluation difficult in choosing one visualization over another. In an attempt to overcome the above-mentioned limitations, we measure the effectiveness of graph visualizations from a cognitive load perspective. Human memory as an information processing system and recent results from cognitive load research are reviewed first. The construct of cognitive load in the context of graph visualization is proposed and discussed. A model of user task performance, mental effort and cognitive load is proposed thereafter to further reveal the interacting relations between these three concepts. A cognitive load measure called mental effort is introduced and this measure is further combined with traditional performance measures into a single multi-dimensional measure called visualization efficiency. The proposed model and measurements are tested in a user study for validity. Implications of the cognitive load considerations in graph visualization are discussed. (C) 2009 Palgrave Macmillan.

Huang, X, and W Lai. 2006, "Clustering Graphs for Visualization Via Node Similarities." Journal of Visual Languages \& Computing 17(3):225-53.

Graph visualization is commonly used to visually model relations in many areas. Examples include Web sites, CASE tools, and knowledge representation. When the amount of information in these graphs becomes too large, users, however, cannot perceive all elements at the same time. A clustered graph can greatly reduce visual complexity by temporarily replacing a set of nodes in clusters with abstract nodes. This paper proposes a new approach to clustering graphs. The approach constructs the node similarity matrix of a graph that is derived from a novel metric of node similarity. The linkage pattern of the graph is thus encoded into the similarity matrix, and then one obtains the hierarchical abstraction of densely linked subgraphs by applying the k-means algorithm to the matrix. A heuristic method is developed to overcome the inherent drawbacks of the k-means algorithm. For clustered graphs we present a multilevel multi-window approach to hierarchically drawing them in different abstract level views with the purpose of improving their readability. The proposed approaches demonstrate good results in our experiments. As application examples, visualization of part of Java class diagrams and Web graphs are provided. We also conducted usability experiments on our algorithm and approach. The results have shown that the hierarchically clustered graph used in our system can improve user performance for certain types of tasks.

Huang, Z, et al. 2006, "Expertise Visualization: An Implementation and Study Based on Cognitive Fit Theory." Decision Support Systems 42(3):1539-57.

Expertise management systems are being widely adopted in organizations to manage tacit knowledge. These systems have successfully applied many information technologies developed for document management to support collection, processing, and distribution of expertise information. In this paper, we report a study on the potential of applying visualization techniques to support more effective and efficient exploration of the expertise information space. We implemented two widely applied dimensionality reduction visualization techniques, the self-organizing map (SOM) and multidimensional scaling (MDS), to generate compact but distorted (due to the dimensionality reduction) map visualizations for an expertise data set. We tested cognitive fit theory in our context by comparing the SOM and MDS displays with a standard table display for five tasks selected from a low-level, domain-independent visual task taxonomy. The experimental results based on a survey data set of research expertise of the business school professors suggested that using both SOM and MDS visualizations is more efficient than using the table display for the associate, compare, distinguish, and cluster tasks, but not the rank task. Users 
generally achieved comparable effectiveness for all tasks using the tabular and map displays in our study. (c) 2006 Elsevier B.V. All rights reserved.

Hubmann-Haidvogel, A, A Scharl, and A Weichselbraun. 2009, "Multiple Coordinated Views for Searching and Navigating Web Content Repositories." Information Sciences 179(12):1813-21.

The advantages and positive effects of multiple coordinated views on search performance have been documented in several studies. This paper describes the implementation of multiple coordinated views within the Media Watch on Climate Change, a domain-specific news aggregation portal available at www.ecoresearch.net/climate that combines a portfolio of semantic services with a visual information exploration and retrieval interface. The system builds contextualized information spaces by enriching the content repository with geospatial, semantic and temporal annotations, and by applying semi-automated ontology learning to create a controlled vocabulary for structuring the stored information. Portlets visualize the different dimensions of the contextualized information spaces, providing the user with multiple views on the latest news media coverage. Context information facilitates access to complex datasets and helps users navigate large repositories of Web documents. Currently, the system synchronizes information landscapes, domain ontologies, geographic maps, tag clouds and just-in-time information retrieval agents that suggest similar topics and nearby locations.

Hummel, M, et al. 2010, "Iris: Illustrative Rendering for Integral Surfaces." IEEE Transactions on Visualization and Computer Graphics 16(6):1319-28.

Integral surfaces are ideal tools to illustrate vector fields and fluid flow structures. However, these surfaces can be visually complex and exhibit difficult geometric properties, owing to strong stretching, shearing and folding of the flow from which they are derived. Many techniques for non-photorealistic rendering have been presented previously. It is, however, unclear how these techniques can be applied to integral surfaces. In this paper, we examine how transparency and texturing techniques can be used with integral surfaces to convey both shape and directional information. We present a rendering pipeline that combines these techniques aimed at faithfully and accurately representing integral surfaces while improving visualization insight. The presented pipeline is implemented directly on the GPU, providing real-time interaction for all rendering modes, and does not require expensive preprocessing of integral surfaces after computation.

Humphrey, A, C Derrick, and BR Tibbitts. 2010, "Gem : Graphical Explorer of Mpi Programs Categories and Subject Descriptors." In 5th ACM Symposium on Software Visualization (SOFTVIS), pp. 217-18.

Hundhausen, CD, SA Douglas, and JT Stasko. 2002, "A Meta-Study of Algorithm Visualization Effectiveness." Journal of Visual Languages \& Computing 13(3):259-90.

Algorithm visualization (AV) technology graphically illustrates how algorithms work. Despite the intuitive appeal of the technology, it has failed to catch on in mainstream computer science education. Some have attributed this failure to the mixed results of experimental studies designed to substantiate AV technology's educational effectiveness. However, while several integrative reviews of AV technology have appeared, none has focused specifically on the software's effectiveness by analyzing this body of experimental studies as a whole. In order to better understand the effectiveness of AV technology, we present a systematic meta-study of 24 experimental studies. We pursue two separate analyses: an analysis ofindependent variables, in which we tie each study to a particular guiding learning theory in an attempt 
to determine which guiding theory has had the most predictive success; and an analysis of dependent variables, which enables us to determine which measurement techniques have been most sensitive to the learning benefits of AV technology. Our most significant finding is that how students use AV technology has a greater impact on effectiveness than what AV technology shows them. Based on our findings, we formulate an agenda for future research into AV effectiveness.

Hung, C, and C-F Tsai. 2008, "Market Segmentation Based on Hierarchical Self-Organizing Map for Markets of Multimedia on Demand." Expert Systems with Applications 34(1):780-87.

Customer relationship management (CRM) aims at understanding and measuring the true value of customers. Market segmentation is a general method for successful CRM. This paper focuses on approaches that provide a human manager with a visualized decision making tool for market segmentation. We propose a novel market segmentation approach, namely the hierarchical self-organizing segmentation model (HSOS), for dealing with a real-world data set for market segmentation of multimedia on demand in Taiwan. HSOS is able to give a human manager a general idea of market segmentation step by step, which can be considered as a potential alternative approach to other hierarchical cluster approaches for market segmentation.

Huo, JW, W Cowan, and Acm. 2008, "Comprehending Boolean Queries." In Symposium on Applied Perception in Graphics and Visualization, pp. 179-86.

Data selection is an integral part of information visualization. It almost universally incorporates Boolean logic in some way, despite the known difficulties of Boolean concepts for users. As a result many different visual interfaces have been proposed for specifying or displaying Boolean queries. In general they are tested experimentally, and though the results are usually promising, they normally show only that one interface is better or worse than another, but not why this is so. This paper describes a new experimental methodology for evaluating query interfaces, which uses stimulus onset asynchrony to separate different aspects of query comprehension. We used it to evaluate a new visual query interface based on Karnaugh maps and discovered that there are two qualitatively different approaches to comprehension, deductive and inductive. The Karnaugh map representation scales extremely well with query complexity, and the experiments show that its good scaling properties occur because it strongly facilitates inductive comprehension.

Hur, I, and JS Yi. 2009, "Simulsort: Multivariate Data Exploration through an Enhanced Sorting Technique." In 13th International Conference on Human-Computer Interaction, pp. 684-93.

Sorting is one of the well-understood and widely-used interaction techniques. Sorting has been adopted in many software applications and supports various cognitive tasks. However, when used in analyzing multi-attribute data in a table, sorting, appears to be limited. When a table is sorted by a column, it rearranges the whole table, so the insights gained through the previous sorting arrangements of another Column are often difficult to retain. Thus, this study proposed an alternative interaction technique, called "SimulSort." By sorting all of the columns simultaneously. SimulSort helps users see an overview of the data at a glance. Additional interaction techniques, such as highlighting and zooming, were also employed to alleviate the drawbacks of SimulSort. A within-subject controlled study with 15 participants was conducted to compare SimulSort and the typical sorting feature. The results showed typical sorting and SimulSort work with comparable efficiency and effectiveness for most of the tasks. Sorting more effectively supports understanding correlation and reading corresponding values. and 
SimulSort shows the potential to more effectively support tasks that need multi-attribute analyses. The implications of the results and planned future work are discussed as well.

Hurter, C, and S Conversy. 2010, "Exploration of Aircraft Trails by Air Traffic Experts." In IEEE Conference on Information Visualization.

Hurter, C, and S Conversy. 2008, "Towards Characterizing Visualizations." In 15th International Workshop on Interactive Systems, pp. 287-93.

The ability to characterize visualizations would bring several benefits to the design process. It would help designers to assess their designs, reuse existing designs in new contexts, communicate with other designers and write compact and unambiguous specifications. The research described in this paper is an initial effort to develop a theory-driven approach to the characterization of visualizations. We examine the Card and Mackinlay characterization tool and we show its limitations when it comes to performing a complete characterization.

Hurtienne, J, et al. 2010, "Physical Gestures for Abstract Concepts: Inclusive Design with Primary Metaphors." Interacting with Computers 22(6):475-84.

Designers in inclusive design are challenged to create interactive products that cater for a wide range of prior experiences and cognitive abilities of their users. But suitable design guidance for this task is rare. This paper proposes the theory of primary metaphor and explores its validity as a source of design guidance. Primary metaphor theory describes how basic mental representations of physical sensorimotor experiences are extended to understand abstract domains. As primary metaphors are subconscious mental representations that are highly automated, they should be robustly available to people with differing levels of cognitive ability. Their proposed universality should make them accessible to people with differing levels of prior experience with technology. These predictions were tested for 12 primary metaphors that predict relations between spatial gestures and abstract interactive content. In an empirical study, 65 participants from two age groups (young and old) were asked to produce two-dimensional touch and three-dimensional free-form gestures in response to given abstract keywords and spatial dimensions of movements. The results show that across age groups in $92 \%$ of all cases users choose gestures that confirmed the predictions of the theory. Although the two age groups differed in their cognitive abilities and prior experience with technology, overall they did not differ in the amount of metaphor-congruent gestures they made. As predicted, only small or zero correlations of metaphor-congruent gestures with prior experience or cognitive ability could be found. The results provide a promising step toward inclusive design guidelines for gesture interaction with abstract content on mobile multitouch devices.

Hushyar, A. 2009, "Network Traffic Clustering and Geographic Visualization." Vol M.S., pp. 50. San Jose State University.

The exploration and analysis of large databases of information are an ever- challenging task as digital data acquisition continues to progress. The discipline of data mining has often been employed to extract structure and patterns from the underlying dataset. In addition, new research in the field of information visualization is being applied to the same challenge. Visual models engage the invaluable pattern processing abilities of the human brain which leads to new areas of insight otherwise undetected. This research applies the benefits of both data mining and information visualization to the specific problem of traffic analysis on computer networks. This is an important issue as it relates to the ability to understand 
diverse behavior on the network and provide many fundamental services. For example, distinct traffic classifications and associated traffic volumes facilitate capacity-planning initiatives. Furthermore, accurate categorization of network traffic can be leveraged by quality of service offerings and, at the same time, lend itself to efficient security analysis. In this research, an example of a data processing pipeline is described that incorporates both data mining and visualization techniques to cluster network flows and project the traffic records on a geographic display.

Hussain, F, and J Cowell. 2002, "Character Recognition of Arabic and Latin Scripts." In IEEE International Conference on Information Visualization, pp. 51-56.

The goal to produce effective optical character recognition (OCR) methods has led to the development of a number of algorithms. The purpose of these is to take the handwritten or printed text and to translate it into a corresponding digital form. The multitude requirements and developments are well represented in the literature (I.S.I. Abuhaiba et al., 1994: C.Y. Suen, 1986). The primary objective of the paper is to provide an insight into a robust system which has been successfully developed and employed to recognise Latin and Arabic characters and whose workings has been described previoulsy (J. Cowell and F. Hussain, 2000). The focus is to discuss the main components used in the multi-stage system, paying particular attention to the normalisation process used for orientation and size for a given bitmapped character. The effectiveness of the approach is demonstrated through its workings for the Arabic and Latin case, both for characters and numbers

Hussain, F, B Zalik, and S Kolmanic. 2000, "Intelligent Digitisation of Arabic Characters." In IEEE International Conference on Information Visualization, pp. 337-42.

There are a number of initiatives aimed at the introduction of the digital age to the Arab world. A leading company in this area is Sakhr Software, who have just launched their programme (SakhrMedia) to support the learning process in local educational institutions. The objective is to meet the greater requirement of computerising both the techniques and the knowledge which has thus far been developed alongside traditional platforms. In this regard efforts have been made to computerise Arabic typography and language (often referred to as Arabisation). This is vitally important not just to meet everyday needs, but also to harmonise for the Arab audience interaction with worldwide accepted application packages, as well as the Internet. The purpose of this paper is to revisit the digitising process (sometimes called rasterisation) of the most valued aspects of a language, its character set. Using a geometric modelling approach, a computer system has been developed to extract and store features of Arabic fonts. Such a process lends itself to fast reconstruction and rendering of characters, and for text recognition techniques. The discussion provides an insight into the workings of the system, with view to assisting the digitisation process for Arabic

Hvam, L, and K Ladeby. 2007, "An Approach for the Development of Visual Configuration Systems." Computers \& Industrial Engineering 53(3):401-19.

How can a visual configuration system be developed to support the specification process 1 in companies that manufacture customer tailored products? This article focuses on how visual configuration systems can be developed. The approach for developing visual configuration systems has been developed by Centre for Product Modelling (CPM) at The Technical University of Denmark. The approach is based on experiences from a visualization project in co-operation between CPM and the global provider of power protection American Power Conversion (APC). The visual configuration system was developed in 
2001-2002 and has during its operation since the beginning of 2003 delivered promising results. The leadtime for approval of quotations has been reduced and the percentages of first-time-completed and correct configuration sessions are increased thanks to an increased user-interaction, caused by the visualization of the product in the visual configuration system.

Hyungeun, J, and R Jung-hee. 2010, "Placegram: A Diagrammatic Map for Personal Geotagged Data Browsing." Visualization and Computer Graphics, IEEE Transactions on 16(2):221-34.

Geotagging personal data such as photos and videos are continuously becoming easier and more popular. Nevertheless, browsing such data on general purpose maps can be difficult, due to the frequent zoom and pan operations as well as visual components unnecessary for the task. This paper presents Placegram, a compact diagrammatic map visualization for personal geotagged data browsing based on cognitive map theories. An evaluation using real-life data sets shows that the speed of finding and pointing to places from the participants' own data increased by a factor of 2.1-2.9, and the number of interesting places discovered from others' data within a time limit increased by 48.8 percent in Placegram compared to a general purpose map. Placegram was even slightly faster than a simple text list, while at the same time, preserving the geographic senses of direction and location. Subjective ratings and comments from participants support these results, indicating that Placegram is significantly preferred over both a general map and a text list.

Hyunmo, K, et al. 2008, "Interactive Entity Resolution in Relational Data: A Visual Analytic Tool and Its Evaluation." Visualization and Computer Graphics, IEEE Transactions on 14(5):999-1014.

Databases often contain uncertain and imprecise references to real-world entities. Entity resolution, the process of reconciling multiple references to underlying real-world entities, is an important data cleaning process required before accurate visualization or analysis of the data is possible. In many cases, in addition to noisy data describing entities, there is data describing the relationships among the entities. This relational data is important during the entity resolution process; it is useful both for the algorithms which determine likely database references to be resolved and for visual analytic tools which support the entity resolution process. In this paper, we introduce a novel user interface, D-Dupe, for interactive entity resolution in relational data. D-Dupe effectively combines relational entity resolution algorithms with a novel network visualization that enables users to make use of an entity's relational context for making resolution decisions. Since resolution decisions often are interdependent, D-Dupe facilitates understanding this complex process through animations which highlight combined inferences and a history mechanism which allows users to inspect chains of resolution decisions. An empirical study with 12 users confirmed the benefits of the relational context visualization on the performance of entity resolution tasks in relational data in terms of time as well as users' confidence and satisfaction.

Inder, R, and J Stader. 1995, "Sustaining Interaction in Database Query." In Sixth International Conference on Human-Computer Interaction, pp. 711-16.

Current research on database systems breaks the problem into two halves-formulating a query and presenting the results. This paper suggests that there is a third "half" which deserves attention in its own right: sustaining the interaction. 
Ingram, S, et al. 2010, "Dimstiller : Workflows for Dimensional Analysis and Reduction." In IEEE Symposium on Visual Analytics Science \& Technology, pp. 3-10.

Inselberg, A. 2001, "Visualization and Knowledge Discovery for High Dimensional Data." In $2^{\text {nd }}$ International Workshop on User Interfaces to Data Intensive Systems, pp. 5-24.

The goal of the article is to present a multidimensional visualization methodology and its applications to visual and automatic knowledge discovery. Visualization provides insight through images and can be considered as a collection of application specific mappings: ProblemDomain\&rarr;VisuaLRange. For the visualization of multivariate problems, a multidimensional system of parallel coordinates (\|-coords) is constructed which induces a one-to-one mapping between subsets of $\mathrm{N}$-space and subsets of 2-space. The result is a rigorous methodology for doing and seeing $\mathrm{N}$-dimensional geometry. We start with an overview of the mathematical foundations where it is seen that from the display of high-dimensional datasets, the search for multivariate relations among the variables is transformed into a $2 \mathrm{D}$ pattern recognition problem. This is the basis for the application to visual knowledge discovery which is illustrated in the second part with a real dataset of VLSI production. Then a recent geometric classifier is presented and applied to 3 real datasets. The results compared to those of 23 other classifiers have the least error. The algorithm has quadratic computational complexity in the size and number of parameters, provides comprehensible and explicit rules, does dimensionality selection, and orders these variables so as to optimize the clarity of separation between the designated set and its complement. Finally a simple visual economic model of a real country is constructed and analyzed in order to illustrate the special strength of $\|$-coords in modeling multivariate relations by means of hypersurfaces

Ippolito, M, and RD Tweney. 1996, "The Inception of Insight." in The Nature of Insight, eds. RJ Sternberg and JE Davidson, pp. 433-62. MIT Press.

Isaak, MI, and MA Just. 1996, "Constraints on Thinking in Insight and Invention." in The Nature of Insight, eds. RJ Sternberg and JE Davidson, pp. 281-325. MIT Press.

Isenberg, P, and S Carpendale. 2007, "Interactive Tree Comparison for Co-Located Collaborative Information Visualization." Visualization and Computer Graphics, IEEE Transactions on 13(6):1232-39.

Isenberg, P, et al. 2010, "An Exploratory Study of Co-Located Collaborative Visual Analytics around a Tabletop Display." In IEEE Symposium on Visual Analytics Science \& Technology, pp. 179-86.

Isenberg, P, et al. 2008, "Grounded Evaluation of Information Visualizations." In Conference on BEyond time and errors: novel evaLuation methods for Information Visualization pp. 1-8.

We introduce grounded evaluation as a process that attempts to ensure that the evaluation of an information visualization tool is situated within the context of its intended use. We discuss the process and scope of grounded evaluation in general, and then describe how qualitative inquiry may be a beneficial approach as part of this process. We advocate for increased attention to the field of qualitative inquiry early in the information visualization development life cycle, as it tries to achieve a richer understanding by using a more holistic approach considering the interplay between factors that influence visualizations, their development, and their use. We present three case studies in which we successfully used observational techniques to inform our understanding of the visual analytics process in groups, medical diagnostic reasoning, and visualization use among computational linguists 
Ishikawa, Y, and M Hasegawa. 2007, "T-Scroll: Visualizing Trends in a Time-Series of Documents for Interactive User Exploration." In 11th European Conference on Digital Libraries (ECDL 2007), pp. 23546.

On the Internet, a large number of documents such as news articles and online journals are delivered everyday. We often have to review 14 major topics and topic transitions from a large time-series of documents, but it requires much time and effort to browse and analyze the target documents. We have therefore developed an information visualization system called T-Scroll (Trend/Topic-Scroll) to visualize the transition of topics extracted from those documents. The system takes periodical outputs of the underlying clustering system for a time-series of documents then visualizes the relationships between clusters as a scroll. Using its interaction facility, users can grasp the topic transitions and the details of topics for the target time period. This paper describes the idea, the functions, the implementation, and the evaluation of the T-Scroll system.

Islam, S, J Krinke, and D Binkley. 2010, "Dependence Cluster Visualization." In 5th ACM Symposium on Software Visualization (SOFTVIS), pp. 93-102.

Ito, S, and S Kunifuji. 2000, "Supporting Conversational Awareness in Text-Based Conferencing System." In 4th International Conference on Knowledge-Based Intelligent Engineering Systems and Allied Technologies, pp. 221-24.

We describe in this paper awareness support for the Text-based Conferencing System. As a disincentive factor of socially-oriented communications on the Text-based Conferencing System, the lack of visual and auditory cues concerning conversational situations is pointed out. We have developed an application of "COnversational Awareness Supporting Environment (COASE)", which supports awareness in conversational situations by extracting awareness information from the messaging history and by visualizing conversational situations for users. Through our evaluation, we learnt that COASE makes it easier to recognize the conversational situation and facilitates socially-oriented communication.

Iwata, M, et al. 2006, "Implementation and Evaluation of Programming Environment Using Real Objects for Children." In 10th International Conference on Information Visualization, pp. 817-22.

We developed a programming environment that promotes logic development in children. Dealing with the real materials in their play environments is important for children using this programming environment. Based on our analysis, we propose a concept model and the user interface for a play environment for children. We confirmed the effectiveness of the programming environment experimentally

Izquierdo, E, and V Guerra. 2002, "Video-Based Camera Registration for Augmented Reality." In IEEE International Conference on Information Visualisation, pp. 499-503.

A video-based approach for camera scene registration in augmented reality systems is presented. The presented technique relies on the definition of a model, which is derived from an appropriate parametric linear optimization problem. The optimal parameters are sought in the solution space defined by physically meaningful constraints. Solving the underlying regularized linear problem, we expect to overcome the major shortcoming observed in image-based augmented reality and telepresence systems: 
the extreme lack of robustness due to the ill-posed nature of the calibration problem. Several computer experiments have been conducted in order to assess the performance of the introduced technique

Jacobs, MK, and RL Dominowski. 1981, "Learning to Solve Insight Problems." Bulletin of the Psychonomic Society 17(4):171-74.

A series of seven insight problems was given to 28 male and 28 female college students. Each problem required that an object be used in a novel manner to reach solution, but both the critical object and its required use varied across problems. Subjects showed moderate improvement in performance on the second half of the problem series. Males solved more quickly than females; relatively stable and predictable individual differences in performance were observed. Results support the conclusions that insight problems form a class involving common abilities and that general transfer effects can occur if sufficient practice is given.

Jacquemin, C, H Folch, and S Nugier. 2006, "Ocean: 2 1/2d Interactive Visual Data Mining of Text Documents." In IEEE Conference on Information Visualization, pp. 383-88.

OCEAN is a tool for a posteriori visual data mining that uses the output of a text miner to help users better explore a document space. Clustered documents are transformed into a hierarchical 3D representation analog to reconfigurable disk trees. An intermediary document representation allows for interface customization and offers a generic approach to 3D structuring of text miner outputs. An interactive exploration is offered that avoids tree manipulation and provides local 2 frac12D view of the disks. A user evaluation is reported that outlines the benefit of controlled document hierarchy traversal and 2 frac12D document layout

Jahiruddin, M Abulaish, and L Dey. 2010, "A Concept-Driven Biomedical Knowledge Extraction and Visualization Framework for Conceptualization of Text Corpora." Journal of Biomedical Informatics 43(6):1020-35.

A number of techniques such as information extraction, document classification, document clustering and information visualization have been developed to ease extraction and understanding of information embedded within text documents. However, knowledge that is embedded in natural language texts is difficult to extract using simple pattern matching techniques and most of these methods do not help users directly understand key concepts and their semantic relationships in document corpora, which are critical for capturing their conceptual structures. The problem arises due to the fact that most of the information is embedded within unstructured or semi-structured texts that computers can not interpret very easily. In this paper, we have presented a novel Biomedical Knowledge Extraction and Visualization framework, BioKEVis to identify key information components from biomedical text documents. The information components are centered on key concepts. BioKEVis applies linguistic analysis and Latent Semantic Analysis (LSA) to identify key concepts. The information component extraction principle is based on natural language processing techniques and semantic-based analysis. The system is also integrated with a biomedical named entity recognizer, ABNER, to tag genes, proteins and other entity names in the text. We have also presented a method for collating information extracted from multiple sources to generate semantic network. The network provides distinct user perspectives and allows navigation over documents with similar information components and is also used to provide a comprehensive view of the collection. The system stores the extracted information components in a structured repository which is integrated with a query-processing module to handle biomedical queries over text documents. We have also 
proposed a document ranking mechanism to present retrieved documents in order of their relevance to the user query.

Jailani, N, et al. 2008, "Secure and Auditable Agent-Based E-Marketplace Framework for Mobile Users." Computer Standards \& Interfaces 30(4):237-52.

Mobile agent based e-marketplace auction trading requires a secure and auditable system with a solid framework to support it. In investigating the requirements, it transpired that there is a lack of such a standardised framework. While mobility helps in avoiding network latency, particularly in increasing fairness in applications with bounded response times such as trading of auctions, it nevertheless raises issues concerning security, privacy and trust in protecting personal confidential information, managing and regulating legitimate trading and payment processing. These issues are of paramount importance and must be taken into consideration when designing a framework for modelling an auditable e-marketplace for mobile users. This also implies that there is also an underlying need to provide mobile users with simple, transparent and unobtrusive user interface. This paper proposes a framework that accommodates these requirements through protocol scenarios and highlight further research work that need to be performed.

Jain, R. 2007, "Incorporating Concept Ontology for Hierarchical Video Classification, Annotation, and Visualization." IEEE Transactions on Multimedia 9(5):939-57.

Most existing content-based video retrieval (CBVR) systems are now amenable to support automatic low-level feature extraction, but they still have limited effectiveness from a user's perspective because of the semantic gap. Automatic video concept detection via semantic classification is one promising solution to bridge the semantic gap. To speed up SVM video classifier training in high-dimensional heterogeneous feature space, a novel multimodal boosting algorithm is proposed by incorporating feature hierarchy and boosting to reduce both the training cost and the size of training samples significantly. To avoid the interlevel error transmission problem, a novel hierarchical boosting scheme is proposed by incorporating concept ontology and multitask learning to boost hierarchical video classifier training through exploiting the strong correlations between the video concepts. To bridge the semantic gap between the available video concepts and the users' real needs, a novel hyperbolic visualization framework is seamlessly incorporated to enable intuitive query specification and evaluation by acquainting the users with a good global view of large-scale video collections. Our experiments in one specific domain of surgery education videos have also provided very convincing results.

Jaiswal, A, DJ Miller, and P Mitra. 2010, "Uninterpreted Schema Matching with Embedded Value Mapping under Opaque Column Names and Data Values." IEEE Transactions on Knowledge and Data Engineering 22(2):291-304.

Schema matching and value mapping across two heterogeneous information sources are critical tasks in applications involving data integration, data warehousing, and federation of databases. Before data can be integrated from multiple tables, the columns and the values appearing in the tables must be matched. The complexity of the problem grows quickly with the number of data attributes/columns to be matched and due to multiple semantics of data values. Traditional research has tackled schema matching and value mapping independently. We propose a novel method that optimizes embedded value mappings to enhance schema matching in the presence of opaque data values and column names. In this approach, the fitness objective for matching a pair of attributes from two schemas depends on the value mapping function for 
each of the two attributes. Suitable fitness objectives include the euclidean distance measure, which we use in our experimental study, as well as relative (cross) entropy. We propose a heuristic local descent optimization strategy that uses sorting and two-opt switching to jointly optimize value mappings and attribute matches. Our experiments show that our proposed technique outperforms earlier uninterpreted schema matching methods, and thus, should form a useful addition to a suite of (semi) automated tools for resolving structural heterogeneity.

Jakobsen, MR, and K Hornbaek. 2009, "Fisheyes in the Field: Using Method Triangulation to Study the Adoption and Use of a Source Code Visualization." In 27th Annual CHI Conference on Human Factors in Computing Systems, pp. 1579-88.

Information visualizations have been shown useful in numerous laboratory studies, but their adoption and use in real-life tasks are curiously under-researched. We present a field study of ten programmers who work with an editor extended with a fisheye view of source code. The study triangulates multiple methods (experience sampling, logging, thinking aloud, and interviews) to describe how the visualization is adopted and used. At the concrete level, our results suggest that the visualization was used as frequently as other tools in the programming environment. We also propose extensions to the interface and discuss features that were not used in practice. At the methodological level, the study identifies contributions distinct to individual methods and to their combination, and discusses the relative benefits of laboratory studies and field studies for the evaluation of information visualizations.

Jalote-Parmar, A, et al. 2010, "Cognitive Processes as Integrative Component for Developing Expert Decision-Making Systems: A Workflow Centered Framework." Journal of Biomedical Informatics 43(1):60-74.

The development of expert decision-making systems, which improve task performance and reduce errors within an intra-operative clinical workspace, is critically dependent on two main aspects: (a) Analyzing the clinical requirements and cognitive processes within the workflow and (b) providing an optimal context for accurate situation awareness through effective intra-operative information visualization. This paper presents a workflow centered framework and its theoretical underpinnings to design expert decision-making systems. The framework integrates knowledge of the clinical workflow based on the requirements within the clinical workspace. Furthermore, it builds upon and integrates the theory of situation awareness into system design to improve decision-making. As an application example, this framework has been used to design an intra-operative visualization system (IVS), which provides image guidance to the clinicians to perform minimally invasive procedure. An evaluative study, comparing the traditional ultrasound guided procedure with the new developed IVS, has been conducted with expert intervention radiologists and medical students. The results reveal significant evidence for improved decision-making when using the IVS. Therefore, it can be stated that this study demonstrates the benefits of integrating knowledge of cognitive processes into system development to support clinical decision-making and hence improvement of task performance and prevention of errors. (C) 2009 Elsevier Inc. All rights reserved. 
James, W, and W Elm. 2002, Supporting Operators of Next Generation Satellite Operations Centers: Providing Revolutionary Visualizations for Autonomous Operations, Variable Presentation Sizes and onCall Expertise. Technical Rpt. AFRL-HE-WP-TR-2002-0136, AEGIS RESEARCH CORP FALLS CHURCH VA.

Jamieson, R, and V Alexandrov. 2007, "A Data Forest: Multi-Dimensional Visualization." In $11^{\text {th }}$ International Conference on Information Visualization, pp. 293-300.

As terabyte datasets become the norm, the focus has shifted away from our ability to produce and store ever larger amounts of data, onto its utilization. It is becoming increasingly difficult to gain meaningful insights into the data produced. Also many forms of the data we are currently producing cannot easily fit into traditional visualization methods. This paper presents a new and novel visualization technique based on the concept of a data forest. Our data forest has been designed to be used with virtual reality (VR) as its presentation method. VR is a natural medium for investigating large datasets. Our approach can easily be adapted to be used in a variety of different ways, from a stand alone single user environment to large multi-user collaborative environments. A test application is presented using multidimensional data to demonstrate the concepts involved.

Jang, J, et al. 2005, "Punctuated Simplification of Man-Made Objects." The Visual Computer 22(2):13645.

We present a simplification algorithm for manifold polygonal meshes of plane-dominant models. Models of this type are likely to appear in man-made environments. While traditional simplification algorithms focus on generality and smooth meshes, the approach presented here considers a specific class of man-made models. By detecting and classifying edge loops on the mesh and providing a guided series of binary mesh partitions, our approach generates a series of simplified models, each of which better respects the semantics of these kinds of models than conventional approaches do. A guiding principle is to eliminate simplifications that do not make sense in constructed environments. This, coupled with the concept of "punctuated simplification", leads to an approach that is both efficient and delivers high visual quality. Comparative results are given.

Jänicke, S, et al. 2010, "Visualization of Graph Products." IEEE Transactions on Visualization and Computer Graphics 16(6):1082-9.

Graphs are a versatile structure and abstraction for binary relationships between objects. To gain insight into such relationships, their corresponding graph can be visualized. In the past, many classes of graphs have been defined, e.g., trees, planar graphs, directed acyclic graphs, and visualization algorithms were proposed for these classes. Although many graphs may only be classified as "general" graphs, they can contain substructures that belong to a certain class. Archambault proposed the TopoLayout framework: rather than draw any arbitrary graph using one method, split the graph into components that are homogeneous with respect to one graph class and then draw each component with an algorithm best suited for this class. Graph products constitute a class that arises frequently in graph theory, but for which no visualization algorithm has been proposed until now. In this paper, we present an algorithm for drawing graph products and the aesthetic criterion graph product's drawings are subject to. We show that the popular High-Dimensional Embedder approach applied to cartesian products already respects this aestetic criterion, but has disadvantages. We also present how our method is integrated as a new 
component into the TopoLayout framework. Our implementation is used for further research of graph products in a biological context.

Jankun-Kelly, TJ, K-L Ma, and M Gertz. 2007, "A Model and Framework for Visualization Exploration." IEEE Transactions on Visualization and Computer Graphics 13(2):357-69.

Visualization exploration is the process of extracting insight from data via interaction with visual depictions of that data. Visualization exploration is more than presentation; the interaction with both the data and its depiction is as important as the data and depiction itself. Significant visualization research has focused on the generation of visualizations (the depiction); less effort has focused on the exploratory aspects of visualization (the process). However, without formal models of the process, visualization exploration sessions cannot be fully utilized to assist users and system designers. Toward this end, we introduce the P-Set Model of Visualization Exploration for describing this process and a framework to encapsulate, share, and analyze visual explorations. In addition, systems utilizing the model and framework are more efficient as redundant exploration is avoided. Several examples drawn from visualization applications demonstrate these benefits. Taken together, the model and framework provide an effective means to exploit the information within the visual exploration process.

Janssen, J, G Erkens, and PA Kirschner. 2010, in press, "Group Awareness Tools: It's What You Do with It That Matters." Computers in Human Behavior.

This study examined the effect of using a group awareness tool on online collaboration. Furthermore, we examined whether the effect of using a group awareness tool on online collaboration is mediated by group awareness (i.e., students' awareness of their group members' levels of participation). To answer these questions, we determined how often and how long 107 secondary education students used the Participation-tool (PT), a group awareness tool designed to visualize group members' relative contribution to the online collaborative process. Our analyses show that duration of PT use (how long students displayed the tool on their screens) significantly predicted group members' participation in the online dialogue, their participation when writing collaborative texts, equality of participation within the group, and coordination and regulation of activities in the relational space (i.e., discussing the collaboration process with group members). No effect of using the PT on group performance was found. Mediation analyses showed that the effect of using the PT is only partially mediated by group awareness: an indirect effect of using the PT, via enhanced awareness of participation, on student participation during chat discussions and the collaborative writing process was found.

Jarupathirun, S, and FM Zahedi. 2007, "Dialectic Decision Support Systems: System Design and Empirical Evaluation." Decision Support Systems 43(4):1553-70.

Many complex and unstructured decisions are hindered by a lack of clear understanding of various underlying assumptions and perspectives involved in the decision process. At present, the traditional decision support systems (DSS) pay little attention to the elicitation of underlying assumptions and perspectives in dealing with complex issues. We argue that the Socratic dialectic inquiry is an effective method for dealing with unstructured problems that are complex and require the involvement of different perspectives in DSS. In this paper, we propose a design for Dialectic Decision Support Systems (DDSS), in which dialectical processes are integrated with traditional DSS in order to provide support for individual decision makers. We then formulate a conceptual model for identifying factors that contribute to the efficacy of DDSS in comparison to traditional DSS. The empirical test of the model supports the 
superior efficacy of the DDSS and identifies factors that contribute to it. The contributions of this research are in generating support for stimulating critical thinking, dealing with complex decision issues and identifying creative solutions.

Javed, W, and N Elmqvist. 2010, "Stack Zooming for Multi-Focus Interaction in Time-Series Data Visualization." In Pacific Visualization Symposium, pp. 33-40.

Information visualization shows tremendous potential for helping both expert and casual users alike make sense of temporal data, but current time series visualization tools provide poor support for comparing several foci in a temporal dataset while retaining context and distance awareness. We introduce a method for supporting this kind of multi-focus interaction that we call stack zooming. The approach is based on the user interactively building hierarchies of 1D strips stacked on top of each other, where each subsequent stack represents a higher zoom level, and sibling strips represent branches in the visual exploration. Correlation graphics show the relation between stacks and strips of different levels, providing context and distance awareness among the focus points. The zoom hierarchies can also be used as graphical histories and for communicating insights to stakeholders. We also discuss how visual spaces that support stack zooming can be extended with annotation and local statistics computations that fit the hierarchical stacking metaphor.

Javed, W, B McDonnel, and N Elmqvist. 2010, "Graphical Perception of Multiple Time Series." IEEE Transactions on Visualization and Computer Graphics 16(6):927-34.

Line graphs have been the visualization of choice for temporal data ever since the days of William Playfair (1759-1823), but realistic temporal analysis tasks often include multiple simultaneous time series. In this work, we explore user performance for comparison, slope, and discrimination tasks for different line graph techniques involving multiple time series. Our results show that techniques that create separate charts for each time series--such as small multiples and horizon graphs--are generally more efficient for comparisons across time series with a large visual span. On the other hand, shared-space techniques--like standard line graphs--are typically more efficient for comparisons over smaller visual spans where the impact of overlap and clutter is reduced.

Jayatilaka, B. 2003, "An Epistemological Taxonomy for Knowledge Management Systems Analysis." 36th Annual Hawaii International Conference on System Sciences, 2003. Proceedings of the:10 pp.$10 \mathrm{pp}$.

Jean, F, and AB Albu. 2010, "Vipers : Visual Prototyping Environment for Real-Time Imaging Systems." In 5th ACM Symposium on Software Visualization (SOFTVIS), pp. 211-12.

Jeong, DH, et al. 2008, "Interactive Visual Analysis of Time-Series Microarray Data." in The Visual Computer, Vol 24, pp. 1053-66. Springer-Verlag, Berlin.

Estimating dynamic regulatory pathways using DNA microarray time-series can provide invaluable information about the dynamic interactions among genes and result in new methods of rational drug design. Even though several purely computational methods have been introduced for DNA pathway analysis, most of these techniques do not provide a fully interactive method to explore and analyze these dynamic interactions in detail, which is necessary to obtain a full understanding. In this paper, we present a unified modeling and visual approach focusing on visual analysis of gene regulatory pathways over 
time. As a preliminary step in analyzing the gene interactions, the method applies two different techniques, a clustering algorithm and an auto regressive (AR) model. This approach provides a successful prediction of the dynamic pathways involved in the biological process under study. At this level, these pure computational techniques lack the transparency required for analysis and understanding of the gene interactions. To overcome the limitations, we have designed a visual analysis method that applies several visualization techniques, including pixel-based gene representation, animation, and multidimensional scaling (MDS), in a new way. This visual analysis framework allows the user to quickly and thoroughly search for and find the dynamic interactions among genes, highlight interesting gene information, show the detailed annotations of the selected genes, compare regulatory behaviors for different genes, and support gene sequence analysis for the interesting genes. In order to enhance these analysis capabilities, several methods are enabled, providing a simple graph display, a pixel-based gene visualization technique, and a relation-displaying technique among gene expressions and gene regulatory pathways.

Jeong, DH, et al. 2008, "Evaluating the Relationship between User Interaction and Financial Visual Analysis." In IEEE Symposium on Visual Analytics Science \& Technology, pp. 83-90.

It has been widely accepted that interactive visualization techniques enable users to more effectively form hypotheses and identify areas for more detailed investigation. There have been numerous empirical user studies testing the effectiveness of specific visual analytical tools. However, there has been limited effort in connecting a userpsilas interaction with his reasoning for the purpose of extracting the relationship between the two. In this paper, we present an approach for capturing and analyzing user interactions in a financial visual analytical tool and describe an exploratory user study that examines these interaction strategies. To achieve this goal, we created two visual tools to analyze raw interaction data captured during the user session. The results of this study demonstrate one possible strategy for understanding the relationship between interaction and reasoning both operationally and strategically.

Jeong, DH, et al. 2008, "Evaluating the Relationship between User Interaction and Financial Visual Analysis." In IEEE Symposium on Visual Analytics Science and Technology, pp. 83-90.

It has been widely accepted that interactive visualization techniques enable users to more effectively form hypotheses and identify areas for more detailed investigation. There have been numerous empirical user studies testing the effectiveness of specific visual analytical tools. However, there has been limited effort in connecting a user's interaction with his reasoning for the purpose of extracting the relationship between the two. In this paper, we present an approach for capturing and analyzing user interactions in a financial visual analytical tool and describe an exploratory user study that examines these interaction strategies. To achieve this goal, we created two visual tools to analyze raw interaction data captured during the user session. The results of this study demonstrate one possible strategy for understanding the relationship between interaction and reasoning both operationally and strategically. C2008 IEEE.

Jeong, DH, et al. 2010, "Comparative Evaluation of Two Interface Tools in Performing Visual Analytics Tasks." In BELIV'10: Beyond time and errors: novel evaluation methods for Information Visualization, April 10-11, 2010.

In this paper, we performed a comparative evaluation to show the effectiveness of two interface tools, one a floating text-based menu (Floating-Menu) and the other a more interactive iconic tool (InteractiveIcon). We evaluated the use and human performance of both tools within one highly interactive visual 
analytics system. During task performance we tracked completion times, task errors, and captured coarsegrained interactive behaviors.

Jeong, DH, et al. 2010, "A Continuous Analysis Process between Desktop and Collaborative Visual Analytics Environments." In IEEE Symposium on Visual Analytics Science \& Technology, pp. 231-32.

Jeong, DH, et al. 2009, "Ipca: An Interactive System for Pca-Based Visual Analytics." Computer Graphics Forum 28(3):767-74.

Principle Component Analysis (PCA) is a widely used mathematical technique in many fields for factor and trend analysis, dimension reduction, etc. However, it is often considered to be a "black box" operation whose results are difficult to interpret and sometimes counter-intuitive to the user. In order to assist the user in better understanding and utilizing PCA, we have developed a system that visualizes the results of principal component analysis using multiple coordinated views and a rich set of user interactions. Our design philosophy is to support analysis of multivariate datasets through extensive interaction with the PCA output. To demonstrate the usefulness of our system, we performed a comparative user study with a known commercial system, SAS/INSIGHT's Interactive Data Exploration. Participants in our study solved a number of high-level analysis tasks with each interface and rated the systems on ease of learning and usefulness. Based on the participants' accuracy, speed, and qualitative feedback, we observe that our system helps users to better understand relationships between the data and the calculated eigenspace, which allows the participants to more accurately analyze the data. User feedback suggests that the interactivity and transparency of our system are the key strengths of our approach.

Jeong, W-K, et al. 2010, "Interactive Histology of Large-Scale Biomedical Image Stacks." IEEE Transactions on Visualization and Computer Graphics 16(6):1386-95.

Histology is the study of the structure of biological tissue using microscopy techniques. As digital imaging technology advances, high resolution microscopy of large tissue volumes is becoming feasible; however, new interactive tools are needed to explore and analyze the enormous datasets. In this paper we present a visualization framework that specifically targets interactive examination of arbitrarily large image stacks. Our framework is built upon two core techniques: display-aware processing and GPUaccelerated texture compression. With display-aware processing, only the currently visible image tiles are fetched and aligned on-the-fly, reducing memory bandwidth and minimizing the need for time-consuming global pre-processing. Our novel texture compression scheme for GPUs is tailored for quick browsing of image stacks. We evaluate the usability of our viewer for two histology applications: digital pathology and visualization of neural structure at nanoscale-resolution in serial electron micrographs.

Jern, M, et al. 2006, "European Research Forum Panel Session - Envisioning Research Challenges in Visual Analytics - Panel Organizers: European Research Forum." In 10th International Conference on Information Visualization, pp. 5-7.

Visual Analytics is the science of analytical reasoning supported by interactive visual interfaces. People use visual analytics tools and techniques to synthesize information; derive, insight from massive, dynamic, and often conflicting data; detect the expected and discover the unexpected, provide timely, defensible, and understandable assessments; and communicate assessments effectively for action. The issues stimulating this body of research provide a grand challenge in science: turning information 
overload into the opportunity of the decade. Visual analytics requires interdisciplinary science beyond traditional scientific and information visualization to include statistics, data mining, knowledge and discovery techhologies, cognitive science and human-computer interaction, production and presentation, and more. An important research agenda "Illuminating the Path" provides recommendations for the next generation suite of visual analytics technologies and is available at http://nvac.pnl.gov/agenda.stm

Jern, M, et al. 2003, "Coordinated Views in Dynamic Interactive Documents." In International Conference on Coordinated and Multiple Views in Exploratory Visualization, pp. 95-101.

Traditional documents are characterized by the paper medium, presented in hierarchical chapter structures, passive for the reader and basically linear readable and normally restricted to static items such as text, imagery and sometimes animations. Integrating existing visualization methods with innovative documentation technology can increase the quality of information in technical documents. We have developed dynamic and interactive documents that incorporate not only text but also the entire interactive data visualisation and navigation process. Authors can produce electronic documents that collaborate and share data, analysis, visualization parameters, information and insight while distributed over Internet, using intuitive visual navigation techniques. Using multiple coordinated views in a dynamic document will further help the reader to explore complex, multivariate data and discover their relationships. Visual data navigation is provided through interactive $2 \mathrm{D}$ and $3 \mathrm{D}$ visualisation components based on scalable and customisable, multilayer component architecture with a small footprint suitable for Web distribution. A "Viewer- client-side plug-in, responsible for data interactivity and visualization, is distributed as freeware to allow free distribution of dynamic documents over am, network.

Jianu, R. 2010, "Exploring Brain Connectivity with Two-Dimensional Neural Maps." In IEEE Conference on Information Visualization, pp. 12-13.

Jianu, R, and DH Laidlaw. 2010, "Poster : Visualizing Protein Interaction Networks as Google Maps." In IEEE Conference on Information Visualization, pp. 1-2.

Jin, J, and P Szekely. 2010, "Interactive Querying of Temporal Data Using a Comic Strip Metaphor." In IEEE Symposium on Visual Analytics Science \& Technology, pp. 163-70.

Jing, L, JJ van Wijk, and JB Martens. 2009, "Evaluation of Symbol Contrast in Scatterplots." In Pacific Visualization Symposium, pp. 97-104.

Symbols are frequently used to represent data objects in visualization. An appropriate contrast between symbols is a precondition that determines the efficiency of a visual analysis process. We study the contrast between different types of symbols in the context of scatterplots, based on user testing and a quantitative model for symbol contrast. In total, 32 different symbols were generated by using four sizes, two classes (polygon-and asterisk shaped), and four categories of rotational symmetry; and used three different tasks. From the user test results an internal separation space is established for the symbol types under study. In this space, every symbol is represented by a point, and the visual contrasts defined by task performance between the symbols are represented by the distances between the points. The positions of the points in the space, obtained by multidimensional scaling (MDS), reveal the effects of different visual feature scales. Also, larger distances imply better symbol separation for visual tasks, and therefore indicate appropriate choices for symbols. The resulting configurations are discussed, and a number of 
patterns in the relation between properties of the symbols and the resulting contrast are identified. In short we found that the size effect in the space is not linear and more dominant than shape effect.

Jinwook, S, and B Shneiderman. 2006, "Knowledge Discovery in High-Dimensional Data: Case Studies and a User Survey for the Rank-by-Feature Framework." Visualization and Computer Graphics, IEEE Transactions on 12(3):311-22.

Knowledge discovery in high-dimensional data is a challenging enterprise, but new visual analytic tools appear to offer users remarkable powers if they are ready to learn new concepts and interfaces. Our three-year effort to develop versions of the hierarchical clustering explorer (HCE) began with building an interactive tool for exploring clustering results. It expanded, based on user needs, to include other potent analytic and visualization tools for multivariate data, especially the rank-by-feature framework. Our own successes using HCE provided some testimonial evidence of its utility, but we felt it necessary to get beyond our subjective impressions. This paper presents an evaluation of the hierarchical clustering explorer (HCE) using three case studies and an e-mail user survey $(n=57)$ to focus on skill acquisition with the novel concepts and interface for the rank-by-feature framework. Knowledgeable and motivated users in diverse fields provided multiple perspectives that refined our understanding of strengths and weaknesses. A user survey confirmed the benefits of HCE, but gave less guidance about improvements. Both evaluations suggested improved training methods

Jiyang, C, et al. 2007, "Visualizing Web Navigation Data with Polygon Graphs." In 11th International Conference on Information Visualization, pp. 232-37.

As the volume of digitally accessible information grows, there is increasing pressure on the development of data visualization methods to enable humans to interpret that data. We provide a description of our WebViz system, as a tool to visualize both the structure and usage of web sites. We illustrate the use of our visualization paradigm by introducing polygonal graphs layered on top of our adaptation of radial disk trees. In our system, the structure of a web segment is rendered as a radial tree, and usage data can be extracted and layered as polygonal graphs. By interactively creating and adjusting these layers, a user can develop real time insight into the data. We present the system, show the idea of interactive visual operators, and provide some examples that help show the value of the specific visualization techniques, as well as the interactive use of those techniques.

Jo, H, and JH Ryu. 2010, "Placegram: A Diagrammatic Map for Personal Geotagged Data Browsing." IEEE Transactions on Visualization and Computer Graphics 16(2):221-34.

Geotagging personal data such as photos and videos are continuously becoming easier and more popular. Nevertheless, browsing such data on general purpose maps can be difficult, due to the frequent zoom and pan operations as well as visual components unnecessary for the task. This paper presents Placegram, a compact diagrammatic map visualization for personal geotagged data browsing based on cognitive map theories. An evaluation using real-life data sets shows that the speed of finding and pointing to places from the participants' own data increased by a factor of 2.1-2.9, and the number of interesting places discovered from others' data within a time limit increased by 48.8 percent in Placegram compared to a general purpose map. Placegram was even slightly faster than a simple text list, while at the same time, preserving the geographic senses of direction and location. Subjective ratings and comments from participants support these results, indicating that Placegram is significantly preferred over both a general map and a text list. 
Joel, et al. 2010, "Interactive Vector Field Feature Identification." IEEE Transactions on Visualization and Computer Graphics 16(6):1560-8.

We introduce a flexible technique for interactive exploration of vector field data through classification derived from userspecified feature templates. Our method is founded on the observation that, while similar features within the vector field may bespatially disparate, they share similar neighborhood characteristics. Users generate feature-based visualizations by interactivelyhighlighting well-accepted and domain specific representative feature points. Feature exploration begins with the computation ofattributes that describe the neighborhood of each sample within the input vector field. Compilation of these attributes forms a representation of the vector field samples in the attribute space. We project the attribute points onto the canonical 2D plane to enableinteractive exploration of the vector field using a painting interface. The projection encodes the similarities between vector field pointswithin the distances computed between their associated attribute points. The proposed method is performed at interactive rates forenhanced user experience and is completely flexible as showcased by the simultaneous identification of diverse feature types.

Johansson, J, et al. 2008, "Perceiving Patterns in Parallel Coordinates: Determining Thresholds for Identification of Relationships." Information Visualization 7(2):152-62.

This article presents a study that investigates the ability of humans to perceive relationships (patterns) in parallel coordinates, an ability that is crucial to the use of this popular visualization technique. It introduces a visual quality metric, acceptable distortions of patterns, which establishes the level of noise that may be present in data while allowing accurate identification of patterns. This metric was used to assess perceptual performance of standard 2D parallel coordinates and multi-relational 3D parallel coordinates in two experiments. In multi-relational 3D parallel coordinates the axes are placed on a circle with a focus axis in the centre, allowing a simultaneous analysis between the focus variable and all other variables. The experiments aimed to determine the maximum number of variables that can be, from a user's point of view, efficiently used in a multi-relational 3D parallel coordinates display and to present a first attempt to study users' ability to analyse noisy data in parallel coordinates. The results show that, in terms of the acceptable level of noise in data, a multi-relational 3D parallel coordinates visualization having 11 axes (variables) is as efficient as standard 2D parallel coordinates. Visualizing a larger number of variables would possibly require a greater amount of manipulation of the visualization and thus be less efficient. Information Visualization (2008) 7, 152-162. doi: 10.1057/palgrave.ivs.9500166

Johansson, J, et al. 2003, "Visual Analysis Based on Algorithmic Classification." In 7th International Conference on Information Visualization, pp. 86-93.

Extracting actionable insight from large high dimensional data sets, and its use for more effective decision-making, has become a pervasive problem across many fields in research and industry. We describe an investigation of the application of tightly coupled statistical and visual analysis techniques to this task. The approach we choose is "unsupervised learning" where we investigate the advantages offered by close coupling of the self-organizing map algorithm with new combinations of visualization components and techniques for interactivity. 
Johansson, J, et al. 2005, "Revealing Structure within Clustered Parallel Coordinates Displays." In IEEE Symposium on Information Visualization, pp. 125-32.

In order to gain insight into multivariate data, complex structures must be analysed and understood. Parallel coordinates is an excellent tool for visualizing this type of data but has its limitations. This paper deals with one of its main limitations - how to visualize a large number of data items without hiding the inherent structure they constitute. We solve this problem by constructing clusters and using high precision textures to represent them. We also use transfer functions that operate on the high precision textures in order to highlight different aspects of the cluster characteristics. Providing predefined transfer functions as well as the support to draw customized transfer functions makes it possible to extract different aspects of the data. We also show how feature animation can be used as guidance when simultaneously analysing several clusters. This technique makes it possible to visually represent statistical information about clusters and thus guides the user, making the analysis process more efficient.

Johansson, J, TSS Neset, and BO Linner. 2010, "Evaluating Climate Visualization: An Information Visualization Approach." In 14th International Conference on Information Visualization, pp. 156-61.

To meet the growing demand of communicating climate science and policy research, the interdisciplinary field of climate visualization has increasingly extended its traditional use of 2D representations and techniques from the field of scientific visualization to include information visualization for the creation of highly interactive tools for both spatial and abstract data. This paper provides an initial discussion on the need and design of evaluations for climate visualization. We report on previous experiences and identify how evaluation methods commonly used in information visualization can be used in climate visualization to increase our understanding of visualization techniques and tools.

Johansson, P, et al. 2006, "How Something Can Be Said About Telling More Than We Can Know: On Choice Blindness and Introspection." Consciousness and Cognition 15(4):673-92.

The legacy of Nisbett and Wilson's classic article, Telling More Than We Can Know: Verbal Reports on Mental Processes (1977), is mixed. It is perhaps the most cited article in the recent history of consciousness studies, yet no empirical research program currently exists that continues the work presented in the article. To remedy this, we have introduced an experimental paradigm we call choice blindness [Johansson, P., Hall, L., Sikström, S., \& Olsson, A. (2005). Failure to detect mismatches between intention and outcome in a simple decision task. Science, 310(5745), 116-119.]. In the choice blindness paradigm participants fail to notice mismatches between their intended choice and the outcome they are presented with, while nevertheless offering introspectively derived reasons for why they chose the way they did. In this article, we use word-frequency and latent semantic analysis (LSA) to investigate a corpus of introspective reports collected within the choice blindness paradigm. We contrast the introspective reasons given in non-manipulated vs. manipulated trials, but find very few differences between these two groups of reports.

Johansson, S, et al. 2009, "Interactive Exploration of Ingredient Mixtures Using Multiple Coordinated Views." In 4th Information Visualization Conference, pp. 210-18.

The complex nature of multivariate data sets calls for high interactive performance and intuitive metaphors. A specific type of multivariate data is where the variables sum lip to a constant, here defined 
as multicomponent data. This application paper presents an interactive application for analysis of modelled multicomponent data. The aim is to find high performance variable combinations that fulfil some requested properties. The application is based on coordinated views that include parallel coordinates, a tertiary diagram, a 2D scatter plot and a line plot. It supports numerous interaction techniques enabling fast analysis of complex patterns in multicomponent data sets. The application is developed in collaboration with researchers within the fields of statistics and chemistry. An informal usability evaluation indicates that the interactive nature of the application clearly facilitates the analysis process.

Johnson, P, et al. 2002, "Visualization of Web Retrieval Results." In 15th International Conference on Computer Applications in Industry and Engineering (CAINE-2002), pp. 33-36.

Information visualization can be an effective solution to support users' search tasks to obtain desired insight from an expansive information workspace. This research has developed a visualization methodology in which Web retrieval results are transformed into intuitive visual representations. This visualization system shows distribution pattern of search terms, structural overview of the Web pages, and the characteristics of the Web resources simultaneously. Through this visual expression, users can instantly recognize related resources from; a collection of possibilities, but can also pinpoint specific segments of a resource instead of having to scan through all of it.. An experimental study has been conducted to compare this visualization method to traditional commercial search engines, and subjects have shown a high degree of satisfaction using the visual approach in presenting the search results.

Johnson, P, and S Marsland. 2010, "Beat : A Tool for Visualizing the Execution of Object Orientated Concurrent Programs." In 5th ACM Symposium on Software Visualization (SOFTVIS), pp. 225-26.

Johnson-Eilola, J, and SA Selber. 2007, "Plagiarism, Originality, Assemblage." Computers and Composition 24(4):375-403.

Although students work and live in a remix culture, composition pedagogy does not always value the discursive practices of that culture, especially when it comes to producing written work for academic contexts. The reasons for these views are historically determined and tied, at least in part, to relatively traditional notions of authorship and creativity. But "writers" in other contexts, both disciplinary and popular, have developed interesting and useful remix approaches that can aid invention, leverage intellectual and physical resources, and dramatize the social dimensions of composing in this day and age. These approaches, however, ask teachers to reconsider taken-for-granted assumptions about plagiarism and originality.

Johnson-Laird, PN. 1983. Mental Models: Towards a Cognitive Science of Language, Inference, and Consciousness. Harvard University Press, Cambridge, MA.

Jones, J, et al. 2008, "Visualizing Uncertainty for Geographical Information in Global Terrorism Database." In SPIE Defense + Security, pp. 69830E.

Presenting information on a geopolitical map can offer powerful insight into a problem by leveraging an individual's innate capacity to discover patterns and to use map-related cues to incorporate pre-existing knowledge. This mode of presentation is not without its flaws, however, as the act of placing information at specific coordinates can imply a false sense of the data's geo-spatial certainty. Traditional uncertainty 
visualization techniques, such as those that change primitive attributes or employ animation, can create large amounts of clutter or actively distract when visualizing geospatially uncertain events within large datasets. To effectively identify geo-spatial trends within the Global Terrorism Database of the START Center, we have developed a novel usage of squarified treemaps that maintains the strengths of traditional map-viewing but incorporates some measure of data verity.

Joorabchi, ME, ME Joorabchi, and CD Shaw. 2010, "Simon Fraser University ( Sfu ) - Tableau Vast 2010 Mini Challenge 2." In IEEE Symposium on Visual Analytics Science \& Technology, pp. 2-3.

Joorabchi, ME, et al. 2010, "Enron Case Study : Analysis of Email Behavior Using Emailtime." In IEEE Symposium on Visual Analytics Science \& Technology, pp. 235-36.

Joorabchi, ME, J-d Yim, and CD Shaw. 2010, "Emailtime : Visual Analytics of Emails." In IEEE Symposium on Visual Analytics Science \& Technology, pp. 233-34.

Jordan, AG. 2008, "Frontiers of Research and Future Directions in Information and Communication Technology." Technology in Society 30(3-4):388-96.

Information and communication technology (ICT), characterized by continual innovation and rapid technological change, is having a tremendous impact on society. Research and development in ICT are being conducted throughout the technology sector, and fundamental research is being carried out in many university departments of electronics, computer science, and engineering. This paper deals with frontiers of research and trends in selected areas of ICT, including computer hardware, microelectronics, and semiconductor devices and materials--areas that are leading the innovations in ICT. It briefly describes emerging technological developments, and concludes with advances in software engineering.

Jourdan, F, and G Melancon. 2003, "A Tool for Metabolic and Regulatory Pathways Visual Analysis." In 10th Annual Conference on Visualization and Data Analysis, pp. 46-55.

The research activity in bio-informatics has now reached a new phase, called post-genomics. It aims at the description of gene products as part of global processes in cells. In this research area, the various tasks to be conducted by biologists call for methods inspired from knowledge extraction and representation, and from information visualization. We describe a system devoted to the visualization of metabolic pathways. This set of biological reactions describe product transformations in the cell. The analysis of various pathway visualization tools led us to qualitative assertions. First, it is essential that the visualization environment preserves the drawing conventions borrowed from biology. Second, it seems important to offer an environment in which the user can navigate while preserving cognitive continuity. Our system focuses on these interactive and navigational issues. It offers mechanisms such as interactive color mapping and semantic zooming of pathways through various levels of details. Our tool also aims at helping biologists in the analysis of experimental results measuring gene expression in various biological processes. Although our efforts have focused on the visualization of metabolic pathways, our system should help to visualize, analyze and discover other types of biological pathways (e.g., regulatory pathways). 
Juarez-Espinosa, O. 2003, "Caeva: Cognitive Architecture to Evaluate Visualization Applications." In $7^{\text {th }}$ International Conference on Information Visualization, pp. 589-94.

We describe a cognitive architecture to evaluate visualization applications (CAEVA). This architecture requires gathering domain knowledge to adapt existing evaluation models to new domains and applications. This architecture has two main components: one for interoperability and one for cognitive models. The interoperability component uses client-server protocols. The cognitive component includes three subcomponents: knowledge about visualization, knowledge about the data domain, and knowledge about data analysis strategies. CAEVA was used to evaluate the visualizations generated to compare environmental products. The cognitive model is written in ACT-R and simulates how human beings use visualizations to perform data analysis tasks. The data produced by the simulation model is validated with empirical data obtained from human subjects performing the same task. The simulation model produces traces that allow researchers to analyze the strategies followed by the users in the task. The data produced by the model allowed us to study the learning process of visualization users.

Juarez-Espinosa, O. 2003, "Caeva: Cognitive Architecture to Evaluate Visualization Applications." In Proceedings of the Seventh International Conference on Information Visualization (IV'03).

Juhel, J. 1991, "Spatial Abilities and Individual Differences in Visual Information Processing." Intelligence 15(1):117-37.

This study explores individual differences in perfromance in visual memory and recognition tasks as a function of performance in spatial tests. One hundred students underwent a battery of spatial tests and laboratory tasks. Factor analysis conducted on the data followed by a Schmid-Leiman transformation (Schmid \& Leiman, 1957) support (a) the validity of the distinction between a spatial visualization factor and a speeded rotation factor, and (b) the assumption of a relatively low overlap between spatial tests and cognitive tasks. Different groups of subjects were then contrasted according to visual memory and spatial visualization dimensions: Study of mean latencies of these groups in visual-spatial computerized tasks established that efficiency of encoding and information organization processes were reflected by the first dimension. The second dimension would particularly correspond with the quality of information processes as well with strategic selections.

Jung, E-C, and K Sato. 2010, "Methodology for Context-Sensitive System Design by Mapping Internal Contexts into Visualization Mechanisms." Design Studies 31(1):26-45.

Visualization implies the internal mental processes of constructing internal images to interpret the world and make decisions in a situation. It is important for designers to consider users' internal processes when creating visualization tools. This research introduces the methodology of Context-Sensitive Visualization (CSV) which incorporates users' internal contexts into interactive product design by mapping out information onto the context models. In order to implement the CSV methodology, 1) representation of context models and 2) layer-based visualization mechanisms are suggested. A case study focusing on car navigation devices is conducted. A car navigation simulator with two different versions, non-CSV-based and CSV-based, is developed by utilizing the suggested methods. Usability tests are conducted to demonstrate and evaluate the proposed methodology. 
Jurgensmann, S, and H-J Schulz. 2010, "Poster : A Visual Survey of Tree Visualization." In IEEE Conference on Information Visualization.

Jusufi, I. 2010, "Poster : Interactive Navigation in Interconnected Biochemical Pathways." In IEEE Conference on Information Visualization.

Kadivar, N, et al. 2009, "Capturing and Supporting the Analysis Process." In IEEE Symposium on Visual Analytics Science \& Technology, pp. 131-38.

Visual analytics tools provide powerful visual representations in order to support the sense-making process. In this process, analysts typically iterate through sequences of steps many times, varying parameters each time. Few visual analytics tools support this process well, nor do they provide support for visualizing and understanding the analysis process itself. To help analysts understand, explore, reference, and reuse their analysis process, we present a visual analytics system named CzSaw (See-Saw) that provides an editable and re-playable history navigation channel in addition to multiple visual representations of document collections and the entities within them (in a manner inspired by Jigsaw [24]). Conventional history navigation tools range from basic undo and redo to branching timelines of user actions. In CzSaw's approach to this, first, user interactions are translated into a script language that drives the underlying scripting-driven propagation system. The latter allows analysts to edit analysis steps, and ultimately to program them. Second, on this base, we build both a history view showing progress and alternative paths, and a dependency graph showing the underlying logic of the analysis and dependency relations among the results of each step. These tools result in a visual model of the sensemaking process, providing a way for analysts to visualize their analysis process, to reinterpret the problem, explore alternative paths, extract analysis patterns from existing history, and reuse them with other related analyses.

Kaempf, C. 2004, "Visualizing Technical Information;a Cultural Critique." Professional Communication, IEEE Transactions on 47(2):152-54.

Käki, M, and A Aula. 2008, "Controlling the Complexity in Comparing Search User Interfaces Via User Studies." Information Processing \& Management 44(1):82-91.

Over time, researchers have acknowledged the importance of understanding the users' strategies in the design of search systems. However, when involving users in the comparison of search systems, methodological challenges still exist as researchers are pondering on how to handle the variability that human participants bring to the comparisons. This paper present methods for controlling the complexity of user-centered evaluations of search user interfaces through within-subjects designs, balanced task sets, time limitations, pre-formulated queries, cached result pages, and through limiting the users' access to result documents. Additionally, we will present our experiences in using three measures - search speed, qualified search speed, and immediate accuracy - to facilitate the comparison of different search systems over studies.

Käkölä, TK. 1995, "Increasing the Interpretive Flexibility of Information Systems through Embedded Application Systems." Accounting, Management and Information Technologies 5(1):79-102.

The conceptual structure of most computer-based information systems reflects a dualism of technology. During the development phase, part of the work-domain related knowledge is formalized and 
encoded in the software, making it difficult for users to reflect upon and use this knowledge. This dualism deters the interpretive flexibility of information systems. In this paper, I propose the Embedded Systems Approach, a redesign of the conceptual structure of software systems that lets users acknowledge the constructed, dual nature of information systems. In this approach, applications are embedded in extended support systems that make the organization of work and the coordinating role of information systems explicit. It also helps users handle breakdowns. The resulting systems are called Embedded Application Systems. I describe the XTEND2 prototype to illustrate the approach and to inspire commercial implementations.

Kamel, NN. 1999, "A Unified Characterisation for Shared Multimedia Cscw Workspace Designs." Information and Software Technology 41(1):1-14.

The target of this study is synchronous multimedia computer systems for co-operative work, which makes extensive use of shared workspaces, supporting both visual and audio presentations. This article develops a formal method for describing shared CSCW workspace designs through a unifying multidimensional characterisation scheme. The scheme consists of a system of taxonomic characters (TCs), and a formal notation to specify the inherent interdependencies among the design choices. This article introduces the notion, and discusses the issues of completeness and coherence of the system. The scheme is then applied to two aspects of Multimedia CSCW: shared workspaces and floor control. Particular attention is paid to provide a unified view of both the audio and visual components, by showing how they can both be treated uniformly using the same characterisaton. Finally, the notion of control shadowing for shared workspaces is presented and is described using a special notation, the notation of control whizzies.

Kampanya, N, et al. 2004, "Citiviz: A Visual User Interface to the Citidel System." In 8th International European Conference on Research and Advanced Technology for Digital Libraries(ECDL 2004), pp. 122-33.

The Digital Library (DL) field is one of the most promising areas of application for information visualization technology. In this paper, we propose a visual user interface tool kit for digital libraries, to deliver an overview of document sets, with support for interactive direct manipulation. Our system, Citiviz, employs a dynamic hyperbolic tree to display hierarchical relationships among documents, based on where their topics fit into the ACM classification system. Also, Citiviz provides an interactive, animated 2-dimensional scatter plot. With it, users may gain insight by changing various parameters, or may directly jump to a particular document based on its label or location. According to a preliminary evaluation, our system shows advantages in performance and user preference relative to traditional text based DL web interfaces.

Kandogan, E, and B Shneiderman. 2003, "Elastic Windows: A Hierarchical Multi-Window World-Wide Web Browser." in The Craft of Information Visualization, Readings and Reflections, eds. BB Benjamin and S Ben, pp. 211-19. Morgan Kaufmann, San Francisco.

Summary The World-Wide Web is becoming an invaluable source for the information needs of many users. However, current browsers are still primitive, in that they do not support many of the navigation needs of users, as indicated by user studies. They do not provide an overview and a sense of location in the information structure being browsed. Also they do not facilitate organization and filtering of information nor aid users in accessing already visited pages without high cognitive demands. In this 
paper, a new browsing interface is proposed with multiple hierarchical windows and efficient multiple window operations. It provides a flexible environment where users can quickly organize, filter, and restructure the information on the screen as they reformulate their goals. Overviews can give the user a sense of location in the browsing history as well as provide fast access to a hierarchy of pages.

Kang, H, and B Shneiderman. 2006, "Exploring Personal Media: A Spatial Interface Supporting UserDefined Semantic Regions." Journal of Visual Languages \& Computing 17(3):254-83.

Graphical mechanisms for spatially organizing personal media data could enable users to fruitfully apply their conceptual models. This paper introduces Semantic regions, an innovative way for users to construct display representations of their conceptual models by drawing regions on 2D space and specifying the semantics for each region. Then users can apply personal categorizations to personal media data using the fling-and-flock metaphor. This allows personal media to be dragged to the spatially organized display and automatically grouped according to time, geography, family trees, groups of friends, or other spatially organized display representations of conceptual models. The prototype implementation for semantic regions, MediaFinder, was refined based on two small usability tests for usage and construction of user-defined conceptual models.

Kang, HM, et al. 2008, "Interactive Entity Resolution in Relational Data: A Visual Analytic Tool and Its Evaluation." IEEE Transactions on Visualization and Computer Graphics 14(5):999-1014.

Databases often contain uncertain and imprecise references to real-world entities. Entity resolution, which is the process of reconciling multiple references to underlying real-world entities, is an important data cleaning process required before accurate visualization or analysis of the data is possible. In many cases, in addition to noisy data describing entities, there is data describing the relationships among the entities. This relational data is important during the entity resolution process; it is useful both for the algorithms that determine likely database references to be resolved and for visual analytic tools that support the entity resolution process. In this paper, we introduce a novel user interface, D-Dupe, for interactive entity resolution in relational data. D-Dupe effectively combines relational entity resolution algorithms with a novel network visualization that enables users to make use of an entity's relational context for making resolution decisions. We describe resolution strategies based on pairs or sets of references and show appropriate visualizations for each. Since resolution decisions often are interdependent, D-Dupe facilitates understanding this complex process through animations that highlight combined inferences and a history mechanism that allows users to inspect chains of resolution decisions. An empirical study with 12 users confirmed the benefits of the relational context visualization on the performance of entity resolution tasks in relational data in terms of time as well as users' confidence and satisfaction.

Kang, S, I Pourang, and B Li. 2005, "An Evaluation of Content Browsing Techniques for Hierarchical Space-Filling Visualizations." In IEEE Symposium on Information Visualization, pp. 81-88.

Space-filling visualizations, such as the TreeMap, are well suited for displaying the properties of nodes in hierarchies. To browse the contents of the hierarchy, the primary mode of interaction is by drilling down through many successive layers. In this paper we introduce a distortion algorithm based on fisheye and continuous zooming techniques for browsing data in the TreeMap representation. The motivation behind the distortion approach is for assisting users to rapidly browse information displayed in the TreeMap without opening successive layers of the hierarchy. Two experiments were conducted to 
evaluate the new approach. In the first experiment $(\mathrm{N}=20)$ the distortion approach is compared to the drill down method. Results show that subjects are quicker and more accurate in locating targets of interest using the distortion method. The second experiment $(\mathrm{N}=12)$ evaluates the effectiveness of the two approaches in a task requiring context, we define as the context browsing task. The results show that subjects are quicker and more accurate in locating targets with the distortion technique in the context browsing task.

Kang, YA, C Gorg, and J Stasko. 2010, "Pragmatic Challenges in the Evaluation of Interactive Visualization Systems." In BELIV'10: Beyond time and errors: novel evaluation methods for Information Visualization, April 10-11, 2010.

In a recent study we examined the utility of an interactive visualization system for performing investigative analysis, as compared to other more traditional methods of analysis. We used a variety of evaluation techniques, both quantitative and qualitative in nature. In this position paper we present a number of pragmatic issues that arose during the study, many of which we had encountered before. Other researchers surely will need to confront many of these same issues in their own evaluations of interactive visualization systems.

Kang, Y-a, C Gorg, and J Stasko. 2009, "Evaluating Visual Analytics Systems for Investigative Analysis: Deriving Design Principles from a Case Study." In IEEE Symposium on Visual Analytics Science and Technology, pp. 139-46.

Kang, Y-A, C Görg, and J Stasko. 2010, "How Can Visual Analytics Assist Investigative Analysis? Design Implications from an Evaluation." IEEE transactions on visualization and computer graphics Preprint.

Despite the growing number of systems providing visual analytic support for investigative analysis, few empirical studies of the potential benefits of such systems have been conducted, particularly controlled, comparative evaluations. Determining how such systems foster insight and sensemaking is important for their continued growth and study, however. Furthermore, studies that identify how people use such systems and why they benefit (or not) can help inform the design of new systems in this area. We conducted an evaluation of the visual analytics system Jigsaw employed in a small investigative sensemaking exercise, and we compared its use to three other more traditional methods of analysis. Sixteen participants performed a simulated intelligence analysis task under one of the four conditions. Experimental results suggest that Jigsaw assisted participants to analyze the data and identify an embedded threat. We describe different analysis strategies used by study participants and how computational support (or the lack thereof) influenced the strategies. We then illustrate several characteristics of the sensemaking process identified in the study and provide design implications for investigative analysis tools based thereon. We conclude with recommendations on metrics and techniques for evaluating visual analytics systems for investigative analysis. 
Kapler, T, and W Wright. 2005, "Geotime Information Visualization." Information Visualization 4(2):136-46.

Karabeg, A, MN Akkok, and K Kristensen. 2004, "Towards a Language for Talking About Information Visualization Aimed at Presentation on the Web." In 8th International Conference on Information Visualisation, pp. 930-37.

Considering variety, quantity and quality of information presentations and representations on the web two research directions emerge for us: the first one has to do with foundations of visual literacy, development of visual languages that will facilitate visual reasoning in more general setting than in problem solving and the other one has to do with the complexity of design process itself. These two directions are not separate, as visual reasoning is a fundamental attribute of design, it is a functionality inherent in visual representations, but has to be rendered by design. This implies a need for design principles whereby visual reasoning can be built into a visual representation, which in turn requires the identification of these sound design principles and a language for talking about them. In this study, we define some of the basic concepts like visual immediacy, visual impetus and visual impedance that may form the basis of the necessary vocabulary. We then discuss types of visual reasoning, extending the goal of visual reasoning from problem solving alone to a more generic goal that includes the kind of reasoning involved in visual metaphors, visual analogies, and visual associations, which all act as organizers of thinking. We then proceed towards trying to summarize, systematize and add new principles and guidelines towards better presentation of information for the web.

Karpenko, O, et al. 2010, "Exploded View Diagrams of Mathematical Surfaces." IEEE Transactions on Visualization and Computer Graphics 16(6):1311-8.

We present a technique for visualizing complicated mathematical surfaces that is inspired by handdesigned topological illustrations. Our approach generates exploded views that expose the internal structure of such a surface by partitioning it into parallel slices, which are separated from each other along a single linear explosion axis. Our contributions include a set of simple, prescriptive design rules for choosing an explosion axis and placing cutting planes, as well as automatic algorithms for applying these rules. First we analyze the input shape to select the explosion axis based on the detected rotational and reflective symmetries of the input model. We then partition the shape into slices that are designed to help viewers better understand how the shape of the surface and its cross-sections vary along the explosion axis. Our algorithms work directly on triangle meshes, and do not depend on any specific parameterization of the surface. We generate exploded views for a variety of mathematical surfaces using our system.

Kasik, DJ, et al. 2009, "Data Transformations and Representations for Computation and Visualization." Information Visualization 8(4):275-85.

At the core of successful visual analytics systems are computational techniques that transform data into concise, human comprehensible visual representations. The general process often requires multiple transformation steps before a final visual representation is generated. This article characterizes the complex raw data to be analyzed and then describes two different sets of transformations and representations. The first set transforms the raw data into more concise representations that improve the performance of sophisticated computational methods. The second transforms internal representations into visual representations that provide the most benefit to an interactive user. The end result is a computing 
system that enhances an end user's analytic process with effective visual representations and interactive techniques. While progress has been made on improving data transformations and representations, there is substantial room for improvement.

Kasprowski, R. 2004, "Humanizing Technology: Report of the 2003 Asist Annual Meeting." Serials Review 30(1):69-74.

Katifori, A, et al. 2006, "A Comparative Study of Four Ontology Visualization Techniques in Protege: Experiment Setup and Preliminary Results." In IEEE Conference on Information Visualization, pp. 41723.

The continuing need for more effective information retrieval has lead to the creation of the notions of the semantic Web and personalized information management, areas of study that very often employ ontologies to represent the semantic context of a domain. Consequently, the need for effective ontology visualization for design, management and browsing has arisen. There are several ontology visualizations available through the existing ontology management tools, but not as many evaluations to determine their advantages and disadvantages and their suitability for various ontologies and user groups. This work presents the preliminary results of an evaluation of four visualization methods in Protege

Katona, G. 1940. Organizing and Memorizing: Studies in the Psychology of Learning and Teaching. Columbia University Press, New York, NY.

Kawaguchi, T, and W Sunayama. 2008, "Visual Support System for Report Distinctiveness Evaluation." In IEEE International Conference on System, Man, and Cybernetic, pp. 295-300.

In recent years, as the Internet has grown, electronic reports have come to be used in educational organizations such as universities. Though reports written by hand must be evaluated by hand, electronic reports can be rated by computer. There are two major criteria in rating reports, correctness and distinctiveness. Correctness is rated by absolute criteria and distinctiveness is rated by relative criteria. Relative evaluation is difficult because raters should memorize all contents of submitted reports to provide objective rates. In addition, electronic data are easily copied or exchanged by students. This paper presents a report evaluation support system with which raters can compare each report and give objective rates for distinctiveness. This system evaluates each report by objective similarity criteria and visualizes them in a two-dimensional interface as the calculated distinctiveness order. Experimental results show the system is valid and effective for estimating associations between reports.

Kawamura, T, et al. 2010, "A High-Quality Sampling Technique of Pbvr for Unstructured Hexahedral Mesh Data." In IEEE Conference on Information Visualization, pp. 2-3.

Kay, P Hash, and D Dryer. 1995, "The Task, Interaction, and Display (Tid) Taxonomy for Human-Virtual Environment Interaction." in Advances in Human Factors/Ergonomics, eds. K Ogawa and M Hirohiko, Vol 20, pp. 499-504. Elsevier.

A taxonomy is proposed that classifies virtual environment tasks according to the type of task, user interaction, and display (TID) that evoke efficient human task performance. The TID can assist virtual environment designers by guiding and directing their design efforts. 
Keahey, TA. 2001, "Getting Along: Composition of Visualization Paradigms." In IEEE Symposium on Information Visualization, pp. 37-40.

Keefe, DF. 2010, "Integrating Visualization and Interaction Research to Improve Scientific Workflows." IEEE Computer Graphics and Applications 30(2):8-13.

Visualization research and interaction research have been connected for some time, and there's a growing trend toward integrating the two. Four examples illustrate the potential and challenges of this integration: 3D selection techniques in brain visualizations, interactive multiview scientific visualizations, fluid pen- and touch-based interfaces for visualization, and modeling human performance in interactive visualization-related tasks. Three goals (improving accuracy, linking multiple visualization strategies, and making data analysis more "fluid") serve as a guide for future interactive-visualization research targeted at improving visualization tools' impact on scientific workflows. (C) 2010 IEEE.

Keefe, DF, et al. 2008, "Scientific Sketching for Collaborative Vr Visualization Design." IEEE Transactions on Visualization and Computer Graphics 14(4):835-47.

We present four studies investigating tools and methodologies for artist-scientist-technologist collaboration in designing multivariate virtual reality (VR) visualizations. Design study 1 identifies the promise of 3D interfaces for rapid VR design and also establishes limitations of the particular tools tested with respect to precision and support for animation. Design study 2 explores animating artist-created visualization designs with scientific 3D fluid flow data. While results captured an accurate sense of flow that was advantageous as compared to the results of study 1 , the potential for visual exploration using the design tools tested was limited. Design study 3 reveals the importance of a new 3D interface that overcomes the precision limitation found in study 1 while remaining accessible to artist collaborators. Drawing upon previous results, design study 4 engages collaborative teams in a design process that begins with traditional paper sketching and moves to animated interactive VR prototypes "sketched" by designers in VR using interactive 3D tools. Conclusions from these four studies Identify Important characteristics of effective artist-accessible VR visualization design tools and lead to a proposed formalized methodology for successful collaborative design that we expect to be useful in guiding future collaborations. We call this proposed methodology Scientific Sketching. C 2008 IEEE.

Keim, D, et al. 2008, "Visual Analytics: Definition, Process, and Challenges." in Information Visualization, Human-Centered Issues and Perspectives, pp. 154-75. Springer-Verlag, Berlin.

Visual analytics combines automated analysis techniques with interactive visualizations for an effective understanding, reasoning and decision making on the basis of very large and complex data sets. The goal of visual analytics is the creation of tools and techniques to enable people to: synthesize information and derive insight from massive, dynamic, ambiguous, and often conflicting data, detect the expected and discover the unexpected, provide timely, defensible, and understandable assessments and communicate assessment effectively for action.Visual analytics integrates information and scientific visualization with data management and data analysis technology, as well as human perception and cognition research. 
Keim, D, et al., ed.^eds. 2010. Mastering the Information Age: Solving Problems with Visual Analytics. EuroGraphics, Goslar, Germany.

Keim, DA. 2002, "Information Visualization and Visual Data Mining." Visualization and Computer Graphics, IEEE Transactions on 8(1):1-8.

Never before in history has data been generated at such high volumes as it is today. Exploring and analyzing the vast volumes of data is becoming increasingly difficult. Information visualization and visual data mining can help to deal with the flood of information. The advantage of visual data exploration is that the user is directly involved in the data mining process. There are a large number of information visualization techniques which have been developed over the last decade to support the exploration of large data sets. In this paper, we propose a classification of information visualization and visual data mining techniques which is based on the data type to be visualized, the visualization technique, and the interaction and distortion technique. We exemplify the classification using a few examples, most of them referring to techniques and systems presented in this special section

Keim, DA, and HP Kriegel. 1994, "Visdb: Database Exploration Using Multidimensional Visualization." Computer Graphics and Applications, IEEE 14(5):40-49.

Discusses how the VisDB system supports the query specification process by representing the result visually. The main idea behind the system stems from the view of relational database tables as sets of multidimensional data where the number of attributes corresponds to the number of dimensions. In such a view, it is often unclear. In this system, each display pixel represents one database item. Pixels are arranged and colored to indicate the item's relevance to a user query and to give a visual impression of the resulting data set

Keim, DA, and HP Kriegel. 1996, "Visualization Techniques for Mining Large Databases: A Comparison." Knowledge and Data Engineering, IEEE Transactions on 8(6):923-38.

Visual data mining techniques have proven to be of high value in exploratory data analysis, and they also have a high potential for mining large databases. In this article, we describe and evaluate a new visualization-based approach to mining large databases. The basic idea of our visual data mining techniques is to represent as many data items as possible on the screen at the same time by mapping each data value to a pixel of the screen and arranging the pixels adequately. The major goal of this article is to evaluate our visual data mining techniques and to compare them to other well-known visualization techniques for multidimensional data: the parallel coordinate and stick-figure visualization techniques. For the evaluation of visual data mining techniques, the perception of data properties counts most, while the CPU time and the number of secondary storage accesses are only of secondary importance. In addition to testing the visualization techniques using real data, we developed a testing environment for database visualizations similar to the benchmark approach used for comparing the performance of database systems. The testing environment allows the generation of test data sets with predefined data characteristics which are important for comparing the perceptual abilities of visual data mining techniques 
Keim, DA, HP Kriegel, and T Seidl. 1993, "Visual Feedback in Querying Large Databases." In IEEE Conference on Visualization, pp. 158-65.

In this paper, we describe a database query system that provides visual relevance feedback in querying large databases. The goal of our system is to support the query specification process by using each pixel of the display to represent one data item of the database. By arranging and coloring the pixels according to their relevance for the query, the user gets a visual impression of the resulting data set. Using sliders for each condition of the query, the user may change the query dynamically and receives immediate feedback by the visual representation of the resulting data set. By using multiple windows for different parts of a complex query, the user gets visual feedback for each part of the query and, therefore, will easier understand the overall result. The system may be used to query any database that contains tens of thousands to millions of data items, but it is especially helpful to explore large data sets with an unknown distribution of values and to find the interesting hot spots in huge amounts of data. The direct feedback allows to visually display the influence of incremental query refinements and, therefore, allows a better, easier and faster query specification

Keim, DA, et al. 2006, "Challenges in Visual Data Analysis." In 10th International Conference on Information Visualization, pp. 9-16.

In today's applications data is produced at unprecedented rates. While the capacity to collect and store new data grows rapidly, the ability to analyze these data volumes increases at much lower pace. This gap leads to new challenges in the analysis process, since analysts, decision makers, engineers, or emergency response teams depend on information "concealed" in the data. The emerging field of visual analytics focuses on handling massive, heterogenous, and dynamic volumes of information through integration of human judgement by means of visual representations and interaction techniques in the analysis process. Furthermore, it is the combination of related research areas including visualization, data mining, and statistics that turns visual analytics into a promising field of research. This paper aims at providing an overview of visual analytics, its scope and concepts, and details the most important technical research challenges in the field

Keim, DA, F Mansmann, and J Thomas. 2009, "Visual Analytics: How Much Visualization and How Much Analytics?" SIGKDD Explor. Newsl. 11(2):5-8.

The term Visual Analytics has been around for almost five years by now, but still there are on-going discussions about what it actually is and in particular what is new about it. The core of our view on Visual Analytics is the new enabling and accessible analytic reasoning interactions supported by the combination of automated and visual analysis. In this paper, we outline the scope of Visual Analytics using two problem and three methodological classes in order to workout the need for and purpose of Visual Analytics. By examples of analytic reasoning interaction, the respective advan- tages and disadvantages of automated and visual analysis methods are explained leading to a glimpse into the future of how Visual Analytics methods will enable us to go beyond what is possible when separately using the two methods.

Keim, DA, et al. 2003, "Pushing the Limit in Visual Data Exploration: Techniques and Applications." In 26th Annual German Conference on Artificial Intelligence, pp. 37-51.

With the rapid growth in size and number of available databases, it is necessary to explore and develop new methods for analysing the huge amounts of data. Mining information and interesting 
knowledge from large databases has been recognized by many researchers as a key research topic in database systems and machine learning, and by many industrial companies as an important area with an opportunity of major revenues. Analyzing the huge amount (usually tera-bytes) of data obtained from large databases such as credit card payments, telephone calls, environmental records, census demographics, however, a very difficult task. Visual Exploration and Visual Data Mining techniques apply human visual perception to the exploration of large data sets and have proven to be of high value in exploratory data analysis. Presenting data in an interactive, graphical form often opens new insights, encouraging the formation and validation of new hypotheses to the end of better problem-solving and gaining deeper domain knowledge. In this paper we give a short overview of visual exploration techniques and present new results obtained from applying PixelBarCharts in sales analysis and internet usage management.

Keim, DA, C Panse, and M Sips. 2003, "Visual Data Mining of Large Spatial Data Sets." In

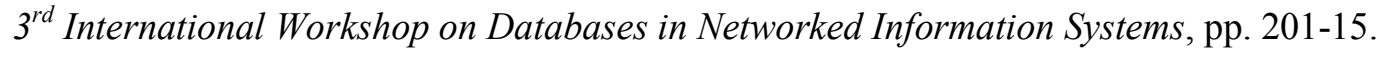

Extraction of interesting knowledge from large spatial databases is an important task in the development of spatial database systems. Spatial data mining is the branch of data mining that deals with spatial (location) data. Analyzing the huge amount (usually tera-bytes) of spatial data obtained from large databases such as credit card payments, telephone calls, environmental records, census demographics etc. is, however, a very difficult task. Visual data mining applies human visual perception to the exploration of large data sets. Presenting data in an interactive, graphical form often fosters new insights, encouraging the formation and validation of new hypotheses to the end of better problem-solving and gaining deeper domain knowledge. In this paper we give a short overview of visual data mining techniques, especially the area of analyzing spatial data. We provide some examples for effective visualizations of spatial data in important application areas such as consumer analysis, e-mail traffic analysis, and census demographics.

Keim, DA, et al. 2004, "Pixel Based Visual Data Mining of Geo-Spatial Data." Computers \& Graphics 28(3):327-44.

In many application domains, data is collected and referenced by its geo-spatial location. Spatial data mining, or the discovery of interesting patterns in such databases, is an important capability in the development of database systems. A noteworthy trend is the increasing size of data sets in common use, such as records of business transactions, environmental data and census demographics. These data sets often contain millions of records, or even far more. This situation creates new challenges in coping with scale. For data mining of large data sets to be effective, it is also important to include humans in the data exploration process and combine their flexibility, creativity, and general knowledge with the enormous storage capacity and computational power of today's computers. Visual data mining applies human visual perception to the exploration of large data sets. Presenting data in an interactive, graphical form often fosters new insights, encouraging the formation and validation of new hypotheses to the end of better problem-solving and gaining deeper domain knowledge. In this paper we give a short overview of visual data mining techniques, especially for analyzing geo-spatial data. We provide examples for effective visualizations of geo-spatial data in important application areas such as consumer analysis and census demographics. (C) 2004 Elsevier Ltd. All rights reserved. 
Keke, C, and L Ling. 2003, "A Visual Framework Invites Human into the Clustering Process." In $15^{\text {th }}$ International Conference on Scientific and Statistical Database Management, pp. 97-106.

Clustering is a technique commonly used in scientific research. The task of clustering inevitably involves human participation - the clustering is not finished when the computer/algorithm finishes but the user has evaluated, understood and accepted the patterns. This defines a human involved "clusteringanalysis/evaluation" iteration. Instead of neglecting this human involvement, we provide a visual framework (VISTA) with all power of algorithmic approaches (since their result can be visualized), and in addition we allow the user to steer/monitor/refine the clustering process with domain knowledge. The visual-rendering result also provides a precise pattern for fast post-processing.

Kellen, V, S Chan, and XW Fang. 2007, "Facilitating Conditional Probability Problems with Visuals." In 12th International Conference on Human-Computer Interaction (HCI International 2007), pp. 63-71.

In tasks such as disease diagnosis, interpretation of evidence in criminal trials and management of security and risk data, people need to process conditional probabilities to make critical judgments and decisions. As dual-coding theory and the cognitive theory of multimedia learning (CTML) would predict, visual representations (VRs) should aid in these tasks. Conditional probability problems are difficult and require subjects to build a mental model of set inclusion relationships to solve them. Evidence from neurological research confirms that mental model construction relies on visual spatial processing. Prior research has shown conflicting accounts of whether visuals aid in these problems. Prior research has also revealed that individuals differ in their ability to perform spatial processing tasks. Do visuals help solve these problems? Do visualization interface designers need to take into account the nuances of spatial processing and individual differences? This study uses a $3 \times 2$ factorial design to determine the relationship between subject's spatial abilities (high or low) and visual and text representations on user performance and satisfaction.

Keller, R, et al. 2006, "Two Sides of the Story: Visualising Products and Processes in Engineering Design." In 10th International Conference on Information Visualization, pp. 362-67.

This paper describes research on the use of multiple views for modelling products and processes in the design of complex products. Single visual representations of design models only provide a limited perspective, hiding important information from the designer. Based on an industrial case study with an engine company, the utility of multiple views in analysing model data is demonstrated. The case study showed an industrial need for improved visualisation techniques, as the currently used method -Gantt charts of design tasks - did not provide enough information in order to properly steer the design process and predict process behaviour. This approach of using a variety of different visualisation techniques together with improved techniques for modelling and simulating design processes provides insight into the hidden dependencies between the design artefact (the product) and its design process

Keller, T, et al. 2006, "Information Visualizations for Knowledge Acquisition: The Impact of Dimensionality and Color Coding." Computers in Human Behavior 22(1):43-65.

Information visualizations - interactive graphical representations of large amounts of abstract data which do not have a natural visual representation - have mainly been used to support information retrieval. This article investigates whether information visualizations are also suitable for fostering knowledge acquisition as well as how information visualizations, from a cognitive perspective, have to be 
designed to be efficient learning tools. An experimental study provided evidence that information visualizations support knowledge acquisition. In addition, with regard to the appropriate design, the empirical results showed that two-dimensional information visualizations are better suited for supporting processes of knowledge acquisition than three-dimensional ones and that color-coded information visualizations slightly increase performance in a knowledge test compared to monochromatic ones.

Keller, T, and SO Tergan. 2005, "Visualizing Knowledge and Information: An Introduction." In International Workshop on Visual Artefacts for the Organization of Information and Knowledge, Searching for Synergies, pp. 1-23.

Visualization has proven to be an effective strategy for supporting users in coping with complexity in knowledge- and information-rich scenarios. Up to now, however, information visualization and knowledge visualization have been distinct research areas, which have been developed independently of each other. This book aims toward bringing both approaches together and looking for synergies, which may be used for fostering learning, instruction, and problem solving. This introductory article seeks to provide a conceptual framework and a preview of the contributions of this volume. The most important concepts referred to in this book are defined and a conceptual rationale is provided as to why visualization may be effective in fostering, processing and managing knowledge and information. The basic ideas underlying knowledge visualization and information visualization are outlined. The preview of each approach addresses its basic concept, as well as how it fits into the conceptual rationale of the book. The contributions are structured according to whether they belong to one of the following basic categories: "Background", "Knowledge Visualization", "Information Visualization", and "Synergies".

Kemp, Z. 2005, "Chapter 25 - a Knowledge-Based Collaborative Environment for Geovisualization: Ontologies for Multiple Perspectives on Distributed Data Resources." in Exploring Geovisualization, eds. D Jason, MM Alan and K Menno-Jan, pp. 495-512. Elsevier, Oxford.

Summary This chapter contributes to the theme "connecting people, data and resources" of the ICA research agenda on visualization and virtual environments. It focuses on the semantic capabilities required to enable sharing of distributed, disparate data resources within a geo-scientific community. The chapter presents the rationale for underlying semantics to be integrated into the interface in a computational environment where the data are multi-dimensional and the geovisualization requirements open-ended. The concept of ontological hierarchies in space, time and scientific dimensions is presented using illustrative examples. The conceptual design of the interface and the architecture of the prototype system are described and examples presented. The chapter also describes other scientific visualization systems and reflects upon the underlying issues and problems.

Kenderdine, S. 2007, "Somatic Solidarity, Magical Realism and Animating Popular Gods: Place-Hampi "Where Intensities Are Felt"." In 11th International Conference Information Visualization, pp. 402-08.

Place-Hampi is an embodied theatre of participation in the drama of Hindu mythology focused at the most significant archaeological, historical and sacred locations of the World Heritage site Vijayanagara (Hampi), South India. The research described in this paper examines a history of several scopic regimes observable in Indian aesthetic traditions (principally those associated with chromolithography) and their socio-cultural implications-to articulate the somatic engagement inherent to encounters with the mythological deities in image form. Information derived from the examination of diverse scopic regimes has been used to guide the decisions in symbolic logic and high level cognitive programming of computer 
graphic characters that help co-evolve the narrative engagement between intangible heritage of 'place' and participants. Place- Hampi investigates experiences of encounter-as tangible knowledge that has implications for immersive heritage visualizations for diverse cultural audiences.

Keramopoulos, E, P Pouyioutas, and T Ptohos. 1999, "The User's View Level of the Goql Graphical Query Language." In 3rd International Conference on Information Visualisation, pp. 81-86.

The paper addresses issues related to the design of a graphical query mechanism that can act as an interface to any object-oriented database system (OODBS), in general, and the object model of ODMG 2.0 , in particular. In the paper a brief literature survey of related work is given, and an analysis methodology that allows the evaluation of such languages is proposed. Moreover, the user's view level of a new graphical query language, namely GOQL (Graphical Object Query Language), for ODMG 2.0 is presented. The user's view level provides a graphical schema that does not contain any of the perplexing details of an object-oriented database schema, and it also provides a foundation for a graphical interface that can support ad-hoc queries for object-oriented database applications. We illustrate, using an example, the user's view level of GOQL

Kerren, A. 2010, "3d Kiviat Diagrams for the Interactive Analysis of Software Metric Trends." In $5^{\text {th }}$ ACM Symposium on Software Visualization (SOFTVIS), pp. 203-04.

Keselman, A, et al. 2008, "Developing Informatics Tools and Strategies for Consumer-Centered Health Communication." Journal of the American Medical Informatics Association 15(4):473-83.

As the emphasis on individuals' active partnership in health care grows, so does the public's need for effective, comprehensible consumer health resources. Consumer health informatics has the potential to provide frameworks and strategies for designing effective health communication tools that empower users and improve their health decisions. This article presents an overview of the consumer health informatics field, discusses promising approaches to supporting health communication, and identifies challenges plus direction for future research and development. The authors' recommendations emphasize the need for drawing upon communication and social science theories of information behavior, reaching out to consumers via a range of traditional and novel formats, gaining better understanding of the public's health information needs, and developing informatics solutions for tailoring resources to users' needs and competencies. This article was written as a scholarly outreach and leadership project by members of the American Medical Informatics Association's Consumer Health Informatics Working Group.

Kessell, A, and B Tversky. 2008, "Cognitive Methods for Visualizing Space, Time, and Agents." in Diagrammatic Representation and Inference, pp. 382-84. Springer-Verlag, Berlin.

Visualizations of space, time, and agents (or objects) are ubiquitous in science, business, and everyday life, from weather maps to scheduling meetings. Effective communications, including visual ones, emerge from use in the field, but no conventional visualization form has yet emerged for this confluence of information. The real-world spiral of production, comprehension, and use that fine-tunes communications can be accelerated in the laboratory. Here we do so in search of effective visualizations of space, time, and agents. Users' production, preference, and performance aligned to favor matrix representations with time as rows or columns and space and agents as entries. Both the diagram type and the technique have broader applications. 
Khalifa, M, and V Liu. 2008, "Semantic Network Representation of Computer-Mediated Discussions: Conceptual Facilitation Form and Knowledge Acquisition." Omega 36(2):252-66.

Prior research on computer-mediated discussions examined their effects on knowledge acquisition without considering the role of the discussion representation. In this study, we investigate and compare the effect of semantic network discussion representations on knowledge acquisition to that of the threaded representations featured in most traditional discussion forums. Furthermore, we identify, define and operationalize a new, i.e., conceptual facilitation (validation of the conceptual organization of the discussion), assessing its role in knowledge acquisition at different levels of restrictiveness. The empirical results of a field experiment indicate that semantic network discussion representations enable the acquisition of more complex and better-integrated knowledge structures than threaded discussion representations. Conceptual facilitation forms entailing different levels of restrictiveness are also found to play a significant role. As the level of conceptual facilitation restrictiveness decreases, knowledge acquisition improves. Our findings empirically demonstrate the importance of accounting for discussion representation as a contingency factor in explaining group discussion processes and outcomes. To practitioners, our study provides empirical evidence on the advantages of semantic networks over threaded representations as an alternative mode for computer-mediated discussion representations. We also suggest guidelines for the selection of appropriate conceptual facilitation for discussion forums intended for knowledge acquisition.

Khan, Y, Z Xu, and M Stigant. 2003, "Virtual Reality for Neuropsychological Diagnosis and Rehabilitation: A Survey." In 7th International Conference on Information Visualization, pp. 158-63.

Recently the considerable potential of virtual reality has been recognized for the scientific study, diagnosis and treatment in the field of mental healthcare. It provides a unique opportunity to provide a natural interface and to mimic actual challenges faced by impaired subjects in their lives. In spite of certain limitations, some work has emerged which provides guidelines for future research efforts. A shortlist of such applications includes assessment and treatment of phobias; obsessive compulsive disorders, posttraumatic stress disorders and hyperactive attention deficit syndromes. We highlight these recent and ongoing research projects and compare this unique paradigm with conventional nonVR techniques by discussion of potential benefits and possible shortcomings. Due to the nature of research, ethical and human factors cannot be over emphasized. We also describe the limitations of VR in the field of practical psychology as media hype has over sold its potential. With recent advances in technology, it is not unrealistic to hope that VR will soon be a part of a clinician's armoury.

Khoury, M, and R Wenger. 2010, "On the Fractal Dimension of Isosurfaces." IEEE Transactions on Visualization and Computer Graphics 16(6):1198-205.

A (3D) scalar grid is a regular $\$ n_{-} 1 \times$ x $\_2 \times n \_3 \$$ grid of vertices where each vertex $v$ is associated with some scalar value \$s_v\$.; Applying trilinear interpolation, the scalar grid determines a scalar function $g$ where $\$ g(v)=s_{-} v \$$ for each grid vertex v. An isosurface with ;isovalue $s$ is a triangular mesh which approximates the level set $\$ \mathrm{~g}^{\wedge}\{-1\}(\& \# 945 ;) \$$. The fractal dimension of an isosurface represents the growth ; in the isosurface as the number of grid cubes increases. We dene and discuss the fractal isosurface dimension. Plotting the fractal ; dimension as a function of the isovalues in a data set provides information about the isosurfaces determined by the data set. We present statistics on the average fractal dimension of 60 publicly available benchmark data sets. We also show the fractal dimension is highly correlated with topological noise in the benchmark data sets, measuring the topological noise by the 
number of connected components in the isosurface. Lastly, we present a formula predicting the fractal dimension as a function of noise and validate the formula with experimental results.

Kieliszewski, C, et al. 2007, "A Visualization Solution for the Analysis and Identification of Workforce Expertise." In Symposium on Human Interface held at the HCI International 2007, pp. 317-26.

Keeping sight of the enterprise's workforce strengthens the entire business by helping to avoid poor decision-making and lowering the risk of failure in problem-solving. It is critical for large-scale, global enterprises to have capabilities to quickly identify subject matter experts (SMEs) to staff teams or to resolve domain-specific problems. This requires timely understanding of the kinds of experience and expertise of the people in the firm for any given set of skills. Fortunately, a large portion of the information that is needed to identify SMEs and knowledge communities is embedded in many structured and unstructured data sources. Mining and understanding this information requires non-linear processes to interact with automated tools; along with visualizations of different interrelated data to enable exploration and discovery. This paper describes a visualization solution coupled with an interactive information analytics technique to facilitate the discovery and identification of workforce experience and knowledge community capacity.

Kienle, HM, and HA Müller. 2010, "Chapter 5 - the Tools Perspective on Software Reverse Engineering: Requirements, Construction, and Evaluation." in Advances in Computers, ed. VZ Marvin, Vol 79, pp. 189-290. Elsevier.

Software reverse engineering is a subdiscipline of software engineering, striving to provide support for the comprehension of software systems by creating suitable representations of the system in another form or higher level of abstraction. In order to be effective, reverse engineering needs tool support, which provides functionality to extract low-level facts from the systems, to analyze and generate knowledge about the systems, and to visualize that knowledge so that reverse engineers are able to comprehend the aspects of the system that they are interested in effectively. This chapter explores the issue of building tools for reverse engineering. Since tools are an important part of conducting research in reverse engineering, it is worthwhile to reflect upon the state of tool building with the goal to advance upon it-and thus to advance reverse engineering research as a whole. We tackle this goal by looking at the issue of tools through a set of lenses. The purpose of each lens is to focus on a critical topic for tool building by surveying the current state-of-the-art and identifying challenges that need to be addressed. In this chapter we discuss three lenses, namely (1) requirements for reverse engineering tools, (2) construction of reverse engineering tools, and (3) evaluation of reverse engineering tools. The first lens identifies a number of generic quality attributes that reverse engineering tools should strive to meet. The second lens approaches tools from the observation that since tool building is a key activity in research, it should be conducted in an effective and rather predictable manner. The third lens looks at the role that tools play in supporting the evaluation of reverse engineering research. While each lens looks at the topic from a different perspective, taken together they provide a holistic picture of tool building in the reverse engineering domain.

Kienle, HM, and HA Müller. 2010, "Rigi--an Environment for Software Reverse Engineering, Exploration, Visualization, and Redocumentation." Science of Computer Programming 75(4):247-63.

The Rigi environment is a mature research tool that provides functionality to reverse engineer software systems. With Rigi large systems can be analyzed, interactively explored, summarized, and 
documented. This is supported with parsers to extract information from source code, an exchange format to store extracted information, analyses to transform and abstract information, a scripting language and library to automate the process, and a visualization engine to interactively explore and manipulate information in the form of typed, directed, hierarchical graphs. In this paper we describe Rigi's main components and functionalities, and assess its impact on reverse engineering research. Furthermore, we discuss Rigi's architecture and design decisions that led to a decoupling of major functionalities, and enable tool extensibility, interoperability and end-user programmability.

Kihm, J, et al. 2010, "Epidetector : Characterization of Epidemic Outbreak." In IEEE Symposium on Visual Analytics Science \& Technology, pp. 2-3.

Kim, B. 2003, "Experimental Evaluation of Query Fingerprinting with Embedded Search Term Markers." in Computational Science and Its Applications - Iccsa 2003, Pt 2, Proceedings, eds. V Kumar, et al., Vol 2668, pp. 532-41.

Previous studies have shown that visualization is an effective solution in finding relevant information from a huge collection of information. This paper presents experimental results of an information visualization system, which presents retrieved articles through visual abstraction. In order to address the difficulties in interpreting the color codes, the Query Fingerprinting system has been reinforced with embedded search term markings on the color codes. For a non-biased evaluation of the developed algorithms, a series of experiments has been executed on TREC-5 text collection with their corresponding queries and relevance judgments. The results show that the participants who utilized the developed method in judging the relevancy of articles showed better performance while maintaining the accuracy than the users who utilized a system based on the traditional approach. The participants also have shown a high degree of satisfaction with the search term markings in understanding the distributional patterns of queries. The experiment acknowledges the feasibility of the Query Fingerprinting with embedded search term markers as a search-supporting tool, which assists users' decision-making processes and lessens the search activities.

Kim, C. 2009, "Spatial Data Mining, Geovisualization." in International Encyclopedia of Human Geography, eds. K Rob and T Nigel, pp. 332-36. Elsevier, Oxford.

Geovisualization in spatial data mining is one of the main methods that has recently been the subject of knowledge discovery research in geographic information science. Geovisualization is often referred to as knowledge discovery in that it produces previously unseen patterns from a larger set of data. Due to the increase in geospatial data, any techniques that can shift through large sets of data quickly and efficiently are in high demand. Geovisualization uses visual representations to facilitate thinking, understanding, and knowledge construction about human and physic environments, at geographic scales of measurement. It augments human visual ability in perceiving high complex structures, and detecting, exploring, and exploiting significant patterns. It integrates scientific visualization with traditional cartography, and can be utilized at data pre-processing, spatial data mining, and knowledge construction. The main purpose of geovisualization, however, is on insight rather than maps. Research needs in geovisualization are extensive as follows: geovisulation in spatiotemporal databases, the automated discovery of spatial knowledge, geovisualization in remote-sensing data and spatial object-oriented databases, effective geovisualizations of spatial relationships, and efficient geocomputation. 
Kim, HN. 2008, "The Phenomenon of Blogs and Theoretical Model of Blog Use in Educational Contexts." Computers \& Education 51(3):1342-52.

Many educators have attempted to implement a blog in educational contexts to enhance the communication environment among students and teachers. However, it is uncertain as to why traditional computer-mediated communication (CMC) applications should be replaced with blogs. It is time to comprehensively explore the effects of educational blogs by considering the CMC tools. This paper reviews prior studies and develops a model for the use of blogs in educational contexts by taking into account socio-technical systems theory. The model contributes to interactivity, an open system, a visualization tool, and a decentralized environment of online communication circumstance.

Kim, J, et al. 2008, "Multimedia for Future Health--Smart Medical Home." in Biomedical Information Technology, pp. 497-512. Academic Press, Burlington.

Summary With recent advances in multimedia technology, its impact toward information technology in biomedicine is ever increasing [1-3]. Multimedia technologies are enabling more comprehensive and intuitive uptake of information in a wide range of Welds that have a direct impact on our life, particularly in entertainment, education, work, and health. Systems and services have been developed to harness the advantages of multimedia technology, which ranges from video-conferencing, online shopping in virtual environments, video-on-demand services and E-learning to remote healthcare $[1,4,5]$. The core components behind these multimedia technologies are human-centered multimedia services, which combine many Welds of information technology including computing, telecommunication, databases, mobile devices, sensors, and virtual/ augmented reality systems. Human-centered multimedia services are built upon three key research pillars as shown in Figure 23.1. These are (1) human-computer interaction (HCI); (2) multimedia delivery; and (3) multimedia data management. HCI (e.g., via the use of keyboard/mouse input devices) is the initial component of the multimedia information Xow with the responsibility of generating outputs by interpreting inputs from the users. Multimedia delivery systems (e.g., the Internet) are responsible for transparent information delivery (e.g., streaming video) from sources to destinations. Finally, the multimedia data management components facilitate information access (e.g., browsing, retrieval, and indexing).

Kim, S, H Hagh-Shenas, and V Interrante. 2004, "Conveying Shape with Texture: Experimental Investigations of Texture's Effects on Shape Categorization Judgments." Visualization and Computer Graphics, IEEE Transactions on 10(4):471-83.

We describe the results of two comprehensive controlled observer experiments intended to yield insight into the following question: If we could design the ideal texture pattern to apply to an arbitrary smoothly curving surface in order to enable its 3D shape to be most accurately and effectively perceived, what would the characteristics of that texture pattern be? We begin by reviewing the results of our initial study in this series, which were presented at the 2003 IEEE Symposium on Information Visualization, and offer an expanded analysis of those findings. We continue by presenting the results of a follow-on study in which we sought to more specifically investigate the separate and combined influences on shape perception of particular texture components, with the goal of obtaining a clearer view of their potential information carrying capacities. In each study, we investigated the observers' ability to identify the intrinsic shape category of a surface patch (elliptical, hyperbolic, cylindrical, or flat) and its extrinsic surface orientation (convex, concave, both, or neither). In our first study, we compared performance under eight different texture type conditions, plus two projection conditions (perspective or orthographic) 
and two viewing conditions (head-on or oblique). We found that: 1) shape perception was better facilitated, in general, by the bidirectional "principal direction grid" pattern than by any of the seven other patterns tested; 2) shape type classification accuracy remained high under the orthographic projection condition for some texture types when the viewpoint was oblique; 3 ) perspective projection was required for accurate surface orientation classification; and 4) shape classification accuracy was higher when the surface patches were oriented at a (generic) oblique angle to the line of sight than when they were oriented (in a nongeneric pose) to face the viewpoint straight on. In our second study, we compared performance under eight new t- - exture type conditions, redesigned to facilitate gathering insight into the cumulative effects of specific individual directional components in a wider variety of multidirectional texture patterns. We found that shape classification accuracy was equivalently good under a variety of test patterns that included components following either the first or first and second principal directions, in addition to other directions, suggesting that a principal direction grid texture is not the only possible "best option" for enhancing shape representation.

Kim, S, et al. 2008, "Mobile Analytics for Emergency Response and Training." Information Visualization $7(1): 77-88$.

During emergency response events, situational awareness is critical in effectively managing and safeguarding civilians and in-field personnel. To better support both command center controllers and in-field operators, we have developed a mobile visual analytics tool to help enhance situational awareness and support rapid decision making. Our mobile visual analytics tool consists of a 2D/3D visualization component, which shows personnel-related information, situational and static scene-related information, integrated multi media playback functionality for personnel outfitted with cameras, and fastforward/rewind capabilities for reviewing events. Our current system has been employed in the evaluation of two different scenarios: a simulated evacuation of The Station nightclub fire that occurred in Rhode Island during 2003 and a testing exercise for a rescue operation in an elementary school. Our system has been deployed on a Dell Axim X51v PDA, an OQO 02, and on a Sprint PCS VisionSM smart device

Kim, SJ, et al. 2010, "Interactive Visualization of Hyperspectral Images of Historical Documents." IEEE Transactions on Visualization and Computer Graphics 16(6):1441-8.

This paper presents an interactive visualization tool to study and analyze hyperspectral images (HSI) of historical documents. This work is part of a collaborative effort with the Nationaal Archief of the Netherlands (NAN) and Art Innovation, a manufacturer of hyperspectral imaging hardware designed for old and fragile documents. The NAN is actively capturing HSI of historical documents for use in a variety of tasks related to the analysis and management of archival collections, from ink and paper analysis to monitoring the effects of environmental aging. To assist their work, we have developed a comprehensive visualization tool that offers an assortment of visualization and analysis methods, including interactive spectral selection, spectral similarity analysis, time-varying data analysis and visualization, and selective spectral band fusion. This paper describes our visualization software and how it is used to facilitate the tasks needed by our collaborators. Evaluation feedback from our collaborators on how this tool benefits their work is included. 
Kim, SY, et al. 2007, "Visual Analytics on Mobile Devices for Emergency Response." In Symposium on Visual Analytics, pp. 35-42.

During emergency response events, situational awareness is critical in effectively managing and safeguarding civilians and in-field personnel. To better support both command center controllers and in-field operators, we have developed a mobile visual analytics tool to help enhance situational awareness and support rapid decision making. Our mobile visual analytics tool consists of a 2D/3D visualization component, which shows personnel-related information, situational and static scene-related information, integrated multi media playback functionality for personnel outfitted with cameras, and fastforward/rewind capabilities for reviewing events. Our current system has been employed in the evaluation of two different scenarios: a simulated evacuation of The Station nightclub fire that occurred in Rhode Island during 2003 and a testing exercise for a rescue operation in an elementary school

Kim, YJ, and CM Hoffmann. 2003, "Enhanced Battlefield Visualization for Situation Awareness." Computers \& Graphics 27(6):873-85.

We present tools to visualize density, clustering, and lethality assessment that help a military commander achieve situation awareness on the battlefield. For the density computation, we provide a geometry-based, image-based, and hybrid approach to tackle the problem. We choose proximity-based clustering as a suitable definition for our application. Based on this clustering definition, we show an efficient computational method based on the Delaunay triangulation. Finally, we present a probabilistic model for the lethality assessment and an efficient way to compute it using hardware support where available. We also explain how to deliver visual information to the human visual system effectively. The principles of human visual perception, such as preattentive and Gestalt perceptual processing, play an important role in this work.

Kimani, S, et al. 2004, "Vidamine: A Visual Data Mining Environment." Journal of Visual Languages \& Computing 15(1):37-67.

That the already vast and ever-increasing amounts of data still do present formidable challenges to effective and efficient acquisition of knowledge is by no means an exaggeration. The knowledge discovery process entails more than just the application of data mining strategies. There are many other aspects including, but not limited to: planning, data pre-processing, data integration, evaluation and presentation. The human-vision channel is capable of recognizing and understanding data at an instant. Effective visual strategies can be used to tap the outstanding human visual channel in extracting useful information from data. Unlike is the case with most research efforts, the exploitation should be employed not just at the beginning or at the end of the knowledge discovery process but across the entire discovery process. In essence, this calls for the development of an effective user/visual component, the development of an overall framework that can support the entire discovery process/all discovery phases, and the strategic placement of the visual component in that framework. Key issues of this component will be the open architecture, allowing extensions and adaptations to specific mining environments, and the precise semantics and syntax, allowing an optimal integration between the presentation and the computation. 
Kincaid, R. 2010, "Signallens: Focus+Context Applied to Electronic Time Series." IEEE Transactions on Visualization and Computer Graphics 16(6):900-7.

Electronic test and measurement systems are becoming increasingly sophisticated in order to match the increased complexity and ultra-high speed of the devices under test. A key feature in many such instruments is a vastly increased capacity for storage of digital signals. Storage of $\$ 10^{\wedge} 9 \$$ time points or more is now possible. At the same time, the typical screens on such measurement devices are relatively small. Therefore, these instruments can only render an extremely small fraction of the complete signal at any time. SignalLens uses a Focus + Context approach to provide a means of navigating to and inspecting low-level signal details in the context of the entire signal trace. This approach provides a compact visualization suitable for embedding into the small displays typically provided by electronic measurement instruments. We further augment this display with computed tracks which display time-aligned computed properties of the signal. By combining and filtering these computed tracks it is possible to easily and quickly find computationally detected features in the data which are often obscured by the visual compression required to render the large data sets on a small screen. Further, these tracks can be viewed in the context of the entire signal trace as well as visible high-level signal features. Several examples using real-world electronic measurement data are presented, which demonstrate typical use cases and the effectiveness of the design.

King-Smith, D, et al. 2009, "An Affordable Wearable Video System for Emergency Response Training." Proceedings of SPIE 7256(1):72560E-60E-12.

Many emergency response units are currently faced with restrictive budgets that prohibit their use of advanced technology-based training solutions. Our work focuses on creating an affordable, mobile, stateof-the-art emergency response training solution through the integration of low-cost, commercially available products. The system we have developed consists of tracking, audio, and video capability, coupled with other sensors that can all be viewed through a unified visualization system. In this paper we focus on the video sub-system which helps provide real time tracking and video feeds from the training environment through a system of wearable and stationary cameras. These two camera systems interface with a management system that handles storage and indexing of the video during and after training exercises. The wearable systems enable the command center to have live video and tracking information for each trainee in the exercise. The stationary camera systems provide a fixed point of reference for viewing action during the exercise and consist of a small Linux based portable computer and mountable camera. The video management system consists of a server and database which work in tandem with a visualization application to provide real-time and after action review capability to the training system.

Kinnaird, P, and M Romero. 2010, "Focus Groups for Functional Infovis Prototype Evaluation: A Case Study." In BELIV'10: Beyond time and errors: novel evaluation methods for Information Visualization, April 10-11, 2010.

In this position paper, we describe our experience conducting a focus group for evaluating an Information Visualization system prototype. We concentrate on the method used and how it differs from traditional focus group methodology. Our position is that Information Visualization system prototypes provide exceptional grounds for customized focus group methodologies due to the exploratory nature of many of the tasks these systems are designed to support. 
Kira, A, DM Nichols, and M Apperley. 2009, "Human Communication in Customer-Agent-Computer Interaction: Face-to-Face Versus over Telephone." Computers in Human Behavior 25(1):8-20.

Customer service can be provided over various communication modes, such as face-to-face, telephone, email or websites. In this paper we examine a setting where a service, such as travel planning, is provided to a customer through a human agent, either face-to-face or via telephone. Specifically, the setting requires three entities, a customer who has approached a business, a representative for the organisation and a computer which the representative uses to support the task. Two experiments were conducted to investigate how the two human entities interact over two different communication modes (face-to-face and telephone) when there is also a computer involved in the interaction. The results showed a significantly shorter task completion time via telephone. There was also a difference in the style of communication, with face-to-face having more single activities (such as talking only), while when using the telephone there was more doubling up in activities (talking while also searching on the computer). There was only a small difference in subjective satisfaction. The results suggest that telephone interaction, although containing fewer communication cues (such as body language), is not necessarily an impoverished mode. Telephone interaction is less time consuming and more task-focused.

Kirschenbauer, S. 2005, "Chapter 18 - Applying "True 3d" Techniques to Geovisualization: An Empirical Study." in Exploring Geovisualization, eds. D Jason, MM Alan and K Menno-Jan, pp. 363-87. Elsevier, Oxford.

Summary "True 3D" techniques have a fairly long history but have been comprehensively adapted to geovisualization only recently. True 3D describes techniques such as stereoscopic displays, anaglyphs, immersive workbenches and holograms, in which the visualized third dimension appears either behind or in front of the display plane. Inspired by developments in technology, the variety of true 3D representations means that this has become an increasingly important research topic. Yet, this particular domain is lacking in fundamental theory. This chapter gives an overview of true 3D techniques applied to geovisualization and reviews relevant issues such as 3D perception. An empirical study was conducted, investigating the impact on different users of the true third dimension realized by an autostereoscopic display. The experiment may be used as an example of how a structured investigation can offer deeper insights into the map-reading process in order to expose beneficial properties of a true 3D technique and its stereo-image, respectively. The results are discussed as they relate to the usefulness of such true 3D visualizations. Three characteristics are found to be particularly relevant to successful map reading when using true 3D displays of spatial information. These include a human user's visual capacities, their level of experience and the purpose of the visualization.

Kirschner, P, and P Gerjets. 2006, "Instructional Design for Effective and Enjoyable Computer-Supported Learning." Computers in Human Behavior 22(1):1-8.

This special issue is dedicated to a number of efforts being made by researchers - primarily in Europe - to make learning more effective AND more enjoyable. The basis of this Special Issue lies in a conference organized by two Special Interest Groups (Instructional Design (SIG 6) and Learning and Instruction with Computers (SIG 7)) of the European Association for Research in Learning and Instruction (EARLI) held July 2004 at the Knowledge Media Research Center in Tuebingen, Germany (http://www.iwm-kmrc.de). 
Kirste, T, and H Schumann. 2001, "Intelligent Interactive Assistance and Mobile Multimedia Computing." Computers \& Graphics 25(4):551-53.

Kjelldahl, L, and BS Santos. 2004, "Visual Perception in Computer Graphics Courses." Computers \& Graphics 28(3):451-56.

Visual perception and the human visual system are important for the judgements of the results in computer graphics. So it is important to address this in computer graphics courses. We describe the experiences of including those features into graphics courses and from our experiences we give examples and proposals as to how an introduction to perception can be done. A bibliography with comments is also included in the paper.

Kjellin, A, et al. 2010, "Different Levels of 3d: An Evaluation of Visualized Discrete Spatiotemporal Data in Space-Time Cubes." Information Visualization 9(2):152-64.

New technologies and techniques allow novel kinds of visualizations and different types of 3D visualizations are constantly developed. We propose a categorization of $3 \mathrm{D}$ visualizations and, based on this categorization, evaluate two versions of a space-time cube that show discrete spatiotemporal data. The two visualization techniques used are a head-tracked stereoscopic visualization ('strong 3D') and a static monocular visualization ('weak 3D'). In terms of effectiveness and efficiency the weak 3D visualization is as good as the strong 3D and thus the need for advanced 3D visualizations in these kinds of tasks may not be necessary. Information Visualization (2010) 9, 152-164. doi:10.1057/ivs.2009.8; published online 11 June 2009

Klein, B, et al. 2004, "Enabling Flow - a Paradigm for Document-Centered Personal Information Spaces." in Proceedings of the Eighth Iasted International Conference on Artificial Intelligence and Soft Computing, pp. 187-92.

We introduce a futuristic vision of a Document-centered Personal Information Space. However, even without the futuristic part of the vision, the underlying paradigm can instantly improve the conditions for a phenomenon at work, called flow. Flow provides those who enter into it, with a high efficiency and satisfaction at work. The vision and its required preconditions are considered a paradigm, for which a corresponding framework is provided, broken down into a structure of four main elements.

Klein, P, et al. 2003, "Metadata Visualisation with Vismeb." In 7th International Conference on Information Visualization (IV 2003), pp. 600-05.

We present a new framework for metadata visualisation systems called VisMeB (visual metadata browser). It is based upon redesign ideas from the IN-SYDER system that were come under extensive evaluations. The aim of our approach is to improve the process of finding relevant information in an intuitive yet multifunctional way. We use a ScatterPlot in combination with a so called SuperTable for visualisation. The two techniques are tightly coupled and present unique possibilities of interaction through the use of visual filters. 
Klimov, D, Y Shahar, and M Taieb-Maimon. 2010, "Intelligent Visualization and Exploration of TimeOriented Data of Multiple Patients." Artificial Intelligence in Medicine 49(1):11-31.

Objective: Clinicians and medical researchers alike require useful, intuitive, and intelligent tools to process large amounts of time-oriented multiple-patient data from multiple sources. For analyzing the results of clinical trials or for quality assessment purposes, an aggregated view of a group of patients is often required. To meet this need, we designed and developed the VISualizatIon of Time-Oriented RecordS (VISITORS) system, which combines intelligent temporal analysis and information visualization techniques. The VISITORS system includes tools for intelligent retrieval, visualization, exploration, and analysis of raw time-oriented data and derived (abstracted) concepts for multiple patient records. To derive meaningful interpretations from raw time-oriented data (known as temporal abstractions), we used the knowledge-based temporal-abstraction method. Methods: The main module of the VISITORS system is an interactive, ontology-based exploration module, which enables the user to visualize raw data and abstract (derived) concepts for multiple patient records, at several levels of temporal granularity; to explore these concepts; and to display associations among raw and abstract concepts. A knowledge-based delegate function is used to convert multiple data points into one delegate value representing each temporal granule. To select the population of patients to explore, the VISITORS system includes an ontology-based temporal-aggregation specification language and a graphical expression-specification module. The expressions, applied by an external temporal mediator, retrieve a list of patients, a list of relevant time intervals, and a list of time-oriented patients' data sets, by using an expressive set of time and value constraints. Results: Functionality and usability evaluation of the interactive exploration module was performed on a database of more than 1000 oncology patients by a group of 10 users five clinicians and five medical informaticians. Both types of users were able in a short time (mean of $2.5+/-$ 0.2 min per question) to answer a set of clinical questions, including questions that require the use of specialized operators for finding associations among derived temporal abstractions, with high accuracy (mean of $98.7+/-2.4$ on a predefined scale from 0 to 100). There were no significant differences between the response times and between accuracy levels of the exploration of the data using different time lines, i.e., absolute (i.e., calendrical) versus relative (referring to some clinical key event). A system usability scale (SUS) questionnaire filled out by the users demonstrated the VISITORS system to be usable (mean score for the overall group: 69.3), but the clinicians' usability assessment was significantly lower than that of the medical informaticians. Conclusions: We conclude that intelligent visualization and exploration of longitudinal data of multiple patients with the VISITORS system is feasible, functional, and usable. (C) 2010 Elsevier B.V. All rights reserved.

Klingner, J, R Kumar, and P Hanrahan. 2008, "Measuring the Task-Evoked Pupillary Response with a Remote Eye Tracker." In Proceedings of the 2008 symposium on Eye tracking research \& applications ETRA'08, pp. 69-69.

The pupil-measuring capability of video eye trackers can detect the task-evoked pupillary response: subtle changes in pupil size which indicate cognitive load. We performed several experiments to measure cognitive load using a remote video eye tracker, which demonstrate two extensions to current research in this area. First, we show that cognitive pupillometry can be extended from head-mounted to remote eye tracking systems. Second, we demonstrate the feasibility of a more fine-grained approach to analyzing pupil size data gathered with an eye tracker, which provides more detail about the timing and magnitude of changes in cognitive load. 
Klippel, A, et al. 2009, "Colour-Enhanced Star Plot Glyphs: Can Salient Shape Characteristics Be Overcome?" Cartographica: The International Journal for Geographic Information and Geovisualization 44(3):217-31.

This article reports two experiments addressing the question of how the shape characteristics of star plot glyphs influence classification tasks and whether additional graphic features such as colour can be used to counterbalance the effects of shape characteristics. In a previous study we found that salient shapes of star plot glyphs, such as "has one spike," influence the classification of the data represented by the glyphs. The shape differences in star plot glyphs are induced by assigning variables to rays in different ways. The first two experiments showed shape influences; we then conducted two follow-up studies to shed more light on the influence of shape. First, to address the question of how the classification of star plot glyphs would be affected if they were stripped of their meaning, participants were asked to group star plot glyphs as shapes. The second study colour-coded the rays of star plot glyphs to focus attention on differences in salient shapes; for example, the general shape characteristic "has one spike" cannot be applied so easily if each spike is a different colour. The results show that colour-enhancing star plot glyphs improves processing speed and reduces the influence of salient shape characteristics.

Klippel, A, F Hardisty, and C Weaver. 2009, "Star Plots: How Shape Characteristics Influence Classification Tasks." Cartography and Geographic Information Science 36(2):149-63.

Klippel, A, et al. 2008, "Wayfinding Choremes 2 . 0 -Conceptual Primitives as a Basis for Translating Natural into Formal Language." In International Workshop on Moving Objects From Natural to Formal Language, pp. 1-11.

Continuous spatial and temporal information is cognitively segmented into meaningful units that we refer to here as conceptual primitives; since we are focusing on routes in street networks, we define these primitives more specifically as wayfinding choremes. For the case of movement patterns in constraining environments such as driving around in street networks the wayfinding choremes are coupled with decision points that can offer alternative path choices or structure and/ or confirm a specific part of the route. To be able to analyze these choremes we have conducted a series of experiments in the past that focused on how a movement pattern can be characterized linguistically. Here, we apply the insights we gained to analyze and interpret linguistically characterized movement patterns to questions of automatically identifying movement patterns in text. That means we are analyzing verbal route directions and identify wayfinding choremes to infer the underlying movement patterns. To support our long term goal of automatic extraction of wayfinding choremes from text documents, we formally specify a taxonomy of wayfinding choremes as a first step for an ontological analysis. We will present the theoretical underpinning, tools, first results, and our perspectives on the linguistic analysis of movement patterns in this contribution. We hope to contribute to a better understanding of how natural language descriptions and formal characterizations can be closer related and can inform the automatic extraction of movement patterns from text.

Klump, RP, and JD Weber. 2002, "Real-Time Data Retrieval and New Visualization Techniques for the Energy Industry Powerworld Corporation Visualization Techniques." In 35th Annual Hawaii International Conference on System Sciences, pp. 712-17.

Effective power system operation requires power system engineers and operators to analyze vast amounts of information. The authors have developed several techniques for visualizing large amounts of 
data including contouring, animated flows, and 3D visualization. These techniques have been completely integrated into PowerWorld Simulator, an offline system study tool for use in the energy industry.

Integrating these visualization techniques with real-time data sources, such as those available in energy control centers, however, has presented a separate challenge to the wide dissemination of these visualization abilities. The paper presents a case study of integrating these abilities with control centers that utilize the Plant Information (PI) System for data storage and archival. Projects have been completed that integrate these visualization techniques into control centers for Commonwealth Edison in Lombard, Illinois and City Water Light and Power in Springfield, Illinois. A separate pilot project has also been completed with the Tennessee Valley Authority.

Knautz, K, S Soubusta, and WG Stock. 2010, "Tag Clusters as Information Retrieval Interfaces." In $43^{\text {rd }}$ Hawaii International Conference on Systems Sciences (HICSS 2010), pp. 1577-86.

The paper presents our design of a next generation information retrieval system based on tag cooccurrences and subsequent clustering We help users getting access to digital data through information visualization in the form of tag clusters Current problems like the absence of interactivity and semantics between tags or the difficulty of adding additional search arguments are solved In the evaluation, based upon SERVQUAL and IT systems quality indicators, we found out that tag clusters are perceived as more useful than tag clouds, are much more trustworthy, and are more enjoyable to use

Knipp, T. 2003, "Creative Performance: Does the Computer Retard Artistic Development?" In $7^{\text {th }}$ International Conference on Information Visualization, pp. 621-25.

Over the last decade, there have been several debates concerning the dominance of technology in academic disciplines such as fine arts and graphic design. Advanced imaging devices, such as fMRI and PET scans, have provided researchers with visual information in the field of brain science that may settle these debates. We outline a research study conducted among undergraduate art students to investigate the level of visual perception and creative development. The purpose is to gather basic information that would merit further scientific investigation. Among the groups studied, the research concluded that artistic traits such as visual memory, perception and a critical eye for detail declined $80 \%$ as compared to research studies conducted in 1944 . We present scientific research in the field of cognition and brain science, suggesting dominant use of technology in the arts retards intellectual and perceptual-motor skills.

Knipp, T. 1999, "Merging Realities: Psychosocial Happenings-a World on Stage." In Third International Conference on Information Visualisation (IV'99), pp. 413-21.

One of the virtues of our changing times is that new media has challenged us to rethink and reexamine our basic presumptions about reality and the reality that is virtually perceived. From the perspective of an electronic media artist, the author presents a body of work (case studies) of performative-like installations comprised of 3D structures integrating video imagery with the reality of the physical, psychological, virtual, and social worlds. These structures are instigators for enticing \&ldquo;social happenings\&rdquo; whereby participants and viewers become subjects from an observational perspective, providing a simulated clinical \&ldquo; case study\&rdquo;. With the influence of theoretical and scientific information in the constructs of mediated environments, perceptions of risk, danger trust, and self-identity are challenged. Using a metaphor to that of a \&ldquo; world on stage\&rdquo;, the works provide a visual, conceptual, and concrete model to illustrate progress from theory to practice 
Kobsa, A. 2001, "An Empirical Comparison of Three Commercial Information Visualization Systems." In IEEE Symposium on Information Visualization (INFOVIS '01), pp. 123-30.

Kobsa, A. 2004, "User Experiments with Tree Visualization Systems." In IEEE Sympoiusm on Information Visualization (INFOVIS '04), pp. 9-16.

This paper describes a comparative experiment with five well-known tree visualization systems, and Windows Explorer as a baseline system. Subjects performed tasks relating to the structure of a directory hierarchy, and to attributes of files and directories. Task completion times, correctness and user satisfaction were measured, and video recordings of subjects' interaction with the systems were made. Significant system and task type effects and an interaction between system and task type were found. Qualitative analyses of the video recordings were thereupon conducted to determine reasons for the observed differences, resulting in several findings and design recommendations as well as implications for future experiments with tree visualization systems

Koc, M. 2010, "Let's Make a Movie: Investigating Pre-Service Teachers' Reflections on Using VideoRecorded Role Playing Cases in Turkey." Teaching and Teacher Education 27(1):95-106.

This study examined the potential consequences of using student-filmed video cases in the study of classroom management in teacher education. Pre-service teachers in groups were engaged in videorecorded role playing to simulate classroom memoirs. Each group shared their video cases and interpretations in a class presentation. Qualitative data collection techniques were used to gather their experiences. Reflection papers written by 97 juniors were analyzed through content analysis and triangulated by group videos and case analysis reports. The results suggest that having pre-service teachers develop and analyze video cases can improve motivation, learning, empathy, and the construction of professional identity.

Koch, S, et al. 2010, in press, "Iterative Integration of Visual Insights During Scalable Patent Search and Analysis." Visualization and Computer Graphics, IEEE Transactions on.

Patents are of growing importance in current economic markets. Analyzing patent information has therefore become a common task for many interest groups. As a prerequisite for patent analysis, extensive search for relevant patent information is essential. Unfortunately, the complexity of patent material inhibits a straightforward retrieval of all relevant patent documents and leads to iterative, time-consuming approaches in practice. Already the amount of patent data to be analyzed poses challenges with respect to scalability. Further scalability issues arise concerning the diversity of users and the large variety of analysis tasks. With 'PatViz', a system for interactive analysis of patent information has been developed addressing scalability at various levels. PatViz provides a visual environment allowing for interactive reintegration of insights into subsequent search iterations, thereby bridging the gap between search and analytic processes. Because of its extensibility, we expect that the approach we have taken can be employed in different problem domains that require high quality of search results regarding their completeness. 
Kock, N. 2007, "Media Naturalness and Compensatory Encoding: The Burden of Electronic Media Obstacles Is on Senders." Decision Support Systems 44(1):175-87.

Compensatory adaptation theory makes two key predictions. On one hand, the theory predicts that electronic communication media in general will pose obstacles to complex communication between collaborators, when compared with the face-to-face medium, which will lead to an increase in cognitive effort and communication ambiguity. On the other hand, the theory also predicts that those obstacles will be met with compensatory adaptation, whereby electronic communication users will attempt to make up for the obstacles by modifying their communication behavior. This will in turn lead to a reduction in communication fluency. This study extends compensatory adaptation theory by also predicting that the burden of compensating for electronic communication media obstacles will fall primarily on those who attempt to convey information, as opposed to those who receive it. Those predictions are tested through an experiment involving 230 students, whose data are analyzed through nonparametric tests. All predictions are supported by the data analysis results. The use of a Web-based quasi-synchronous electronic communication medium, when compared with the face-to-face medium, increased perceived cognitive effort by approximately $12 \%$ and perceived communication ambiguity by about $19 \%$. Communication fluency was reduced by about $90 \%$. Perceived compensatory encoding effort (i.e., the effort spent by information givers) was increased by approximately $26 \%$, and perceived compensatory decoding effort (i.e., the information receivers' effort) by a statistically insignificant percentage.

Koh, K, et al. 2010, "Maniwordle: Providing Flexible Control over Wordle." IEEE Transactions on Visualization and Computer Graphics 16(6):1190-7.

Among the multifarious tag-clouding techniques, Wordle stands out to the community by providing an aesthetic layout, eliciting the emergence of the participatory culture and usage of tag-clouding in the artistic creations. In this paper, we introduce ManiWordle, a Wordle-based visualization tool that revamps interactions with the layout by supporting custom manipulations. ManiWordle allows people to manipulate typography, color, and composition not only for the layout as a whole, but also for the individual words, enabling them to have better control over the layout result. We first describe our design rationale along with the interaction techniques for tweaking the layout. We then present the results both from the preliminary usability study and from the comparative study between ManiWordle and Wordle. The results suggest that ManiWordle provides higher user satisfaction and an efficient method of creating the desired "art work," harnessing the power behind the ever-increasing popularity of Wordle.

Kohls, C, and J-G Uttecht. 2009, "Lessons Learnt in Mining and Writing Design Patterns for Educational Interactive Graphics." Computers in Human Behavior 25(5):1040-55.

Design patterns capture the invariant parts of proven designs as solutions to a problem in a specific context. While e-learning patterns have become popular in academic discussion, a large-scale use of these patterns or pattern languages in general cannot yet be observed in the field. One problem is that there are no agreed standards to define, analyze, organize, and evaluate such patterns. Another challenge is to find the right patterns and describe them at an appropriate level of abstraction, granularity, and detail. In this paper, we present a case study on the mining, writing and application of patterns for interactive educational graphics. It focuses on pattern mining and describes how to derive patterns from experience and analysis. Based on schema theory, a method is proposed to find patterns that are shared by other individuals. To improve the quality of written patterns, we consider established methods of pattern 
writing. The aim is to document patterns that are understandable, well-structured, and that capture the expert's intrinsic knowledge about real patterns as precisely as possible. Finally, the benefits and applicability of patterns are discussed.

Koike, H. 1993, "The Role of Another Spatial Dimension in Software Visualization." ACM Transactions on Information Systems (TOIS) 11(3):266-86.

Kok, P, et al. 2010, "Articulated Planar Reformation for Change Visualization in Small Animal Imaging." IEEE Transactions on Visualization and Computer Graphics 16(6):1396-404.

The analysis of multi-timepoint whole-body small animal CT data is greatly complicated by the varying posture of the subjectat different timepoints. Due to these variations, correctly relating and comparing corresponding regions of interest is challenging. In addition, occlusion may prevent effective visualization of these regions of interest. To address these problems, we have developeda method that fully automatically maps the data to a standardized layout of sub-volumes, based on an articulated atlas registration. We have dubbed this process articulated planar reformation, or APR. A sub-volume can be interactively selected for closer inspectionand can be compared with the corresponding sub-volume at the other timepoints, employing a number of different comparative visualization approaches. We provide an additional tool that highlights possibly interesting areas based on the change of bone densitybetween timepoints. Furthermore we allow visualization of the local registration error, to give an indication of the accuracy of theregistration. We have evaluated our approach on a case that exhibits cancer-induced bone resorption.

Komlodi, A, JR Goodall, and WG Lutters. 2004, "An Information Visualization Framework for Intrusion Detection." In CHI 2004, pp. 1743-46.

Komlodi, A, et al. 2005, "User-Centered Look at Glyph-Based Security Visualization." In IEEE Workshop on Visualization for Computer Security, pp. 21-28.

This paper presents the Intrusion Detection toolkit (IDtk), an information Visualization tool for intrusion detection (ID). IDtk was developed through a user-centered design process, in which we idenfiTed design guidelines to support ID users. ID analysts protect their networks by searching for evidence of attacks in ID system output, Trewall and system logs, and other complex, textual data sources. Monitoring and analyzing these sources incurs a heavy cognitive load for analysts. The use of information visualization techniques offers a valuable addition to the toolkit of the ID analyst. Several visualization techniques for ID have been developed, but few usability or Teld studies have been completed to assess the needs of ID analysts and the usability and usefulness of these tools. We intended to Tll this gap by applying a user-centered design process in the development and evaluation of IDtk, a $3 \mathrm{D}$, glyph-based visualization tool that gives the user maximum texibility in setting up how the visualization display represents ID data. The user can also customize whether the display is a simple, high-level overview to support monitoring, or a more complex 3D view allowing for viewing the data from multiple angles and thus supporting analysis and diagnosis. This texibility was found crucial in our usability evaluation. In addition to describing the tool, we report the Tndings of our user evaluation and propose new guidelines for the design of information visualization tools for ID. 
Kong, N, J Heer, and M Agrawala. 2010, "Perceptual Guidelines for Creating Rectangular Treemaps." IEEE Transactions on Visualization and Computer Graphics 16(6):990-8.

Treemaps are space-filling visualizations that make efficient use of limited display space to depict large amounts of hierarchical data. Creating perceptually effective treemaps requires carefully managing a number of design parameters including the aspect ratio and luminance of rectangles. Moreover, treemaps encode values using area, which has been found to be less accurate than judgments of other visual encodings, such as length. We conduct a series of controlled experiments aimed at producing a set of design guidelines for creating effective rectangular treemaps. We find no evidence that luminance affects area judgments, but observe that aspect ratio does have an effect. Specifically, we find that the accuracy of area comparisons suffers when the compared rectangles have extreme aspect ratios or when both are squares. Contrary to common assumptions, the optimal distribution of rectangle aspect ratios within a treemap should include non-squares, but should avoid extremes. We then compare treemaps with hierarchical bar chart displays to identify the data densities at which length-encoded bar charts become less effective than area-encoded treemaps. We report the transition points at which treemaps exhibit judgment accuracy on par with bar charts for both leaf and non-leaf tree nodes. We also find that even at relatively low data densities treemaps result in faster comparisons than bar charts. Based on these results, we present a set of guidelines for the effective use of treemaps and suggest alternate approaches for treemap layout.

Korakakis, G, et al. 2009, "3d Visualization Types in Multimedia Applications for Science Learning: A Case Study for 8th Grade Students in Greece." Computers \& Education 52(2):390-401.

This research aims to determine whether the use of specific types of visualization (3D illustration, 3D animation, and interactive 3D animation) combined with narration and text, contributes to the learning process of 13- and 14- years-old students in science courses. The study was carried out with 212 th grade students in Greece. This exploratory study utilizes three different versions of an interactive multimedia application called "Methods of separation of mixtures", each one differing from the other two in a type of visuals. The results indicate that multimedia applications with interactive $3 \mathrm{D}$ animations as well as with 3D animations do in fact increase the interest of students and make the material more appealing to them. The findings also suggest that the most obvious and essential benefit of static visuals (3D illustrations) is that they leave the time control of learning to the students and decrease the cognitive load.

Kosara, R. 2010, "Poster : Indirect Multi-Touch Interaction for Brushing in Parallel Coordinates." In IEEE Symposium on Informaiton Visualization (INFOVIS '10).

Kosara, R. 2007, "Visualization Criticism - the Missing Link between Information Visualization and Art." In 11th International Conference on Information Visualization, pp. 631-36.

Classifications of visualization are often based on technical criteria, and leave out artistic ways of visualizing information. Understanding the differences between information visualization and other forms of visual communication provides important insights into the way the field works, though, and also shows the path to new approaches. We propose a classification of several types of information visualization based on aesthetic criteria. The notions of artistic and pragmatic visualization are introduced, and their properties discussed. Finally, the idea of visualization criticism is proposed, and its rules are laid out. Visualization criticism bridges the gap between design, art, and technical/pragmatic information 
visualization. It guides the view away from implementation details and single mouse clicks to the meaning of a visualization.

Kosara, R, F Bendix, and H Hauser. 2006, "Parallel Sets: Interactive Exploration and Visual Analysis of Categorical Data." IEEE Transactions on Visualization and Computer Graphics 12(4):558-68.

Categorical data dimensions appear in many real-world data sets, but few visualization methods exist that properly deal with them. Parallel Sets are a new method for the visualization and interactive exploration of categorical data that shows data frequencies instead of the individual data points. The method is based on the axis layout of parallel coordinates, with boxes representing the categories and parallelograms between the axes showing the relations between categories. In addition to the visual representation, we designed a rich set of interactions. Parallel Sets allow the user to interactively remap the data to new categorizations and, thus, to consider more data dimensions during exploration and analysis than usually possible. At the same time, a metalevel, semantic representation of the data is built. Common procedures, like building the cross product of two or more dimensions, can be performed automatically, thus complementing the interactive visualization. We demonstrate Parallel Sets by analyzing a large CRM data set, as well as investigating housing data from two US states.

Koshman, S. 2005, "Testing User Interaction with a Prototype Visualization-Based Information Retrieval System." Journal of the American Society for Information Science and Technology 56(8):824-33.

The VIBE (Visual Information Browsing Environment) prototype system, which was developed at Molde College in Norway in conjunction with researchers at the University of Pittsburgh, allows users to evaluate documents from a retrieved set that is graphically represented as geometric icons within one screen display. While the formal modeling behind VIBE and other information visualization retrieval systems is well known, user interaction with the system is not. This investigation tested the designer assumption that VIBE is a tool for a smart (expert) user and asked: What are the effects of the different levels of user expertise upon VIBE usability? Three user groups including novices, online searching experts, and VIBE system experts totaling 31 participants were tested over two sessions with VIBE. Participants selected appropriate features to complete tasks, but did not always solve the tasks correctly. Task timings improved over repeated use with VIBE and the nontypical visually oriented tasks were resolved more successfully than others. Statistically significant differences were not found among all parameters examined between novices and online experts. The VIBE system experts provided the predicted baseline for this study and the VIBE designer assumption was shown to be correct. The study's results point toward further exploration of cognitive preattentive processing, which may help to understand better the novice/expert paradigm when testing a visualized interface design for information retrieval.

Koshman, S. 2006, "Visualization-Based Information Retrieval on the Web." Library \& Information Science Research 28(2):192-207.

The application of visualization techniques to information retrieval (IR) has resulted in the development of innovative systems and interfaces that are now available for public use. Visualization tools have emerged in research environments and more recently on the Web to retrieve information. Questions arise in regard to the utility of Web-based IR visualization tools for assisting users not only in manipulating search output, but also in managing the information retrieval process. To understand how Web-based visualization tools enable visual information retrieval, this article reviews some of the human 
perceptual theory behind the graphical interface of information visualization systems, analyzes iconic representations and information density on visualization displays, and examines information retrieval tasks that have been used in visualization system user research. This article is timely since it addresses new technologies for Web information retrieval and discusses future information visualization user research directions.

Koshman, S, and L Chi-Jung. 2009, "Comparing Visualization Techniques to Structure Collaborative Concepts." In 5th International Conference on Collaborative Computing: Networking, Applications and Worksharing, pp. 1-8.

Much research has been devoted to collaborative tools for writing, group communication, reviewing, managing projects, and developing network infrastructures to support sharing activities. However the creation and display of foundational collaborative concepts that manage group work has not been paid significant attention. Information visualization potentially addresses the progression of distributed concept building and presentation by visually representing group-generated concepts. This paper analyzes current visualization-based techniques including tag clouds, node-link diagrams, and mapping structures in a web-based environment. The analysis contributes to exploring the use of visualization tools for creating group concept structures that organize ideas for documents, projects, or specifications in academia and industry. A comparative features table is built and used as a basis for presenting an initial interface design to develop a collaborative visualization tool for concept management. Implications for future work include visualization system interface design, user evaluation, and adaptation to mobile collaborative visualization environments.

Kostelnick, C. 2007, "The Visual Rhetoric of Data Displays: The Conundrum of Clarity." Professional Communication, IEEE Transactions on 50(4):280-94.

The visual rhetoric of data displays (e.g., charts, graphs, maps) has changed profoundly over the past 50 years as a result of research in display techniques, the application of traditional and emerging rhetorical approaches, and the democratizing effects of data design technology. Perhaps in no other visual realm than data design is the notion of clarity more critical or more contested. Indeed the ascendancy of rhetorical approaches was initiated by the perceptual/cognitive science of data design, which in seeking to identify optimal display techniques, fostered a concern for ethics and evoked the universality and minimalism of modernist aesthetics. The rhetoric of adaptation, which emphasizes the variability of audiences, purposes, and situational contexts, rendered clarity contingent and mutable-a moving target that requires constant attention. Social rhetoric considered data design as a collective construct, tethering clarity to visual discourse communities, convention-building, cultural values, and power. The concept of clarity has been further reoriented by the rhetoric of participation, which is fostered by interactive digital design that enables users to adapt displays according to their needs and interests.

Kotranza, A, et al. 2009, "Real-Time in-Situ Visual Feedback of Task Performance in Mixed Environments for Learning Joint Psychomotor-Cognitive Tasks." In 8th IEEE International Symposium on Mixed and Augmented Reality, pp. 125-34.

This paper proposes an approach to mixed environment training of manual tasks requiring concurrent use of psychomotor and cognitive skills. To train concurrent use of both skill sets, the learner is provided real-time generated, in-situ presented visual feedback of her performance. This feedback provides reinforcement and correction of psychomotor skills concurrently with guidance in developing cognitive 
models of the task. The general approach is presented: 1) Sensors placed in the physical environment detect in real-time a learner's manipulation of physical objects. 2) Sensor data is input to models of task performance which output quantitative measures of the learner's performance. 3) Pre-defined rules are applied to transform the learner's performance data into visual feedback presented in real-time and in-situ with the physical objects being manipulated. With guidance from medical education experts, we have applied this approach to a mixed environment for learning clinical breast exams (CBEs). CBE belongs to a class of tasks that require learning multiple cognitive elements and task-specific psychomotor skills. Traditional approaches to learning CBEs and other joint psychomotor-cognitive tasks rely on extensive one-on-one training with an expert providing subjective feedback. By integrating real-time visual feedback of learners' quantitatively measured CBE performance, a mixed environment for learning CBEs provides on-demand learning opportunities with more objective, detailed feedback than available with expert observation. The proposed approach applied to learning CBEs was informally evaluated by four expert medical educators and six novice medical students. This evaluation highlights that receiving realtime in-situ visual feedback of their performance provides students an advantage, over traditional approaches to learning CBEs, in developing correct psychomotor and cognitive skills.

Koua, EL, and M-J Kraak. 2005, "Chapter 33 - Evaluating Self-Organizing Maps for Geovisualization." in Exploring Geovisualization, eds. D Jason, MM Alan and K Menno-Jan, pp. 627-43. Elsevier, Oxford.

Summary Information spaces are increasingly used to visualize complex data. With geospatial data specifically, there is a growing need for more flexible, accurate and usable information spaces to support exploratory analysis. Here, we present a prototype exploratory geovisualization environment based on a usability framework. The environment implements a self-organizing map neural network algorithm, and relates spatial analysis, datamining and knowledge discovery methods for the extraction of patterns. Some graphical representations are then used to portray extracted patterns in a visual form that contribute to improved understanding of the derived structures and the geographical processes. As part of the design and development process, a usability evaluation plan is proposed to assess ways in which users interpret these graphical representations and their appropriateness for exploratory visual analysis. The ultimate goal of the proposed evaluation strategy is to improve the visual design of the representations.

Kovács, B. 2010, "A Generalized Model of Relational Similarity." Social Networks 32(3):197-211.

This paper introduces two principles for relational similarity, and based on these principles it proposes a novel geometric representation for similarity. The first principle generalizes earlier measures of similarity such as Pearson-correlation and structural equivalence: while correlation and structural equivalence measure similarity by the extent to which the actors have similar relationships to other actors or objects, the proposed model views two actors similar if they have similar relationships to similar actors or objects. The second principle emphasizes consistency among similarities: not only are actors similar if they have similar relationships to similar objects, but at the same time objects are similar if similar actors relate to them similarly. We examine the behavior of the proposed similarity model through simulations, and re-analyze two classic datasets: the Davis et al. (1941) data on club membership and the roll-call data of the U.S. Senate. We find that the generalized model of similarity is especially useful if (1) the dimensions of comparison are not independent, or (2) the data are sparse, or (3) the boundaries between clusters are not clear. 
Kozlowski, SWJ. 1998, "Training and Developing Adaptive Teams: Theory, Principles, and Research." in Making Decisions under Stress: Implications for Individual and Team Training, eds. JA Cannon-Bowers and E Salas, pp. 115-53. American Psychological Associaiton, Washington, DC.

Kraak, M-J. 2003, "Geovisualization Illustrated." ISPRS Journal of Photogrammetry and Remote Sensing 57(5-6):390-99.

In recent years, the visualization of geospatial data has undergone dramatic and important developments. Next to static maps, nowadays, immersive and highly interactive virtual environments can be used to explore and present dynamic geospatial data. Additionally, the World Wide Web has developed into a prominent medium to disseminate geospatial data and maps. In visualizing geospatial data, methods and techniques from fields, such as scientific visualization and information visualization, are applied because of the large volumes of data at hand. This has accumulated in what is known as geovisualization--the use of visual geospatial displays to explore data and through that exploration to generate hypotheses, develop problem solutions and construct knowledge. Maps and other linked graphics play a key role in this process. The objective of this paper is to demonstrate the usefulness of geovisualization and, in particular, how alternative graphic representations can stimulate the visual thought process. This is demonstrated by applying geovisualization techniques to Minard's well-known map of Napoleon's 1812 campaign into Russia, the "Carte figurative des pertes successives en hommes de l'Armee Française dans la campagne de Russie 1812-1813" (http://www.itc.nl/personal/kraak/1812/).

Kraak, MJ. 2009, "Geovisualization." in International Encyclopedia of Human Geography, eds. K Rob and T Nigel, pp. 468-80. Elsevier, Oxford.

Recent developments in information and communication technology (ICT) have introduced many new opportunities, and have influenced many scientific disciplines in application of their methods and techniques. From a mapping perspective, this includes cartography and related disciplines like scientific visualization, image analysis and remote sensing, information visualization, exploratory data analysis, visual analytics, and GI Science. Interactivity and dynamics are prominent keywords and allow one not only to apply maps and diagrams to present-known facts but also to analyze and explore unknown data. The environment in which the maps and diagrams are used has also changed and often includes coordinated multiple views display via the Internet. This allows for simultaneous alternative views of the data and stimulates visual thinking, resulting in geovisualization.

Kraak, MJ. 2006, "Playing with Maps - Explore, Discover, Learn, Categorize, Model, Analyse, Explain, Present Geographic and Non-Geographic Data." In 10th International Conference on Information Visualization (IV06), pp. 291-96.

Maps are especially known for their capability to provide insight in geographic patterns and trends. Maps do this well because they only present a selection of the complex reality and visualize it in an abstract way. But today they have many more roles to play. They should also be seen as flexible interface to geospatial data, since they offer interaction with the data behind the visual representation and additionally maps are instruments that encourage exploration. As such they are used to stimulate (visual) thinking about geospatial patterns, relationships, and trends. The context where maps like this operate is the world of Geovisualization which can be described as a loosely bounded domain that addresses the visual exploration, analysis, synthesis and presentation of geospatial data by integrating approaches from disciplines including cartography with those from scientific visualization, image analysis, information 
visualization, exploratory data analysis, visual analytics, and GIScience. Contact with all those disciplines has enriched the world of maps but have also stimulated others to use the map (metaphor) to visualize nongeographic data. The discussion will illustrated the new and exiting role maps can play to visualize geographic and non-geographic data in combination with other visual means.

Krasser, S, et al. 2005, "Real-Time and Forensic Network Data Analysis Using Animated and Coordinated Visualization." In 6th Annual IEEE SMC Information Assurance Workshop, pp. 42-49.

Rapidly detecting and classifying malicious activity contained within network traffic is a challenging problem exacerbated by large datasets and functionally limited manual analysis tools. Even on a small network, manual analysis of network traffic is inefficient and extremely time consuming. Current machine processing techniques, while fast, suffer from an unacceptable percentage of false positives and false negatives. To complement both manual and automated analysis of network traffic, we applied information visualization techniques to appropriately and effectively bring the human into the analytic loop. This paper describes the implementation and lessons learned from the creation of a novel network traffic visualization system capable of both realtime and forensic data analysis. Combining the strength of link analysis using parallel coordinate plots with the time-sequence animation of scatter plots, we examine a 2D and 3D coordinated display that provides insight into both legitimate and malicious network activity. Our results indicate that analysts can rapidly examine network traffic and detect anomalies far more quickly than with manual tools.

Kriglstein, S. 2010, "Owl Ontology Visualization: Graphical Representations of Properties on the Instance Level." In 14th International Conference on Informaiton Visualization, pp. 92-97.

For several ontology applications, a combination of classes with their instances, their properties on the class level and on the instance level are from interest. However, the focus of most visualization approaches is on the hierarchical and non-hierarchical relationships on the class level. This paper presents an approach to visualizing datatype properties and object properties on the instance level. For this purpose, three different layouts were developed for the ontology visualization tool Knoocks. Furthermore, the paper discusses results of an evaluation that was motivated to identify which one of these layout versions was preferred by the users. The evaluation should also reveal if the concept of the representation of the properties was understandable for them.

Kriglstein, S, and R Motschnig-Pitrik. 2008, "Knoocks: New Visualization Approach for Ontologies." In 12th International Conference Information Visualisation, pp. 163-68.

Ontologies are becoming popular in various communities. They give users the possibilities to understand, exchange, analyze or share knowledge of a specific domain. However ontologies can be very large and complex and therefore visualizations should help the user to understand and manipulate ontologies easily. Most visualizations concentrate on the structure Of ontologies. For users, instances are often more interesting, because they represent the real world objects. This paper presents Knoocks (Knowledge Blocks) as a visualization approach which focuses on instances related to their structure. An evaluation which compared Knoocks with Jambalaya and CropCircles showed its benefits in visualizing instances. 
Krishnan, M, et al. 2007, "Scalable Visual Analytics of Massive Textual Datasets." In IEEE International Parallel and Distributed Processing Symposium, pp. 1-10.

This paper describes the first scalable implementation of a text processing engine used in visual analytics tools. These tools aid information analysts in interacting with and understanding large textual information content through visual interfaces. By developing a parallel implementation of the text processing engine, we enabled visual analytics tools to exploit cluster architectures and handle massive datasets. The paper describes key elements of our parallelization approach and demonstrates virtually linear scaling when processing multi-gigabyte data sets such as Pubmed. This approach enables interactive analysis of large datasets beyond capabilities of existing state-of-the art visual analytics tools.

Kristensson, PO, et al. 2009, "An Evaluation of Space Time Cube Representation of Spatiotemporal Patterns." Visualization and Computer Graphics, IEEE Transactions on 15(4):696-702.

Space time cube representation is an information visualization technique where spatiotemporal data points are mapped into a cube. Information visualization researchers have previously argued that space time cube representation is beneficial in revealing complex spatiotemporal patterns in a data set to users. The argument is based on the fact that both time and spatial information are displayed simultaneously to users, an effect difficult to achieve in other representations. However, to our knowledge the actual usefulness of space time cube representation in conveying complex spatiotemporal patterns to users has not been empirically validated. To fill this gap, we report on a between-subjects experiment comparing novice users' error rates and response times when answering a set of questions using either space time cube or a baseline 2D representation. For some simple questions, the error rates were lower when using the baseline representation. For complex questions where the participants needed an overall understanding of the spatiotemporal structure of the data set, the space time cube representation resulted in on average twice as fast response times with no difference in error rates compared to the baseline. These results provide an empirical foundation for the hypothesis that space time cube representation benefits users analyzing complex spatiotemporal patterns.

Kruk, SR, B McDaniel, and Acm. 2008, "Rendering Navigation and Information Space with Honeycomb (Tm)." In 26th Annual CHI Conference on Human Factors in Computing Systems, pp. 1793-96.

The growing amount of available information poses challenges not only in the process of information retrieval. The usability of the rendered search process and results can be increased by appropriate visualization techniques or new interaction paradigms, or both. In this article we present the HoneyComb (TM) paradigm, an information visualization style that aims to render and manage large quantities of information items. We describe the design objectives and the prototype of HC (TM). Finally, we present a short evaluation of the $\mathrm{HC}$ (TM) paradigm in the context of search and browsing.

Kuchar, OA, et al. 2006, "Isn't It About Time? [Data Visualization]." IEEE Computer Graphics and Applications 26(3):80-83.

Time plays a key role in all aspects of the intelligence analysis process, from data ingest through analysis methods to the cognitive processes that create intelligence products. However, the concept of time is difficult to grasp and not yet fully understood. In this article, we briefly discuss why time is difficult to grasp, the need for visualizations and interactions to deal with time, and some thoughts about a temporal analytic discourse. Overall, time cannot be left as an afterthought when developing 
visualizations. We should consider time as a first-class object in its own right, rather than an implicit attribute, and we must make it as interactively manipulable as any other object

Kudikyala, UK, and RB Vaughn. 2005, "Software Requirement Understanding Using Pathfinder Networks: Discovering and Evaluating Mental Models." Journal of Systems and Software 74(1):101-08.

Understanding and communicating user requirements in a software requirement analysis effort is very important. Misunderstandings of user requirements between software developers and users, will cause problems in terms of satisfying user needs, defects, cost and schedule during the software development process. This paper proposes a new technique that has the ability to represent the mental models of the user and developer communities as network representations using Pathfinder networks. Graphs (mental models) are generated for each of the user and developer groups and compared for similarities/dissimilarities using a graph similarity metric. This paper overviews how this technique is used to categorize requirements and to identify ambiguous and duplicate requirements. We also propose to extend this technique to enhance communication and reduce misunderstanding surrounding the user requirements during the requirement analysis phase.

Kühl, T, et al. 2011, "Can Differences in Learning Strategies Explain the Benefits of Learning from Static and Dynamic Visualizations?" Computers \& Education 56(1):176-87.

The effects of dynamic and static visualizations in understanding physical principles of fish locomotion were investigated. Seventy-five students were assigned to one of three conditions: a text-only, a text with dynamic visualizations, or a text with static visualizations condition. During learning, subjects were asked to think aloud. Learning outcomes were measured by tests assessing verbal factual knowledge, pictorial recall as well as transfer. Learners in the two visualization conditions outperformed those in the text-only condition for transfer and pictorial recall tasks, but not for verbal factual knowledge tasks. Analyses of the think-aloud protocols revealed that learners had generated more inferences in the visualization conditions as opposed to the text-only condition. These results were mirrored by students' self-reported processing demands. No differences were observable between the dynamic and the static condition concerning any of the learning outcome measures. However, think-aloud protocols revealed an illusion of understanding when learning with dynamic as opposed to static visualizations. Furthermore, learners with static visualizations tended to play the visualizations more often. The results stress the importance of not only using outcome-oriented, but also process-oriented approaches to gain deeper insight into learning strategies when dealing with various instructional materials.

Kuhn, A, and D Erni. 2010, "Embedding Spatial Software Visualization in the Ide : An Exploratory Study." In 5th ACM Symposium on Software Visualization (SOFTVIS), pp. 113-22.

Kuhn, GR, MM Oliveira, and LAF Fernandes. 2008, "An Efficient Naturalness-Preserving ImageRecoloring Method for Dichromats." IEEE Transactions on Visualization and Computer Graphics 14(6):1747-54.

We present an efficient and automatic image-recoloring technique for dichromats that highlights important visual details that would otherwise be unnoticed by these individuals. While previous techniques approach this problem by potentially changing all colors of the original image, causing their results to look unnatural to color vision cleficients, our approach preserves, as much as possible, the image's original colors. Our approach is about three orders of magnitude faster than previous ones. The 
results of a paired-comparison evaluation carried out with fourteen color-vision deficients (CVDs) indicated the preference of our technique over the state-of-the-art automatic recoloring technique for dichromats. When considering information visualization examples, the subjects tend to prefer our results over the original images. An extension of our technique that exaggerates color contrast tends to be preferred when CVDs compared pairs of scientific visualization images. These results provide valuable information for guiding the design of visualizations for color-vision deficients.

Kules, B, and B Shneiderman. 2008, "Users Can Change Their Web Search Tactics: Design Guidelines for Categorized Overviews." Information Processing \& Management 44(2):463-84.

Categorized overviews of web search results are a promising way to support user exploration, understanding, and discovery. These search interfaces combine a metadata-based overview with the list of search results to enable a rich form of interaction. A study of 24 sophisticated users carrying out complex tasks suggests how searchers may adapt their search tactics when using categorized overviews. This mixed methods study evaluated categorized overviews of web search results organized into thematic, geographic, and government categories. Participants conducted four exploratory searches during a 2-hour session to generate ideas for newspaper articles about specified topics such as "human smuggling." Results showed that subjects explored deeper while feeling more organized, and that the categorized overview helped subjects better assess their results, although no significant differences were detected in the quality of the article ideas. A qualitative analysis of searcher comments identified seven tactics that participants reported adopting when using categorized overviews. This paper concludes by proposing a set of guidelines for the design of exploratory search interfaces. An understanding of the impact of categorized overviews on search tactics will be useful to web search researchers, search interface designers, information architects and web developers.

Kulik, I, KS Hornsby, and ID Bishop. 2011, "Modeling Geospatial Trend Changes in Vegetation Monitoring Data." Computers, Environment and Urban Systems 35(1):45-56.

In a constantly changing environment, monitoring supports analysis and understanding of many types of change. This paper is concerned specifically with monitoring of vegetation and describes the development and application of a formal model that supports the analysis of spatiotemporal changes in the recorded attributes of a forest/heathland environment. Typically, the monitoring points are not ideally distributed in time or space. The proposed analytical techniques are designed to deal with incomplete data sets and to reveal abnormal changes or transitions. These transitions can potentially be linked to causal events which may have not been otherwise recorded. This work distinguishes five key change types as a basis for 25 transition types present in time series of vegetation data. These are distilled from the data using a set of transition point analysis methods including spatiotemporal neighborhood and trend sequence analysis. In addition, cross-comparisons between vegetation attributes, based on the identified transitions, are illustrated. A prototype GIS-based tool VeMonA provides an analytical environment for time series data obtained through vegetation monitoring and supports understanding of dynamic geospatial ecosystems.

Kumar, M, et al. 2008, "Improving the Accuracy of Gaze Input for Interaction." In Proceedings of the 2008 symposium on Eye tracking research \& applications - ETRA '08, pp. 65-65.

Using gaze information as a form of input poses challenges based on the nature of eye movements and how we humans use our eyes in conjunction with other motor actions. In this paper, we present three 
techniques for improving the use of gaze as a form of input. We first present a saccade detection and smoothing algorithm that works on real-time streaming gaze information. We then present a study which explores some of the timing issues of using gaze in conjunction with a trigger (key press or other motor action) and propose a solution for resolving these issues. Finally, we present the concept of Focus Points, which makes it easier for users to focus their gaze when using gaze-based interaction techniques. Though these techniques were developed for improving the performance of gaze-based pointing, their use is applicable in general to using gaze as a practical form of input.

Kumar, P, N Subramanian, and K Zhang. 2008, "Evaluation of Information Visualization Tools Using the Nfr Approach." In 10th International Conference on Visual Information Systems, pp. 44-55.

Determination of quality of information Visualization (infovis) tools will assist practitioners ill industry and academia to choose the appropriate tools, for their needs. Infovis tools are widely used to visualize data-intensive. applications so that quick decisions may be taken. In this paper we use a novel technique, the NFR Approach, to determine quality of infovis tools. The NFR Approach considers visualizability as an important non-functional requirement, (NFR) for infovis tools and evaluates visualizability to determine the quality of these tools. The chief artifact that the NFR Approach employs for evaluation is the softgoal interdependency graph (SIG) wherein factors affecting visualizability are related to tool features by means of contributions that come in four flavors. Well-defined propagation rules help determine a qualitative score for visualizability based oil the contributions. The chief advantages of the NFR Approach include rationale for the scores, flexibility to accommodate different definitions for visualizability, and historical record-keeping. The NFR Approach is validated by applying the Approach to three infovis tools - Prefuse, Tom Sawyer, and GnuPlot.

Kumas, AC, and S Srinivas. 2010, "Concept Lattice Reduction Using Fuzzy K-Means Clustering." Expert Systems with Applications 37(3):2696-704.

During the design of concept lattices, complexity plays a major role in computing all the concepts from the huge incidence matrix. Hence for reducing the size of the lattice, methods based on matrix decompositions like SVD are available in the literature. However, SVD computation is known to have large time and memory requirements. In this paper, we propose a new method based on Fuzzy K-Means clustering for reducing the size of the concept lattices. We demonstrate the implementation of proposed method on two application areas: information retrieval and information visualization.

Kuniavsky, M. 2010, "Chapter 17 - Augmentations and Mashups." in Smart Things, pp. 253-71. Morgan Kaufmann, Boston.

Kurlander, D. 1988, "Editable Graphical Histories." In IEEE Workshop on Visual Languages, pp. 127-34.

Graphical interfaces typically provide their users with little idea of a session's history, except insofar as it is reflected in the current state of the system. If undo and redo commands are provided, they are often the only way to review the actions performed, cycling through them in sequence. We introduce the notion of an editable graphical history that can allow the user to review and modify the actions performed with a graphical interface. We have designed a testbed system that creates a series of automatically-generated panels that depict in chronological order the important events in the history of a user's session with Chimera, a graphical editor. Our system uses heuristics to determine the contents of each panel and the 
actions that it illustrates. The user may scroll through the sequence of panels, reviewing actions at different levels of detail, and selectively undoing, modifying, and redoing previous actions.

Kurtz, CF, and DJ Snowden. 2009, "The New Dynamics of Strategy Sense-Making in a Complex and Complicated World." IBM Systems Journal 42(3):462-83.

Kuttel, M, et al. 2006, "Techniques for Visualization of Carbohydrate Molecules." Journal of Molecular Graphics and Modelling 25(3):380-88.

Standard molecular visualizations, such as the classic ball-and-stick model, are not suitable for large, complex molecules because the overall molecular structure is obscured by the atomic detail. For proteins, the more abstract ribbon and cartoon representations are instead used to reveal large scale molecular conformation and connectivity. However, there is currently no accepted convention for simplifying oligoand polysaccharide structures. We introduce two novel visualization algorithms for carbohydrates, incorporated into a visualization package, CarboHydra. Both algorithms highlight the sugar rings and backbone conformation of the carbohydrate chain, ignoring ring substituents. The first algorithm, termed PaperChain, emphasizes the type and conformation of the carbohydrate rings. The second, Twister, emphasizes the relative orientation of the rings. We further include two rendering enhancements to augment these visualizations: silhouettes edges and a translucent overlay of the ball-and-stick atomic representation. To demonstrate their utility, the algorithms and visualization enhancements are here applied to a variety of carbohydrate molecules. User evaluations indicate that they present a more useful view of carbohydrate structure than the standard ball-and-stick representation. The algorithms were found to be complementary, with PaperChain particularly effective for smaller carbohydrates and Twister useful at larger scales for highlighting the backbone twist of polysaccharides.

Lam, H. 2008, "A Framework of Interaction Costs in Information Visualization." IEEE Transactions on Visualization and Computer Graphics 14(6):1149-56.

Interaction cost is an important but poorly understood factor in visualization design. We propose a framework of interaction costs inspired by Norman's Seven Stages of Action to facilitate study. From 484 papers, we collected 61 interaction-related usability problems reported in 32 user studies and placed them into our framework of seven costs: (1) Decision costs to form goals; (2) System-power costs to form system operations; (3) Multiple input mode costs to form physical sequences; (4) Physical-motion costs to execute sequences; (5) Visual-cluttering costs to perceive state; (6) View-change costs to interpret perception; (7) State-change costs to evaluate interpretation. We also suggested ways to narrow the gulfs of execution (2-4) and evaluation (5-7) based on collected reports. Our framework suggests a need to consider decision costs (1) as the gulf of goal formation.

Lam, HLM. 2008, "Visual Exploratory Analysis of Large Data Sets : Evaluation and Application." in Computer Science, Vol PhD. University of British Columbia.

Large data sets are difficult to analyze. Visualization has been proposed to assist exploratory data analysis (EDA) as our visual systems can process signals in parallel to quickly detect patterns. Nonetheless, designing an effective visual analytic tool remains a challenge. This challenge is partly due to our incomplete understanding of how common visualization techniques are used by human operators during analyses, either in laboratory settings or in the workplace. This thesis aims to further understand how visualizations can be used to support EDA. More specifically, we studied techniques that display 
multiple levels of visual information resolutions (VIRs) for analyses using a range of methods. The first study is a summary synthesis conducted to obtain a snapshot of knowledge in multiple-VIR use and to identify research questions for the thesis: (1) low-VIR use and creation; (2) spatial arrangements of VIRs. The next two studies are laboratory studies to investigate the visual memory cost of image transformations frequently used to create low-VIR displays and overview use with single-level data displayed in multiple-VIR interfaces. For a more well-rounded evaluation, we needed to study these techniques in ecologically-valid settings. We therefore selected the application domain of web session log analysis and applied our knowledge from our first three evaluations to build a tool called Session Viewer. Taking the multiple coordinated view and overview + detail approaches, Session Viewer displays multiple levels of web session log data and multiple views of session populations to facilitate data analysis from the high-level statistical to the low-level detailed session analysis approaches. Our fourth and last study for this thesis is a field evaluation conducted at Google Inc. with seven session analysts using Session Viewer to analyze their own data with their own tasks. Study observations suggested that displaying web session logs at multiple levels using the overview + detail technique helped bridge between high-level statistical and low-level detailed session analyses, and the simultaneous display of multiple session populations at all data levels using multiple views allowed quick comparisons between session populations. We also identified design and deployment considerations to meet the needs of diverse data sources and analysis styles.

Lammarsch, T. 2010, "Facets of Time - Making the Most of Time's Structure in Interactive Visualization." Vol PhD. Vienna University of Technology, Krems, Austria.

Lamy, JB, et al. 2008, "An Iconic Language for the Graphical Representation of Medical Concepts." Bmc Medical Informatics and Decision Making 8.

Background: Many medication errors are encountered in drug prescriptions, which would not occur if practitioners could remember the drug properties. They can refer to drug monographs to find these properties, however drug monographs are long and tedious to read during consultation. We propose a two-step approach for facilitating access to drug monographs. The first step, presented here, is the design of a graphical language, called VCM. Methods: The VCM graphical language was designed using a small number of graphical primitives and combinatory rules. VCM was evaluated over 11 volunteer general practitioners to assess if the language is easy to learn, to understand and to use. Evaluators were asked to register their VCM training time, to indicate the meaning of VCM icons and sentences, and to answer clinical questions related to randomly generated drug monograph-like documents, supplied in text or VCM format. Results: VCM can represent the various signs, diseases, physiological states, life habits, drugs and tests described in drug monographs. Grammatical rules make it possible to generate many icons by combining a small number of primitives and reusing simple icons to build more complex ones. Icons can be organized into simple sentences to express drug recommendations. Evaluation showed that VCM was learnt in 2 to 7 hours, that physicians understood $89 \%$ of the tested VCM icons, and that they answered correctly to $94 \%$ of questions using VCM ( versus $88 \%$ using text, $p=0.003$ ) and 1.8 times faster $(\mathrm{p}<0.001)$. Conclusion: VCM can be learnt in a few hours and appears to be easy to read. It can now be used in a second step: the design of graphical interfaces facilitating access to drug monographs. It could also be used for broader applications, including the design of interfaces for consulting other types of medical document or medical data, or, very simply, to enrich medical texts. 
Landwich, P, et al. 2009, "Supporting Patent Retrieval in the Context of Innovation-Processes by Means of Information Dialogue." World Patent Information 31(4):315-22.

Innovations are an essential factor of competition for manufacturing companies in technical industries. Patent information plays an important role for innovation-processes and innovators in the knowledge management. The combination of cross-organizational spread information and resources from patent databases and digital libraries is necessary in order to gain profit for innovation experts. The major challenge is to overcome the current information deficit and to fulfill the information need of the experts in the innovation-process. In this paper we first present in detail three innovation scenarios to derive challenges on advanced information systems which support an information dialogue but also the complete search process. Then we define essential conditions of a search task and from that we derive the elementary information sets and activities in the next step. An example shows the applicability and utility of the formalization described and shows how the activities fill up the user's information dialogue context. During the formalization we apply a cognitive walk-through on a patent database. We will use the Daffodil-system as an experimental system for further development and evaluation of the above described framework.

Lange, CFJ, MAM Wijns, and MRV Chaudron. 2007, "Supporting Task-Oriented Modeling Using Interactive Uml Views." Journal of Visual Languages \& Computing 18(4):399-419.

The UML is a collection of 13 diagram notations to describe different views of a software system. The existing diagram types display model elements and their relations. Software engineering is becoming more and more model-centric, such that software engineers start using UML models for more tasks than just describing the system. Tasks such as analysis or prediction of system properties require additional information such as metrics of the UML model or from external sources, e.g., a version control system. In this paper we identify tasks of model-centric software engineering and information that is required to fulfill these tasks. We propose views to visualize the information to support fulfilling the tasks. This paper reports on a large-scale controlled experiment to validate the usefulness of the proposed views that are implemented in our MetricView Evolution tool. The results of the experiment with 100 participants are statistically significant and show that the correctness of comprehension is improved by $4.5 \%$ and that the time needed is reduced by $20 \%$.

Lanzenberger, M, et al. 2003, "Applying Information Visualization Techniques to Capture and Explore the Course of Cognitive Behavioral Therapy." In ACM Symposium on Applied Computing (SAC '03), pp. 268-74.

Lanzenberger, M, S Miksch, and M Pohl. 2005, "Exploring Highly Structured Data: A Comparative Study of Stardinates and Parallel Coordinates." In Ninth International Conference on Information Visualisation (IV'05), pp. 312-20.

Comparing different information visualization (InfoVis) techniques is a challenging task and a necessary step to reach the users and their tasks. We evaluated the effectiveness in visualizing psychotherapeutic data of two InfoVis techniques, namely the stardinates and the parallel coordinates by a comparative study with 22 participants. Based on three research questions we interpret the results of our study in order to derive statements on both visualization techniques. We evaluated (1) the time participants were engaged in testing our material; (2) the number of correct answers; (3) subjects' statements, which were categorized by the type of statement; and (4) the subjects' key statements in 
comparison to those defined by an expert. Our empirical results indicate that the stardinates are a more appropriate method for interpreting such highly structured data in detail whereas parallel coordinates show advantages for gaining information at the first glance.

Lanzenberger, M, S Miksch, and M Pohl. 2003, "The Stardinates - Visualizing Highly Structured Data." In Seventh International Conference on Information Visualization, pp. 47-52.

The Stardinates are a novel interactive information visualization (InfoVis) technique, which aims at visualizing highly structured data. They represent some Gestalt principles very well, especially the principles of Closure and 'Pragnanz'. As a consequence, Stardinates form very distinct and memorable patterns, which make abstraction and aggregation much easier. We give a formal description of the Stardinates as a basis for implementation. Furthermore, we show an application by visualizing psychotherapeutic data derived from a clinical study on anorectic girls.

Lanzenberger, M, and J Sampson. 2007, "Human-Mediated Visual Ontology Alignment." in Human Interface and the Management of Information: Interacting in Information Environments, Pt 2, Proceedings, eds. MJ Smith and G Salvendy, Vol 4558, pp. 394-403.

We develop a multiple-view tool called AlViz, which aims at supporting the ontology alignment process visually. Combing views on several levels of abstraction, the tool tries to make the 'relatedness' between entities accessible. Based on a literature study we identified relevant phases emerging in ontology alignment. We extended a general alignment framework in order to reflect the adoption of visualization techniques. This framework builds the background for our user study. We evaluate visual ontology alignment with AlViz in three stages: (1) Participative software development, (2) usability evaluation, and (3) utility study. The evaluation methods proved viable even though our study design is challenging.

Lanzenberger, M, J Sampson, and M Rester. 2010, "Ontology Visualization: Tools and Techniques for Visual Representation of Semi-Structured Meta-Data." Journal of Universal Computer Science 16(7):1036-54.

Ontologies are used to represent a variety of domain knowledge and data collections, scopes, viewpoints and linked heterogeneous information sources. They range from simple topologies to highly structured knowledge bases with complex relations. When mapping or aligning two or more ontologies an efficient user support is needed so that the users can understand the prerequisites and the consequences of the alignments. Information Visualization techniques can help to facilitate user understanding of the ontology alignment results. In general, a lot of work in visualization of ontologies exist. We found an enormous number of ontology visualization tools by a literature study. Many of them apply graph visualization but there are other approaches as well. We have identified interesting solutions for dealing with the complexity of large ontologies. Ontology engineering, ontology mapping and alignment can benefit from Information Visualization. Our collection is a starting point to demonstrate the usefulness of Information Visualization techniques, however, a detailed evaluation would be the next step to consolidate this research area and help to boost the adoption of ontologies in common Web applications. 
Lanzenberger, M, J Sampson, and M Rester. 2009, "Visualization in Ontology Tools." In International Conference on Complex, Intelligent and Software Intensive Systems, pp. 705-11.

With a literature study we found an enormous number of ontology visualization tools. Many of them apply graph visualization but there are other approaches as well. We have identified interesting solutions for dealing with the complexity of large ontologies. Ontology engineering, ontology mapping and alignment can benefit from Information Visualization. Our collection is a starting point to demonstrate the usefulness of Information Visualization techniques, however, a detailed evaluation would be the next step to consolidate this research area and help to boost the adoption of ontologies in common Web applications.

Lanzenberger, M, et al. 2009. Visualization in Ontology Tools.

With a literature study we found an enormous number of ontology visualization tools. Many of them apply graph visualization but there are other approaches as well. We have identified interesting solutions for dealing with the complexity of large ontologies. Ontology engineering, ontology mapping and alignment can benefit from Information Visualization. Our collection is a starting point to demonstrate the usefulness of Information Visualization techniques, however, a detailed evaluation would be the next step to consolidate this research area and help to boost the adoption of ontologies in common Web applications.

Larkin, JH, and HA Simon. 1987, "Why a Diagram Is (Sometimes) Worth Ten Thousand Words." Cognitive Science 11(1):65-100.

We distinguish diagrammatic from sentential paper-and-pencil representations of information by developing alternative models of information-processing systems that are informationally equivalent and that can be characterized as sentential or diagrammatic. Sentential representations are sequential, like the propositions in a text. Diagrammatic representations are indexed by location in a plane. Diagrammatic representations also typically display information that is only implicit in sentential representations and that therefore has to be computed, sometimes at great cost, to make it explicit for use. We then contrast the computational efficiency of these representations for solving several illustrative problems in mathematics and physics. When two representations are informationally equivalent, their computational efficiency depends on the information-processing operators that act on them. Two sets of operators may differ in their capabilities for recognizing patterns, in the inferences they can carry out directly, and in their control strategies (in particular, the control of search). Diagrammatic and sentential representations support operators that differ in all of these respects. Operators working on one representation may recognize features readily or make inferences directly that are difficult to realize in the other representation. Most important, however, are differences in the efficiency of search for information and in the explicitness of information. In the representations we call diagrammatic, information is organized by location, and often much of the information needed to make an inference is present and explicit at a single location. In addition, cues to the next logical step in the problem may be present at an adjacent location. Therefore problem solving can proceed through a smooth traversal of the diagram, and may require very little search or computation of elements that had been implicit. 
Lau, A, and AV Moere. 2007, "Towards a Model of Information Aesthetics in Information

Visualization." In 11th International COnference on Information Visualization.

Lau, HYK, LKY Chan, and RHK Wong. 2008, "A Vr-Based Visualization Framework for Effective Information Perception and Cognition." In Conference on Human System Interactions, pp. 197-202.

An information visualization framework is proposed that considers key human factors for effective complex data perception and cognition. Virtual reality (VR) technology with data transformation heuristics are deployed in building the framework where an interactive VR-based 3-D information visualization platform is developed. The framework is applied to develop a visualization system for an express cargo handling centre where users is able to effectively perceive operation details and carry out timely decision making.

Laube, P, et al. 2007, "Movement Beyond the Snapshot - Dynamic Analysis of Geospatial Lifelines." Computers, Environment and Urban Systems 31(5):481-501.

Geographical Information Science is challenged by an unprecedented increase in the availability of tracking data related to human and animal movement, typically captured through location-aware portable devices such as GPS receivers. Capture of trajectory data at fine temporal and spatial granularities has allowed with the representation of detailed geospatial lifelines, opening new options for analysis. In this respect we propose a dynamic perspective to analysis which, in contrast to summary trajectory statistics on speed, motion azimuth or sinuosity, that refers to the variability of motion properties throughout the developing lifeline. Four specific lifeline context operators are identified in this paper: [']instantaneous', [']interval', [']episodal' and [']total'. Using this framework, we discuss standardisations that integrate the extended set of motion descriptors within various temporal and spatial frames of reference and the proposed lifeline context operators and standardisations are illustrated using high resolution trajectory data obtained from homing pigeons carrying miniature global positioning devices.

Law, AJ, DG Aliaga, and A Majumder. 2010, "Projector Placement Planning for High Quality Visualizations on Real-World Colored Objects." IEEE Transactions on Visualization and Computer Graphics 16(6):1633-41.

Many visualization applications benefit from displaying content on real-world objects rather than on a traditional display(e.g., a monitor). This type of visualization display is achieved by projecting precisely controlled illumination from multiple projectorsonto the real-world colored objects. For such a task, the placement of the projectors is critical in assuring that the desiredvisualization is possible. Using ad hoc projector placement may cause some appearances to suffer from color shifting due toinsufficient projector light radiance being exposed onto the physical surface. This leads to an incorrect appearance and ultimately toa false and potentially misleading visualization. In this paper, we present a framework to discover the optimal position andorientation of the projectors for such projection-based visualization displays. An optimal projector placement should be able toachieve the desired visualization with minimal projector light radiance. When determining optimal projector placement, objectvisibility, surface reflectance properties, and projector-surface distance and orientation need to be considered. We first formalize atheory for appearance editing image formation and construct a constrained linear system of equations that express when a desirednovel appearance or visualization is possible given a geometric and surface reflectance model of the physical surface. Then, weshow how to apply this constrained system in an adaptive search to efficiently discover the optimal projector placement whichachieves the desired 
appearance. Constraints can be imposed on the maximum radiance allowed by the projectors and theprojectors' placement to support specific goals of various visualization applications. We perform several real-world and simulatedappearance edits and.

Leach, SM, et al. 2009, "Biomedical Discovery Acceleration, with Applications to Craniofacial Development." PLoS Computational Biology 5(3).

The profusion of high-throughput instruments and the explosion of new results in the scientific literature, particularly in molecular biomedicine, is both a blessing and a curse to the bench researcher. Even knowledgeable and experienced scientists can benefit from computational tools that help navigate this vast and rapidly evolving terrain. In this paper, we describe a novel computational approach to this challenge, a knowledge-based system that combines reading, reasoning, and reporting methods to facilitate analysis of experimental data. Reading methods extract information from external resources, either by parsing structured data or using biomedical language processing to extract information from unstructured data, and track knowledge provenance. Reasoning methods enrich the knowledge that results from reading by, for example, noting two genes that are annotated to the same ontology term or database entry. Reasoning is also used to combine all sources into a knowledge network that represents the integration of all sorts of relationships between a pair of genes, and to calculate a combined reliability score. Reporting methods combine the knowledge network with a congruent network constructed from experimental data and visualize the combined network in a tool that facilitates the knowledge-based analysis of that data. An implementation of this approach, called the Hanalyzer, is demonstrated on a large-scale gene expression array dataset relevant to craniofacial development. The use of the tool was critical in the creation of hypotheses regarding the roles of four genes never previously characterized as involved in craniofacial development; each of these hypotheses was validated by further experimental work.

Lee, B, et al. 2006, "Treeplus: Interactive Exploration of Networks with Enhanced Tree Layouts." IEEE Transactions on Visualization and Computer Graphics 12(6):1414-26.

Despite extensive research, it is still difficult to produce effective interactive layouts for large graphs. Dense layout and occlusion make food webs, ontologies, and social networks difficult to understand and interact with. We propose a new interactive Visual Analytics component called TreePlus that is based on a tree-style layout. TreePlus reveals the missing graph structure with visualization and interaction while maintaining good readability. To support exploration of the local structure of the graph and gathering of information from the extensive reading of labels, we use a guiding metaphor of "Plant a seed and watch it grow." It allows users to start with a node and expand the graph as needed, which complements the classic overview techniques that can be effective at (but often limited to) revealing clusters. We describe our design goals, describe the interface, and report on a controlled user study with 28 participants comparing TreePlus with a traditional graph interface for six tasks. In general, the advantage of TreePlus over the traditional interface increased as the density of the displayed data increased. Participants also reported higher levels of confidence in their answers with TreePlus and most of them preferred TreePlus. 
Lee, B, et al. 2006, "Task Taxonomy for Graph Visualization." In Proceedings of the 2006 AVI workshop on BEyond time and errors novel evaluation methods for information visualization - BELIV'06, pp. 5-5.

Lee, B, et al. 2010, "Sparkclouds: Visualizing Trends in Tag Clouds." IEEE Transactions on Visualization and Computer Graphics 16(6):1182-9.

Tag clouds have proliferated over the web over the last decade. They provide a visual summary of a collection of texts by visually depicting the tag frequency by font size. In use, tag clouds can evolve as the associated data source changes over time. Interesting discussions around tag clouds often include a series of tag clouds and consider how they evolve over time. However, since tag clouds do not explicitly represent trends or support comparisons, the cognitive demands placed on the person for perceiving trends in multiple tag clouds are high. In this paper, we introduce SparkClouds, which integrate sparklines [23] into a tag cloud to convey trends between multiple tag clouds. We present results from a controlled study that compares SparkClouds with two traditional trend visualizations\&\#8212;multiple line graphs and stacked bar charts\&\#8212; as well as Parallel Tag Clouds [4]. Results show that SparkClouds ability to show trends compares favourably to the alternative visualizations.

Lee, B, et al. 2010, "Fast High-Quality Volume Ray Casting with Virtual Samplings." IEEE Transactions on Visualization and Computer Graphics 16(6):1525-32.

Volume ray-casting with a higher order reconstruction filter and/or a higher sampling rate has been adopted in direct volume rendering frameworks to provide a smooth reconstruction of the volume scalar and/or to reduce artifacts when the combined frequency of the volume and transfer function is high. While it enables high-quality volume rendering, it cannot support interactive rendering due to its high computational cost. In this paper, we propose a fast high-quality volume ray-casting algorithm which effectively increases the sampling rate. While a ray traverses the volume, intensity values are uniformly reconstructed using a high-order convolution filter. Additional samplings, referred to as virtual samplings, are carried out within a ray segment from a cubic spline curve interpolating those uniformly reconstructed intensities. These virtual samplings are performed by evaluating the polynomial function of the cubic spline curve via simple arithmetic operations. The min max blocks are refined accordingly for accurate empty space skipping in the proposed method. Experimental results demonstrate that the proposed algorithm, also exploiting fast cubic texture filtering supported by programmable GPUs, offers renderings as good as a conventional ray-casting algorithm using high-order reconstruction filtering at the same sampling rate, while delivering $2.5 \mathrm{x}$ to $3.3 \mathrm{x}$ rendering speed-up.

Lee, H, and H Park. 2010, "Genetracer : Gene Sequence Analysis of Disease Mutations Vast 2010 Mini Challenge 3 Award : Excellent Process Explanation." In IEEE Symposium on Visual Analytics Science \& Technology, pp. 291-92.

Lee, J, J Forlizzi, and SE Hudson. 2008, "Iterative Design of Move: A Situationally Appropriate Vehicle Navigation System." International Journal of Human-Computer Studies 66(3):198-215.

Drivers need assistance when navigating an unfamiliar route. In-vehicle navigation systems have improved in recent years due to the technology advances, but are sometimes problematic because of information overload while driving. To address the attentional demands of reading a map while driving, we have developed the maps optimized for vehicular environments (MOVE) in-car navigation display, which provides situationally appropriate navigation information to the driver through optimization of map 
information. In this paper, we describe the iterative design and evaluation process that shaped the MOVE system. We describe early map reading and navigation studies that led to early designs for our system. We present a study on visual search tasks that refined the renditions used for the system. Finally, we present a study on the effectiveness of several variations of a contextually optimized route map visualization with a desktop steering system. The result of this study shows that MOVE's contextually optimized navigation information can reduce the driver's perceptual load significantly. Our laboratory experiment shows that the total map display fixation time was decreased six-fold, and the number of glances to interpret the map display were decreased about threefold, when comparing the contextually optimized display to a static display.

Lee, KC, and N Chung. 2008, "Empirical Analysis of Consumer Reaction to the Virtual Reality Shopping Mall." Computers in Human Behavior 24(1):88-104.

The Internet shopping mall has received wide attention from researchers and practitioners due to the fact that it is one of the most killing applications customers can find on the Internet. Though numerous studies have been performed on various issues of the Internet shopping mall, some research issues relating to the user interface of VR (virtual reality) shopping malls still await further empirical investigation. The objective of this study is to investigate whether the user interface of the VR shopping mall positively affects customer satisfaction in comparison with the ordinary shopping mall. For this purpose, we developed a prototype of the VR shopping mall for which the user interface consists of both 3D graphics and an avatar, using it as an experimental medium. 102 valid questionnaires were gathered from active student users of the ordinary shopping mall, and two research hypotheses were then tested to prove whether the three explanatory variables such as convenience, enjoyment, quality assurance improve in the VR shopping mall, and whether customer satisfaction is also significantly enhanced in the VR shopping mall in comparison with the ordinary shopping mall. Additionally, we conducted the PLS (partial least square) analysis to test whether the customer satisfaction is explained significantly by the three explanatory variables or not.

Lee, MD, MA Butavicius, and RE Reilly. 2003, "Visualizations of Binary Data: A Comparative Evaluation." International Journal of Human-Computer Studies 59(5):569-602.

Data visualization has the potential to assist humans in analysing and comprehending large volumes of data, and to detect patterns, clusters and outliers that are not obvious using non-graphical forms of presentation. For this reason, data visualizations have an important role to play in a diverse range of applied problems, including data exploration and mining, information retrieval, and intelligence analysis. Unfortunately, while various different approaches are available for data visualization, there have been few rigorous evaluations of their effectiveness. This paper presents the results of three controlled experiments comparing the ability of four different visualization approaches to help people answer meaningful questions for binary data sets. Two of these visualizations, Chernoff faces and star glyphs, represent objects using simple icon-like displays. The other two visualizations use a spatial arrangement of the objects, based on a model of human mental representation, where more similar objects are placed nearer each other. One of these spatial displays uses a common features model of similarity, while the other uses a distinctive features model. The first experiment finds that both glyph visualizations lead to slow, inaccurate answers being given with low confidence, while the faster and more confident answers for spatial visualizations are only accurate when the common features similarity model is used. The second experiment, which considers only the spatial visualizations, supports this finding, with the common features approach again producing more accurate answers. The third experiment measures human 
performance using the raw data in tabular form, and so allows the usefulness of visualizations in facilitating human performance to be assessed. This experiment confirms that people are faster, more confident and more accurate when an appropriate visualization of the data is made available.

Lee, Y-L, S-L Hwang, and Eric. 2005, "Reducing Cognitive Workload of a Computer-Based Procedure System." International Journal of Human-Computer Studies 63(6):587-606.

The use of procedure systems is an important safety management strategy in coping with emergency or abnormal situations in a process control system. With the digitalization trend in these complex and large-scale systems, most aspects of a process control system are also computerized. In addition to the primary tasks, operators now have to do extra secondary tasks when using the computerized systems. In this research, three design features aimed to reduce the cognitive workload are evaluated on our research platform, SimCBP and SimPlant. These two systems work in tandem to simulate a Computer-Based Procedure (CBP) system and a simplified nuclear power plant. From the results of the experiments, the design of embedded controls/parameters is found to be efficient but its counterpart has implications for the design of training materials. Navigation aid, although not statistically significant, is important because of the subjective responses and the need of cross-referencing. The simplified flowchart display format, like other researches on the use of this format, revealed mixed results. Implications and directions for future studies are also proposed.

Lehmann, DJ, and H Theisel. 2010, "Discontinuities in Continuous Scatter Plots." IEEE Transactions on Visualization and Computer Graphics 16(6):1291-300.

The concept of continuous scatterplot (CSP) is a modern visualization technique. The idea is to define a scalar density value based on the map between an n-dimensional spatial domain and an m-dimensional data domain, which describe the CSP space. Usually the data domain is two-dimensional to visually convey the underlying, density coded, data. In this paper we investigate kinds of map-based discontinuities, especially for the practical cases $n=m=2$ and $n=3 \mid m=2$, and we depict relations between them and attributes of the resulting CSP itself. Additionally, we show that discontinuities build critical line structures, and we introduce algorithms to detect them. Further, we introduce a discontinuitybased visualization approach\&\#8212; called contribution map (CM)\&\#8212; which establishes a relationship between the CSP's data domain and the number of connected components in the spatial domain. We show that CMs enhance the CSP-based linking \&\#x0026; brushing interaction. Finally, we apply our approaches to a number of synthetic as well as real data sets.

Leide, JE, et al. 2003, "Visualization Schemes for Domain Novices Exploring a Topic Space: The Navigation Classification Scheme." Information Processing \& Management 39(6):923-40.

In this article and two other articles which conceptualize a future stage of the research program (Leide, Cole, Large, \& Beheshti, submitted for publication; Cole, Leide, Large, Beheshti, \& Brooks, in preparation), we map-out a domain novice user's encounter with an IR system from beginning to end so that appropriate classification-based visualization schemes can be inserted into the encounter process. This article describes the visualization of a navigation classification scheme only. The navigation classification scheme uses the metaphor of a ship and ship's navigator traveling through charted (but unknown to the user) waters, guided by a series of lighthouses. The lighthouses contain mediation interfaces linking the user to the information store through agents created for each. The user's agent is the cognitive model the user has of the information space, which the system encourages to evolve via 
interaction with the system's agent. The system's agent is an evolving classification scheme created by professional indexers to represent the structure of the information store. We propose a more systematic, multidimensional approach to creating evolving classification/indexing schemes, based on where the user is and what she is trying to do at that moment during the search session.

Leishi, Z, J Kuljis, and L Xiaohui. 2008, "Information Visualization for DNA Microarray Data Analysis: A Critical Review." Systems, Man, and Cybernetics, Part C: Applications and Reviews, IEEE

Transactions on 38(1):42-54.

Graphical representation may provide effective means of making sense of the complexity and sheer volume of data produced by DNA microarray experiments that monitor the expression patterns of thousands of genes simultaneously. The ability to use ldquoabstractrdquo graphical representation to draw attention to areas of interest, and more in-depth visualizations to answer focused questions, would enable biologists to move from a large amount of data to particular records they are interested in, and therefore, gain deeper insights in understanding the microarray experiment results. This paper starts by providing some background knowledge of microarray experiments, and then, explains how graphical representation can be applied in general to this problem domain, followed by exploring the role of visualization in gene expression data analysis. Having set the problem scene, the paper then examines various multivariate data visualization techniques that have been applied to microarray data analysis. These techniques are critically reviewed so that the strengths and weaknesses of each technique can be tabulated. Finally, several key problem areas as well as possible solutions to them are discussed as being a source for future work.

Lengler, R. 2007, "How to Induce the Beholder to Persuade Himself: Learning from Advertising Research for Information Visualization." In 11th International Conference on Information Visualization (IV 2007), pp. 382-92.

Research on the interplay of cognition and effect in the interpretation of visuals has made significant theoretical advances. This literature review highlights three themes which have emerged in advertising research. First it looks at the determinants constituting and influencing 'likeability'- the best predictor of advertisement effectiveness. Second it presents evidence how emotional advertising frames and shapes perception and how it could influence persuasion. Finally it discusses the role of 'co-authoring' in the interpretation of visuals and how it does affect persuasion over the emotional route.

Lengler, R. 2006, "Identifying the Competencies of 'Visual Literacy' - a Prerequisite for Knowledge Visualization." In International Conference on Information Visualization (IV '06), pp. 232-36.

Visual representations are becoming more important for teaching, learning and communicating. This paper argues that a more profound understanding of the "vision competencies" constituting "visual literacy" will enable practitioners and researchers alike to produce more effective knowledge visualizations. The main contribution of this paper is to identify some of the core competencies of a visually literate person, by examining the abilities tested in visual intelligence tests 
Lengler, R, and AV Moere. 2009, "Guiding the Viewer's Imagination: How Visual Rhetorical Figures Create Meaning in Animated Infographics." In 13th International Conference Information Visualisation, pp. 585-91.

Some visualizations need not only adequately represent information; their presentation needs also to be effective, leading to a behavior change in the viewer. Rhetorical figures, like metaphors, are said to increase the effectiveness of a message. This position paper first reviews the literature on why metaphors are more persuasive. Second, it presents four distinct meaning creation operations as one dimension of a future classification system. Third, it explains the properties of the meaning creation operations on four state-of-the-art works from infographics. Fourth, it presents an analysis of published empirical studies from the field of advertising, and provides advice on how to best use them in practice.

Leroy, G, et al. 2007, "An End User Evaluation of Query Formulation and Results Review Tools in Three Medical Meta-Search Engines." International Journal of Medical Informatics 76(11-12):780-89.

Purpose Retrieving sufficient relevant information online is difficult for many people because they use too few keywords to search and search engines do not provide many support tools. To further complicate the search, users often ignore support tools when available. Our goal is to evaluate in a realistic setting when users use support tools and how they perceive these tools.Methods We compared three medical search engines with support tools that require more or less effort from users to form a query and evaluate results. We carried out an end user study with 23 users who were asked to find information, i.e., subtopics and supporting abstracts, for a given theme. We used a balanced within-subjects design and report on the effectiveness, efficiency and usability of the support tools from the end user perspective.Conclusions We found significant differences in efficiency but did not find significant differences in effectiveness between the three search engines. Dynamic user support tools requiring less effort led to higher efficiency. Fewer searches were needed and more documents were found per search when both query reformulation and result review tools dynamically adjust to the user query. The query reformulation tool that provided a long list of keywords, dynamically adjusted to the user query, was used most often and led to more subtopics. As hypothesized, the dynamic result review tools were used more often and led to more subtopics than static ones. These results were corroborated by the usability questionnaires, which showed that support tools that dynamically optimize output were preferred.

Lespinats, S, et al. 2009, "Rankvisu: Mapping from the Neighborhood Network." Neurocomputing 72(1315):2964-78.

Most multidimensional scaling methods focus on the preservation of dissimilarities to map high dimensional items in a low-dimensional space. However, the mapping function usually does not consider the preservation of small dissimilarities as important, since the cost is small with respect to the preservation of large dissimilarities. As a consequence, an item's neighborhoods may be sacrificed for the benefit of the overall mapping. We have subsequently designed a mapping method devoted to the preservation of neighborhood ranks rather than their dissimilarities: RankVisu. A mapping of data is obtained in which neighborhood ranks are as close as possible according to the original space. A comparison with both metric and non-metric MDS highlights the pros (in particular, cluster enhancement) and cons of RankVisu. 
Leung, YK, and MD Apperley. 1994, "A Review and Taxonomy of Distortion-Oriented Presentation Techniques." ACM Transactions on Computer-Human Interaction 1(2):126-60.

Lex, A, et al. 2010, "Comparative Analysis of Multidimensional, Quantitative Data." IEEE Transactions on Visualization and Computer Graphics 16(6):1027-35.

When analyzing multidimensional, quantitative data, the comparison of two or more groups of dimensions is a commontask. Typical sources of such data are experiments in biology, physics or engineering, which are conducted in different configurationsand use replicates to ensure statistically significant results. One common way to analyze this data is to filter it using statistical methodsand then run clustering algorithms to group similar values. The clustering results can be visualized using heat maps, which showdifferences between groups as changes in color. However, in cases where groups of dimensions have an a priori meaning, it is notdesirable to cluster all dimensions combined, since a clustering algorithm can fragment continuous blocks of records. Furthermore,identifying relevant elements in heat maps becomes more difficult as the number of dimensions increases. To aid in such situations, we have developed Matchmaker, a visualization technique that allows researchers to arbitrarily arrange and compare multiple groupsof dimensions at the same time. We create separate groups of dimensions which can be clustered individually, and place them in anarrangement of heat maps reminiscent of parallel coordinates. To identify relations, we render bundled curves and ribbons betweenrelated records in different groups. We then allow interactive drill-downs using enlarged detail views of the data, which enable in-depthcomparisons of clusters between groups. To reduce visual clutter, we minimize crossings between the views. This paper concludeswith two case studies. The first demonstrates the value of our technique for the comparison of clustering algorithms. In the second,biologists use our system to investigate why certain strains of mice develop liver disease while others remain healthy, informallyshowing the efficacy of our system when analyzing multidimensional data containing distinct groups of dimensions.

Li, B, X Xue, and J Fan. 2007, "A Robust Incremental Learning Framework for Accurate Skin Region Segmentation in Color Images." Pattern Recognition 40(12):3621-32.

In this paper, we propose a robust incremental learning framework for accurate skin region segmentation in real-life images. The proposed framework is able to automatically learn the skin color information from each test image in real-time and generate the specific skin model (SSM) for that image. Consequently, the SSM can adapt to a certain image, in which the skin colors may vary from one region to another due to illumination conditions and inherent skin colors. The proposed framework consists of multiple iterations to learn the SSM, and each iteration comprises two major steps: (1) collecting new skin samples by region growing; (2) updating the skin model incrementally with the available skin samples. After the skin model converges (i.e., becomes the SSM), a post-processing can be further performed to fill up the interstices on the skin map. We performed a set of experiments on a large-scale real-life image database and our method observably outperformed the well-known Bayesian histogram. The experimental results confirm that the SSM is more robust than static skin models.

Li, J, JB Martens, and J J. 2010, "Judging Correlation from Scatterplots and Parallel Coordinate Plots." Information Visualization 9(1):13-30.

Scatterplots and parallel coordinate plots (PCPs) that can both be used to assess correlation visually. In this paper, we compare these two visualization methods in a controlled user experiment. More 
specifically, 25 participants were asked to report observed correlation as a function of the sample correlation under varying conditions of visualization method, sample size and observation time. A statistical model is proposed to describe the correlation judgment process. The accuracy and the bias in the judgments in different conditions are established by interpreting the parameters in this model. A discriminability index is proposed to characterize the performance accuracy in each experimental condition. Moreover, a statistical test is applied to derive whether or not the human sensation scale differs from a theoretically optimal (that is, unbiased) judgment scale. Based on these analyses, we conclude that users can reliably distinguish twice as many different correlation levels when using scatterplots as when using PCPs. We also find that there is a bias towards reporting negative correlations when using PCPs. Therefore, we conclude that scatterplots are more effective than parallel plots in supporting visual correlation analysis. C 2010 Macmillan Publishers Ltd.

Li, Q. 2009, "Variable-Scale Representation of Road Networks on Small Mobile Devices." Computers \& Geosciences 35(11):2185-90.

A method is proposed for the adaptive multi-scale representation of road networks for location-based service applications. The method is able to automatically set a feasible scale according to geographic scope, the complexity of the road network, and the distance to the viewer. Moreover, the method achieves multi-scale representations of road networks on a display screen. The key steps of the method and the initial experimental studies undertaken to evaluate its feasibility are described.

Li, Q, and C North. 2003, "Empirical Comparison of Dynamic Query Sliders and Brushing Histograms." In 9th Annual IEEE Symposium on Information Visualization (INFOVIS 2003), pp. 147-53.

Dynamic queries facilitate rapid exploration of information by real-time visual display of both query formulation and results. Dynamic query sliders are linked to the main visualization to filter data. A common alternative to dynamic queries is to link several simple visualizations, such as histograms, to the main visualization with a brushing interaction strategy. Selecting data in the histograms highlights that data in the main visualization. We compare these two approaches in an empirical experiment on DataMaps, a geographic data visualization tool. Dynamic query sliders resulted in better performance for simple range tasks, while brushing histograms was better for complex trend evaluation and attribute relation tasks. Participants preferred brushing histograms for understanding relationships between attributes and the rich information they provided.

Li, S-T, and W-C Chang. 2009, "Exploiting and Transferring Presentational Knowledge Assets in R\&D Organizations." Expert Systems with Applications 36(1):766-77.

Nowadays, how to exploit and transfer the value of knowledge assets effectively has been the primary challenge faced by global R\&D organizations. Surprisingly, there seems to be little argument about managing presentational knowledge assets, which are widely used by knowledge workers to present their ideas, proposals, findings and reports. This paper develops the management model and related IT enabling tools that support users to better exploit and transfer presentational knowledge assets. The solution integrates text extractor, slideshow generator, knowledge repository, content-based retrieval and ontology-enabled search engine along with the goal of portraying the search results in a visual navigation form. The performance satisfaction of the proposed system was proved statistically by conducting the user's survey of effectiveness and usability. This solution has demonstrated to be a feasible way for better managing contents, prompting cognitive learning, improving presentation production and presentational 
knowledge transformation, and consequently facilitates the value leverage of the presentational knowledge assets.

Lian, X, and MX Zhou. 2010, "Understanding Text Corpora with Multiple Facets." In IEEE Symposium on Visual Analytics Science \& Technology, pp. 99-106.

Liang, et al. 2010, "A New Concentric-Circle Visualization of Multi-Dimensional Data and Its Application in Network Security." Journal of Visual Languages \& Computing 21(4):194-208.

With the rapid growth of networked data communications in size and complexity, network administrators today are facing more challenges to protect their networked computers and devices from all kinds of attacks. This paper proposes a new concentric-circle visualization method for visualizing multi-dimensional network data. This method can be used to identify the main features of network attacks, such as DDoS attack, by displaying their recognizable visual patterns. To reduce the edge overlaps and crossings, we arrange multiple axes displayed as concentric circles rather than the traditional parallel lines. In our method, we use polycurves to link values (vertexes) rather than polylines used in parallel coordinate approach. Some heuristics are applied in our new method in order to improve the readability of views. We discuss the advantages as well as the limitations of our new method. In comparison with the parallel coordinate visualization, our approach can reduce more than $15 \%$ of the edge overlaps and crossings. In the second stage of the method, we have further enhanced the readability of views by increasing the edge crossing angle. Finally, we introduce our prototype system: a visual interactive network scan detection system called CCScanViewer. It is based on our new visualization approach and the experiments have showed that the new approach is effective in detecting attack features from a variety of networking patterns, such as the features of network scans and DDoS attacks.

Liang, H-N, and K Sedig. 2010, "Can Interactive Visualization Tools Engage and Support Pre-University Students in Exploring Non-Trivial Mathematical Concepts?" Computers \& Education 54(4):972-91.

Many students find it difficult to engage with mathematical concepts. As a relatively new class of learning tools, visualization tools may be able to promote higher levels of engagement with mathematical concepts. Often, development of new tools may outpace empirical evaluations of the effectiveness of these tools, especially in educational contexts. This seems to be the case with educational visualization tools. Much evidence about the effectiveness of these tools appears to be more suggestive than based on empirical evaluations. In this paper, we attempt to fill this gap and provide empirical evidence for the use of visualization tools in supporting exploratory and other learning-related activities. In particular, we aim to investigate whether visualization tools can be used to engage pre-university students in exploring nontrivial mathematical concepts. We focus particularly on this age group and content domain because of the difficulty these students may encounter when trying to investigate more challenging mathematical concepts. Also, it is during their formative years before university that students' predisposition and likeness towards mathematical ideas are formed. We report in this paper a study assessing whether a visualization tool, whose design was informed explicitly by research from information visualization and human-computer interaction, could engage pre-university students in their exploration and learning of more advanced mathematical concepts. Students who participated in this study came from multiple grade levels and have diverse cognitive and language skills as well as preferences towards mathematics. The results of this study indicate that visualization tools can effectively engage these students and support their exploration of non-trivial mathematical concepts, only if the tool is designed such that it can cater the diverse needs of these students. 
Liao, Q, et al. 2010, "Managing Networks through Context: Graph Visualization and Exploration." Computer Networks 54(16):2809-24.

With the increasing prevalence of multi-user environments in distributed systems, it has become an increasingly challenging task to precisely identify who is doing what on an enterprise network. Current management systems that rely on inference for user identity and application are not capable of accurately reporting and managing a large-scale network due to the coarseness of the collected data or scaling of the collection mechanism. We propose a system that focuses data collection in the form of local context, i.e., the precise user and application associated with a network connection. Through the use of dynamic correlation and novel graph modeling, we developed a visualization tool called ENAVis (the work appeared in earlier form in [1] and received USENIX best paper award). (Enterprise Network Activities Visualization). ENAVis aids a real-world administrator in allowing them to more efficiently manage and gain insight about the connectivity between hosts, users, applications and data access offering significant streamlining of the management process.

Liao, Z, and B Chen. 2010, "Anomaly Detection in Gps Data Based on Visual Analytics." In IEEE Symposium on Visual Analytics Science \& Technology, pp. 51-58.

Liiv, I. 2010, "Towards Information-Theoretic Visualization Evaluation Measure: A Practical Example for Bertin's Matrices." In BELIV'10: Beyond time and errors: novel evaluation methods for Information Visualization, April 10-11, 2010.

The definition and usage of quality metrics for Information Visualization techniques is still an immature field. Several proposals are available but a common view and understanding of this issue is still missing. This paper attempts a first step toward a visual quality metrics systematization, providing a general classification of both metrics and usage purposes. Moreover, the paper explores a quite neglected class of visual quality metrics, namely Feature Preservation Metrics, that allow for evaluating and improving in a novel way the effectiveness of basic Infovis techniques.

Lim, JS, SR Jeong, and SR Schach. 2005, "An Empirical Investigation of the Impact of the ObjectOriented Paradigm on the Maintainability of Real-World Mission-Critical Software." Journal of Systems and Software 77(2):131-38.

Empirical evidence for the maintainability of object-oriented systems is far from conclusive, partly due to the lack of representativeness of the subjects and systems used in earlier studies. We empirically examined this issue for mission-critical software that was currently operational and maintained by software professionals. Two functionally equivalent versions of a credit approval system were maintained, one object oriented (OO) and the other non-object oriented (NOO). We found that the OO group took less time to maintain a greater number of software artifacts than its NOO counterpart. This difference held for all phases of the software development life cycle. This result was due to the usefulness of UML for impact analysis of the OO version, which contributed to effective comprehension and communication. Insufficient design specifications for the NOO version led to ambiguity and costly defects in transferring design solutions to development. Also, the encapsulation of the OO version appeared to reduce mental loads for maintenance tasks and resulted in code reuse. On the other hand, the number of files to be managed increased and, thus, dependency management was required for the $\mathrm{OO}$ version. Furthermore, despite much tuning, the $\mathrm{OO}$ version ran slower than its NOO counterpart. More 
field studies on software professionals are needed to compare contextual factors such as methods, processes, and maintenance tools.

Lim, SW, BS Lee, and AHB Duffy. 2001, "Incremental Modelling of Ambiguous Geometric Ideas (IMagi): Representation and Maintenance of Vague Geometry." Artificial Intelligence in Engineering 15(2):93-108.

During conceptual design, a designer may wish to describe a shape vaguely, either because it is desired that the shape remains flexible or the shape has not yet been defined precisely. Maintaining the vagueness of an early idea until it is sufficiently developed is often of vital importance. However, most existing systems, mainly due to limitations in their modelling capabilities, attempt to interpret and thereby remove the vagueness at the earliest opportunity. This may lead to the loss of considerable information in original concepts or design fixation too early in the design stage. A new approach is needed to represent and maintain vague geometric information and such an approach is presented in this paper.

Lin, MC, and CY Chang. 2005, "The Development of a 3d Visualization Tool on the Topic of Cold Front." In 13th International Conference on Computers in Education (ICCCE 2005), pp. 771-74.

This paper presents the development of a Virtual Environmental learning interface on the topic cold front. Due to limited class time and tight course schedule, teachers are usually bound to providing students with 'instant knowledge' that is constructed and disseminated by professional scientific research. Therefore, students often think inactively and learn passively, resulting in their less retention of knowledge in secondary schools in Taiwan. With the design of a visualization 3D tool proposed by the study, learners will better understand the temperature distribution by measuring and simulating temperature parameters in a 3D/VR environment on their own. They will also be provided with greater opportunities to realize that a cold front is defined as the transition zone where a cold air mass is replacing a warm air mass in a 3D sense. The rationale behind the development this type of system is to show that the learning process should emphasize a model that is created by students themselves instead of by teachers' displaying and students' memorizing.

Lin, Q. 2008, "Techniques of Visualization for Distribution Automation System." In China International Conference on Electricity Distribution, pp. 1-6.

Distribution automation system supervises and manages all the loads, equipments and faults in the area of distribution. Due to a vast area , too many equipments and complex circumstance, the traditional display method can't satisfy the demand of modern distribution automation system. In the traditional distribution automation system we often use one-line diagram, geography diagram, curve drawing and bar chart to display the state of distribution automation system, they're too simple to display the complex and large scale diagrams. It need to be introduced into new techniques to supervise, control and display the running state of distribution automation system. The visualization technique is a new project practical technique which appears along with the computer graphics development in 1990s. It transforms each kind of complex project data as the direct-viewing graph. It's advantageous to people to have a thorough grasp and a correct understanding of the facts. This paper introduces the graph visualization technique, discusses the application of visualization technique in the distribution automation system. In order to analyze data, dig up the connections between data and select suitable method to display, the paper provides a new thinking. The paper introduces all kinds of method of visualization graph, such as: $2 \mathrm{D}, 3 \mathrm{D}$ graph, contour line, gradual change area coloring, pie chart, arrowhead, direction, speed, varying size and 
so on. It describes the method to realize these techniques. The new techniques include: three-dimensional graph technique based on openGL, cartoon technique, the algorithm of contouring, the algorithm of area drawing. The research indicates that it is inefficient to simply display the system data that isn't processed. The visualized vivid graph may express the information which need many data to indicate. Our visualization software not only expresses the system with the visualization graph method, but also carries on the analysis and the exc- avation in the system magnanimous data. It discovers intrinsic relation of the data to reflect the system mode accurately and displays them by the suitable visualization method. The information expressed in the distribution automation system is: state, capacity, margin, phase angle, speed, load distribution, movement tendency, stable region, geographic distribution information and so on. The display of the information is not isolated. It may simultaneously express many kinds of data meaning through one visualization method. Certainly, the time which the visualization technique is used in our country is not very long. Many companies use the overseas software directly. There are few companies to develop their own real-time application visualization software. Our company makes great progress in the research into computer graph technique and applying visualization technique in the realtime distribution automation system. We also have obtained the quite good effect. Along with the scale of our power system unceasing expansion, higher request, the visualization technique must be obtained broad application.

Lin, S, et al. 2010, "Towards Anomaly Comprehension : Using Structural Compression to Navigate Profiling Call-Trees." In 5th International Symposium on Software Visualization.

Lin, X, HD White, and J Buzydlowski. 2003, "Real-Time Author Co-Citation Mapping for Online Searching." Information Processing \& Management 39(5):689-706.

Author searching is traditionally based on the matching of name strings. Special characteristics of authors as personal names and subject indicators are not considered. This makes it difficult to identify a set of related authors or to group authors by subjects in retrieval systems. In this paper, we describe the design and implementation of a prototype visualization system to enhance author searching. The system, called AuthorLink, is based on author co-citation analysis and visualization mapping algorithms such as Kohonen's feature maps and Pathfinder networks. AuthorLink produces interactive author maps in real time from a database of 1.26 million records supplied by the Institute for Scientific Information. The maps show subject groupings and more fine-grained intellectual connections among authors. Through the interactive interface the user can take advantage of such information to refine queries and retrieve documents through point-and-click manipulation of the authors' names.

Lindholm, S, et al. 2010, "Spatial Conditioning of Transfer Functions Using Local Material Distributions." IEEE Transactions on Visualization and Computer Graphics 16(6):1301-10.

In many applications of Direct Volume Rendering (DVR) the importance of a certain material or feature is highly dependent on its relative spatial location. For instance, in the medical diagnostic procedure, the patient's symptoms often lead to specification of features, tissues and organs of particular interest. One such example is pockets of gas which, if found inside the body at abnormal locations, are a crucial part of a diagnostic visualization. This paper presents an approach that enhances DVR transfer function design with spatial localization based on user specified material dependencies. Semantic expressions are used to define conditions based on relations between different materials, such as only render iodine uptake when close to liver. The underlying methods rely on estimations of material distributions which are acquired by weighing local neighborhoods of the data against approximations of 
material likelihood functions. This information is encoded and used to influence rendering according to the user's specifications. The result is improved focus on important features by allowing the user to suppress spatially less-important data. In line with requirements from actual clinical DVR practice, the methods do not require explicit material segmentation that would be impossible or prohibitively timeconsuming to achieve in most real cases. The scheme scales well to higher dimensions which accounts for multi-dimensional transfer functions and multivariate data. Dual-Energy Computed Tomography, an important new modality in radiology, is used to demonstrate this scalability. In several examples we show significantly improved focus on clinically important aspects in the rendered images.

Lipford, HR, et al. 2010, "Helping Users Recall Their Reasoning Process." In IEEE Symposium on Visual Analytics Science \& Technology, pp. 187-94.

Liu, C-L. 2010, "The Impact of Goods-Classification and Landmarks for Spatial Knowledge and GoodsFinding in the Elderly within a 3d Virtual Store." Computers in Human Behavior 26(6):1777-86.

Online shopping has become quite popular since its first arrival on the internet. Although numerous studies have been performed to investigate various issues related to the internet store, some research issues relating to the spatial cognition of the elderly (the fastest growing internet group) when exploring a $3 \mathrm{D}$ virtual store still await further empirical investigation. The objective of this study was to examine how elderly users acquire spatial knowledge in an on-screen virtual store. Specifically, the impact of different types of landmarks on the acquisition of spatial knowledge was examined. In addition, in this study, goods-classification was seen as an implicit landmark associated with the acquisition of spatial knowledge. Therefore, it is worth observing the impact during the location of the goods and examining the combined effect with landmarks. The experimental results indicated that landmarks are important for the elderly as they attempt to locate goods within a 3D virtual store, no matter what types are used. However, landmarks are not the only resources for constructing spatial knowledge in a 3D virtual store; the classification of goods is also a good resource and may be more important than landmarks. In addition, the combined effect of goods-classification and landmarks in a 2D image would be best for the elderly in terms of acquired spatial cognition and the location of goods within a 3D virtual store.

Liu, D, A Raja, and J Vaidyanath. 2007, "Tibor: A Resource-Bounded Information Foraging Agent for Visual Analytics." In IEEE/WIC/ACM International Conference on Intelligent Agent Technology, pp. 349-55.

Visual analytics is the science of applying reasoning and analysis techniques to large, complex realworld data for problem solving using visualizations. Real world knowledge gathering and investigative tasks are very complex because the problem-solving context is constantly evolving, and the data may be incomplete, unreliable and/or conflicting. We describe a mixed-initiative reasoning agent that will assist investigative analysts to choose from and reason about enormous databases of text, imagery, video and Webcast. This agent leverages sequential decision making and an AI blackboard system to support hypothesis tracking and validation in a highly uncertain environment. Resource-bounded control mechanisms enable the agent to reason about the uncertainty. We have also designed a user interface that will enable analysts to gather and sift large amounts of evidence and to collaborate with and, where necessary, to control the agent. 
Liu, D, et al. 2008, "The Role of Blackboard-Based Reasoning and Visual Analytics in Resin's Predictive Analysis." In IEEE/WIC/ACM International Conference on Web Intelligence and Intelligent Agent Technology, pp. 2-5.

Knowledge gathering and investigative tasks in open environments can be very complex because the problem-solving context is constantly evolving, and the data may be incomplete, unreliable and/or conflicting. This paper significantly extends our previous work on a mixed-initiative agent by making it capable of assisting humans in foraging task analysis using AI blackboard-based reasoning, visualizations and a mix-initiative user interface. The agent is equipped with the ability to adapt its processing to available resources, deadlines and its current problem-solving context.

Liu, J, JC Wang, and Y Zhou. 2007, "The Applications of Geo-Referenced Data Visualization Technologies for Gis - Art. No. 67542m." In 15th International Conference on Geoinformatics, pp. M7542-M42.

Geo-referenced data visualization is one of the most important components of geographic information systems. Over the past several years, geospatial data are growing much more in size and complexity than ever before, and researchers are engaged in doing a lot of works to visualize these diverse geospatial data by taking advantage of computer graphics which helps to convey information and amplify cognition and makes possible for more powerful participating exploration and discovery experience. This paper will discuss the related works on visualization for GIS. The first chapter of this paper is an introduction which will present an overview. In the second chapter, we will talk about the geo-virtual environment which closely related to the virtual reality concept. We will focus on representation of urban models. terrain rendering algorithms, and the problems we currently face. For the third part, we will talk about two young but promising fields, which are scientific visualization and information visualization. The brief history and the research issues of these two disciplines will be the main topic. Finally, we will make a outlook on the future works about human-computer interaction, and hardware acceleration.

Liu, XP, et al. 2007, "Constraint Information Visualization Methodology for Cooperative Design." in Computer Supported Cooperative Work in Design Iii, eds. W Shen, et al., Vol 4402, pp. 63-72.

It is difficult to manage constraint information in cooperative design due to its variety and changeability. The concept of Constraint Information Visualization (CIV) in cooperative design and CIV framework comprising the CIV methodology are put forward to control cooperative design flow efficiently, detect constraint conflicts early and negotiate the contradiction under the support of visualization. On the basis of classification and representation of constraint information, this paper discusses the importance of CIV and its formalized description for cooperative design. The procedure of visual mapping and harmony evaluation is given in details. The main step of visual mapping is divided into classification mapping, structure and harmony mapping and detailed property mapping. Visual Mapping implements mapping from constraint information to visual representation with corresponding mapping modes. The concepts of local harmony and global harmony are also proposed for cooperation. An application of CIV verifies CIV methodology, which will direct a new way to implement complicated constraints management in cooperative design. 
Liu, Y, and G Salvendy. 2006, "Design and Evaluation of Visualization Support to Facilitate Association Rules Modeling." International Journal of Human-Computer Interaction 21(1):15-38.

Association rules mining is a popular data mining modeling tool. It discovers interesting associations or correlation relationships among a large set of data items, showing attribute values that occur frequently together in a given dataset. Despite their great potential benefit, current association rules modeling tools are far from optimal. This article studies how visualization techniques can be applied to facilitate the association rules modeling process, particularly what visualization elements should be incorporated and how they can be displayed. Original designs for visualization of rules, integration of data and rule visualizations, and visualization of rule derivation process for supporting interactive visual association rules modeling are proposed in this research. Experimental results indicated that, compared to an automatic association rules modeling process, the proposed interactive visual association rules modeling can significantly improve the effectiveness of modeling, enhance understanding of the applied algorithm, and bring users greater safisfaction with the task. The proposed integration of data and rule visualizations can significantly facilitate understanding rules compared to their nonintegrated counterpart.

Liu, Y, and G Salvendy. 2007, "Design and Evaluation of Visualization Support to Facilitate Decision Trees Classification." International Journal of Human-Computer Studies 65(2):95-110.

The loosely coupled relationships between visualization and analytical data mining (DM) techniques represent the majority of the current state of art in visual data mining; DM modeling is typically an automatic process with very limited forms of guidance from users. A conceptual model of the visualization support to DM modeling process and a novel interactive visual decision tree (IVDT) classification process have been proposed in this paper, with the aim of exploring humans' pattern recognition ability and domain knowledge to facilitate the knowledge discovery process. An IVDT for categorical input attributes has been developed and experimented on 20 subjects to test three hypotheses regarding its potential advantages. The experimental results suggested that, compared to the automatic modeling process as typically applied in current decision tree modeling tools, IVDT process can improve the effectiveness of modeling in terms of producing trees with relatively high classification accuracies and small sizes, enhance users' understanding of the algorithm, and give them greater satisfaction with the task.

Liu, Z, et al. 2010, "Data Ingestion and Evidence Marshalling in Jigsaw." In IEEE Symposium on Visual Analytics Science \& Technology, pp. 271-72.

Liu, Z, and B Lee. 2010, "Netclinic : Interactive Visualization to Enhance Automated Fault Diagnosis in Enterprise Networks." In IEEE Symposium on Visual Analytics Science \& Technology, pp. 131-38.

Liu, Z, NJ Nersessian, and JT Stasko. 2008, "Distributed Cognition as a Theoretical Framework for Information Visualization." IEEE Transactions on Visualization and Computer Graphics 14(6):1173-80.

Even though information visualization (InfoVis) research has matured in recent years, it is generally acknowledged that the field still lacks supporting, encompassing theories. In this paper, we argue that the distributed cognition framework can be used to substantiate the theoretical foundation of InfoVis. We highlight fundamental assumptions and theoretical constructs of the distributed cognition approach, based on the cognitive science literature and a real life scenario. We then discuss how the distributed cognition framework can have an impact on the research directions and methodologies we take as InfoVis 
researchers. Our contributions are as follows. First, we highlight the view that cognition is more an emergent property of interaction than a property of the human mind. Second, we argue that a reductionist approach to study the abstract properties of isolated human minds may not be useful in informing InfoVis design. Finally we propose to make cognition an explicit research agenda, and discuss the implications on how we perform evaluation and theory building. (C) 2008 IEEE.

Liu, Z, and J Stasko. 2010, "Mental Models, Visual Reasoning and Interaction in Information Visualization: A Top-Down Perspective." Visualization and Computer Graphics, IEEE Transactions on 16(6):999-1008.

Although previous research has suggested that examining the interplay between internal and external representations can benefit our understanding of the role of information visualization (InfoVis) in human cognitive activities, there has been little work detailing the nature of internal representations, the relationship between internal and external representations and how interaction is related to these representations. In this paper, we identify and illustrate a specific kind of internal representation, mental models, and outline the high-level relationships between mental models and external visualizations. We present a top-down perspective of reasoning as model construction and simulation, and discuss the role of visualization in model based reasoning. From this perspective, interaction can be understood as active modeling for three primary purposes: external anchoring, information foraging, and cognitive offloading. Finally we discuss the implications of our approach for design, evaluation and theory development

Liu, ZC, NJ Nersessian, and JT Stasko. 2008, "Distributed Cognition as a Theoretical Framework for Information Visualization." IEEE Transactions on Visualization and Computer Graphics 14(6):1173-80.

Even though information visualization (InfoVis) research has matured in recent years, it is generally acknowledged that the field still lacks supporting, encompassing theories. In this paper, we argue that the distributed cognition framework can be used to substantiate the theoretical foundation of InfoVis. We highlight fundamental assumptions and theoretical constructs of the distributed cognition approach, based on the cognitive science literature and a real life scenario. We then discuss how the distributed cognition framework can have an impact on the research directions and methodologies we take as InfoVis researchers. Our contributions are as follows. First, we highlight the view that cognition is more an emergent property of interaction than a property of the human mind. Second, we argue that a reductionist approach to study the abstract properties of isolated human minds may not be useful in informing InfoVis design. Finally we propose to make cognition an explicit research agenda, and discuss the implications on how we perform evaluation and theory building.

Lobenhofer, EK, et al. 2006, "Application of Visualization Tools to the Analysis of Histopathological Data Enhances Biological Insight and Interpretation." Toxicologic Pathology 34(7):921-28.

Gene expression profiling, metabolomic screens, and other high-dimensional methods have become an integral part of many biological investigations. To facilitate interpretation of these data, it is important to have detailed phenotypic data - including histopathology - to which these data can be associated, or anchored. However, as the amount of phenotypic data increases, associations within and across these data can be difficult to visualize and interpret. We have developed an approach for categorizing and clustering biologically related histopathological diagnoses to facilitate their visualization, thereby increasing the possibility of identifying associations and facilitating the comparison with other data streams. In this study, we utilize histopathological data generated as part of a standardized toxicogenomics compendium 
study to generate composite histopathological scores and to develop visualizations that facilitate biological insight. The validity of this approach is illustrated by the identification of transcripts that correlate with the pathology diagnoses that comprise the categories of "response to hepatocellular injury" and "repair." This approach is broadly applicable to studies in which histopathology is used to phenotypically anchor other data, and results in visualizations that facilitate biological interpretation and the identification of associations and relationships within the data.

Lohse, GL. 1997, "Chapter 6 - Models of Graphical Perception." in Handbook of Human-Computer Interaction, eds. GH Marting, KL Thomas and VP Prasad, pp. 107-35. North-Holland, Amsterdam.

Summary Graphical perception investigates the mechanisms by which humans perceive, interpret, use, and communicate graphical information. Models of graphical perception explain how people process information from graphs and heighten our understanding of the underlying psychological processes. This knowledge facilitates the design of graphics by providing robust quantitative predictions for evaluating alternative graphic designs or for automating the design of graphics entirely. Algorithmic models of graphical perception and cognition enable quantitative predictions about the effectiveness of a graph for a specific task. The ultimate goal for future versions of commercial graphics software is to automate graphic design processes in a manner that is transparent to the user. Embedded intelligence would reduce the amount of time users spend creating graphs and presentations. Furthermore, many casual users could then create graphics that follow certain rules for effective graphic presentation.

Lopes, AA, et al. 2007, "Visual Text Mining Using Association Rules." Computers \& Graphics 31(3):316-26.

In many situations, individuals or groups of individuals are faced with the need to examine sets of documents to achieve understanding of their structure and to locate relevant information. In that context, this paper presents a framework for visual text mining to support exploration of both general structure and relevant topics within a textual document collection. Our approach starts by building a visualization from the text data set. On top of that, a novel technique is presented that generates and filters association rules to detect and display topics from a group of documents. Results have shown a very consistent match between topics extracted using this approach to those actually present in the data set.

Lopes, RB, et al. 2010, "Information Visualization in Facility Location and Vehicle Routing Decisions." In 14th International Conference on Information Visualization, pp. 110-15.

Facility location and vehicle routing are amongst the most important logistic decisions in today's organizations. These aspects are intertwined and, in some cases, should be addressed in an integrated way (giving rise to the location-routing approach). A decision support tool that can make easier the visualization (and editing) of information regarding these problems is becoming increasingly important as: it enables to further understand the problem at hand; and, at the same time, it fosters better communication of the decisions in a way easier to understand by the general public. This paper presents some concepts for information visualization on the problems arisen by the aforementioned decisions, which have been incorporated in a decision support tool and tested using usability evaluation methods. 
Loureiro da Costa, A, and G Bittencour. 1998, "Dynamic Social Knowledge: A Cognitive Multi-Agent System Cooperation Strategy." In International Conference on Multi Agent Systems, pp. 415-16.

The adopted cooperation strategy is one of the most important aspects to make an agent community achieve a common goal. Basically there are two kinds of cooperation strategies for cognitive multi agent systems: contract net protocol and negotiation. Contract net protocol requires a small amount of knowledge about the other agents in the community, but it is not possible to persuade another agent to perform a given task or to join some agents in an agent group to perform a given task during a cooperation process. On the other hand, negotiation allows an agent to communicate directly with another agent. This direct communication allows agreements when the optimal solution is not available, but the required amount of social knowledge and the communication overhead needed to update it is much greater than in the contract net protocol. A new cooperation strategy for open cognitive autonomous multi agent systems, called Dynamic Social Knowledge is proposed. This strategy combines the advantages of both contract net protocol and negotiation, and makes intensive use of rule based reasoning

Lowe, A, RW Jones, and MJ Harrison. 2001, "The Graphical Presentation of Decision Support Information in an Intelligent Anaesthesia Monitor." Artificial Intelligence in Medicine 22(2):173-91.

This contribution examines the graphical presentation of decision support information generated by an intelligent monitor, named Sentinel, developed for use during anaesthesia. Clinicians make diagnoses in real-time during operations by examining clinically significant trends in multiple signals. Sentinel attempts to mimic this decision process by using a system of fuzzy trend templates. Sentinel's implementation of fuzzy trend templates is capable of providing the dual fuzzy measures of belief and plausibility, which are derived from the theory of evidence. It is thus capable of generating fairly rich diagnostic decision support information. However, for Sentinel to be effective, the visual presentation of this information must be intuitive to the anaesthetist, who may not be familiar with the theory of evidence. This paper discusses techniques that are being evaluated to meet the requirements of the Sentinel anaesthesia monitor. Specifically, the paper presents methods for highlighting clinically significant trends in physiological (or derived) signals by superimposing a coloured band on the signal that reflects fuzzy output from the intelligent monitor. This paper also discusses the intuitive graphical presentation of binary diagnostic fuzzy measures, including their further interpretation and presentation as crisp "alarm" and "warning" conditions.

Lowrie, T, and CM Diezmann. 2011, "Solving Graphics Tasks: Gender Differences in Middle-School Students." Learning and Instruction 21(1):109-25.

The capacity to solve tasks that contain high concentrations of visual-spatial information, including graphs, maps and diagrams, is becoming increasingly important in educational contexts as well as everyday life. This research examined gender differences in the performance of students solving graphics tasks from the Graphical Languages in Mathematics (GLIM) instrument that included number lines, graphs, maps and diagrams. The participants were 317 Australian students (169 males and 148 females) aged 9-12 years. Boys outperformed girls on graphical languages that required the interpretation of information represented on an axis and graphical languages that required movement between two- and three-dimensional representations (generally Map language). 
Lu, A, et al. 2007, "Example-Based Volume Illustrations." In IEEE Conference on Information Visualization, pp. 655-62.

Scientific illustrations use accepted conventions and methodologies to effectively convey object properties and improve our understanding. We present a method to illustrate volume datasets by emulating example illustrations. As with technical illustrations, our volume illustrations more clearly delineate objects, enrich details, and artistically visualize volume datasets. For both color and scalar 3D volumes, we have developed an automatic color transfer method based on the clustering and similarities in the example illustrations and volume sources. As an extension to 2D Wang tiles, we provide a new, general texture synthesis method for Wang cubes that solves the edge discontinuity problem. We have developed a 2D illustrative slice viewer and a GPU-based direct volume rendering system that uses these non-periodic 3D textures to generate illustrative results similar to the 2D examples. Both applications simulate scientific illustrations to provide more information than the original data and visualize objects more effectively, while only requiring simple user interaction.

Lu, A, R Maciejewski, and DS Ebert. 2010, "Volume Composition and Evaluation Using Eye-Tracking Data." ACM Transactions on Applied 6(4):1-22.

This article presents a method for automating rendering parameter selection to simplify tedious user interaction and improve the usability of visualization systems. Our approach acquires the important/interesting regions of a dataset through simple user interaction with an eye tracker. Based on this importance information, we automatically compute reasonable rendering parameters using a set of heuristic rules, which are adapted from visualization experience and psychophysical experiments. A user study has been conducted to evaluate these rendering parameters, and while the parameter selections for a specific visualization result are subjective, our approach provides good preliminary results for general users while allowing additional control adjustment. Furthermore, our system improves the interactivity of a visualization system by significantly reducing the required amount of parameter selections and providing good initial rendering parameters for newly acquired datasets of similar types.

Lu, X-J, M-X Ding, and Y-K Wang. 2009, "A New Pseudo-Color Transform for Fibre Masses Inspection of Industrial Images." Acta Automatica Sinica 35(3):233-38.

In this paper, we propose a novel pseudo-color approach which can effectively mark all different particulars while it has the ability to divide foreign pieces with indiscernible brightness in fibre masses. Hence, it yields highly separate color images for this kind of industrial images. Our approach aims at constructing an adaptive fuzzy rule based on a feature space of gradients, gray values, local entropies, and local features from scalable morphological filters. Due to the fact that the method is based on a full contextual information, it can handle small changes in images more adaptively than classical pseudo-color methods. And it is perfectly suitable to distinguish impurities mixed in fibre masses, as tested in a simulating cotton inspection system and demonstrated by several cotton sample images.

Lu, Y-H. 2006, "Resource-Driven Content Adaptation." In Computational Imaging IV Conference, pp. 111.

Recent trends have created new challenges in the presentation of multimedia information. First, large, high-resolution video displays are increasingly popular. Meanwhile, many mobile devices, such as PDAs and mobile telephones, can display images and videos on small screens. One obvious issue is that content 
designed for a large display is inappropriate for a small display. Moreover, wireless bandwidth and battery lifetime are precious resources for mobile devices. In order to provide useful content across systems with di erent resources, we propose \resource-driven content adaptation" by augmenting the content with metadata that can be used to display or render the content based on the available resources. We are investigating several problems related to resource-driven content adaptation. These include: adaptation of the presented content based on available resources| display resolution, bandwidth, processor speed, quality of services, and energy. Content adaptation may add or remove information based on available resources. Adaptive content can utilize resources more e ectively but also present challenges in resource management, content creation, transmission, and user perception.

Luboschik, M, A Radloff, and H Schumann. 2010, "Poster : Using Non-Photorealistic Rendering Techniques for the Visualization of Uncertainty." In IEEE Conference on Information Visualization.

Luboschik, M, H Schumann, and H Cords. 2008, "Particle-Based Labeling: Fast Point-Feature Labeling without Obscuring Other Visual Features." IEEE Transactions on Visualization and Computer Graphics 14(6):1237-44.

In many information visualization techniques, labels are an essential part to communicate the visualized data. To preserve the expressiveness of the visual representation, a placed label should neither occlude other labels nor visual representatives (e.g., icons, lines) that communicate crucial information. Optimal, non-overlapping labeling is an NP-hard problem. Thus, only a few approaches achieve a fast non-overlapping labeling in highly interactive scenarios like information visualization. These approaches generally target the point-feature label placement (PFLP) problem, solving only label-label conflicts. This paper presents a new, fast, solid and flexible 2D labeling approach for the PFLP problem that additionally respects other visual elements and the visual extent of labeled features. The results (number of placed labels, processing time) of our particle-based method compare favorably to those of existing techniques. Although the esthetic quality of non-real-time approaches may not be achieved with our method, it complies with practical demands and thus supports the interactive exploration of information spaces. In contrast to the known adjacent techniques, the flexibility of our technique enables labeling of dense point clouds by the use of non-occluding distant labels. Our approach is independent of the underlying visualization technique, which enables us to demonstrate the application of our labeling method within different information visualization scenarios.

Lunzer, A, and K Hornbæk. 2010, "Chapter 14 - Subjunctive Interfaces for the Web." in No Code Required: Giving Users Tools to Transform the Web, eds. C Allen, et al., pp. 267-85. Morgan Kaufmann, Boston.

Summary Abstract The data resources and applications accessible through today's Web offer tremendous opportunities for exploration: ask a slightly different question, receive a correspondingly different answer. However, typical browser-based mechanisms for accessing the Web only enable users to pose one such question at a time, placing a heavy operational and cognitive burden on any user who wants to explore and compare alternatives. A subjunctive-interface approach may reduce this burden. Subjunctive interfaces support the setting up, viewing, and adjustment of multiple scenarios in parallel, allowing side-by-side instead of temporally separated viewing, and more efficient iteration through alternatives. We have implemented a spreadsheet-inspired environment where end users can program and use their own Web-access applications that include such multiscenario support. In this chapter we describe three modes of use of this environment - parallel retrieval, coordinated manipulation, and 
tentative composition - and explain how these may help to alleviate typical challenges in Web-based tasks. At the same time, we acknowledge that the increased scope for exploration made possible through this environment can itself present a form of cognitive burden to users, and we outline our plans for evaluating the impact of this effect.

Luo, D, et al. 2009, "Eventriver: Interactive Visual Explorations of Streaming Text." Symposium A Quarterly Journal In Modern Foreign Literatures 28(3):1-8.

In this paper, we introduce NewsLab, an exploratory visualization approach for the analysis of large scale broadcast news video collections containing many thousands of news stories over extended periods of time. A river metaphor is used to depict the thematic changes of the news over time. An interactive lens metaphor allows the playback of fine-grained video segments selected through the river overview. Multi-resolution navigation is supported via a hierarchical time structure as well as a hierarchical theme structure. Themes can be explored hierarchically according to their thematic structure, or in an unstructured fashion using various ranking criteria. A rich set of interactions such as filtering, drilldown/roll-up navigation, history animation, and keyword based search are also provided. Our case studies show how this set of tools can be used to find emerging topics in the news, compare different broadcasters, or mine the news for topics of interest.

Luo, H, et al. 2008, "Integrating Multi-Modal Content Analysis and Hyperbolic Visualization for LargeScale News Video Retrieval and Exploration." Signal Processing: Image Communication 23(7):538-53.

In this paper, we have developed a novel scheme to achieve more effective analysis, retrieval and exploration of large-scale news video collections by performing multi-modal video content analysis and synchronization. First, automatic keyword extraction is performed on news closed captions and audio channels to detect the most interesting news topics (i.e., keywords for news topic interpretation), and the associations among these news topics (i.e., contextual relationships among the news topics) are further determined according to their co-occurrence probabilities. Second, visual semantic items, such as human faces, text captions, video concepts, are extracted automatically by using our semantic video analysis techniques. The news topics are automatically synchronized with the most relevant visual semantic items. In addition, an interestingness weight is assigned for each news topic to characterize its importance. Finally, a novel hyperbolic visualization scheme is incorporated to visualize large-scale news topics according to their associations and interestingness. With a better global overview of large-scale news video collections, users can specify their queries more precisely and explore large-scale news video collections interactively. Our experiments on large-scale news video collections have provided very positive results.

Luo, H, et al. 2006, Integrating Semantic Video Understanding and Knowledge Visualization for LargeScale News Video Exploration. Technical Rpt. CVC-UNCC-07-01, UNC-Charlotte.

In this paper, we have developed a novel framework to enable more effective visual analysis and exploration of large-scale news videos via knowledge visualization. A novel interestingness measurement for video news reports is proposed to enable analysts and general audiences to find news stories of interest at first glance and catch the valuable knowledge in large-scale video news databases. Keyframes, keywords and their relations are automatically extracted from news video clips and visually represented according to their interestingness measurement. Our techniques for intelligent news video analysis have the capacity to enable more effective visualization and exploration of large-scale news videos. Our 
visualization-based news video analysis and exploration system is very useful for analysts and general audiences to quickly find the news stories of interest from large-scale news videos extracted from many channels.

Luo, H, et al. 2007, "Analyzing Large-Scale News Video Databases to Support Knowledge Visualization and Intuitive Retrieval." In IEEE Symposium on Visual Analytics Science and Technology, pp. 107-14.

In this paper, we have developed a novel framework to enable more effective investigation of largescale news video database via knowledge visualization. To relieve users from the burdensome exploration of well-known and uninteresting knowledge of news reports, a novel interestingness measurement for video news reports is presented to enable users to find news stories of interest at first glance and capture the relevant knowledge in large-scale video news databases efficiently. Our framework takes advantage of both automatic semantic video analysis and human intelligence by integrating with visualization techniques on semantic video retrieval systems. Our techniques on intelligent news video analysis and knowledge discovery have the capacity to enable more effective visualization and exploration of largescale news video collections. In addition, news video visualization and exploration can provide valuable feedback to improve our techniques for intelligent news video analysis and knowledge discovery.

Luo, H, et al. 2006, "Exploring Large-Scale Video News Via Interactive Visualization." In IEEE Symposium on Visual Analytics Science \& Technology, pp. 75-82.

In this paper, we have developed a novel visualization framework to enable more effective visual analysis of large-scale news videos, where keyframes and keywords are automatically extracted from news video clips and visually represented according to their interestingness measurement to help audiences rind news stories of interest at first glance. A computational approach is also developed to quantify the interestingness measurement of video clips. Our experimental results have shown that our techniques for intelligent news video analysis have the capacity to enable more effective visualization of large-scale news videos. Our news video visualization system is very useful for security applications and for general audiences to quickly find news topics of interest from among many channels

Luo, H, et al. 2008, "Incorporating Feature Hierarchy and Boosting to Achieve More Effective Classifier Training and Concept-Oriented Video Summarization and Skimming." ACM Transactions on Multimedia Computing, Communications, and Applications 4(1):1-25.

For online medical education purposes, we have developed a novel scheme to incorporate the results of semantic video classification to select the most representative video shots for generating conceptoriented summarization and skimming of surgery education videos. First, salient objects are used as the video patterns for feature extraction to achieve a good representation of the intermediate video semantics. The salient objects are defined as the salient video compounds that can be used to characterize the most significant perceptual properties of the corresponding real world physical objects in a video, and thus the appearances of such salient objects can be used to predict the appearances of the relevant semantic video concepts in a specific video domain. Second, a novel multi-modal boosting algorithm is developed to achieve more reliable video classifier training by incorporating feature hierarchy and boosting to dramatically reduce both the training cost and the size of training samples, thus it can significantly speed up SVM (support vector machine) classifier training. In addition, the unlabeled samples are integrated to reduce the human efforts on labeling large amount of training samples. Finally, the results of semantic 
video classification are incorporated to enable concept-oriented video summarization and skimming. Experimental results in a specific domain of surgery education videos are provided.

Luo, Q, et al. 2008, "Human Action Detection Via Boosted Local Motion Histograms." Machine Vision and Applications 21(3):377-89.

This paper presents a novel learning method for human action detection in video sequences. The detecting problem is not limited in controlled settings like stationary background or invariant illumination, but studied in real scenarios. Spatio-temporal volume analysis for actions is adopted to solve the problem. To develop effective representation while remaining resistant to background motions, only motion information is exploited to define suitable descriptors for action volumes. On the other hand, action models are learned by using boosting techniques to select discriminative features for efficient classification. This paper also shows how the proposed method enables learning efficient action detectors, and validates them on publicly available datasets.

Luximon, A, VG Duffy, and W Zhang. 2001, "Performance Differences in a Cross-Cultural Comparison of Voice Enhanced Interface." International Journal of Industrial Ergonomics 28(3-4):133-42.

The increasing power of computer hardware is enabling the user interface to enter a new dimension, where voice enhanced interfaces can remove the barrier between people and the machine for some users. In this study the traditional keyboard and mouse interface (TI) was compared with voice enhanced interface (VEI). An automatic semester grade calculation software was developed in visual basic and enhanced with a "trainable" voice recognition software for Cantonese and English. Results show that there are significant differences in task completion time, number of errors and satisfaction for the traditional keyboard interface and the voice enhanced interface. In addition, contrary to our intuition, native Cantonese speakers had more voice input errors when speaking Cantonese than English. Though voice enhanced interfaces have many potential applications, consideration should be given to the differences between English and spoken languages that are based on tones such as Cantonese. It appears that further development in voice recognition technology is required in order to make voice input widely usable for the Chinese speaking computer users. Relevance to industry Voice input may seem the ultimate input method, since hands free data entry can allow tremendous flexibility. However, the results of this study indicate that, in industry, voice input should be used with caution, especially when tonal languages or safety are involved.

Luzzardi, PRG, et al. 2004, "An Extended Set of Ergonomic Criteria for Information Visualization Techniques." in Proceedings of the Seventh Iasted International Conference on Computer Graphics and Imaging, ed. MH Hamza, pp. 236-41. ACTA Press.

Several information visualization techniques have been developed in the last years due to the need of representing and analyzing the huge amount of data generated by several applications or made available through the World Wide Web. These techniques are usually interactive and provided as part of a graphical user interface. Information visualization techniques are usually reported showing their use in experimental situations, employing some kind of analysis. Recently, studies have specifically addressed the evaluation of such techniques. This paper reports results from the definition and application of specific criteria for the evaluation of information visualization techniques. The criteria encompass the evaluation of both visual representations and interaction mechanisms, within the framework of usability testing methods. 
MacEachren, AM. 2005, "Chapter 22 - Moving Geovisualization toward Support for Group Work." in Exploring Geovisualization, eds. D Jason, MM Alan and K Menno-Jan, pp. 445-61. Elsevier, Oxford.

Summary This chapter provides a conceptual framework for research and practice in geovisualization and for visualization as a facilitator for group work with geospatial information. This framework identifies three primary functions for visual representations as a vehicle to support group work. First, visual representations can act as the object of collaboration, thus as an entity to discuss, create, or manipulate. Second, visualization can provide support for dialogue (about information, plans, methods, strategies, or decisions). Third, visual representation can provide support for coordinated activity (thus for compiling information, carrying out plans, or executing decisions). This chapter addresses each function in a section that sketches the state of the art and identifies some of the key research questions that need attention. Examples from recent and ongoing research in the GeoVISTA Center at Penn State University are used to illustrate the issues discussed. This chapter concludes with a call for multidisciplinary collaboration to address issues raised so that we can take full advantage of advances in technology that promise to enable distributed and collaborative work.

MacEachren, AM. 2001, "An Evolving Cognitive-Semiotic Approach to Geographic Visualization and Knowledge Construction." Information Design Journal 10(1):26-36.

Maceachren, AM, and M-J Kraak. 1997, "Exploratory Cartographic Visualization: Advancing the Agenda." Computers \& Geosciences 23(4):335-43.

An approach to the visualization of georeferenced data is presented. This approach is rooted in cartography and emphasizes the use of visual methods in research and decision making. Several definitions proposed within cartography are considered and the links between "cartographic" visualization and scientific visualization more generally are discussed. From this base, a perspective on visualization is articulated in which attention is directed to the goals for use of maps and related georeferenced displays. We argue that a use-based approach is needed in order to develop information processing environments appropriate to distinct stages of scientific research and decision making. The paper concludes by proposing a set of research problems that are prompted by taking a use-based approach to visualization, and then outlining the selection and context of the papers in this special issue.

MacEachren, AM, et al. 2006, "Building a Geocollaboratory: Supporting Human-Environment Regional Observatory (Hero) Collaborative Science Activities." Computers, Environment and Urban Systems $30(2): 201-25$.

Collaboratories have been defined as centers without walls, virtual places where teams of scientists can undertake coordinated research. As part of the Human-Environment Regional Observatory (HERO) infrastructure project, we have been developing a geocollaboratory to support work by geographically distributed scientists about geographic problems. Our specific focus is on science teams developing and applying protocols for long-term study of the local and regional scale human impacts of global environmental change. The HERO geocollaboratory includes web and other Internet-based tools to enable same-time and different-time (thus synchronous and asynchronous) different-place collaboration. Methods and tools have been developed to support (1) synchronous distributed meetings that include video links and shared visual display of geospatial information; (2) asynchronous perspective comparison and consensus building activities; and (3) long-term information sharing and knowledge development. This paper introduces the research effort, sketches the conceptual framework within which the 
geocollaboratory is being developed, outlines progress thus far in the three collaboratory components listed above, and discusses our experiences using these tools for distributed science as well as our plans for continued development. We direct specific attention to three web-based, collaborative tools we have developed in support of components 2 and 3 above: an e-Delphi tool (supporting sharing and comparing of expert opinions), a concept-mapping tool that supports building, sharing, and comparing concept relationship diagrams linked to formal ontologies, and a web portal (called Codex) that provides a personal workspace, mechanisms for forming groups and accessing group resources, and methods for encoding knowledge objects that include geographic referencing.

Maciejewski, R, WS Cleveland, and LT Glickman. 2007, "Lahva : Linked Animal-Human Health Visual Analytics." In IEEE Symposium on Visual Analytics Science and Technology, pp. 27-34.

Coordinated animal-human health monitoring can provide an early warning system with fewer false alarms for naturally occurring disease outbreaks, as well as biological, chemical and environmental incidents. This monitoring requires the integration and analysis of multi-field, multi-scale and multisource data sets. In order to better understand these data sets, models and measurements at different resolutions must be analyzed. To facilitate these investigations, we have created an application to provide a visual analytics framework for analyzing both human emergency room data and veterinary hospital data. Our integrated visual analytic tool links temporally varying geospatial visualization of animal and human patient health information with advanced statistical analysis of these multi-source data. Various statistical analysis techniques have been applied in conjunction with a spatio-temporal viewing window. Such an application provides researchers with the ability to visually search the data for clusters in both a statistical model view and a spatio-temporal view. Our interface provides a factor specification/filtering component to allow exploration of causal factors and spread patterns. In this paper, we will discuss the application of our linked animal-human visual analytics (LAHVA) tool to two specific case studies. The first case study is the effect of seasonal influenza and its correlation with different companion animals (e.g., cats, dogs) syndromes. Here we use data from the Indiana Network for Patient Care (INPC) and Banfield Pet Hospitals in an attempt to determine if there are correlations between respiratory syndromes representing the onset of seasonal influenza in humans and general respiratory syndromes in cats and dogs. Our second case study examines the effect of the release of industrial wastewater in a community through companion animal surveillance.

Maciejewski, R, et al. 2008, "Companion Animals as Sentinels for Community Exposure to Industrial Chemicals: The Fairburn, Ga, Propyl Mercaptan Case Study." Public health reports (Washington, D.C. : 1974) 123(3):333-42.

OBJECTIVES: This study utilized the electronic medical records of six veterinary hospitals (operated by Banfield, The Pet Hospital) in the vicinity of Fairburn, Georgia, to assess the health of dogs and cats following the unintentional release of propyl mercaptan from a waste-processing facility. METHODS: Standardized electronic medical records were used to define clinical syndromes (eye inflammation, gastrointestinal, respiratory, fever, general weakness/change in mental state) in dogs and cats. The frequency and geographic distribution of each syndrome was evaluated before, during, and after the chemical release, using control charts, density maps, change in average mean distance from a suspected point source of chemical release, space-time statistics, and autoregressive integrated moving averages. RESULTS: No consistent pattern of change in syndromic events was observed following the suspected release of propyl mercaptan. Some syndromes, including respiratory syndrome in cats, gastrointestinal syndrome in dogs, and eye inflammation syndrome in both cats and dogs, showed a change in time and 
spatial patterns following the release of propyl mercaptan into the community. These changes were consistent with clinical signs observed in people during a previous propyl mercaptan release in California as well as the release in Fairburn. CONCLUSIONS: A systematic review of electronic medical records of dogs and cats exposed to release of propyl mercaptan showed no conclusive and consistent evidence of adverse health effects. Methods for the use of medical records of pets for detecting environmental hazards require further development and evaluation.

Maciejewski, R, et al. 2008, "Measuring Stipple Aesthetics in Hand-Drawn and Computer-Generated Images." IEEE Computer Graphics and Applications 28(2):62-74.

When people compare a computer-generated illustration to a hand-drawn illustration of the same object, they usually perceive differences. This seems to indicate that the two kinds of images follow different aesthetic principles. To explore and explain these differences, the authors compare texture stippling in hand-drawn and computer-generated illustrations, using image-processing analysis techniques.

Maciejewski, R, et al. 2008, "Situational Awareness and Visual Analytics for Emergency Response and Training." In IEEE Conference on Technologies for Homeland Security, pp. 252-56.

Many emergency response units are currently faced with restrictive budgets which prohibit their use of technology both in training and in real-world situations. Our work focuses on creating an affordable, mobile, state-of-the-art emergency response test-bed through the integration of low-cost, commercially available products. We have developed a command, control, communications, surveillance and reconnaissance system that will allow small-unit exercises to be tracked and recorded for evaluation purposes. Our system can be used for military and first responder training providing the nexus for decision making through the use of computational models, advanced technology, situational awareness and command and control. During a training session, data is streamed back to a central repository allowing commanders to evaluate their squads in a live action setting and assess their effectiveness in an after-action review. In order to effectively analyze this data, an interactive visualization system has been designed in which commanders can track personnel movement, view surveillance feeds, listen to radio traffic, and fast-forward/rewind event sequences. This system provides both 2-D and 3-D views of the environment while showing previously traveled paths, responder orientation and activity level. Both stationary and personnel-worn mobile camera video feeds may be displayed, as well as the associated radio traffic.

Maciejewski, R, S Rudolph, and SJ Grannis. 2008, "The Day-of-the-Week Effect: A Study across the Indiana Public Health Emergency Surveillance System." Advances in Disease Surveillance 5:44.

This paper investigates the existence of the day-ofthe- week (DoW) effect across twenty-six hospitals within the Indiana Public Health Emergency Surveillance System (PHESS) [1]. We will consider both the impact of each DoW and the impact of individual hospitals. BACKGROUND Complex

Maciejewski, R, et al. 2008, "A Visual Analytics Toolkit for Evaluating Potential Syndromic Outbreaks." Advances in Disease Surveillance 5(2):115.

This paper presents a toolkit designed to aid in the assessment of disease outbreak by visualizing spatiotemporal trends and interactively displaying detailed statistical data 
Maciejewski, R, et al. 2008, "Understanding Syndromic Hotspots - a Visual Analytics Approach." In 2008 IEEE Symposium on Visual Analytics Science and Technology, pp. 35-42.

Maciejewski, R, et al. 2010, "A Visual Analytics Approach to Understanding Spatiotemporal Hotspots." IEEE Transactions on Visualization and Computer Graphics 16(2):205-20.

As data sources become larger and more complex, the ability to effectively explore and analyze patterns among varying sources becomes a critical bottleneck in analytic reasoning. Incoming data contain multiple variables, high signal-to-noise ratio, and a degree of uncertainty, all of which hinder exploration, hypothesis generation/exploration, and decision making. To facilitate the exploration of such data, advanced tool sets are needed that allow the user to interact with their data in a visual environment that provides direct analytic capability for finding data aberrations or hotspots. In this paper, we present a suite of tools designed to facilitate the exploration of spatiotemporal data sets. Our system allows users to search for hotspots in both space and time, combining linked views and interactive filtering to provide users with contextual information about their data and allow the user to develop and explore their hypotheses. Statistical data models and alert detection algorithms are provided to help draw user attention to critical areas. Demographic filtering can then be further applied as hypotheses generated become fine tuned. This paper demonstrates the use of such tools on multiple geospatiotemporal data sets.

Maciel, M, et al. 2008, "The Impact of Multiple Coordinated Views on the Visual Data Exploration and Analysis." In 12th International Conference on Information Visualization, pp. 113-19.

The use of information visualization tools that support multiple coordinated views for data exploration and analysis has become more frequent. However, few usability studies have focused this group of tools. The goal of this paper is to perform usability experiments with tools supporting multiple coordinated views on the same screen, in separate screens and tools that support each technique individually. To ensure a fair evaluation, a complexity-based task taxonomy was developed. The tools were therefore used to answer questions of the same level of complexity.

Mackey, P, et al. 2005, "Dynamic Visualization of Graphs with Extended Labels." In IEEE Symposium on Information Visualization, pp. 73-80.

The paper describes a novel technique to visualize graphs with extended node and link labels. The lengths of these labels range from a short phrase to a full sentence to an entire paragraph and beyond. Our solution is different from all the existing approaches that almost always rely on intensive computational effort to optimize the label placement problem. Instead, we share the visualization resources with the graph and present the label information in static, interactive, and dynamic modes without the requirement for tackling the intractability issues. This allows us to reallocate the computational resources for dynamic presentation of real time information. The paper includes a user study to evaluate the effectiveness and efficiency of the visualization technique. 
Mackinlay, JD. 2002, "Opportunities for Information Visualization." Computer Graphics and Applications, IEEE 20(1):22-23.

Maheswara, G, JS Bradbury, and C Collins. 2010, "Tie : An Interactive Visualization of Thread Interleavings." In 5th ACM Symposium on Software Visualization (SOFTVIS), pp. 215-16.

Mahmoud, AH, A Clayden, and C Higgins. 1999, "A Comparative Study of Environmental Cognition in a Real Environment and Its Vrml Simulation (Virtual Reality Modelling Language)." In Third International Conference on Information Visualisation (IV'99), pp. 208-13.

The paper investigates the acquisition of environmental cognitive knowledge in a real world and its desktop VRML simulation. It focuses on the effect of design background and gender on spatial cognition in both displays. A post-rest-only control-group design is used examine to what extent a desktop VRML simulation provides users with cognitive data that is comparable to real world experience. Results indicate that there is a between-group agreement and disagreement depending on the typology of space.

Participants using desktop VRML could provide cognitive distance estimations that are equivalent to their counterparts in real world. Design background did have a significant effect on spatial cognition in real world, however it did not show a remarkable effect in desktop VRML. Gender has affected height estimation in the real world, however it did not show any effect in desktop VRML

Mahyar, N, A Sarvghad, and M Tory. 2010, "A Closer Look at Note Taking in the Co-Located Collaborative Visual Analytics Process." In IEEE Symposium on Visual Analytics Science \& Technology, pp. 171-78.

Malandrino, D, G Palmieri, and V Scarano. 2007, "Visualizing Processes on the Web." Journal of Visual Languages \& Computing 18(6):592-612.

In this paper, we describe 3WPS, a framework to build distributed systems that are able to monitor and interact with a process through a 3D interface that is accessible via the World Wide Web (WWW). The 3WPS is easily configurable, easily adaptable to different processes with high reuse of its software components and its distributed architecture leverages on off-the-shelf components of the WWW infrastructure such as Java applets and Virtual Reality Modeling Language (VRML) browsers. We describe the characteristics of 3WPS framework by mainly focusing on the issue of programmability and by contextually providing an example tour of its usage.

Malik, A, et al. 2010, "Vaccinated - Visual Analytics for Characterizing a Pandemic Spread." In IEEE Symposium on Visual Analytics Science \& Technology, pp. 281-82.

Manber, U. 1997, "The Use of Customized Emphasis in Text Visualization." In IEEE Conference on Information Visualisation, pp. 132-38.

The paper presents a technique to allow quick evaluation of the relevance of documents to one's interests. It is designed primarily for presenting documents on the World Wide Web, but it can also be used in other situations. The idea is to automatically highlight selected keywords in Web pages as they are shown. Users maintain lists of keywords of interest to them. At any given time, a user can activate any such list and from then on all words in that list will be highlighted automatically, transparently, and efficiently in all documents. Users can change lists at any time and also control several other highlighting 
parameters (such as the number of selected words). Extensions of this scheme, where documents are automatically changed in other ways based on readers' preferences, are also discussed

Manco, G, C Pizzuti, and D Talia. 2004, "Eureka!: An Interactive and Visual Knowledge Discovery Tool." Journal of Visual Languages \& Computing 15(1):1-35.

Visualization techniques may guide the data mining process since they provide effective support for data partitioning and visual inspection of results, especially when high dimensional data sets are considered. In this paper we describe Eureka!, an interactive, visual knowledge discovery tool for analyzing high dimensional numerical data sets. The tool combines a visual clustering method, to hypothesize meaningful structures in the data, and a classification machine learning algorithm, to validate the hypothesized structures. A two-dimensional representation of the available data allows users to partition the search space by choosing shape or density according to criteria they deem optimal. A partition can be composed by regions populated according to some arbitrary form, not necessarily spherical. The accuracy of clustering results can be validated by using different techniques (e.g., a decision tree classifier) included in the mining tool.

Mandic, M, and A Kerne. 2004, "Famailiar \& Intimacy-Based Email Visualization." In IEEE Symposium on Information Visualization, pp. p14-p14.

Email has developed into one of the most extensively used computer applications. Email interfaces, on the other hand, have gone through very few transformations since their inception, and as the growing volumes of email data accumulate in users' email boxes, these interfaces fail to provide effective message handling and browsing support. Saved email messages provide not only a vast record of one's electronic past, but also a potential source of valuable insights into the structure and dynamics of one's social network. In this paper, we present faMailiar, a novel email visualization that draws upon email's inherently personal character by using intimacy as a key visualization parameter. The program presents a visualization of email use over time. faMailiar facilitates navigation through large email collections, enabling the user to discover communication rhythms and patterns.

Mane, KK, and K Borner. 2007, "Computational Diagnostics: A Novel Approach to Viewing Medical Data." In International Conference on Coordinated and Multiple Views in Exploratory Visualization 2007, pp. 27-32.

A transition from traditional paper-based medical records to electronic health records is underway. The resulting massive amounts of electronic patient records offer tremendous potential to personalize patient diagnosis and treatment. In this paper, we discuss a computational diagnostics tool that uses digital medical records to help physicians gain better insight about a patient's medical condition. The paper details different interactive features of the tool and discusses the tool's potential in the practice of evidence-based medicine and for advancing patient diagnosis practices. 
Mann, G, and J Russell. 2010, "Deriving Structured Intelligence from Text : Supporting a Document Analysis Methodology." In IEEE Symposium on Visual Analytics Science \& Technology, pp. 2-3.

Mann, TM. 1999, "Visualization of Www-Search Results." In 10th International Workshop on Database and Expert Systems Applications, pp. 264-68.

The idea of Information Visualization is to get insights into great amounts of abstract data. Especially document sets found by searching the World Wide Web are a special challenge. The paper gives a short overview on the variety of possible visualizations for this application area. The presented ideas are grouped by using the four phase framework of information seeking. Crucial factors for the success of visualizations are discussed. An approach is presented to use alternative simple visualizations grouped around the traditional result-list, for the usage with a local meta web search engine

Mansmann, F, et al. 2007, "Visual Analysis of Network Traffic for Resource Planning, Interactive Monitoring, and Interpretation of Security Threats." Visualization and Computer Graphics, IEEE Transactions on 13(6):1105-12.

The Internet has become a wild place: malicious code is spread on personal computers across the world, deploying botnets ready to attack the network infrastructure. The vast number of security incidents and other anomalies overwhelms attempts at manual analysis, especially when monitoring service provider backbone links. We present an approach to interactive visualization with a case study indicating that interactive visualization can be applied to gain more insight into these large data sets. We superimpose a hierarchy on IP address space, and study the suitability of Treemap variants for each hierarchy level. Because viewing the whole IP hierarchy at once is not practical for most tasks, we evaluate layout stability when eliding large parts of the hierarchy, while maintaining the visibility and ordering of the data of interest.

Mansmann, F, and S Vinnik. 2006, "Interactive Exploration of Data Traffic with Hierarchical Network Maps." Visualization and Computer Graphics, IEEE Transactions on 12(6):1440-49.

Network communication has become indispensable in business, education and government. With the pervasive role of the Internet as a means of sharing information across networks, its misuse for destructive purposes, such as spreading malicious code, compromising remote hosts, or damaging data through unauthorized access, has grown immensely in the recent years. The classical way of monitoring the operation of large network systems is by analyzing the system logs for detecting anomalies. In this work, we introduce hierarchical network map, an interactive visualization technique for gaining a deeper insight into network flow behavior by means of user-driven visual exploration. Our approach is meant as an enhancement to conventional analysis methods based on statistics or machine learning. We use multidimensional modeling combined with position and display awareness to view source and target data of the hosts in a hierarchical fashion with the ability to interactively change the level of aggregation or apply filtering. The interdisciplinary approach integrating data warehouse technology, information visualization and decision support brings about the benefit of efficiently collecting the input data and aggregating over very large data sets, visualizing the results and providing interactivity to facilitate analytical reasoning 
Mao, R, et al. 2003, "Global up-Regulation of Chromosome 21 Gene Expression in the Developing Down Syndrome Brain." Genomics 81(5):457-67.

Down syndrome (DS) results from complete or partial triplication of human chromosome 21. It is assumed that the neurological and other symptoms are caused by the overexpression of genes on chromosome 21, but this hypothesis has not yet been assessed on a chromosome-wide basis. Here we show that expression of genes localized to chromosome 21 is globally up-regulated in human fetal trisomy 21 cases, both in cerebral cortex extracts and in astrocytic cell lines cultured from cerebral cortex. This abnormal regulation of gene expression is specific to chromosome 21 . Our data describe transcriptional changes that are specific to many genes assigned to chromosome 21 and do not directly measure the clinical phenotype of DS. However, it is possible that these gene expression changes ultimately relate to the phenotypic variability of DS.

Maranto, G, and M Barton. 2010, "Paradox and Promise: Myspace, Facebook, and the Sociopolitics of Social Networking in the Writing Classroom." Computers and Composition 27(1):36-47.

This article situates current theoretical, rhetorical, and ethical analyses of the net's most prominent social networking sites, MySpace and Facebook. It also discusses the implications of bringing these web sites into the classroom, comparing how students, teachers, and administrators use (and abuse) these spaces. Both MySpace and Facebook privilege a discourse based on the construction and representation of an identity. Rather than assert unique identities, these sites ask users to label and classify themselves according to many criteria, including age, religion, political leanings, hobbies, and interests. Users can then list others who share these labels or interests and request to "add them as friends." MySpace and Facebook emphasize categories and aspects of popular culture that teenagers find important. They remediate the traditions of high school for the Web and by doing so greatly extend their reach. Many writing instructors wonder how these sites can be used to teach writing. How users represent themselves online could help students understand postmodern logics of identity construction and political engagement. However, there are dangers for teachers who create their own profiles and add their students as "friends." Like chat and email, these forums undercut concepts of more conventional rhetorical spaces. They both contribute to and undermine student and faculty ethos, although students may not appreciate that their profiles might have a lasting negative impact. Despite the public nature of most profiles, users often denounce these "invasions" as blatant violations of their privacy. Perhaps teachers and scholars should work to protect the integrity of these spaces.

Marchand, P, et al. 2004, "Implementation and Evaluation of a Hypercube-Based Method for Spatiotemporal Exploration and Analysis." ISPRS Journal of Photogrammetry and Remote Sensing 59(12):6-20.

This paper presents the results obtained with a new type of spatiotemporal topological dimension implemented within a hypercube, i.e., within a multidimensional database (MDDB) structure formed by the conjunction of several thematic, spatial and temporal dimensions. Our goal is to support efficient SpatioTemporal Exploration and Analysis (STEA) in the context of Automatic Position Reporting System (APRS), the worldwide amateur radio system for position report transmission. Mobile APRS stations are equipped with GPS navigation systems to provide real-time positioning reports. Previous research about the multidimensional approach has proved good potential for spatiotemporal exploration and analysis despite a lack of explicit topological operators (spatial, temporal and spatiotemporal). Our project implemented such operators through a hierarchy of operators that are applied to pairs of instances of 
objects. At the top of the hierarchy, users can use simple operators such as "same place", "same time" or "same time, same place". As they drill down into the hierarchy, more detailed topological operators are made available such as "adjacent immediately after", "touch during" or more detailed operators. This hierarchy is structured according to four levels of granularity based on cognitive models, generalized relationships and formal models of topological relationships. In this paper, we also describe the generic approach which allows efficient STEA within the multidimensional approach. Finally, we demonstrate that such an implementation offers query run times which permit to maintain a "train-of-thought" during exploration and analysis operations as they are compatible with Newell's cognitive band (query runtime $<10$ s) (Newell, A., 1990. Unified theories of cognition. Harvard University Press, Cambridge MA, 549 p.).

Marchesin, S, et al. 2010, "View-Dependent Streamlines for 3d Vector Fields." IEEE Transactions on Visualization and Computer Graphics 16(6):1578-86.

This paper introduces a new streamline placement and selection algorithm for 3D vector fields. Instead of considering the problem as a simpl feature search in data space, we base our work on the observation that most streamline fields generate a lot of self-occlusion which prevents proper visualization. In order to avoid this issue, we approach the problem in a view-dependent fashion and dynamically determine a set of streamlines which contributes to data understanding without cluttering the view. Since our technique couples flow characteristic criteria and view-dependent streamline selection we are able achieve the best of both worlds: relevant flow description and intelligible, uncluttered pictures. We detail an efficient GPU implementation of our algorithm, show comprehensive visual results on multiple datasets and compare our method with existing flow depiction techniques. Our results show that our technique greatly improves the readability of streamline visualizations on different datasets without requiring user intervention.

Marchionini, G, and X Mu. 2003, "User Studies Informing E-Table Interfaces." Information Processing \& Management 39(4):561-79.

In this paper, we describe a series of user studies that were used to advance understanding of how people use electronic tables (E-tables) and inform the development of a web-based statistical table browser for use by non-specialists. Interviews and focus groups with providers, intermediaries, and nonspecialist end users; transaction log analysis; and e-mail content analysis were used to develop a user-task taxonomy for government statistical data. These studies were the basis for a prototype web-based interface for browsing statistical tables. Two usability studies with 23 subjects and two eye-tracking studies with 21 subjects were conducted with this interface and paper, PDF, and spreadsheet interfaces. The results of the needs assessment, prototype development, and user studies provide a foundation for understanding E-tables in general and guiding continued design of interfaces for E-tables.

Marchionini, G, Y Song, and R Farrell. 2009, "Multimedia Surrogates for Video Gisting: Toward Combining Spoken Words and Imagery." Information Processing \& Management 45(6):615-30.

Good surrogates that allow people to quickly derive the gist of videos without taking the time to view the full video are crucial to video retrieval and browsing systems. Although there are many kinds of textual and visual surrogates used in video retrieval systems, there are few audio surrogates in practice. To evaluate the effectiveness of audio surrogates alone and in combination with one kind of visual surrogate, fast forwards, a user study with 48 participants was conducted. The study investigated the 
effects of manually and automatically generated spoken keywords and spoken descriptions, using a textto-speech synthesizer, on six specific video gisting tasks. Results demonstrate that manually generated spoken descriptions are better than both manually generated spoken keywords and fast forwards for video gisting. Both spoken keywords, whether manually or automatically generated, and fast forwards are better than automatically extracted descriptions. High quality spoken summaries were found very effective for video gisting. Combining fast forwards with either type of spoken text was not significantly better than any of the individual spoken surrogates; however, the visual elements added subjective value to the user experience. Adding spoken descriptions or keywords as surrogates to video retrieval and browsing systems is recommended.

Marghescu, D. 2007, "User Evaluation of Multidimensional Data Visualization Techniques for Financial Benchmarking." In European Conference on Information Management and Evaluation, pp. 341-56.

In this paper, we investigate the use of information visualization techniques for getting insight into multidimensional financial data. In particular, we focus on exploring different multidimensional data visualization techniques with respect to their effectiveness in solving a financial problem, namely financial competitor benchmarking. Financial competitor benchmarking is concerned with comparing the financial performance of different companies competing in the same market, industry, country or region. We investigate the extent to which different multidimensional visualization techniques are effective in revealing interesting patterns in financial performance data. For this purpose, we conducted a user evaluation study in which nine multidimensional data visualization techniques were assessed. The assessment concerns the extent to which users of these techniques are capable of discovering interesting patterns in multidimensional financial data, patterns associated with the problem of financial benchmarking. These patterns are identified as outlier detection, clustering, cluster description, class description and data comparison. The visualization techniques under analysis are: multiple line graphs, permutation matrix, survey plot, scatter plot matrix, parallel coordinates, treemap, principal components analysis, Sammon's mapping and the Self-Organizing Maps. The evaluation method consists in questionnaire-based data collection and analysis. We obtained answers from 12 students who agreed upon participating in this study. The evaluation we have conducted is useful especially in the early stage of the development of a visualization system, because it helps in the process of selection of most appropriate techniques for solving certain tasks.

Mark, G, K Carpenter, and A Kobsa. 2003, "A Model of Synchronous Collaborative Information Visualization." In 7th International Conference on Information Visualization, pp. 373-81.

We describe a model of the process by which people solve problems using information visualization systems. The model was based on video analysis of forty dyads who performed information visualization tasks in an experiment. We examined the following variables: focused questions vs. free data discovery, remote vs. collocated collaboration, and systems judged to have high and low transparency. The model describes the stages of reasoning and generating solutions with visual data. We found the model to be fairly robust across task type, collaborative setting, and system type, though subtle differences were found. We propose that system transparency can support some stages of the process, and that support is needed in the last stage to help users translate their findings from visual to written representations. 
Marsh, SL, J Dykes, and F Attilakou. 2006, "Evaluating a Geovisualization Prototype with Two Approaches: Remote Instructional Vs. Face-to-Face Exploratory." In IEEE International Conference on Information Visualization, pp. 310-15.

Two evaluations of a prototype designed to help expert users visualize key census statistics are conducted. The results yielded are compared in terms of usability issues, task completion (interaction) and ideation facilitated. Ways in which this information may be affected by the use of different data collection techniques, participants and tasks are considered. We report differences in the results of the evaluations in each of the three areas and suggest that flexible and non-disruptive methods be used to investigate whether geovisualization tools can support knowledge construction. We recommend using exploratory tasks, employing 'think aloud' strategies, requiring users to suggest and explain hypotheses and using screen capture to contextualise the data collected

Martinez-moyano, I, et al. 2006, "Modeling the Emergence of Insider Threat Vulnerabilities." In Winter Simulation Conference, pp. 562-68.

In this paper, we present insights generated by modeling the emergence of insider threat vulnerabilities in organizations. In our model, we integrate concepts from social judgment theory, signal detection theory, and the cognitive psychology of memory and belief formation. With this model, we investigate the emergence of vulnerabilities (especially that are insider-driven) in complex systems characterized by high levels of feedback complexity, multiple actors, and the presence of uncertainty in the judgment and decision processes. We use the system dynamics method of computer simulation to investigate the consequences caused by changes to the model's assumptions. We find that the emergence of vulnerability can be an endogenous process and that leverage points to reduce this vulnerability involve improvement in information acquisition, information management, and the training of personnel in judgment and decision-making techniques

Martins, SB, et al. 2008, "Evaluation of an Architecture for Intelligent Query and Exploration of TimeOriented Clinical Data." Artificial Intelligence in Medicine 43(1):17-34.

Objective: Evaluate KNAVE-II, a knowledge-based framework for visualization, interpretation, and exploration of longitudinal clinical data, clinical concepts and patterns. KNAVE-II mediates queries to a distributed temporal-abstraction architecture(IDAN), which uses a knowledge-based problem-solving method specializing in on-the-fly computation of clinical queries. Methods: A two-phase, balanced crossover study to compare efficiency and satisfaction of a group of clinicians when answering queries of variable complexity about time-oriented clinical data, typical for oncology protocols, using KNAVE-II, versus standard methods: both paper charts and a popular electronic spreadsheet (ESS) in Phase 1; an ESS in Phase II. The measurements included the time required to answer and the correctness of answer for each query and each complexity category, and for all queries, assessed versus a predetermined gold standard set by a domain expert. User satisfaction was assessed by the Standard Usability Score (SUS) tool-specific questionnaire and by a "Usability of Toot Comparison" comparative questionnaire developed for this study. Results: In both evaluations, subjects answered higher-complexity queries significantly faster using KNAVE-II than when using paper charts or an ESS up to a mean of $255 \mathrm{~s}$ difference per query versus the ESS for hard queries $(p=0.0003)$ in the second evaluation. Average correctness scores when using KNAVE-II versus paper charts, in the first phase, and the ESS, in the second phase, were significantly higher over all queries. In the second evaluation, $91.6 \%(110 / 120)$ of all of the questions asked within queries of all levels produced correct answers using KNAVE-II, opposed to 
only $57.5 \%(69 / 120)$ using the ESS ( $<<0.0001)$. User satisfaction with KNAVE-II was significantly superior compared to using either a paper chart or the ESS $(p=0.006)$. Clinicians ranked KNAVE-II superior to both paper and the ESS. Conclusions: An evaluation of the functionality and usability of KNAVE-II and its supporting knowledge-based temporal-mediation architecture has produced highly encouraging results regarding saving of physician time, enhancement of accuracy of clinical assessment, and user satisfaction. (c) 2008 Elsevier B.V. All rights reserved.

Marzano, R. 2000, Designing Effective Projects : Thinking Skills Frameworks Marzano’s New Taxonomy. Technical.

Robert Marzano, respected educational researcher, has proposed what he calls A New Taxonomy of Educational Objectives (2000). Developed to respond to the shortcomings of the widely used Bloom's Taxonomy and the current environment of syllabus guidelines-based instruction, Marzano's model of thinking skills incorporates a wider range of factors that affect how students think and provides a more research-based theory to help teachers improve their students' thinking. Marzano's New Taxonomy is made up of three systems and the Knowledge Domain, all of which are important for thinking and learning. The three systems are the Self-System, the Metacognitive System, and the Cognitive System. When faced with the option of starting a new task, the Self-System decides whether to continue the current behavior or engage in the new activity; the Metacognitive System sets goals and keeps track of how well they are being achieved; the Cognitive System processes all the necessary information, and the Knowledge Domain provides the content.

Masseroli, M, et al. 2006, "He@Lthco-Op: A Web-Based System to Support Distributed Healthcare CoOperative Work." Computers in Biology and Medicine 36(2):109-27.

Healthcare is characterized by close collaboration and information sharing among many distinct actors, who co-operate for the patient care in different temporal moments, also at a distance. In this context, availability to care givers of all relevant patient health data and of specific healthcare cooperative work supporting tools is fundamental for best patient treatment. We designed and implemented He@lthCo-op, a web-based modular system supporting co-operative work and patient information secure sharing among healthcare personnel also from remotely located sites. He@lthCo-op enables easily gathering, storing, and accessing patient clinical and personal data anytime and from anywhere an Internet connection is available.

Masud, L, et al. 2010, "From Data to Knowledge - Visualizations as Transformation Processes within the Data-Information-Knowledge Continuum." In 14th International Conference Information Visualisation, pp. 445-49.

In this paper we suggest a different approach that considers visualizations in the wider domain of communication and defines a model capable of taking into account the context in which visualizations act as communication tools. In this perspective we consider visualizations as transformation processes within the Data-Information-Knowledge (DIK) continuum. In the paper we discuss the continuum, and apply the transformation process model to the main disciplines of visualization. Visualizations represent powerful cognitive tools that surround our everyday life. By doing this we are able to think about visualization from a multidisciplinary perspective, exploring the role of visualizations in design practice, as artifacts that are used in facing problems of various degrees of complexity and nature. The contribution of the 
model is mainly addressed to orientate and nurture the reflective practice and to formalize the strategic more than the technical role of visualizations in the design discipline.

Matkovic, K, et al. 2010, "Interactive Visual Analysis of Multiple Simulation Runs Using the Simulation Model View: Understanding and Tuning of an Electronic Unit Injector." IEEE Transactions on Visualization and Computer Graphics 16(6):1449-57.

Multiple simulation runs using the same simulation model with different values of control parameters generate a large data set that captures the behavior of the modeled phenomenon. However, there is a conceptual and visual gap between the simulation model behavior and the data set that makes data analysis more difficult. We propose a simulation model view that helps to bridge that gap by visually combining the simulation model description and the generated data. The simulation model view provides a visual outline of the simulation process and the corresponding simulation model. The view is integrated in a Coordinated Multiple Views ;(CMV) system. As the simulation model view provides a limited display space, we use three levels of details. We explored the use of the simulation model view, in close collaboration with a domain expert, to understand and tune an electronic unit injector (EUI). We also developed analysis procedures based on the view. The EUI is mostly used in heavy duty Diesel engines. We were mainly interested in understanding the model and how to tune it for three different operation modes: low emission, low consumption, and high power. Very positive feedback from the domain expert shows that the use of the simulation model view and the corresponding; analysis procedures within a CMV system represents an effective technique for interactive visual analysis of multiple simulation runs.

Matsushita, M, E Maeda, and T Kato. 2004, "An Interactive Visualization Method of Numerical Data Based on Natural Language Requirements." International Journal of Human-Computer Studies 60(4):469-88.

This paper proposes an interactive visualization method to support the exploration of data in decisionmaking and problem solving. Since this method employs an asymmetric communication mode, i.e., taking queries and requests expressed in a natural language as input and answering them with statistical charts, it can convert the normally tedious repetitive human-computer interaction into a felicitous dialogue. This is because the natural language interface allows users to articulate their requests directly and intuitively, and charts and graphics have many benefits when analysing a large amount of data in order to determine overall characteristics or to resolve user questions. The proposed method resolves the conundrum that the appropriateness of a chart depends on the context. In this method, two factors are considered in choosing chart type so as to satisfy the user requirement represented in a natural language: the type of chart displayed and the type of user utterance. Our proposed method allows the data to be visualized interactively to match the changes in the user's viewpoint without interrupting the thinking process.

Matzen, LE, et al. 2010, "Proposed Working Memory Measures for Evaluating Information Visualization Tools." In BELIV'10: Beyond time and errors: novel evaluation methods for Information Visualization, April 10-11, 2010.

The current information visualization literature highlights design and evaluation processes that are highly variable and situation dependent, which raises at least two broad challenges. First, lack of a standardized evaluation criterion leads to costly re-designs for each task and specific user community. Second, this inadequacy in criterion validation raises significant uncertainty regarding visualization 
outputs and their related decisions, which may be especially troubling in high consequence environments like those of intelligence analysts. We seek ways to standardize the "apples and oranges" of the extant situation through tools based upon general principles of human cognition. Theoretically, information visualization tools enable the user to see information in a way that should attenuate the user's memory load and increase the user's task-available cognitive resources. By using general cognitive abilities, like available working memory resources, as a dependent measure, we propose standardized evaluative capabilities can be generalized across contexts, tasks, and user communities.

Maxion, RA, and RW Reeder. 2005, "Improving User-Interface Dependability through Mitigation of Human Error." International Journal of Human-Computer Studies 63(1-2):25-50.

Security may be compromised when humans make mistakes at the user interface. Cleartext is mistakenly sent to correspondents, sensitive files are left unprotected, and erroneously configured systems are left vulnerable to attackers. Such mistakes may be blamed on human error, but the regularity of human error suggests that mistakes may be preventable through better interface design. Certain userinterface constructs drive users toward error, while others facilitate success. Two security-sensitive user interfaces were evaluated in a laboratory user study: the Windows XP file-permissions interface and an alternative interface, called Salmon, designed in accordance with an error-avoiding principle to counteract the misleading constructs in the XP interface. The alternative interface was found to be more dependable; it increased successful task completion by up to $300 \%$, reduced commission of a class of errors by up to $94 \%$, and provided a nearly $3 \times$ speed-up in task completion time. Moreover, users spent less time searching for information with the alternative interface, and a greater proportion of time on essential task steps. An explanatory theory in its early stages of development is presented.

May, T, J Davey, and J Kohlhammer. 2010, "Combining Statistical Independence Testing, Visual Attribute Selection and Automated Analysis to Find Relevant Attributes for Classification." In IEEE Symposium on Visual Analytics Science \& Technology, pp. 239-40.

Mayer, RE. 1996, "The Search for Insight: Grappling with Gestalt Psychology's Unanswered Questions." in The Nature of Insight, eds. RJ Sternberg and JE Davidson, pp. 3-32. MIT Press.

Mayerich, D. 2010, "Gpu-Based Dynamic Tubular Grids for Sparse Volume Rendering." In IEEE Conference on Information Visualization, pp. 3-4.

Mayr, E, M Smuc, and H Risku. 2010, "Many Roads Lead to Rome. Mapping Users' Problem Solving Strategies." In BELIV'10: Beyond time and errors: novel evaluation methods for Information Visualization, April 10-11, 2010.

Especially in ill-defined problems like complex, real-world tasks more than one way leads to a solution. Until now, the evaluation of information visualizations was often restricted to measuring outcomes only (time and error) or insights into the data set. A more detailed look into the processes which lead to or hinder task completion is provided by analyzing users' problem solving strategies. A study illustrates how they can be assessed and how this knowledge can be used in participatory design to improve a visual analytics tool. In order to provide the users a tool which functions as a real scaffold, it should allow them to choose their own path to Rome. We discuss how evaluation of problem solving strategies can shed more light on the users' "exploratory minds". 
Mazza, R, and A Berre. 2007, "Focus Group Methodology for Evaluating Information Visualization Techniques and Tools." In 11th International Conference on Information Visualization (IV 2007), pp. $74-$ 78.

This paper proposes a structured methodology that uses focus group interviews to evaluate Information Visualization techniques and tools. Focus groups are particularly suitable for the collection of qualitative data from users, and allow researchers to uncover unexpected problems that cannot be identified through analytical evaluations or controlled experiments. The approach relies on open-ended questions to explore user attitudes, feelings, and beliefs, but also indicates a number of questions designed to explore specific cognitive tasks related to Information Visualization systems. We argue that focusing the discussion on cognitive tasks allows for the generation of user comments that are more effective than informal and unstructured interviews.

Mazza, R, and V Dimitrova. 2007, "Coursevis: A Graphical Student Monitoring Tool for Supporting Instructors in Web-Based Distance Courses." International Journal of Human-Computer Studies 65(2):125-39.

This paper presents CourseVis, a system that takes a novel approach of using Web log data generated by course management systems (CMSs) to help instructors become aware of what is happening in distance learning classes. Specifically, techniques from information visualization (IV) are employed to graphically render complex, multidimensional student tracking data. Several graphical representations are generated to help distance learning instructors get a better understanding of social, behavioural, and cognitive aspects related to learners. The evaluation of CourseVis shows that it can help instructors to quickly identify tendencies in their classes and discover individuals that might need special attention. This suggests that the effectiveness of CMSs can be improved by integrating IV techniques to generate appropriate graphical representations, similar to those produced in CourseVis.

Mazza, R, and V Dimitrova. 2003, "Coursevis: Externalising Student Information to Facilitate Instructors in Distance Learning." In 11th International Conference on Artificial Intelligence in Education (AI-ED 2003), pp. 279-86.

In this paper we present an approach for externalising student information to facilitate instructors involved in distance learning. Information visualization techniques have been used in CourseVis - a tool which obtains tracking data from course management systems, transforms the data into a form convenient for processing, and generates graphical representations that can be explored by course instructors to examine social, cognitive, and behavioural aspects of distance students. CourseVis is presented in the paper, and several examples of pictorial representations generated by the tool are discussed.

Mazza, R, and V Dimitrova. 2005, "Generation of Graphical Representations of Student Tracking Data in Course Management Systems." In Ninth International Conference on Information Visualisation (IV'05), pp. 253-58.

An approach of employing information visualisation to develop systems that facilitate instructors in Web-based distance learning is presented here. The paper describes a tool, called CourseVis, that uses multidimensional student tracking data collected by CMS and generates graphical representations that can be used by instructors to gain an understanding of what is happening in distance learning classes. The work followed a systematic approach that started from collecting the instructors' needs, produced some 
appropriate graphical representations of student tracking data, and evaluated the effectiveness, efficiency and usefulness of the proposed representations. The evaluation has shown that with CourseVis the instructors can identify tendencies in their classes, quickly discover individuals that need special attention, and are able to provide better support to their students.

McCrickard, DS, et al. 2003, "Establishing Tradeoffs That Leverage Attention for Utility: Empirically Evaluating Information Display in Notification Systems." International Journal of Human-Computer Studies 58(5):547-82.

Designing and evaluating notification systems represents an emerging challenge in the study of human-computer interaction. Users rely on notification systems to present potentially interruptive information in an efficient and effective manner to enable appropriate reaction and comprehension. Little is known about the effects of these systems on ongoing computer tasks. As the research community strives to understand information design suitable for opposing usage goals, few existing efforts lend themselves to extensibility. However, three often conflicting design objectives are interruption to primary tasks, reaction to specific notifications, and comprehension of information over time. Based on these competing parameters, we propose a unifying research theme for the field that defines success in notification systems design as achieving the desirable balance between attention and utility. This paradigm distinguishes notification systems research from traditional HCI by centering on the limitations of the human attention system. In a series of experiments that demonstrate this research approach and investigate use of animated text in secondary displays, we describe two empirical investigations focused on the three critical parameters during a browsing task. The first experiment compares tickering, blasting, and fading text, finding that tickering text is best for supporting deeper comprehension, fading best facilitates reaction, and, compared to the control condition, none of the animated displays are interruptive to the browsing task. The second experiment investigates fading and tickering animation in greater detail with similar tasks--at two different speeds and sizes. Here, we found smaller displays allowed better reaction but were more interruptive, while slower displays provides increased comprehension. Overall, the slow fade appears to be the best secondary display animation type tested. Focusing research and user studies within this field on critical parameters such as interruption, reaction, and comprehension will increase cohesion among design and evaluation efforts for notification systems.

McCrickard, DS, M Czerwinski, and L Bartram. 2003, "Introduction: Design and Evaluation of Notification User Interfaces." International Journal of Human-Computer Studies 58(5):509-14.

Notification systems attempt to deliver current, important information to the computer screen in an efficient and effective manner. All notification systems require that the user attends to them to at least some degree if they are to succeed. Examples of notification systems include instant messaging systems, system and user status updates, email alerts and news and stock tickers. The benefits of notification systems are numerous, including rapid availability of important information, access to nearly instantaneous communication and heightened awareness of the availability of personal contacts. While the popularity of these systems has skyrocketed in recent years, the effects of incoming notifications on ongoing computing tasks have been relatively unexplored. The investigation of the costs, benefits and the optimal display of instant messages and all notifications in the context of desktop or mobile computing tasks falls in the general arena of psychological research on alerting and disruptions, but also requires research contributions from design, computer science and information visualization. To date, much of the psychological research on interruption leverages theoretical task constructions. In this special issue, we 
focus on the nature of interruptions such as messaging while computing and how to optimize the user experience.

McFadzean, J, NG Cross, and JH Johnson. 1999, "An Analysis of Architectural Visual Reasoning in Conceptual Sketching Via Computational Sketch Analysis (Csa)." In Third International Conference on Information Visualisation (IV'99), pp. 258-65.

Visual reasoning in design is facilitated by sketching. This research investigates how designers sketch, specifically analysing the physical details of mark making. It relates the graphical representations to the abstract cognitive processes of architectural design. A new form of protocol analysis has been developed using video and computer records of designers' sketching activity. The analysis of the resulting data compares the designer's retrospective commentary and interpretations of the sketching activity with the computer's record of that activity. The analysis will lead to a greater understanding of the relationships between 'Design Events' and 'Graphical Events' and thus how the notational activity of sketching supports the cognitive activity of conceptual design

McGuffin, MJ, and JM Robert. 2010, "Quantifying the Space-Efficiency of 2d Graphical Representations of Trees." Information Visualization 9(2):115-40.

A mathematical evaluation and comparison of the space-efficiency of various 2D graphical representations of tree structures is presented. As part of the evaluation, a novel metric called the mean area exponent is introduced that quantifies the distribution of area across nodes in a tree representation, and that can be applied to a broad range of different representations of trees. Several representations are analyzed and compared by calculating their mean area exponent as well as the area they allocate to nodes and labels. Our analysis inspires a set of design guidelines as well as a few novel tree representations that are also presented. Information Visualization (2010) 9, 115-140. doi:10.1057/ivs.2009.4; published online 21 May 2009

McQuaid, MJ, et al. 1999, "Multidimensional Scaling for Group Memory Visualization." Decision Support Systems 27(1-2):163-76.

We describe an attempt to overcome information overload through information visualization -- in a particular domain, group memory. A brief review of information visualization is followed by a brief description of our methodology. We discuss our system, which uses multidimensional scaling (MDS) to visualize relationships between documents, and which we tested on 60 subjects, mostly students. We found three important (and statistically significant) differences between task performance on an MDSgenerated display and on a randomly generated display. With some qualifications, we conclude that MDS speeds up and improves the quality of manual classification of documents and that the MDS display agrees with subject perceptions of which documents are similar and should be displayed together.

Mecella, M, et al. 2006, "Access Control Enforcement for Conversation-Based Web Services." In 15th International Conference on World Wide Web, pp. 257-57.

Service Oriented Computing is emerging as the main approach to build distributed enterprise applications on the Web. The widespread use of Web services is hindered by the lack of adequate security and privacy support. In this paper, we present a novel framework for enforcing access control in conversation-based Web services. Our approach takes into account the conversational nature of Web 
services. This is in contrast with existing approaches to access control enforcement that assume a Web service as a set of independent operations. Furthermore, our approach achieves a tradeoff between the need to protect Web service's access control policies and the need to disclose to clients the portion of access control policies related to the conversations they are interested in. This is important to avoid situations where the client cannot progress in the conversation due to the lack of required security requirements. We introduce the concept of k-trustworthiness that defines the conversations for which a client can provide credentials maximizing the likelihood that it will eventually hit a final state.

Medani, D, G Haggard, and K Computers. 2010, "Graph Works - Pilot Graph Theory Visualization Tool." In 5th ACM Symposium on Software Visualization (SOFTVIS), pp. 205-06.

Medina, D, J Wood, and C Poly. 2010, "Real-Time Visualizations of Ocean Data Collected by the Norus Glider." In IEEE Conference on Information Visualization.

Meier, HA, et al. 2008, "Visualization of Particle Interactions in Granular Media." Visualization and Computer Graphics, IEEE Transactions on 14(5):1110-25.

Interaction between particles in so-called granular media, such as soil and sand, plays an important role in the context of geomechanical phenomena and numerous industrial applications. A two scale homogenization approach based on a micro and a macro scale level is briefly introduced in this paper. Computation of granular material in such a way gives a deeper insight into the context of discontinuous materials and at the same time reduces the computational costs. However, the description and the understanding of the phenomena in granular materials are not yet satisfactory. A sophisticated problemspecific visualization technique would significantly help to illustrate failure phenomena on the microscopic level. As main contribution, we present a novel 2D approach for the visualization of simulation data, based on the above outlined homogenization technique. Our visualization tool supports visualization on micro scale level as well as on macro scale level. The tool shows both aspects closely arranged in form of multiple coordinated views to give users the possibility to analyze the particle behavior effectively. A novel type of interactive rose diagrams was developed to represent the dynamic contact networks on the micro scale level in a condensed and efficient way.

Meng, H, and D Li. 2006, "Chapter 11 - Multilingual Spoken Dialog Systems." in Multilingual Speech Processing, pp. 399-447. Academic Press, Burlington.

Summary Spoken dialog systems (SDS) are becoming increasingly pervasive in our everyday lives for information access. Efforts devoted to the design and development of SDS aim to bring the right information to the right people at the right time for a diversity of application domains, such as finance, air travel, train schedules, and weather. These domains typically involve dynamic information of recurring interests to the user. SDS encompasses a suite of speech and language technologies to offer a conversational interface to dynamic information, including speech recognition, natural language understanding, dialog modeling, and speech synthesis. Hence, the user can present queries to the system by speaking naturally, and the SDS can respond in real time in synthetic speech. Numerous commercial SDS have been deployed for multiple languages. For example, the Speech Works1 voice-activated stocktrading system enables callers to get real-time stock and market information, place orders, and trade stocks at any time. The entire human-computer interaction is conducted over a fixed-line/mobile telephone channel--that is, in a screenless setting. An example is provided in Table 11.1 to illustrate the directed nature of the dialog interactions, in which the system elicits a series of information attributes 
from the user in a scripted order. Interactions of this style are often known as directed dialogs or systeminitiative dialogs. The system is always in control to guide the dialog at each step and constrain possible user input to a small set of options. In this way, the system is able to attain a decent level of robustness in recognizing and interpreting user input in order to achieve a high task-completion rate for informational transactions. However, the ease of use of these human-computer spoken-language interfaces is partially sacrificed due to constraints in interactivity. Recent research efforts in the field strive to relax the constraints in system-initiative dialogs.

Merrill, MD. "Knowledge Objects and Mental Models." In, pp. 244-46.

Merrill, MD. 2000, "Knowledge Objects and Mental Models." In Proceedings International Workshop on Advanced Learning Technologies. IWALT 2000. Advanced Learning Technology: Design and Development Issues, pp. 244-46.

This paper describes knowledge components that are thought to be appropriate and suficient to precisely describe certain types of cognitive subject matter content (knowledge). It also describes knowledge structures that show the relationships among these knowledge components and among other knowledge objects. It suggests that a knowledge structure is a form of schema such as those that leamers use to represent knowledge in memory. A mental model is a schema plus cognitive processes for manipulating and modifying the knowledge stored in a schema. We suggested processes that enable learners to manipulate the knowledge components of conceptual network knowledge structures for purposes of generalization, and concept elaboration. We further suggested processes that enable learners to manipulate the knowledge components of process knowledge structures (PEAnets) for purposes of explanation, prediction, and trouble shooting. The hypothesis of this paper is that knowledge components and knowledge structures, such as those described in this paper, could serve as meta mental models that would enable learners to more easily acquire conceptual and causal networks and their associated processes. The resulting specific mental models would facilitate their ability to solve problems of conceptualization and interpretation.

Merrill, MD. 2000, "Knowledge Objects and Mental Models, Presentation at Utah State University."

Mesarina, M, et al. 2009, "Evaluating a Personal Communication Tool: Sidebar." in Human-Computer Interaction, Pt I, ed. JA Jacko, Vol 5610, pp. 490-99.

By more closely integrating email with the web we aim to bring organization to email and more collaboration to the web. To this end we developed the Sidebar, a web-browser plug which displays email messages which link to the currently displayed URL. We conducted longitudinal studies on two versions of Sidebar to observe the usage of Sidebar and determine if it improves communications productivity. We found that providing an email summary in Sidebar resulted in raised awareness of the email collaborations, increased serendipitous discovery of information, and resulted in higher reported communication productivity. This paper Summarizes Sidebar's operation, describes the user studies, and presents conclusions. 
Metcalfe, J. 1986, "Feeling of Knowing in Memory and Problem Solving." Journal of Experimental Psychology: Learning, Memory, and Cognition 12:288-94.

This study investigates feelings of knowing for problem solving and memory. In Experiment 1 subjects judged their feelings of knowing to trivia questions they had been unable to answer, then performed a multiple-choice recognition test. In a second task, subjects gave feeling-of-knowing judgments for "insight" problems to which they did not immediately know the answers. Later, they were given $5 \mathrm{~min}$ to solve each problem. In contrast to the positive correlation found in the memory task, the feeling-of-knowing rank ordering of insight problems did not relate to problem solution. Experiment 2 provided a replication of Experiment 1 with a generation memory technique rather than a multiple-choice recognition test. Both experiments showed that although people could predict memory performance reasonably well, predictive metacognitions were nonexistent for the problems. The data are interpreted as implying that insight problems do involve a sudden illumination, and that illumination cannot be predicted in advance.

Metcalfe, J. 1986, "Premonitions of Insight Predict Impending Error." Journal of Experimental Psychology: Learning, Memory, and Cognition 12(4):623-34.

Five experiments explored the dynamic metacognitions that accompany the problem- and anagramsolving processes. Subjects repeatedly rated how "warm" or close they were to solution. High feelings of warmth before an answer indicated that the answer would be incorrect. Moderately low warmth ratings characterized correct responses. The data suggest that the high warmth ratings may result from a process wherein subjects convince themselves that an inelegant but plausible (wrong) answer is correct. No gradual rationalization process precedes the correct response to insight problems. The warmth-rating data also indicate that when the correct answer was given to the problems and anagrams used in this study, there was usually a subjectively catastrophic insight process.

Mete, HO, and ZB Zabinsky. 2008, "Preparing for Disasters : Medical Supply Location and Distribution." In ISCRAM Conference 2005, pp. 1-14.

Mete, HO, ZB Zabinsky, and W Kohn. 2008, "Optimization of Enterprises Modeled by Rules." In 2008 Industrial Engineering Research Conference.

Meunier, JG, D Forest, and I Biskri. 2005, "Chapter 43 - Classification and Categorization in ComputerAssisted Reading and Text Analysis." in Handbook of Categorization in Cognitive Science, eds. C Henri and L Claire, pp. 955-78. Elsevier Science Ltd, Oxford.

Summary Automated computer classification and categorization have been successfully applied in the field of document retrieval and text mining. These methods are increasingly used in the field of computerassisted reading and analysis of texts (CARAT) in the field of the humanities and social sciences. Here, the primary aim is not to tag the document for better recall, but to help a specialized reader and analyst to navigate through the themes, concepts, and content of a textual corpus according to a particular research objective. This chapter presents some of the methods and applications of these techniques. 
Meyer, E, et al. 2010, "Improving Literacy and Metacognition with Electronic Portfolios: Teaching and Learning with Epearl." Computers \& Education 55(1):84-91.

Can an electronic portfolio that is both a multimedia container for student work and a tool to support key learning processes have a positive impact on the literacy practices and self-regulated learning skills of students? This article presents the findings of a yearlong study conducted in three Canadian provinces during the 2007-2008 school year initially involving 32 teachers and 388 students. Due to varying levels of implementation our final data set included 14 teachers and 296 students. Using a non-equivalent pretest/post-test design, we found that grade 4-6 students who were in classrooms where the teacher provided regular and appropriate use of the electronic portfolio tool ePEARL (i.e., medium-high implementation condition, $\mathrm{n}=7$ classrooms and 121 students), compared to control students ( $\mathrm{n}=7$ classrooms and 175 students) who did not use ePEARL, showed significant improvements $(\mathrm{p}<.05)$ in their writing skills on a standardized literacy measure (i.e., the constructed response subtest of the Canadian Achievement Test4th ed.) and certain metacognitive skills measured via student self-report. The results of this study indicate that teaching with ePEARL has positive impacts on students' literacy and self-regulated learning skills when the tool is used regularly and integrated into classroom instruction.

Meyer, M, et al. 2010, "Multeesum : A Tool for Comparative Spatial and Temporal Gene Expression Data." IEEE Transactions on Visualization and Computer Graphics 16(6):908-17.

Meyer, M, T Munzner, and H Pfister. 2009, "Mizbee: A Multiscale Synteny Browser." Visualization and Computer Graphics, IEEE Transactions on 15(6):897-904.

In the field of comparative genomics, scientists seek to answer questions about evolution and genomic function by comparing the genomes of species to find regions of shared sequences. Conserve dsyntenic blocks are an important biological data abstraction for indicating regions of shared sequences. The goal of this work is to show multiple types of relationships at multiple scales in a way that is visually comprehensible in accordance with known perceptual principles. We present a task analysis for this domain where the fundamental questions asked by biologists can be understood by a characterization of relationships into the four types of proximity/location, size, orientation, and similarity/strength, and the four scales of genome, chromosome, block, and genomic feature. We also propose a new taxonomy of the design space for visually encoding conservation data. We present MizBee, a multiscale synteny browser with the unique property of providing interactive side-by-side views of the data across the range of scales supporting exploration of all of these relationship types. We conclude with case studies from two biologists who used MizBee to augment their previous automatic analysis work flow, providing anecdotal evidence about the efficacy of the system for the visualization of syntenic data, the analysis of conservation relationships, and the communication of scientific insights.

Meyer, RD, and D Cook. 2000, "Visualization of Data." Current Opinion in Biotechnology 11(1):89-96.

Data visualization has developed in several directions: theoretical; methodological; and in new application areas. Advances include the development of a grammar of graphics, deeper understanding of human perception and implications for graphical layout, and better approaches to visualizing multidimensional data and large data sets. Gene expression is a notable new application area for visualization of large data sets. 
Michelitsch, G, G Welling, and M Ott. 1998, "A User-Centric View of Qos-Aware Communication." Computer Communications 21(15):1310-19.

Recent technological advances coupled with new trends in using today's computing and communication infrastructure have raised new challenges in designing user interfaces for distributed multimedia applications. It has become essential to present the idea of QoS to the user, not only due to limitations of the infrastructure, but also because of the need to control user-machine interface bandwidth. In addition, a need for a tighter integration of communication and collaboration has become apparent. In this paper, we motivate the need for QoS support in the user-interface by considering shared virtual spaces, a unified communication paradigm for collaboration. We present a user-interface framework which provides the user with implicit and explicit control of quality of service, and describe our experience in designing and implementing such systems.

Migut, M, and M Worring. 2010, "Visual Exploration of Classification Models for Risk Assessment." In IEEE Symposium on Visual Analytics Science \& Technology, pp. 11-18.

Miller, GA. 1956, "The Magical Number Severn, Plus or Minus Two: Some Limits on Our Capacity to Process Information." Psychological Review 63:81-96.

Miller, SG. 2000, "A Proposal for the Development of a Study to Determine the Effectiveness of a Creativity-Centered Unit of Study in the Advancement of Spatial Abilities of American and Japanese Female Engineering Students." In IEEE International Conference on Information Visualization, pp. 25557.

The purpose of this project is the development, evaluation and implementation of a creativitycentered unit of study in the advancement of spatial abilities of American and Japanese female engineering students. This project is envisioned as a partnership between the author and colleagues in universities in Japan. The benefits will include international cooperation between Japanese universities and Purdue University in developing spatial ability research. Female engineering students will also hopefully benefit from the findings of this research. This paper discusses the project and the theoretical foundation upon which it was based. The experimental treatment will consist of exposure or no exposure to a unit of study in creativity in an engineering graphics course

Min, C, et al. 2009, "Data, Information, and Knowledge in Visualization." Computer Graphics and Applications, IEEE 29(1):12-19.

In visualization, we use the terms data, information and knowledge extensively, often in an interrelated context. In many cases, they indicate different levels of abstraction, understanding, or truthfulness. For example, "visualization is concerned with exploring data and information," "the primary objective in data visualization is to gain insight into an information space," and "information visualization" is for "data mining and knowledge discovery." In other cases, these three terms indicate data types, for instance, as adjectives in noun phrases, such as data visualization, information visualization, and knowledge visualization. These examples suggest that data, information, and knowledge could serve as both the input and output of a visualization process, raising questions about their exact role in visualization. 
Mirel, B. 2004, "Design Strategies and Choices for Optimizing the Mix." in Interaction Deisgn for Complex Problem Solving: Developing Useful and Usable Software, pp. 231-70. Morgan Kaufmann, Burlington.

Mirel, B. 2004, "Introduction." in Interaction Deisgn for Complex Problem Solving: Developing Useful and Usable Software, pp. xxvii-xlii. Morgan Kaufmann, Burlington.

Mirel, B. 1999, "Usability Test Results for Information Visualizations: Determinants of Usefulness for Complex Business Problems." in Human-Computer Interaction - Interact '99, pp. 70-78.

Designers know too little about how users conduct realistic business analyses with interactive graphics. This article reports the results of two usability tests that assessed visualizations for use-incontext and realistic tasks. Results show that meanings in visualized displays are socially negotiated and that users - even novices do ad hoc analysis for any inquiries that are the least bit unstructured. Results also show that users have integrated views of core problem-solving activities that they expect the visualizations to support. These results suggest a user model and designs for utility that would address it. To support ad hoc analysis, visualizations need to make it easy for users to create variables and new fields an the fly and to help users learn the right graphics to use for specific types of problems. For users' concepts of tasks, programs have to provide feedback on interactions and display states so that users may weave discrete actions into an integrated whole. Programs also have to offer memory aids and reporting capabilities to help users keep track of where they have been, what they know, and what they still need to discover. Only contextual and scenario-based testing can uncover these utility-based issues, all of which are crucial to users' mastery of visual querying.

Mirel, B. 2004, "What Makes Complex Problem Solving Complex?" in Interaction Deisgn for Complex Problem Solving: Developing Useful and Usable Software, pp. 3-29. Morgan Kaufmann, Burlington.

Mirhaji, P. 2009, "Public Health Surveillance Meets Translational Informatics: A Desiderata." JALA Journal of the Association for Laboratory Automation 14(3):157-70.

"Public health surveillance (PHS) is the ongoing and systematic collection, analysis, interpretation, and dissemination of data regarding a health-related event for use in public health action to reduce morbidity and mortality and to improve health." As information technology gains acceptance as a core element of public health practice, many approaches to the design of PHS systems have been proposed, much has been spent implementing them, and expectations have been high. Unfortunately, the systems implemented so far have been criticized as having not met expectations, especially in the domain of early detection and bioterrorism readiness, or so-called syndromic surveillance (The term "syndromic surveillance" applies to monitoring health-related data that precede diagnosis to signal a sufficient probability of a case or an outbreak that warrants public health response.). There are no fully established frameworks to enable seamless interoperability, information sharing, and collaboration among PHS stakeholders and the technological and infrastructural requirements to fulfill the grand vision of initiatives such as the Public Health Information Network and National Health Information Network are poorly investigated and documented. In this article, we examine the current state of the conceptualization, design, analysis, and implementation of PHS systems from a translational informatics perspective. Although most examples in this article are informed by the needs of public health preparedness (syndromic and bioterrorism detection and response), we believe the framework we introduce is generalizable and applicable to the broader context of PHS systems. We also apply concepts from 
cognitive science and knowledge engineering to suggest directions for improvement and further research. (C) 2009 The Association for Laboratory Automation.

Mirhaji, P, et al. 2004, "Public Health Situation Awareness, Towards a Semantic Approach." in Multisensor, Multisource Information Fusion: Architectures, Algorithms, and Applicatons 2004, ed. BV Dasarathy, Vol 5434, pp. 339-50. SPIE.

Introduction: We propose a knowledge-based public health situation awareness system. The basis for this system is an explicit representation of public health situation awareness concepts and their interrelationships. This representation is based upon the users' (public health decision makers) cognitive model of the world, and optimized towards the efficacy of performance and relevance to the public health situation awareness processes and tasks. In our approach, explicit domain knowledge is the foundation for interpretation of public health data, as apposed to conventional systems where the statistical methods are the essence of the processes. Objectives: To develop a prototype knowledge-based system for public health situation awareness and to demonstrate the utility of knowledge intensive approaches in integration of heterogeneous information, eliminating the effects of incomplete and poor quality surveillance data, uncertainty in syndrome and aberration detection and visualization of complex information structures in public health surveillance settings, particularly in the context of bioterrorism (BT) preparedness.

Methods: The system employs the Resource Definition Framework (RDF) and additional layers of more expressive languages to explicate the knowledge of domain experts into machine interpretable and computable problem-solving modules that can then guide users and computer systems in sifting through the most "relevant" data for syndrome and outbreak detection and investigation of root cause of the event. Results: The Center for Biosecurity and Public Health Informatics Research is developing a prototype knowledge-based system around influenza, which has complex natural disease patterns, many public health implications, and is a potential agent for bioterrorism. Conclusions: The preliminary data from this effort may demonstrate superior performance in information integration, syndrome and aberration detection, information access through information visualization, and cross-domain investigation of the root causes of public health events.

Mirhaji, P, et al. 2004, "Knowledge Based Public Health Situation Awareness." in Sensors, and Command, Control, Communications, and Intelligence(C31) Technologies for Homeland Security and Homeland Defense Iii, Pts 1 and 2, ed. EM Carapezza, Vol 5403, pp. 198-209. SPIE.

Mitchell, J, M Francke, and D Eng. 2007, "Illustrative Rendering in Team Fortress 2." In 5th International Symposium on Non-photorealistic Animation and Rendering, pp. 48-56.

We present a set of artistic choices and novel real-time shading techniques which support each other to enable the unique rendering style of the game Team Fortress 2. Grounded in the conventions of early 20th century commercial illustration, the look of Team Fortress 2 is the result of tight collaboration between artists and engineers. In this paper, we will discuss the way in which the art direction and technology choices combine to support artistic goals and gameplay constraints. In addition to achieving a compelling style, the shading techniques are designed to quickly convey geometric information using rim highlights as well as variation in luminance and hue, so that game players are consistently able to visually "read" the scene and identify other players in a variety of lighting conditions. 
Miyadera, Y, et al. 2008, "Labchart: A Support System for Collaborative Research Activities in University Laboratories and Its Practical Evaluations." In IEEE International Conference on Information Visualization, pp. 169-78.

This paper describes a support system for collaborative research activities in university laboratories. The system includes (1) a research information transition graph (RITG) to visually express research information and the relationships among the information for personal management, and (2) a method that enables collaborative research activities and knowledge sharing on a large scale by converting an RITG into a common view with specified constraints on redrawing. These issues are related to the solving of a trade-off problem between the management of research information based on personal viewpoints and the sharing of such information on a large scale. Furthermore this paper describes the results of the system evaluation through application of it. Furthermore this paper describes the results of the system evaluation through application of it.

Moere, AV. 2004, "Information Flocking: Time-Varying Data Visualization Using Boid Behaviors." In 8th International Conference on Information Visualisation, pp. 409-14.

This paper presents a significant extension of the information flocking concept and algorithms originally presented by Proctor \& Winter [1]. It introduces a novel way of visualizing time-varying datasets using the emergent characteristics of self-organization and dynamic behavior simulation. The current prototype uses both spatial clustering and behavioral animation to represent temporal data similarities by simulating the time-varying evolution of dynamic datasets. Instead of presenting exact data values, the way how the data values change over time is being visualized. In addition, the current information flocking method is capable of visualizing short-term temporal events or long-term timevarying data evolutions by automatically generating different recognizable motion typologies. This research will show how artificial life principles have been merged with the field of information visualization. Several aspects of the original information flocking algorithms have been improved to incorporate the real-time evaluation of continuous dynamic data value streams and to generate multiple stable, recognizable atomic as well as collective dynamic behaviors that reflect time-varying dataset changes and relative data value evolutions. The main information flocking principles are demonstrated through a visualization of historical stock market quotes.

Moere, AV. 2004, "Time-Varying Data Visualization Using Information Flocking Boids." In 10th Annual IEEE Symposium on Information Visualization (InfoVis 2004), pp. 97-104.

This research demonstrates how principles of self-organization and behavior simulation can be used to represent dynamic data evolutions by extending the concept of information flocking, originally introduced by Proctor \& Winter [1], to time-varying datasets. A rule-based behavior system continuously controls and updates the dynamic actions of individual, three-dimensional elements that represent the changing data values of reoccurring data objects. As a result, different distinguishable motion types emerge that are driven by local interactions between the spatial elements as well as the evolution of timevarying data values. Notably, this representation technique focuses on the representation of dynamic data alteration characteristics, or how reoccurring data objects change over time, instead of depicting the exact data values themselves. In addition, it demonstrates the potential of motion as a useful information visualization cue. The original information flocking approach is extended to incorporate time-varying datasets, live database querying, continuous data streaming, real-time data similarity evaluation, automatic shape generation and more stable flocking algorithms. Different experiments prove that 
information flocking is capable of representing short-term events as well as long-term temporal data evolutions of both individual and groups of time-dependent data objects. An historical stock market quote price dataset is used to demonstrate the algorithms and principles of time-varying information flocking.

Mokhtari, M, et al. 2010, "Visual Tools for Dynamic Analysis of Complex Situations." In IEEE Symposium on Visual Analytics Science \& Technology, pp. 241-42.

Moloney, J. 2006, "Augmented Reality Visualisation of the Built Environment to Support Design Decision Making." In IEEE International Conference on Information Visualization, pp. 687-92.

This paper reviews current approaches to design visualization in the built environment and establishes a gap in theory and practice. While it has been acknowledged for some time that the temporal component is an important aspect of visualisation there has been minimal attempts to integrate this approach into design practice. We articulate an approach to visualization which we term 'temporal context'. This approach has two aspects: (1) the evaluation of designs in terms of spatial sequence and multiple viewpoints over time; (2) perceptual change over time due to changing light and atmospheric conditions. Building on our previous research in the use of video game technology to facilitate design, we evaluate the potential of augmented reality (AR) technology to address temporal context. We describe proposals for two innovative screen based AR systems, which incorporate a multi-camera capture mechanism, a video data base and a unique illumination data capture approach

Monson, EE, G Chen, and R Brady. 2010, "Data Representation and Exploration with Geometric Wavelets." In IEEE Symposium on Visual Analytics Science \& Technology, pp. 243-44.

Moons, J, and Carlos. 2009, "Rationale Behind the Design of the Eduvisor Software Visualization Component." Electronic Notes in Theoretical Computer Science 224:57-65.

The EduVisor software visualization component is a new pedagogical tool specifically developed to address some wide-spread problems in teaching object-oriented technology to novice programmers. The visualization tool is integrated in a world-class IDE, and shows the students the structure of their own creations at runtime. EduVisor is based on a solid grounding in literature and over 25 years of combined experience in teaching a CS1 course. With this component we have set the goal of helping our students progress faster through the most difficult initial stages of programming.

Mora, B, et al. 2009, "Visualization and Computer Graphics on Isotropically Emissive Volumetric Displays." Computer 15(2):221-33.

The availability of commodity volumetric displays provides ordinary users with a new means of visualizing 3D data. Many of these displays are in the class of isotropically emissive light devices, which are designed to directly illuminate voxels in a 3D frame buffer, producing $\mathrm{x}$-ray-like visualizations. While this technology can offer intuitive insight into a 3D object, the visualizations are perceptually different from what a computer graphics or visualization system would render on a $2 \mathrm{D}$ screen. This paper formalizes rendering on isotropically emissive displays and introduces a novel technique that emulates traditional rendering effects on isotropically emissive volumetric displays, delivering results that are much closer to what is traditionally rendered on regular 2D screens. Such a technique can significantly broaden the capability and usage of isotropically emissive volumetric displays. Our method takes a 3D data set or object as the input, creates an intermediate light field, and outputs a special 3D volume data set 
called a lumi-volume. This lumi-volume encodes approximated rendering effects in a form suitable for display with accumulative integrals along unobtrusive rays. When a lumi-volume is fed directly into an isotropically emissive volumetric display, it creates a 3D visualization with surface shading effects that are familiar to the users. The key to this technique is an algorithm for creating a 3D lumi-volume from a 4D light field. In this paper, we discuss a number of technical issues, including transparency effects due to the dimension reduction and sampling rates for light fields and lumi-volumes. We show the effectiveness and usability of this technique with a selection of experimental results captured from an isotropically emissive volumetric display, and we demonstrate its potential capability and scalability with computersimulated high-resolution results

Moreau, L, et al. 2007, The Open Provenance Model. Technical, University of Southampton.

Morgado, L, and K Kahn. 2008, "Towards a Specification of the Toontalk Language." Journal of Visual Languages \& Computing 19(5):574-97.

ToonTalk is a child-oriented programming language whose environment is an animated virtual world, with objects that children can pick up and use as in a game, such as birds, trucks, and robots, providing direct child-oriented metaphors for programming constructs. Actions performed by a programmer's avatar with these objects are both code and coding. ToonTalk is a powerful system, not just a "toy" system: it is based upon concurrent constraint programming languages, and programs written in languages such as Flat Guarded Horn Clauses and Flat Concurrent Prolog can be straight-forwardly constructed in ToonTalk. However, there is not a specification of ToonTalk, for ready implementation in other environments. We propose that the ToonTalk language lies not in the animations displayed by the current environment, but on the actions performed by the programmer with virtual world objects; we present a description and analysis of the methods the ToonTalk language provides to programmers for expressing programs.

Mori, N. 1996, "A Factor Related to the Strength of an "Aha!" Experience." Japanese Psychological Research 38(2):85-89.

Morris, S, et al. 2002, "Diva: A Visualization System for Exploring Document Databases for Technology Forecasting." Computers \& Industrial Engineering 43(4):841-62.

Database Information Visualization and Analysis system (DIVA) is a computer program that helps perform bibliometric analysis of collections of scientific literature and patents for technology forecasting. Documents, drawn from the technological field of interest, are visualized as clusters on a two dimensional map, permitting exploration of the relationships among the documents and document clusters and also permitting derivation of summary data about each document cluster. Such information, when provided to subject matter experts performing a technology forecast, can yield insight into trends in the technological field of interest. This paper discusses the document visualization and analysis process: acquisition of documents, mapping documents, clustering, exploration of relationships, and generation of summary and trend information. Detailed discussion of DIVA exploration functions is presented and followed by an example of visualization and analysis of a set of documents about chemical sensors. (C) 2002 Published by Elsevier Science Ltd. 
Morrison, A, M Bell, and M Chalmers. 2009, "Visualisation of Spectator Activity at Stadium Events." In 13th IEEE International Conference on Information Visualization, pp. 219-26.

Recent advances in mobile device technology have opened up new possibilities in enhancing the experience of spectators at stadium-based sporting events. In creating novel applications for use in such settings, designers must be aware of the current practices of spectators and of features of the environment at such events that novel applications may seek to exploit. This work forms an early part of the Designing the Augmented Stadium project. Data sets have been collected from spectators, logging the results of Bluetooth scans alongside GPS location. This paper presents an information visualization tool that can be used in the analysis and exploration of this data, to provide insight into the activities of spectators, the relationship between an individual spectator and the crowd as a whole and the suitability of stadium environments for applications based on infrastructure such as mobile ad hoc networks (MANETs) and wireless mesh networking. Various visualization tools are described and example cases are illustrated, using several real-world data sets recorded at football matches.

Morse, E, M Lewis, and KA Olsen. 2000, "Evaluating Visualizations: Using a Taxonomic Guide." Int. J. Hum.-Comput. Stud. 53(5):637-62.

Although visualizations are components of many information interfaces, testing of these visual elements is rarely undertaken except as a part of overall usability testing. For this reason, it is unclear what role, if any, visualizations actually perform. Our method involves the creation of simple visual prototypes and task sets based on a visual taxonomy which allows testing of the visualization in isolation from the rest of the system. By de"ning tests using a visual taxonomy rather than customary tasks from the application domain, our method circumvents the problems of restricting evaluation of newer more capable systems to only those tasks which might be accomplished with older, less capable ones. This paper will discuss methods for exhaustively testing the capabilities of a visualization by mapping from a domain-independent taxonomy of visual tasks to a speci"c domain, i.e., information retrieval. Experimental results are presented illustrating this approach to determining the role visualizations may play in supporting users in information-seeking environments. Our methods could easily be extended to other domains including data visualization.

Morse, EL, et al. 1998, "Evaluation of Text, Numeric and Graphical Presentations for Information Retrieval Interfaces: User Preference and Task Performance Measures." In IEEE International Conference on Systems, Man, and Cybernetics - Intelligent Systems for Humans in a Cyberworld (SMC 98), pp. 1026-31.

Information retrieval has a long history of dealing with printed materials, More recent work has involved the development of experimental visual interfaces to support users' attempts to access appropriate documents. This research has matured to the point that usability studies and evaluation of approaches to information visualization are needed to guide further development. The reported studies examine the use of alternative document visualizations in tightly controlled settings. Five types of interface representations were defined, including ordered text, ordered icons, a table format, a x-y graph format and a novel spring-based visualization. To assess the relative utility of the various interfaces, we have chosen to apply two kinds of measures: performance on information retrieval tasks and user preference rankings of the interfaces. The results show that performance is strikingly different across the range of interface types with the ordered icon list and text list producing the best results. Users' preferences, however, indicated that the textual format was the least desirable, while both of the 
visualization methods, i.e., icon list and spring-based visual, were preferred. We conclude that performance is more easily and accurately measured and that preferences of users can not be used alone to determine the utility of interfaces.

Mounir, et al. 2010, "A User-Centered Approach for the Design and Implementation of Kdd-Based Dss: A Case Study in the Healthcare Domain." Decision Support Systems 50(1):64-78.

In this article we propose an approach for a decision support system (DSS) based on Knowledge Discovery from Databases (KDD). In such system, user must be involved throughout the decision-making process. In consequence we propose the integration of a Human-Computer Interaction (HCI) model into the development of DSS process based on KDD. The approach we propose is based on two systems development methods--the Unified Process (UP) from Software Engineering and the U model from HCI. In this article, we describe our combined approach (UP/U) and the way we used it to develop a DSS in a medical field.

Mukherjea, S, and JD Foley. 1995, "Visualizing the World-Wide Web with the Navigational View Builder." Computer Networks and ISDN Systems 27(6):1075-87.

Overview diagrams are one of the best tools for orientation and navigation in hypermedia systems. However, constructing effective overview diagrams is a challenging task. This paper describes the Navigational View Builder, a tool which allows the user to interactively create useful visualizations of the information space. It uses four strategies to form effective views. These are binding, clustering, filtering and hierarchization. These strategies use a combination of structural and content analysis of the underlying space for forming the visualizations. This paper discusses these strategies and shows how they can be applied for forming visualizations for the World-Wide Web.

Mukhopadhyay, S, M Palakal, and K Maddu. 2010, "Multi-Way Association Extraction and Visualization from Biological Text Documents Using Hyper-Graphs: Applications to Genetic Association Studies for Diseases." Artificial Intelligence in Medicine 49(3):145-54.

Objectives Biological research literature, as in many other domains of human endeavor, represents a rich, ever growing source of knowledge. An important form of such biological knowledge constitutes associations among biological entities such as genes, proteins, diseases, drugs and chemicals, etc. There has been a considerable amount of recent research in extraction of various kinds of binary associations (e.g., gene-gene, gene-protein, protein-protein, etc.) using different text mining approaches. However, an important aspect of such associations (e.g., "gene A activates protein B") is identifying the context in which such associations occur (e.g., "gene A activates protein B in the context of disease C in organ D under the influence of chemical E"). Such contexts can be represented appropriately by a multi-way relationship involving more than two objects (e.g., objects A, B, C, D, E) rather than usual binary relationship (objects A and B).Methods Such multi-way relations naturally lead to a hyper-graph representation of the knowledge rather than a binary graph. The hyper-graph based multi-way knowledge extraction from biological text literature represents a computationally difficult problem (due to its combinatorial nature) which has not received much attention from the Bioinformatics research community. In this paper, we describe and compare two different approaches to such multi-way hypergraph extraction: one based on an exhaustive enumeration of all multi-way hyper-edges and the other based on an extension of the well-known A Priori algorithm for structured data to the case unstructured textual data. We also present a representative graph based approach towards visualizing these genetic 
association hyper-graphs. Results Two case studies are conducted for two biomedical problems (related to the diseases of lung cancer and colorectal cancer respectively), illustrating that the latter approach (using the text-based A Priori method) identifies the same hyper-edges as the former approach (the exhaustive method), but at a much less computational cost. The extracted hyper-relations are presented in the paper as cognition-rich representative graphs, representing the corresponding hyper-graphs.Conclusions The text-based A Priori algorithm is a practical, useful method to extract hyper-graphs representing multi-way associations among biological objects. These hyper-graphs and their visualization using representative graphs can provide important contextual information for understanding gene-gene associations relevant to specific diseases.

Muller, H, et al. 2010, "Interactive Patient Records." In 14th International IEEE Conference on Information Visualization, pp. 252-57.

Researches in humanities and social sciences indicate that people using more visual information would be more creative and benefit from the power of the human mind to a higher degree. Therefore information should not only be recorded as static text. It is rather conceivable to construct new visual languages that are not based on letters but on icons. We developed a set of medical symbols that can be used in visual representations of medical records and that may also be used to build complex medical statements. Each of the basic symbols is available in 4 abstraction levels, which can be combined in a 'visual sentence'. Complex visual representations are recognized more easily than simplified versions, while on the other hand the simple version can be perceived and memorized in a faster way than complex signs. To prove this hypothesis and to evaluate the perception of the symbols in a medical context we created a web-based evaluation tool covering two perception tests. With the help of the symbol library we developed a visual language for patient records and an interactive browser.

Muller, L, et al. 2006, "Creating Affective Visualisations for a Physiologically Interactive Artwork." In 10th International Conference on Information Visualization, pp. 651-57.

This paper describes an action research project based on the process of designing the visualisation of heart and breath rate data for the interactive artwork Cardiomorphologies. The project aimed to realise the affective goals of the artist as closely as possible by studying the audience experience of the visualisations and incorporating the findings into an iterative design process. The qualities of richness and ambiguity were found to contribute to the achievement of the artists' two major aims: 1) to create a sense of integrated physical and mental engagement with the work and 2) to create a reflective state in which participants consider correlations between their thoughts and specific physiological states

Müller, T, S Grottel, and D Weiskopf. 2010, "Special Relativistic Visualization by Local Ray Tracing." IEEE Transactions on Visualization and Computer Graphics 16(6):1243-50.

Special relativistic visualization offers the possibility of experiencing the optical effects of traveling near the speed of light, including apparent geometric distortions as well as Doppler and searchlight effects. Early high-quality computer graphics images of relativistic scenes were created using offline, computationally expensive CPU-side 4D ray tracing. Alternate approaches such as image-based rendering and polygon-distortion methods are able to achieve interactivity, but exhibit inferior visual quality due to sampling artifacts. In this paper, we introduce a hybrid rendering technique based on polygon distortion and local ray tracing that facilitates interactive high-quality visualization of multiple objects moving at relativistic speeds in arbitrary directions. The method starts by calculating tight image-space footprints for 
the apparent triangles of the 3D scene objects. The final image is generated using a single image-space ray tracing step incorporating Doppler and searchlight effects. Our implementation uses GPU shader programming and hardware texture filtering to achieve high rendering speed.

Muller, W, and H Schumann. 2003, "Visualization Methods for Time-Dependent Data-an Overview." In Winter Simulation Conference, pp. 737-45 Vol.1.

Visualization has been successfully applied to analyse time-dependent data for a long time now. Lately, a number of new approaches have been introduced, promising more effective graphs especially for large datasets and multi-parameter data. In this paper, we give an overview on the visualization of timeseries data and the available techniques. We provide a taxonomy and discuss general aspects of timedependent data. After an overview on conventional techniques we discuss techniques for analysing timedependent multivariate data sets in more detail. After this, we give an overview on dynamic presentation techniques and event-based visualization.

Munzner, T. 2009, "A Nested Model for Visualization Design and Validation." IEEE Transactions on Visualization and Computer Graphics 15(6):921-28.

We present a nested model for the visualization design and validation with four layers: characterize the task and data in the vocabulary of the problem domain, abstract into operations and data types, design visual encoding and interaction techniques, and create algorithms to execute techniques efficiently. The output from a level above is input to the level below, bringing attention to the design challenge that an upstream error inevitably cascades to all downstream levels. This model provides prescriptive guidance for determining appropriate evaluation approaches by identifying threats to validity unique to each level. We also provide three recommendations motivated by this model: authors should distinguish between these levels when claiming contributions at more than one of them, authors should explicitly state upstream assumptions at levels above the focus of a paper, and visualization venues should accept more papers on domain characterization.

\section{Munzner, T, et al. 2006, "Nih-Nsf Visualization Research Challenges Report Summary." Computer} Graphics and Applications, IEEE 26(2):20-24.

The US National Science Foundation (NSF) convened a panel to report on the potential of visualization as a new technology. The NSF and US National Institutes of Health (NIH) convened the Visualization Research Challenges (VRC) Executive Committee to write a new report. Here, we summarize that new VRC report. We explore the state of the field, examine the potential impact of visualization on areas of national and international importance, and present our findings and recommendations for the future of our growing discipline. Our audience is twofold: the supporters, sponsors, and application users of visualization research on the one hand, and researchers and practitioners in visualization on the other. We direct our discussion toward solving key problems of national interest and helping this work's sponsors to concentrate resources to the greatest effect. Our findings and recommendations reflect information gathered from visualization and applications scientists during two workshops on VRC, as well as input from the larger visualization community. 
Murphy-Hill, E, and AP Black. 2010, "An Interactive Ambient Visualization for Code Smells." In 5th ACM Symposium on Software Visualization (SOFTVIS), pp. 5-14.

Musa, PF. 2000, "A Comparative Study of Task Domain Analysis to Enhance Organizational Knowledge Management: Systems Thinking and Goldratt's Thinking Process." in Department of Business Administration, Vol PhD Dissertation, pp. 225 pp. Texas Tech University.

Myers, C, and D Duke. 2010, "A Map of the Heap : Revealing Design Abstractions in Runtime Structures." In 5th ACM Symposium on Software Visualization (SOFTVIS), pp. 63-71.

Myers, C, and D Duke. 2010, "Poster : More Is More - Enriched Graphs to Aid Navigation." In IEEE Conference on Information Visualization.

Nafari, M, and C Weaver. 2010, "Poster : Translating Cross-Filtered Queries into Questions." In IEEE Symposium on Visual Analytics Science \& Technology, pp. 245-46.

Nakakoji, K, A Takashima, and Y Yamamoto. 2001, "Cognitive Effects of Animated Visualization in Exploratory Visual Data Analysis." In 5th International Conference on Information Visualization, pp. 7784.

The goal of this research is to study the role and effects of the use of animated information visualization in the early stages of exploratory data analysis tasks. Despite the existence of a large body of research on information visualization, there is little known regarding how and when one should use and how to interact with animated visualization to help explore data. By animated visualization, we mean a type of information visualization technique that produces autonomous motions of representations. This research explored the issue from two aspects: what cognitive effects animated information visualization has, and what interactions people have with animated visualization when exploring data. We conducted two user studies to investigate each aspect, and identified research challenges for designing an interactive animated information visualization environment that supports the early stages of exploratory data analysis. These findings help us further study how to extend the notions developed in spatial visualization to temporal visualization - e.g., what focus + context means when applied to the time dimension in animated visualization

Nakakoji, K, and Y Yamamoto. 2003, "Toward a Taxonomy of Interaction Design Techniques for Externalising in Creative Work." In HCII 2003, pp. 1258-62.

Externalizing, the act of creating and modifying an external representation, plays a crucial role in creative design work. Whether a tool supports externalizing in a designer's desirable manner depends not only on what the tool does but also on its interaction design; how a user interacts with the tool through what representations. As an initial attempt to the development of taxonomy of interaction design techniques for tools that support externalizing, this paper examines seven tools for externalizing to illustrate why and how fine-grained interaction influences the process of externalizing. 1 Externalizing in Creative Work The goal of our research has been to design and develop conceptual frameworks and computational tools that support creative design work (Nakakoji et al. 2000)(Yamamoto et al. 2000)(Fischer, Nakakoji 1994). This paper focuses on a designer's externalization processes, and uses a word externalizing to refer to the act of creating and modifying an external representation. Tools for 
externalizing provide a designer a space for the act of externalizing, supporting the designer's creative process and amplifying their creative ability.

Nakamura, S, et al. 2005, "A Visualization Method of Relations among Knowledge-Information for Research Activities." In 11th International Conference on Parallel and Distributed Systems (ICPADS '05), pp. 702-08 Vol. 1.

In research activities, it is quite important task to classify and arrange "knowledge-information", which consists of research papers, presentation materials of the seminars, memorandums of idea, advice from colleagues, and so on. However, it is extremely difficult to manage knowledge-information systematically and to grasp the process of research. Therefore, this paper proposes the visualization method of relations among knowledge-information. At first, classification of knowledge-information and relations among them are performed. After that, details of method, which represents knowledgeinformation and relations among them as nodes and edges of a graph respectively, are described. Moreover, design and implementation of the support system is described. Finally, an experiment with the support system and evaluation based on the results of it are reported.

Nakatsu, RT, and I Benbasat. 2003, "Improving the Explanatory Power of Knowledge-Based Systems: An Investigation of Content and Interface-Based Enhancements." Ieee Transactions on Systems Man and Cybernetics Part a-Systems and Humans 33(3):344-57.

This research, investigates techniques that can improve the explanatory power of knowledge-based systems (KBS). Two types of enhancements are considered. First, deep explanations are textual descriptions that explain the underlying domain principles Associated with system recommendations. Second, graphical hierarchies are used to represent the structure of the KBS rule base so that end-users can more easily visualize how a KBS reaches system recommendations. The experimental method was employed to investigate the effectiveness of the deep explanations and the hierarchic models. A 2 x 2 factorial design was used: one factor, interface type, is whether a subject interacted with a hierarchic interface or an informationally-equivalent flat interface; the second factor, explanations provision, is whether a subject was provided with deep explanation support or not. Multiple methods of measurements were employed to understand the effects of different treatments in the study. Problem-solving performance, as assessed by a series of tasks, and execution time were measured. Short answer questions were administered to all Subjects to understand user preferences. Finally, to gain A richer understanding of problem-solving processes, all subject actions were captured in a computer log. From these logs, problem-solving strategies were reconstructed and analyzed. We found support for the benefits associated with interfaces having greater explanatory power according to the task-technology fit perspective; problem-solving performance was improved on certain tasks, and the correct problem-solving strategies were more likely to be adopted by subjects when they were provided with the appropriate support.

Nakazato, M, L Manola, and TS Huang. 2003, "Imagegrouper: A Group-Oriented User Interface for Content-Based Image Retrieval and Digital Image Arrangement." Journal of Visual Languages \& Computing 14(4):363-86.

In content-based image retrieval (CBIR), experimental (trial-and-error) query with relevance feedback is essential for successful retrieval. Unfortunately, the traditional user interfaces are not suitable for trying different combinations of query examples. This is because first, these systems assume query examples are always added incrementally. Second, the query and the result display are done on the same 
workspace. Once the user removes an image from the query examples, the image may disappear from the user interface. In addition, it is difficult to combine the result of different queries. In this paper, we propose a new interface for Content-based image retrieval named ImageGrouper. ImageGrouper is a Group-Oriented user interface in that all operations are done by creating groups of images. This approach has several advantages. First, the users can interactively compare different combinations of the query examples by dragging and grouping the images on the workspace (Query-by-Group). Because the query results are displayed on another pane, the user can quickly review the results and modify the query. Combining different queries is also easy. Furthermore, the concept of "Image Groups" is also applied for annotating and organizing many images. The Annotation-by-Groups method relieves the user of tedious task of annotating textual information on a large number of images. This method realizes a hierarchical annotation of the images as well as Bulk Annotation. The Organize-by-Group method lets the users manipulate the image groups as "Photo Albums" to organize the images. Finally, the usability of the system is compared with the traditional user interfaces. By incorporating the lessons from the experiments, the usability of ImageGrouper is further improved.

Nation, DA, et al. 2003, "Visualizing Websites Using a Hierarchical Table of Contents Browser: Webtoc." in The Craft of Information Visualization: Readings and Reflections, eds. BB Bederson and B Shneiderman, pp. 199-210. Morgan Kaufmann, San Francisco.

Summary A method is described for visualizing the contents of a Web site with a hierarchical table of contents using a Java program and applet called WebTOC. The automatically generated expand/contract table of contents provides graphical information indicating the number of elements in branches of the hierarchy as well as individual and cumulative sizes. Color can be used to represent another attribute such as file type and provide a rich overview of the site for users and managers of the site. Early results from user studies suggest that WebTOC is easily learned and can assist users in navigating websites.

Nelson, B, and B Haimes. 2010, "Gpu-Based Interactive Pixel-Exact Cut-Surface Extraction from HighOrder Finite Element Fields." In IEEE International Conference on Information Visualization.

Nene, H. 2010, "A Statistical Approach to Measuring Alignment of Genetic Sequences and Determining Critical Mutations in a Viral Gene Sequence." In IEEE Symposium on Visual Analytics Science \& Technology, pp. 289-90.

Nesbitt, KV, and C Friedrich. 2002, "Applying Gestalt Principles to Animated Visualizations of Network Data." In 6th International Conference on Information Visualisation, pp. 737-43.

Graphs are a commonly used data structure for representing relational information. Drawings of these structures, as node and link diagrams, can provide a useful visualization of the underlying abstract data. This makes drawings of graphs a useful tool in information visualization. Indeed graph drawing has been applied in many application areas including software engineering, knowledge management and for depicting communication networks. The spatial layout can help the user build up a cognitive model or 'mental map' of the information structure. Many automatic algorithms for producing drawings of a graph have been implemented. In many domains it is also common for the underlying information to be dynamic and this means the graph drawing must be updated. Unfortunately, even small changes to the underlying data can result ire dramatic changes to the final drawing and this means the user may totally lose their previous 'mental map'. Animation between the two versions of the layout is one approach that can assist the user to make the transition between the two drawings. We have been examining how to 
apply the Gestalt principles of organisation to this animation phase. The aim is to assist the user in understanding the structural and visual changes that have occurred in the layout. Results of that work are described here with relevant examples.

Neville, K, et al. 2008, "The Procurement Woes Revisited." Intelligent Systems, IEEE 23(1):72-75.

The set of people who are frustrated every day by badly designed information technology is very large. So is the set of people whose dollars pay for the badly designed technology. A conservative estimate ranges in the billions for the cost of large-scale information systems that end up collecting dust because they're not properly human-centered. Yes, billions and still counting-that's the scary part. Within this large set of frustrated customers (see the sidebar "When Systems Development Neglects Human Considerations") is a subset whose job it is to do something about this situation. That subset includes policymakers, program managers, and systems engineers. It also includes a sub-subset comprising cognitive systems engineers, ethnographers, and many others who, in one vernacular or another, advocate human-centered computing. We must show that intelligent technologies-those designed to interact with humans or play a role in the cognitive work conducted in sociotechnical work systems-are usable, useful, and understandable.

Newby, GB. 2001, "Empirical Study of a 3d Visualization for Information Retrieval Tasks." Journal of Intelligent Information Systems 18(1):31-53.

There are many challenges to visualizing information including choosing between 2D and 3D interfaces, navigation and interaction methods, and selecting an appropriate level of detail. Visualizing information retrieval (IR) search results, including Web search engine results, poses additional challenges, notably the determination of appropriate relative locations for terms and document in a visual display. Latent Semantic Indexing (LSI) and related techniques offer approaches to visualizing relations among terms and documents. In this work, "information space" is presented as a framework for discussing relations among terms and documents, and a technique related to LSI is utilized to generate information spaces from IR search results. This paper provides an overview of more than three decades of work on information visualization, identifying several trends and some relatively unexplored areas. An experimental evaluation of a prototype interface for visualizing IR results is described. Results indicate that the 3D navigation interface for IR search results was usable, but that subjects had difficulty with some aspects. Further study and development of 2D and 3D methods for interacting with retrieval search results is suggested.

Newell, A, and HA Simon. 1963, "Gps: A Program That Simulates Human Thought." in Computers and Thought, eds. EA Feigenbaum and J Feldman. MacGraw Hill, New York.

Ng, KK, and EJ Delp. 2009, "New Models for Real-Time Tracking Using Particle Filtering." In Visual Communications and Image Processing Conference, pp. 1-12.

This paper presents new methods for efficient object tracking in video sequences using multiple features and particle filtering. A histogram-based framework is used to describe the features. Histograms are useful because have the property that they allow changes in the object appearance while the histograms remain the same. Particle filtering is used because it is very robust for non-linear and nonGaussian dynamic state estimation problems and performs well when clutter and occlusions are present. Color histogram based particle filtering is the most common method used for object tracking. However, a 
single feature tracker loses track easily and can track the wrong object. One popular remedy for this problem is using multiple features. It has been shown that using multiple features for tracking provides more accurate results while increasing the computational complexity. In this paper we address these problems by describing an efficient method for histogram computation. For better tracking performance we also introduce a new observation likelihood model with dynamic parameter setting. Experiments show our proposed method is more accurate and more efficient then the traditional color histogram based particle filtering.

Nguyen, GP, and M Worring. 2008, "Optimization of Interactive Visual-Similarity-Based Search." ACM Transactions on Multimedia Computing Communications and Applications 4(1).

At one end of the spectrum, research in interactive content-based retrieval concentrates on machine learning methods for effective use of relevance feedback. On the other end, the information visualization community focuses on effective methods for conveying information to the user. What is lacking is research considering the information visualization and interactive retrieval as truly integrated parts of one content-based search system. In such an integrated system, there are many degrees of freedom like the similarity function, the number of images to display, the image size, different visualization modes, and possible feedback modes. To base the optimal values for all of those on user studies is unfeasible. We therefore develop search scenarios in which tasks and user actions are simulated. From there, the proposed scheme is optimized based on objective constraints and evaluation criteria. In such a manner, the degrees of freedom are reduced and the remaining degrees can be evaluated in user studies. In this article, we present a system that integrates advanced similarity based visualization with active learning. We have performed extensive experimentation on interactive category search with different image collections. The results using the proposed simulation scheme show that indeed the use of advanced visualization and active learning pays off in all of these datasets.

Nguyen, MX, et al. 2003, "Inspire: An Interactive Image Assisted Non-Photorealistic Rendering System." In 11th Pacific Conference on Computer Graphics and Applications, pp. 472-76.

We present a GPU supported interactive non-photorealistic rendering system, INSPIRE, which performs feature extraction in both image space, on intermediately rendered images, and object space, on models of various representations, e.g., point, polygon, or hybrid models, without needing connectivity information. INSPIRE obtains interactive NPR rendering with most styles of existing NPR systems, but offers more flexibility on model representations and compromises little on rendering speed.

Nguyen, QV, and ML Huang. 2004, "Hierarchical Information Visualization Using Enccon Model." In IASTED International Conference on Software Engineering, pp. 129-35.

This paper describes a new efficient approach for visualizing large hierarchical information. Our technique is based on the connection + enclosure visualization model [1] from which the area division is used for the recursive positioning of nodes, while a node-link diagram is still drawn to present the entire hierarchical structure. We inherit the advantages of Space-Optimized (SO) Tree technique [1] that can enhance the usability of display space by using area division. However, we replace a set of polygons used in SO Tree by a set of rectangles for the area division. This not only decreases the computation cost in calculating geometrical polygons, but also greatly reduces the human perceptual and cognitive loads spent on understanding the underlying hierarchical structure. We use semantic zooming technique to enlarge a 
particular viewing area and filter out the rest of structure that is less interested. The navigation is accommodated by animation in order to preserve the mental map [2].

Nielsen, J. 1994, "Heuristic Evaluation." in Usability Inspection Methods, eds. J Nielsen and RL Mack. John Wiley \& Sons, New York.

Nielsen, J. 1994. Usability Engineering. Morgan Kaufmann, San Francisco.

Nikander, J, J Helminen, and A Korhonen. 2009, "Experiences on Using Trakla2 to Teach Spatial Data Algorithms." Electronic Notes in Theoretical Computer Science 224:77-88.

This paper reports on the results of a two year project in which visual algorithm simulation exercises were developed for a spatial data algorithms course. The success of the project is studied from several point of views, i.e., from developer's, teachers's, and student's perspective. The amount of work, learning outcomes, and feasibility of the system has been estimated based on the data gathered during the project. The results are encouraging, which motivates to extend the concept also for other courses in the future.

Nivala, A-M, L., and T Sarjakoski. 2007, "Usability Methods' Familiarity among Map Application Developers." International Journal of Human-Computer Studies 65(9):784-95.

Developments in hardware and software have led to new innovative methods for visualising geospatial data. At the same time user-centred design (UCD) and usability engineering methods have a fundamental role in designing applications for new technical environments, which involve entirely new ways of interacting. However, applying methods from other research disciplines may not always be straightforward, as the product developers have to operate in a challenging interdisciplinary field. The aim of this study was to find out how usability engineering is currently included in the development of map services. Seven companies developing different types of map applications in Finland were interviewed. The results support the suitability of usability engineering for map application design, since by including the usability approach into the product design, while simultaneously taking into account the individuality and diversity of users and their tasks together with the characteristics of the maps, application developers are more likely to design products that have a higher quality of use. This study identifies the main occasions when the usability approach could be most beneficial. Furthermore, the benefits and challenges of including usability approaches in map application design are discussed. Preliminary ideas on what usability means in the context of map applications are also given. Finally, the importance for providing a basis for the further development of application-specific guidelines and techniques is addressed.

Nkambou, R, P Fournier-Viger, and EM Nguifo. 2011, "Learning Task Models in Ill-Defined Domain Using an Hybrid Knowledge Discovery Framework." Knowledge-Based Systems 24(1):176-85.

Domain experts should provide Intelligent Tutoring Systems (ITS) with relevant domain knowledge that enable it to guide the learner during problem-solving learning activities. However, for ill-defined domains this knowledge is hard to define explicitly. Our hypothesis is that knowledge discovery (KD) techniques can be used to extract problem-solving task models from the recorded usage of expert, intermediate and novice learners. This paper proposes a procedural-knowledge acquisition framework based on a combination of sequential pattern mining and association rules discovery techniques. The framework has been implemented and is used to discover new meta-knowledge and rules in a given domain which then extend domain knowledge and serve as problem space, allowing the Intelligent 
Tutoring System to guide learners in problem-solving situations. Preliminary experiments have been conducted using the framework as an alternative to a path-planning problem solver in CanadarmTutor.

Noah, SA, S Yaakob, and S Shahar. 2009, "Application of Information Visualization Techniques in Representing Patients' Temporal Personal History Data." In 1st International Visual Informatics Conference, pp. 168-79.

The anthropometries and nutrients records of patients are usually vast in quantity, complex and exhibit temporal features. Therefore, the information acceptance among users will become blur and give cognitive burden if such data is not displayed using effective techniques. The aim of this study is to apply, use and evaluate Information Visualization (IV) techniques for displaying the Personal History Data (PHD) of patients for dietitians during counseling sessions. Since PHD values change consistently with the counseling session, our implementation mainly focused on quantitative temporal data such as Body Mass Index (BMI), blood pressure and blood glucose readings. This data is mapped into orientation circle type of visual representation, whereas data about medicinal and supplement intake are mapped into timeline segment which is based on the thickness of lines as well as the colors. A usability testing has been conducted among dietitians at Faculty of Allied Health Sciences, UKM. The result of the testing has shown that the use of visual representations capable of summarising complex data which ease the dietitian task of checking the PHD.

Noble, RA, and GJ Clapworthy. 1999, "Direct Manipulation of Surfaces Using Nurbs-Based Free-Form Deformations." In IEEE International Conference on Information Visualization, pp. 238-43.

The paper demonstrates how NURBS-based Free-Form Deformations (NFFDs) can be used to generate and sculpt NURBS based objects in a way that avoids many of the limitations of ordinary FFDs. A system is described in which the user interacts with the NFFD through a set of direct acting tools, removing the need to manipulate the mesh directly. As the user applies the tools to the object, the NFFD is updated automatically. Objects are represented as isosurfaces of the NFFD volume, giving a good insight into the way an NFFD would deform a general object

Nocke, T, et al. 2003, "Information Visualization Supporting Modelling and Evaluation Tasks for Climate Models." In 36th Winter Simulation Conference, pp. 763-71.

Information visualization exploits the phenomenal abilities of human perception to identify structures by presenting abstract data visually, allowing an intuitive exploration of data to get insight, to draw conclusions and to interact directly with the data. The specification, analysis and evaluation of complex models and simulated model data can benefit from information visualization techniques by obtaining visual support for different tasks. This paper presents an approach that combines modelling and visualization functionality to support the modelling process. Based on this general approach, we have developed and implemented a framework that allows to combine a variety of models with statistical and analytical operators as well as with visualization methods. We present several examples in the context of climate modelling. 
Noh, T-G, et al. 2010, "Learning the Emergent Knowledge from Annotated Blog Postings." Web Semantics: Science, Services and Agents on the World Wide Web 8(4):329-39.

Emergent knowledge does not come from a particular document or a particular knowledge source, but comes from a collection of documents or knowledge sources. This paper proposes a system which combines social web content and semantic web technology to process the emergent knowledge from the blogosphere. The proposed system regards blog postings as experiences of people on particular topics. By annotating postings in the selected domains with ontology vocabularies, the system collects experiences from various people into an ontology about people and experiences. The system processes this ontology with semantic rules to find the emergent knowledge. Users can access previously unavailable facts, concepts and trends which are emerging from social web content by using the proposed system.

Norman, DA. 1988, "Psychology of Everyday Action." in The Design of Everyday Things pp. 45-46. Basic Books, New York.

Norman, DA. 1983, "Some Observations on Mental Models." in Mental Models, eds. D Dentner and AL Stevens, pp. 7-14. Lawrence Erlbaum Associates, Hillsdale, NJ.

North, C. 2006, "Toward Measuring Visualization Insight." IEEE Computer Graphics and Applications 26(3):6-9.

Recent visualization research literature has paid an increasing amount of attention to evaluating visualizations. It seems an appropriate time to reopen the question about what the ultimate purpose of visualization is and how it should be evaluated. One potential claim is: the purpose of visualization is insight. The purpose of visualization evaluation is to determine to what degree visualizations achieve this purpose. If this claim is true, then evaluating visualizations should seek to determine how well visualizations generate insight. Measuring insight would enable the direct comparison of visualization design alternatives, or the comparison against an insight goal. This article examines the capability of the controlled experiment method to measure insight

North, C, and BEN Shneiderman. 2000, "Snap-Together Visualization: Can Users Construct and Operate Coordinated Visualizations?" International Journal of Human-Computer Studies 53(5):715-39.

Multiple coordinated visualizations enable users to rapidly explore complex information. However, users often need unforeseen combinations of coordinated visualizations. Snap-together visualization (Snap) enables users to rapidly and dynamically construct coordinated-visualization interfaces, customized for their data, without programming. Users U001load data into desired visualizations, then construct coordinations between them for brushing and linking, overview and detail view, drill down, etc. Snap formalizes a conceptual model of visualization coordination based on the relational data model. Visualization developers can easily Snap-enable their independent visualizations using a simple API. Empirical evaluation reveals benefits, cognitive issues and usability concerns with coordination concepts and Snap. Two user studies explore coordination construction and operation. Data-savvy users successfully, enthusiastically and rapidly constructed powerful coordinated-visualization interfaces of their own. Operating an overview-and-detail coordination reliably improved user performance by $30-80 \%$ over detail-only and uncoordinated interfaces for most tasks. 
Nowell, L, E Hetzler, and T Tanasse. 2001, "Change Blindness in Information Visualization: A Case Study." In 7th IEEE Symposium on Information Visualization (INFOVIS 2001), pp. 15-22.

Change blindness occurs when people do not notice changes in visible elements Of 0 scene. If people use on information visualization system to compare document collection subsets partitioned by their timestamps, change blindness makes it impossible for them to recognize even very major changes, let alone minor ones. Me describe theories from cognitive science that account for the change blindness phenomenon, as well as solutions developed for two visual analysis tools, a dot plot (SPIRE Galaxies) and landscape (ThemeView(TM)) visualizations.

Nowell, L, R Schulman, and D Hix. 2002, "Graphical Encoding for Information Visualization: An Empirical Study." In IEEE Symposium on Information Visualization, pp. 43-50.

Research in several areas provides scientific guidance for use of graphical encoding to convey information in an information visualization display. By graphical encoding we mean the use of visual display elements such as icon color, shape, size, or position to convey information about objects represented by the icons. Literature offers inconclusive and often conflicting viewpoints, including the suggestion that the effectiveness of a graphical encoding depends on the type of data represented. Our empirical study suggests that the nature of the users' perceptual task is more indicative of the effectiveness of a graphical encoding than the type of data represented.

Noy, P. 2005, "Chapter 12 - Signature Exploration, a Means to Improve Comprehension and Choice within Complex Visualization Processes: Issues and Opportunities." in Exploring Geovisualization, eds. D Jason, MM Alan and K Menno-Jan, pp. 243-63. Elsevier, Oxford.

Summary All fields of visualization have, to some extent, to deal with visualizing high-dimensional data. In projecting such complex data into 2- or 3D or in the use of novel representations that allow direct mapping of greater numbers of attributes, two problems must be addressed: choice of appropriate display and understanding of the resultant graphic. At the same time, the general context is of an ever-expanding set of visualization tools available to increasingly varied types of users, so that it is becoming increasingly important to establish techniques to support user comprehension. This chapter provides some thoughts on this subject and outlines a proposed concept to provide a framework for developing techniques to support user choice and understanding in visualizations of complex data -- signature exploration. Some example software has been developed that illustrates the techniques. Signature exploration involves the use of specially constructed datasets that are known in some sense by the user. The datasets are used to explore the signatures of different visual representations and mathematical transformations. The chapter defines and illustrates signature exploration, together with five proposed techniques for the use of constructed data: generic dataset provision; user-construction of data; querying; insertion of landmarks; elicitation and application of feedback data. Two new interaction forms are described: brushing between data table and representation; synthetic additions to real-world datasets to provide the user with calibration of the visual depiction. Whilst each of these techniques needs further empirical evaluation, this overview provides sufficient description for developers to incorporate specific aspects easily. Thus the chapter is a general discussion of relevant issues and the suggestion of some specific techniques for increasing comprehension of visualization systems. 
Ntuen, CA, EH Park, and K Gwang-Myung. 2010, "Designing an Information Visualization Tool for Sensemaking." International Journal of Human-Computer Interaction 26(2-3):189-205.

The study presents the AVALANCHE visualization test-bed for sensemaking in illstructured problem domains. AVALANCHE allows the users to develop and frame hypotheses, analyze the hypotheses in the experimental domain, and provide cases for simulation experiments. The visualization and sensemaking support module in AVALANCHE provides human-computer interface and visualization supports.

Validation experiments using groups aided with visualization and support tools and groups with no aiding was performed on two open-ended sensemaking cases provided by a military subject matter expert. Statistical analyses revealed mean performance differences in plan accuracy, plan time, and number of cue prompts between aided and unaided groups across task scenarios. In general, the aided group had the highest mean plan outcome accuracy, low planning time, and the least number of prompts. The intention is to extend the study to collaborative sensemaking tasks to address the effects of negotiation on team planning time, cue prompting frequencies, and different types of cue prompting modalities. (C) Taylor \& Francis Group, LLC.

O'Brien, T, et al. 2010, "Gremlin: An Interactive Visualization Model for Analyzing Genomic Rearrangements." IEEE Transactions on Visualization and Computer Graphics 16(6):918-26.

In this work we present, apply, and evaluate a novel, interactive visualization model for comparative analysis of structural variants and rearrangements in human and cancer genomes, with emphasis on data integration and uncertainty visualization. To support both global trend analysis and local feature detection, this model enables explorations continuously scaled from the high-level, complete genome perspective, down to the low-level, structural rearrangement view, while preserving global context at all times. We have implemented these techniques in Gremlin, a genomic rearrangement explorer with multiscale, linked interactions, which we apply to four human cancer genome data sets for evaluation. Using an insight-based evaluation methodology, we compare Gremlin to Circos, the state-of-the-art in genomic rearrangement visualization, through a small user study with computational biologists working in rearrangement analysis. Results from user study evaluations demonstrate that this visualization model enables more total insights, more insights per minute, and more complex insights than the current stateof-the-art for visual analysis and exploration of genome rearrangements.

O'Connell, TA, and YY Choong. 2008, "Metrics for Measuring Human Interaction with Interactive Visualizations for Information Analysis." In 26th Annual CHI Conference on Human Factors in Computing Systems, pp. 1493-96.

There is a lack of widely-accepted metrics for evaluating analysts' experiences with interactive visualizations (IV) for information analysis. We report an approach for developing analyst-centered IV metrics that is built upon understanding the workplace needs and experiences of information analysts with respect to IVs. We derive metrics from human-computer interaction heuristics, specializing the metrics to address the characteristics of IVs and analysts. When there are no existing heuristics, analysts' needs and experiences inform new heuristics. 
O'Donnell, R, A Dix, and LJ Ball. 2007, "Exploring the Pietree for Representing Numerical Hierarchical Data." In 20th Annual Conference of the British-HCI-Group, pp. 239-54.

This paper describes the first full implementation and evaluation of an area-based tree visualization known as the PieTree. The PieTree was first proposed in papers in 1998 and 2000 but has never been fully implemented and evaluated. Informal evaluation was used to enhance the usability of the PieTree and compare it with the more well-known TreeMap. A controlled experiment considered parallel views' effect on task performance time. There were substantial differences between kinds of tasks and in participants' styles of use. Whilst suggesting that further development of PieTrees is worthwhile the experiments underline the importance of careful task fit.

Oelke, D, et al. 2008, "Visual Evaluation of Text Features for Document Summarization and Analysis." In IEEE Symposium on Visual Analytics Science \& Technology, pp. 75-82.

Thanks to the Web-related and other advanced technologies, textual information is increasingly being stored in digital form and posted online. Automatic methods to analyze such textual information are becoming inevitable. Many of those methods are based on quantitative text features. Analysts face the challenge to choose the most appropriate features for their tasks. This requires effective approaches for evaluation and feature-engineering.

Oelke, D, A Stoffel, and DA Keim. 2010, "Visual Readability Analysis : How to Make Your Writings Easier to Read." In IEEE Symposium on Visual Analytics Science \& Technology, pp. 123-30.

Oesterling, P, et al. 2010, "Two-Stage Framework for a Topology-Based Projection and Visualization of Classified Document Collections." In IEEE Symposium on Visual Analytics Science \& Technology, pp. 91-98.

Ogao, PJ, and MJ Kraak. 2002, "Defining Visualization Operations for Temporal Cartographic Animation Design." International Journal of Applied Earth Observation and Geoinformation 4(1):23-31.

Cartographic animation has emerged as a potentially effective visualization technique that has an intuitive power in representing dynamic geographical phenomena through its ability to show interrelations amongst geospatial data's components, location, attribute and time. Whereas cartographic animation has prominently featured in communicating geospatial information, their use as tools for visual exploration has been hampered by lack of the necessary functionality that is capable of allowing users to interact with the dynamic display. In this paper, we outline an approach that defines visualizations operations or basic visual actions that implement a viewer's task of exploration and characterization of geospatial structures in data or phenomena. The defined operations go along to reinforcing the quest in enabling users to perceive relationships and be able to manipulate geospatial data using more efficient visual tools while keeping low on cognitive demands.

Ogata, H, et al. 1999, "Knowledge Awareness Map for Open-Ended and Collaborative Learning on World Wide Web." In 7th International Conference on Computers in Education (ICCE 99), pp. 319-26.

This paper proposes an open-ended and collaborative learning environment, called SharlokII. In this environment, the learners provide and share individual knowledge and other knowledge on the World Wide Web and discuss about them. This paper describes knowledge awareness map and its design, 
implementation and evaluation The map visualizes the relationship between the shared knowledge and the current and past interactions of learners. The map plays a very important role of finding peer helpers, and inducing collaboration. In this map, a mediator agent recommends suitable collaborators who can help the problem solving. SharlokII has been developed and used it experimentally.

Ogawa, M. 2010, "Software Evolution Storylines." In 5th ACM Symposium on Software Visualization (SOFTVIS), pp. 35-41.

Ogiela, MR, and R Tadeusiewicz. 2010, "Towards New Classes of Cognitive Vision Systems." In International Conference on Complex, Intelligent and Software Intensive Systems, pp. 851-55.

This publication describes two new classes of specialised information systems: UBIAS and EUBIAS. Such systems belong to the group of cognitive reasoning systems and are designed for the semantic analysis of complex patterns having the form of biomedical images. These systems are predecessors of a new generation of intelligent systems for understanding medical image data and using this data as biometric characteristics.

Oh, JY, and H Hua. 2008, "Usability of Multi-Scale Interfaces for 3d Workbench Displays." PresenceTeleoperators and Virtual Environments 17(5):415-40.

We consider that multi-scale visualization interfaces support users to view different levels of scales simultaneously and to understand large-scale, complex 3D information in 3D display environments. This article presents a user evaluation on three multi-scale interfaces on a 3D workbench display: focus + context $(f+c)$, fixed $f+c$, and overview + detail $(o+d)$. The interfaces differ in terms of (1) window arrangement and (2) positioning of detailed information relative to the user. Our goal is to identify the effect of these interface differences in large scale information visualization on a 3D workbench. To address the usability of the interfaces for a wide range of applications, we designed two tasks that differ by the level of information integration and cognitive demand. The evaluation results suggest that focusbased interfaces (i.e., the $\mathrm{f}+\mathrm{c}$ and fixed $\mathrm{f}+\mathrm{c}$ interfaces) are useful for tasks that require tight coupling between information layers and the $\mathrm{o}+\mathrm{d}$ interface is useful for tasks performed in a densely populated information space. In terms of interface design on a 3D workbench, it is important to provide an up-close view of the current region of interest for fast scene navigation and an easy way to change viewing direction to see the 3D information from more comfortable directions. The detailed design guidelines based on the evaluation analysis are presented in this article.

Ohene-Djan, J, A Sammon, and R Shipsey. 2006, "Colour Spectrum's of Opinion: An Information Visualisation Interface for Representing Degrees of Emotion in Real Time." In IEEE International Conference on Information Visualization, pp. 80-88.

In this paper we present an information visualisation interface that enables a user to input a real-time continuous flow of their predominant emotion incorporating degrees of uncertainty relative to other choices. The novel equilateral triangle-based interface allows users to express opinions on temporal media presentations such as television advertisements, video and musical compositions. The continuous flow of information generated by the interface is mapped into a colour spectrum which reveals a user's developing opinion of the presentation. Such a colour spectrum not only provides a visual snapshot to enhance statistical interpretations of user input but also provides valuable insight into when, how and with what degree of certainty opinions were developed and changed over time. The real-time emotional spectrum 
tool (REST) is being developed to answer a need in the commercial research market. It is intended for use in gathering opinions from online audiences

Ohsawa, Y. 2008, "Plenary Abstracts Chance Discovery and Chance Creation : A Data-Visualization Approach to Value Sensitive Innovation the University of Tokyo." In Second Asia International Conference on Modelling \& Simulation.

Summary form only given. A scenario is a meaningful sequence of events. For the decision of human, it is essential to create valuable scenarios of the future and choose one to realize. This talk is composed of two topics: (1) computational methods for visualizing a scenario map: a figure visualizing the relations between events, on which humans can think about scenarios. The obtained scenarios can be regarded as patterns of event occurrences in the past or beneficial plans to be executed in the future. (2) Human's competencies for sensing a (or a sequence of) valuable event, and new criteria for evaluating the value of forthcoming events. We have been studying this talent from the viewpoint of cognitive science and business management

Ohwada, H, and F Mizoguchi. 2003, "Integrating Information Visualization and Retrieval for Www Information Discovery." Theoretical Computer Science 292(2):547-71.

An important technology in knowledge discovery is to access the desired information from the large amount of data stored on the WWW. At present, such information can be accessed by a browser itself or by using a keyword search function. However, browsing is a time consuming task where a user must access individual pages one by one. Furthermore, in keyword searches, it is difficult for users to provide reasonable keywords in knowledge discovery processes. This paper outlines an approach for integrating information visualization and retrieval into WWW information discovery. In this approach, the link structure of a web site is displayed in a 3-D hyperbolic tree in which the height of a node (corresponding to a web page) within the tree indicates a user's "interest" for each page. Here, interest is calculated by a fitting function between a page and a user-supplied query (nested keywords). This measure can be used to filter uninteresting pages, reducing the size of the link structure. Furthermore, each web page is modeled as semi-structured data and can also be displayed as a hyperbolic tree in which the result of query evaluation is visible. Such functions are incorporated within our browser, allowing us to interactively discover desired pages from a large web site. We selected typical web sites to show the performance of the proposed method with improved accuracy and efficiency in WWW information discovery. Here, accuracy indicates how surely the user accesses his/her desired documents, and efficiency indicates how quickly the user reaches the documents. (C) 2002 Elsevier Science B.V. All rights reserved.

Omote, H, and K Sugiyama. 2007, "Method for Visualizing Complicated Structures Based on Unified Simplification Strategy." IEICE Transactions on Information and Systems E90D(10):1649-56.

In this paper, we present a novel force-directed method for automatically drawing intersecting compound mixed graphs (ICMGs) that can express complicated relations among elements such as adjacency, inclusion, and intersection. For this purpose, we take a strategy called unified simplification that can transform layout problem for an ICMG into that for an undirected graph. This method is useful for various information visualizations. We describe definitions, aesthetics, force model, algorithm, evaluation, and applications. 
Ong, J, and E Remolina. 2010, "Graph Arrays and Timelines for Genetic Sequence Analysis Vast Mini Challenge 3." In IEEE Symposium on Visual Analytics Science \& Technology.

Ong, J, and E Remolina. 2010, "Graph Arrays for Disease Outbreak Analysis Vast Mini Challenge 2." In IEEE Symposium on Visual Analytics Science \& Technology.

Ortiz, S. 2010, "Use Cases of Impure, an Information Interface." In IEEE International Conference on Information Visualization, pp. 2-4.

Osawa, N. 2006, "Tactile Glyphs for Palpation of Relationships." In 10th International Conference on Information Visualization, pp. 575-84.

We propose the use of tactile glyphs to represent abstract information such as hierarchical relationships. A tactile glyph uses vibro-tactile feedback to the fingers and palm of the hand. By comparing two tactile glyphs, one for each hand, one can understand the relationships between the information that the glyphs represent. The use of tactile glyph comparison does not require remembering complex stimulus patterns; it only needs that some simple rules are learned. Although a limited number of stimuli can be provided to one hand, techniques for mapping hierarchical information to a pattern and using a sequence of tactile feedback enable the representation of relationships in a large hierarchy. Experimental evaluations were conducted to clarify the features of the tactile glyphs. The experimental evaluations suggested that the tactile glyphs can be used to represent hierarchical relationships and for palpation of relationships

Osbeck, LM. 2009, "Transformations in Cognitive Science: Implications and Issues Posed." Journal of Theoretical \& Philosophical Psychology 29(1):16-33.

Cognitive science currently offers models of cognition that depart substantively from those of information processing models and classical artificial intelligence, while it embraces methods of inquiry that include case-based, ethnographic, and philosophical methods. To illustrate, five overlapping approaches that constitute departures from classical representational cognitive science are briefly discussed in this paper: dynamical cognition, situated cognition, embodied cognition, extended mind theory, and integrative cognition. Critical responses to these efforts from members of the self-proclaimed cognitive science orthodoxy are also summarized. The paper then discusses ethical and epistemological implications arising from the "new" cognitive science and from critical responses to it and considers the broader importance of this literature for theoretical and philosophical psychology.

Ostermann, FO. 2010, "Digital Representation of Park Use and Visual Analysis of Visitor Activities." Computers, Environment and Urban Systems 34(6):452-64.

Urban public parks can serve an important function by contributing to urban citizens' quality of life. At the same time, they can be the location of processes of displacement and exclusion. Despite this ambiguous role, little is known about actual park use patterns. To learn more about park use in three parks in Zurich, Switzerland, extensive data on visitor activities was collected using a new method based on direct recording via a portable GIS solution. Then, the data was analyzed using qualitative and quantitative methods. This paper examines whether geographic visualization of these data can help domain experts like landscape designers and park managers to assess park use. To maximize accessibility, the visualizations are made available through a web-interface of a common, off-the-shelf GIS. The 
technical limitations imposed by this choice are critically assessed, before the available visualization techniques are evaluated in respect to the needs and tasks of practitioners with limited knowledge on spatial analysis and GIS. Key criteria are each technique's level of abstraction and graphical complexity. The utility and suitability of the visualization techniques is characterized for the distinct phases of exploration, analysis and synthesis. The findings suggest that for a target user group of practitioners, a combination of dot maps showing the raw data and surface maps showing derived density values for several attributes serves the purpose of knowledge generation best.

Otto, HE, F Kimura, and KG Mueller. 2005, "Overcoming Complexity and Tradition-Related Shortcomings of Enabling Technologies: Solutions for Advanced Information Displays in Lca." In 4th International Symposium on Environmentally Conscious Design and Inverse Manufacturing (EcoDesign 05), pp. 712-19.

In practice, enabling technologies, along with digital models and computer-aided/computer-based systems, are still not sufficiently integrated to make life cycle modeling and life cycle assessment methods and technologies suitable tools for designers and engineers. For example, life cycle assessment is becoming more and more an integral tool in today's product design and design evaluation during analysis and optimization. However, approaches and tools that still feature old fashioned paradigms and structures in critical areas such as human-computer interaction and information representation are somewhat counter-productive, considering the increase of both the complexity of the problem and the quantities of data to be handled. In this paper we aim at presenting, in a transparent way, the motivations, structure, and consequences of one approach leading to solutions which overcome such obstacles. This approach also supports the application and successful translation into practice of one modern methodology which is central for the cradle-to-grave evaluation used to improve environmentally conscious products and service designs.

Otto, HE, KG Mueller, and F Kimura. 2003, "Efficient Information Visualization in Lca: Approach and Examples." International Journal of Life Cycle Assessment 8(5):259-65.

Aim, Scope and Background. The data-intensive nature of life cycle assessment (LCA), even for noncomplex products, quickly leads to the utilization of various methods of representing the data in forms other than written characters. Up until now, traditional representations of life cycle inventory (LCI) data and environmental impact analysis (EIA) results have usually been based on 2D and 3D variants of simple tables, bar charts, pie charts and $\mathrm{x} / \mathrm{y}$ graphs. However, these representation methods do not sufficiently address aspects such as representation of life cycle inventory information at a glance, filtering out data while summarizing the filtered data (so as to reduce the information load), and representation of data errors and uncertainty. Main Features. This new information representation approach with its glyphbased visualization method addresses the specific problems outlined above, encountered when analyzing LCA and EIA related information. In particular, support for multi-dimensional information representation, reduction of information load, and explicit data feature propagation are provided on an interactive, computer-aided basis. Results. Three-dimensional, interactive geometric objects, so called OM-glyphs, were used in the visualization method introduced, to represent LCA-related information in a multi-dimensional information space. This representation is defined by control parameters, which in turn represent spatial, geometric and retinal properties of glyphs and glyph formations. All relevant analysis scenarios allowed and valid can be visualized. These consist of combinations of items for the material and energy inventories, environmental items, life cycle phases and products, or their parts and components. Individual visualization scenarios, once computed and rendered on a computer screen, can then 
interactively be modified in terms of visual viewpoint, size, spatial location and detail of data represented, as needed. This helps to increase speed, efficiency and quality of the assessment performance, while at the same time considerably reducing mental load due to the more structured manner in which information is represented to the human expert. Conclusions. The previous paper in this series discussed the motivation for a new approach to efficient information visualization in LCA and introduced the essential basic principles. This second paper offers more insight into and discussion on technical details and the framework developed. To provide a means for better understanding the visualization method presented, examples have been given. The main purpose of the examples, as already indicated, is to demonstrate and make transparent the mapping of LCA related data and their contexts to glyph parameters. Those glyph parameters, in turn, are used to generate a novel form of sophisticated information representation which is transparent, clear and compact, features which cannot be achieved with any traditional representation scheme. Outlook. Final technical details of this approach and its framework will be presented and discussed in the next paper. Theoretical and practical issues related to the application of this visualization method to the computed life cycle inventory data of an actual industrial product will also be discussed in this next paper.

Overby, D, J Keyser, and J Wall. 2010, "Asynchronous View-Dependent Data Retrieval for Interactive out-of-Core Terrain Visualization." In IEEE International Conference on Information Visualization.

Oxman, R. 1998, "Computational Support for Visual Thinking in Design Ideation." In IEEE International Conference on Information Visualization, pp. 192-95.

A CDM (cognitive design medium) is defined as a medium which supports the cognitive nature of design in computerized environments. We review the theoretical background and demonstrate the basis upon which an interactive counterpart can be achieved in supporting the cognitive capabilities of the designer, such as visual thinking. The approach is elaborated and illustrated by experimental work in the area of conceptual design. We define three phenomena related to visual thinking in design which are characteristic of conceptual design: ideation, re-representation, and the evocation of generic representations. Finally, we demonstrate through examples how interactive computational environments can support these cognitive design phenomena

Oxman, R. 2004, "Think-Maps: Teaching Design Thinking in Design Education." Design Studies 25(1):63-91.

A pedagogical framework for design learning and design teaching termed Think-Maps is introduced, presented and demonstrated. In this framework, domain knowledge becomes explicit as a significant component to be taught and transferred in design education. The Think-Maps framework proposes that by constructing a conceptual map that reflects one's thinking in a domain, we make explicit the knowledge learned. The learner constructs structured representations of concepts and their relationships to other concepts and fills these structures with the content of the specific design domain or design task. This resulting structured representation of knowledge can later be accessed and expanded in additional processes of design thinking. Web-Pad -- a computational tool that implements these ideas is presented and illustrated. Web-Pad is used for organizing and representing conceptual maps of a specific domain. The Think-Maps framework and the Web-Pad tool are demonstrated in an educational environment. 
Ozcelik, E, et al. 2009, "An Eye-Tracking Study of How Color Coding Affects Multimedia Learning." Computers \& Education 53(2):445-53.

Color coding has been proposed to promote more effective learning. However, insufficient evidence currently exists to show how color coding leads to better learning. The goal of this study was to investigate the underlying cause of the color coding effect by utilizing eye movement data. Fifty-two participants studied either a color-coded or conventional format of multimedia instruction. Eye movement data were collected during the study. The results indicate that color coding increased retention and transfer performance. Enhancement of learning by color coding was due to efficiency of locating corresponding information between illustration and text. Color coding also attracted attention of learners to perceptually salient information.

Paci, F, and S Universit. 2008, "Verification of Access Control Requirements in Web Services Choreography." In IEEE International Conference on Services Computing, pp. 5-12.

Web services choreography is used to design peer-to-peer applications where each peer is potentially a Web service. It defines the required behavior of participating Web services along with their interactions through message exchanges. Implementing a complex system described by a choreography requires selecting actual Web services whose individual behaviors are compatible with the overall behavior described by the choreography. Although the selected Web services implement the specified behavior, they may not be able to interact due to the policies they enforce to protect their resources. A Web service' resource can be an operation or a credential type to be submitted to be able to invoke an operation. In this paper, we propose a novel approach to determine at design time whether a choreography can be implemented by a set of Web services based on their access control policies and the disclosure policies regulating the release of their credentials. We model both Web services and Web services choreography as transition systems and represent Web services credential disclosure policies as directed graphs. We then verify that all possible conversations of the Web services choreography can be implemented by matching credential disclosure policies of the invoker Web service with the access control policy of the Web services being invoked. We propose a resource release graph to enable this verification

Pajarinen, J, J Peltonen, and MA Uusitalo. 2010, in press, "Fault Tolerant Machine Learning for Nanoscale Cognitive Radio." Neurocomputing.

We introduce a machine learning based classifier that identifies free radio channels for cognitive radio. The architecture is designed for nanoscale implementation, under nanoscale implementation constraints; we do not describe all physical details but believe future physical implementation to be feasible. The system uses analog computation and consists of cyclostationary feature extraction and a radial basis function network for classification. We describe a model for nanoscale faults in the system, and simulate experimental performance and fault tolerance in recognizing WLAN signals, under different levels of noise and computational errors. The system performs well under expected non-ideal manufacturing and operating conditions. 
Pan, C-c. 2008, "Vast 2008 Wiki Editors Mini Challenge - Identifying Social Networks Using Wiki . Viz." In IEEE Symposium on Visual Analytics Science \& Technology.

Pan, CC, et al. 2007, "Vast 2007 Contest Texplorer." In IEEE Symposium on Visual Analytics Science \& Technology, pp. 243-44.

TexPlorer is an integrated system for exploring and analyzing vast amount of text documents. The data processing modules of TexPlorer consist of named entity extraction, entity relation extraction, hierarchical clustering, and text summarization tools. Using time line tool, tree-view, table-view, and concept maps, TexPlorer provides visualizations from different aspects and allows analysts to explore vast amount of text documents efficiently.

Pan, CC, P Mitra, and AR Ganguly. 2007, "Spatio-Temporal Analysis on Fema Situation Updates with Automated Information Extraction." In IEEE Symposium on Visual Analytics Science \& Technology.

Pan, C-C, et al. 2007, "Textplorer: An Application Supporting Text Analysis." In IEEE Symposium on Visual Analytics Science \& Technology, pp. 205-06.

TexPlorer is an integrated system for exploring and analyzing large amounts of text documents. The data processing modules of TexPlorer consist of named entity extraction, entity relation extraction, hierarchical clustering, and text summarization tools. Using a timeline tool, tree-view, table-view, and concept maps, TexPlorer provides an analytical interface for exploring a set of text documents from different perspectives and allows users to explore vast amount of text documents efficiently.

Pan, C-C, and P Mitra. 2007, "Femarepviz: Automatic Extraction and Geo-Temporal Visualization of Fema National Situation Updates." In IEEE Symposium on Visual Analytics Science \& Technology, pp. $11-18$.

An architecture for visualizing information extracted from text documents is proposed. In conformance with this architecture, a toolkit, FemaRepViz, has been implemented to extract and visualize temporal, geospatial, and summarized information from FEMA national update reports. Preliminary tests have shown satisfactory accuracy for FEMARepViz. A central component of the architecture is an entity extractor that extracts named entities like person names, location names, temporal references, etc.

FEMARepViz is based on FactXtractor, an entity-extractor that works on text documents. The information extracted using FactXtractor is processed using GeoTagger, a geographical name disambiguation tool based on a novel clustering-based disambiguation algorithm. To extract relationships among entities, we propose a machine-learning based algorithm that uses a novel stripped dependency tree kernel. We illustrate and evaluate the usefulness of our system on the FEMA National Situation Updates. Daily reports are fetched by FEMARepViz from the FEMA website, segmented into coherent sections and each section is classified into one of several known incident types. We use concept Vista, Google maps and Google earth to visualize the events extracted from the text reports and allow the user to interactively filter the topics, locations, and time-periods of interest to create a visual analytics toolkit that is useful for rapid analysis of events reported in a large set of text documents. 
Panse, C, et al. 2006, "Visualization of Geo-Spatial Point Sets Via Global Shape Transformation and Local Pixel Placement." Visualization and Computer Graphics, IEEE Transactions on 12(5):749-56.

In many applications, data is collected and indexed by geo-spatial location. Discovering interesting patterns through visualization is an important way of gaining insight about such data. A previously proposed approach is to apply local placement functions such as PixelMaps that transform the input data set into a solution set that preserves certain constraints while making interesting patterns more obvious and avoid data loss from overplotting. In experience, this family of spatial transformations can reveal fine structures in large point sets, but it is sometimes difficult to relate those structures to basic geographic features such as cities and regional boundaries. Recent information visualization research has addressed other types of transformation functions that make spatially-transformed maps with recognizable shapes. These types of spatial-transformation are called global shape functions. In particular, cartogram-based map distortion has been studied. On the other hand, cartogram-based distortion does not handle point sets readily. In this study, we present a framework that allows the user to specify a global shape function and a local placement function. We combine cartogram-based layout (global shape) with PixelMaps (local placement), obtaining some of the benefits of each toward improved exploration of dense geo-spatial data sets

Pantuwong, N. 2010, "3d Curve-Skeleton Extraction Using a Skeleton-Growing Algorithm." In IEEE International Conference on Information Visualization, pp. 4-5.

Paradis, S, et al. 2002, "A Pragmatic Cognitive System Engineering Approach to Model Dynamic Human Decision-Making Activities in Intelligent and Automated Systems." In RTO HFM Symposium on The Role of Humans in Intelligent and Automated Systems.

Pardue, JH, et al. 2009, "Look-Ahead and Look-Behind Shortcuts in Large Item Category Hierarchies: The Impact on Search Performance." Interacting with Computers 21(4):235-42.

Websites use shortcuts to facilitate navigation of large hierarchies of item categories. Two common types of shortcuts used for this purpose are location breadcrumbs and down-to-child/up-to-parent links; frequently both are employed simultaneously. The combined used of these shortcuts provide proximal cues which enable the user to look-ahead and look-behind in the navigational structure. In this study, the impact of shortcut usage on search performance on a known-item search task is estimated. A controlled experiment was conducted using a realistic hypertext hierarchy of item categories. The results indicate that greater use of shortcuts decreases both time on task and lostness for the user, and that the decrease is associated with increased depth in the hierarchy. These findings provide insight into possible performance trade-offs involved in website designs that include look-ahead shortcuts for navigating large item category hierarchies.

Park, A. 2007, "Intended Use Evaluation Approach for Information Visualization." in Computer Science, Vol M.S. Virginia Polytechnic Institute and State University.

Information visualization is applied in many fields to gain faster insights with lighter user cognitive loads in analyzing large sets of data. As more products are being introduced each year, how can one select the most effective tool or representation form for the task? There are a number of information visualization evaluation methods currently available. However, these evaluation methods are often limited by the appropriateness of the tool for a given domain since they are not evaluating according to tools 
intended use. Current methods conduct evaluations in a laboratory environment with benchmark tasks and often with field data sets not aligned with the intended use of the tools. The absence of realistic data sets and routine tests reduces the effectiveness of the evaluation in terms of the appropriateness of the tool for a given domain. Intended use evaluation approach captures the key activities that will use the visual technology to calibrate the evaluation criteria toward these first-order needs. This research thesis presents the results from an investigation into an intended use evaluation approach and its effectiveness of measuring domain specific information visualization tools. In investigating the evaluation approach, criteria for the intelligence analysis community have been developed for demonstration purposes. While the observations from this research are compelling for the intelligence community, the principles of the evaluation approach should apply to a wider range of visualization technologies. All the design rationale and processes were captured in this thesis. This thesis presents a design process of developing criteria and measuring five intelligence analysis visual analytic tools. The study suggests that in selecting and/or evaluating visual analytic tools, a little up front effort to analyze key activities regarding the domain field will be beneficial. Such analysis can substantially reduce evaluation time and necessary effort throughout a longer period of time.

Park, Y, and J Park. 2010, "Disk Diagram: An Interactive Visualization Technique of Fuzzy Set Operations for the Analysis of Fuzzy Data." Information Visualization 9(3):220-32.

Fuzzy set refers to the data set which does not have separate, distinct clusters, and they contain data elements whose membership degrees are between 0.0 and 1.0. Many fuzzy sets exist in the real world, and one of the important issues is to make a decision from the fuzzy sets using visual analytics tools by extracting information in the data set intuitively. To analyze the element data in fuzzy sets, the visualization of fuzzy sets needs to show an overview of the data with membership degree and the relationship among the sets. In this article, we suggest an interactive visualization technique of fuzzy set operations, called Disk Diagram, which offers distribution of fuzzy data and two scenarios to allow users to interpret inter-dependency among fuzzy sets. A Disk Diagram enables to depict complexity of fuzzy sets by showing the degree of resemblance between the sets with the layout of star coordinates. This article describes the use of a Disk Diagram with two different data sets such as fuzzy disease set and terror related words set. Lastly, we report the results of heuristic evaluation to show that our technique supports visual perception, usability, and knowledge discovery process in the areas of visual representation and interaction. Information Visualization (2010) 9, 220-232. doi:10.1057/ivs.2010.3

Parker, BC, and F Myrick. 2009, "A Critical Examination of High-Fidelity Human Patient Simulation within the Context of Nursing Pedagogy." Nurse Education Today 29(3):322-29.

Summary The use of high-fidelity human patient simulators (HPS) have been embraced by nursing education programs in the development of immersive clinical simulations despite the lack of research into a pedagogy or educational philosophy appropriate to guide this technology-based learning tool. In this article, we explore this approach to clinical teaching through a critical examination of the application of behaviorist and constructivist pedagogy to high-fidelity scenario-based simulation sessions. Practical guidelines for developing simulation-based learning sessions that reflect both philosophical paradigms are provided. Consideration is also given to societal trends such as the digital revolution and the incoming millennial generation who represent the aptitude of the modern nursing student to utilize high-fidelity realistic and immersive simulation. Depending on the desired goal of simulator utilization, the nurse educator may want to draw on constructivism or behaviorism or a blend of both educational philosophies to best meet the needs of the adult learner. 
Parnin, C, C Görg, and S Rugaber. 2010, "Codepad : Interactive Spaces for Maintaining Concentration in Programming Environments." In 5th ACM Symposium on Software Visualization (SOFTVIS), pp. 15-24.

Parush, A, and D Berman. 2004, "Navigation and Orientation in 3d User Interfaces: The Impact of Navigation Aids and Landmarks." International Journal of Human-Computer Studies 61(3):375-95.

This study examined how users acquire spatial cognition in 3D user interfaces depicting an on-screen virtual environment. The study was divided into two main phases: learning and a test of learning transfer. The learning phase consisted of participants directly navigating (search for objects) in the on-screen virtual environment using one of two navigation aids: a visual map or a route list. In addition, there were two virtual environments, one with landmarks and the other without landmarks. Learning transfer was examined by testing both navigation and orientation tasks (relative-direction pointing) in the environment without the use of the navigation aids. Findings show that while the initial navigation with a map appeared to be harder, with longer navigation times and more navigation steps than with a route list, this difference became insignificant at the end of the learning phase. Moreover, performance degradation upon removal of the navigation aids was less for those that navigated with a map as compared to route list. A similar pattern was found for the impact of landmarks. Initial navigation with landmarks appeared to be harder than without landmarks, but this difference became insignificant at the end of the learning phase. Moreover, performance degradation upon removal of the navigation aid was less for those that navigated with landmarks as compared to no landmarks. Finally, the combined impact of both the navigation aid used in the learning and the presence of landmarks was primarily evident in the orientation task. Relative direction pointing was better for those who learnt with a map without landmarks, or with route list with landmarks. The findings are discussed in terms of the impact of navigations aids and landmarks on the acquisition of route and survey knowledge in spatial cognition. In addition, some gender differences are discussed in terms of different strategies in spatial cognition acquisition.

Parush, A, A Hod, and A Shtub. 2007, "Impact of Visualization Type and Contextual Factors on Performance with Enterprise Resource Planning Systems." Computers \& Industrial Engineering 52(1):133-42.

We studied the use of two types of graphic information visualization to support human operators performing tasks using enterprise resource planning systems (ERP). We compared the original display design of a commercial ERP system with a prototype information visualization design. A simulated supply chain was used to test the hypothesis that graphical visualization can improve the performance of the human operator using ERP systems for supply chain management. Tasks from the production and purchasing domains were tested in the experiment in which experienced and inexperienced human operators had to perform tasks of an easy, moderate, and difficult nature. Analysis of the results revealed that the two types of graphical visualization significantly reduced response time, particularly with inexperienced users performing difficult tasks. Our results imply that some graphic information visualization displays for ERP systems can increase the probability of a successful implementation and enhance the capabilities of the human operators.

Pattath, A, et al. 2006, "Interactive Visualization and Analysis of Network and Sensor Data on Mobile Devices." In IEEE Symposium on Visual Analytics Science \& Technology, pp. 83-90.

Mobile devices are rapidly gaining popularity due to their small size and their wide range of functionality. With the constant improvement in wireless network access, they are an attractive option not 
only for day to day use. but also for in-field analytics by first responders in widespread areas. However, their limited processing, display, graphics and power resources pose a major challenge in developing effective applications. Nevertheless, they are vital for rapid decision making in emergencies when combined with appropriate analysis tools. In this paper, we present an efficient, interactive visual analytic system using a PDA to visualize network information from Purdue's Ross-Ade Stadium during football games as an example of in-held data analytics combined with text and video analysis. With our system, we can monitor the distribution of attendees with mobile devices throughout the stadium through their access of information and association/disassociation from wireless access points, enabling the detection of crowd movement and event activity. Through correlative visualization and analysis of synchronized video (instant replay video) and text information (play statistics) with the network activity, we can provide insightful information to network monitoring personnel, safety personnel and analysts. This work provides a demonstration and testbed for mobile sensor analytics that will help to improve network performance and provide safety personnel with information for better emergency planning and guidance

Pattath, A, et al. 2008, "Real Time Scalable Visual Analysis on Mobile Devices." In Conference on Multimedia on Mobile Devices 2008.

Interactive visual presentation of information can help an analyst gain faster and better insight from data. When combined with situational or context information, visualization on mobile devices is invaluable to in-field responders and investigators. However, several challenges are posed by the formfactor of mobile devices in developing such systems. In this paper, we classify these challenges into two broad categories - issues in general mobile computing and issues specific to visual analysis on mobile devices. Using NetworkVis and Infostar as example systems, we illustrate some of the techniques that we employed to overcome many of the identified challenges. NetworkVis is an OpenVC-based real-time network monitoring and visualization system developed for Windows Mobile devices. Infostar is a flashbased interactive, real-time visualization application intended to provide attendees access to conference information. Linked time-synchronous visualization, stylus/button-based interactivity, vector graphics, overview-context techniques, details-on-demand and statistical information display are some of the highlights of these applications.

Pattath, A, et al. 2009, "Contextual Interaction for Geospatial Visual Analytics on Mobile Devices." In Multimedia on Mobile Devices Conference 2009, pp. 1-12.

Limited display area creates unique challenges for information presentation and user exploration of data on mobile devices. Traditional scrolling, panning and zooming interfaces pose signi cant cognitive burdens on the user to assimilate the new context after each interaction. To overcome these limitations, we examine the uses of $\backslash$ focus + context" techniques, speci cally for performing visual analytic tasks with geospatial data on mobile devices. In particular, we adapted the translucency-based \focus + context" technique called \blending lens" to mobile devices. The adaptation enhances the lens functionalities with dynamically changing features based on users' navigation intentions, for mobile interaction. We extend the concept of \spatial context" of this method to include relevant semantic content to aid spatial navigation and analytical tasks such as nding related data. With these adaptations, the lens can be used to view spatially clustered results of a search query, related data based on various proximity functions (such as distance, category and time) and other correlative information for immediate in- eld analysis, all without losing the current geospatial context. 
Paulovich, FV, CT Silva, and LG Nonato. 2010, "Two-Phase Mapping for Projecting Massive Data Sets." IEEE Transactions on Visualization and Computer Graphics 16(6):1281-90.

Most multidimensional projection techniques rely on distance (dissimilarity) information between data instances to embed high-dimensional data into a visual space. When data are endowed with Cartesian coordinates, an extra computational effort is necessary to compute the needed distances, making multidimensional projection prohibitive in applications dealing with interactivity and massive data. The novel multidimensional projection technique proposed in this work, called Part-Linear Multidimensional Projection (PLMP), has been tailored to handle multivariate data represented in Cartesian highdimensional spaces, requiring only distance information between pairs of representative samples. This characteristic renders PLMP faster than previous methods when processing large data sets while still being competitive in terms of precision. Moreover, knowing the range of variation for data instances in the high-dimensional space, we can make PLMP a truly streaming data projection technique, a trait absent in previous methods.

Pauly, T, et al. 2009, "Keeping Pace with Technology through the Development of an Intuitive Data Fusion, Management, Analysis Visualization Software Solution." In OCEANS 2009 Conference - Europe, pp. 1-8.

A significant challenge facing researchers is the bridging of domains between the natural and physical sciences through the integration, analysis \&amp; visualization of multivariate environmental data from a variety of sources. Typically the temporal, visual and analytical components are not well handled within a single software application or by existing tools, and the final products are often difficult to share with collaborating researchers and managers. To address these issues, Myriax has launched Eonfusion; the next generation of geospatial analysis software which provides a state-of-the-art 4D-analysis environment that is intuitive and readily extensible. The software significantly enhances the ease with which scientists can integrate diverse data types (raster, vector and media) and share methods across disciplines. The powerful 4D-visualization interface also incorporates a range of analysis and plotting tools as well as a time-navigation capability that allow users to explore the temporal evolution of spatial structure and resolved relationships. Its revolutionary fusion capability allows data at different temporal or spatial scales to be reconciled, attributes merged and topological relationships identified. The software is customizable, through an integrated coding environment for algorithm implementation and an application programming interface (API), to facilitating the development of modules. It is ideal for both undertaking complex analyses and communicating syntheses. This paper introduces the concepts behind fusion of irregular vector data in Eonfusion. Multiple data sets, including modeled and sampled data, can be fused into a single set with shared coordinates, enabling the discovery of topological relationships between coincident data items. These relationships also allow data attributes to be directly compared and transferred between data sets. A corresponding process also allows raster data attributes to be directly compared with vector data a- ttributes through a raster mapping form of fusion. Both the raster and vector data sets can be visualized together, and the attribute transfer results displayed and used for further analysis. Vector data is introduced to the application as sets of spatial features (e.g., points, lines or polygons). Attributes may vary from vertex to vertex across a spatial feature (vertex attributes, e.g., $x, y$, $z, t$ ), or may be constant across a feature (feature attributes). Fusion typically takes place in a space defined by a subset of common vertex attributes. The implementation is sufficiently general to use any such collection of attributes, for example temperature or mean volume backscatter. A simple yet powerful example is the fusion of video data with camera trajectory data for ground truthing acoustic data. This architecture enables user-positioned "probes" in scenes on the camera trajectory line to locate the 
corresponding video frames. Along track acoustic integration data can also be fused with the trajectory data, providing tools for seabed classification or combined net/acoustic studies. This example represents the simple application of a powerful set of tools for oceanographic and ecological studies. Eonfusion was developed with the support of leading researchers around the world to meet the demands of the latest marine research tools, technology and methodology. Eonfusion moves the technology horizon far beyond simultaneous 4D visualization of coincident data, to the elegant integration of data via fusion. The ability to fuse data from multiple sources in a versatile 4-dimensional visualization environment provides new opportunities for observation insights into marine ecosystems. It will ultimately yield the analysis methodologies of the future.

Pauw, WD, and S Heisig. 2010, "Zinsight : A Visual and Analytic Environment for Exploring Large Event Traces." In 5th ACM Symposium on Software Visualization (SOFTVIS), pp. 143-52.

Payne, SJ. 2003, "Users Mental Models: The Very Ideas." in Hci Models, Theories and Frameworks: Toward a Multidisciplinary Science, ed. JM Carroll, pp. 135-56. Morgan Kaufmann, San Francisco.

Pea, RD. 1987, "Socializing the Knowledge Transfer Problem." International Journal of Educational Research 11(6):639-63.

A central issue in acquiring knowledge is its appropriate transfer beyond the contexts and contents of first acquisition. In contrast to dominant "common elements" transfer theory, an interpretive perspective is developed, according to which "appropriate transfer" is a concept socioculturally rather than objectively defined. "Elements" perceived by the thinker as common between the current and a prior situation are not given in the nature of things but "read" in terms of the thinker's culturally-influenced categorization system, of problem types. A synthesis of cognitive research findings identifies specific features of thinking-skills instruction effective for promoting transfer. These include learning about and practicing knowledge application in multiple contexts of use, constructively participating in bridging instruction across school and nonschool problem situations, thinking and self-management skills taught within domains, and synergistic integration of the learning of different subjects. Recommendations are made for developing new learning technologies that build upon these conditions for enhancing knowledge transfer.

Peffer, JE, W Elm, and SS Potter. 2006, Reconciling Differences among Multiple Experts: A Framework for Developing a Common Ground. Technical, Resilient Cognitive Solutions, San Diego, CA.

Peffer, JE, et al. 2006, Decision-Centered Command and Control: Designing Large Scale Decision Support Environments to Enable Effective Team Decision Making. Technical, Resilient Cognitive Solutions, San Diego, CA.

Peffer, JE, et al. 2008, How Costly Is Your C2 Coordination? Assessing the Coordination Requirements within Command and Control. Technical, Resilient Cognitive Solutions, San Diego, CA.

Pellegrino, D, et al. 2008, "Grand Challenge Award: Data Integration Visualization and Collaboration in the Vast 2008 Challenge." In IEEE Symposium on Visual Analytics Science \& Technology, pp. 197-98.

The VAST 2008 Challenge consisted of four heterogeneous synthetic data sets each organized into separate mini-challenges. The Grand Challenge required integrating the raw data from these four data sets as well as integrating results and findings from team members working on specific mini-challenges. 
Modeling the problem with a semantic network provided a means for integrating both the raw data and the subjective findings.

Perer, A, and B Shneiderman. 2009, "Integrating Statistics and Visualization for Exploratory Power: From Long-Term Case Studies to Design Guidelines." IEEE computer graphics and applications 29(3):39-51.

Perer, A, and B Shneiderman. 2008, "Integrating Statistics and Visualization: Case Studies of Gaining Clarity During Exploratory Data Analysis." In 26th Annual CHI Conference on Human Factors in Computing Systems, pp. 265-74.

Although both statistical methods and visualizations have been used by network analysts, exploratory data analysis remains a challenge. We propose that a tight integration of these technologies in an interactive exploratory tool could dramatically speed insight development. To test the power of this integrated approach, we created a novel social network analysis tool, SocialAction, and conducted four long-term case studies with domain experts, each working on unique data sets with unique problems. The structured replicated case studies show that the integrated approach in SocialAction led to significant discoveries by a political analyst, a bibliometrician, a healthcare consultant, and a counter-terrorism researcher. Our contributions demonstrate that the tight integration of statistics and visualizations improves exploratory data analysis, and that our evaluation methodology for long-term case studies captures the research strategies of data analysts.

Perera, S, D Hobbs, and D Moore. 1999, "Visual Metaphors to Enhance Hypermedia." In IEEE International Conference on Information Visualization, pp. 484-89.

The paper argues that whilst hypermedia systems potentially have a major role to play in education, there is the danger that they tend to engender cognitive and navigational overhead in their users, which in turn detracts from their educational value. A potentially valuable means of ameliorating this difficulty is to use metaphors to aid the user as they interact with the system. The value of metaphors in overcoming the problems of cognitive overloading and navigational overhead is discussed and an experimental study proposed to investigate the design and deployment of suitable metaphors in this context

Perez, C, and A de Antonio. 2004, "3d Visualization of Text Collections: An Experimental Study to Assess the Usefulness of 3d." In 8th International Conference on Information Visualisation, pp. 317-23.

Previous studies about the use of 3D models for information search have not shown a clear advantage over 2D or textual user interfaces. This paper describes an experimental study focused on initial overview and information browsing tasks, that we consider to be more adequate to take advantage of 3D exploration. To this end, a 3D Explorer prototype was developed and tested by experimental subjects. The $3 \mathrm{D}$ usability evaluation provided interesting design concepts and feedback, while the statistical evaluation of results showed significant speed advantages over a more classic interface, and a good potential for future improvements. 
Perkins, C. 2009, "Mapping, Philosophy." in International Encylcopedia of Human Geography, eds. K Rob and T Nigel, pp. 385-97. Elsevier, Oxford.

Mapping is, at once, ontological and epistemological. It creates objects, is a set of practices, and is also underpinned by ideas associated with different philosophical positions. These philosophies are complex and interwoven, their popularity changing over time and space. Most people hold the common sense view that mapping is a neutral technology describing an objective world [']out there', but the scientific philosophies that underpin this view are mutable. Different justifications for a rational approach have been employed in Robinsonian cartography, cartographic communication, analytical cartography, behavioral and cognitive psychological approaches, visualization, representation, and realist approaches to mapping. Social-theoretical alternatives to science are also being advanced to rethink mapping. Critical and humanistic philosophies offer new ways of thinking about the medium. Social constructivist theory, and structuralist critique informed by psychoanalysis, linguistics, and the Marxist dialectic have challenged the power of cartographic reason. Phenomenological alternatives such as esthetics, and hermeneutic approaches have also challenged scientific hegemony, while more recent post-structural approaches imagine mapping as discourse, or power-knowledge. Nonrepresentational theory has also recently challenged philosophical orthodoxies, encouraging a turn toward action, performance, affect, and relational thinking. There is little evidence yet that the contradictions between these philosophical positions will be resolved, or that change will cease.

Perkins, DN. 1996, "Insight in Minds and Genes." in The Nature of Insight, eds. RJ Sternberg and JE Davidson, pp. 495-534. MIT Press.

Persson, O. 2010, "Identifying Research Themes with Weighted Direct Citation Links." Journal of Informetrics 4(3):415-22.

In this study direct citations are weighted with shared references and co-citations in an attempt to decompose a citation network of articles on the subject of library and information science. The resulting maps have much in common with author co-citation maps that have been previously presented. However, using direct citations yields somewhat more detail in terms of detecting sub-domains. Reducing the network down to the strongest links of each article yielded the best results in terms of a high number of clusters, each with a substantial number of articles similar in content.

Perugini, S. 2010, "Supporting Multiple Paths to Objects in Information Hierarchies: Faceted Classification, Faceted Search, and Symbolic Links." Information Processing \& Management 46(1):2243.

We present three fundamental, interrelated approaches to support multiple access paths to each terminal object in information hierarchies: faceted classification, faceted search, and web directories with embedded symbolic links. This survey aims to demonstrate how each approach supports users who seek information from multiple perspectives. We achieve this by exploring each approach, the relationships between these approaches, including tradeoffs, and how they can be used in concert, while focusing on a core set of hypermedia elements common to all. This approach provides a foundation from which to study, understand, and synthesize applications which employ these techniques. This survey does not aim to be comprehensive, but rather focuses on thematic issues. 
Perugini, S, and N Ramakrishnan. 2003, "Personalizing Interactions with Information Systems." in Advances in Computers, Vol 57, pp. 323-82. Elsevier.

Personalization constitutes the mechanisms and technologies necessary to customize information access to the end-user. It can be defined as the automatic adjustment of information content, structure, and presentation tailored to the individual. In this chapter, we study personalization from the viewpoint of personalizing interaction. The survey covers mechanisms for information-finding on the web, advanced information retrieval systems, dialog-based applications, and mobile access paradigms. Specific emphasis is placed on studying how users interact with an information system and how the system can encourage and foster interaction. This helps bring out the role of the personalization system as a facilitator which reconciles the user's mental model with the underlying information system's organization. Three tiers of personalization systems are presented, paying careful attention to interaction considerations. These tiers show how progressive levels of sophistication in interaction can be achieved. The chapter also surveys systems support technologies and niche application domains.

Peters, G. 2007, "Aesthetic Primitives of Images for Visualization." In 11th International Conference on Information Visualization, pp. 316-25.

Images play an important role in visualization. As users are more willing to adopt a product if it evokes pleasurable feelings the aesthetic appeal of interfaces becomes more important. Thus, there is a growing need to generate also images which appear aesthetically to the user. Starting with the modularities of the human visual system, we derive six dimensions of visual aesthetics. For each dimension we explore, inspired by principles of the visual arts and insights of cognitive neuroscience, which pecularities of the dimensions are particularly adequate for an aesthetic impression. Accompanied by a fair number of image examples, these considerations result in an easy to understand guideline for computer scientists and interface designers how to deal with images in terms of aesthetics.

Peterson, CR, and LR Beach. 1967, "Man as an Intuitive Statistician." Psychological Bulletin 68(1):2946.

CONSIDERS EXPERIMENTAL RESEARCH THAT HAS USED PROBABILITY THEORY AND STATISTICS AS A FRAMEWORK WITHIN WHICH TO STUDY HUMAN STATISTICAL INFERENCE. EXPERIMENTS HAVE INVESTIGATED ESTIMATES OF PROPORTIONS, MEANS, VARIANCES, AND CORRELATIONS, BOTH OF SAMPLES AND OF POPULATIONS. IN SOME EXPERIMENTS, PARAMETERS OF POPULATIONS WERE STATIONARY; IN OTHERS, THE PARAMETERS CHANGED OVER TIME. THE EXPERIMENTS ALSO INVESTIGATED THE DETERMINATION OF SAMPLE SIZE AND TRIAL-BY-TRIAL PREDICTIONS OF EVENTS TO BE SAMPLED FROM A POPULATION. IN GENERAL, THE RESULTS INDICATE THAT PROBABILITY THEORY AND STATISTICS CAN BE USED AS THE BASIS FOR PSYCHOLOGICAL MODELS THAT INTEGRATE AND ACCOUNT FOR HUMAN PERFORMANCE IN A WIDE RANGE OF INFERENTIAL TASKS.

Petersson, H, et al. 2009, "Web-Based Interactive 3d Visualization as a Tool for Improved Anatomy Learning." Anatomical Sciences Education 2(2):61-68.

Despite a long tradition, conventional anatomy education based on dissection is declining. This study tested a new virtual reality (VR) technique for anatomy learning based on virtual contrast injection. The 
aim was to assess whether students value this new three-dimensional (3D) visualization method as a learning tool and what value they gain from its use in reaching their anatomical learning objectives. Several 3D vascular VR models were created using an interactive segmentation tool based on the "virtual contrast injection" method. This method allows users, with relative ease, to convert computer tomography or magnetic resonance images into vivid 3D VR movies using the OsiriX software equipped with the CMIV CTA plug-in. Once created using the segmentation tool, the image series were exported in Quick Time Virtual Reality (QTVR) format and integrated within a web framework of the Educational Virtual Anatomy (EVA) program. A total of nine QTVR movies were produced encompassing most of the major arteries of the body. These movies were supplemented with associated information, color keys, and notes. The results indicate that, in general, students' attitudes towards the EVA-program were positive when compared with anatomy textbooks, but results were not the same with dissections. Additionally, knowledge tests suggest a potentially beneficial effect on learning. Anat Sci Ed 2:61-68, 2009. (C) 2009 American Association of Anatomists.

Petkos, G, K Moustakas, and D Tzovaras. 2010, "Discovering Mutation Paths in Sets of Genetic Sequences and Determining Critical Mutations." In IEEE Symposium on Visual Analytics Science \& Technology.

Petre, M. 2010, "Mental Imagery and Software Visualization in High-Performance Software Development Teams." Journal of Visual Languages \& Computing 21(3):171-83.

This paper considers the relationship between mental imagery and software visualization in professional, high-performance software development. It presents overviews of four empirical studies of professional software developers in high-performing teams: (1) expert programmers' mental imagery, (2) how experts externalize their mental imagery as part of teamwork, (3) experts' use of commercially available visualization software, and (4) what tools experts build themselves, how they use the tools they build for themselves, and why they build tools for themselves. Through this series of studies, the paper provides insight into a relationship between how experts reason about and imagine solutions, and their use of and requirements for external representations and software visualization. In particular, it provides insight into how experts use visualization in reasoning about software design, and how their requirements for the support of design tasks differ from those for the support of other software development tasks. The paper draws on theory from other disciplines to explicate issues in this area, and it discusses implications for future work in this field.

Pezanowski, S, and B Tomaszewski. 2007, "An Open Geospatial Standards-Enabled Google Earth Application to Support Crisis Management." in Geomatics Solutions for Disaster Management, pp. 22538, University Park, PA.

Google Earth (GE) and related open geospatial technologies have changed both the accessibility of and audience for geospatial information dramatically. Through data rich applications with easy to use interfaces, these technologies bring personalized geospatial information directly to the non-specialist. When coupled with open geospatial data standards, such as Web Map Services (WMS), Web Features Services (WFS), and GeoRSS, the resulting web-based technologies have the potential to assimilate heterogeneous data from distributed sources rapidly enough to support time-critical activities such as crisis response. Although the ability to view and interact with data in these environments is important, this functionality alone is not sufficient for the demands of crisis response activity. For example, GE's standard version currently lacks geoanalysis capabilities such as geographic buffering and topology 
functions. In this paper, we present development of the "Google Earth Dashboard" (GED), a web-based interface powered by open geospatial standards and designed for supplementing and enhancing the geospatial capabilities of GE. The GED allows users to create custom maps through WMS layer addition to GE and perform traditional GIS analysis functions. Utility of the GED is presented in a usecase scenario where GIS operations implemented to work with GE are applied to support crisis management activities. The GED represents an important first step towards combining the ubiquity of GE and geospatial standards into an easy-to-use, data rich, geo-analytically powerful environment that can support crisis management activity.

Pfautz, J, and E Roth. 2006, "Using Cognitive Engineering for System Design and Evaluation: A Visualization Aid for Stability and Support Operations." International Journal of Industrial Ergonomics 36(5):389-407.

Cognitive engineering offers a principled approach to the design and development of human-centered systems. The cognitive engineering process integrates an analysis of the cognitive and collaborative demands of a user engaged in work with an iterative design, implementation, and evaluation approach to developing support tools. In this paper, we illustrate this process in the context of a specific design application: a tool to support military intelligence analyses in stability and support operations (SASO), called the stability and support operations visualization aid (SASOVA). As part of the cognitive engineering process, we performed a cognitive task analysis that uncovered the sources of complexity in the military intelligence support of SASO and identified opportunities to provide more effective aiding. Working in close collaboration with domain experts, we developed aiding concepts and implemented a rapid prototype of the SASOVA system. We then evaluated the prototype using military analysts with extensive SASO experience as study participants. The results of the evaluation supported the viability of the design concepts while uncovering additional requirements for effective decision-aiding. In this paper, the SASOVA development and evaluation process is used to illustrate the benefits of the cognitive engineering process as well as the issues and trade-offs that arise in the cognitive engineering of complex systems. Relevance to Industry This work presents an approach to user-centered system design that can be applied to many types of complex, human-in-the-loop system development. An identification and description of key issues and tradeoffs are included to support the application of this approach.

Pfitzner, D, V Hobbs, and D Powers. 2001, "A Unified Taxonomic Framework for Information Visualization." In 2nd Australian Institute of Computer Ethics Conference (AICE 2000), pp. 57-66.

We present a taxonomy for Information Visualization (IV) that characterizes it in terms of data, task, skill and context, as well as a number of dimensions that relate to the input and output hardware, the software tools, as well as user interactions and human perceptual abilities. We illustrate the utility of the taxonomy by focusing particularly on the information retrieval task and the importance of taking into account human perceptual capabilities and limitations. Although the relevance of Psychology to IV is often recognised, we have seen relatively little translation of psychological results and theory to practical IV applications. This paper targets the better development of information visualizations through the introduction of a framework delineating the major factors in interface development. We believe that higher quality visualizations will result from structured developments that take into account these considerations and that the framework will also serve to assist the development of effective evaluation and assessment processes. 
Pham, T, et al. 2010, "Visualization of Diversity in Large Multivariate Data Sets." Visualization and Computer Graphics, IEEE Transactions on 16(6):1053-62.

Understanding the diversity of a set of multivariate objects is an important problem in many domains, including ecology, college admissions, investing, machine learning, and others. However, to date, very little work has been done to help users achieve this kind of understanding. Visual representation is especially appealing for this task because it offers the potential to allow users to efficiently observe the objects of interest in a direct and holistic way. Thus, in this paper, we attempt to formalize the problem of visualizing the diversity of a large (more than 1000 objects), multivariate (more than 5 attributes) data set as one worth deeper investigation by the information visualization community. In doing so, we contribute a precise definition of diversity, a set of requirements for diversity visualizations based on this definition, and a formal user study design intended to evaluate the capacity of a visual representation for communicating diversity information. Our primary contribution, however, is a visual representation, called the Diversity Map, for visualizing diversity. An evaluation of the Diversity Map using our study design shows that users can judge elements of diversity consistently and as or more accurately than when using the only other representation specifically designed to visualize diversity.

Phan, D, et al. 2007, "Visual Analysis of Network Flow Data with Timelines and Event Plots." In VizSEC 2007, pp. 85-99.

This paper describes Isis, a system that uses progressive multiples of timelines and event plots to support the iterative investigation of intrusions by experienced analysts using network flow data. The visual representations have been designed to make temporal relationships apparent, allow visual classification of events with dynamic brushing, and enable users to organize their visualizations to reveal traffic structure and patterns by reordering rows. Isis combines visual affordances with SQL to provide a flexible tool for investigation. We present an annotated case study using anonymized data of a real intrusion that demonstrates the features of Isis.

Phan, D, A Paepcke, and T Winograd. 2007, "Progressive Multiples for Communication-Minded Visualization." In Graphics Interface Conference, pp. 225-25.

This paper describes a communication-minded visualization called progressive multiples that supports both the forensic analysis and presentation of multidimensional event data. We combine ideas from progressive disclosure, which reveals data to the user on demand, and small multiples [21], which allows users to compare many images at once. Sets of events are visualized as timelines. Events are placed in temporal order on the x-axis, and a scalar dimension of the data is mapped to the $\mathrm{y}$-axis. To support forensic analysis, users can pivot from an event in an existing timeline to create a new timeline of related events. The timelines serve as an exploration history, which has two benefits. First, this exploration history allows users to backtrack and explore multiple paths. Second, once a user has concluded an analysis, these timelines serve as the raw visual material for composing a story about the analysis. A narrative that conveys the analytical result can be created for a third party by copying and reordering timelines from the history. Our work is motivated by working with network security administrators and researchers in political communication. We describe a prototype that we are deploying with administrators and the results of a user study where we applied our technique to the visualization of a simulated epidemic. 
Phan, D, et al. 2005, "Flow Map Layout." In IEEE International Conference on Information Visualization, pp. 219-24.

Cartographers have long used flow maps to show the movement of objects from one location to another, such as the number of people in a migration, the amount of goods being traded, or the number of packets in a network. The advantage of flow maps is that they reduce visual clutter by merging edges. Most flow maps are drawn by hand and there are few computer algorithms available. We present a method for generating flow maps using hierarchical clustering given a set of nodes, positions, and flow data between the nodes. Our techniques are inspired by graph layout algorithms that minimize edge crossings and distort node positions while maintaining their relative position to one another. We demonstrate our technique by producing flow maps for network traffic, census data, and trade data.

Piegorsch, WW, SL Cutter, and F Hardisty. 2007, "Benchmark Analysis for Quantifying Urban Vulnerability to Terrorist Incidents." Risk analysis : an official publication of the Society for Risk Analysis 27(6):1411-25.

We describe a quantitative methodology to characterize the vulnerability of U.S. urban centers to terrorist attack, using a place-based vulnerability index and a database of terrorist incidents and related human casualties. Via generalized linear statistical models, we study the relationships between vulnerability and terrorist events, and find that our place-based vulnerability metric significantly describes both terrorist incidence and occurrence of human casualties from terrorist events in these urban centers. We also introduce benchmark analytic technologies from applications in toxicological risk assessment to this social risk/vulnerability paradigm, and use these to distinguish levels of high and low urban vulnerability to terrorism. It is seen that the benchmark approach translates quite flexibly from its biological roots to this social scientific archetype.

Pike, W, R May, and A Turner. 2007, "Supporting Knowledge Transfer through Decomposable Reasoning Artifacts." In 40th Annual Hawaii International Conference on System Sciences, pp. 204c-04c.

Technology to support knowledge transfer and cooperative inquiry must offer its users the ability to effectively interpret knowledge structures produced by collaborators. Communicating the reasoning processes that underlie a finding is one method for enhancing interpretation, and can result in more effective evaluation and application of shared knowledge. In knowledge management tools, interpretation is aided by creating knowledge artifacts that can expose their provenance to scrutiny and that can be transformed into diverse representations that suit their consumers' perspectives and preferences. We outline the information management needs of inquiring communities characterized by hypothesis generation tasks, and propose a model for communication, based in theories of hermeneutics, semiotics, and abduction, in which knowledge structures can be decomposed into the lower-level reasoning artifacts that produced them. We then present a proof-of-concept implementation for an environment to support the capture and communication of analytic products, with emphasis on the domain of intelligence analysis

Pike, WA, et al. 2008, "The Scalable Reasoning System: Lightweight Visualization for Distributed Analytics." In IEEE Symposium on Visual Analytics Science \& Technology, pp. 131-38.

A central challenge in visual analytics is the creation of accessible, widely distributable analysis applications that bring the benefits of visual discovery to as broad a user base as possible. Moreover, to support the role of visualization in the knowledge creation process, it is advantageous to allow users to 
describe the reasoning strategies they employ while interacting with analytic environments. We introduce an application suite called the scalable reasoning system (SRS), which provides Web-based and mobile interfaces for visual analysis. The service-oriented analytic framework that underlies SRS provides a platform for deploying pervasive visual analytic environments across an enterprise. SRS represents a ldquolightweightrdquo approach to visual analytics whereby thin client analytic applications can be rapidly deployed in a platform-agnostic fashion. Client applications support multiple coordinated views while giving analysts the ability to record evidence, assumptions, hypotheses and other reasoning artifacts. We describe the capabilities of SRS in the context of a real-world deployment at a regional law enforcement organization.

Pike, Wa, et al. 2007, "Scalable Visual Reasoning: Collaboration Thorugh Distributed Analysis." In International Symposium on Collaborative Technologies and Systems, pp. 24-32.

We present a visualization environment called the scalable reasoning system (SRS) that provides a suite of tools for the collection, analysis, and dissemination of reasoning products. This environment is designed to function across multiple platforms, bringing the display of visual information and the capture of reasoning associated with that information to both mobile and desktop clients. The service-oriented architecture of SRS facilitates collaboration and interaction between users regardless of their location or platform. Visualization services allow data processing to be centralized and analysis results to be collected from distributed clients in real time. We use the concept of ldquoreasoning artifactsrdquo to capture the analytic value attached to individual pieces of information and collections thereof, helping to fuse the foraging and sense-making loops in information analysis. Reasoning structures composed of these artifacts can be shared across platforms while maintaining references to the analytic activity (such as interactive visualization) that produced them.

Pike, WA, et al. 2009, "The Science of Interaction." Information Visualization 8(4):263-74.

There is a growing recognition within the visual analytics community that interaction and inquiry are inextricable. It is through the interactive manipulation of a visual interface - the analytic discourse - that knowledge is constructed, tested, refined and shared. This article reflects on the interaction challenges raised in the visual analytics research and development agenda and further explores the relationship between interaction and cognition. It identifies recent exemplars of visual analytics research that have made substantive progress toward the goals of a true science of interaction, which must include theories and testable premises about the most appropriate mechanisms for human-information interaction. Seven areas for further work are highlighted as those among the highest priorities for the next 5 years of visual analytics research: ubiquitous, embodied interaction; capturing user intentionality; knowledge-based interfaces; collaboration; principles of design and perception; interoperability; and interaction evaluation. Ultimately, the goal of a science of interaction is to support the visual analytics and human-computer interaction communities through the recognition and implementation of best practices in the representation and manipulation of visual displays. Information Visualization (2009) 8, 263-274. doi:10.1057/ivs.2009.22

Piringer, H, et al. 2009, "A Multi-Threading Architecture to Support Interactive Visual Exploration." Visualization and Computer Graphics, IEEE Transactions on 15(6):1113-20.

During continuous user interaction, it is hard to provide rich visual feedback at interactive rates for datasets containing millions of entries. The contribution of this paper is a generic architecture that ensures 
responsiveness of the application even when dealing with large data and that is applicable to most types of information visualizations. Our architecture builds on the separation of the main application thread and the visualization thread, which can be cancelled early due to user interaction. In combination with a layer mechanism, our architecture facilitates generating previews incrementally to provide rich visual feedback quickly. To help avoiding common pitfalls of multi-threading, we discuss synchronization and communication in detail. We explicitly denote design choices to control trade-offs. A quantitative evaluation based on the system VI S P L ORE shows fast visual feedback during continuous interaction even for millions of entries. We describe instantiations of our architecture in additional tools.

Pirolli, P. 2003, "Exploring and Finding Information." in Hci Models, Theories, and Frameworks: Toward a Multidisciplinary Science, ed. MC John, pp. 157-91. Morgan Kaufmann, San Francisco.

Summary This chapter will discuss recent theories that address how people forage for information in their environment. The emergence of the global information ecology has created enormous pressures for users who seek useful information. Understanding how people adapt to these pressures has led to the development of information-foraging theory and the notion of users following information scent. Information-foraging theory is grounded in computational theories of human cognition and optimal foraging theories from biology. Applications of the theory to a novel browsing system (called Scatter/Gather) and the World Wide Web illustrate the utility of the approach.

Pirolli, P, and S Card. 2005, "The Sensemaking Process and Leverage Points for Analyst Technology as Identified through Cognitive Task Analysis." In Conference on Intelligence Analysis, pp. 1-6.

There are a relatively few open literature reports that provide empirical descriptive studies of intelligence analysis and that link these into the context of expertise and work. This paper, based on first results from a cognitive task analysis and verbal protocols give a broad brush description of intelligence analysis as an example of sensemaking. It then suggests some possible leverage points where technology might be applied.

Pirolli, P, SK Card, and M M. 2001, "Visual Information Foraging in a Focus+ Context Visualization." In SIGCHI Conference on Human Factors in Computing Systems, pp. 506-13.

Plaisant, C. 2004, "The Challenge of Information Visualization Evaluation." In Working Conference on Advanced Visual Interfaces (AVI'04), pp. 109-16.

As the field of information visualization matures, the tools and ideas described in our research publications are reaching users. The reports of usability studies and controlled experiments are helpful to understand the potential and limitations of our tools, but we need to consider other evaluation approaches that take into account the long exploratory nature of users tasks, the value of potential discoveries or the benefits of overall awareness. We need better metrics and benchmark repositories to compare tools, and we should also seek reports of successful adoption and demonstrated utility.

Plaisant, C. 2005, "Chapter 3 - Information Visualization and the Challenge of Universal Usability." in Exploring Geovisualization, eds. D Jason, MM Alan and K Menno-Jan, pp. 53-82. Elsevier, Oxford.

Summary Information Visualization aims to provide compact graphical presentations and user interfaces for interactively manipulating large numbers of items. We present a simple "data by tasks 
taxonomy" then discuss the challenges of providing universal usability, with example applications using geo-referenced data. Information Visualization has been shown to be a powerful visual thinking or decision tool but it is becoming important for services to reach and empower every citizen. Technological advances are needed to deal with user diversity (age, language, disabilities, etc.) but also with the variety of technology used (screen size, network speed, etc.) and the gaps in user's knowledge (general knowledge, knowledge of the application domain, of the interface syntax or semantic). We present examples that illustrate how those challenges can be addressed.

Plaisant, C, D Carr, and B Shneiderman. 1995, "Image-Browser Taxonomy and Guidelines for Designers." Software, IEEE 12(2):21-32.

In many applications users must browse large images. Most designers merely use two onedimensional scroll bars or ad hoc designs for two-dimensional scroll bars. However, the complexity of two-dimensional browsing suggests that more careful analysis, design, and evaluation might lead to significant improvements. Our exploration of existing 2D browsers has led us to identify many features and a wide variety of tasks performed with the browsers. We introduce an informal specification technique to describe 2D browsers and a task taxonomy, suggest design features and guidelines, and assess existing strategies. We focus on the tools to explore a selected image and so do not cover techniques to browse a series of images or to browse large-image databases

Plaisant, C, JD Fekete, and G Grinstein. 2008, "Promoting Insight-Based Evaluation of Visualizations: From Contest to Benchmark Repository." Visualization and Computer Graphics, IEEE Transactions on 14(1):120-34.

Information visualization (InfoVis) is now an accepted and growing field, but questions remain about the best uses for and the maturity of novel visualizations. Usability studies and controlled experiments are helpful, but generalization is difficult. We believe that the systematic development of benchmarks will facilitate the comparison of techniques and help identify their strengths under different conditions. We were involved in the organization and management of three InfoVis contests for the 2003, 2004, and 2005 IEEE InfoVis Symposia, which requested teams to report on insights gained while exploring data. We give a summary of the state of the art of evaluation in InfoVis, describe the three contests, summarize their results, discuss outcomes and lessons learned, and conjecture the future of visualization contests. All materials produced by the contests are archived in the InfoVis benchmark repository.

Plaisant, C, G Grinstein, and J Scholtz. 2009, "Visual-Analytics Evaluation." Computer Graphics and Applications, IEEE 29(3):16-17.

Visual analytics (VA) is the science of analytical reasoning facilitated by interactive visual interfaces. Assessing VA technology's effectiveness is challenging because VA tools combine several disparate components, both low and high level, integrated in complex interactive systems used by analysts, emergency responders, and others. These components include analytical reasoning, visual representations, computer-human interaction techniques, data representations and transformations, collaboration tools, and especially tools for communicating the results of their use. VA tool users' activities can be exploratory and can take place over days, weeks, or months. Users might not follow a predefined or even linear work flow. They might work alone or in groups. To understand these complex behaviors, an evaluation can target the component level, the system level, or the work environment level, and requires realistic data and tasks. Traditional evaluation metrics such as task completion time, number of errors, or recall and 
precision are insufficient to quantify the utility of VA tools, and new research is needed to improve our VA evaluation methodology.

Plaisant, C, et al. 2008, "Evaluating Visual Analytics at the 2007 Vast Symposium Contest." Computer Graphics and Applications, IEEE 28(2):12-21.

In this article, we report on the contest's data set and tasks, the judging criteria, the winning tools, and the overall lessons learned in the competition. We believe that by organizing these contests, we're creating useful resources for researchers and are beginning to understand how to better evaluate VA tools.

Competitions encourage the community to work on difficult problems, improve their tools, and develop baselines for others to build or improve upon. We continue to evolve a collection of data sets, scenarios, and evaluation methodologies that reflect the richness of the many VA tasks and applications.

Plaisant, C, J Grosjean, and BB Bederson. 2002, "Spacetree: Supporting Exploration in Large Node Link Tree, Design Evolution and Empirical Evaluation." In IEEE Symposium on Information Visualization (InfoVis 2002), pp. 57-64.

We present a novel tree browser that builds on the conventional node link tree diagrams. It adds dynamic rescaling of branches of the tree to best fit the available screen space, optimized camera movement, and the use of preview icons summarizing the topology of the branches that cannot be expanded. In addition, it includes integrated search and filter functions. This paper reflects on the evolution of the design and highlights the principles that emerged from it. A controlled experiment showed benefits for navigation to already previously visited nodes and estimation of overall tree topology.

Plaue, C. 2009, "Exploring and Visualizing the Impact of Multiple Shared Displays on Collocated Meeting Practices." Vol Ph.D., pp. 225-25. Georgia Institute of Technology, United States -- Georgia.

A tremendous amount of information is produced in the world around us, both as a product of our daily lives and as artifacts of our everyday work. An emerging area of Human-Computer Interaction (HCI) focuses on helping individuals manage this flood of information. Prior research shows that multiple displays can improve an individual user's ability to deal with large amounts of information, but it is unclear whether these advantages extend for teams of people. This is particularly relevant as more employees are spending large portions of their workdays in meetings My contribution to HCI research is empirical fieldwork and laboratory studies investigating how multiple shared displays improve aspects of teamwork. In particular, I present an insight-based evaluation method for analyzing how teams collaborate on a data-intensive sensemaking task. Using this method, I show how the presence and location of multiple shared displays impacted the meeting process with respect to performance, collaboration, and satisfaction. I also illustrate how multiple shared displays engaged team members who might not have otherwise contributed to the collaboration process. Finally, I present Mimosa, a software tool developed to visualize large volumes of time series data. Mimosa combines aspects of information visualization with data analysis, facilitating a deep and iterative exploration of relationships within large datasets. 
Pohl, M, and P Purgathofer. 2000, "Hypertext Authoring and Visualization." International Journal of Human-Computer Studies 53(5):809-25.

Visualizing information structure plays an increasing role in hypertext systems. Empirical evidence shows that such visualizations support the users or readers of hypertext systems. The following study investigates whether such information visualizations in the form of two-dimensional graphical overview maps also support the writers of hypertext documents. It also seems plausible to assume that the process of writing text changes when graphical overview maps integrated into hypertext systems are used. We also analysed the relationship between the authoring process and the character of the finished documents. In general, it can be said that visualizing the information structure inherent in a document plays an important role in hypertext authoring. It was the only innovative feature of the hypertext authoring system we developed which was used intensively by almost all the authors. In contrast to that, the finished documents were rather traditional. Authors did not exploit the novel features of hypertext/hypermedia systems like, e.g., introducing pictures, movies or sounds. On the basis of the analysis of the hypertext authoring process guidelines can be formulated to support writers of hypertext documents.

Pohl, M, M Rester, and S Wiltner. 2007, "Usability and Transferability of a Visualization Methodology for Medical Data." In 3rd Symposium of the Workgroup Human-Computer Interaction and Usability Engineering of the Austrian Computer Society, pp. 171-84.

Information Visualization (InfoVis) techniques can offer a valuable contribution for the examination of medical data. We successfully developed an InfoVis application - Gravi - for the analysis of questionnaire data stemming from the therapy of anorectic young women. During the development process, we carefully evaluated Gravi in several stages. In this paper, we describe selected results from the usability evaluation, especially results from qualitative studies. The results indicate that Gravi is easy to use and intuitive. The subjects of the two studies described here especially liked the presentation of time-oriented data and the interactivity of the system. In the second study, we also found indication that Gravi can be used in other areas than the one it was developed for.

Pohl, M, S Wiltner, and S Miksch. 2010, "Exploring Information Visualization - Describing Different Interaction Patterns." In BELIV'10: Beyond time and errors: novel evaluation methods for Information Visualization, April 10-11, 2010.

Interactive Information Visualization methods engage users in exploratory behavior. Detailed information about such processes can help developers to improve the design of such methods. The following study which is based on software logging describes patterns of such behavior in more detail. Subjects in our study engaged in some activities (e.g., adding data, changing form of visualization) significantly more than in others. They adapted their activity patterns to different tasks, but not fundamentally so. In addition, subjects adopted very systematic sequences of actions. These sequences were quite similar across the whole sample, thus indicating that such sequences might reflect specific problem solving behavior. Davidson's [7] framework of problem solving behavior is used to interpret the results. More research is necessary to show whether similar interaction patterns can be found for the usage of other InfoVis methodologies as well. 
Poliakov, AV, et al. 2005, "Server-Based Approach to Web Visualization of Integrated ThreeDimensional Brain Imaging Data." Journal of the American Medical Informatics Association 12(2):14051.

The authors describe a client-server approach to three-dimensional (3-D) visualization of neuroimaging data, which enables researchers to visualize, manipulate, and analyze large brain imaging datasets over the Internet. All computationally intensive tasks are done by a graphics server that loads and processes image volumes and 3-D models, renders 3-D scenes, and sends the renderings back to the client. The authors discuss the system architecture and implementation and give several examples of client applications that allow visualization and analysis of integrated language map data from single and multiple patients.

Polys, NF, DA Bowman, and C North. 2011, "The Role of Depth and Gestalt Cues in Information-Rich Virtual Environments." International Journal of Human-Computer Studies 69(1-2):30-51.

Managing the layout of multi-dimensional visualizations is a crucial concern for the development of effective visual analytic interfaces. In these environments, heterogeneous and multi-dimensional information must be structured and combined into data representations that demand low cognitive resources but yield accurate mental models and insights. In this paper, we use Information-Rich Virtual Environments (IRVE) to articulate crucial tradeoffs in the use of Depth and Gestalt cues in text label layouts. We present a design space and evaluation methodology to explore the usability effects of these tradeoffs and collect results from a series of user studies. These lessons are posed as a set of design guidelines to aid developers of new, advantageous interfaces and specifications.

Polys, NF, et al. 2004, "Snap2diverse: Coordinating Information Visualizations and Virtual Environments." In Conference on Visualization and Data Analysis 2004, pp. 189-200.

The field of Information Visualization is concerned with improving how users perceive, understand, and interact with visual representations of abstract information. Immersive Virtual Environments (VEs) excel at a greater comprehension of spatial information. This project addresses the intersection of these two fields known as Information-Rich Virtual Environments (IRVEs) where perceptually realistic information, such as models and scenes, are enhanced with abstract information, such as text, numeric data, hyperlinks, or multimedia resources. IRVEs present a number of important design challenges including the management, coordination, and display of interrelated perceptual and abstract information. We describe a set of design issues for this type of integrated visualization and demonstrate a coordinated, multiple-views approach to support 2D and 3D visualization interactions such as overview, navigation, details-on-demand, and brushing-and-linking. In the CAVE, spatial information in a VE is interactively linked to embedded visualizations of related abstract information. Software architecture issues are discussed with details of our implementation applied to the domain of chemical information visualization. Lastly, we subject our system to an informal usability evaluation and identify usability issues with interaction and navigation that guides future work in these environments.

Portet, F, et al. 2009, "Automatic Generation of Textual Summaries from Neonatal Intensive Care Data." Artificial Intelligence 173(7-8):789-816.

Effective presentation of data for decision support is a major issue when large volumes of data are generated as happens in the Intensive Care Unit (ICU). Although the most common approach is to present 
the data graphically, it has been shown that textual summarisation can lead to improved decision making. As part of the BabyTalk project, we present a prototype, called BT-45, which generates textual summaries of about 45 minutes of continuous physiological signals and discrete events (e.g.,: equipment settings and drug administration). Its architecture brings together techniques from the different areas of signal processing, medical reasoning, knowledge engineering, and natural language generation. A clinical offward experiment in a Neonatal ICU (NICU) showed that human expert textual descriptions of NICU data lead to better decision making than classical graphical visualisation, whereas texts generated by BT-45 lead to similar quality decision-making as visualisations. Textual analysis showed that BT-45 texts were inferior to human expert texts in a number of ways, including not reporting temporal information as well and not producing good narratives. Despite these deficiencies, our work shows that it is possible for computer systems to generate effective textual summaries of complex continuous and discrete temporal clinical data.

Potesnak, K. 1989, "Mental Models: Helping Users Understand Software." IEEE Software 6(5):85-86, 88.

Potter, SS, RW Ball, and WC Elm. 1996, "Supporting Aeromedical Evacuation Planning through Information Visualization." In 3rd Symposium on Human Interaction with Complex Systems (HICS '96), pp. 208-15.

One of the key elements in the design of a cooperative decision support system (with a human and an automated system jointly working toward a solution) is the information visualization requirements. A variety of research efforts have shown how typical automated systems tend to remove the human partner from the decision-making role and into a supervisory role. However, enhanced visualization tools can promote interaction with the automated system and keep the human partner \&ldquo;in the loop\&rdquo; as the central decision maker. This paper describes several problem areas with respect to the development of a global medical regulation and evacuation command and control system and approaches to improve the user's ability to collaborate with this automated system to jointly construct a solution through enhanced visualization techniques. These techniques focus on supporting information needs to enhance the operator's ability to sift through a data-intense problem domain, gain an understanding of the state of the world, and comprehend complex solutions provided by the automated system

Potter, SS, and W Elm. 2007, "Adapting C2 to the 21st Century." In 12th ICCRTS.

Potter, SS, W Elm, and JW Gualtieri. 2006, "Making Sense of Sensemaking: Requirements of a Cognitive Analysis to Support C2 Decision Support System Design." In 2006 CCRTS.

Conducting a Cognitive Analysis to adequately support a follow-on design effort for innovative decision support tools is a tall order and requires specific properties to exist in the CTA in order to be successful. This paper outlines requirements for analytical methodologies to satisfy this need. These requirements are based on several premises. The first premise is that a representation shapes cognition and collaboration, which guide behavior. This is known as the Representation Effect (Norman, 1993). The representation effect summarizes a widespread psychological result that the content and context of a problem representation (i.e., its reflection in the design of user interface) can radically alter a decisionmaker's responses. Thus, how a command and control system is designed influences the cognitive work that needs to be accomplished, either improving or degrading sense making performance. The second premise is that the representation must reflect the essential characteristics of the work domain. That is, the relationship (or mapping) between the visual structure established by a particular representation and the 
underlying constraints and relationships within the work domain itself is fundamental to the decisionmaker's effectiveness when using the visualization. Without an explicit specification of this mapping, it is impossible to determine if the visualizations are supporting user's needs as intended, or, making the supported task more difficult (Zhang, 1997). Woods (1991) has called this the Mapping Principle.

Potter, SS, et al. 2007, Bridging the Gap between Cognitive Analysis and Effective Decision Aiding. Technical.

Potter, SS, et al. 2001, The Development of a Computer-Aided Cognitive Systems Engineering Tool to Facilitate the Design of Advanced Decision Support Systems. Technical Rpt. AFRL-HE-WP-TR-20010125.

Potter, SS, W Elm, and JS Tittle. 2007, Evaluating the Resilience of a Human-Computer DecisionMaking Team: A Methodology for Decision-Centered Testing. Technical.

Potter, SS, JW Gualtieri, and W Elm. 2003, Case Studies: Applied Cognitive Work Analysis in the Design of Innovative Decision Support. Technical.

Potter, SS, EM Roth, and W Elm. 1998, Toward the Development of a Computer-Aided Cognitive Engineering Tool to Facilitate the Development of Advanced Decision Support Systems for Information Warfare Domains. Technical Rpt. AFRL-HE-WP-TR-1998-0004.

Potter, SS, et al. 2000, "Bootstrapping Multiple Converging Cognitive Task Analysis Techniques for System Design." in Cognitive Task Analysis, pp. 317-40. Lawrence Erlbaum Associates, Inc., Publishers, Mahwah, NJ.

---. 1998, "A Framework for Integrating Cognitive Task Analysis into the System Development Process." In 42nd Annual Meeting of the Human Factors and Ergonomics Society, pp. 395-99.

This paper describes a process that orchestrates different types of specific cognitive task analysis (CTA) techniques to provide design relevant CTA results and integrates CTA results into the software development process. Two fundamental premises underlie the approach. First, CTA is more than the application of any single CTA technique. Instead, developing a meaningful understanding of a field of practice relies on multiple converging techniques in a bootstrapping process. The important issue from a CTA perspective is to evolve a model of the interconnections between the demands of the domain, the strategies and knowledge of practitioners, the cooperative interactions across human and machine agents, and how artifacts shape these strategies and coordinative activities across a series of different specific techniques. Second, since CTA is a means to support the design of computer-based artifacts that enhance human and team performance, CTA must be integrated into the software and system development process. Thus, the vision of CTA as an initial, self-contained technique that is handed-off to system designers is reconceived as an incremental process of uncovering the cognitive demands imposed on the operator(s) by the complexities and constraints of the domain

Potzsch, S, et al. 2006, "Visualization of Lattice-Based Protein Folding Simulations." In 10th International Conference on Information Visualization (IV06), pp. 89-94.

Analysis of the spatial structure of proteins including folding processes is a challenge for modem bioinformatics. Due to limited experimental access to folding processes, computer simulations are a 
standard approach. Since realistic continuous (all-atom) simulations are far too expensive, lattice based protein folding simulations are a common coarse-graining. In this paper we present a visualization toolfor lattice based protein folding simulations. The system is based on Shneiderman's mantra "Overview first, zoom and filter details on demand" and uses a collection of information visualization techniques including multiple views, focus+context and table lenses which have been tailored towards our data. We demonstrate the potential of information visualization techniques for providing insight into such simulations.

Poulovassilis, A, and SG Hild. 2001, "Hyperlog: A Graph-Based System for Database Browsing, Querying, and Update." Knowledge and Data Engineering, IEEE Transactions on 13(2):316-33.

Hyperlog is a declarative, graph based language that supports database querying and update. It visualizes schema information, data, and query output as sets of nested graphs, which can be stored, browsed, and queried in a uniform way. Thus, the user need only be familiar with a very small set of syntactic constructs. Hyperlog queries consist of a set of graphs that are matched against the database. Database updates are supported by means of programs consisting of a set of rules. The paper discusses the formulation, evaluation, expressiveness, and optimization of Hyperlog queries and programs. We also describe a prototype implementation of the language and we compare and contrast our approach with work in a number of related areas, including visual database languages, graph based data models, database update languages, and production rule systems

Pousman, Z, JT Stasko, and M Mateas. 2007, "Casual Information Visualization: Depictions of Data in Everyday Life." IEEE Transactions on Visualization and Computer Graphics 13:1145-52.

Information visualization has often focused on providing deep insight for expert user populations and on techniques for amplifying cognition through complicated interactive visual models. This paper proposes a new subdomain for infovis research that complements the focus on analytic tasks and expert use. Instead of work-related and analytically driven infovis, we propose Casual Information Visualization (or Casual Infovis) as a complement to more traditional infovis domains. Traditional infovis systems, techniques, and methods do not easily lend themselves to the broad range of user populations, from expert to novices, or from work tasks to more everyday situations. We propose definitions, perspectives, and research directions for further investigations of this emerging subfield. These perspectives build from ambient information visualization [32], social visualization, and also from artistic work that visualizes information [41]. We seek to provide a perspective on infovis that integrates these research agendas under a coherent vocabulary and framework for design. We enumerate the following contributions. First, we demonstrate how blurry the boundary of infovis is by examining systems that exhibit many of the putative properties of infovis systems, but perhaps would not be considered so. Second, we explore the notion of insight and how, instead of a monolithic definition of insight, there may be multiple types, each with particular characteristics. Third, we discuss design challenges for systems intended for casual audiences. Finally we conclude with challenges for system evaluation in this emerging subfield. 2007 IEEE.

Pouyioutas, P, et al. 2006, "Redesigning the User Interface of the Interlearning Software - Graphic Design Meets Computer Science." In IEEE International Conference on Information Visualization, pp. 811-34.

In this paper we present the redesign of the user interface of the InterLearning software. InterLearning is an in-house developed e-learning software that has been in use for the last 3 years at our College. The software was developed by a team of computer scientists. InterLearning has been evaluated by both 
students and faculty since its development, in terms of the functionality that it offers. It has subsequently been enhanced based on the feedback of the users. The software has only started to be used by some Design faculty and students this year. Our experience from its use within this Department has revealed the need for redesigning its interface. Both students and faculty have expressed some negative views with regards to the screen layouts, menus, icons, colours, textual representations and fonts used. Based on this feedback, we have redesigned the interface taking also into consideration feedback obtained during the redesign stages from some Design faculty members and Design students. This paper presents the results of the evaluation of the existing interface by faculty members and students and describes the new interface designed by a team of graphic designers based on the feedback obtained

Prabhat, et al. 2008, "A Comparative Study of Desktop, Fishtank, and Cave Systems for the Exploration of Volume Rendered Confocal Data Sets." IEEE Transactions on Visualization and Computer Graphics 14(3):551-63.

We present a participant study that compares biological exploration tasks using volume renderings of laser confocal microscopy data across three environments which vary in level of immersion. For the tasks, data, and visualization approach used in our study, we found that subjects qualitatively preferred and quantitatively performed better in environments with greater levels of immersion. Subjects performed real-world biological data analysis tasks that emphasized understanding spatial relationships including characterizing the general features in a volume, identifying co-located features, and reporting geometric relationships such as whether clusters of cells were coplanar. After analyzing data in each environment, subjects were asked to choose which environment they wanted to analyze additional data sets in - subjects uniformly selected the Cave environment. (C) 2006 IEEE.

Prabni, J-S, T Ropinski, and K Hinrichs. 2010, "Uncertainty-Aware Guided Volume Segmentation." IEEE Transactions on Visualization and Computer Graphics 16(6):1358-65.

Prabowo, R, and M Thelwall. 2008, "Finding and Tracking Subjects within an Ongoing Debate." Journal of Informetrics 2(2):107-27.

This paper describes a new algorithm for finding and tracking different subjects within an ongoing debate. The algorithm finds blocks of co-occurring terms, representing subjects, including blocks for which the term co-occurrence pattern forms a ring topology. We used short online debate forum data and longer summary bulletins to assess the extent to which the algorithm could correctly detect subjects, according to the judgements of human evaluators. The results show that it could normally detect subjectshifting and track different subjects over time in online debate forums and with adjustments could find subjects in bulletins, but could not track the subjects in the bulletins because the interlinking between subjects was too dense in the longer documents.

Prasolova-Førland, E. 2008, "Analyzing Place Metaphors in 3d Educational Collaborative Virtual Environments." Computers in Human Behavior 24(2):185-204.

In the recent years, the usage of three dimensional (3D) collaborative virtual environments (CVEs) for educational purposes has increased. The metaphors behind the design of virtual places are quite diverse, from replication of real universities to art museums and scientific labs. This paper reports the results of a case study where the students of our university, as a part of their course assignment, analyzed place metaphors used in a range of 3D educational CVEs vs. the corresponding educational goals. The 
students suggested a design for a virtual campus representing the Norwegian University of Science and Technology (NTNU). The results of this study provide some suggestions concerning the characterization of different design features in educational CVEs and the suitability of such features for different educational goals. Also, a preliminary set of design guidelines for an ideal virtual campus representing a real university is presented. Finally, some challenges associated with using 3D CVEs in various educational situations are discussed.

Preim, B. 2010, "Clinical Impact of the Tumor Therapy Manager." In IEEE International Conference on Information Visualization.

Pretorius, AJ. 2005, "Visual Analysis for Ontology Engineering." Journal of Visual Languages \& Computing 16(4):359-81.

An ontology may be decomposed into a layer of binary fact types and a layer of application specific constraints imposed on these fact types. An ontology base is a large set of binary fact types called lexons. This paper presents LexoVis, a lexon visualization tool that addresses the inherent size and scale of ontology bases. LexoVis facilitates the analysis of lexons by providing an ordered visual representation. This representation offers overview and detail by employing the graphical fisheye view. Different ordering and clustering heuristics incorporated in LexoVis lead to insights not explicit in text-based representations of lexons.

Pretorius, AJ, and J J. 2009, "What Does the User Want to See? What Do the Data Want to Be." Information Visualization 8(3):153-66.

Information visualization is a user-centered design discipline. In this article we argue, however, that designing information visualization techniques often requires more than designing for user requirements. Additionally, the data that are to be visualized must also be carefully considered. An approach based on both the user and their data is encapsulated by two questions, which we argue information visualization designers should continually ask themselves: What does the user want to see? and What do the data want to be? As we show by presenting cases, these two points of departure are mutually reinforcing. By focusing on the data, new insight is gained into the requirements of the user, and vice versa, resulting in more effective visualization techniques. (C) 2009 Palgrave Macmillan.

Price, Ba, IS Small, and RM Baecker. 1992, "A Taxonomy of Software Visualization." In Proceedings of the Twenty-Fifth Hawaii International Conference on System Sciences, pp. 597-606.

Software visualization is the use of interactive computer graphics, typography, graphic design, animation, and cinematography to enhance the interface between the software engineer or the computer science student and their programs. Although several taxonomies of software visualization have been proposed, they use few dimensions and do not span the space of important distinctions between systems. The authors propose a novel and systematic taxonomy of six areas making up thirty characteristic features of software visualization technology. The taxonomy is presented and illustrated in terms of its application to seven systems of historic importance and technical interest. 
Pritzkau, A, et al. 2010, "Poster : Scattering and Jittering : Using Real and Illusionary Motion for Better Visual Scatterplot Analysis." In IEEE International Conference on Information Visualization.

Proulx, P, A Khamisa, and R Harper. 2010, "Integrated Visual Analytics Workflow with Geotime and Nspace Vast 2010 Mini Challenge 1 Award : Outstanding Analysis and Accuracy." In IEEE Symposium on Visual Analytics Science \& Technology, pp. 273-74.

Proulx, P, et al. 2006, "Avian Flu Case Study with Nspace and Geotime." In IEEE Symposium on Visual Analytics, Science \& Technology, pp. 27-34.

GeoTime and nSpace are new analysis tools that provide innovative visual analytic capabilities. This paper uses an epidemiology analysis scenario to illustrate and discuss these new investigative methods and techniques. In addition, this case study is an exploration and demonstration of the analytical synergy achieved by combining GeoTime's geo-temporal analysis capabilities, with the rapid information triage, scanning and sense-making provided by nSpace. A fictional analyst works through the scenario from the initial brainstorming through to a final collaboration and report. With the efficient knowledge acquisition and insights into large amounts of documents, there is more time for the analyst to reason about the problem and imagine ways to mitigate threats. The use of both nSpace and GeoTime initiated a synergistic exchange of ideas, where hypotheses generated in either software tool could be crossreferenced, refuted, and supported by the other tool

Pulsifer, PL, et al. 2005, "Chapter 20 - the Development of the Cybercartographic Atlas of Antarctica." in Modern Cartography Series, ed. DRF Taylor, Vol 4, pp. 461-90. Academic Press.

This chapter discusses the ongoing development of a Web-based Atlas of the Antarctic region entitled the Cybercartographic Atlas of Antarctica (The Atlas). An overview of the design, development, and ongoing implementation of The Atlas is presented. Central to The Atlas development approach is the extensive analysis of the needs of a general public target user group. Through user needs analysis (UNA), specifications are established and prototypes used to test concepts before expensive development tasks are carried out. Constant feedback between process stages is built into this iterative User-Centred Design (UCD) approach. The UCD results provide specifications for many aspects of cartographic design, including interface elements, usage context, and information architecture. The chapter presents preliminary interface designs built on content supported by a prototype mediator-based system architecture. The concluding sections discuss emerging research challenges and directions in terms of cartographic representation, atlas design, and systems development.

Qi, Y, et al. 2008, "Viscrm: A Social Network Visual Analytic Tool to Enhance Customer Relationship Management." In IEEE International Conference on Service Operationa and Logistics, and Informatics, pp. 825-30.

As acquiring and retaining the most profitable customers are challenging tasks of service providers, various CRM tools are used to support these processes. Traditional CRM methods focus on various customer profitability models in different scenarios based on their past profit contribution. Social network analysis provides a natural way to understand the relationships between customers; however, this method has seldom been used in customer relationship management. In this paper, we propose a visual analytic tool-VisCRM to analyze the graph features in customer relationship network. Based on the Visual Analytics Mantra, users can get the properties of the whole network as well as the communication 
patterns of certain customer with VisCRM. Furthermore, customers' ranking scores could be got with different statistical algorithms, and a combined ranking score could also be got for each customer by setting different weights to these properties. After choosing a valuable customer, in order to get a deeper insight into customer's communication patterns, people can explore the features of certain customer's egocentric social network visually. To evaluate the effect of VisCRM, we will explore different mobile call networks in telecom services in the case studies.

Qian, CZ, VY Chen, and RF Woodbury. 2006, "Near: Visualizing Information Relations in Multimedia Repository A.Vi.Re." In Conference of the World-Academy-of-Science-Engineering-and-Technology, pp. 216-21.

This paper describes the NEAR (Navigating Exhibitions, Annotations and Resources) panel, a novel interactive visualization technique designed to help people navigate and interpret groups of resources, exhibitions and annotations by revealing hidden relations such as similarities and references. NEAR is implemented on A-VI-RE, an extended online information repository. A-VI-RE supports a semistructured collection of exhibitions containing various resources and annotations. Users are encouraged to contribute, share, annotate and interpret resources in the system by building their own exhibitions and annotations. However, it is hard to navigate smoothly and efficiently in A-VI-RE because of its high capacity and complexity. We present a visual panel that implements new navigation and communication approaches that support discovery of implied relations. By quickly scanning and interacting with NEAR, users can see not only implied relations but also potential connections among different data elements. NEAR was tested by several users in the A-VI-RE system and shown to be a supportive navigation tool. In the paper, we further analyze the design, report the evaluation and consider its usage in other applications.

Qian, W, et al. 2010, "Interactive Demo : Visually Analyzing Text Collections with Multiple Facets." In IEEE International Conference on Information Visualization, pp. 1-2.

Qin, H, P-LP Rau, and G Salvendy. 2010, "Effects of Different Scenarios of Game Difficulty on Player Immersion." Interacting with Computers 22(3):230-39.

This study investigates the effects of game difficulty on player's immersion. Key factors in this study are difficulty of direction changes, including three directions (up and down, down and up, and continuously increasing) and difficulty of rate changes, with three rates (slow, medium, and fast). An experiment was conducted with 48 participants, each playing the same experimental games with different difficulty of direction or rate changes. The results indicate that the players have better immersion when the difficulty changes up and down than when it changes down and up or when the difficulty is continuously increased. And the participants have better immersion when the difficulty changes at a medium rate than when it changes slowly or fast.

Qing, L, and C North. 2003, "Empirical Comparison of Dynamic Query Sliders and Brushing Histograms." In IEEE Symposium on Information Visualization, pp. 147-53.

Dynamic queries facilitate rapid exploration of information by real-time visual display of both query formulation and results. Dynamic query sliders are linked to the main visualization to filter data. A common alternative to dynamic queries is to link several simple visualizations, such as histograms, to the main visualization with a brushing interaction strategy. Selecting data in the histograms highlights that 
data in the main visualization. We compare these two approaches in an empirical experiment on DataMaps, a geographic data visualization tool. Dynamic query sliders resulted in better performance for simple range tasks, while brushing histograms was better for complex trend evaluation and attribute relation tasks. Participants preferred brushing histograms for understanding relationships between attributes and the rich information they provided.

Qishi, W, et al. 2009, "Visualization of Security Events Using an Efficient Correlation Technique." In IEEE Symposium on Computational Intelligence in Cyber Security (CICS '09), pp. 61-68.

The timely and reliable data transfer required by many networked applications necessitates the development of comprehensive security solutions to monitor and protect against an increasing number of malicious attacks. However, providing complete cyber space situation awareness is extremely challenging because of the lack of effective translation mechanisms from low-level situation information to high-level human cognition for decision making and action support. We propose an adaptive cyber security monitoring system that integrates a number of component techniques to collect time-series situation information, perform intrusion detection, keep track of event evolution, characterize and identify security events, and present a visual representation in order to provide comprehensive situational view so that corresponding defense actions can be taken in a timely and effective manner. We explore the principles of designing and applying appropriate visualization techniques for situation monitoring by defining graphical representations of security events. This differs from the traditional rule-based pattern matching techniques in that security events in the proposed system are represented as forms of correlation networks using random matrix theory and identified through the computation of network similarity measurement. The events and corresponding event types are visualized using a stemplot to show location and quantity. Extensive simulation results on event identification illustrate the efficacy of the proposed system.

Quek, FKH. 1995, "Eyes in the Interface." Image and Vision Computing 13(6):511-25.

Computer vision has a significant role to play in the human-computer interaction (HCI) devices of the future. All computer input devices serve one essential purpose. They transduce some motion or energy from a human agent into machine useable signals. One may therefore think of input devices as the [']perceptual organs' by which computers sense the intents of their human users. We outline the role computer vision will play, highlight the impediments to the development of vision-based interfaces, and propose an approach for overcoming these impediments. Prospective vision research areas for HCI include human face recognition, facial expression interpretation, lip reading, head orientation detection, eye gaze tracking three-dimensional finger pointing, hand tracking, hand gesture interpretation and body pose tracking. For vision-based interfaces to make any impact, we will have to embark on an expansive approach, which begins with the study of the interaction modality we seek to implement. We illustrate our approach by discussing our work on vision-based hand gesture interfaces. This work is based on information from such varied disciplines as semiotics, anthropology, neurophysiology, neuropsychology and psycholinguistics. Concentrating on communicative (as opposed to manipulative) gestures, we argue that interpretation of a large number of gestures involves analysis of image dynamics to identify and characterize the gestural stroke, locating the stroke extrema in ordinal 3D space, and recognizing the hand pose at stroke extrema. We detail our dynamic image analysis algorithm which enforces our constraints: directional variance, spatial cohesion, directional cohesion and path cohesion. The clustered vectors characterize the motion of a gesturing hand. 
Quigley, AJ, and F Bodea. 2010, "Chapter 1 - Face-to-Face Collaborative Interfaces." in Human-Centric Interfaces for Ambient Intelligence, eds. A Hamid, Delgado and Augusto, pp. 3-32. Academic Press, Oxford.

Summary The presentation of information as a large display and the use of such displays to support collaboration in face-to-face activities have long been commonplace. Computationally enhanced displays relying on the form factor of whiteboards, surfaces, tables, benches, and desks now afford face-to-face computer-supported interaction and collaboration not possible with classical desktop or mobile computing. This chapter provides an introduction to research and developments in multitouch input technologies that can be used to realize large interactive tabletop or "surface user interfaces." Such hardware systems, along with supporting software, allow for applications that can be controlled through direct touch or multitouch. Further, a review of gestural interactions and design guidelines for surface user interface design for collaboration is also provided.

Quintana, C, J Krajcik, and E Soloway. 2003, "Issues and Approaches for Developing Learner-Centered Technology." in Advances in Computers, Vol 57, pp. 271-321. Elsevier.

As computing technology has evolved, there has been significant research and a range of approaches exploring the use of computers for learning. Traditionally, the prevalent approach when developing software has been a user-centered approach that focuses on software usability to help experts in some practice effectively engage in their work. However, in recent years, researchers have come to understand that a different approach--a learner-centered approach--is needed to design tools that help novices in some practice do and learn the work involved in that practice. Rather than solely focusing on the conceptual gulfs that lie between tool users and their tools, learner-centered tools primarily focus on addressing the conceptual gulf between a learner's novice expertise in some practice and the expertise of an expert in that practice. In this chapter, we discuss some major approaches that have been considered for learnercentered technologies by describing software based on behaviorist, information processing, and social constructivist approaches. Expanding on the latter two approaches, we will focus on two types of learnercentered software. We will look at intelligent tutoring systems and how they oversee a learner's work to provide assistance when learners encounter problems as they are working on certain tasks. We will also look at scaffolded software that incorporates specific functionalities and user interface features to support learners as they use the tool to engage in new activity. For each type of software, we will discuss the issues, approaches, and tradeoffs involved in designing, implementing, and evaluating the software given its learner-centered focus. Finally, we will consider emerging wireless, handheld technologies, to discuss the new kinds of functionalities offered by these technologies, their possible impact on learning, and the challenges that developers face in creating educationally effective software given the constraints of these technologies.

Quirin, A, et al. 2010, "Graph-Based Data Mining: A New Tool for the Analysis and Comparison of Scientific Domains Represented as Scientograms." Journal of Informetrics 4(3):291-312.

The creation of some kind of representations depicting the current state of Science (or scientograms) is an established and beaten track for many years now. However, if we are concerned with the automatic comparison, analysis and understanding of a set of scientograms, showing for instance the evolution of a scientific domain or a face-to-face comparison of several countries, the task is titanically complex as the amount of data to analyze becomes huge and complex. In this paper, we aim to show that graph-based data mining tools are useful to deal with scientogram analysis. Subdue, the first algorithm proposed in the 
graph mining area, has been chosen for this purpose. This algorithm has been customized to deal with three different scientogram analysis tasks regarding the evolution of a scientific domain over time, the extraction of the common research categories substructures in the world, and the comparison of scientific domains between different countries. The outcomes obtained in the developed experiments have clearly demonstrated the potential of graph mining tools in scientogram analysis.

Rabelo, E, et al. 2008, "Information Visualization: Which Is the Most Appropriate Technique to Represent Data Mining Results?" In International Conference on Computational Intelligence for Modelling, Control and Automation, pp. 1228-33.

Data mining $(\mathrm{DM})$ is a research area that has been contributing in the search for implicit knowledge that can give support to decision making. However, the analysis of the results obtained by using the techniques of DM is not an easy task. Such difficulty can be minimized, by using techniques of information visualization. But the issue is: which will be the most appropriate technique for each type of result to be analyzed? To help in the choice of techniques, this paper presents an evaluation of techniques for information visualization. Such task was accomplished by using the method known as Analysis of Characteristics, having as result the analysis on the effectiveness when using techniques of geometric and iconographical information visualization, mainly in relation to the results obtained with the application of K-means algorithm.

Radburn, R, et al. 2010, "Discovery Exhibition : Using Spatial Treemaps in Local Authority Decision Making and Reporting." In IEEE Symposium on Information Visualization, pp. 3-6.

Rae, A. 2009, "From Spatial Interaction Data to Spatial Interaction Information? Geovisualisation and Spatial Structures of Migration from the 2001 Uk Census." Computers, Environment and Urban Systems 33(3):161-78.

In modern GIS applications, flow mapping and geodynamics are not particularly well represented. This is somewhat surprising given the potential of such approaches to shed light on the spatial characteristics associated with the movement of people and goods. Despite the attention of a select number of researchers whose contributions have been significant, however, the subject matter remains underdeveloped and the full potential of spatial interaction data remains largely unrealised. Therefore, this paper represents an attempt to popularise the use of flow mapping within a contemporary GIS, with a view to aiding and enhancing decision support at a range of different spatial scales. It discusses advances in flow mapping, proposes an approach to mapping a large migration matrix from the United Kingdom's 2001 census, and considers the ways in which this can add value to the understanding of geographical patterns of residential mobility. Subsequently, it considers possible dissemination methods via new developments in web-based geospatial technologies before offering some general principles and identifying future challenges. The findings indicate that it is now entirely feasible to map large spatial interaction datasets with common GIS software, that this has the benefit of producing instant, query-led information, and that the knowledge derived from this could be used to inform all kinds of local, regional and national policy formulation and evaluation. 
Raij, AB, and BC Lok. 2008, "Ipsviz: An after-Action Review Tool for Human-Virtual Human Experiences." In 15th Annual IEEE Virtual Reality 2008 Conference, pp. 91-98.

This paper proposes after-action review (AAR) with human-virtual human $(\mathrm{H}-\mathrm{VH})$ experiences. $\mathrm{H}-$ $\mathrm{VH}$ experiences are seeing increased use in training for real-world, $\mathrm{H}-\mathrm{H}$ experiences. To improve training, the users of H-VH experiences need to review, evaluate, and get feedback on them. AAR enables users to review their $\mathrm{H}-\mathrm{VH}$ interaction, evaluate their actions, and receive feedback on how to improve future real-world, H-H experiences. The Interpersonal Scenario Visualizer (IPSViz), an AAR tool for HVH experiences, is presented. IPSViz allows medical students to review their interactions with VH patients. To enable review, IPSViz generates spatial, temporal, and social visualizations of $\mathrm{H}-\mathrm{VH}$ interactions. Visualizations are generated by treating the interaction as a set of signals. Interaction signals are captured, logged, and processed to generate visualizations for review, evaluation and feedback. In a study $(\mathrm{N}=27)$, reviewing the visualizations helped students become self-aware of their actions with a virtual human and gain insight into how to improve interactions with real humans.

Rainer, A. 2010, "Representing the Behaviour of Software Projects Using Multi-Dimensional Timelines." Information and Software Technology 52(11):1217-28.

Context There are few empirical studies in the empirical software engineering research community that describe software projects, at the level of the project, as they progress over time.Objective To investigate how to coherently represent a large volume of qualitative and quantitative data on a range of project-level attributes as those attributes change over time.Method Develop a modelling technique, multi-dimensional timelines (MDTs) and undertake a preliminary appraisal of the technique using examples from a longitudinal case study of a project at IBM Hursley Park.Results MDTs can represent project-level attributes as they change over time, provided these attributes, and the empirical data about them, can be located in time (an analytical requirement) and can be represented in terms of the simple geometrical structures of points, lines and planes (a graphical requirement). Changes in attributes are documented at the point in time at which the change occurs. There are a number of ways in which an attribute can be represented on the MDT: as a quantitative time series, as an event, as an event with label containing complex qualitative information, or as a schedule. The MDT technique is currently not capable of representing relationships between different attributes e.g., a causal relationship.Conclusion The initial appraisal of MDTs is encouraging, but further work is needed on the development of the MDT technique and on its evaluation.

Raja, A, and A Goel. 2007, "Introspective Self-Explanation in Analytical Agents." In AAMAS 2007 Workshop on on Metareasoning in Agent-based Systems, pp. 76-91.

There is a critical and urgent need for automated analytical agents operating in complex domains to provide meta-level explanations of their reasoning and conclusions. In this paper, we identify the principles for designing analytical agents that can explain their reasoning and justify their conclusions at di erent levels of abstractions to potential human customers with varying goals. We also analyze the goals of users of an automated agent for investigative analysis along the dimensions of why, what, how and when, and develop a taxonomy of human goals that will leverage the explanations generated by the agent. 
Rasmussen, J, and AM Pejtersen. 1990, Taxonomy for Cognitive Work Analysis. Technical Rpt. Riso-M2871, Riso National Laboratiry, Roskilde, Denmark.

Ray, WC. 2010, "A Visual Analytics Approach to Identifying Protein Structural Constraints." In IEEE Symposium on Visual Analytics Science \& Technology, pp. 249-50.

Redpath, R, and B Srinivasan. 2003, "Criteria for a Comparative Study of Visualization Techniques in Data Mining." in Intelligent Systems Design and Applications, eds. A Abraham, K Franke and M Koppen, pp. 609-20.

The paper considers the relationship of information visualization tools to the data mining process. The types of structures that may be of interest to data mining experts in data sets are outlined. The performance of a particular visualization technique in revealing those structures and supporting the subsequent steps in the process needs to be based on a number of criteria. The criteria for performing an evaluation are suggested and explained. A division into two main groups is suggested; criteria that relate to interface issues and criteria that relate to the characteristics of the data set. An example application of some of the criteria is made.

Rees, K. 2010, "Periscopic Visualizes Symptomatology of Pandemic." In IEEE Symposium on Visual Analytics Science \& Technology, pp. 283-84.

Reid, EF, and H Chen. 2007, "Mapping the Contemporary Terrorism Research Domain." International Journal of Human-Computer Studies 65(1):42-56.

A systematic view of terrorism research to reveal the intellectual structure of the field and empirically discern the distinct set of core researchers, institutional affiliations, publications, and conceptual areas can help us gain a deeper understanding of approaches to terrorism. This paper responds to this need by using an integrated knowledge-mapping framework that we developed to identify the core researchers and knowledge creation approaches in terrorism. The framework uses three types of analysis: (a) basic analysis of scientific output using citation, bibliometric, and social network analyses, (b) content map analysis of large corpora of literature, and (c) co-citation analysis to analyse linkages among pairs of researchers. We applied domain visualization techniques such as content map analysis, block-modeling, and co-citation analysis to the literature and author citation data from the years 1965 to 2003. The data were gathered from ten databases such as the ISI Web of Science. The results reveal: (1) the names of the top 42 core terrorism researchers (e.g., Brian Jenkins, Bruce Hoffman, and Paul Wilkinson) as well as their institutional affiliations; (2) their influential publications; (3) clusters of terrorism researchers who work in similar areas; and (4) that the research focus has shifted from terrorism as a low-intensity conflict to a strategic threat to world powers with increased focus on Osama Bin Laden.

Reif, R, et al. 2010, "Evaluation of an Augmented Reality Supported Picking System under Practical Conditions." Computer Graphics Forum 29(1):2-12.

Order picking is one of the most important process steps in logistics. Because of their flexibility, human beings cannot be replaced by machines. But if workers, in order, picking systems are equipped with a head-mounted display, Augmented Reality can improve the information visualization. In this paper, the development of such a system-called Pick-by-Vision-is presented. The system is evaluated in a user study performed in a real storage environment. Important logistics figures as well as the subjective 
strain were measured. The results show that a Pick-by-Vision system can considerably improve industrial order picking processes.

Reilly, D, K Inkpen, and Acm. 2007, "White Rooms and Morphing Don't Mix: Setting and the Evaluation of Visualization Techniques." In Conference on Human Factors in Computing Systems, pp. 111-20.

The results presented in this paper illustrate how a specific map visualization technique is sensitive to setting: a comparative evaluation of the technique gives conflicting results depending on where it takes place. While prior research has explored the impact of factors other than basic visual perception on visualization techniques, relatively little attention has been directed toward the physical setting in which the technique is used. We present results from a study involving 120 participants, comparing the effectiveness of two different geovisualization techniques in promoting recall of map layout. Recall was shown to be sensitive to setting, such that one technique in particular was more effective in a noisy public space than in a controlled, 'white-room' environment. The results have implications for the validation and measurement of information visualization techniques as a whole, and in particular for those employing motion as a communicative attribute.

Reilly, DF, and KM Inkpen. 2004, "Map Morphing: Making Sense of Incongruent Maps." In 30th Graphics Interface Conferernce (GI 2004), pp. 231-38.

Map morphing is an interactive visualization technique that provides a user-controlled, animated translation from one map to another. Traditionally, overlay mechanisms are used to present layers of information over a single projection. Map morphing provides a way to relate maps with significant spatial and schematic differences. This paper presents the morphing technique and the results of a comparative evaluation of map morphing against standard ways of presenting related maps. Our results demonstrate that map morphing provides additional information that can be used to effectively relate maps. In particular, significantly more tasks were completed correctly using the morphing interface than either a windowed or an inset interface.

Reiter, E, et al. 2005, "Choosing Words in Computer-Generated Weather Forecasts." Artificial Intelligence 167(1-2):137-69.

One of the main challenges in automatically generating textual weather forecasts is choosing appropriate English words to communicate numeric weather data. A corpus-based analysis of how humans write forecasts showed that there were major differences in how individual writers performed this task, that is, in how they translated data into words. These differences included both different preferences between potential near-synonyms that could be used to express information, and also differences in the meanings that individual writers associated with specific words. Because we thought these differences could confuse readers, we built our SumTime-Mousam weather-forecast generator to use consistent datato-word rules, which avoided words which were only used by a few people, and words which were interpreted differently by different people. An evaluation by forecast users suggested that they preferred SumTime-Mousam's texts to human-generated texts, in part because of better word choice; this may be the first time that an evaluation has shown that nlg texts are better than human-authored texts. 
Remco, C, et al. 2007, "Legible Cities: Focus-Dependent Multi-Resolution Visualization of Urban Relationships." Visualization and Computer Graphics, IEEE Transactions on 13(6):1169-75.

Numerous systems have been developed to display large collections of data for urban contexts; however, most have focused on layering of single dimensions of data and manual calculations to understand relationships within the urban environment. Furthermore, these systems often limit the user's perspectives on the data, thereby diminishing the user's spatial understanding of the viewing region. In this paper, we introduce a highly interactive urban visualization tool that provides intuitive understanding of the urban data. Our system utilizes an aggregation method that combines buildings and city blocks into legible clusters, thus providing continuous levels of abstraction while preserving the user's mental model of the city. In conjunction with a 3D view of the urban model, a separate but integrated information visualization view displays multiple disparate dimensions of the urban data, allowing the user to understand the urban environment both spatially and cognitively in one glance. For our evaluation, expert users from various backgrounds viewed a real city model with census data and confirmed that our system allowed them to gain more intuitive and deeper understanding of the urban model from different perspectives and levels of abstraction than existing commercial urban visualization systems.

Ren, L, et al. 2010, "Doi-Wave: A Focus Plus Context Interaction Technique for Networks Based on Attention-Reactive Interface." In International Conference on Visual Information Communications, pp. 85-94.

This paper presents DOI-Wave, a Focus+Context technique for interactive exploration of networks, whose layout depends dynamically on the user's degree-of-interest (DOI). DOI-Wave uses the hierarchical clustered graph paradigm, in which, the view of a graph is dynamically determined by the selection of a single node as the focus node. The layout algorithm could change the view of graph animatedly, as the selected focus node is changed, according to each node's DOI value computed by a DOI function. The Focus+Context paradigm in DOI-Wave, from user's perspective, makes it easy to understand the relationships between the focus node and the other nodes. And the hierarchical cluster paradigm, which adapts to display space dynamically, leads to simultaneously clear and compact visualization. We have applied DOI-Wave to visualizing and exploring social networks. Evaluation results show DOI-Wave is effective.

Renshaw, JA, et al. 2004, "Understanding Visual Influence in Graph Design through Temporal and Spatial Eye Movement Characteristics." Interacting with Computers 16(3):557-78.

We describe an experiment in which the eye movements of participants, carrying out tasks using two contrasting graph designs, were recorded by means of a remote eye tracking device. A variety of eye movement properties were measured and analysed both temporally and spatially. Both graph designs were based on specific psychological theories and established graph design guidelines. One incorporated attributes thought likely to enhance usability, the other included attributes likely to have the opposite effect. The results demonstrate that the design and location of a graph's legend and its spatial relationship to the data area are extremely important in determining a graph's usability. The incorporation of these and other design features may promote or detract from perceptual proximity and therefore influence a display's usability. The paper demonstrates that this influence is reflected in eye movement patterns, which can be readily monitored by means of a remote eye tracking system, and that a relatively simple temporal analysis of the results can give important insights as to how the usability of visual displays has been influenced. 
Rester, M, and M Pohl. 2006, "Methods for the Evaluation of an Interactive Infovis Tool Supporting Exploratory Reasoning Processes." In AVI workshop on BEyond time and errors: novel evaluation methods for information visualization (BELIV'06), pp. 1-6.

Developing Information Visualization (InfoVis) techniques for complex knowledge domains makes it necessary to apply alternative methods of evaluation. In the evaluation of Gravi++ we used several methods and studied different user groups. We developed a reporting system yielding data about the insights the subjects gained during the exploration. It provides complex information about subjects' reasoning processes. Log files are valuable for time-dependent analysis of cognitive strategies. Focus groups provide a different view on the process of gaining insights. We assume that our experiences with all these methods can also be applied in similar evaluation studies on InfoVis techniques for complex data.

Rester, M, et al. 2007, "Evaluating an Infovis Technique Using Insight Reports." In 11th International Conference on Information Visualization (IV 2007), pp. 693-700.

The evaluation of Information Visualization (InfoVis) techniques can help to identify specific strengths and weaknesses of these methods:, The following article describes the results of an empirical study assessing the contribution of an interactive InfoVis method based on a spring metaphor (GRAVI), Exploratory,Data Analysis (EDA) and Machine Learning (ML) to ease understanding. The application domain is the psychotherapeutic treatment of anorectic young women. The three methods are supposed to support the therapists in finding the variables which influence success or failure of the therapy. To conduct the evaluation we developed a report system which helped subjects to formulate and document in a self-directed manner the insights they gained when using the three methods. The results indicate that the three methods are complementary and should be used in conjunction.

---. 2007, "Mixing Evaluation Methods for Assessing the Utility of an Interactive Infovis Technique." In 12th International Conference on Human-Computer Interaction (HCI International 2007), pp. 604-13.

We describe the results of an empirical study comparing an interactive Information Visualization (InfoVis) technique called Gravi++ (GRAVI), Exploratory Data Analysis (EDA) and Machine Learning (ML). The application domain is the psychotherapeutic treatment of anorectic young women. The three techniques are supposed to support the therapists in finding the variables which influence success or failure in therapy. To evaluate the utility of the three techniques we developed on the one hand a report system which helped subjects to formulate and document in a self-directed manner the insights they gained when using the three techniques. On the other hand, focus groups were held with the subjects. The combination of these very different evaluation methods prevents jumping to false conclusions and enables for an comprehensive assessment of the tested techniques. The combined results indicate that the three techniques (EDA, ML, and GRAVI) are complementary and therefore should be used in conjunction.

Rex. 2003, "Cartography, Virtual Reality, and the Internet: Integrating Abstract Models of the Environment Via the Internet." in Maps and the Internet, ed. P Michael, pp. 359-70. Elsevier Science, Oxford.

Summary The use of maps to develop public awareness for environmental issues is a significant research question for cartographers. With the development of multimedia cartography and virtual reality, cartography can develop a perceptual experience that will immerse map-readers into the physical 
environment. This case study demonstrates how to integrate abstract map representations within virtual reality displays. The Little Sac River was mapped and augmented with QuickTime scenes for distribution through an interactive Internet mapping service. By adding maps into virtual reality, an enhanced perceptual experience may lead to an improved understanding of the environment and the effects of human action.

Ribarsky, W. 2009, "Toward Effective Insight Management in Visual Analytics Systems." In 2009 IEEE Pacific Visualization Symposium, pp. 49-56.

Ribarsky, W, et al. 2009, Visual Analysis of Urban Terrain Dynamics. Technical Rpt. CVC-UNCC-0611, University of North Carolina.

Modern urban areas are places of continuous change. Over periods of time of months, buildings may be torn down and new ones started; streets can be altered and new ones constructed; railways or other means of urban transport may change. The models of urban areas must be able to accommodate this change. This is especially so since models are significantly higher resolution than previously and cover wider areas. Now models can typically have imagery and elevation data at resolutions of 1 foot to 1 meter with certain features (on key buildings, for example) that may be at higher resolution. At these resolutions and for certain applications, even small changes can be noteworthy. In addition, urban terrain models can come from many sources. These include varieties of sensors such as LIDAR, satellite imagery, airborne oblique photography, ground-based depth and appearance fields, SARS, and so on. Automated, semiautomated, or by-hand techniques are used to reconstruct the urban model. In the latter case, users may use 3D design software to create individual building or streetscape models from combinations of photographs, measurements, and building plans. A comprehensive, dynamic model should be able to handle contributions from any of these sources. In many cases, such as urban planning, civil engineering, or military applications, a lower resolution model of an urban area of interest, will be augmented with higher resolution data, which may come from another sensing source than the original data. These data must be embedded into the context of the existing model and often there is not time (nor for certain applications should it be necessary) to reconstruct the whole model based on the new data.

Ribarsky, W, B Fisher, and WM Pottenger. 2009, "Science of Analytical Reasoning." Information Visualization 8(4):254-62.

There has been progress in the science of analytical reasoning and in meeting the recommendations for future research that were laid out when the field of visual analytics was established. Researchers have also developed a group of visual analytics tools and methods that embody visual analytics prin- ciples and attack important and challenging real-world problems. However, these efforts are only the beginning and much study remains to be done. This article examines the state of the art in visual analytics methods and reasoning and gives examples of current tools and capabilities. It shows that the science of visual analytics needs interdisciplinary efforts, indicates some of the disci- plines that should be involved and presents an approach to how they might work together. Finally, the article describes some gaps, opportunities and future directions in developing new theories and models that can be enacted in methods and design principles and applied to significant and complex prac- tical problems and data. 
Riche, NH. 2010, "Beyond System Logging: Human Logging for Evaluating Information Visualization." In BELIV '10: Beyond time and errors: novel evaluation methods for Information Visualization, April 1011, 2010.

Riche, NH, and T Dwyer. 2010, "Untangling Euler Diagrams." IEEE Transactions on Visualization and Computer Graphics 16(6):1090-9.

In many common data analysis scenarios the data elements are logically grouped into sets. Venn and Euler style diagrams are a common visual representation of such set membership where the data elements are represented by labels or glyphs and sets are indicated by boundaries surrounding their members. Generating such diagrams automatically such that set regions do not intersect unless the corresponding sets have a non-empty intersection is a difficult problem. Further, it may be impossible in some cases if regions are required to be continuous and convex. Several approaches exist to draw such set regions using more complex shapes, however, the resulting diagrams can be difficult to interpret. In this paper we present two novel approaches for simplifying a complex collection of intersecting sets into a strict hierarchy that can be more easily automatically arranged and drawn (Figure 1). In the first approach, we use compact rectangular shapes for drawing each set, attempting to improve the readability of the set intersections. In the second approach, we avoid drawing intersecting set regions by duplicating elements belonging to multiple sets. We compared both of our techniques to the traditional non-convex region technique using five readability tasks. Our results show that the compact rectangular shapes technique was often preferred by experimental subjects even though the use of duplications dramatically improves the accuracy and performance time for most of our tasks. In addition to general set representation our techniques are also applicable to visualization of networks with intersecting clusters of nodes.

Riekki, J, et al. 2003, "Genie of the Net, an Agent Platform for Managing Services on Behalf of the User." Computer Communications 26(11):1188-98.

We will present an agent-based architecture, Genie of the Net, for context-aware systems that are used as components of an intelligent environment and manage services on behalf of the user. We will also discuss the need for knowledge representations. Furthermore, we will present information management and event scheduling as our primary application area and discuss the application we are working on: the Home Notice Board, which presents information and schedules events for family members. We will discuss the first prototype, the Family Calendar, that we have already completed and demonstrated. For this prototype, we have implemented a general agent platform and agents specialized in scheduling events. Furthermore, we will present an extension to the first prototype, Neon Digipainting. This prototype will be a combined changing art work and information channel, which will enhance communication between family members.

Rilling, J, and SP Mudur. 2005, "3d Visualization Techniques to Support Slicing-Based Program Comprehension." Computers \& Graphics 29(3):311-29.

Graphic visuals derived from reverse engineered source code have long been recognized for their impact on improving the comprehensibility of structural and behavioral aspects of large software systems and their source code. A number of visualization techniques, primarily graph-based, do not scale. Some other proposed techniques based on 3D metaphors tend to obscure important structural relationships in the program. Multiple views displayed in overlapping windows are suggested as a possible solution, which more often than not results in problems of information overload and cognitive discontinuity. In this paper, 
we first present a comprehensive survey of related work in program comprehension and software visualization, and follow it up with a detailed description of our research which uses program slicing for deriving program structure-based attributes and 3D-metaball-based rendering techniques to help visualization-based analysis of source code structure. Metaballs, a 3D modeling technique, has already found extensive use for representing complex organic shapes and structural relationships in biology and chemistry. We have developed a metaball software visualization system in Java3D, named MetaViz. As proof of concept, using MetaViz, we demonstrate the creation of 3D visuals that are intuitively comprehensible and communicate information about relative component complexity and coupling among components and therefore enhance comprehension of the program structure.

Rimey, RD, and DS Bolme. 2007, "Re-Using Millions of Visualizations - Art. No. 649504." In Conference on Visualization and Data Analysis 2007, pp. 49504-04.

Our goal is to enable an individual analyst to utilize and benefit from millions of visualization instances created by a community of analysts. A visualization instance is the combination of a specific set of data and a specific configuration of a visualization providing a visual depiction of that data. As the variety and number of visualization techniques and tools continues to increase, and as users increasingly adopt these tools, more visualization instances will be created (today, perhaps only viewed for a moment and thrown away) during the solution of analysis tasks. This paper discusses what fraction of these visualization instances are worth keeping and why, and argues that keeping more (or all) visualization instances has high value and very low cost. Even if a small fraction is retained the result over time is still a large number of visualization instances and the issue remains, how can users utilize them? This paper describes what new functionality users need to utilize all those visualization instances, illustrated by examples using an information workspace tool based on zoomable user interface principles. The paper concludes with a concise set of principles for future analysis tools that utilize spatial organization of large numbers of visualization instances.

Risch, J, et al. 1996, "Interactive Information Visualization for Exploratory Intelligence Data Analysis." In Virtual Reality Annual International Symposium, pp. 230-38,69.

This paper describes an experimental exploratory information visualization system under development at PNNL for integrating multimedia data and visualizing the contents of large multimedia databases. Our system prototype, named Starlight, represents multimedia corpora graphically as collections of icons in 3-space. Each icon in the information display represents an individual element of a multimedia database. The proximity of any two icons in the display is an indication of their general similarity, providing fast access to thematically related information. We extend the effectiveness of this approach by coupling it with a linkage display system to enable the simultaneous visualization of database structure and contents at multiple levels of abstraction. The utility of the system is further extended by enabling a variety of graphical and text-based interactions with the information display. The system enables the interactive generation of multiple simultaneous \&ldquo;views\&rdquo; into multimedia databases, providing intelligence analysts with the potential to process large multimedia data volumes quickly and effectively 
Risch, JS, et al. 1997, "The Starlight Information Visualization System." In IEEE Conference on Information Visualization, pp. 42-49.

STARLIGHT is an example of a new class of information system expressly designed around visualization-oriented user-interface. Incorporating more traditional information storage and retrieval technologies into its design, the STARLIGHT system also enables the integrated use of multiple, concurrent visualization techniques to support comparison of content and interrelationship information levels of abstraction simultaneously. This powerful new form of information analysis eases cognitive workloads by providing a visual context for the information under study. Originally developed for intelligence analysis applications, the STARLIGHT software is intended to support the rapid, concurrent analysis of complex multimedia information, including structured and unstructured text, geographic information, and digital imagery. The system uses novel 3-D visualization techniques that interactively generate easily understandable representations of explicit and implicit relationships contained in information collections of various types. This paper describes the general theory behind our approach, and the design and features of a Windows NT-based operational system

Riveiro, M. 2007, "Evaluation of Uncertainty Visualization Techniques for Information Fusion." In 10th International Conference on Information Fusion, pp. 623-30.

This paper highlights the importance of uncertainty visualization in information fusion, reviews general methods of representing uncertainty and presents perceptual and cognitive principles from Tufte, Chambers and Bertin as well as users experiments documented in the literature. Examples of uncertainty representations in information fusion are analyzed using these general theories. These principles can be used in future theoretical evaluations of existing or newly developed uncertainty visualization techniques before usability testing with actual users.

Riveiro, M, and G Falkman. 2010, "Supporting the Analytical Reasoning Process in Maritime Anomaly Detection: Evaluation and Experimental Design." In 14th International Conference on Information Visualization, pp. 170-78.

Despite the growing number of systems providing visual analytic support for investigative analysis, few empirical studies include investigations on the analytical reasoning process that needs to be supported. In this paper, we present an approach to evaluate the ability of certain visual representations from an integrated visual-computational environment to support the completion of representative tasks. The problem area studied is the detection and identification of anomalous vessels and situations while monitoring maritime traffic data. This paper presents: (1) a brief review of current evaluation methodologies within information visualization and visual analytics, (2) an analysis of operator's analytical reasoning process (derived from field work in maritime control centers and a literature review on analytical reasoning theories), (3) a list of representative tasks for usability evaluation and (4) an approach to evaluate the use of normal behavioral models representations during the detection process.

Rivest, S, et al. 2005, "Solap Technology: Merging Business Intelligence with Geospatial Technology for Interactive Spatio-Temporal Exploration and Analysis of Data." ISPRS Journal of Photogrammetry and Remote Sensing 60(1):17-33.

To support their analytical processes, today's organizations deploy data warehouses and client tools such as OLAP (On-Line Analytical Processing) to access, visualize, and analyze their integrated, 
aggregated and summarized data. Since a large part of these data have a spatial component, better client tools are required to take full advantage of the geometry of the spatial phenomena or objects being analyzed. With this regard, Spatial OLAP (SOLAP) technology offers promising possibilities. A SOLAP tool can be defined as "a type of software that allows rapid and easy navigation within spatial databases and that offers many levels of information granularity, many themes, many epochs and many display modes synchronized or not: maps, tables and diagrams" [Bédard, Y., Proulx, M.J., Rivest, S., 2005. Enrichissement du OLAP pour l'analyse géographique: exemples de réalisation et différentes possibilités technologiques. In: Bentayeb, F., Boussaid, O., Darmont, J., Rabaseda, S. (Eds.), Entrepôts de Données et Analyse en ligne, RNTI B_1. Paris: Cépaduès, pp. 1-20]. SOLAP tools offer a new user interface and are meant to be client applications sitting on top of multi-scale spatial data warehouses or datacubes. As they are based on the multidimensional paradigm, they facilitate the interactive spatio-temporal exploration of data. The purpose of this paper is to discuss how SOLAP concepts support spatio-temporal exploration of data and then to present the geovisualization, interactivity, and animation features of the SOLAP software developed by our research group. This paper first reviews the general concepts behind OLAP and SOLAP systems. This is followed by a discussion of how these SOLAP concepts support spatio-temporal exploration of data. In the subsequent section, SOLAP software is introduced along with features that enable geovisualization, interactivity and animation.

Roberts, D, et al. 2010, "Centrifuge : Characterization of Pandemic Spread Vast 2010 Mini Challenge 2." In IEEE Symposium on Visual Analytics Science \& Technology, pp. 2-3.

\section{Roberts, JC. 1998, "On Encouraging Multiple Views for Visualization." In IEEE Conference on} Information Visualization, pp. 8-14.

Visualization enables 'seeing the unseen', and provides new insight into the underlying data. However, users far too easily believe or rely on a single representation of the data; this view may be a favourite method, the simplest to perform, or a method that has always been used. A single representation may generate a misinterpretation of the information or provide a situation where the user is missing the 'richness' of the data content. By displaying the data in multiple ways a user may understand the information through different perspectives, overcome possible misinterpretations and perform interactive investigative visualization through correlating the information between views. Thus, the use of multiple views of the same information should be encouraged. We believe the visualization system itself should actively encourage the generation of multiple views by providing appropriate tools to aid in this operation. We present and categorise issues for encouraging multiple views and provide a framework for the generation, management and manipulation of such views

Roberts, S, A Parush, and G Lindgaard. 2005, "Chapter 10 - Cognitive Theories and Aids to Support Navigation of Multimedia Information Space." in Modern Cartography Series, ed. DRF Taylor, Vol 4, pp. 231-56. Academic Press.

Navigation in information cyberspace presents difficulties for users leading to reduced satisfaction. How navigation can be facilitated is not well understood, particularly with complex systems involving spatial or geo-referenced information such as in cybercartographic products. There is extensive research on the way people navigate and orient themselves successfully in real and virtual environments, which could provide insight regarding ways to aid navigation and orientation in information cyberspace. This chapter discusses the similarities and differences of navigation and orientation between real/virtual spaces and cyberspace in terms of: (1) acquisition and representation of space; (2) tasks; and (3) navigation aids. 
The degree of generalization between these two spaces is discussed and serves as a basis for a conceptual framework, as well as guidelines, to support navigation in information cyberspaces.

Robertson, G, et al. 2002, "Polyarchy Visualization: Visualizing Multiple Intersecting Hierarchies." In SIGCHI conference on Human factors in computing systems: Changing our world, changing ourselves pp. 423-30.

We describe a new information structure composed of multiple intersecting hierarchies, which we call Polyarchies. Visualizing polyarchies enables use of novel views for discovery of relationships which are very difficult using existing hierarchy visualization tools. This paper will describe the visualization design and system architecture challenges as well as our current solutions. A Mid-Tier Cache architecture is used as a "polyarchy server" which supports a novel web-based polyarchy visualization technique, called Visual Pivot. A series of five user studies guided iterative design of Visual Pivot.

Robertson, G, SK Card, and JD Mackinlay. 1989, "The Cognitive Coprocessor Architecture for Interactive User Interfaces." In 2nd annual ACM SIGGRAPH symposium on User interface software and technology, pp. 10-18.

Robertson, G, et al. 2008, "Effectiveness of Animation in Trend Visualization." Visualization and Computer Graphics, IEEE Transactions on 14(6):1325-32.

Animation has been used to show trends in multi-dimensional data. This technique has recently gained new prominence for presentations, most notably with Gapminder Trendalyzer. In Trendalyzer, animation together with interesting data and an engaging presenter helps the audience understand the results of an analysis of the data. It is less clear whether trend animation is effective for analysis. This paper proposes two alternative trend visualizations that use static depictions of trends: one which shows traces of all trends overlaid simultaneously in one display and a second that uses a small multiples display to show the trend traces side-by-side. The paper evaluates the three visualizations for both analysis and presentation. Results indicate that trend animation can be challenging to use even for presentations; while it is the fastest technique for presentation and participants find it enjoyable and exciting, it does lead to many participant errors. Animation is the least effective form for analysis; both static depictions of trends are significantly faster than animation, and the small multiples display is more accurate.

Robertson, GG, SK Card, and JD Mackinlay. 1993, "Information Visualization Using 3d Interactive Animation." Communications of the ACM 36(4):57-71.

Robertson, GG, T Chilimbi, and B Lee. 2010, "Allocray : Memory Allocation Visualization for Unmanaged Languages." In 5th ACM Symposium on Software Visualization (SOFTVIS), pp. 43-52.

Robertson, GG, JD Mackinlay, and SK Card. 1991, "Cone Trees: Animated 3d Visualizations of Hierarchical Information." In SIGCHI conference on Human factors in computing systems: Reaching through technology, pp. 189-94.

Robinson, AC. 2008, "Collaborative Synthesis of Visual Analytic Results." In IEEE Symposium on Visual Analytics Science \& Technology, pp. 67-74.

Visual analytic tools allow analysts to generate large collections of useful analytical results. We anticipate that analysts in most real world situations will draw from these collections when working 
together to solve complicated problems. This indicates a need to understand how users synthesize multiple collections of results. This paper reports the results of collaborative synthesis experiments conducted with expert geographers and disease biologists. Ten participants were worked in pairs to complete a simulated real-world synthesis task using artifacts printed on cards on a large, paper-covered workspace. Experiment results indicate that groups use a number of different approaches to collaborative synthesis, and that they employ a variety of organizational metaphors to structure their information. It is further evident that establishing common ground and role assignment are critical aspects of collaborative synthesis. We conclude with a set of general design guidelines for collaborative synthesis support tools.

Robinson, AC, and G Center. 2007, "Synthesizing Results in Geovisualization." In 23rd International Cartographic Conference, pp. 1-9.

The transition from geovisual analysis to presentation involves an intermediate stage called synthesis. In this stage of analysis, analysts collect and combine results to generalize their discoveries. Despite its presence in most theories of geovisualization design, synthesis is a topic that has remained largely unexplored in geovisualization research. This paper examines the use of synthesis in geovisualization theory and the definitions that have been used to describe it as a stage of analytical work. Several potential interface metaphors are proposed for supporting synthesis. These designs are inspired by tools and techniques that are commonly used by analysts outside of current geovisualization environments. Finally, a set of key research questions are suggested for future work to understand and characterize geovisual synthesis.

Robinson, AC, et al. 2007, "Geovisual Analytics and Crisis Management." In 4th International ISCRAM Conference, pp. 173-79.

Increasing data heterogeneity, fragmentation and volume, coupled with complex connections among specialists in disaster response, mitigation, and recovery situations demand new approaches for information technology to support crisis management. Advances in visual analytics tools show promise to support time-sensitive collaboration, analyti-cal reasoning, problem solving and decision making for crisis management. Furthermore, as all crises have geospa-tial components, crisis management tools need to include geospatial data representation and support for geographic contextualization of location-specific decision-making throughout the crisis. This paper provides an introduction to and description of Geovisual Analytics applied to crisis management activity. The goal of Geovisual Analytics in this context is to support situational awareness, problem solving, and decision making using highly interactive, vis-ual environments that integrate multiple data sources that include georeferencing. We use an emergency support function example to discuss how recent progress in Geovisual Analytics can address the issues a crisis can present.

Robinson, AC, and C Weaver. 2006, "Re-Visualization : Interactive Visualization of the Process of Visual Analysis." In Workshop on Visual Analytics and Spatial Decision Support at GIScience 2006, pp. $1-21$.

Despite the analytical power of geographic visualization, it remains difficult for users of these tools to save, re-use, or re-visit what they have discovered. Most current systems are limited to providing screen captures and can only save basic data and layout settings. Since the interactivity of geovisualization is so critical to its utility, there is currently a need for new methods to capture and make use of this rich set of interactions. This paper describes Re-Visualization, a method that can capture and re-use analysis 
sessions. Low-level state changes are collected during analysis and saved into sessions. These sessions can then be replayed in a way that allows users to review and explore their interactions. During analysis, users can leave breadcrumbs behind that mark portions of the analysis for follow-up. We outline design guidelines for a toolkit called ReVise that implements Re-Visualization. Finally, we describe our substantial progress toward implementing ReVise in the Improvise visualization environment.

Rodgers, J. 2010, "Poster : Visualizing Residential Resource Use : A Framework for Design." In IEEE International Conference on Information Visualization.

Rodgers, P. 2005, "Chapter 7 - Graph Drawing Techniques for Geographic Visualization." in Exploring Geovisualization, eds. D Jason, MM Alan and K Menno-Jan, pp. 143-58. Elsevier, Oxford.

Summary Complex data consisting of related items often occurs in Geography, and is frequently represented as a graph. Examples include travel routes, regional borders, population flow and commerce. The field of graph drawing provides an effective group of techniques to tackle the problem of visualizing such data. This chapter provides a broad background to graph drawing methods and discusses possible future applications of this area to geovisualization.

Rodrigues, JF, et al. 2006, "Reviewing Data Visualization: An Analytical Taxonomical Study." In IEEE International Conference on Information Visualization, pp. 713-20.

This paper presents an analytical taxonomy that can suitably describe, rather than simply classify, techniques for data presentation. Unlike previous works, we do not consider particular aspects of visualization techniques, but their mechanisms and foundational vision perception. Instead of just adjusting visualization research to a classification system, our aim is to better understand its process. For doing so, we depart from elementary concepts to reach a model that can describe how visualization techniques work and how they convey meaning

Rogers, Y, and S Price. 2009, "Chapter 1 - How Mobile Technologies Are Changing the Way Children Learn." in Mobile Technology for Children, pp. 1-22. Morgan Kaufmann, Boston.

Rojo, C, W Xu, and K Mueller. 2010, "Street Light View : Enriching Navigable Panoramic Street View Maps with Informative Illumination Thumbnails." In IEEE International Conference on Information Visualization, pp. 1-2.

Roman, GC, and KC Cox. 1993, "A Taxonomy of Program Visualization Systems." Computer 26(12):1124.

Roman, P, M Lazarov, and A Majumder. 2010, "A Scalable Distributed Paradigm for Multi-User Interaction with Tiled Rear Projection Display Walls." IEEE Transactions on Visualization and Computer Graphics 16(6):1623-32.

We present the first distributed paradigm for multiple users to interact simultaneously with large tiled rear projection display walls. Unlike earlier works, our paradigm allows easy scalability across different applications, interaction modalities, displays and users. The novelty of the design lies in its distributed nature allowing well-compartmented, application independent, and application specific modules. This enables adapting to different 2D applications and interaction modalities easily by changing a few application specific modules. We demonstrate four challenging 2D applications on a nine projector 
display to demonstrate the application scalability of our method: map visualization, virtual graffiti, virtual bulletin board and an emergency management system. We demonstrate the scalability of our method to multiple interaction modalities by showing both gesture-based and laser-based user interfaces. Finally, we improve earlier distributed methods to register multiple projectors. Previous works need multiple patterns to identify the neighbors, the configuration of the display and the registration across multiple projectors in logarithmic time with respect to the number of projectors in the display. We propose a new approach that achieves this using a single pattern based on specially augmented QR codes in constant time. Further, previous distributed registration algorithms are prone to large misregistrations. We propose a novel radially cascading geometric registration technique that yields significantly better accuracy. Thus, our improvements allow a significantly more efficient and accurate technique for distributed self-registration of multi-projector display walls.

Romero, C, and S Ventura. 2007, "Educational Data Mining: A Survey from 1995 to 2005." Expert Systems with Applications 33(1):135-46.

Currently there is an increasing interest in data mining and educational systems, making educational data mining as a new growing research community. This paper surveys the application of data mining to traditional educational systems, particular web-based courses, well-known learning content management systems, and adaptive and intelligent web-based educational systems. Each of these systems has different data source and objectives for knowledge discovering. After preprocessing the available data in each case, data mining techniques can be applied: statistics and visualization; clustering, classification and outlier detection; association rule mining and pattern mining; and text mining. The success of the plentiful work needs much more specialized work in order for educational data mining to become a mature area.

Romero, C, S Ventura, and E García. 2008, "Data Mining in Course Management Systems: Moodle Case Study and Tutorial." Computers \& Education 51(1):368-84.

Educational data mining is an emerging discipline, concerned with developing methods for exploring the unique types of data that come from the educational context. This work is a survey of the specific application of data mining in learning management systems and a case study tutorial with the Moodle system. Our objective is to introduce it both theoretically and practically to all users interested in this new research area, and in particular to online instructors and e-learning administrators. We describe the full process for mining e-learning data step by step as well as how to apply the main data mining techniques used, such as statistics, visualization, classification, clustering and association rule mining of Moodle data. We have used free data mining tools so that any user can immediately begin to apply data mining without having to purchase a commercial tool or program a specific personalized tool.

Rook, FW, and ML Donnell. 1993, "Human Cognition and the Expert System Interface: Mental Models and Inference Explanations." IEEE Transactions on Systems, Man, and Cybernetics 23(6):1649-61.

Rosario, M Hammar, and V Scarano. 2005, "A System for Virtual Directories Using Euler Diagrams." Electronic Notes in Theoretical Computer Science 134:33-53.

In this paper, we describe how to use Euler Diagrams to represent virtual directories. i.e., collection of files that are computed on demand and satisfy a number of constraints. We, then, briefly describe the state of VennFS project that is currently modified to include this new capability. In particular, we show a data structure designed to answer queries about a given Euler Diagram and its sets. The data structure 
EulerTree described here is based on the R-Tree (see [Pankaj K. Agarwal, Mark de Berg, Joachim Gudmundsson, Mikael Hammar and Herman J. Haverkort, Box-trees and R-trees with near-optimal query time, in: Symposium on Computational Geometry, 2001, pp. 124-133]), a data structure designed for answering range queries over a family of shapes in the 2-dimensional space.

Rosario, GE, et al. 2004, "Mapping Nominal Values to Numbers for Effective Visualization." Information Visualization 3(2):80-95.

Data sets with a large number of nominal variables, some with high cardinality, are becoming increasingly common and need to be explored. Unfortunately, most existing visual exploration displays are designed to handle numeric variables only. When importing data sets with nominal values into such visualization tools, most solutions to date are rather simplistic. Often, techniques that map nominal values to numbers do not assign order or spacing among the values in a manner that conveys semantic relationships. Moreover, displays designed for nominal variables usually cannot handle high cardinality variables well. This paper addresses the problem of how to display nominal variables in general-purpose visual exploration tools designed for numeric variables. Specifically, we investigate (1) how to assign order and spacing among the nominal values, and (2) how to reduce the number of distinct values to display. We propose that nominal variables be pre-processed using a distance-quantification-classing (DQC) approach before being imported into a visual exploration tool. In the distance step, we identify a set of independent dimensions that can be used to calculate the distance between nominal values. In the quantification step, we use the independent dimensions and the distance information to assign order and spacing among the nominal values. In the classing step, we use results from the previous steps to determine which values within a variable are similar to each other and thus can be grouped together. Each step in the DQC approach can be accomplished by a variety of techniques. We extended the XmdvTool package to incorporate this approach. We evaluated our approach on several data sets using a variety of evaluation measures.

Rose, AF, et al. 2005, "Using Qualitative Studies to Improve the Usability of an Emr." Journal of Biomedical Informatics 38(1):51-60.

The adoption of electronic medical records (EMRs) and user satisfaction are closely associated with the system's usability. To improve the usability of a results management module of a widely deployed web-based EMR, we conducted two qualitative studies that included multiple focus group and field study sessions. Qualitative research can help focus attention on user tasks and goals and identify patterns of care that can be visualized through task modeling exercises. Findings from both studies raised issues with the amount and organization of information in the display, interference with workflow patterns of primary care physicians, and the availability of visual cues and feedback. We used the findings of these studies to recommend design changes to the user interface of the results management module.

Rossi, F, and N Villa-Vialaneix. 2010, "Optimizing an Organized Modularity Measure for Topographic Graph Clustering: A Deterministic Annealing Approach." Neurocomputing 73(7-9):1142-63.

This paper proposes an organized generalization of Newman and Girvan's modularity measure for graph clustering. Optimized via a deterministic annealing scheme, this measure produces topologically ordered graph clusterings that lead to faithful and readable graph representations based on clustering induced graphs. Topographic graph clustering provides an alternative to more classical solutions in which a standard graph clustering method is applied to build a simpler graph that is then represented with a 
graph layout algorithm. A comparative study on four real world graphs ranging from 34 to 1133 vertices shows the interest of the proposed approach with respect to classical solutions and to self-organizing maps for graphs.

Rosson, MB, and JM Carroll. 2002, "Chapter 4 - Information Design." in Usability Engineering: Scenario-Based Development of Human-Computer Interaction, pp. 109-57. Morgan Kaufmann, San Francisco.

Roth, EM, et al. 2002, "Scenario Development for Decision Support System Evaluation." In Annual Meeting of the Human Factors and Ergonomics Society, pp. 357-61.

This paper introduces a methodology for developing scenarios representative of the cognitive and collaborative challenges inherent in a domain of practice for evaluating decision support systems (DSS). Explicit links are made between particular aspects of the DSS and specific cognitive and collaborative demands they are intended to support. The effectiveness of the DSS in supporting performance can then be systematically probed by creating scenarios that are informed by an understanding of individual and team cognitive processing factors, fundamental relationships within the domain, and known complicating factors that can arise in the domain to challenge cognitive and collaborative performance. This paper introduces a set of explicit artifacts to systematically create such scenarios to provide feedback on the viability of the DSS design concepts (e.g., are the hypothesized positive impacts of the DSS realized?), as well as feedback on additional unanticipated requirements for support

Roth, EM, et al. 1987, "Providing on-Line Advice for a Dynamic Control Task: A Case Study in Intelligent Support System Design." In 31st Annual Meeting of the Human Factors Society, pp. 36-40.

The article describes an intelligent manual feedwater control station (IMFCS) that provides on-line expert guidance for a process control task: control of feedwater during power plant startup and operation. The IFMCS provides control action advice derived from analysis of the performance of expert operators. It represents a novel approach to advisory systems in that (a) the IFMCS does not attempt to replicate in detail the surface activities of domain experts, but rather abstracts the cognitive competencies required for expert performance and provides a domain representation that promotes those competencies; (b) the advice takes an analogue graphic display form; (c) a common frame of reference is established between machine advisor and human practitioner that integrates advise and 'explanation' avoiding problems associated with opaque advice

Roth, RE, et al. 2008, "Web-Based Geovisualization and Geocollaboration: Applications to Public Health." In 2008 Joint Statistical Meeting, Invited Session on Web Mapping, pp. 2-5.

This paper describes the design and implementation of three web-based geovisualization and geocollaboration applications developed for the domain of public health. Each was implemented using Web 2.0 architecture. First, the Pennsylvania Cancer Atlas is a web-based geovisualization tool for the exploration of countylevel cancer incidence rates using multiple interactive and live-linked statistical representations. Second, the Health GeoJunction is a web-based geovisualization and geocollaboration tool for geographic, temporal, and attribute filtering of articles from the PubMed medical research database. Finally, the Geo-Explication Web Portal is a webbased geocollaboration tool for the annotation and dissemination of geovisualization tools, training materials, and analysis artifacts collected from the application of geovisualization tools and techniques. 
Rousseau, R, et al. 2005, "Decision-Centered Testing (Dct): Evaluating Joint Human-Computer Cognitive Work." In 49th Annual Meeting of the Human Factors and Ergonomics Society, pp. 322-26.

In order to test the effectiveness of a human operator paired with a decision support system, it is necessary to complement current testing practices addressing software validation, human performance, and usability. Decision Centered Testing (DCT) aims at testing the effectiveness of operators teamed with Decision Support Systems (DSS) in any challenging work domain. DCT is grounded in a Cognitive Systems Engineering (CSE) framework, where the concept of a joint cognitive system (JCS) is central. DCT aims at evaluating the decision-making effectiveness across identified 'error prone' regions in the JCS structure. A description of the DCT Methodology with an illustration taken from an initial application of the methodology is presented. In this application, insights from the DCT methodology enabled the definition of appropriate test metrics and the construction of unique test scenarios to exercise the decision-making effectiveness. From this application, it can be concluded that following the DCT Methodology facilitated the construction of an evaluation framework for assessing JCS net decisionmaking effectiveness.

Roussinov, DG, and H Chen. 1999, "Document Clustering for Electronic Meetings: An Experimental Comparison of Two Techniques." Decision Support Systems 27(1-2):67-79.

In this article, we report our implementation and comparison of two text clustering techniques. One is based on Ward's clustering and the other on Kohonen's Self-organizing Maps. We have evaluated how closely clusters produced by a computer resemble those created by human experts. We have also measured the time that it takes for an expert to "clean up" the automatically produced clusters. The technique based on Ward's clustering was found to be more precise. Both techniques have worked equally well in detecting associations between text documents. We used text messages obtained from group brainstorming meetings.

Ruan, H, et al. 2010, "Exploring the Inventor' S Paradox : Applying Jigsaw to Software Visualization." In 5th ACM Symposium on Software Visualization (SOFTVIS), pp. 83-92.

Rudolph, S, A Savikhin, and DS Ebert. 2009, "Finvis: Applied Visual Analytics for Personal Financial Planning." In IEEE Symposium on Visual Analytics Science \& Technology, pp. 195-202.

FinVis is a visual analytics tool that allows the non-expert casual user to interpret the return, risk and correlation aspects of financial data and make personal finance decisions. This interactive exploratory tool helps the casual decision-maker quickly choose between various financial portfolio options and view possible outcomes. FinVis allows for exploration of inter-temporal data to analyze outcomes of short-term or long-term investment decisions. FinVis helps the user overcome cognitive limitations and understand the impact of correlation between financial instruments in order to reap the benefits of portfolio diversification. Because this software is accessible by non-expert users, decision-makers from the general population can benefit greatly from using FinVis in practical applications. We quantify the value of FinVis using experimental economics methods and find that subjects using the FinVis software make better financial portfolio decisions as compared to subjects using a tabular version with the same information. We also find that FinVis engages the user, which results in greater exploration of the dataset and increased learning as compared to a tabular display. Further, participants using FinVis reported increased confidence in financial decision-making and noted that they were likely to use this tool in practical application. 
Ruppert, T. 2010, "A Radial Visualization Tool for Depicting Hierarchically Structured Video Content." In IEEE Symposium on Visual Analytics Science \& Technology, pp. 251-52.

Russell, AD, C-Y Chiu, and T Korde. 2009, "Visual Representation of Construction Management Data." Automation in Construction 18(8):1045-62.

Construction projects are associated with voluminous and often unstructured data sets, generated in support of construction management functions. Project managers face the challenge of making meaningful deductions from this data. A central contribution of this paper is that visual analytics can provide a means of analyzing data from various dimensions of a project to extract information in aid of decision making and helping to explain reasons for performance to date. Questions posed relate to the role of visual analytics in the execution and post-construction phases of a project, data representations and transformations of specific interest, and the kinds of visual representations and interactions that provide useful insights to management personnel, help explain performance, or assist with communication. Principles of designing effective visual analytics solutions for various construction management functions and applicable to the associated analytic reasoning tasks, data representations and transformations, and visual representations including relevant interaction features are described. Emphasis is placed on the choice of visual representations along with discussion of approaches for validating the usefulness of the visual analytics solutions proposed. The notion of context dimensions and performance dimensions for representing construction projects is introduced as part of the formulation of visual representation designs. To demonstrate the application of the concepts presented, data sets from two different projects were used to produce visual representations helpful for analytical reasoning about change order management data. A detailed assessment is given of several of the images presented in terms of strengths and weaknesses, and interaction features desired. The findings of the paper in terms of principles, concepts and lessons learned should prove helpful to those wishing to apply visual analytics to a broad range of construction management functions.

Russell, DM, et al. 2009, "Sensemaking Workshop Chi 2009." In 27th International Conference Extended Abstracts on Human Factors in Computing Systems pp. 4751-54.

How does one make sense of a large or complex task? By the term-sensemaking? we mean the processes people go through to frame, collect, organize and structure information to help understand a problem. Sensemaking is what people do to get from the earliest phases of an information collecting and organizing task to the conclusion. Sensemaking tasks are commonplace, and this workshop is dedicated to understanding the range of sensemaking behaviors and systems that can support sensemaking.

Russell, S, A Gangopadhyay, and V Yoon. 2008, "Assisting Decision Making in the Event-Driven Enterprise Using Wavelets." Decision Support Systems 46(1):14-28.

This paper discusses issues related to data-driven decision support systems in event-driven enterprises and proposes Discrete Wavelet Transformation (DWT) as a method to improve these systems. DWT is proposed as a method of data reduction that reduces the effects of excessive data, enabling better visualization and scalability, while preserving patterns, trends, and surprises in the data. A procedural model for using a data-driven decision support system that integrates DWT is presented. Finally, the procedural model is evaluated in experiments based on real event-driven data from a large telecommunications company. 
Rusu, A, and R Docimo. 2009, "Securing the Web Using Human Perception and Visual Object Interpretation." In 4th Information Visualization Conference, pp. 613-18.

Being motivated by the need for usable Web security systems, we present in this paper new imagebased CAPTCHA systems that overcome the weaknesses of commercial CAPTCHAs. We describe Tree and Shape CAPTCHAs in addition to handwritten CAPTCHAs that feature visual objects transformed according to specific principles of cognitive psychology. These transformations, in addition to other security measures we describe, ensure that our CAPTCHAs are easily interpretable by humans but not by machines. We seek not only to provide useful CAPTCHAs, but to offer important insights into such fields as Information Visualization, Human-Computer Interaction, etc. Early testing results indicate that users find our CAPTCHAs an attractive alternative to text-based CAPTCHAs and are readily able to solve them based on cognitive abilities. Conversely, machine recognition is low due to the current inability to make use of these cognitive aids, thus making our CAPTCHAs a viable solution for cyber security.

Ruthruff, JR, et al. 2005, "Interactive, Visual Fault Localization Support for End-User Programmers." Journal of Visual Languages \& Computing 16(1-2):3-40.

End-user programmers are writing an unprecedented number of programs, primarily using languages and environments that incorporate a number of interactive and visual programming techniques. To help these users debug these programs, we have developed an entirely visual, interactive approach to fault localization. This paper presents the approach. We also present the results of a think-aloud study that examined interactive, human-centric issues that arise in end-user debugging using a fault localization approach. Our results provide insights into the contributions such approaches can make to the end-user debugging process.

Saad, A, G Hamarneh, and T Möller. 2010, "Exploration and Visualization of Segmentation Uncertainty Using Shape and Appearance Prior Information." IEEE Transactions on Visualization and Computer Graphics 16(6):1366-75.

We develop an interactive analysis and visualization tool for probabilistic segmentation in medical imaging. The originality of our approach is that the data exploration is guided by shape and appearance knowledge learned from expert-segmented images of a training population. We introduce a set of multidimensional transfer function widgets to analyze the multivariate probabilistic field data. These widgets furnish the user with contextual information about conformance or deviation from the population statistics. We demonstrate the user's ability to identify suspicious regions (e.g., tumors) and to correct the misclassification results. We evaluate our system and demonstrate its usefulness in the context of static anatomical and time-varying functional imaging datasets.

Sadeghian, P, et al. 2006, "The Frequent Wayfinding-Sequence (Fws) Methodology: Finding Preferred Routes in Complex Virtual Environments." International Journal of Human-Computer Studies 64(4):35674.

Advances in computing techniques, as well as the reduction in the cost of technology, have made possible the viability and spread of complex virtual environments (VEs). However, efficient navigation within these environments remains problematic for the user. Several research projects have shown that users of VEs are often disoriented and have extreme difficulty completing navigational tasks.

Furthermore, there is often more than one route to get to a specified destination. Novice users often lack 
the spatial knowledge needed to pick an appropriate route due to the deficiency of experience with the system. A number of navigation tools such as maps, 3-D thumbnails, trails, and personal agents have been proposed. The introduction of these tools have met with some degree of success, but most researchers agree that new techniques need to be developed to aid users efficiently navigate within complex VEs. In this paper, we propose the frequent wayfinding-sequence (FWS) methodology that uses a modified sequence mining technique to discover a model of routes taken by experienced users of a VE. The model is used to build an interface that provides navigation assistance to novice users by recommending routes. We conducted both real world and simulation experiments using our methodology. Results from the real world experiment suggest that the FWS approach has the potential to improve the user's navigation performance and the quality of the human-computer interaction. Our simulation studies showed that our approach is scalable, efficient, and able to find useful route models for complex VEs.

Saito, T, et al. 2005, "Two-Tone Pseudo Coloring: Compact Visualization for One-Dimensional Data." In IEEE Symposium on Information Visualization, pp. 173-80.

A new pseudo coloring technique for large scale one-dimensional datasets is proposed. For visualization of a large scale dataset, user interaction is indispensable for selecting focus areas in the dataset. However, excessive switching of the visualized image makes it difficult for the user to recognize overview/ detail and detail/ detail relationships. The goal of this research is to develop techniques for visualizing details as precisely as possible in overview display. In this paper, visualization of a onedimensional but very large dataset is considered. The proposed method is based on pseudo coloring, however, each scalar value corresponds to two discrete colors. By painting with two colors at each value, users can read out the value precisely. This method has many advantages: it requires little image space for visualization; both the overview and details of the dataset are visible in one image without distortion; and implementation is very simple. Several application examples, such as meteorological observation data and train convenience evaluation data, show the effectiveness of the method.

Sakamoto, R, and S Kunifuji. 2000, "Collaborative World Wide Web Browsing System through Supplement of Awareness." In 4th International Conference on Knowledge-Based Intelligent Engineering Systems and Allied Technologies, pp. 233-36.

The difficulty in finding required information on the hyper-media is widely recognized. The awareness in the computer mediated communication is also considered to be important for collaborative work. We propose in this paper a collaborative browsing system that supplements awareness information and try to resolve the difficulty of information retrieval by inquiring and monitoring other users, which are the actions frequently taken when a user is faced with a problem. In this study, we built and evaluated prototype to see the system is effective. As the result of the evaluation, we found that the environment in which we can get information of WWW browsing awareness enables us to feel the others' presence and to grasp what the others are now doing. Additionally, many subjects answered that they could browse the web pages, which they did not know before. They also answered that they could increase the efficiency of browsing. We make a proposal about this collaboration browsing and inform results of experiments on its availability. 
Salom, P, et al. 2009, "Dynamic Picking System for 3d Seismic Data: Design and Evaluation." International Journal of Human-Computer Studies 67(7):551-60.

In the framework of data interpretation for petroleum exploration, this paper contributes two contributions for visual exploration aiming to manually segment surfaces embedded in volumetric data. Resulting from a user-centered design approach, the first contribution, dynamic picking, is a new method of viewing slices dedicated to surface tracking, i.e., fault-picking, from 3D large seismic data sets. The proposed method establishes a new paradigm of interaction breaking with the conventional 2D slices method usually used by geoscientists. Based on the $2 \mathrm{D}+$ time visualization method, dynamic picking facilitates localizing of faults by taking advantage of the intrinsic ability of the human visual system to detect dynamic changes in textured data. The second, projective slice, is a focus +context visualization technique that offers the advantage of facilitating the anticipation of upcoming slices over the sloping 3D surface. From the reported experimental results, dynamic picking leads to a good compromise between fitting precision and completeness of picking while the projective slice significantly reduces the amount of workload for an equivalent level of precision.

Samiei, M, J Dill, and A Kirkpatrick. 2004, "Ezmail: Using Information Visualization Techniques to Help Manage Email." In 8th International Conference on Information Visualisation, pp. 477-82.

Originally designed as a messaging application, email is now used for a wide range of functions. Email clients however have not progressed correspondingly to support users with email management. This paper describes the design of EzMail, an email visualization tool that runs in conjunction with an email client and creates a multi-view interface to assist with email management. Our primary focus is to group and visualize messages as components of threads to provide contextual information and conversational history. We have developed visualizations for individual messages, message properties, and messages in a thread. Message annotation is incorporated, along with a capability for replying simultaneously to several messages. A small user survey and a user study provided information regarding users' management of their messages and threads, and a comparative evaluation of EzMail with respect to Microsoft Outlook.

Sanderson, A, et al. 2010, "Analysis of Recurrent Patterns in Toroidal Magnetic Fields." IEEE Transactions on Visualization and Computer Graphics 16(6):1431-40.

In the development of magnetic confinement fusion which will potentially be a future source for low cost power, physicists must be able to analyze the magnetic field that confines the burning plasma. While the magnetic field can be described as a vector field, traditional techniques for analyzing the field's topology cannot be used because of its Hamiltonian nature. In this paper we describe a technique developed as a collaboration between physicists and computer scientists that determines the topology of a toroidal magnetic field using fieldlines with near minimal lengths. More specifically, we analyze the Poincar\&\#x00E9; map of the sampled fieldlines in a Poincar\&\#x00E9; section including identifying critical points and other topological features of interest to physicists. The technique has been deployed into an interactiveparallel visualization tool which physicists are using to gain new insight into simulations of magnetically confined burning plasmas. 
Sanfilippo, A, et al. 1999, "Building a Human Information Discourse Interface to Uncover Scenario Content." In 2005 International Conference on Intelligence Analysis, pp. 1-6.

Software environments for intelligence analysis need to leverage scenario-based analytical processes to help analysts achieve a more comprehensive view of competing hypotheses in their risk assessments. While current solutions offer some of the tools and functionality required for such advancement, there still is no integrated set of capabilities that addresses the extraction and manipulation of scenario content from unstructured intelligence data sources. We propose to fill this gap through the development of a visual interactive environment for event analytics that integrates text mining, discourse analysis and visualization capabilities to support scenario manipulation and generation processes.

Sanfilippo, A, et al. 2007, "A Layered Dempster-Shafer Approach to Scenario Construction and Analysis." In IEEE Intelligence and Security Informatics, pp. 95-102.

Sanfilippo, a, et al. 2005, "An Adaptive Visual Analytics Platform for Mobile Devices." In ACM/IEEE Supercomputing Conference, pp. 74-74.

We present the design and implementation of InfoStar, an adaptive visual analytics platform for mobile devices such as PDAs, laptops, Tablet PCs and mobile phones. InfoStar extends the reach of visual analytics technology beyond the traditional desktop paradigm to provide ubiquitous access to interactive visualizations of information spaces. These visualizations are critical in addressing the knowledge needs of human agents operating in the field, in areas as diverse as business, homeland security, law enforcement, protective services, emergency medical services and scientific discovery. We describe an initial real world deployment of this technology, in which the InfoStar platform has been used to offer mobile access to scheduling and venue information to conference attendees at Supercomputing 2004.

Santos, BS, et al. 2004, "Visual Application for the Analysis of Web-Based Information Systems Usage: A Preliminary Usability Evaluation." In 8th International Conference on Information Visualisation, pp. 812-18.

This paper presents a general description of the methods used in the on-going evaluation of a Visualizer, which is a sub-component of the Web Log Visual Analysis System. We are trying to evaluate some aspects of the user interface and visualization techniques implemented as part of the prototype. Observation and querying techniques were used with two types of users. A general description of those users and methods is presented Preliminary results were encouraging and provided new ideas and information that will, eventually, allow a more complete and formal evaluation of our application.

Sanyal, J, et al. 2010, "Noodles: A Tool for Visualization of Numerical Weather Model Ensemble Uncertainty." IEEE Transactions on Visualization and Computer Graphics 16(6):1421-30.

Numerical weather prediction ensembles are routinely used for operational weather forecasting. The members of these ensembles are individual simulations with either slightly perturbed initial conditions or different model parameterizations, or occasionally both. Multi-member ensemble output is usually large, multivariate, and challenging to interpret interactively. Forecast meteorologists are interested in understanding the uncertainties associated with numerical weather prediction; specifically variability between the ensemble members. Currently, visualization of ensemble members is mostly accomplished 
through spaghetti plots of a single mid-troposphere pressure surface height contour. In order to explore new uncertainty visualization methods, the Weather Research and Forecasting (WRF) model was used to create a 48-hour, 18 member parameterization ensemble of the 13 March 1993 "Superstorm". A tool was designed to interactively explore the ensemble uncertainty of three important weather variables: watervapor mixing ratio, perturbation potential temperature, and perturbation pressure. Uncertainty was quantified using individual ensemble member standard deviation, inter-quartile range, and the width of the $95 \%$ confidence interval. Bootstrapping was employed to overcome the dependence on normality in the uncertainty metrics. A coordinated view of ribbon and glyph-based uncertainty visualization, spaghetti plots, iso-pressure colormaps, and data transect plots was provided to two meteorologists for expert evaluation. They found it useful in assessing uncertainty in the data, especially in finding outliers in the ensemble run and therefore avoiding the WRF parameterizations that lead to these outliers. Additionally, the meteorologists could identify spatial regions where the uncertainty was significantly high, allowing for identification of poorly simulated storm environments and physical interpretation of these model issues.

Saraiya, P, C North, and K Duca. 2010, "Comparing Benchmark Task and Insight Evaluation Methods on Timeseries Graph Visualizations." In BELIV'10: Beyond time and errors: novel evaluation methods for Information Visualization, April 10-11, 2010.

A study to compare two different empirical research methods for evaluating visualization tools is described: the traditional benchmark-task method and the insight method. The methods were compared using different criteria such as: the conclusions about the visualization tools provided by each method, the time participants spent during the study, the time and effort required to analyze the resulting empirical data, and the effect of individual differences between participants on the results. The studies used three graph visualization alternatives to associate bioinformatics microarray timeseries data to pathway graph vertices, based on popular approaches used in existing bioinformatics software.

Saraiya, P, C North, and K Duca. 2004, "An Evaluation of Microarray Visualization Tools for Biological Insight." In IEEE Symposium on Information Visualization, pp. 1-8.

High-throughput experiments such as gene expression microarrays in the life sciences result in large datasets. In response, a wide variety of visualization tools have been created to facilitate data analysis. Biologists often face a dilemma in choosing the best tool for their situation. The tool that works best for one biologist may not work well for another due to differences in the type of insight they seek from their data. A primary purpose of a visualization tool is to provide domain-relevant insight into the data. Ideally, any user wants maximum information in the least possible time. In this paper we identify several distinct characteristics of insight that enable us to recognize and quantify it. Based on this, we empirically evaluate five popular microarray visualization tools. Our conclusions can guide biologists in selecting the best tool for their data, and computer scientists in developing and evaluating visualizations

Saraiya, P, C North, and K Duca. 2005, "An Insight-Based Methodology for Evaluating Bioinformatics Visualizations." IEEE Transactions on Visualization and Computer Graphics 11(4):443-56.

High-throughput experiments, such as gene expression microarrays in the life sciences, result in very large data sets. In response, a wide variety of visualization tools have been created to facilitate data analysis. A primary purpose of these tools is to provide biologically relevant insight into the data. Typically, visualizations are evaluated in controlled studies that measure user performance on 
predetermined tasks or using heuristics and expert reviews. To evaluate and rank bioinformatics visualizations based on real-world data analysis scenarios, we developed a more relevant evaluation method that focuses on data insight. This paper presents several characteristics of insight that enabled us to recognize and quantify it in open-ended user tests. Using these characteristics, we evaluated five microarray visualization tools on the amount and types of insight they provide and the time it takes to acquire it. The results of the study guide biologists in selecting a visualization tool based on the type of their microarray data, visualization designers on the key role of user interaction techniques, and evaluators on a new approach for evaluating the effectiveness of visualizations for providing insight. Though we used the method to analyze bioinformatics visualizations, it can be applied to other domains.

Saraiya, P, et al. 2006, "An Insight-Based Longitudinal Study of Visual Analytics." IEEE Transactions on Visualization and Computer Graphics 12(6):1511-22.

Visualization tools are typically evaluated in controlled studies that observe the short-term usage of these tools by participants on preselected data sets and benchmark tasks. Though such studies provide useful suggestions, they miss the long-term usage of the tools. A longitudinal study of a bioinformatics data set analysis is reported here. The main focus of this work is to capture the entire analysts process that an analyst goes through from a raw data set to the insights sought from the data. The study provides interesting observations about the use of visual representations and interaction mechanisms provided by the tools, and also about the process of insight generation in general. This deepens our understanding of visual analytics, guides visualization developers in creating more effective visualization tools in terms of user requirements, and guides evaluators in designing future studies that are more representative of insights sought by users from their data sets.

Saraiya, PB. 2006, "Insight-Based Studies for Pathway and Microarray Visualization Tools." Vol PhD, pp. 198-98. Virginia Polytechnic Institute and State University.

Pathway diagrams, similar to the graph diagrams using a node-link representation, are used by biologists to represent complex interactions at the molecular level in living cells. The recent shift towards data-intensive bioinformatics and systems-level science has created a strong need for advanced pathway visualization tools that support exploratory data analysis. User studies suggest that an important requirement for biologists is the need to associate microarray data to pathway diagrams. A design space for visualization tools that allow analysis of microarray data in pathway context was identified for a systematic evaluation of the visualization alternatives. The design space is divided into two dimensions. Dimension 1 is based on the method used to overlay data attributes onto pathway nodes. The three possible approaches are: overlay of data on pathway nodes one data attribute at a time by manipulating a visual property (e.g., color) of the node, along with sliders or some such mechanism to animate the pathway for other timepoints. In another approach data from all the attributes in data can be overlaid simultaneously by embedding small charts (e.g., line charts or heatmap) into pathway nodes. The third approach uses miniature version of the pathways-as-glyph view for each attribute in the data. Dimension 2 decides if additional view besides pathway diagrams were used. These pathway visualizations are often linked to other type of visualization methods (e.g., parallel co-ordinates) using the concept of brushing and linking. The visualization alternatives from pathway + microarray data design space were evaluated by conducting two independent user studies. Both the studies used timeseries datasets. The first study used visualization alternatives from both dimension 1 and dimension 2 . The results suggest that the method to overlay multidimensional data on pathway nodes has a non trivial influence on accuracy of participants responses, whereas the number of visualizations affect participants performance time for 
pre-selected tasks. The second study used visualization alternatives from dimension 1 that focuses on method used to overlay data attributes on pathway nodes. The study suggests that participants using pathway visualization that display data one attribute at a time on nodes have more controlled performance for all type of tasks as compared to the participants using other alternatives. Participants using pathway visualization that display data in node-as-glyphs view have better performance for tasks that require analysis for a single node, and identifying outlier nodes. Whereas, pathway visualizations with pathwaysas-glyph view provide better performance on tasks that require analysis of overall changes in the pathway, and identifying interesting timepoints in the data. An insight-based method was designed to evaluate visualization tools for real world biologists data analysis scenarios. The insight-based method uses different quantifiable characteristics of an insight that can be measured uniformly across participants. These characteristics were identified based on observations of the participants analyzing microarray data in a pilot study. The insight-based method provides an alternative to traditional task-based methods. This is especially helpful for evaluating visualization tools on large and complicated datasets where designing tasks can be difficult. Though, the insight-based method was developed to empirically evaluate visualization tools for short term studies, the method can also be used in real world longitudinal studies that analyzes the usage of visualization tools by the intended end-users.

Sarathy, S, K Shujaee, and K Cannon. 2000, "Visualization of Large Complex Datasets Using Virtual Reality." In International Conference on Information Technology: Coding and Computing, pp. 522-26.

Major advances in high performance computing software and hardware have made it possible for designers and analysts to obtain detailed information about the behavior of complex systems. However, this has lead to a proliferation of vast amounts of data which is beyond the ability of humans to easily comprehend using traditional visualization methods. The method presented combines emerging visualization tools with data filtering and data mining techniques. This method uses virtual reality (VR) coupled with multimedia techniques to display information within an environment that allows non-expert users to understand and interpret the data. A prototype visualization tool was developed under a NASA SBIR Phase II effort. This paper builds on the results of this work and suggests a visualization framework that can be used to examine, comprehend and interpret results in any complex data set, particularly those used in support of decision making activities

Sarter, NB, and DD Woods. 1991, "Situation Awareness: A Critical but Ill-Defined Phenomenon." International Journal of Aviation Psychology 1:45-57.

Satava, RM, and SB Jones. 1998, "Laparoscopic Surgery: Transition to the Future." Urologic Clinics of North America 25(1):93-102.

In no other time in history has such a rapid transition to the future occurred. What had been a century of evolution from the Industrial Age to the Information Age has, over the past decade, become a revolution. Laparoscopic surgery, which provided the "wake-up call to the information age" as the leading edge technology, has become the accepted standard of medical practice; now even more advanced technologies promise further improvements in the practice of medicine. Many authors have written about revolutionary times; however, there are revolutions and then there are revolutions. The first great revolution in medicine occurred in surgery in the late 1800s when the giants in medicine still strode the earth. Although many pioneers contributed to the final remodeling of medicine, there were few visionaries who truly understood the magnitude of change and were able to give birth to the new discipline of surgery. Among the more notable were Bilroth, Lister, Virchow, and Morton. This disparate 
group never worked together, but it was the spontaneous integration of their research and clinical skills that made modern surgery possible. It was the convergence of their visions and technologies over a short time that enabled surgery, not any single event. Bilroth brought the new skills and surgical instruments, Lister the asepsis, Virchow the pathology, and Moore the anesthesia. Without the synergy of all areas, modern surgery would not have occurred. Ancient myth, mysticism, and even some empirical fact over thousands of years of medicine had proved that the sanctity of the human body was inviolate to the knife-that a patient could not be operated upon and survive. Yet the scientific tools of the Industrial Age enabled the impossible, and science gave birth to surgery. In a relatively short period the foundations of surgery were laid for the next generation of pioneers to lead--the clinicians who exploited the technologies and advanced the art of surgery. There have been numerous startling discoveries since these early beginnings and many remarkable advances. These have been noteworthy in their own right, but none changed the entire foundation of medicine as did the inception of surgery. The understanding of shock, cardiopulmonary bypass and cardiac surgery, and transplantation have all had enormous impact on the practice of surgery, but they have fostered the development of a new niche, splintering off a new specialty without changing the fabric of medicine. Other new areas have been created, in infectious diseases and chemotherapy, yet these are evolutions of the ancient art of the pharmacopoeia. It is interesting, but obvious, that the changes that led to the birth of surgery were contingent on the discoveries that ushered in the Industrial Age. And just as the Industrial Age is waning, so too is the golden age of surgery. The Industrial Age is being replaced by the Information Age, and conventional surgery is being replaced by a host of minimally invasive therapies and noninvasive procedures. Because we are currently in the middle of this transition, it is unclear now how the next generation of medicine and surgery will appear, although trends in the technologies are toward low-power, miniaturized, low-cost yet highly "intelligent" systems that eventually will transform surgery from minimally invasive into noninvasive procedures whose development will depend on the emerging Information Age technologies. This is not to say that surgeons will no longer perform open or minimally invasive surgical procedures in the future, but rather that "conventional" surgery will recede to a niche, and noninvasive procedures will predominate. Laparoscopic (or minimal access) surgery is not an end-point; rather, it is a transitional phase between the radical approach of "open" surgery and the emerging forms of noninvasive imageguided procedures. But it was the seminal event of laparoscopic surgery that triggered the wake-up call to the Information Age--the realization that a revolution is occurring and that physicians must extend their hor zons to discover the direction toward the future. In order to have a revolution, all facets of a discipline must be affected, not just a single specialty such as surgery. A revolution must also reflect the same changes that are occurring in other scientific areas and in society as a whole. It must be consistent with the global predictions that are proffered, such as The Third Wave of Alvin Toffler,19 MegaTrends of John Naisbitt,13 and, most important, Being Digital of Nicholas Negroponte.14 The former two authors gave us a peek into the power and magnitude of the revolution, but it was Negroponte's concept of "bits instead of atoms" that completed the concept. He emphasized that what we did previously on a daily basis has required using actual physical objects (atoms); whereas the new technologies emphasize using information (bits) to accomplish the same task. His classic example is that up to and including the Industrial Age, information in documents and letters was mailed physically from point to point (sending atoms across the United States), whereas during the Information Age the same information is sent by fax (bits) at a much faster rate and at lower cost. In translating this to the medical world, I will refer to "information equivalents," which are electronic or digital representations of real physical world objects or actions. The magnitude of the importance of the Information Age for medicine was revealed during a National Science Foundation workshop on Medical Applications of Virtual Reality in 1994, where the question was asked, "How much of what a physician does on a daily basis is really information 
management?" If you use the most liberal interpretation, the answer is about $80 \%$ to $90 \%$. For example, during laparoscopic surgery, the surgeon looks not at the actual organs but at the video monitor (electronic image or information equivalent of the organs). When surgery is complete and the patient is visited in the recovery room, the surgeon glances at the physiologic monitor for the blood pressure, pulse, and other vital signs (equivalent of sense of touch). The visit is recorded into the electronic or computer medical record (rather than being written on paper). Radiographs, CT scans, and other images are all becoming digital (instead of film, microscope slides), and our entire surgical education process is incorporating computer-aided instruction, multimedia, and even virtual reality for simulation and training. Laboratory experiments in telesurgery have converted our hand motions to electronic signals, such that when the surgeon's hand moves, the electronic signal (information) is sent to the tip of the instrument, and the scalpel cuts. By making this mental leap of interacting with information as a substitute for real-world objects, the physician gains the capability to do things not possible in the physical world. For example, by using Doppler ultrasound to display the false color images on a video monitor we have given surgeons the long dreamed-of capability to "see into the body with x-ray vision," which in this case is ultrasound vision of actual blood flow. Although we usually view the images on a video monitor, Jonathan Prince 2 has created one of the first three-dimensional (3-D) true suspended holographic images (hologram) for anatomic visualization, and this will soon be a full 3-D representation of the human body. With the imaginative concept of information equivalents, the challenge is to discover in daily practice ways of enhancing capabilities for patient care. The potential of this innovative approach to medicine can be best illustrated by the results of a "blue sky" brainstorming session in late 1995. This rudimentary idea is referred to as the "Doorway to the Future" and touches upon how information equivalents tie together the fabric of medicine. The session was inspired by the many technologies under investigation, and integrated them into a meaningful system of complementary technologies. The following scenario, based on advanced technologies currently under investigation in the laboratory is used to illustrate how the future could be 20,50 , or perhaps 100 years from now. A patient enters a physician's office, passing through a doorway, the frame of which contains many scanning devices, from CT scan to MRI to ultrasound to near infrared and others. These scanners record anatomic, physiologic, and biochemical (like the pulse oximeters) data. When the patient sits down next to the physician, a full 3-D holographic image appears suspended on the desktop--a visual integration of the information acquired just a minute before by the scanners. When the patient expresses a complaint of pain over the right flank, the physician can rotate the image, remove various layers, and query the representation of the patient's liver or kidney about the LDH, SGOT, alkaline phosphatase, serum creatinine, or other relevant information. This information and more is stored in each pixel of the patient's representative image (a "medical avatar") such that the image of each structure and organ (such as the liver) stacks up into a "deep pixel" all the relevant information about the structure. Each pixel contains not only anatomic data but also biochemical, physiologic, and historical data, so that needed information can be found directly from the image without searching through volumes of written medical records or a prolonged computer database search. Should a problem or disease be discovered, the image can be used immediately for patient education, instantly explaining to the patient on their own avatar what the problem might be. If a surgical problem is discovered, this same image can be used by the surgeon for preoperative planning or imported into a surgical simulator to practice a variety of different approaches to a difficult surgical procedure that will be performed later. At the time 
Sato, S, K Misue, and J Tanaka. 2008, "Readable Representations for Large-Scale Bipartite Graphs." In 12th International Conference on Knowledge-Based Intelligent Information and Engineering Systems, pp. 831-38.

Bipartite graphs appear in various scenes in the real world., and visualizing these graphs helps improve our understanding of network structures. The amount of information that is available to us has increased dramatically in recent, years, and it is therefore necessary to develop a drawing technique that corresponds to large-scale graphs. fit this paper, we describe drawing methods to make large-scale bipartite graphs easy to read. We propose two techniques: "node contraction drawing", which involves collecting similar nodes and drawing them as one node, and "isosimilarity contour drawing," which puts clusters into all outlined area. We developed interactive user interfaces for the drawing methods and conducted all evaluation experiment to demonstrate the effectiveness of the proposed techniques.

Savikhin, A, R Maciejewski, and DS Ebert. 2008, "Applied Visual Analytics for Economic DecisionMaking." In IEEE Symposium on Visual Analytics Science \& Technology, pp. 107-14.

This paper introduces the application of visual analytics techniques as a novel approach for improving economic decision making. Particularly, we focus on two known problems where subjectspsila behavior consistently deviates from the optimal, the Winnerpsilas and Loserpsilas Curse. According to economists, subjects fail to recognize the profit-maximizing decision strategy in both the Winnerpsilas and Loserpsilas curse because they are unable to properly consider all the available information. As such, we have created a visual analytics tool to aid subjects in decision making under the Acquiring a Company framework common in many economic experiments. We demonstrate the added value of visual analytics in the decision making process through a series of user studies comparing standard visualization methods with interactive visual analytics techniques. Our work presents not only a basis for development and evaluation of economic visual analytic research, but also empirical evidence demonstrating the added value of applying visual analytics to general decision making tasks.

Scannapieco, M, et al. 2006, "Privacy Preserving Schema and Data Matching." In ACM SIGMOD international conference on Management of data, pp. 653-64.

In many business scenarios, record matching is performed across different data sources with the aim of identifying common information shared among these sources. However such need is often in contrast with privacy requirements concerning the data stored by the sources. In this paper, we propose a protocol for record matching that preserves privacy both at the data level and at the schema level. Specifically, if two sources need to identify their common data, by running the protocol they can compute the matching of their datasets without sharing their data in clear and only sharing the result of the matching. The protocol uses a third party, and maps records into a vector space in order to preserve their privacy. Experimental results show the efficiency of the matching protocol in terms of precision and recall as well as the good computational performance.

Schaal, S. 2010, "Cognitive and Motivational Effects of Digital Concept Maps in Pre-Service Science Teacher Training." Procedia - Social and Behavioral Sciences 2(2):640-47.

University teaching deals with complex and miscellaneous knowledge and higher education should focus on sustained conceptual understanding of relevant topics. Guidance on how to construct adequate knowledge structures can support meaningful learning. But lectures, for instance, often are structured in 
linear sequence by a number of slides and are thus biased towards linear representations of knowledge that are not appropriate for most scientific disciplines. In this study, a framework for concept maps was developed to complement lectures in human biology online. The course was aimed at student teachers at the undergraduate level. The work is based on recent theoretical research on the use of multimedia in learning environments and e-learning, knowledge structures perspectives of teaching and learning as well as theoretical assumptions of self-determination in educational processes. Each session (14 in total, e.g., human respiratory and circulatory system, physiology of senses, human anatomy) was supplemented by a digital concept map providing relevant concepts connected with linking words and continuative material attached via hyperlinks (e.g., images, web links, animations, video). Following each single lecture, students had free access to the concept maps to reinforce the latest topics and to prepare for the final examination. The objective of the study was to examine if the use of complementary concept maps (i) influences achievement and (ii) motivational variables. The students' $(\mathrm{N}=171)$ concept map use was logged along with a documentation of their achievement and miscellaneous variables regarding their motivation (e.g., interest/ enjoyment, perceived competence, effort/ importance, value/ usefulness). The logfile-data allowed distinguishing learners according to the frequency and duration of their concept map use. Additionally, computer-related self-efficacy was documented as a moderating factor. Results reveal the benefit of additional concept maps for achievement and positive motivational aspects, moderating factors are discussed. The emphasize of further research should be on students' active engagement in structuring their individual learning by constructing adequate concept maps themselves, especially in science education courses.

Scharl, A. 2001, "Explanation and Exploration Visualizing the Topology of Web Information Systems." International Journal of Human-Computer Studies 55(3):239-58.

Innovation substantially reduces the practical value of traditional communication models. This paper examines the role of conceptual, user-centric modelling of web information systems as a primary means of standardized visual communication between and within organizations. It presents the development and potential of the extended World Wide Web Design Technique as a visual, consistent, and semantically rich language to share knowledge about content and structure of both planned and deployed systems ("Explanation"). As web information systems represent semantic networks in themselves, it is only natural to leverage their semantics to provide analytical tools and intuitive user interfaces. Visual frameworks based on the extended World Wide Web Design Technique enable interactive visualization of the users' access patterns. Limited, statistically oriented representations of commercially available webtracking software are enhanced by a map-like view based on the system's unique topology. When integrated into the user interface via multiple, tightly coupled views, such automatically generated site maps help users to explore the available navigation space ("Exploration").

Schneidewind, A, P Neumann, and I Schmitt. 2004, "An Approach to Visualize Image Retrieval Results." In Conference on Computer Vision and Pattern Recognition Workshop, pp. 148-48.

Many current content-based image retrieval systems suffer from poor result presentation. A sophisticated visualization can be used to identify differences between human perception of similarity and system-determined similarity. Analyzing such discrepancies is a prerequisite for a system trimmed towards the user's comprehension of the underlying retrieval process. The aim of the visualization techniques presented in this paper is to provide a tool to analyze a mismatch between the user's perception and the system's calculation of similarity. We combine techniques of visual image retrieval and information visualization to acquire insight into the extracted feature data. In our project we implemented 
visualization techniques to present feature data on three different levels of abstraction. We discuss our experiences when working with a Data Table, a Parallel Coordinate Plot, and a Color Space Plot.

Schneidewind, J, M Sips, and DA Keim. 2006, "Pixnostics: Towards Measuring the Value of Visualization." In IEEE Symposium on Visual Analytics Science \& Technology, pp. 199-206.

During the last two decades a wide variety of advanced methods for the visual exploration of large data sets have been proposed. For most of these techniques user interaction has become a crucial element, since there are many situations in which a user or an analyst has to select the right parameter settings from among many or select a subset of the available attribute space for the visualization process, in order to construct valuable visualizations that provide insight, into the data and reveal interesting patterns. The right choice of input parameters is often essential, since suboptimal parameter settings or the investigation of irrelevant data dimensions make the exploration process more time consuming and may result in wrong conclusions. In this paper we propose a novel method for automatically determining meaningful parameter- and attribute settings based on the information content of the resulting visualizations. Our technique called Pixnostics, in analogy to Scagnostics (Wilkinson et al., 2005), automatically analyses pixel images resulting from diverse parameter mappings and ranks them according to the potential value for the user. This allows a more effective and more efficient visual data analysis process, since the attribute/parameter space is reduced to meaningful selections and thus the analyst obtains faster insight into the data. Real world applications are provided to show the benefit of the proposed approach

Scholtz, J. 2006, "Beyond Usability: Evaluation Aspects of Visual Analytic Environments." In IEEE Symposium on Visual Analytics Science \& Technology, pp. 145-50.

A new field of research, visual analytics, has been introduced. This has been defined as "the science of analytical reasoning facilitated by interactive visual interfaces" (Thomas and Cook, 2005). Visual analytic environments, therefore, support analytical reasoning using visual representations and interactions, with data representations and transformation capabilities, to support production, presentation, and dissemination. As researchers begin to develop visual analytic environments, it is advantageous to develop metrics and methodologies to help researchers measure the progress of their work and understand the impact their work has on the users who work in such environments. This paper presents five areas or aspects of visual analytic environments that should be considered as metrics and methodologies for evaluation are developed. Evaluation aspects need to include usability, but it is necessary to go beyond basic usability. The areas of situation awareness, collaboration, interaction, creativity, and utility are proposed as the five evaluation areas for initial consideration. The steps that need to be undertaken to develop systematic evaluation methodologies and metrics for visual analytic environments are outlined

Scholtz, J. 2010, "Developing Qualitative Metrics for Visual Analytic Environments." In BELIV'10: Beyond time and errors: novel evaluation methods for Information Visualization, April 10-11, 2010.

In this paper, we examine reviews for the entries to the 2009 Visual Analytics Science and Technology (VAST) Challenge. By analyzing these reviews we gained a better understanding of what is important to our reviewers, both visualization researchers and professional analysts. This is a bottom up approach to the development of heuristics to use in the evaluation of visual analytic environments. The meta-analysis and the results are presented in this paper. 
Scholtz, J, et al. 2009, "Visual Analytics Technology Transition Progress." Information Visualization 8(4):294-301.

The authors provide a description of the transition process for visual analytic tools and contrast this with the transition process for more traditional software tools. This paper takes this difference into account and describes a user-oriented approach to technology transition including a discussion of key factors that should be considered and adapted to each situation. The progress made in transitioning visual analytic tools in the past 5 years is described and challenges that remain are enumerated. Information Visualization (2009) 8, 294-301. doi:10.1057/ivs.2009.20

Schooler, JW, M Fallshore, and SM Fiore. 1996, "Epilogue : Putting Insight into Perspective." in The Nature of Insight, eds. RJ Sternberg and JE Davidson, pp. 559-87. MIT Press.

Schroeder, M. 2005, "Chapter 24 - Intelligent Information Integration: From Infrastructure through Consistency Management to Information Visualization." in Exploring Geovisualization, eds. D Jason, MM Alan and K Menno-Jan, pp. 477-94. Elsevier, Oxford.

Summary Information agents collect information from various locations, prepare the information and visualize the result to the user. This information integration process needs to address three different aspects: First, the infrastructure to facilitate information integration; second, data preparation and consistency management; and third, Information Visualization. For these three aspects, we present current trends such as Grid computing, the semantic Web and visual datamining and we outline ways in which these technologies apply to geovisualization.

Schuff, D, O Turetken, and J D'Arcy. 2006, "A Multi-Attribute, Multi-Weight Clustering Approach to Managing "E-Mail Overload"." Decision Support Systems 42(3):1350-65.

The increasing volume of electronic mail communication threatens to cause a state of "e-mail overload" where the volume of messages exceeds individuals' capacity to process them. To address this problem, this study extends the application of hierarchical clustering to the domain of e-mail. We report on the design and development of a system that applies a multi-weight, multi-attribute clustering approach to a collection of messages. We found strong evidence that clustering messages improves users' ability to locate messages compared to an ordered list, and promising (though weaker) evidence of even greater improvement when given the ability to adjust attribute weights.

Schultz, T, and GL Kindlmann. 2010, "Superquadric Glyphs for Symmetric Second-Order Tensors." IEEE Transactions on Visualization and Computer Graphics 16(6):1595-604.

Symmetric second-order tensor fields play a central role in scientific and biomedical studies as well as in image analysis and feature-extraction methods. The utility of displaying tensor field samples has driven the development of visualization techniques that encode the tensor shape and orientation into the geometry of a tensor glyph. With some exceptions, these methods work only for positive-definite tensors (i.e., having positive eigenvalues, such as diffusion tensors). We expand the scope of tensor glyphs to all symmetric second-order tensors in two and three dimensions, gracefully and unambiguously depicting any combination of positive and negative eigenvalues. We generalize a previous method of superquadric glyphs for positive-definite tensors by drawing upon a larger portion of the superquadric shape space, supplemented with a coloring that indicates the quadratic form (including eigenvalue sign). We show that 
encoding arbitrary eigenvalue magnitudes requires design choices that differ fundamentally from those in previous work on traceless tensors that arise in the study of liquid crystals. Our method starts with a design of 2-D tensor glyphs guided by principles of scale-preservation and symmetry, and creates 3-D glyphs that include the 2-D glyphs in their axis-aligned cross-sections. A key ingredient of our method is a novel way of mapping from the shape space of three-dimensional symmetric second-order tensors to the unit square. We apply our new glyphs to stress tensors from mechanics, geometry tensors and Hessians from image analysis, and rate-of-deformation tensors in computational fluid dynamics.

Sebrechts, MM, et al. 1999, "Visualization of Search Results: A Comparative Evaluation of Text, 2d, and 3d Interfaces." In 22nd International Conference on Research and Development in Information Retrieval, pp. 3-10.

Although there have been many prototypes of visualization in support of information retrieval, there has been little systematic evaluation that distinguishes the benefits of the visualization per se from that of various accompanying features. The current study focuses on such an evaluation of NIRVE, a tool that supports visualization of search results. Insofar as possible, functionally equivalent $3 \mathrm{D}, 2 \mathrm{D}$, and text versions of NIRVE were implemented. Nine novices and six professional users completed a series of information-seeking tasks on a set of retrieved documents. There were high interface costs for the 3D visualization, although those costs decreased substantially with experience. Performance was best when the tool's properties matched task demands; only under the right combination of task, user, and interface did $3 \mathrm{D}$ visualization result in performance comparable to functionally matched $2 \mathrm{D}$ and textual tools.

Sedig, K, and J Morey. 2005, "17 - a Descriptive Framework for Designing Interaction for Visual Abstractions." in Studies in Multidisciplinarity, ed. M Grant, Vol 2, pp. 239-54. Elsevier.

This chapter propses a descriptive framework for categorisation and characterisation of the different forms of interaction with visual abstractions (VAs). Abstract visual representations play an important role in assisting human reasoning, thinking, and understanding processes. There are different forms of designing interaction with these representations. The goal of this chapter is to provide a descriptive framework to guide the designers and evaluators of cognitive tools to determine the appropriate forms of interaction that can facilitate the understanding of abstract concepts, patterns, structures and processes. The framework is described and substantiated using a number of VAs that represent and communicate mathematical ideas.

Sedig, K, et al. 2005, "18 - Visualising, Interacting and Experimenting with Lattices Using a Diagrammatic Representation." in Studies in Multidisciplinarity, ed. M Grant, Vol 2, pp. 255-68. Elsevier.

This chapter presents the K-lattice machine (KLM), a tool that allows users to visualise, interact, and experiment with $\mathrm{K}$ lattices using a diagrammatic representation. $\mathrm{K}$ lattices are a subset of $2 \mathrm{D}$ lattices that can be represented as state-transition diagrams. KLM allows users to explore the relationship between K lattices and their state-transition diagram representations to gain insight into the structure of these lattices.

Sedig, K, S Rowhani, and H-N Liang. 2005, "Designing Interfaces That Support Formation of Cognitive Maps of Transitional Processes: An Empirical Study." Interacting with Computers 17(4):419-52.

Many conditions, phenomena, and concepts are of a transitional nature. Transitional processes involve change from one form to another, such as biological, chemical, and geological metamorphoses. 
Transitional processes take place in time-space and are not always easy to encode, communicate, and understand. The purpose of this research is to investigate how to design human-computer interfaces that support users in their formation of cognitive maps of transitional processes. To conduct this investigation, geometric shapes were used as the testbed, and their metamorphic transformations were captured and communicated using three different interface styles: temporally stacked, spatially distributed, and spatiotemporal. The usability and effectiveness of each interface was evaluated. The results of the study indicate that the spatio-temporal interface is the most effective of the three interfaces. The findings of this research imply that many kinds of transitional processes, such as rock metamorphoses, historical changes, or economical processes, may best be explored and understood using spatio-temporal interfaces.

Sedlmair, M, et al. 2008, "A Dual-View Visualization of in-Car Communication Processes." In 12th International Conference Information Visualisation 2008, pp. 157-62.

With the increasing complexity of in-car communication architectures, their diagnostics have become essential for automotive development and maintenance. In order to help engineers to detect and analyze the potential sources and consequences of errors, it is crucial to provide both comprehensive and detailed insight into the communication processes and their contexts. Two important aspects of these are the dependencies and correlations between onboard functions. In this paper we present a dual-view visualization for exploring the functional dependency chains of in-car communication processes. One view presents the dependencies of hardware components using a space filling approach similar to a treemap, whereas the other view displays the functional correlations as an interactive sequence chart. The views are coupled via color coding and show the dependencies of an interactively selectable functional unit. In an expert evaluation, we assessed the benefits of using this visualization technique for in-car communication diagnostics with very positive results.

Sedlmair, M, et al. 2010, "Evaluating Information Visualization in Large Companies: Challenges, Experiences and Recommendations." In BELIV'10: Beyond time and errors: novel evaluation methods for Information Visualization, April 10-11, 2010.

We examine the process and some implications of evaluating information visualization in a large company setting. While several researchers have addressed the difficulties of evaluating information visualizations with regards to changing data, tasks, and visual encodings, considerably less work has been published on the difficulties of evaluation within specific work contexts. In this paper, we specifically focus on the challenges arising in the context of large companies with several thousand employees. We present a collection of evaluation challenges, discuss our own experiences conducting information visualization evaluation within the context of a large automotive company, and present a set of recommendations derived from our experiences. The set of challenges and recommendations can aid researchers and practitioners in preparing and conducting evaluations of their products within a large company setting.

Sedlmair, M, et al. 2009, "User-Centered Development of a Visual Exploration System for in-Car Communication." In 10th International Symposium on Smart Graphics, pp. 105-16.

Modern premium automobiles are equipped with an increasing number of Electronic Control Units (ECUs). These ECUs are interconnected and form a complex network to provide a wide range of advanced vehicle functionality. Analyzing the flow of messages in this network and tracking down problems has become a major challenge for automotive engineers. By observing their working practices, 
we found that the tools they currently use are mostly, text-based and largely fail to provide correlations among the enormous amount of data. We established requirements for a more appropriate (visual) toot set. We followed a user-centered approach to design several visualizations for in-car communication processes, each with a clear purpose and application scenario. Then we used low-fidelity prototypes to evaluate our ideas and to identify the "working" designs. Based on this selection, we finally implemented a prototype and conducted an expert evaluation which revealed the emergence of a novel mental model for thinking about and discussing in-car communication processes.

Segel, E, and J Heer. 2010, "Narrative Visualization: Telling Stories with Data." IEEE Transactions on Visualization and Computer Graphics 16(6):1139-48.

Data visualization is regularly promoted for its ability to reveal stories within data, yet these $\& \# 8220$; data stories\&\#8221; differ in important ways from traditional forms of storytelling. Storytellers, especially online journalists, have increasingly been integrating visualizations into their narratives, in some cases allowing the visualization to function in place of a written story. In this paper, we systematically review the design space of this emerging class of visualizations. Drawing on case studies from news media to visualization research, we identify distinct genres of narrative visualization. We characterize these design differences, together with interactivity and messaging, in terms of the balance between the narrative flow intended by the author (imposed by graphical elements and the interface) and story discovery on the part of the reader (often through interactive exploration). Our framework suggests design strategies for narrative visualization, including promising under-explored approaches to journalistic storytelling and educational media.

Sehyun, K, K Sungye, and Y Kyung-Hyun. 2000, "A Study on the Ray-Tracing Acceleration Technique Based on the Zf-Buffer Algorithm " In Fourth International Conference on Information Visualisation (IV'00), pp. 393-98.

In this work, we propose the ZF-buffer algorithm in order to accelerate the intersection test of ray tracing. The ZF-buffer is used in the preprocessing of ray tracing and records the pointer that points to a parent face of a depth value ( $\mathrm{z}$ value) of an object determined in the Z-buffer. As a result, the face that intersects with the first ray can be determined easily by using the pointer stored in the F-buffer. Though the ZF-buffer and vista-buffer resemble each other, the difference between the two methods is that what ZF-buffer records is not bounding volume but the pointer of a displayable face. We applied the

Seifert, CM, et al. 1996, "Demystification of Cognitive Insight: Opportunistic Assimilation and the Prepared-Mind Perspective." in The Nature of Insight, eds. RJ Sternberg and JE Davidson. MIT Press.

Senge, PM. 1990. The Fifth Discipline: The Art and Practice of the Learning Organization. Doubleday Currency, New York.

Seo, J. 2005, "Information Visualization Design for Multidimensional Data: Integrating the Rank-byFeature Framework with Hierarchical Clustering." in Computer Science, Vol Ph.D., pp. 227-27. University of Maryland, College Park.

Interactive exploration of multidimensional data sets is challenging because: (1) it is difficult to comprehend patterns in more than three dimensions, and (2) current systems are often a patchwork of graphical and statistical methods leaving many researchers uncertain about how to explore their data in an 
orderly manner. This dissertation offers a set of principles and a novel rank-by-feature framework that could enable users to better understand multidimensional and multivariate data by systematically studying distributions in one (1D) or two dimensions (2D), and then discovering relationships, clusters, gaps, outliers, and other features. Users of this rank-by-feature framework can view graphical presentations (histograms, boxplots, and scatterplots), and then choose a feature detection criterion to rank ID or 2D axis-parallel projections. By combining information visualization techniques (overview, coordination, and dynamic query) with summaries and statistical methods, users can systematically examine the most important 1D and 2D axis-parallel projections. This research provides a number of valuable contributions: (a) Graphics, Ranking, and Interaction for Discovery (GRID) principles--a set of principles for exploratory analysis of multidimensional data, which are summarized as: (1) study $1 \mathrm{D}$, study $2 \mathrm{D}$, then find features (2) ranking guides insight, statistics confirm. GRID principles help users organize their discovery process in an orderly manner so as to produce more thorough analyses and extract deeper insights in any multidimensional data application. (b) Rank-by-feature framework--a user interface framework based on the GRID principles. Interactive information visualization techniques are combined with statistical methods and data mining algorithms to enable users to orderly examine multidimensional data sets using ID and 2D projections. (c) The design and implementation of the Hierarchical Clustering Explorer (HCE), an information visualization tool available at www.cs.umd.edu/hcil/hce. HCE implements the rank-by-feature framework and supports interactive exploration of hierarchical clustering results to reveal one of the important features--clusters. (d) Validation through case studies and user surveys: Case studies with motivated experts in three research fields and a user survey via emails to a wide range of HCE users demonstrated the efficacy of HCE and the rank-by-feature framework. These studies also revealed potential improvement opportunities in terms of design and implementation.

Seo, J, and B Shneiderman. 2006, "Knowledge Discovery in High-Dimensional Data: Case Studies and a User Survey for the Rank-by-Feature Framework." IEEE Transactions on Visualization and Computer Graphics 12(3):311-22.

Knowledge discovery in high- dimensional data is a challenging enterprise, but new visual analytic tools appear to offer users remarkable powers if they are ready to learn new concepts and interfaces. Our three-year effort to develop versions of the Hierarchical Clustering Explorer (HCE) began with building an interactive tool for exploring clustering results. It expanded, based on user needs, to include other potent analytic and visualization tools for multivariate data, especially the rank-by-feature framework. Our own successes using HCE provided some testimonial evidence of its utility, but we felt it necessary to get beyond our subjective impressions. This paper presents an evaluation of the Hierarchical Clustering Explorer ( HCE) using three case studies and an e-mail user survey $(\mathrm{n}=57)$ to focus on skill acquisition with the novel concepts and interface for the rank-by-feature framework. Knowledgeable and motivated users in diverse fields provided multiple perspectives that refined our understanding of strengths and weaknesses. A user survey confirmed the benefits of HCE, but gave less guidance about improvements. Both evaluations suggested improved training methods.

Seong, Y, and CS Nam. 2008, "Capturing Judgment Policy on Customers' Creditworthiness: A Lens Model and Sdt Approach." International Journal of Industrial Ergonomics 38(7-8):593-600.

Designing an intervention program to train human operator's decision-making process and subsequently to improve their performance requires a thorough understanding of the relationships between the environment, the human operator, and cues on which decisions are based. Understanding these relationships provides opportunities to make better decisions when human operators encounter 
novel situations. This study investigated the operator's decision-making process from the lens model perspective and signal detection approach, in which operators make judgments on customers' creditworthiness using three types of information such as (1) days that accounts were unpaid, (2) probability of the customers damaging the institution based on the customers' credit histories, and (3) case-based reasoning (CBR) scores. Results showed that the operators were conservative, which resulted in extremely low performance while maintaining a good level of domain knowledge. Analyses of the relative cue weights also showed that the aggregated judgment strategy represented by the cue weights was similar to the validity of the cues to the ecology, reflected in the high level of policy matching (rm). Specifically, the probability and the days cues were more and equally emphasized over the CBR score.Relevance to industry Capturing the human operator's judgment policy can be used to identify and develop customized training needs for individual operator to make better judgments on customers' creditworthiness or the environment of interest in general.

Serenko, A. 2010, "The Development of an Ai Journal Ranking Based on the Revealed Preference Approach." Journal of Informetrics 4(4):447-59.

This study presents a ranking of 182 academic journals in the field of artificial intelligence. For this, the revealed preference approach, also referred to as a citation impact method, was utilized to collect data from Google Scholar. This list was developed based on three relatively novel indices: h-index, g-index, and hc-index. These indices correlated almost perfectly with one another (ranging from 0.97 to 0.99 ), and they correlated strongly with Thomson's Journal Impact Factors (ranging from 0.64 to 0.69 ). It was concluded that journal longevity (years in print) is an important but not the only factor affecting an outlet's ranking position. Inclusion in Thomson's Journal Citation Reports is a must for a journal to be identified as a leading A+ or A level outlet. However, coverage by Thomson does not guarantee a high citation impact of an outlet. The presented list may be utilized by scholars who want to demonstrate their research output, various academic committees, librarians and administrators who are not familiar with the AI research domain.

Serfaty, D, et al. 1997, "The Decision-Making Expertise of Battle Commanders." in Naturalistic Decision Making, eds. CE Zsambok and G Klein, pp. 233-46. Lawrence Elrbaum Associates, Mahwah, NJ.

Serrano, E, et al. 2010, "Debugging Complex Software Systems by Means of Pathfinder Networks." Information Sciences 180(5):561-83.

This paper introduces a new methodology based on the use of Pathfinder networks (PFNETs) for the debugging of multi-agent systems (MASs). This methodology is specifically designed to develop a forensic analysis (i.e., a debugging process performed on previously recorded data of the MAS run) of MASs showing complex tissues of relationships between agents (i.e., a high complexity in their social level). Like previous works in the field of forensic analysis of MASs, our approach is performed by considering displays of the system activity which aim to be understandable by human beings. These displays allow us to understand the social behavior of the system, discover emergent behaviors, and debug possible undesirable behaviors. However, it is well known that the visualization of information in a humanly comprehensible way becomes a complex task when large amounts of information have to be represented, as is the case of the social behavior of large-scale MASs. Our methodology tackles this problem through the use of PFNETs, which are considered to reduce the data complexity in order to obtain simple representations that show only the most important global interactions in the system. In addition, the proposed methodology is customizable thanks to the use of two thresholds allowing the user 
to define the desired specificity level in the display. The proposal is illustrated with a detailed case study considering a complex customer-seller MAS.

Shahar, Y, and C Cheng. 2000, "Model-Based Visualization of Temporal Abstractions." Computational Intelligence 16(2):279-306.

We describe a new conceptual methodology and related computational architecture called Knowledge-based Navigation of Abstractions for Visualization and Explanation (KNAVE). KNAVE is a domain-independent framework specific to the task of interpretation, summarization, visualization, explanation, and interactive exploration, in a context-sensitive manner, of time-oriented raw data and the multiple levels of higher level, interval-based concepts that can be abstracted from these data. The KNAVE domain-independent exploration operators are based on the relations defined in the knowledgebased temporal-abstraction problem-solving method, which is used to abstract the data, and thus can directly use the domain-specific knowledge base on which that method relies. Thus, the domain-specific semantics are driving the domain-independent visualization and exploration processes, and the data are viewed through a filter of domain-specific knowledge. By accessing the domain-specific temporalabstraction knowledge base and the domain-specific time-oriented database, the KNAVE modules enable users to query for domain-specific temporal abstractions and to change the focus of the visualization, thus reusing for a different task (visualization and exploration) the same domain model acquired for abstraction purposes. We focus here on the methodology, but also describe a preliminary evaluation of the KNAVE prototype in a medical domain. Our experiment incorporated seven users, a large medical patient record, and three complex temporal queries, typical of guideline-based care, that the users were required to answer and/or explore. The results of the preliminary experiment have been encouraging. The new methodology has potentially broad implications for planning, monitoring, explaining, and interactive data mining of time-oriented data.

Shahar, Y, et al. 2006, "Distributed, Intelligent, Interactive Visualization and Exploration of TimeOriented Clinical Data and Their Abstractions." Artificial Intelligence in Medicine 38(2):115-35.

SummaryObjectives We present KNAVE-II, an intelligent interface to a distributed architecture specific to the tasks of query, knowledge-based interpretation, summarization, visualization, interactive exploration of large numbers of distributed time-oriented clinical data, and dynamic sensitivity analysis of these data. KNAVE-II main contributions to the fields of temporal reasoning and intelligent user interfaces are: (1) the capability for interactive computation and visualization of domain specific temporal abstractions, supported by ALMA - a computational engine that applies the domain knowledge base to the clinical time-oriented database. (2) Semantic (ontology-based) navigation and exploration of the data, knowledge, and temporal abstractions, supported by the IDAN mediator, a distributed architecture that enables runtime access to domain-specific knowledge bases that are maintained by expert physicians.Methods and materials KNAVE-II was designed according to 12 requirements that were defined through iterative cycles of design and user-centered evaluation. The complete architecture has been implemented and evaluated in a cross-over study design that compared the KNAVE-II module versus two existing methods: paper charts and an Excel electronic spreadsheet. A small group of clinicians answered the same queries, using the domain of oncology and a set of 1000 patients followed after bone-marrow transplantation. Results The results show that users are able to perform medium to hard difficulty level queries faster and more accurately by using KNAVE-II than paper charts and Excel. Moreover, KNAVE-II was ranked first in preference by all users, along all usability dimensions.Conclusions Initial evaluation of KNAVE-II and its supporting knowledge based temporal- 
mediation architecture, by applying it to a large data base of patients monitored several years after bone marrow transplantation (BMT), has produced highly encouraging results.

Shang, Y, W Ruml, and MPJ Fromherz. 2006, "Positioning Using Local Maps." Ad Hoc Networks 4(2):240-53.

It is often useful to know the positions of nodes in a network. However, in a large network it is impractical to build a single global map. In this paper, we present a new approach for distributed localization called Positioning using Local Maps (PLM). Given a path between a starting node and a remote node we wish to localize, the nodes along the path each compute a map of their local neighborhood. Adjacent nodes then align their maps, and the relative position of the remote node can then be determined in the coordinate system of the starting node. Nodes with known positions can easily be incorporated to determine absolute coordinates. We instantiate the PLM framework using the previously proposed MDS-MAP(P) algorithm to generate the local maps. Through simulation experiments, we compare the resulting algorithm, which we call MDS-MAP(D), with existing distributed methods and show improved performance on both uniform and irregular topologies.

Shanker, SG. 1995, "The Nature of Insight." Minds and Machines 5(4):561-81.

Shapiro, M, et al. 2008, "A Virtual Look at Epstein-Barr Virus Infection: Simulation Mechanism." Journal of Theoretical Biology 252(4):633-48.

Epstein-Barr virus (EBV) is an important human pathogen that establishes a life-long persistent infection and for which no precise animal model exists. In this paper, we describe in detail an agent-based model and computer simulation of EBV infection. Agents representing EBV and sets of B and T lymphocytes move and interact on a three-dimensional grid approximating Waldeyer's ring, together with abstract compartments for lymph and blood. The simulation allows us to explore the development and resolution of virtual infections in a manner not possible in actual human experiments. Specifically, we identify parameters capable of inducing clearance, persistent infection, or death.

Sharko, J, et al. 2007, "Heat Map Visualizations Allow Comparison of Multiple Clustering Results and Evaluation of Dataset Quality: Application to Microarray Data." In 11th International Conference on Information Visualization, pp. 521-26.

Since clustering algorithms are heuristic, multiple clustering algorithms applied to the same dataset will typically not generate the same sets of clusters. This is especially true for complex datasets such as those from microarray time series experiments. Two such microarray datasets describing gene expression activities from regenerating newt forelimbs at various times following limb amputation were used in this study. A cluster stability matrix, which shows the number of times two genes appear in the same cluster, was generated as a heat map. This was used to evaluate the overall variation among the clustering algorithms and to identify similar clusters. A comparison of the cluster stability matrices for two related microarray experiments with different levels of precision was shown to be an effective basis for comparing the quality of the two sets of experiments. A pairwise heat map was generated to show which pairs of clustering algorithms grouped the data into similar clusters. 
Shaw, CD, and MZ Baraghoush. 2010, "Imas and Genetic Sequences : Tracing the Mutations of a Disease Vast 2010 Mini Challenge 3." In IEEE Symposium on Visual Analytics Science \& Technology.

Shaw, R-S. 2010, "A Study of Learning Performance of E-Learning Materials Design with Knowledge Maps." Computers \& Education 54(1):253-64.

This research investigated the application of knowledge maps in e-learning materials design and hypothesized that knowledge maps would be more effective than e-learning in general at improving the performance and satisfaction of e-learning. In order to test the hypotheses, we conducted an experiment with 175 participants and randomly assigned them into knowledge map-based and browse-based groups. Both groups of participants needed to acquire specific skills and knowledge to write the target ADO.NET program. In the end of each training session subjects received an assessment to understand their learning score, satisfaction level, and computer self-efficacy. Our statistical analysis result showed that knowledge map-based learning group outperformed browse-based group in these three measurements. Thus, the proposed hypotheses were supported. We concluded that if knowledge map-based materials design approach were employed novice users would acquire the ADO.NET programming in a more effective manner. In addition, the satisfaction and computer self-efficacy of users could be improved substantially as a result of knowledge map-based materials design approach. Thus, we propose that if knowledge maps are employed to adequately present the relationships of learning contents that knowledge could be better understood and learning performance could be improved.

Shaw, RS, et al. 2009, "The Impact of Information Richness on Information Security Awareness Training Effectiveness." Computers \& Education 52(1):92-100.

In recent years, rapid progress in the use of the internet has resulted in huge losses in many organizations due to lax security. As a result, information security awareness is becoming an important issue to anyone using the Internet. To reduce losses, organizations have made information security awareness a top priority. The three main barriers to information security awareness are: (1) general security awareness, (2) employees' computer skills, and (3) organizational budgets. Online learning appears a feasible alternative to providing information security awareness and countering these three barriers. Research has identified three levels of security awareness: perception, comprehension and projection. This paper reports on a laboratory experiment that investigates the impacts of hypermedia, multimedia and hypertext to increase information security awareness among the three awareness levels in an online training environment. The results indicate that: (1) learners who have the better understanding at the perception and comprehension levels can improve understanding at the projection level; (2) learners with text material perform better at the perception level; and (3) learners with multimedia material perform better at the comprehension level and projection level. The results could be used by educators and training designers to create meaningful information security awareness materials.

Shen, L, S Kim, and AJ Saykin. 2009, "Fourier Method for Large-Scale Surface Modeling and Registration." Computers \& Graphics 33(3):299-311.

Spherical harmonic (SPHARM) description is a powerful Fourier shape modeling method for processing arbitrarily shaped but simply connected 3D objects. As a highly promising method, SPHARM has been widely used in several domains including medical imaging. However, its primary use has been focused on modeling small or moderately sized surfaces that are relatively smooth, due to challenges related to its applicability, robustness and scalability. This paper presents an enhanced SPHARM 
framework that addresses these issues and show that the use of SPHARM can expand into broader areas. In particular, we present a simple and efficient Fourier expansion method on the sphere that enables largescale modeling, and propose a new SPHARM registration method that aims to preserve the important homological properties between 3D models. Although SPHARM is a global descriptor, our experimental results show that the proposed SPHARM framework can accurately describe complicated graphics models and highly convoluted 3D surfaces and the proposed registration method allows for effective alignment and registration of these 3D models for further processing or analysis. These methods greatly enable the potential of applying SPHARM to broader areas such as computer graphics, medical imaging, $\mathrm{CAD} / \mathrm{CAM}$, bioinformatics, and other related geometric modeling and processing fields.

Shen, Y, SK Ong, and AYC Nee. 2010, "Augmented Reality for Collaborative Product Design and Development." Design Studies 31(2):118-45.

This paper presents the application of Augmented Reality (AR) to support concurrent collaborative product design among members of a multi-disciplinary team. A client/server framework has been developed to enable users in a distributed environment to carry out product design collaboratively. An intuitive interface, consisting of virtual and tangible interfaces, and a tri-layer model representation scheme have been designed and developed to support solid modeling and collaborative design activities in the AR-based environment. Using AR technology, the users wearing head-mounted devices can move about in a physical 3D space to view a part that is being designed from different angles and perspectives. The users can observe the design effects in real-time as the modifications are being made in the 3D space.

Shen, Y, SK Ong, and AYC Nee. 2008, "Product Information Visualization and Augmentation in Collaborative Design." Computer-Aided Design 40(9):963-74.

In this paper, a collaborative system for product information visualization and augmentation is presented. The developed system allows the users, who can be remotely distributed, to view a product model, which is a geometric representation of the product, from different perspectives. They can choose to view design and product history, such as previous modification processes and feature information of the product independently. The product models displayed to the users are immediately updated after any design modifications have been made to the CAD model. Product features being discussed can be highlighted to draw the users' attention. In addition, modifications can be displayed dynamically for the users to evaluate the design effect. The product history document module in the system provides a userfriendly interface for retrieving design records. After a specific record has been chosen, the related product model is displayed, and it can be aligned with the current product model for the ease of comparison and evaluation. The feature information of the product is displayed using virtual "floating" annotations linked to the related features. A user interface to enter annotations is provided, and the annotations entered by different users can be shared in real time. A cluster-based greedy algorithm is implemented to avoid overlapping annotations in the field of view. (C) 2008 Elsevier Ltd. All rights reserved. 
Shen, Z, and N Sundaresan. 2010, "Trail Explorer : Understanding User Experience in Webpage Flows." In IEEE Conference on Information Visualization.

Shen, ZQ, and KL Ma. 2008, "Mobivis: A Visualization System for Exploring Mobile Data." In IEEE Pacific Visualisation Symposium, pp. 175-82.

The widespread use of mobile devices brings opportunities to capture large-scale, continuous information about human behavior. Mobile data has tremendous value, leading to business opportunities, market strategies, security concerns, etc. Visual analytics systems that support interactive exploration and discovery are needed to extracting insight from the data. However, visual analysis of complex socialspatial-temporal mobile data presents several challenges. We have created MobiVis, a visual analytics tool, which incorporates the idea of presenting social and spatial information in one heterogeneous network. The system supports temporal and semantic filtering through an interactive time chart and ontology graph, respectively, such that data subsets of interest can be isolated for close-up investigation. "Behavior rings," a compact radial representation of individual and group behaviors, is introduced to allow easy comparison of behavior patterns. We demonstrate the capability of MobiVis with the results obtained from analyzing the MIT Reality Mining dataset.

Sheppard, SRJ, and JD Salter. 2004, "Landscape and Planning | the Role of Visualization in Forest Planning." in Encyclopedia of Forest Sciences, ed. B Jeffery, pp. 486-98. Elsevier, Oxford.

Shi, L, et al. 2010, "Visworks Text and Network Visual Analytics." In IEEE Symposium on Visual Analytics Science \& Technology, pp. 269-70.

Shi, Z, et al. 2007, "Msminer--a Developing Platform for Olap." Decision Support Systems 42(4):201628.

Since the early 1970s, decision support systems (DSS) have evolved significantly. In this paper, the design and implementation of MSMiner, a developing platform for DSS, is introduced. The system is constructed on a data warehouse and integrated with a number of data mining algorithms. It is well suited for on-line analytical processing (OLAP). The characteristics of MSMiner include the ability to support multiple data sources and data mining strategies, additional organizational flexibility in regard to data and mining strategies, and the powerful expansibility of data mining tasks.

Shihong, H, et al. 2009, "Knowledge-Based Evaluation of Nursing Care Practice." In IEEE International Conference on Information Reuse \& Integration, pp. 171-74.

Providing people with a complete and responsive healthcare solution requires a multi-tiered health service delivery system. One aspect in the healthcare hierarchy is the need for care provided by nurses. Nursing care and observation provide the basis for nurses to communicate their practice with others in the healthcare system. It is necessary to capture and manage knowledge of nursing care to improve the quality nursing practice. This paper proposes a novel knowledge-based decision support system for nurses to capture and manage nursing practice, and further, to monitor nursing care quality, as well as to test aspects of an electronic health record for recording and reporting nursing practice. As part of ongoing collaborative research of the Nursing School and the Department of Computer Science, a prototype toolset was developed to capture and manage nursing practice in order to improve the quality of care. A case study is presented to demonstrate the toolset used by nurses in a local hospital environment. 
Shimoyama, K, et al. 2007, "An Approach for Multi-Objective Robust Optimization Assisted by Response Surface Approximation and Visual Data-Mining." In IEEE Congress on Evolutionary Computation, pp. 2413-20.

A new approach for multi-objective robust design optimization has been proposed and applied to a real-world design problem with a large number of objective functions. The present approach is assisted by response surface approximation and visual data-mining, which results in two major gains regarding computational time and data interpretation. The Kriging model for response surface approximation can realize accurate predictions of robustness measures, and dramatically reduces the computational time for objective function evaluation. In addition, the use of self-organizing maps as a data-mining technique allows visualization of complicated design information between optimality and robustness of design in a comprehensible two-dimensional form. Therefore, the extraction and interpretation of trade-off relations between optimality and robustness of design, and also the location of sweet-spots in the design space, can be performed in a comprehensive manner.

Shive, J, and G Francis. 2008, "Applying Models of Visual Search to Map Display Design." International Journal of Human-Computer Studies 66(2):67-77.

We explored how to use a computational model of visual search to design a map of a mall directory. We parameterized the Guided Search model [Wolfe, J.M., 1994. Guided Search 2.0: a revised model of visual search. Psychonomic Bulletin and Review 1(2), 202-238] for a task involving visual search of a target store in a map. The resulting model was then used to choose color assignments for all the elements of the display that would result in the fastest average search time for the display and search tasks. These predicted optimized color assignments were then tested empirically. The empirical data closely matched the predicted pattern of search times. We conclude that computational models of visual search are sophisticated enough to contribute to the development of optimized map designs, discuss some limitations of the current models, and suggest directions for further development.

Shneiderman, B. 1997. Designing the User Interface, 3rd Ed. Addison-Wesley, Reading, MA.

Shneiderman, B. 1996, "The Eyes Have It: A Task by Data Type Taxonomy for Information Visualizations." In IEEE Symposium on Visual Languages, pp. 336-43.

A useful starting point for designing advanced graphical user interfaces is the visual information seeking Mantra: overview first, zoom and filter, then details on demand. But this is only a starting point in trying to understand the rich and varied set of information visualizations that have been proposed in recent years. The paper offers a task by data type taxonomy with seven data types (one, two, three dimensional data, temporal and multi dimensional data, and tree and network data) and seven tasks (overview, zoom, filter, details-on-demand, relate, history, and extracts)

Shneiderman, B. 2003, "The Eyes Have It: A Task by Data Type Taxonomy for Information Visualizations." in The Craft of Information Visualization: Readings and Reflections, eds. BB Bederson and B Shneiderman, pp. 364-71. Morgan Kaufmann, San Francisco.

Summary A useful starting point for designing advanced graphical user interfaces is the Visual Information-Seeking Mantra: overview first, zoom and filter, then details on demand. But this is only a starting point in trying to understand the rich and varied set of information visualizations that have been 
proposed in recent years. This paper offers a task by data type taxonomy with seven data types (one-, two, three-dimensional data, temporal and multi-dimensional data, and tree and network data) and seven tasks (overview, zoom, filter, details-on-demand, relate, history, and extracts).

Shneiderman, B. 2004, "Facilitating Understanding of Information Visualizations: Emerging Principles and Examples." In Eighth International Conference on Information Visualisation (IV'04), pp. 5-5.

Summary form only given. The enthusiasm for information visualization has generated a wide variety of interesting tools for multi-dimensional, hierarchical, and other kinds of visualizations. However, some designs are readily accepted as understandable and useful, while others are perceived as confusing and useless. Controlled studies have begun to sort of some of the issues, but the insights of designers and usability tests are contributing interesting cognitive hypotheses for researchers and practical guidelines for developers. This paper offers examples of what works and what doesn't with a preliminary set of principles that might have wide applicability.

Shneiderman, B. 2002, "Inventing Discovery Tools: Combining Information Visualization with Data Mining." Information Visualization 1(1):5-12.

The growing use of information visualization tools and data mining algorithms stems from two separate lines of research. Information visualization researchers believe in the importance of giving users an overview and insight into the data distributions, while data mining researchers believe that statistical algorithms and machine learning can be relied on to find the interesting patterns. This paper discusses two issues that influence design of discovery tools: statistical algorithms vs visual data presentation, and hypothesis testing vs exploratory data analysis. The paper claims that a combined approach could lead to novel discovery tools that preserve user control, enable more effective exploration, and promote responsibility

Shneiderman, B. 2003, "Supporting Creativity with Advanced Information-Abundant User Interfaces." in The Craft of Information Visualization: Readings and Reflections, eds. BB Bederson and B Shneiderman, pp. 372-77. Morgan Kaufmann, San Francisco.

Summary A challenge for human-computer interaction researchers and user interface designers is to construct information technologies that support creativity. This ambitious goal can be attained if designers build on an adequate understanding of creative processes.

Shneiderman, B, and C Plaisant. 2006, "Strategies for Evaluating Information Visualization Tools: MultiDimensional in-Depth Long-Term Case Studies." In AVI workshop on BEyond time and errors: novel evaluation methods for information visualization (BELIV'06).

After an historical review of evaluation methods, we describe an emerging research method called Multi-dimensional In-depth Long-term Case Studies (MILCs) which seems well adapted to study the creative activities that users of information visualization systems engage in. We propose that the efficacy of tools can be assessed by documenting 1) usage (observations, interviews, surveys, logging etc.) and 2) expert users' success in achieving their professional goals. We summarize lessons from related ethnography methods used in HCI and provide guidelines for conducting MILCs for information visualization. We suggest ways to refine the methods for MILCs in modest sized projects and then envision ambitious projects with 3-10 researchers working over 1-3 years to understand individual and 
organizational use of information visualization by domain experts working at the frontiers of knowledge in their fields.

Shrinivasan, YB, and JJ van Wijk. 2008, "Supporting Exploration Awareness for Visual Analytics." In IEEE Symposium on Visual Analytics Science and Technology, pp. 185-86.

While exploring data using information visualization, analysts try to make sense of the data, build cases, and present them to others. However, if the exploration is long or done in multiple sessions, it can be hard for analysts to remember all interesting visualizations and the relationships among them they have seen. Often, they will see the same or similar visualizations, and are unable to recall when, why and how they have seen something similar. Recalling and retrieving interesting visualizations are important tasks for the analysis processes such as problem solving, reasoning, and conceptualization. In this paper, we argue that offering sup- port for thinking based on past analysis processes is important, and present a solution for this.

Shrinivasan, YB, and JJ van Wijk. 2009, "Supporting Exploration Awareness in Information Visualization." IEEE Computer Graphics and Applications 29(5):34-43.

When users want to continue an analysis performed in the past, done by themselves or by a collaborator, they need an overview of what has been done and found so far. Such an overview helps them to gain a shared knowledge about each others' analysis strategy and continue the analysis. We aim to support users in this process, and thereby support their exploration awareness. We present an information visualization framework with three linked processes: overview, search and retrieve for this purpose. First, we present a user's information interest model that captures key aspects of the exploration process. Exploration overview, and keyword and similarity based search mechanisms are designed based on these key aspects. A metadata view is used to visualize the search results and help users to retrieve specific visualizations from past analysis. Finally, we present three case studies and discuss the support offered by the framework for developing exploration awareness.

Shrinivasan, YB, and JJ van Wijk. 2008, "Supporting the Analytical Reasoning Process in Information Visualization." In 26th Annual SIGCHI Conference on Human Factors in Computing Systems, pp. 123746.

This paper presents a new information visualization framework that supports the analytical reasoning process. It consists of three views - a data view, a knowledge view and a navigation view. The data view offers interactive information visualization tools. The knowledge view enables the analyst to record analysis artifacts such as findings, hypotheses and so on. The navigation view provides an overview of the exploration process by capturing the visualization states automatically. An analysis artifact recorded in the knowledge view can be linked to a visualization state in the navigation view. The analyst can revisit a visualization state from both the navigation and knowledge views to review the analysis and reuse it to look for alternate views. The whole analysis process can be saved along with the synthesized information. We present a user study and discuss the perceived usefulness of a prototype based on this framework that we have developed. 
Shrinivasan, YB, and JJ van Wijk. 2007, "Vispad: Integrating Visualization, Navigation and Synthesis." In IEEE Symposium on Visual Analytics Science \& Technology, pp. 209-10.

We present a newframework -VisPad - to support the user to revisit the visual exploration process, and to synthesize and disseminate information. It offers three integrated views. The data view allows the user to interactively explore the data. The navigation view captures the exploration process. It enables the user to revisit any particular state and reuse it. The knowledge view enables the user to record his/her findings and the relations between these finding

Shu, G, N Avis, and O Rana. 2008, "Bringing Semantics to Visualization Services." Advances in Engineering Software 39(6):514-20.

This paper firstly reviews the related visualization taxonomies, and discusses the need for ontology for visualization. It then presents the design of the ontology for visualization, which aims to provide more semantics for the discovery of visualization services, and gives the detailed description of the ontology's components. In the end, the paper illustrates how to use the ontology in the portal for discovery visualization services, which is being developed.

Sidjanin, P. 1998, "Visualisation of Gis Data in Vr Related to Cognitive Mapping of Environment." In IEEE Conference on Information Visualization, pp. 339-49.

The main concepts of a design tool and its testing supported by visualisation of GIS data in a VR system are described. The design tool should improve architectural design with respect to analysis and improving existing and planned urban environments regarding several quality criteria, especially those associated with visual aspects. Preconditions for defining the design tool's purpose are the determination of the \&ldquo; well-situated\&rdquo; urban elements, their impact on cognitive mapping, and the exploitation of this knowledge on cognitive mapping for the improvement of urban environments. Cognitive mapping is a kind of representation of schematic knowledge that a person has about familiar environments. A cognitive map is stored information or knowledge concerning the purpose and function of the environment. This leads to the conclusion that an urban environment design which takes the process of cognitive mapping into consideration, will be experienced by most people in the same way. Investigations of this process result in a theoretical model of elements of urban environments, their relationships and their dependencies. The theoretical platform of the tool is based on design theory, cognitive science and computer science. Design theory and cognitive science are used to develop the theoretical model. This theoretical model together with computer science are the basis for tool development

Siirtola, H. 2003, "Combining Parallel Coordinates with the Reorderable Matrix." In International Conference on Coordinated and Multiple Views in Exploratory Visualization, pp. 63-74.

There is evidence that the highlighting of the same data items in different views will improve the proficiency of knowledge acquisition tasks. Making the connection for the user will reduce the cognitive load of switching from one view to another and allows making observations that would not be possible from the two separate views. However, making the connection between conceptually different views might not be so straightforward if the view types are complex and different enough. This paper compares two conceptually different information visualization techniques called parallel coordinate plots and the Reorderable Matrix, and considers how the two representations could be combined. We report an 
experiment where the participants performed tasks with an application having a Reorderable Matrix and parallel coordinates views to the same data, with linking and without linking. It was found out that although the view linking initially slows the task performance, it will accelerate learning and it is well received by the users.

Siirtola, H, and K-J Räihä. 2006, "Interacting with Parallel Coordinates." Interacting with Computers 18(6):1278-309.

Parallel coordinate visualizations have a reputation of being difficult to understand, expert-only representations. We argue that this reputation may be partially unfounded, because many of the parallel coordinate browser implementations lack essential features. This paper presents a survey of current interaction techniques for parallel coordinate browsers and compares them to the visualization design guidelines in the literature. In addition, we report our experiences with parallel coordinate browser prototypes, and describe an experiment where we studied the immediate usability of parallel coordinate visualizations. In the experiment, 16 database professionals performed a set of tasks both with the SQL query language and a parallel coordinate browser. The results show that although the subjects had doubts about the general usefulness of the parallel coordinate technique, they could perform the tasks more efficiently with a parallel coordinate browser than with their familiar query language interface.

Simonton, DK. 1996, "Foresight in Insight? A Darwinian Answer." in The Nature of Insight, eds. RJ Sternberg and JE Davidson, pp. 465-94. MIT Press.

Simunic, K. 2003, "Visualization of Stock Market Charts." In 11th International Conference on Computer Graphics, Visualization and Computer Vision 2003, pp. 129-32.

With this work in progress we propose a visualization system for stock market charts. Insight into stock charts is important in technical stock market analysis where exclusively the chart shape is considered in decision making. We focus on clustering existing chart shapes. Clustering delivers representative charts representing a set of similar charts. In our work, we generate a $2 \mathrm{D}$ map of these representative charts and implement tools like zooming, levels of details and selection. Thus, we present a new approach of automatically generating the whole picture of the stock market dynamics.

Skogen, MGR. 2006, "An Investigation into the Subjective Experience of Icons: A Pilot Study." In 10th International Conference on Information Visualization, pp. 368-73.

In electronic applications, the graphical user interface ("GUI") contains information on many levels of visualization. The user interacts with the computer by manipulation of graphic elements on the screen, the smallest unit being the icon. This paper describes a pilot test conducted with eight students at the Norwegian University of Science and Technology (NTNU). The hypothesis is that simpler, less visually complicated icons will be understood (thus picked up) first. The more immediately communicative an icon is, the faster the response, hence, the lesser the cognitive load. An unconventional test method was used: students were asked to place user-interface icons from daily computer-use (e.g., "HOME") on a dual-axis matrix, based on their immediate reaction. Through the pilot test, the author attempted to discover the relationship between 1) visual interpretation of icons and 2) the speed in which they are understood. Results showed that there is a consistency in how the icons were interpreted visually. The results also demonstrated a relationship between an icon's design and the order in which it was picked up. Future studies will target these issues more specifically 
Skupin, A. 2009, "Discrete and Continuous Conceptualizations of Science: Implications for Knowledge Domain Visualization." Journal of Informetrics 3(3):233-45.

Visual depiction of the structure and evolution of science has been proposed as a key strategy for dealing with the large, complex, and increasingly interdisciplinary records of scientific communication. While every such visualization assumes the existence of spatial structures within the system of science, new methods and tools are rarely linked to thorough reflection on the underlying spatial concepts. Meanwhile, geographic information science has adopted a view of geographic space as conceptualized through the duality of discrete objects and continuous fields. This paper argues that conceptualization of science has been dominated by a view of its constituent elements (e.g., authors, articles, journals, disciplines) as discrete objects. It is proposed that, like in geographic information science, alternative concepts could be used for the same phenomenon. For example, one could view an author as either a discrete object at a specific location or as a continuous field occupying all of a discipline. It is further proposed that this duality of spatial concepts can extend to the methods by which low-dimensional geometric models of high-dimensional scientific spaces are created and used. This can result in new methods revealing different kinds of insights. This is demonstrated by a juxtaposition of two visualizations of an author's intellectual evolution on the basis of either a discrete or continuous conceptualization.

Skupin, A. 2000, "From Metaphor to Method: Cartographic Perspectives on Information Visualization." In IEEE Symposium on Information Visualization (INFOVIS 2000), pp. 91-97.

By virtue of their spatio-cognitive abilities, humans are able to navigate through geographic space as well as meaningfully communicate geographic information represented in cartographic form. The current dominance of spatial metaphors in information visualization research is the result of the realization that those cognitive skills also have value in the exploration and analysis of nongeographic information. While mapping or landscape metaphors are routinely used in this field there is a noticeable lack of consideration for existing cartographic expertise. This is especially appal-ent whenever problematic issues are encountered, such as graphic complexity or feature labeling. There are a number of areas in which a cartographic outlook could provide a valuable perspective. This paper discusses how geographic and cartographic notions may influence the design of visualizations for textual information spaces. Map projections, generalization, feature labeling and map design issues are discussed.

Skupin, A. 2002, "On Geometry and Transformation in Map-Like Information Visualization." In 2nd International Workshop on Visual Interfaces to Digital Libraries held at the Joint Conference on Digital Libraries (JCDL), pp. 161-70.

A number of visualization techniques have been put forward that implement a map metaphor to display abstract, non-georeferenced information. This paper refers to these as map-like information visualizations that are distinguished from other information visualization approaches in a number of ways. It interprets some of the principles underlying these techniques within a framework informed by geographic information science (GIScience). Recent geographic efforts in this research area have linked ideas about the nature of geographic information to cognitive schemata proposed by cognitive linguists. This paper draws on the arguments that have emerged from those efforts regarding the nature and usefulness of geographic metaphors. It proposes to discuss particular projection techniques, like multidimensional scaling or self-organizing maps, with reference to the geometric primitives they employ. These primitives will drive the choice of geometric and symbolic transformations that are 
necessary to achieve a particular visualization. Designers of map-like visualizations are thus challenged to seriously consider the implications of particular computational techniques and the consequences of symbolization choices.

Skupin, A, and SI Fabrikant. 2003, "Spatialization Methods: A Cartographic Research Agenda for NonGeographic Information Visualization." Cartography and Geographic Information Science 30(2):99-120.

Slingsby, A. 2010, "Designing Visual Analytics Systems for Disease Spread and Evolution." In IEEE Symposium on Visual Analytics Science \& Technology, pp. 285-86.

Slingsby, A, J Dykes, and J Wood. 2010, "Poster : Tweeting Visualizations for Collaborative Visual Analysis." In IEEE International Conference on Information Visualization, pp. 1-2.

Slingsby, A, J Dykes, and J Wood. 2008, "Using Treemaps for Variable Selection in Spatio-Temporal Visualisation." Information Visualization 7(3-4):210-24.

We demonstrate and reflect upon the use of enhanced treemaps that incorporate spatial and temporal ordering for exploring a large multivariate spatio-temporal data set. The resulting data-dense views summarise and simultaneously present hundreds of space-, time-, and variable-constrained subsets of a large multivariate data set in a structure that facilitates their meaningful comparison and supports visual analysis. Interactive techniques allow localised patterns to be explored and subsets of interest selected and compared with the spatial aggregate. Spatial variation is considered through interactive raster maps and high-resolution local road maps. The techniques are developed in the context of 42.2 million records of vehicular activity in a $98 \mathrm{~km}(2)$ area of central London and informally evaluated through a design used in the exploratory visualisation of this data set. The main advantages of our technique are the means to simultaneously display hundreds of summaries of the data and to interactively browse hundreds of variable combinations with ordering and symbolism that are consistent and appropriate for space- and time- based variables. These capabilities are difficult to achieve in the case of spatio-temporal data with categorical attributes using existing geovisualisation methods. We acknowledge limitations in the treemap representation but enhance the cognitive plausibility of this popular layout through our two-dimensional ordering algorithm and interactions. Patterns that are expected (e.g., more traffic in central London), interesting (e.g., the spatial and temporal distribution of particular vehicle types) and anomalous (e.g., low speeds on particular road sections) are detected at various scales and locations using the approach. In many cases, anomalies identify biases that may have implications for future use of the data set for analyses and applications. Ordered treemaps appear to have potential as interactive interfaces for variable selection in spatio-temporal visualisation. Information Visualization (2008) 7, 210-224. doi:

$10.1057 /$ palgrave.ivs. 9500185

Slingsby, A, et al. 2007, "Interactive Tag Maps and Tag Clouds for the Multiscale Exploration of Large Spatio-Temporal Datasets." In 11th International Conference Information Visualization, pp. 497-504.

'Tag clouds' and 'tag maps' are introduced to represent geographically referenced text. In combination, these aspatial and spatial views are used to explore a large structured spatio-temporal data set by providing overviews and filtering by text and geography. Prototypes are implemented using freely available technologies including Google Earth and Yahoo! 's Tag Map applet. The interactive tag map and tag cloud techniques and the rapid prototyping method used are informally evaluated through successes and limitations encountered. Preliminary evaluation suggests that the techniques may be useful 
for generating insights when visualizing large data sets containing geo-referenced text strings. The rapid prototyping approach enabled the technique to be developed and evaluated, leading to geovisualization through which a number of ideas were generated. Limitations of this approach are reflected upon. Tag placement, generalisation and prominence at different scales are issues which have come to light in this study that warrant further work.

Slingsby, A, and J Wood. 2010, "Discovery Exhibition : Making Hurricane Track Data Accessible." In IEEE Conference on Information Visualization.

Slocum, TA, et al. 2001, "Cognitive and Usability Issues in Geovisualization." Cartography and Geographic Information Science 28(1):61-75.

Smidts, C, and A Mosleh. 1995, "A Taxonomy and Root-Cause Analysis of Human Cognitive Behavior Based on a Cognitive Model." In Annual Reliability and Maintainability Symposium 1995 Proceedings, pp. 303-14.

A taxonomy of human cognitive behavior has been developed which will help classify human cognitive errors and is a first stcp in the collection of human error data from actual events. This taxonomy is based on a cognitive model for accident situations in nuclear power plants called the IDA (Information, Decision, and Action) Cognitive Model. IDA essentially focuses on aspects of problem solving and decision making. However, observation of information, crew communication, and distribution of tasks are also addressed even if in a lesser extent. The basic elements of the model include the Mental State (engine of the problem solving and decision making process), the Goals generated by the opcrators, and the Strategies used to solve them. We present the taxonomy and apply it on an example: the Pressurizer Spray Valve Failure Event of Crystal River - 3 nuclear power plant. 1.

Smith, SM. 1996, "Getting into and out of Mental Ruts : A Theory of Fixation , Incubation , and Insight." in The Nature of Insight, eds. RJ Sternberg and JE Davidson, pp. 229-52. MIT Press.

Smolnik, S, L Nastansky, and T Knieps. 2003, "Mental Representations and Visualization Processes in Organizational Memories." In Seventh International Conference on Information Visualization (IV'03), pp. 568-75.

Visual metaphors describe cognitive processes in every part of life and function as a connection between what we see and what we think. Mental activities and perceptional interactions of the real world are often interdependent and occur mutually. Cognition is enhanced by the use of the real world, in particular the use of cognitive artifacts or physical inventions (S. K. Card, et al., 1999). We describe the process from perception to information, explain different models of mental representations, and introduce Chi's visualization process. We use these theories and models to introduce and evaluate different visualization tools, which are positioned to explore and navigate a groupware-based organizational memory that is presented as a small case. We point out how the different characteristics and functionalities of these visualization tools contribute to a synergetic match of visual metaphors and cognitive processes. 
Smuc, M, et al. 2009, "To Score or Not to Score? Tripling Insights for Participatory Design." IEEE Computer Graphics and Applications 29(3):29-38.

For evaluating visual-analytics tools, many studies confine to scoring user insights into data. For participatory design of those tools, we propose a three-level methodology to make more out of users' insights. The relational insight organizer (RIO) helps to understand how insights emerge and build on one each other. In recent years, computers have also been used to develop visual methods and tools that further support the data analysis process. With the advent of the emerging field of visual analytics (VA), the underlying concept of visual tools is taken a step further. In essence, VA combines human analytical capabilities with computer processing capacities. In the human-computer interaction process, the user generates new knowledge and gains insights.

Smuc, M, et al. 2008, "Visualizations at First Sight: Do Insights Require Training?" In 4th Symposium of the Workgroup Human-Computer Interaction and Usability Engineering of the Austrian Computer Society on HCI and Usability for Education and Work, pp. 261-80.

Understanding novel visualizations can be a challenge even for experienced users. During iterative usability engineering phases in the DisCō project, visualizations of time-oriented data are explored by domain experts and non-experts. The aim of our study is to analyze the generation of knowledge and understanding by means of visualizations without previous user training. Focusing on applicability in various business domains for personnel planning and time scheduling, we tested mockups of visualizations with a method based on user-reported insights. Results show almost identical behavior of domain experts and non-experts when generating insights into the data from scratch. In the course of working with a visualization, an interchange of insights into the visualization and insights into the data was found. (C) 2008 Springer Berlin Heidelberg.

Smuc, M, E Mayr, and H Risku. 2010, "Is Your User Hunting or Gathering Insights? Identifying Insight Drivers across Domains." In BELIV'10: Beyond time and errors: novel evaluation methods for Information Visualization, April 10-11, 2010.

In recent years, using the number of insights to benchmark visual analytics tools has become a prominent method in the Infovis community. The insight methodology has become a frequently used instrument to measure the performance of tools that are developed for highly specialized purposes for highly specialized domain-experts. But some tools have a wider target group of experts with knowledge in different domains. The utility of the insight-method for other expert user groups without specific domain knowledge has been addressed to a far lesser extent. In a case study we give an illustration of how and where insights from experts with and without domain knowledge differ, and how these findings might enrich the evaluation of visualization tools designed for usage across different domains.

Snyder, C. 2004, "Chapter 13 - the Politics of Paper Prototyping." in Paper Prototyping, pp. 285-318. Morgan Kaufmann, Burlington.

Somerville, J, et al. 2010, "Iraster: A Novel Information Visualization Tool to Explore Spatiotemporal Patterns in Multiple Spike Trains." Journal of Neuroscience Methods 194(1):158-71.

Over the last few years, simultaneous recordings of multiple spike trains have become widely used by neuroscientists. Therefore, it is important to develop new tools for analysing multiple spike trains in order 
to gain new insight into the function of neural systems. This paper describes how techniques from the field of visual analytics can be used to reveal specific patterns of neural activity. An interactive raster plot called iRaster has been developed. This software incorporates a selection of statistical procedures for visualization and flexible manipulations with multiple spike trains. For example, there are several procedures for the re-ordering of spike trains which can be used to unmask activity propagation, spiking synchronization, and many other important features of multiple spike train activity. Additionally, iRaster includes a rate representation of neural activity, a combined representation of rate and spikes, spike train removal and time interval removal. Furthermore, it provides multiple coordinated views, time and spike train zooming windows, a fisheye lens distortion, and dissemination facilities. iRaster is a user friendly, interactive, flexible tool which supports a broad range of visual representations. This tool has been successfully used to analyse both synthetic and experimentally recorded datasets. In this paper, the main features of iRaster are described and its performance and effectiveness are demonstrated using various types of data including experimental multi-electrode array recordings from the ganglion cell layer in mouse retina. iRaster is part of an ongoing research project called VISA (Visualization of Inter-Spike Associations) at the Visualization Lab in the University of Plymouth. The overall aim of the VISA project is to provide neuroscientists with the ability to freely explore and analyse their data. The software is freely available from the Visualization Lab website (see www.plymouth.ac.uk/infovis).

Soomlek, C, and L Benedicenti. 2010, "Operational Wellness Model: A Wellness Model Designed for an Agent-Based Wellness Visualization System." In 2nd International Conference on eHealth, Telemedicine and Social Medicine, pp. 45-50.

This paper introduces an operational wellness model that forms the basis of a wellness visualization system. The operational wellness model is needed to determine a common reference enabling the evaluation of the visualization system. Existing definitions of wellness and wellness evaluation models have limitations and cannot be realized in software. The proposed model comprises the operational definition of wellness and the operational wellness evaluation model. The results generated by the operational wellness model will be used in the wellness visualization system. The model presents numerous advantages: it is extensible, computable, and can be rendered by a computer program.

Sorapure, M. 2010, "Information Visualization, Web 2.0, and the Teaching of Writing." Computers and Composition 27(1):59-70.

With the development of free, online, interactive visualization tools, the field of information visualization--or infovis--is being opened to diverse users and uses, and particularly to novice users who want to visualize personally relevant information. Indeed, Web 2.0 is making infovis increasingly viable as a medium for organizing, exploring, analyzing, and creatively deriving meaning from the deluge of information that we face in our everyday lives. For writing teachers, new developments in information visualization bring valuable opportunities to enhance our students' digital and critical competencies. Projects that ask students to visualize text, personal data, and social data can provide compelling entry points into Web 2.0, as students learn about existing tools and sources of data, create their own visualizations, and then analyze the insight that they and others can gain through seeing data represented visually. Incorporating infovis assignments into writing classes can help us reinvigorate some of our standard assignments, encourage students to think critically about the software they use, and provide new opportunities for the production of digital artifacts. 
Sousa Santos, B, et al. 2002, "Quantifying the Objective Quality of Voxel Based Data Visualizations Produced by a Ray Caster: A Proposal." In IEEE Conference on Information Visualization, pp. 111-16.

A set of parameters to assess the objective quality of visualizations of a voxel-based data set, produced using a ray caster, is proposed as a first step toward the evaluation of the overall quality of these visualizations. Results obtained using synthetic data and a simple implementation of a ray caster are presented. The final goal of this evaluation is the computation of \&ldquo; confidence indices\&rdquo; that could offer the user a \&ldquo;guided visualization\&rdquo; i.e., allow him/her to decide what are the \&ldquo; best\&rdquo; visualizations of a data set

Speckmann, B, and K Verbeek. 2010, "Necklace Maps." IEEE Transactions on Visualization and Computer Graphics 16(6):881-9.

Statistical data associated with geographic regions is nowadays globally available in large amounts and hence automated methods to visually display these data are in high demand. There are several wellestablished thematic map types for quantitative data on the ratio-scale associated with regions: choropleth maps, cartograms, and proportional symbol maps. However, all these maps suffer from limitations, especially if large data values are associated with small regions. To overcome these limitations, we propose a novel type of quantitative thematic map, the necklace map. In a necklace map, the regions of the underlying two-dimensional map are projected onto intervals on a one-dimensional curve (the necklace) that surrounds the map regions. Symbols are scaled such that their area corresponds to the data of their region and placed without overlap inside the corresponding interval on the necklace. Necklace maps appear clear and uncluttered and allow for comparatively large symbol sizes. They visualize data sets well which are not proportional to region sizes. The linear ordering of the symbols along the necklace facilitates an easy comparison of symbol sizes. One map can contain several nested or disjoint necklaces to visualize clustered data. The advantages of necklace maps come at a price: the association between a symbol and its region is weaker than with other types of maps. Interactivity can help to strengthen this association if necessary. We present an automated approach to generate necklace maps which allows the user to interactively control the final symbol placement. We validate our approach with experiments using various data sets and maps.

Speier, C. 2006, "The Influence of Information Presentation Formats on Complex Task Decision-Making Performance." International Journal of Human-Computer Studies 64(11):1115-31.

Understanding the influence of information presentation formats on decision-making effectiveness is an important component of human-computer interaction user interface design. The pervasive nature and ease of use associated with information display formats in widely used personal productivity software suggests that decision-makers are likely to create and/or use documents with both text-based and more visually oriented information displays. Past research has investigated the role of these displays on simple decision tasks; however, empirical research has not extended to more complex tasks, more comparable to the types of tasks decision-makers face every day. Results from the empirical analysis suggest that the relationship between information presentation format and decision performance is moderated by the complexity of the task. More specifically, spatial formats result in superior decision accuracy for simpleand complex-spatial tasks and faster decision time for all tasks except the complex-symbolic task where graphs and tables result in equivalent decision time. 
Spence, B, et al. 1995, "Visualisation for Functional Design." In IEEE Conference on Information Visualization, pp. 4-10.

We present two novel visualisation tools: the Influence Explorer and the Prosection Matrix. These were specifically created to support engineering artifact design and similar tasks in which a set of parameter values must be chosen to lead to acceptable artifact performance. These tools combine two concepts. One is the interactive and virtually immediate responsive display of data in a manner conducive to the acquisition of insight. The other, involving the precalculation of samples of artifact performance, facilitates smooth exploration and optimisation leading to a design decision. The anticipated benefits of these visualisation tools are illustrated by an example taken from electronic circuit design, in which full account must be taken of the uncertainties in parameter values arising from inevitable variations in the manufacturing process.

Spence, R. 2001. Information Visualization. Addison-Wesley Reading, MA.

Spence, R. 2008, "A Science of Interaction - a Multidimensional Canvas." In GRAPP '08, pp. 415-18.

A recently proposed search for a science of interaction will involve the creation of a multidimensional canvas composed of many features: complex perceptual and cognitive processes, the many approaches to interaction design, the many models of interaction and the vast range of interaction modalities. In any attempt to meet this challenge this paper urges emphasis on precise definitions, especially for visualization and interaction, and reports the results of an exploratory study.

Spindler, M, and C Tominski. 2010, "Poster : Towards Making Infovis Views Tangible." In IEEE Conference on Information Visualization.

Spoerri, A. 2004, "Coordinated Views and Tight Coupling to Support Meta Searching." In Second International Conference on Coordinated \& Multiple Views in Exploratory Visualization, pp. 39-48.

This paper addresses how information visualization can support users in the meta search process and some of the design issues that arise. It describes MetaCrystal and its linked tools that enable users to control how to combine and filter the search results by different search engines. A hierarchy of aggregation is employed to provide users with quick insights into the number of documents found by a specific number or combination of search engines. MetaCrystal enables users to visually perform advanced filtering operations, such as complex Boolean constraints. This paper also discusses how to support tight coupling between the filtering controls and tools that operate at different levels of aggregation.

Sprague, DW, and M Tory. 2009, "Motivation and Procrastination: Methods for Evaluating Pragmatic Casual Information Visualizations." Computer Graphics and Applications, IEEE 29(4):86-91.

For casual users, how do goals and incentives interact with visualization usage patterns? Professional race car drivers are almost exclusively concerned about a car's performance, whereas average car owners might be swayed by fuel efficiency, aesthetics, and even color. Similarly, factors other than performance might motivate casual information visualization (InfoVis) users. Outside of work contexts, visualizations serve as cognitive aids, art, propaganda, and even procrastination aids. Out of curiosity, we asked two women with no computer science training to look at the digg visualizations by Stamen design. To our 
surprise, comments changed from "sooo cute" and "I like [the] animation" during the first minute to "annoying" and "cute but not practical" less than five minutes later. Motion rapidly went from being appealing and motivating to being distracting and discouraging. Perhaps simply getting eyes on the screen is insufficient. But what makes a visualization successful in informal contexts, and if we do not know, how do we find out?

Sripada, SG, and G Feng. 2007, "Linguistic Interpretations of Scuba Dive Computer Data." In 11th International Conference Information Visualization (IV'07), pp. 436-41.

Exploratory visualizations of dive computer data are available to the scuba diving community to examine the safety of their recorded dives. In a study involving certified scuba divers on the usage of these visualizations we found that these are not used regularly which agrees well with the suspicions expressed by the medical staff who attend to patients with diving related illnesses. We built a prototype knowledge based system that interprets dive computer data to automatically generate their linguistic interpretations to supplement the existing visualizations. We hypothesized that linguistic interpretations by virtue of their structure communicate safety related messages directly to divers. Our evaluation showed that divers like the idea of integrating linguistic interpretations into existing visualizations. However, the study also showed that linguistic interpretations can be useful only if they are emotionally appropriate to individual divers.

Staller, A, I Informatik, and TU München. 2006, "Merging Domain Knowledge and Task Analysis in an Ontology." in Current Developments in Technology-Assisted Education, Badajoz, Spain. Technological Science Education, Collaborative Learning, Knowledge Management, pp. 1585-89. FORMATEX.

Stapleton, G, and A Delaney. 2008, "Evaluating and Generalizing Constraint Diagrams." Journal of Visual Languages \& Computing 19(4):499-521.

The constraint diagram language was designed to be used in conjunction with the unified modelling language (UML), primarily for placing formal constraints on software models. In particular, constraint diagrams play a similar role to the textual object constraint language (OCL) in that they can be used for specifying system invariants and operation contracts in the context of a UML model. Unlike the OCL, however, constraint diagrams can be used independently of the UML. In this paper, we illustrate a range of intuitive and counter-intuitive features of constraint diagrams and highlight some (potential) expressiveness limitations. The counter-intuitive features are related to how the individual pieces of syntax interact. A generalized version of the constraint diagram language that overcomes the illustrated counter-intuitive features and limitations is proposed. In order to discourage specification readers and writers from overlooking certain semantic information, the generalized notation allows this information to be expressed more explicitly than in the non-generalized case. The design of the generalized notation takes into account five language design principles which are discussed in the paper. We provide a formalization of the syntax and semantics for generalized constraint diagrams. Moreover, we establish a lower bound on the expressiveness of the generalized notation and show that they are at least as expressive as constraint diagrams. 
Stasko, J. 2008, "Visualization for Information Exploration and Analysis." In IEEE Symposium on Visual Languages and Human-Centric Computing, pp. 7-8.

Making sense of data becomes more challenging as the data grows larger and becomes more complex. If a picture truly can be worth a thousand words, then clever visualizations of data should hold promise in helping people with sense-making tasks. I firmly believe that visual representations of data can help people to better explore, analyze, and understand it, thus transforming the data into information. In this talk, I will explain how visualization and visual analytics help people make sense of data and I will provide many such examples. I also will describe my present research into visualization for investigative analysis. This project explores how visual analytics can help investigators examine a large document collection in order to discover embedded stories and narratives scattered across the documents in the collection.

Stasko, J, et al. 2000, "An Evaluation of Space-Filling Information Visualizations for Depicting Hierarchical Structures." International Journal of Human-Computer Studies 53(5):663-94.

A variety of information visualization tools have been developed recently, but relatively little effort has been made to evaluate the effectiveness and utility of the tools. This article describes results from two empirical studies of two visualization tools for depicting hierarchies, in particular, computer file and directory structures. The two tools examined implement space-filling methodologies, one rectangular, the Treemap method, and one circular, the Sunburst method. Participants performed typical file/directory search and analysis tasks using the two tools. In general, performance trends favored the Sunburst tool with respect to correct task performance, particularly on initial use. Performance with Treemap tended to improve over time and use, suggesting a greater learning cost that was partially recouped over time. Each tool afforded somewhat different search strategics, which also appeared to influence performance. Finally, participants strongly preferred the Sunburst tool, citing better ability to convey structure and hierarchy. (C) 2000 Academic Press.

Stasko, J, et al., ed.^eds. 1998. Software Visualization: Programming as a Multimedia Experience. The MIT Press, Cambridge, MA.

Stasko, J, C Gorg, and Z Liu. 2008, "Sensemaking across Text Documents : Human-Centered, Visual Exploration with Jigsaw." In CHI '08 Workshop on Sensemaking, pp. 1-7.

In this article we describe our research on providing techniques and tools for a particular type of sensemaking - finding embedded narratives or plots across large collections of relatively brief text documents. Our approach is decidedly human-centered and we have created the Jigsaw system as an embodiment of these principles. Jigsaw provides multiple visualizations depicting the documents, the entities within them, and connections between the two. Analysts interactively explore the entities and documents as a way to guide document selection for reading, which ultimately is necessary to synthesize the embedded plot. This article describes the challenges in this particular style of sensemaking as well as the ways in which Jigsaw is attempting to overcome those challenges. 
Stasko, J, C Görg, and R Spence. 2008, "Jigsaw: Supporting Investigative Analysis through Interactive Visualization." Information Visualization 7(2):118-32.

Investigative analysts who work with collections of text documents connect embedded threads of evidence in order to formulate hypotheses about plans and activities of potential interest. As the number of documents and the corresponding number of concepts and entities within the documents grow larger, sense-making processes become more and more difficult for the analysts. We have developed a visual analytic system called Jigsaw that represents documents and their entities visually in order to help analysts examine reports more efficiently and develop theories about potential actions more quickly. Jigsaw provides multiple coordinated views of document entities with a special emphasis on visually illustrating connections between entities across the different documents.

Stedmon, AW, et al. 1999, "Old Theories, New Technologies: Cumulative Clutter Effects Using Augmented Reality." In IEEE International Conference on Information Visualization, pp. 132-37.

The paper investigates human cognitive performance when information is presented via augmented reality (AR) and overlaid upon a primary display. Initial results support traditional experimental paradigms of human memory (G.A. Miller, 1956) and comprehension of information (C.D. Wickens, 1992), and have been used to compare AR and standard display formats when used in isolation. Results from these experiments provide a fundamental baseline for cognitive performance with a see-through AR headset. Furthermore the results lay the foundations for more comprehensive trials later in the research programme when the display formats are combined to provide a full AR facility. Consideration is given to the specific effects of cumulative clutter and two experiments are outlined that investigate the effects on target identification. Although the data are still being analysed, further consideration is given to key points addressed in the experimental design. Details of a dedicated Web site are provided where information will be consolidated and provide a basis for developing guidelines for the future development and application of AR technology

Steinbrückner, F, and C Lewerentz. 2010, "Representing Development History in Software Cities." In 5th ACM Symposium on Software Visualization (SOFTVIS), pp. 193-202.

Sternberg, RJ, and TI Lubart. 1996, "Investment Perspective on Creative Insight." in The Nature of Insight, eds. RJ Sternberg and JE Davidson, pp. 535-58.

Stoica, F, and D Simian. 2008, "Approaches to Cognitive Support in Biomedical Knowledge-Based Systems." In 9th WSEAS International Conference on Mathematics and Computeres in Biology and Chemistry, pp. 217-22.

The current Internet already contains vast amounts of biomedical information resources, Such as research articles, images, clinical guidelines, and drug catalogues. Making these resources available in a more structured way is one of the goals of several largescale ontology development efforts. Constructing large knowledge biomedical models is a cognitively challenging process. In order to assist people working with these models and tools, this paper proposes considering the tools used in light of the cognitive support they provide. A tool's usefulness is ultimately dependent upon its utility relating to cognition: i.e., to thinking, reasoning, and creating. Cognitive Support describes those elements of a tool which aid human reasoning and understanding. For the put-pose of this paper, has been used GandrKB (Gene annotation data representation), an ontology and knowledge base describing gene functions 
enabling biologists to annotate (multiple) genes on Affymetrix Microarrays. In the paper are investigated several tools which provide cognitive support using information visualization to construct advanced visual interfaces.

Stoll, J, et al. 2007, "Exploiting the User: Adapting Personas for Use in Security Visualization Design." In VizSec: Workshop on Visualization for Computer Security, pp. 1-14.

professional information analysts professional information analysts ...spend lots of time exploring their data before they can perform productive analysis. ...need a faster way to get a sense of the data. ...need a faster way to see meaningful changes. first look problem

Stolte, C, et al. 1999, "Visualizing Application Behavior on Superscalar Processors." In IEEE Symposium on Information Visualization, pp. 10-17,141.

The advent of superscalar processors with out-of-order execution makes it increasingly difficult to determine how well an application is utilizing the processor and how to adapt the application to improve its performance. We describe a visualization system for the analysis of application behavior on superscalar processors. Our system provides an overview-plus-detail display of the application's execution. A timeline view of pipeline performance data shows the overall utilization of the pipeline. This information is displayed using multiple time scales, enabling the user to drill down from a high-level application overview to a focus region of hundreds of cycles. This region of interest is displayed in detail using an animated cycle-by-cycle view of the execution. This view shows how instructions are reordered and executed and how functional units are being utilized. Additional context views correlate instuctions in this detailed view with the relevant source code for the application. This allows the user to discover the root cause of the poor pipeline utilization and make changes to the application to improve its performance. This visualization system can be easily configured to display a variety of processor models and configurations. We demonstrate it for both the MXS and MMIX processor models

Stone, LM, et al. 1994, "Visualizing Data: Is Virtual Reality the Key?" In IEEE Conference on Information Visualization, pp. 410-13.

A visualization goal is to simplify the analysis of large-quantity, numerical data by rendering the data as an image that can be intuitively manipulated. The question the article addresses is whether or not virtual reality techniques are the cure-all to the dilemma of visualizing increasing amounts of data. It determines the usefulness of techniques available today and in the near future that will ease the problem of visualizing complex data. In regards to visualization, the article discusses characteristics of virtual reality systems, data in three-dimensional environments, augmented reality, and virtual reality market opportunities

Storey, MAD, FD Fracchia, and HA Müller. 1999, "Cognitive Design Elements to Support the Construction of a Mental Model During Software Exploration." Journal of Systems and Software 44(3):171-85.

The scope of software visualization tools which exist for the navigation, analysis and presentation of software information varies widely. One class of tools, which we refer to as Software exploration tools, provides graphical representations of static software structures linked to textual views of the program source code and documentation. This paper describes a hierarchy of cognitive issues which should be 
considered during the design of a software exploration tool. The hierarchy of cognitive design elements is derived through the examination of program comprehension cognitive models. Examples of how existing tools address each of these issues are provided. In addition, this paper demonstrates how these cognitive design elements may be applied to the design of an effective interface for software exploration.

Storey, M-AD, FD Fracchia, and HA MÜLler. 1999, "Customizing a Fisheye View Algorithm to Preserve the Mental Map." Journal of Visual Languages \& Computing 10(3):245-67.

Frequently, large knowledge bases are represented by graphs. Many visualization tools allow users or other applications to interact with and adjust the layouts of these graphs. One layout adjustment problem is that of showing more detail without eliding parts of the graph. Approaches based on a fisheye lens paradigm seem well suited to this task. However, many of these techniques are non-trivial to implement and their distortion techniques often cannot be altered to suit different graph layouts. When distorting a graph layout, it is often desirable to preserve various properties of the original graph in an adjusted view. Pertinent properties may include straightness of lines, graph topology, orthogonalities and proximities. However, it is normally not possible to preserve all of the original properties of the graph layout. The type of layout and its application should be considered when deciding which properties to preserve or distort. This paper describes a fisheye view algorithm which can be customized to suit various different graph layouts. In contrast to other methods, the user can select which properties of the original graph layout to preserve in an adjusted view. The technique is demonstrated through its application to visualizing structures in large software systems.

Storey, MD. 1999, "Cognitive Design Elements to Support the Construction of a Mental Model During Software Visualization School of Computing Science." Journal of Systems and Software 44(3):171-85.

The scope of software visualization tools which exist for the navigation, analysis and presentation of software information varies widely. One class of tools, which we refer to as Software exploration tools, provides graphical representations of static software structures linked to textual views of the program source code and documentation. This paper describes a hierarchy of cognitive issues which should be considered during the design of a software exploration tool. The hierarchy of cognitive design elements is derived through the examination of program comprehension cognitive models. Examples of how existing tools address each of these issues are provided. In addition, this paper demonstrates how these cognitive design elements may be applied to the design of an effective interface for software exploration.

Strobelt, H, M Heilig, and O Deussen. 2010, "Interactive Demo : Stay in Touch with Infovis Visualizing Document Collections with Document Cards." In IEEE Conference on Information Visualization.

Strothotte, T, and S Schlechtweg. 2002, "Introduction." in Non-Photorealistic Computer Graphics: Modelling, Rendering, and Animation, pp. 1-30. Morgan Kaufmann, San Francisco.

Stryker, M, I Turton, and AM MacEachren. 2008, "Health Geojunction: Geovisualization of News and Scientific Publications to Support Situation Awareness." In Workshop on Geospatial Visual Analytics at GIScience '08, pp. 1-4.

The Health GeoJunction application is a web portal that extracts information from scientific literature, public health reports, and news feeds to support geographically contextualized searches and the discovery 
of cross-connections between documents through a mapbased interface of coordinated views. Text extracted from documents can be queried and represented as visual artifacts within a map, timeline, or an extended tag cloud. These linked views enable the user to progressively filter a collection of documents and provide an intuitive means for expressing queries in terms of the conceptual dimensions of location, time, and theme. In this paper we focus specifically on aspects of the Health GeoJunction client development, including support for the space-time-attribute perspective, information visualization and cartographic visual representations, and access to computational reasoning and information extraction tools on the server. To guide development, we have created scenarios for situation awareness and knowledge construction within a visual analytic environment for an analyst assessing the evolution of Avian Influenza as a public health threat from the exploration of RSS (Real Simple Syndication) feeds from the World Health Organization (WHO), World Animal Health Organization (OIE) reports on outbreak incidents, and the PubMed database of biomedical scientific abstracts.

Stuart, L, D Marocco, and A Cangelosi. 2005, "Information Visualization for Knowledge Extraction in Neural Networks." In 15th International Conference on Artificial Neural Networks (ICANN 2005), pp. 515-20.

In this paper, a user-centred innovative method of knowledge extraction in neural networks is described. This is based on information visualization techniques and tools for artificial and natural neural systems. Two case studies are presented. The first demonstrates the use of various information visualization methods for the identification of neuronal structure (e.g., groups of neurons that fire synchronously) in spiking neural networks. The second study applies similar techniques to the study of embodied cognitive robots in order to identify the complex organization of behaviour in the robot's neural controller.

Stuck, ER. 1996, "Detecting and Diagnosing Mistakes in Vision-Based Navigation." Robotics and Autonomous Systems 17(4):259-85.

Robust mobile robot navigation is an open problem. Navigational mistakes are inevitable due to the characteristics of sensors, models, actuators, and natural environments. This paper describes how to detect and diagnose mistakes that autonomous mobile robots make while navigating through large-scale space using vision. Mistakes are perceptual, cognitive, or motor events that lead a robot astray from its intended route. Detecting and diagnosing a mistake involves realizing that this has happened and determining what the mistake was and when it happened. The approach described in this paper handles mistakes explicitly. Mistakes are detected by looking for mismatches between observations and expectations. Detailed symbolic representations of visual information support comparing observations and expectations augmented by a priori knowledge. Mistakes are diagnosed by examining knowledge from a variety of sources, including a history of the mobile robot's observations and actions. A computer program called implements this approach in simulation and provides experimental results that demonstrate the feasibility and potential of the approach.

Su, CY, and TI Wang. 2010, "Construction and Analysis of Educational Assessments Using Knowledge Maps with Weight Appraisal of Concepts." Computers \& Education 55(3):1300-11.

The rapid advance of information and communication technologies (ICT) has important impacts on teaching and learning, as well as on the educational assessment. Teachers may create assessments utilizing some developed assessment software or test authoring tools. However, problems could occur, 
such as neglecting key concepts in the curriculum or having disproportionate course topics distribution, when teachers create assessments or test items. This study proposes a novel approach, which uses knowledge map with appraisal of concept weights and other ICTs, and implements an assessment system KMAAS to help primary school teachers in Taiwan, or elsewhere, create educational assessments properly. When compiling an assessment, KMAAS ensures that teachers can include all important course concepts intended for assessing and maintain correct balance between course concepts among test items. It does so first by analyzing course material of the assessment range and displaying a concept-weightannotated knowledge map which concretize and visualize the importance of and the relationships among concepts in the range. It then analyzes the test sheet which is being complied and displays another similar real-time updated knowledge map containing balance between course concepts among the test items. Teachers may cross-refer to these maps to help them adjust concept balances and even select appropriate test items from test banks. The system has being evaluated in both the accuracy of learning concepts extraction and the degree of user satisfaction, as measured by questionnaires given to the teachers who tested the system. The promising results confirm the feasibility of this system in helping teachers compile their educational assessments easily and precisely. Other results of the formative evaluations on techniques have being used to improve the system in order to make it more effective and efficient. The methodology and technologies KMAAS employed are all well developed and are domain independent, which makes it highly flexible to transfer to other course subject domain too.

Succar, B. 2009, "Building Information Modelling Framework: A Research and Delivery Foundation for Industry Stakeholders." Automation in Construction 18(3):357-75.

Building Information Modelling (BIM) is an expansive knowledge domain within the Architecture, Engineering, Construction and Operations (AECO) industry. To allow a systematic investigation of BIM's divergent fields, its knowledge components must be defined and expanding boundaries delineated. This paper explores some of the publicly available international guidelines and introduces the BIM Framework, a research and delivery foundation for industry stakeholders. This is a ['] scene-setting' paper identifying many conceptual parts (fields, stages, steps and lenses), providing examples of their application and listing some of the Framework's deliverables. This paper also identifies and deploys visual knowledge models and a specialised ontology to represent domain concepts and their relations.

Suebnukarn, S, P Haddawy, and P Rhienmora. 2008, "A Collaborative Medical Case Authoring Environment Based on the Umls." Journal of Biomedical Informatics 41(2):318-26.

In this paper, we present a novel collaborative authoring tool that was designed to allow medical teachers to formalize and visualize their knowledge for medical intelligent tutoring systems. Our goal is to increase the efficiency and effectiveness in creating the domain model representing the problem solutionoften referred to as the bottleneck in developing intelligent tutoring systems. We incorporate the Unified Medical Language System (UMLS) knowledge base to assist the authors in creating the problem solution collaboratively via a videoconferencing platform. The system consists of a shared workspace gathering information visualization and tools necessary for collaborative problem-solving tasks. We found that the authoring tool can be used to effectively elicit the knowledge structure of the domain model. This was achieved in hours compared to months for the conventional paper-based approach. (c) 2007 Elsevier Inc. All rights reserved. 
Suma, E, et al. 2008, "A Sketch-Based Approach for Detecting Common Human Actions." in Advances in Visual Computing, Vol 5358, pp. 418-27. Springer Berlin / Heidelberg, Charlotte, NC.

We present a method for detecting common human actions in video, common to athletics and surveillance, using intuitive sketches and motion cues. The framework presented in this paper is an automated end-to-end system which (1) interprets the sketch input, (2) generates a query video based on motion cues, and (3) incorporates a new content-based action descriptor for matching. We apply our method to a publicly-available video repository of many common human actions and show that a video matching the concept of the sketch is generally returned in one of the top three query results.

Summers, KL, et al. 2003, "An Experimental Evaluation of Continuous Semantic Zooming in Program." In IEEE Symposium on Information Visualization, pp. 155-62.

This paper presents the results of an experiment aimed at investigating how different methods of viewing visual programs affect users' understanding. The first two methods used traditional flat and semantic zooming models of program representation; the third is a new representation that uses semantic zooming combined with blending and proximity. The results of several search tasks performed by approximately 80 participants showed that the new method resulted in both faster and more accurate searches than the other methods.

Sunghee, K, H Hagh-Shenas, and V Interrante. 2003, "Conveying Shape with Texture: An Experimental Investigation of the Impact of Texture Type on Shape Categorization Judgments." In IEEE Symposium on Information Visualization, pp. 163-70.

As visualization researchers, we are interested in gaining a better understanding of how to effectively use texture to facilitate shape perception. If we could design the ideal texture pattern to apply to an arbitrary smoothly curving shape to be most accurately and effectively perceived, what would the characteristics of that texture pattern be? In this paper we describe the results of a comprehensive controlled observer experiment intended to yield insight into that question. Here, we report the results of a new study comparing the relative accuracy of observers' judgments of shape type (elliptical, cylindrical, hyperbolic or flat) and shape orientation (convex, concave, both, or neither) for local views of boundary masked quadric surface patches under six different principal direction texture pattern conditions plus two texture conditions (an isotropic pattern and a non-principal direction oriented anisotropic pattern), under both perspective and orthographic projection conditions and from both head-on and oblique viewpoints. Our results confirm the hypothesis that accurate shape perception is facilitated to a statistically significantly greater extent by some principal direction texture patterns than by others. Specifically, we found that, for both views, under conditions of perspective projection, participants more often correctly identified the shape category and the shape orientation when the surface was textured with the pattern that contained oriented energy along both the first and second principal directions only than in the case of any other texture condition. Patterns containing markings following only one of the principal directions, or containing information along other directions in addition to the principal directions yielded poorer performance overall. 
Sutcliffe, A, B Gault, and J-E Shin. 2005, "Presence, Memory and Interaction in Virtual Environments." International Journal of Human-Computer Studies 62(3):307-27.

An experimental study is described, comparing presence, memory, and interaction in three different virtual environments: CAVE, Interactive WorkBench, and Reality Room. The aim of the experiment was to investigate possible relationships between these three parameters. It was found that the CAVE was remembered better, had better usability, and provided a better sense of presence to its users.

Sutcliffe, A, et al. 2010, "User Engagement by User-Centred Design in E-Health." Philosophical Transactions of the Royal Society a-Mathematical Physical and Engineering Sciences 368(1926):4209-

24.

This paper describes the application of user-centred design (UCD) methods and a user engagement (UE) approach to a case study development of a visualization tool (ADVISES) to support epidemiological research. The combined UCD/UE approach consisted of scenario-based design, and analysis of the users' tasks and mental model of the domain. Prototyping and storyboarding techniques were used to explore design options with users as well as specifying functionality for two versions of the software to meet the needs of novice and expert users. An evaluation of the prototype was carried out to assess the extent to which the expert model would support public health professionals in their analysis activities. The results of the design exploration requirements analysis study are reported. The implications of scenario-based design exploration, participatory design and user engagement are discussed.

Sutcliffe, AG, M Ennis, and J Hu. 2000, "Evaluating the Effectiveness of Visual User Interfaces for Information Retrieval." International Journal of Human-Computer Studies 53(5):741-63.

An integrated visual thesaurus and results browser to support information retrieval was designed using a task model of information searching. The system provided a hierarchical thesaurus with a results cluster display representing similarity between retrieved documents and relevance ranking using a bullseye metaphor. Latent semantic indexing (LSI) was used as the retrieval engine and to calculate the similarity between documents. The design was tested with two information retrieval tasks. User behaviour, performance and attitude were recorded as well as usability problems. The system had few usability problems and users liked the visualizations, but recall performance was poor. The reasons for poor/good performance were investigated by examining user behaviour and search strategies. Better searchers used the visualizations more effectively and spent longer on the task, whereas poorer performances were attributable to poor motivation, difficulty in assessing article relevance and poor use of system visualizations. The bullseye browser display appeared to encourage limited evaluation of article relevance on titles, leading to poor performance. The bullseye display metaphor for article relevance was understood by users; however, they were confused by the concept of similarity searching expressed as visual clusters. The conclusions from the study are that while visual user interfaces for information searching might seem to be usable, they may not actually improve performance. Training and advisor facilities for effective search strategies need to be incorporated to enhance the effectiveness of visual user interfaces for information retrieval. 
Suvanaphen, E, and JC Roberts. 2004, "Explicit Verses Implicit: An Analysis of a Multiple Search Result Visualization." In 8th International Conference on Information Visualisation, pp. 731-36.

When searching on the web, users often reformulate their queries after viewing the results and viewing some of the pages. After one or two reformulations the user may implicitly realize patterns and relationships between the multiple search results. We believe that these patterns can be used to identify interesting results. We have developed the prototype Search Engine Similarity (SES) tool which explicitly visualizes the similarity between multiple searches. In this paper we describe an experiment to determine whether explicitly visualizing the relationships between multiple searches will let users browse more effectively. Our results show that explicit difference visualizations can enhance the search process for some tasks.

Szymczak, S, W Elm, and JW Gualtieri. 2006, Improved Performance and Reduced Performace Variability of a Joint Cognitive System - a Method of Technology Design. Technical, Resilient Cognitive Solutions.

Szymczak, S, et al. 2006, Operational Design Review of an Envisioned World: Empirical Lessons Learned on Reviewing a Future Cse-Based Design for a Large Scale Dss Environment. Technical, Resilient Cognitive Solutions.

Takacs, B, and L Simon. 2007, "A Clinical Virtual Reality Rehabilitation System for Phobia Treatment." In 11th International Conference Information Visualization (IV'07), pp. 798-806.

This paper describes a unified approach to applying virtual reality for clinical rehabilitation and its uses to administer cognitive, mental and physicals exercises. Our goal is to create a flexible and reconfigurable VR solution that can be readily installed in hospitals and serve all needs of a rehab-unit with ease. We have successfully used this system for treating cognitive deficits and phobias and present details that underline the usability and importance of our solution.

Takatsuka, M, and M Gahegan. 2002, "Geovista Studio: A Codeless Visual Programming Environment for Geoscientific Data Analysis and Visualization." Computers \& Geosciences 28(10):1131-44.

The fundamental goal of the GeoVISTA Studio project is to improve geoscientific analysis by providing an environment that operationally integrates a wide range of analysis activities, including those both computationally and visually based. Improving the infrastructure used in analysis has far-reaching potential to better integrate human-based and computationally based expertise, and so ultimately improve scientific outcomes. To address these challenges, some difficult system design and software engineering problems must be overcome. This paper illustrates the design of a component-oriented system, GeoVISTA Studio, as a means to overcome such difficulties by using state-of-the-art component-based software engineering techniques. Advantages described include: ease of program construction (visual programming), an open (non-proprietary) architecture, simple component-based integration and advanced deployment methods. This versatility has the potential to change the nature of systems development for the geosciences, providing better mechanisms to coordinate complex functionality, and as a consequence, to improve analysis by closer integration of software tools and better engagement of the human expert. Two example applications are presented to illustrate the potential of the Studio environment for exploring and better understanding large, complex geographical datasets and for supporting complex visual and computational analysis. 
Takeda, K, and H Nomiyama. 2000, "View Composition for Digital Libraries." In International Conference on Digital Libraries: Research and Practice, pp. 426-33.

We propose a novel visual interface for digital libraries, which employs a collection of views and a compositional mechanism of views. While the views capture and visualize specific aspects of digital libraries, their compositions can be utilized to give deeper and richer insights into a set of retrieved data. Two types of compositions-superimposition and neighboring-of views are introduced. Some mathematical properties and functions associated with view composition are also described

Talbot, J, and P Hanrahan. 2010, "Adapting Daniel and Wood' S Modeling Approach to Interactive Visual Analytics." In IEEE Symposium on Visual Analytics Science \& Technology, pp. 253-54.

Talbot, J, S Lin, and P Hanrahan. 2010, "An Extension of Wilkinson's Algorithm for Positioning Tick Labels on Axes." IEEE Transactions on Visualization and Computer Graphics 16(6):1036-43.

The non-data components of a visualization, such as axes and legends, can often be just as important as the data itself. They provide contextual information essential to interpreting the data. In this paper, we describe an automated system for choosing positions and labels for axis tick marks. Our system extends Wilkinson\&\#8217;s optimization-based labeling approach to create a more robust, full-featured axis labeler. We define an expanded space of axis labelings by automatically generating additional nice numbers as needed and by permitting the extreme labels to occur inside the data range. These changes provide flexibility in problematic cases, without degrading quality elsewhere. We also propose an additional optimization criterion, legibility, which allows us to simultaneously optimize over label formatting, font size, and orientation. To solve this revised optimization problem, we describe the optimization function and an efficient search algorithm. Finally, we compare our method to previous work using both quantitative and qualitative metrics. This paper is a good example of how ideas from automated graphic design can be applied to information visualization.

Tam, J, and S Greenberg. 2006, "A Framework for Asynchronous Change Awareness in Collaborative Documents and Workspaces." International Journal of Human-Computer Studies 64(7):583-98.

Change awareness is the ability of individuals to track the asynchronous changes made to a collaborative document or graphical workspace by other participants over time. We develop a framework that articulates what change awareness information is critical if people are to track and maintain change awareness. Information elements include: knowing who changed the artifact, what those changes involve, where changes occur, when changes were made, how things have changed and why people made the changes. The framework accounts for people's need to view these changes from different perspectives: an artifact-based view, a person-based view and a workspace-based view. Each information element is further broken down into distinguishing features and matched against these perspectives, e.g., location history within the where category prompts the questions ['] where was this artifact when I left' in the artifact-based view, ['] where in the workspace has a person visited' in the person-based view and [']where have people been in the workspace' in the workspace-based view. The framework can be used both to inform and critique change awareness tools. 
Tanahashi, Y, and Y Wu. 2010, "Analyzing Hospitalization Records of a Pandemic." In IEEE Symposium on Visual Analytics Science \& Technology.

Tanin, E, R Beigel, and B Shneiderman. 1997, "Design and Evaluation of Incremental Data Structures and Algorithms for Dynamic Query Interfaces." In IEEE Symposium on Information Visualization (InfoVis '97), pp. 81-86.

A dynamic query interface (DQI) is a database access mechanism that provides continuous real-time feedback to the user during query formulation. Previous work shows that DQIs are elegant and powerful interfaces to small databases. Unfortunately, when applied to large databases, previous DQI algorithms slow to a crawl. We present a new incremental approach to DQI algorithms and display updates that work well with large databases, both in theory and in practice.

Tanin, E, R Beigel, and B Shneiderman. 1997, "Research Report - Design and Evaluation of Incremental Data Structures and Algorithms for Dynamic Query Interfaces." In IEEE Symposium on Information Visualization (InfoVis 97), pp. 81-86.

Dynamic query interfaces (DQIs) are a recently developed database access mechanism that provides continuous real-time feedback to the user during query formulation. Previous work shows that DQIs are an elegant and powerful interface to small databases. Unfortunately, when applied to large databases, previous DQI algorithms slow to a crawl. We present a new incremental approach to DQI algorithms and display updates that works well with large databases, both in theory and in practice.

Tao, Y, et al. 2004, "Information Visualization Techniques in Bioinformatics During the Postgenomic Era." Drug Discovery Today: BIOSILICO 2(6):237-45.

Information visualization techniques, which take advantage of the bandwidth of human vision, are powerful tools for organizing and analyzing a large amount of data. In the postgenomic era, information visualization tools are indispensable for biomedical research. This paper aims to present an overview of current applications of information visualization techniques in bioinformatics for visualizing different types of biological data, such as from genomics, proteomics, expression profiling and structural studies. Finally, we discuss the challenges of information visualization in bioinformatics related to dealing with more complex biological information in the emerging fields of systems biology and systems medicine.

Tateosian, L, et al. 2010, "Tangeoms: Tangible Geospatial Modeling System." IEEE Transactions on Visualization and Computer Graphics 16(6):1605-12.

We present TanGeoMS, a tangible geospatial modeling visualization system that couples a laser scanner, projector, and a flexible physical three-dimensional model with a standard geospatial information system (GIS) to create a tangible user interface for terrain data. TanGeoMS projects an image of realworld data onto a physical terrain model. Users can alter the topography of the model by modifying the clay surface or placing additional objects on the surface. The modified model is captured by an overhead laser scanner then imported into a GIS for analysis and simulation of real-world processes. The results are projected back onto the surface of the model providing feedback on the impact of the modifications on terrain parameters and simulated processes. Interaction with a physical model is highly intuitive, allowing users to base initial design decisions on geospatial data, test the impact of these decisions in GIS simulations, and use the feedback to improve their design. We demonstrate the system on three 
applications: investigating runoff management within a watershed, assessing the impact of storm surge on barrier islands, and exploring landscape rehabilitation in military training areas.

Taugher, C, and D Keim-Campbell. 2002, "Moving Data-Chaos to a Clarity of Vision: Findings on Information Design from the University of Idaho Department of Art." In Conference on Internet Imaging III, pp. 157-73.

Through a state funded grant initiative, the Department of Art at the University of Idaho has recently founded an information visualization studio. We specialize in interdisciplinary research on developing visualization techniques that allow a transformation of complex data into visual formats while providing clarity and exposing underlying structures. Clear and concise visualization of information makes this understanding more immediate. It also allows for added critical thinking about a subject. For example, in the area of hardware and software design, existing visualizations of structures are often clouded by an engineer's previous knowledge about the subject. They know what they are looking at, but a machine doesn't, nor does an end user. Clear graphics developed by an information designer make it easier to see problems and give parameters that a programmer can then apply to building a better system. We seek to produce visualization systems, methodologies, and examples for the resolution of problems where visual demonstration of concepts and materials enhances, even permits, understanding. We intend to summarize the findings of our project by illustrating and demonstrating techniques, which we have developed, for manipulating raw data into clear graphic presentations of complex systems for decision making. We will also summarize our findings on methods for creating visualizations designed to assist individuals with perceptual or cognitive difficulties.

Taylor, DRF. 2003, "The Concept of Cybercartography." in Maps and the Internet, ed. P Michael, pp. 405-20. Elsevier Science, Oxford.

Summary This paper argues that the increasing use of maps and the Internet requires a new paradigm for cartography. Computer and telecommunications technologies are revolutionizing cartography but the challenges facing the discipline are not only technical but also conceptual. Maps and Mapping will take on new functions in the 21 st century as a process, as an organizational concept and as a product. A new definition of cartography is suggested and the seven major elements of the concept of cybercartography elaborated. Cybercartography is multi-sensory, multimedia, and interactive, applied to a wide variety of subjects, is part of an information package, is compiled by interdisciplinary teams and provides new research partnerships. It involves new ways of engaging and understanding the user and new integrated research frameworks. Research to create two new cybercartographic products, a Cybercartographic Atlas of Antarctica and Canada's Trade with the World, is described. Traditional maps were key to the age of exploration. Cybermaps may equally be a key to navigation in the information era both as a framework to integrate information and a process by which that information can be organized, understood and used. 
Taylor, K, et al. 2010, "Multiviz : Investigations into Arms Dealing Vast 2010 Mini Challenge." In IEEE Symposium on Visual Analytics Science \& Technology.

Taylor Parkins, FLM. 1999, "Visualisation of Construction Costs and Techniques of EmploymentIntensive Road Construction in Developing Areas." In International Conference on Information Visualization, pp. 225-29.

Employment generation in the construction of economically efficient infrastructure can contribute to the alleviation of poverty. Practical Choice of Technique Analysis (COTA-P) is a tool that enables decision makers to estimate the financial and socio-economic costs involved in a construction. COTA-P looks at the construction from an employment-intensive perspective, with the underlying idea to substitute as much labour for machines as is feasible, and to promote the use of local labour in underdeveloped and poor areas. COTA-P consists of a technical screening phase, a financial and socioeconomical screening phase, and thirdly the monitoring and evaluation phase. Each phase analyses the possibility of the enhanced use of labour. COTA-P is programmed in a Windows 32-bit environment, and gives the user maximum user friendliness, while allowing for extensive manipulation of the core variables and parameters, thus enabling the user to analyse various scenarios in a short time

Teets, JM, DP Tegarden, and RS Russell. 2010, "Using Cognitive Fit Theory to Evaluate the Effectiveness of Information Visualizations: An Example Using Quality Assurance Data." Visualization and Computer Graphics, IEEE Transactions on 16(5):841-53.

Cognitive fit theory, along with the proximity compatibility principle, is investigated as a basis to evaluate the effectiveness of information visualizations to support a decision-making task. The task used in this study manipulates varying levels of task complexity for quality control decisions in a high-volume discrete manufacturing environment. The volume of process monitoring and quality control data produced in this type of environment can be daunting. Today's managers need effective decision support tools to sort through the morass of data in a timely fashion to make critical decisions on product and process quality.

Tegarden, DP. 1999, "Business Information Visualization." Communications of the AIS 1(1es):4-4.

Tegarden, DP, and SD Sheetz. 2003, "Group Cognitive Mapping: A Methodology and System for Capturing and Evaluating Managerial and Organizational Cognition." Omega 31(2):113-25.

Organizations would like to capture and merge the perceptions of key individuals into an organizational memory. Various cognitive mapping approaches have been used to identify and capture these perceptions. However, merging the cognitive maps of individuals into a collective cognitive map to represent the shared perceptions has been problematic. Due to the merging problems, the creation of collective cognitive maps is impractical for many organizational situations. In this paper, we describe and demonstrate a cognitive mapping based methodology and system that eliminates the merging problem, supports data collection, and provides data analyses to uncover both individual and collective cognitive maps. 
ten Caat, M, NM Maurits, and J Roerdink. 2007, "Design and Evaluation of Tiled Parallel Coordinate Visualization of Multichannel Eeg Data." IEEE Transactions on Visualization and Computer Graphics 13(1):70-79.

The field of visualization assists data interpretation in many areas, but does not manage all types of data equally well. This holds, in particular, for time-varying multichannel EEG data. No existing method can successfully visualize simultaneous information from all channels in use at all time steps. To address this problem, a new visualization method is presented based on the parallel coordinate method and making use of a tiled organization. This tiled organization employs a two-dimensional row-column representation, rather than a one-dimensional arrangement in columns as used for classical parallel coordinates. The usefulness of the new method, referred to as tiled parallel coordinates (TPC), is demonstrated by a particular type of EEG data. It can be applied to an arbitrary number of time steps, handling the maximum number of channels currently in use. An extensive user evaluation shows that, for a typical EEG assessment task, data evaluation by the TPC method is faster than by an existing clinical EEG visualization method, without loss of information. The generality of the TPC method makes it widely applicable to other time-varying multivariate data types.

Tennekes, M. 2010, "Poster : Application of Treemaps to Business Statistics Analysis." In IEEE Conference on Information Visualization.

Teoh, M Kwan-Liu, and SF Wu. 2003, "A Visual Exploration Process for the Analysis of Internet Routing Data." In IEEE Visualization, pp. 523-30.

The Internet pervades many aspects of our lives and is becoming indispensable to critical functions in areas such as commerce, government, production and general information dissemination. To maintain the stability and efficiency of the Internet, every effort must be made to protect it against various forms of attacks, malicious users, and errors. A key component in the Internet security effort is the routine examination of Internet routing data, which unfortunately can be too large and complicated to browse directly. We have developed an interactive visualization process which proves to be very effective for the analysis of Internet routing data. In this application paper, we show how each step in the visualization process helps direct the analysis and glean insights from the data. These insights include the discovery of patterns, detection of faults and abnormal events, understanding of event correlations, formation of causation hypotheses, and classification of anomalies. We also discuss lessons learned in our visual analysis study.

Teoh, ST, and KL Ma. 2003, "Starclass: Interactive Visual Classification Using Star Coordinates." In 3rd SIAM International Conference on Data Mining, pp. 178-85.

Classification operations in a data-mining task are often performed using decision trees. The visualbased approach to decision tree construction has gained increasing popularity. We present Star-Class, a new interactive visual classification method. This method maps multi-dimensional data to the visual display using star coordinates, allowing the user to interact with the display to create a decision tree. Preliminary evaluation indicates that this new technique is as effective as state-of-the-art algorithmic classification methods, and more effective than the previous visual-based methods. Star-Class also offers additional advantages such as improving the user's understanding of the data. 
Terry, M, and ED Mynatt. 2005, "Enhancing General-Purpose Tools with Multi-State Previewing Capabilities." Knowledge-Based Systems 18(8):415-25.

General-purpose design tools can be applied to a wide variety of design problems, but the large number of unique states they are able to produce makes it difficult to find results most relevant to a specific design problem. Current interfaces exacerbate this problem by offering only a single preview of one potential future state. We introduce multi-state previewing tools to facilitate the process of generating, displaying, navigating, and evaluating multiple, potential future states simultaneously. Multistate previewing tools specifically encode and automate higher-level design practices, such as exploring multiple alternatives, better aligning computer-based tools with design. In this paper, we synthesize a framework for this class of tools by combining and generalizing existing instantiations, then show how this framework can be used to guide the design, implementation, and further research of these tools.

Teutsch, C, et al. 2007, "Adaptive Real-Time Grid Generation from 3d Line Scans for Fast Visualization and Data Evaluation." In 1th International Conference Information Visualization, pp. 177-84.

This paper presents a method for the real-time generation of grids from 3D line scan data for in-line scan previews and the evaluation of large point clouds derived from different 3D-scanners. By exploiting the underlying measuring principles, we generate regular grids for each scan operation even if the sensor movement is non-linear. These grids are finally used for NURBS patch approximations, which enable the determination of higher order features, e.g., curvature and quality evaluations. Experimental results at the example of different point clouds illustrate the effectiveness of our methods in practice.

Teyseyre, AR, and MR Campo. 2009, "An Overview of 3d Software Visualization." IEEE Transactions on Visualization and Computer Graphics 15(1):87-105.

Software visualization studies techniques and methods for graphically representing different aspects of software. Its main goal is to enhance, simplify and clarify the mental representation a software engineer has of a computer system. During many years, visualization in 2D space has been actively studied, but in the last decade, researchers have begun to explore new 3D representations for visualizing software. In this article, we present an overview of current research in the area, describing several major aspects like: visual representations, interaction issues, evaluation methods and development tools. We also perform a survey of some representative tools to support different tasks, i.e., software maintenance and comprehension, requirements validation and algorithm animation for educational purposes, among others. Finally, we conclude identifying future research directions.

Thai, V, S Handschuh, and S Decker. 2008, "Tight Coupling of Personal Interests with MultiDimensional Visualization for Exploration and Analysis of Text Collections." In 12th International Conference Information Visualisation, pp. 221-26.

In this paper, we present an interactive matrix-based multi-dimensional visualization component which enables the users to explore a text collection along different conceptual dimensions. Of importance in our approach are the tight coupling of the users' personal ontologies representing their spheres of interest with the visualization component and the application of barycenter heuristic for edge crossing minimization to enhance its visual display. We also discuss how IVEA, the information visualization tool containing the proposed component, can address the commonly perceived constraints of building a personal ontology from scratch for IVEA to work. (C) 2008 IEEE. 
Thalmann, D. 1984, "An Interactive Data Visualization System." Software-Practice \& Experience 14(3):277-90.

GRAFANA is an interactive program, which allows the user to produce drawings based on data stored on a disk file. The major advantage and originality of the system is that the user can interactively determine the visual effect of a drawing and immediately see it on the screen. Moreover, any drawing produced by GRAFANA can still be edited by a generalpurpose graphics editor. This system allows the user to obtain precisely the right drawing for any book, paper or report.

Theron, R. 2006, "Hierarchical-Temporal Data Visualization Using a Tree-Ring Metaphor." In 6th International Symposium on Smart Graphics, pp. 70-81.

This paper describes a novel and efficient visualization technique intended for hierarchical-temporal data using a tree-ring like layout. Temporal hierarchies appear in numerous fields such as genealogy, evolution taxonomies or time lines. In many cases, state-of-the-art static diagrams are produced in these fields. By using several information visualization strategies, such as focus + context, the tree-ring approach has the ability to visualize and navigate these, potentially complex, hierarchies trough time. Thus, a deeper insight into the problem at hand can be gained.

Thiele, O. 2010, "M-Business and Organizational Behavior Applying Behavioral Theory Ideas to Mobile Interface Design." In International Joint Conference on Computer, Information, Systems Sciences and Engineering, pp. 463-66.

Just ten years ago programming interfaces were built according to technological possibilities. Search results had to be displayed as textual lists, due to a lack of graphical support. Later, when searching for a music album, search results could be enhanced by showing the cover to simplify recognition. Today, mobile devices are mainly used in private contexts (e.g., on the IPhone), corporate use, like Blackberry devices, is just emerging. Whether the same principles apply for corporate as well as private us is questionable, therefore insight from behavioral theory might aid in deriving corporate mobile interfaces by combining these insights and existing approaches from private use. Special attention is given to mobile information visualization. Behavioral Theory has been developed and evaluated for over sixty years. Its main ideas evolve around bounded rationality of the people, decision making processes and conflicting interests. As these are main themes in the offline business world, members of an organization cannot abandon them using mobile devices in the online world. Therefore, taking organizational considerations into account, mobile interface design might be improved.

Thiele, O, P Sutterlin, and L Kaczmirek. 2005, "Evaluation of User Interfaces for Displaying Text Messages on Mobile Devices." In IASTED International Conference on Human-Computer Interaction, pp. 120-25.

More and more information such as short text messages (SMS) or emails is stored on mobile phones. Retrieving the right information at the right time has become a difficult task when using traditional interfaces included in today's cell phones. We created two novel user interfaces (one textual and one graphical) and compared both to the classical interface for retrieving stored SMS messages in a 34participant usability experiment. Both novel interfaces yielded significantly better results than the existing classical interface. Moreover, the results show that the novel textual interface, which groups messages by social contacts, outperforms the complex graphical interface, which displays messages in a chessboard- 
like grid. Both objective and subjective findings are in favor of the textual interface except for one result: The graphical interface is more enjoyable than the textual one.

Thiele, O, and D Thoma. 2007, "Evaluation of Information Visualizations Vs. Language Proficiency." In 2nd IASTED International Conference on Human-Computer Interaction, pp. 199-204.

In this paper we will ask the question of what proficiency in a textual language (e.g., English) has in common with evaluating information visualizations. We admit that this question might not seem completely straightforward. Therefore we will first present how experimental practice led us to this question. We hypothesize that the ability to use a visualization (to speak the visual language) affects both objective and subjective evaluation methods. We propose to transfer the use of language testing and grammar description from linguistics to human computer interaction evaluation.

Thomas, BH, and P Calder. 2005, "Supporting Cartoon Animation Techniques in Direct Manipulation Graphical User Interfaces." Information and Software Technology 47(5):339-55.

If judiciously applied, the techniques of cartoon animation can enhance the illusion of direct manipulation that many human computer interfaces strive to present. In particular, animation can convey a feeling of substance in the objects that a user manipulates, strengthening the sense that real work is being done. This paper describes algorithms and implementation issues to support cartoon style graphical object distortion effects for direct manipulation user interfaces. Our approach is based on suggesting a range of animation effects by distorting the view of the manipulated object. To explore the idea, we added a warping transformation capability to the InterViews user interface toolkit.

Thomas, BH, and V Demczuk. 2002, "Which Animation Effects Improve Indirect Manipulation?" Interacting with Computers 14(3):211-29.

This paper describes an experiment to explore the effectiveness of animation in improving indirect manipulation operations. Indirect manipulation operations are those initiated by command menus and buttons to perform a transformation on a graphical object or set of graphical objects. The particular improvement is an operation's ability to show both what would happen if the operation were committed and what would happen if it were cancelled while an operation is being considered. The experiment required subjects to watch a simple alignment operation for a set of graphical objects. They were then asked to record the original placement of those graphical objects. Each task used one of four visual cues: modified telltale, wiggle, colour, or no visual cue. We found the modified telltale, wiggle, and colour visual effects significantly more effective than no visual feedback for cuing the user as to original position of the graphical objects. The modified telltale and colour effects were significantly more effective than the wiggle effect. The major conclusion drawn from this experiment is the use of visual feedback improves a user's ability to remember the previous position of graphical objects after an alignment operation. 
Thomas, J. 2007, "Visual Analytics: A Grand Challenge in Science - Turning Information Overload into the Opportunity of the Decade [Keynote Address]." In 6th International Asia-Pacific Symposium on Visualization, pp. ix-ix.

Thomas, JJ. 2009, "Taxonomy for Visual Analytics: Seeking Feedback." VAC Views May:6-7.

Thomas, JJ. 2007, "Visual Analytics : Why Now ?" Information Visualization 6:104-07.

This special issue of Information Visualization IVS is focused on recent updated papers presented at the IEEE Symposium on Visual Analytics Science and Technology (VAST) in Baltimore, October 2006. Why is there so much interest in what is being called visual analytics? Let us first address the term visual analytics. Visual analytics is defined as the science of analytical reasoning facilitated by the visual interface. People use visual analytics tools and techniques to synthesize information and derive insight from massive, dynamic, ambiguous, and often conflicting data; detect the expected and discover the unexpected; provide timely, defensible, and understandable assessments; and communicate assessment effectively for action. Visual analytics was defined to represent a new envisioned suite of technologies and resultant field of study by a team of approximately 40 experts with representation from government, academia, industry, and national laboratories. The need for visual analytics was driven by an ever increasing amount of data to analyze; increasing complexity and uncertainty in the data; decreasing amount of time to analyze the data; and a lack of methods, technology, or tools available today or perceived on the horizon.

Thomas, JJ, and KA Cook. 2005. Illuminating the Path: The Research and Development Agenda for Visual Analytics. IEEE.

Illuminating the Path is a call to action for researchers and developers to help safeguard our nation by transforming information overload into insights through visual analytics - the science of analytical reasoning facilitated by interactive visual interfaces. Achieving this will require interdisciplinary, collaborative efforts of researchers from throughout academia, industry, and the national laboratories.

Thomas, JJ, and KA Cook. 2006, "A Visual Analytics Agenda." IEEE Computer Graphics and Applications 26(1):10-3.

In fields ranging from security to systems biology to business, the ability to collect data far outstrips the ability to analyze the data that have been collected. The science of visual analytics is meant to enable analysts of all types to overcome information overload so that they can detect the expected and discover the unexpected from massive, dynamic, conflicting, and incomplete information while rigorously adhering to privacy and security laws and policies. During the past year, a panel of research leaders from academia, industry government, the national laboratory system developed a five year research and development agenda for the field of visual analytics. This article presents a concise summary of that agenda.

Thornson, CA, BF Goldiez, and H Le. 2009, "Predicting Presence: Constructing the Tendency toward Presence Inventory." International Journal of Human-Computer Studies 67(1):62-78.

We used a rational-empirical approach to construct the Tendency toward Presence Inventory (TPI), constructing scales to measure the individual difference human factors hypothesized to predict a person's 
tendency to experience the cognitive state of presence. The initial pool of 105 items was administered to 499 undergraduate psychology students at a university in the southeastern United States in order to empirically validate the underlying factor structure associated with this tendency. Six factors were derived, resembling the original conceptual model. Construct validity and reliability evidence are presented. Future empirical work is needed to explore the criterion and predictive validities of the factors constituting this inventory.

Thorsen, Ø, and C Wallace. 2010, "Understanding Relaxed Memory Consistency through Interactive Visualization." In 5th ACM Symposium on Software Visualization (SOFTVIS), pp. 223-24.

Tiede, D, and S Lang. 2010, "Analytical 3d Views and Virtual Globes -- Scientific Results in a Familiar Spatial Context." ISPRS Journal of Photogrammetry and Remote Sensing 65(3):300-07.

In this paper we introduce analytical three-dimensional (3D) views as a means for effective and comprehensible information delivery, using virtual globes and the third dimension as an additional information carrier. Four case studies are presented, in which information extraction results from very high spatial resolution (VHSR) satellite images were conditioned and aggregated or disaggregated to regular spatial units. The case studies were embedded in the context of: (1) urban life quality assessment (Salzburg/Austria); (2) post-disaster assessment (Harare/Zimbabwe); (3) emergency response

(Lukole/Tanzania); and (4) contingency planning (faked crisis scenario/Germany). The results are made available in different virtual globe environments, using the implemented contextual data (such as satellite imagery, aerial photographs, and auxiliary geodata) as valuable additional context information. Both dayto-day users and high-level decision makers are addressees of this tailored information product. The degree of abstraction required for understanding a complex analytical content is balanced with the ease and appeal by which the context is conveyed.

Tikhonova, A, CD Correa, and K-L Ma. 2010, "Visualization by Proxy: A Novel Framework for Deferred Interaction with Volume Data." IEEE Transactions on Visualization and Computer Graphics 16(6):15519 .

Interactivity is key to exploration of volume data. Interactivity may be hindered due to many factors, e.g., large data size, high resolution or complexity of a data set, or an expensive rendering algorithm. We present a novel framework for visualizing volumedata that enables interactive exploration using proxy images, without accessing the original 3D data. Data exploration using directvolume rendering requires multiple (often redundant) accesses to possibly large amounts of data. The notion of visualization by proxyrelies on the ability to defer operations traditionally used for exploring 3D data to a more suitable intermediate representation forinteraction - proxy images. Such operations include view changes, transfer function exploration, and relighting. While previous workhas addressed specific interaction needs, we provide a complete solution that enables real-time interaction with large data sets andhas low hardware and storage requirements.

Tittle, J, W Elm, and S Potter. 2005, "Functional Requirements for Effective Decision Making in HumanRobot Teams: Lessons Learned from Operational Settings." In 49th Annual Meeting of the Human Factors and Ergonomics Society, pp. 452-56.

Many environments require humans and robots operating together to accomplish complex and dangerous tasks, but technology-centered designs often support robot navigation but not the mission goals 
of the organization using the robot. Urban Search and Rescue (USAR) is a particularly valuable domain to identify general functional requirements for effective HRI, and our purpose in this paper is to demonstrate how a CSE approach can lead to valuable design guidelines that more effectively support decision making within Human-Robot teams. Our analysis of HRI in USAR lead us to identify several important guidelines for supporting effective coordination for Human-Robot teams: including (i) enable individual problem holders to have direct control over point-of-view to facilitate active information seeking, and (ii) create common reference frames on shared imagery so different problem holders can remotely coordinate information and actions. Designs based on these guidelines will support a broad class of coordinated activities between team members.

Tittle, JS, et al. 2006, Designing Large Scale Decision Support Environments to Enable Effective Team Decision Making. Technical.

Tobón, C. 2005, "Chapter 34 - Evaluating Geographic Visualization Tools and Methods: An Approach and Experiment Based Upon User Tasks." in Exploring Geovisualization, eds. D Jason, MM Alan and K Menno-Jan, pp. 645-66. Elsevier, Oxford.

Summary This chapter discusses the contribution that fields such as experimental design and humancomputer interaction can make to geovisualization. In particular, it shows how multiple techniques can be combined not only to evaluate a system's effectiveness, but also to address the complex issue of task definition in geovisualization research. A methodology for defining a task typology is suggested and used to evaluate a geovisualization system. The results from the evaluation suggest that the approach taken can serve to refine existing task characterizations to reflect the cognitive visual operations that spatial data and geovisualizations can support.

Toga, AW, and P Thompson. 1999, "Chapter 1 - an Introduction to Brain Warping." in Brain Warping, pp. 1-26. Academic Press, San Diego.

Tolone, WJ. 2009, "Interactive Visualizations for Critical Infrastructure Analysis." International Journal of Critical Infrastructure Protection 2(3):124-34.

Critical infrastructure analysis often involves overwhelming volumes of complex, heterogeneous, interdependent information. Human judgment is essential to the analysis as insights and understandings are synthesized from information that is often complex, dynamic, incomplete, diverse, conflicting and even deceptive. Yet, our ability to collect information is increasing at rates far beyond our ability to analyze it. Visual analytics-the science of analytical reasoning facilitated by interactive visual interfacescan help analysts obtain better insights and understanding with greater efficiency. This paper discusses the research challenges involved in applying interactive visualization to critical infrastructure analysis. The research challenges are organized around three dimensions that are adapted from metrics proposed by Scholtz (2006) [8] for evaluating human information interaction systems. The challenges are illustrated using examples from the integrated modeling and simulation of critical infrastructures.

Tolosa, JB, et al. 2010, "Interactive Web Environment for Collaborative and Extensible Diagram Based Learning." Computers in Human Behavior 26(2):210-17.

Nowadays there is a growing need of ubiquity for learning, research and development tools, due to the portability and availability problems concerning traditional desktop applications. In this paper, we 
suggest an approach to avoid any further download or installation. The main goal is to offer a collaborative and extensible web environment which will cover a series of domains highly demanded by different kinds of working groups, in which it is crucial to have tools which facilitate the exchange of information and the collaboration among their members. The result of those interactions would be the development of one or several diagrams accessible from any geographical location, independently of the device employed. The environment can be adapted through personalized components, depending on the type of diagram that the user wants to interact with and the users can also create new elements or search and share components with other users of the community. By means of this environment, it will be possible to do research on the usability of collaborative tools for design diagrams, as well as research on the psychology of group interactions, assessing the results coming from the employment of known methodologies, techniques, paradigms or patterns, both at an individual and at a collaborative group level.

Tomaszewski, B. 2007, "Developing Geo-Temporal Context from Implicit Sources with Geovisual Analytics." In ICA Commission on Visualization and Virtual Environments, pp. 1-12.

The key Geovisual Analytics research question addressed in this paper is how knowledge of past situations can be computationally extracted from heterogeneous and implicit information spaces and presented as visual artifacts within an interactive environment to facilitate reasoning about situations from geographic and temporal (or geo-temporal) contextual perspectives. This paper in particular will examine the shared development of geo-temporal context from implicit geospatial and temporal references contained in open-source channels such as the news media to support situation assessment in crisis management activities. The paper presents the "Context Discovery Application" (CDA), which is a prototype collaborative, Geovisual Analytics situation assessment environment that facilitates the development of geographical and temporal context using implicit sources.

Tomaszewski, B. 2010, "Geo-Historical Context Support for Information Foraging and Sensemaking : Conceptual Model , Implementation, and Assessment." In IEEE Symposium on Visual Analytics Science \& Technology, pp. 139-46.

Tomaszewski, B. 2007, "Local Model Semantics, Categories, and External Representation: Towards a Model for Geo-Historical Context." In Sixth International and Interdisciplinary Conference on Modeling and Using Context (CONTEXT'07), pp. 1-12.

Perspectives within social interaction situations are often shaped by geo-historical contexts derived from knowledge of indirectly experienceable phenomena such as geographic scale entities and past events that are communicated through external representations such as maps and historical accounts. Although geo-historical context is important for proving meaning to collaborative situations, no formal approach for modeling geo-historical context exists. This paper will provide a preliminary analysis of how select aspects of Local Model Semantics (LMS), used in conjunction with geographic and historic categories, can be used to theoretically inform the formal modeling of geo-historical context and how external representations affect geographical and historical categories. A brief case-study example will be given to show how geo-visual tools theoretically informed by LMS, geographical and historical categories, and external representation can support the development of geo-historical context from heterogeneous sources in a collaborative setting. 
Tomaszewski, B. 2008, "Producing Geo-Historical Context from Implicit Sources: A Geovisual Analytics Approach." Cartographic Journal, The 45(3):165-81.

Geo-historical context, or GHC, is a contextual setting based on the interconnectedness of phenomena, events, and place across multiple spatial and temporal scales. GHC allows for situations to be understood and reasoned with, often with aid of visual representations such as maps. This paper introduces a conceptual model of GHC that theoretically motivates a Geovisual Analytics application called the Context Discovery Application (CDA), which is also presented. The CDA is designed to aid in the production of geo-historical context by using computational processes to identify and extract potentially relevant context information from heterogeneous, implicit situation information. This information can then be explored through visual interfaces to help users explain and understand the information. A hypothetical humanitarian context analysis case study is used to show how the CDA can be applied to real world problems.

Tomaszewski, B. 2010, in press, "Situation Awareness and Virtual Globes: Applications for Disaster Management." Computers \& Geosciences.

This paper presents research on the use of virtual globes to support the development of disaster event situation awareness in humans via open source information analysis and visualization. The key technology used for this research is the Context Discovery Application (CDA), which is a geovisual analytic environment designed to integrate implicit geographic information with Google Earth(TM). A case study of humanitarian disaster management is used to demonstrate the unique abilities of the CDA and Google EarthTM to support situation awareness. The paper provides some of the first empirical evidence on the utility of the virtual globes to support situation awareness for disaster management using implicit geographic information. The evidence presented was derived from evaluations by disaster management practitioners at the United Nations (UN) ReliefWeb project, an extremely relevant, yet difficult group to access for conducting academic disaster management research. Finally, ideas for future research on developing virtual globe applications to support situation awareness are described.

Tomaszewski, BM, et al. 2007, "Facilitating Situation Assessment through Gir with Multi-Scale Open Source Web Documents." In 4th Workshop on Geographic Information Retrieval, held at CIKM 2007, pp. 95-96.

Topi, H, and W Lucas. 2005, "Mix and Match: Combining Terms and Operators for Successful Web Searches." Information Processing \& Management 41(4):801-17.

This paper presents a detailed analysis of the structure and components of queries written by experimental participants in a study that manipulated two factors found to affect end-user information retrieval performance: training in Boolean logic and the type of search interface. As reported previously, we found that both Boolean training and the use of an assisted interface improved the participants' ability to find correct responses to information requests. Here, we examine the impact of these training and interface manipulations on the Boolean operators and search terms that comprise the submitted queries. Our analysis shows that both Boolean training and the use of an assisted interface improved the participants' ability to correctly utilize various operators. An unexpected finding is that this training also had a positive impact on term selection. The terms and, to a lesser extent, the operators comprising a query were important factors affecting the participants' performance in query tasks. Our findings demonstrate that even small training interventions can improve the users' search performance and 
highlight the need for additional information retrieval research into how search interfaces can provide superior support to today's untrained users of the Web.

Tory, M, and T Moller. 2004, "Human Factors in Visualization Research." IEEE Transactions on Visualization and Computer Graphics 10(1):72-84.

Tory, M, and T Moller. 2004, "Rethinking Visualization: A High-Level Taxonomy." In IEEE Symposium on Information Visualization, pp. 151-58.

We present the novel high-level visualization taxonomy. Our taxonomy classifies visualization algorithms rather than data. Algorithms are categorized based on the assumptions they make about the data being visualized; we call this set of assumptions the design model. Because our taxonomy is based on design models, it is more flexible than existing taxonomies and considers the user's conceptual model, emphasizing the human aspect of visualization. Design models are classified according to whether they are discrete or continuous and by how much the algorithm designer chooses display attributes such as spatialization, timing, colour, and transparency. This novel approach provides an alternative view of the visualization field that helps explain how traditional divisions (e.g., information and scientific visualization) relates and overlap, and that may inspire research ideas in hybrid visualization areas

Tosa, N, and S Matsuoka. 2003, "Recreating Our Selves: Zenetic Computer." In Seventh International Conference on Information Visualization (IV'03), pp. 614-18.

We tried to develop an interactive system that could help us recreate our conscious selves by calling on Buddhist principles, Asian philosophy, and traditional Japanese culture through the inspirational media of ink painting, kimono and haiku. "Recreating our selves" means the process of making the consciousness of our 'daily self' meet that of our 'hidden self' through rediscovering creative resources deep within us that may have been forgotten but still resonate with vital meaning. In other words, this interactive system is based on the effort to meld our consciousness and unconsciousness in complete harmony. It is difficult to achieve this through traditional logic-based interaction. We succeeded in reaching this goal by setting as our target of scientific computing images from the above traditional Japanese media.

Tottenham, LS, et al. 2003, "Female Advantage for Spatial Location Memory in Both Static and Dynamic Environments." Brain and Cognition 53(2):381-83.

A female advantage has previously been found for spatial location tests of object memory. Previous studies have used static, 2-D tasks to test this advantage. This study used a computerized adaptation of the game Concentration to test object location memory in both a static and dynamic array of 24 pairs of line drawings. The dynamic version of this task was used to better reflect the dynamic real world in which we usually use object location memory. Consistent with previous research, we observed a female advantage. This advantage was found to a similar extent in both the static and dynamic versions of the task. The female advantage for object location memory is a concrete advantage in spatial cognition that females show on the Concentration Task, regardless of the nature of the presentation environment. 
Trafton, JG, SB Trickett, and FE Mintz. 2005, "Connecting Internatl and External Representations: Spatial Transformations of Scientific Visualizations." Foundations of Science 10(1):89-106.

Treffner, PJ, and R Barrett. 2004, "Hands-Free Mobile Phone Speech While Driving Degrades Coordination and Control." Transportation Research Part F: Traffic Psychology and Behaviour 7(45):229-46.

Using a closed-circuit driving track environment, we investigated the influence of using a hands-free mobile (or cell) phone on various biomechanical and perceptual factors that underlie the control of driving. Results showed that in three tasks representative of everyday driving conditions, the perceptual control of action was compromised when compared to a control condition where no mobile phone conversation was present. While conversing, critical control actions related to braking were postponed on approach to a corner. During controlled braking, as when approaching a stationary car at a traffic light, the degree of braking was reduced and braking style was altered in a non-optimal manner. During an obstacle avoidance task, car dynamics were affected as a result of the conversation. Interpretation of the results is motivated by the ecological approach to perception-action and the theory of affordances. It is concluded that a driver's sensitivity to prospective information about upcoming events and the associated perception and awareness of what the road environment affords may both significantly be degraded when simultaneously using a hands-free mobile phone. Implications for intervention and policy are discussed.

Treloar, R, and M Jern. 2003, "Applied Visual User Interface Technique in Knowledge Management." In Seventh International Conference on Information Visualization (IV'03), pp. 2-2.

Extracting actionable insight from large high-dimensional data sets, and its use for more effective decision-making, has become a pervasive problem across many application fields in both research and industry. The objective of our presentation is to report on some investigations of this problem covering both these areas. Taking as the problem domain the area of "unsupervised learning", we show that by tightly coupling statistical analysis technique with combinations of visualization components and techniques for interactivity, real-time analysis of multidimensional data can be efficiently made. We give particular attention to the ways in which dynamic visual representations can be used in these contexts to facilitate shared understanding. Our system is implemented and validated in the context of 3D medical imaging knowledge construction, knowledge management and geovisualisation.

Trümper, J, and J Bohnet. 2010, "Understanding Complex Multithreaded Software Systems by Using Trace Visualization." In 5th ACM Symposium on Software Visualization (SOFTVIS), pp. 133-42.

Trutschl, M, G Grinstein, and U Cvek. 2003, "Intelligently Resolving Point Occlusion." In 9th Annual IEEE Symposium on Information Visualization (INFOVIS 2003), pp. 131-36.

Large and high-dimensional data sets mapped to low-dimensional visualizations often result in perceptual ambiguities. One such ambiguity is overlap or occlusion that occurs when the number of records exceeds the number of unique locations in the presentation or when there exist two or more records that map to the same location. To lessen the affect of occlusion, non-standard visual attributes (i.e., shading and/or transparency) are applied, or such records may be remapped to a corresponding jittered location. The resulting mapping efficiently portrays the crowding of records but fails to provide the insight into the relationship between the neighboring records. We introduce a new interactive technique that intelligibly organizes overlapped points, a neural network-based Smart Jittering algorithm. 
We demonstrate this technique on a scatter plot, the most widely used visualization. The algorithm can be applied to other one, two, and multidimensional visualizations which represent data as points, including 3-dimensional scatter plots, RadViz, polar coordinates.

Tsai, B-s. 2003, "Information Landscaping: Information Mapping, Charting, Querying and Reporting Techniques for Total Quality Knowledge Management." Information Processing \& Management 39(4):639-64.

Information landscaping--an integration of information mapping, charting, querying and reporting techniques--has been developed to enable the construction of a total quality knowledge management system focusing on a particular subject information field. The techniques apply five major parameters of the Fuzzy commonality model (FCM) including unionization, quantity, continuity or stability, changeability, and critical probability, to construct a series of information maps (infomaps) and a set of chronological-statistical charts (infocharts). The infomaps and infocharts are used as the blueprints and navigation agents for building and developing a web-based subject experts depository and query-report system. Focusing on the subject experts/expertise, this system enables a researcher to expedite a query search through infomaps (qualitative reference) and infocharts (quantitative reference). The entropy measurement and the entropy constant (the square root of the average entropy measure) are calculated to compare with the critical probability of the FCM. This leads to the finding of a set of regression straight lines and the establishment of an information oscillogram. The tropics (upper limit, middle range, lower limit), and the potential/solstitial population and its growth rate within a subject information domain during a particular time period can be determined. They can effectively and efficiently guide librarians and information professionals towards the construction and the continuous development of an electronic collection. The cultivation of a virtual learning and referencing environment can also be created by utilizing this data.

Tsang, HY, M Tory, and C Swindells. 2010, "Eseetrack Visualizing Sequential Fixation Patterns." IEEE Transactions on Visualization and Computer Graphics 16(6):953-62.

We introduce eSeeTrack, an eye-tracking visualization prototype that facilitates exploration and comparison of sequential gaze orderings in a static or a dynamic scene. It extends current eye-tracking data visualizations by extracting patterns of sequential gaze orderings, displaying these patterns in a way that does not depend on the number of fixations on a scene, and enabling users to compare patterns from two or more sets of eye-gaze data. Extracting such patterns was very difficult with previous visualization techniques. eSeeTrack combines a timeline and a tree-structured visual representation to embody three aspects of eye-tracking data that users are interested in: duration, frequency and orderings of fixations. We demonstrate the usefulness of eSeeTrack via two case studies on surgical simulation and retail store chain data. We found that eSeeTrack allows ordering of fixations to be rapidly queried, explored and compared. Furthermore, our tool provides an effective and efficient mechanism to determine pattern outliers. This approach can be effective for behavior analysis in a variety of domains that are described at the end of this paper.

Tu, Y-N, and J-L Seng. 2009, "Research Intelligence Involving Information Retrieval - an Example of Conferences and Journals." Expert Systems with Applications 36(10):12151-66.

This paper reports a work that was intended to reveal the connection between topics investigated by conference papers and journal papers. This work selected hundreds of papers in data mining and 
information retrieval from well-known databases and showed that the topics covered by conference papers in a year often leads to similar topics covered by journal papers in the subsequent year and vice versa. This study used some existing algorithms and combination of these algorithms to proposed a new detective procedure for the researchers to detect the new trend and get the academic intelligence from conferences and journals. The goal of this research is fourfold: First, the research investigates if the conference papers' themes lead the journal papers'. Second, the research examines how the new research themes can be identified from the conference papers. Third, the research looks at a specific area such as information retrieval and data mining as an illustration. Fourth, the research studies any inconsistencies of the correlation between the conference papers and the journal papers. This study explores the connections between the academic publications. The methodologies of information retrieval and data mining can be exploited to discover the relationships between published papers among all topics. By discovering the connections between conference papers and journal papers, researchers can improve the effectiveness of their research by identifying academic intelligence. This study discusses how conference papers and journal papers are related. The topics of conference papers are identified to determine whether they represent new trend discussed in journal papers. An automatic examination procedure based on information retrieval and data mining is also proposed to minimize the time and human resources required to predict further research developments. This study develops a new procedure and collects a dataset to verify those problems. Analytical results demonstrate that the conference papers submitted to journals papers are similar each year. Conference papers certainly affect the journal papers published over three years. About $87.23 \%$ of data points from papers published in 1991-2007 support our assumption. The research is intended to help researchers identify new trend in their research fields, and focus on the urgent topics. This is particularly valuable for new researchers in their field, or those who wish to perform crossdomain studies.

Tudoreanu, ME. 2003, "Designing Effective Program Visualization Tools for Reducing User's Cognitive Effort." In ACM Symposium on Software Visualization pp. 105-05.

Tufte, ER. 2006. Beautiful Evidence. Graphics Press, Cheshire, CT.

Turdukulov, UD, M-J Kraak, and CA Blok. 2007, "Designing a Visual Environment for Exploration of Time Series of Remote Sensing Data: In Search for Convective Clouds." Computers \& Graphics 31(3):370-79.

Interactive animated images are often the only means to explore large time series of meteorological data sets. However, despite being interactive, animations still lead to information overload. We firstly look at the factors limiting the exploratory use of animations for studies of precipitating cloud and argue that two main factors are responsible for that: data complexity and animation design based on images that mimic reality. Then we present an example of how the current approach to visualize time series of meteorological images can be improved by computational methods, particularly by feature tracking. Next, we describe the visualization environment and discuss the representational, data mining and interactive functionality resulting from such a combination in an environment that is specifically dedicated to visual exploration and analysis of precipitating clouds. 
Turetken, O, and D Schuff. 2007, "The Impact of Context-Aware Fisheye Models on Understanding Business Processes: An Empirical Study of Data Flow Diagrams." Information \& Management 44(1):4052.

We investigated whether a "context-aware" fisheye view can more successfully communicate the information contained in a set of process models (data flow diagrams) than a traditional "context-free" presentation. We conducted two controlled experiments: the first included a simple set of DFDs and tasks that required a basic understanding of the system, while the second involved more detailed views of the same processes, and also a more complex task. Subjects who used the fisheye process models outperformed those using the traditional presentations. This difference was reflected in task performance for all subjects, and in task completion time for inexperienced subjects.

Turetken, O, and R Sharda. 2005, "Clustering-Based Visual Interfaces for Presentation of Web Search Results: An Empirical Investigation." Information Systems Frontiers 7(3):273-97.

The result of a typical web search is often overwhelming. It is very difficult to explore the textual listing of the resulting documents, which may be in the thousands. In order to improve the utility of the search experience, we explore presenting search results through clustering and a zoomable twodimensional map (zoomable treemap). Furthermore, we apply the fisheye view technique to this map of web search clusters to provide details in context. In this study, we report on our evaluation of these presentation features. The particular interfaces evaluated were: (1) a textual list, (2) a zoomable twodimensional map of the clustered results, and (3) a fisheye version of the zoomable two-dimensional map where the results were clustered. We found that subjects completed search tasks faster with the visual interfaces than with the textual interface, and faster with the fisheye interface than just the zoomable interface. Based on the findings, we conclude that there is promise in the use of clustering and visualization with a fisheye zooming capability in the exploration of web search results.

Turetken, O, and R Sharda. 2004, "Development of a Fisheye-Based Information Search Processing Aid (Fispa) for Managing Information Overload in the Web Environment." Decision Support Systems 37(3):415-34.

Information technologies have proliferated at an unprecedented rate to provide access to information across geographical boundaries. However, this proliferation has led to an information overload. Information overload has adverse impacts on information use and decision quality. This research focuses on the overload problem resulting from a web search, and proposes a potential remedy. We develop the requirements of a system that makes use of clustering and visualization for browsing the results of a typical web search. Based on this model, we develop a prototype that visualizes search results by first organizing them into a hierarchy according to their individual contents. This system presents a visual overview of the groups in this hierarchy, and lets the users focus (zoom) on specific groups of interest. One general problem with zooming within hierarchical structures is the separation between the details and the context. To address this problem, we implement a fisheye zooming capability in our system. This paper describes a typology of the various components necessary for addressing the problem and then the proposed solution based upon a fisheye view-based visualization. Next, the specific visualization algorithm and the system implementation are described. We conclude with research questions for further development of such interfaces for presentation of the results from web searches. 
Turton, I. 2008, "A System for the Automatic Comparison of Machine and Human Geocoded Documents." In 2nd international workshop on Geographic information retrieval - GIR '08, pp. 23-23.

Turton, I, M Gahegan, and A Jaiswal. 2007, "Geographic Information Retrieval from Disparate Data Sources." In Geocomputation.

Turton, I, and AM Maceachren. 2008, "Visualizing Unstructured Text Documents Using Trees and Maps." In GIScience 2008, pp. 1-4.

Turton, I, and A Murdoch. 2008, "A Web Based Tool for the Detection and Analysis of Avian Influenza Outbreaks from Internet News Sources." Medical Informatics:2-3.

Tuttle, C, LG Nonato, and C Silva. 2010, "Pedvis: A Structured, Space-Efficient Technique for Pedigree Visualization." IEEE Transactions on Visualization and Computer Graphics 16(6):1063-72.

Public genealogical databases are becoming increasingly populated with historical data and records of the current population's ancestors. As this increasing amount of available information is used to link individuals to their ancestors, the resulting trees become deeper and more dense, which justifies the need for using organized, space-efficient layouts to display the data. Existing layouts are often only able to show a small subset of the data at a time. As a result, it is easy to become lost when navigating through the data or to lose sight of the overall tree structure. On the contrary, leaving space for unknown ancestors allows one to better understand the tree's structure, but leaving this space becomes expensive and allows fewer generations to be displayed at a time. In this work, we propose that the H-tree based layout be used in genealogical software to display ancestral trees. We will show that this layout presents an increase in the number of displayable generations, provides a nicely arranged, symmetrical, intuitive and organized fractal structure, increases the user's ability to understand and navigate through the data, and accounts for the visualization requirements necessary for displaying such trees. Finally, user-study results indicate potential for user acceptance of the new layout.

Tversky, B. 2007, "Gestalts of Thought." in Visual Thought, ed. L Albertazzi, pp. 155-63. Benjamins, Amsterdam.

Tversky, B. in press, "Making Thought Visible Barbara Tversky." in Studying Design Creativity, ed. J Gero. Springer-Verlag, Berlin.

Tversky, B, et al. 2007, "Cognitive Design Principles : From Cognitive Models to Computer Models." in Model-Based Reasoning in Science and Engineering, ed. M L., pp. 1-20. King's College, London.

Tversky, B, JB Morrison, and M Betrancourt. 2002, "Animation: Can It Facilitate?" International Journal of Human-Computer Studies 57(4):247-62.

Graphics have been used since ancient times to portray things that are inherently spatiovisual, like maps and building plans. More recently, graphics have been used to portray things that are metaphorically spatiovisual, like graphs and organizational charts. The assumption is that graphics can facilitate comprehension, learning, memory, communication and inference. Assumptions aside, research on static graphics has shown that only carefully designed and appropriate graphics prove to be beneficial for conveying complex systems. Effective graphics conform to the Congruence Principle according to which the content and format of the graphic should correspond to the content and format of the concepts to be 
conveyed. From this, it follows that animated graphics should be effective in portraying change over time. Yet the research on the efficacy of animated over static graphics is not encouraging. In cases where animated graphics seem superior to static ones, scrutiny reveals lack of equivalence between animated and static graphics in content or procedures; the animated graphics convey more information or involve interactivity. Animations of events may be ineffective because animations violate the second principle of good graphics, the Apprehension Principle, according to which graphics should be accurately perceived and appropriately conceived. Animations are often too complex or too fast to be accurately perceived. Moreover, many continuous events are conceived of as sequences of discrete steps. Judicious use of interactivity may overcome both these disadvantages. Animations may be more effective than comparable static graphics in situations other than conveying complex systems, for example, for real time reorientations in time and space.

Tweedie, L. 1997, "Characterizing Interactive Externalizations." In SIGCHI Conference on Human Factors in Computing Systems, pp. 375-75.

Tyman, J, GP Gruetzmacher, and J Stasko. 2004, "Infovisexplorer." In IEEE Symposium on Information Visualization, pp. r7-r7.

In this paper we briefly describe 3 tools developed to visualize the history of information visualization papers. The visualization consists of a standard 3D scatterplot view enhanced with "bubbles," lines, text, and colors aimed at making comparisons between authors and topics found in the papers. Three components were developed to translate and display raw XML data using OpenGL and Cocoa. We use the visualization tool to perform five tasks and discuss it\&\#146;s weaknesses.

Tyndiuk, F, et al. 2007, "Études Des caractéristiques De l'utilisateur, De la tâche Et de l'interface En réalité Virtuelle." Revue Européenne de Psychologie Appliquée/European Review of Applied Psychology 57(4):225-36.

Résumé Le développement des applications de réalité virtuelle et son incursion dans de nouveaux domaines (formation, loisirs, santés) ont mis en exergue les différences que les utilisateurs peuvent manifester dans leurs performances avec cette nouvelle technologie. Afin de mieux comprendre ces différences interindividuelles, nous avons étudié l'impact des caractéristiques de la tâche, de l'interface et de l'utilisateur sur cette performance. Du point de vue de la tâche, nous nous sommes intéressées aux tâches de manipulation et de locomotion, concernant l'interface, à l'impact de la taille de l'écran (TE) sur la performance et en ce qui concerne l'utilisateur, à l'implication de la mémoire de travail (MT) et des capacités spatiales (CS) dans les performances. Nos résultats montrent un gain de performance sur grand écran quel que soit le type de tâche (comme la locomotion et la manipulation). Ils mettent également en évidence que les CS des utilisateurs prédisent la performance en réalité virtuelle, quelle que soit la tâche, et essentiellement pour les tâches sur petit écran. Les recommandations issues de cette étude incitent à utiliser un grand écran. Celui-ci joue alors le rôle d'aide cognitive permettant de soutenir un haut niveau de performances en réalité virtuelle. The increase of virtual reality application and its use into new fields (training, entertainment and health) point up the difficulty for users to be efficient with this new technology. In order to understand these inter-individual differences, we have studied the impact of the task, the interface and the users' characteristics on this performance. For the task, we have focused on manipulation and travel tasks. For the interface, we have studied the impact of the screen size on the performance and finally with regard to the user, we have focused on the impact of working memory and spatial capacities on performance. Our results show a performance gain on large screen whatever the task. 
They show that the users' spatial capacities predicted the performance, particularly for the tasks realized on a small screen. We conclude that a large screen is a cognitive aid that permits a high level of performance.

Tzeng, FY, and KL Ma. 2005, "Opening the Black Box - Data Driven Visualization of Neural Networks." In IEEE Conference on Information Visualization, pp. 383-90.

Artificial neural networks are computer software or hardware models inspired by the structure and behavior of neurons in the human nervous system. As a powerful learning tool, increasingly neural networks have been adopted by many large-scale information processing applications but there is no a set of well defined criteria for choosing a neural network. The user mostly treats a neural network as a black box and cannot explain how learning from input data was done nor how performance can be consistently ensured. We have experimented with several information visualization designs aiming to open the black box to possibly uncover underlying dependencies between the input data and the output data of a neural network. In this paper, we present our designs and show that the visualizations not only help us design more efficient neural networks, but also assist us in the process of using neural networks for problem solving such as performing a classification task.

Uldall-Espersen, T, E Frøkjær, and K Hornbæk. 2008, "Tracing Impact in a Usability Improvement Process." Interacting with Computers 20(1):48-63.

Analyzing usability improvement processes as they take place in real-life organizations is necessary to understand the practice of usability work. This paper describes a case study where the usability of an information system is improved and a relationship between the improvements and the evaluation efforts is established. Results show that evaluation techniques complemented each other by suggesting different kinds of usability improvement. Among the techniques applied, a combination of questionnaires and Metaphors of Human Thinking (MOT) showed the largest mean impact and MOT produced the largest number of impacts. Logging of real-life use of the system over 6 months indicated six aspects of improved usability, where significant differences among evaluation techniques were found. Concerning five of the six aspects Think Aloud evaluations and the above-mentioned combination of questionnaire and MOT performed equally well, and better than MOT. Based on the evaluations 40 redesign proposals were developed and 30 of these were implemented. Four of the implemented redesigns where considered especially important. These evolved with inspiration from multiple evaluations and were informed by stakeholders with different kinds of expertise. Our results suggest that practitioners should not rely on isolated evaluations. Instead complementing techniques should be combined, and people with different expertise should be involved.

Upstill, TG, BA Nagappan, and N Craswell. 2001, "Visual Clustering of Image Search Results." In 8th Meeting of the Visual Data Exploration and Analysis Conference, pp. 49-59.

This paper presents a novel method for visualizing the results of an image search. Current approaches to visualizing WWW image searches rank results in a linear list and present them as a sorted thumbnail grid. The method outlined in this paper visually clusters images based on the user's search terms. To accomplish this, a flexible image retrieval method which incorporates a combination of content-based and textual image matching is used. A new information visualization is used to display the search results. In our model multiple types of partitioning and querying can occur concurrently, thereby creating a multidimensional display of image properties. The display groups similar images, enabling users to quickly 
scan for the most relevant images. This visualization allows users to exploit the location of images as their guide to what an image contains and use thumbnails to preview potentially relevant images. Through the identification of relevant images users can locate relevant areas in the visualization. It is then possible for users to focus their attention on one area of the visualization using a zooming function. The user's interaction with the system is explored using new evaluation metrics based on Information Foraging theory.

Upton, C, and G Doherty. 2006, "Designing Usable Charts for Complexwork Settings." In IEEE Conference on Information Visualization, pp. 447-52.

Advances in graphing applications, plug-ins and toolkits means that integrating charts and graphs into software is easier than ever before. However, selecting the optimal graphing technique for a workers task remains a difficult challenge. Information visualization experts draw on research from cognitive engineering, perceptual psychology and human computer interaction when designing displays. For the increasing number of developers who are integrating visual displays into applications, there is for a lack of a general methodology that pulls together key activities from these diverse fields. In the absence of such a methodology, it is very difficult for software developers to identify if their choice of representation satisfies both the user's tasks and perceptual limitations. We describe the approach taken in the redesign of an interactive chart used in a high volume manufacturing environment. We show how analyses of the work domain, the data and the users' tasks are all crucial steps in the design process

Upton, C, and G Doherty. 2008, "Extending Ecological Interface Design Principles: A Manufacturing Case Study." International Journal of Human-Computer Studies 66(4):271-86.

Identifying information requirements is a well-understood activity, but the practice of converting data into visual form based on these requirements is less defined. The Ecological Interface Design (EID) framework attempts to bridge this design gap by offering a set of visual design principles. While these principles supply high-level goals to be achieved by the display, they do not describe the design process per se. EID case studies tend to report the work domain analysis, the design solution and the relationship between these two artefacts. Unfortunately, the presentation of a final solution does not reveal the rationale for decisions made during the design process. This, coupled with the complexity of the systems involved, can make it difficult to transfer design knowledge to other work domains. Here a methodology is proposed to guide the design of visual interface components that make up an ecological display. A structured approach for matching requirements to visual form based on work domain analysis, task analysis, scale matching, and data transformations is presented. A case study reveals the rationale behind the redesign of a process control health reporting system using this methodology.

Urquiza-Fuentes, J, and JÁ Velázquez-Iturbide. 2007, "An Evaluation of the Effortless Approach to Build Algorithm Animations with Winhipe." Electronic Notes in Theoretical Computer Science 178:3-13.

The use of algorithm visualizations in computer science education is not a new thing. Although there is a firm belief that graphical representations of algorithms are learning aids, empirical studies show that what is important is what the students do with the animations rather than what they see in them. In this paper we compare to kinds of interaction: viewing animations vs constructing animations. We have conducted a controlled experiment where a group of students $(n=15)$ had to study an algorithm and complete a knowledge test about it and a subjective opinion questionnaire. Students were randomly divided in constructing and viewing groups. Results have been measured by means of learning outcomes, 
efficiency issues and student's subjective opinion. Results significantly evidence that builders obtained better results than viewers.

V., et al. 2008, "A Chorem-Based Approach for Visually Synthesizing Complex Phenomena." Information Visualization 7(3-4):253-64.

When dealing with scenarios referring to complex issues, such as political, economic and demographic problems, the usage of visual metaphors represents a more effective approach in supporting users to locate facts and new patterns. In this paper, we describe a research project whose aim is to investigate a cartographic solution able to represent dynamics, movements and changes that underlie possible problems. The solution we propose is based on the chorem concept. It represents an immediate synthesis of data of interest, and provides expert users with both a global view of objects and phenomena, and an insight into a specific issue. Based on preliminary studies, we first provide a formal definition and classification of chorems in terms of structure and meaning, meant to homogenize chorem construction and usage. Then, a system to generate chorematic maps from available data sets is described and an XML-like language, named ChorML is specified, enabling system modules communication. In order to validate our approach, we exemplify the construction of a chorematic map, which depicts the most significant flows of migrating population in Italy in 2000. Such a synthesis may represent the starting point for further processing tasks aimed to derive spatial analysis data, as well as to support expert users in decision making. Information Visualization (2008) 7, 253-264. doi: 10.1057/palgrave.ivs.9500186

Vaidya, V, et al. 2007, "Building Intuitive 3d Interfaces for Virtual Reality Systems - Art. No. 65091x." In Medical Imaging 2007 Conference, pp. X5091-X91.

An exploration of techniques for developing intuitive, and efficient user interfaces for virtual reality systems. Work seeks to understand which paradigms from the better-understood world of 2D user interfaces remain viable within $3 \mathrm{D}$ environments. In order to establish this anew user interface was created that applied various understood principles of interface design. A user study was then performed where it was compared with an earlier interface for a series of medical visualization tasks.

Valiati, ERA, MS Pimenta, and CMDS Freitas. 2006, "A Taxonomy of Tasks for Guiding the Evaluation of Multidimensional Visualizations." In AVI workshop on BEyond time and errors: novel evaluation methods for information visualization (BELIV'06), pp. 1-6.

The design of multidimensional visualization techniques is based on the assumption that a graphical representation of a large dataset can give more insight to a user, by providing him/her a more intuitive support in the process of exploiting data. When developing a visualization technique, the analytic and exploratory tasks that a user might need or want to perform on the data should guide the choice of the visual and interaction metaphors implemented by the technique. Usability testing of visualization techniques also needs the definition of users' tasks. The identification and understanding of the nature of the users' tasks in the process of acquiring knowledge from visual representations of data is a recent branch in information visualization research. Some works have proposed taxonomies to organize tasks that a visualization technique should support. This paper proposes a taxonomy of visualization tasks, based on existing taxonomies as well as on the observation of users performing exploratory tasks in a multidimensional data set using two different visualization techniques, Parallel Coordinates and RadViz. Different scenarios involving low-level tasks were estimated for the completion of some high-level tasks, and they were compared to the scenarios observed during the users' experiments. 
Valkanova, N, et al. 2010, "Ambientnews: Augmenting Information Discovery in Complex Settings through Aesthetic Design." In 14th International Conference on Information Visualization, pp. 439-44.

Nowadays professional journalists create and edit broadcasting materials inside newsrooms that are characterized by an intense and multivariate flux of information. Within these settings, the journalists have difficulties keeping up with the evolving geopolitical picture of events developing in the world and how it relates to the topics of their interest. In this paper we present AmbientNEWS, an ambient display that visualizes dynamic news content and informs professional journalists on the global picture of events. We argue that the conceptualization and design of its information aesthetics criteria can be grounded in user requirements, and subsequently engaged to augment the awareness of professionals in an environment that is highly crowded with information. We also discuss the evaluation process of ambient information visualization with a focus on aesthetic design aspects. We conclude by summarizing our findings and discuss future work.

van der Heyden, JE, et al. 2001, "Exploring Presentation Methods for Tomographic Medical Image Viewing." Artificial Intelligence in Medicine 22(2):89-109.

This paper explores the presentation of tomographic medical images on a computer screen. Limitations of the computer screen are apparent, as even a very large computer monitor cannot display an entire study consisting of dozens of images at once. Our objective is to propose filmless computer presentation methods for these images, in particular for magnetic resonance images. First, we observe the magnetic resonance image analysis task in the traditional light screen environment where presentation of many images has always been possible. We then propose solutions for meeting requirements in the computer environment. After implementation of these solutions we obtain user feedback on alternatives in order to determine feasibility and preference. Observations reveal three requirement categories: user control of film management, navigation of images and image series, and simultaneous availability of detail and context. We developed a framework of detail-in-context-technique parameters for the purpose of viewing tomographic medical images and presented our solution directions to the radiologists for feedback. Results from the user feedback study support the feasibility of the proposed approaches and clearly indicate the importance of presentation issues in the development of medical imaging viewing systems.

van Ham, F. 2004, "Case Study: Visualizing Visualization." In IEEE Symposium on Information Visualization, pp. r5-r5.

In this case study we attempt to visualize a real-world dataset consisting of 600 recently published information visualization papers and their references. This is done by first creating a global layout of the entire graph that preserves any cluster structure present. We then use this layout as a basis to define a hierarchical clustering. The clusters in this hierarchy are labelled using keywords supplied with the dataset, allowing insight into the clusters semantics.

van Lammeren, R, et al. 2010, "Affective Appraisal of 3d Land Use Visualization." Computers, Environment and Urban Systems 34(6):465-75.

In this paper we discuss the affective appraisal and affective response of users to three different visualization types: colored raster cells, 2D-icon and 3D-icons. For that we developed a dedicated multilayered visualization of current and future land use in the Netherlands, that may allow policy-makers to 
assess and compare land use scenarios. This Google Earth based visualization, abbreviated GESO, facilitates users by means of the three different visualizations of current and future land use. It is often assumed that 3D-visualization improves the cognitive understanding of scenario outcomes. There are many uncertainties, however, about the affective responses to 3D-visualization. A between-subject experiment has been designed to compare viewers' responses to the three types of visualizations on affective appraisals of the environment. 3D-icon visualization elicited the highest affective appraisals and positively influenced perception of the environmental quality. Moreover, the results demonstrated that 2D-icons and 3D-icons, compared with colored raster cells, did not improve the efficiency or accuracy of the participants in this experiment. The results provide evidence that the visualization type may influence the affective appraisal of the environment represented. The need for further research is discussed, especially regarding the question whether these types of visualizations may influence judgement and decision-making in environmental planning.

van Nimwegen, C, and H van Oostendorp. 2009, "The Questionable Impact of an Assisting Interface on Performance in Transfer Situations." International Journal of Industrial Ergonomics 39(3):501-08.

Previous research has shown that a "guided" interface where relevant task information is shown (externalization) can result in worse performance than an "unguided" interface where users have to think more for themselves (internalization). We studied transfer of task performance and whether switching from an "unguided" to a "guided" interface results in better performance than other way around. We also investigated whether the unguided interface enhances performance on a transfer task. In an experiment participants solved a series of problems with a "guided" or a "non-guided" (initial phase). In the transfer phase, they received the opposite interface. The unguided interface resulted in more efficient performance than the guided interface. We attribute this to the fact that the unguided interface provokes more active contemplation. Switching from an unguided to a guided interface had no effect on efficiency, while the other way around, it did. Performance on a transfer task with an unguided interface after working with the guided interface caused worse performance than other combinations. Deeper levels of thought instigated by the unguided interface caused better knowledge and better strategies. This can be important in situations where learning itself is the aim, or in when making errors generates a high cost.Relevance to industry User interface designers should be careful with providing interface cues that give away too much information in safety critical task situations or in situations when learning or transfer is the aim. In these cases it is wise to consider how much information is made externally available on screen.

van Pelt, R, et al. 2010, "Exploration of 4d Mri Blood Flow Using Stylistic Visualization." IEEE Transactions on Visualization and Computer Graphics 16(6):1339-47.

Insight into the dynamics of blood-flow considerably improves the understanding of the complex cardiovascular system and its pathologies. Advances in MRI technology enable acquisition of 4D bloodflow data, providing quantitative blood-flow velocities over time. The currently typical slice-by-slice analysis requires a full mental reconstruction of the unsteady blood-flow field, which is a tedious and highly challenging task, even for skilled physicians. We endeavor to alleviate this task by means of comprehensive visualization and interaction techniques. In this paper we present a framework for preclinical cardiovascular research, providing tools to both interactively explore the 4D blood-flow data and depict the essential blood-flow characteristics. The framework encompasses a variety of visualization styles, comprising illustrative techniques as well as improved methods from the established field of flow visualization. Each of the incorporated styles, including exploded planar reformats, flow-direction highlights, and arrow-trails, locally captures the blood-flow dynamics and may be initiated by an 
interactively probed vessel cross-section. Additionally, we present the results of an evaluation with domain experts, measuring the value of each of the visualization styles and related rendering parameters.

van Schaik, P. 2010, "Using Interactive 3-D Visualization for Public Consultation." Interacting with Computers 22(6):556-68.

3-D models are often developed to aid the design and development of indoor and outdoor environments. This study explores the use of interactive 3-D visualization for public consultation for outdoor environments. Two visualization techniques (interactive 3-D visualization and static visualization) were compared using the method of individual testing. Visualization technique had no effect on the perception of the represented outdoor environment, but there was a preference for using interactive 3-D. Previously established mechanisms for a preference for interactive 3-D visualization in other domains were confirmed in the perceived strengths and weaknesses of visualization techniques. In focus-group discussion, major preferences included provision of more information through interactive 3$\mathrm{D}$ visualization and wider access to information for public consultation. From a users' perspective, the findings confirm the strong potential of interactive 3-D visualization for public consultation.

van Schaik, P, and J Ling. 2006, "The Effects of Graphical Display and Screen Ratio on Information Retrieval in Web Pages." Computers in Human Behavior 22(5):870-84.

Although many web pages consist of blocks of text surrounded by graphics, there is a lack of valid empirical research to aid the design of this type of page [D. Diaper, P. Waelend, Interact. Comput. 13 (2000) 163]. In particular little is known about the influence of animations on interaction with web pages. Proportion, in particular the Golden Section, is known to be a key determinant of aesthetic quality of objects and aesthetics have recently been identified as a powerful factor in the quality of human-computer interaction [N. Tractinsky, A.S. Katz, D. Ikar, Interact. Comput. 13 (2000) 127]. The current study aimed to establish the relative strength of the effects of graphical display and screen ratio of content and navigation areas in web pages, using an information retrieval task and a split-plot experimental research design. Results demonstrated the effect of screen ratio, but a lack of an effect of graphical display on task performance and two subjective outcome measures. However, there was an effect of graphical display on perceived distraction, with animated display leading to more distraction than static display, $\mathrm{t}(64)=2.33$. Results are discussed in terms of processes of perception and attention and recommendations for web page design are given.

van Wijk, JJ. 2006, "Bridging the Gaps." IEEE Computer Graphics and Applications 26(6):6-9.

The aim of visualization is to enable users to obtain insight, and not just to produce images. Hence, if we want to make progress in the field of visualization, close cooperation with users is essential, but such cooperation is not without problems. There are two major gaps to be bridged. First, there's a knowledge gap between the visualization expert and domain expert. The visualization expert must spend much effort to understand what the domain expert is studying and what his or her concerns are. Second, there is an interest gap. A visualization researcher will aim at new methods and results, whereas the domain expert primarily needs a useful tool. This article discusses various modes for cooperation, in view of these gaps. The royal road is to use user-centered design, but this can be time consuming and does not necessarily lead to innovative results. The latter holds even stronger when a visualization expert just acts (or is viewed as) a tool smith. In contrast, a visualization expert can also operate as a computer scientist, and 
focus just on the parts of the puzzle where he or she already has expertise. Finally, the article discusses a purely curiosity driven approach, where the visualization expert acts as domain expert. (C) 2006 IEEE.

van Wijk, JJ. 2006, "Views on Visualization." IEEE Transactions on Visualization and Computer Graphics 12(4):421-32.

The field of visualization is maturing. Many problems have been solved and new directions are sought. In order to make good choices, an understanding of the purpose and meaning of visualization is needed. In this paper, visualization is considered from multiple points of view. First, a technological viewpoint is adopted, where the value of visualization is measured based on effectiveness and efficiency. An economic model of visualization is presented and benefits and costs are established. Next, consequences and limitations of visualization are discussed (including the use of alternative methods, high initial costs, subjectiveness, and the role of interaction). Example uses of the model for the judgment of existing classes of methods are given to understand why they are or are not used in practice. However, such an economic view is too restrictive. Alternative views on visualization are presented and discussed: visualization as an art, visualization as design and, finally, visualization as a scientific discipline.

Van Wijk, JJ, and H Van de Wetering. 1999, "Cushion Treemaps: Visualization of Hierarchical Information." In IEEE Symposium on Information Visualization, pp. 73-78,147.

A new method is presented for the visualization of hierarchical information, such as directory structures and organization structures. Cushion treemaps inherit the elegance of standard treemaps: compact, space-filling displays of hierarchical information, based on recursive subdivision of a rectangular image space. Intuitive shading is used to provide insight in the hierarchical structure. During the subdivision, ridges are added per rectangle, which are rendered with a simple shading model. The result is a surface that consists of recursive cushions. The method is efficient, effective, easy to use and implement, and has a wide applicability

Van Wijk, JJ, and ER Van Selow. 1999, "Cluster and Calendar Based Visualization of Time Series Data." In IEEE Symposium on Information Visualization, pp. 4-9,140.

A new method is presented to get an insight into univariate time series data. The problem addressed is how to identify patterns and trends on multiple time scales (days, weeks, seasons) simultaneously. The solution presented is to cluster similar daily data patterns, and to visualize the average patterns as graphs and the corresponding days on a calendar. This presentation provides a quick insight into both standard and exceptional patterns. Furthermore, it is well suited to interactive exploration. Two applications, numbers of employees present and energy consumption, are presented

Vande Moere, A. 2004, "Information Flocking: Time-Varying Data Visualization Using Boid Behaviors." In 8th International Conference on Information Visualization, pp. 409-14.

This work presents a significant extension of the information flocking concept and algorithms originally presented by G. Proctor and C. Winter (1998). It introduces a novel way of visualizing timevarying datasets using the emergent characteristics of self organization and dynamic behavior simulation. The current prototype uses both spatial clustering and behavioral animation to represent temporal data similarities by simulating the time-varying evolution of dynamic datasets. Instead of presenting exact data values, the way how the data values change over time is being visualized. In addition, the current 
information flocking method is capable of visualizing short-term temporal events or long-term timevarying data evolutions by automatically generating different recognizable motion typologies. This research show how artificial life principles have been merged with the field of information visualization. Several aspects of the original information flocking algorithms have been improved to incorporate the real-time evaluation of continuous dynamic data value streams and to generate multiple stable, recognizable atomic as well as collective dynamic behaviors that reflect time-varying dataset changes and relative data value evolutions. The main information flocking principles are demonstrated through a visualization of historical stock market quotes.

Veeramachaneni, H. 2010, "Body - Buckets of Disease Symptoms for Disease Outbreak Analysis Vast Data Challenge 2010 - Mini Challenge 2." In IEEE Symposium on Visual Analytics Science \& Technology.

Veerasamy, A, and NJ Belkin. 1996, "Evaluation of a Tool for Visualization of Information Retrieval Results." In 19th Annual International ACM SIGIR Conference on Research and Development in Information Retrieval, pp. 85-92.

Reports on the design and evaluation of a visualization tool for information retrieval (IR) systems that aims to help the end user in the following respects. (1) As an indicator of document relevance, the tool graphically provides specific query-related information about individual documents. (2) As a diagnosis tool, it graphically provides aggregate information about the query results that could help in identifying how the different query terms influence the retrieval and ranking of documents. Two different experiments using TREC-4 data were conducted to evaluate the effectiveness of this tool. Results, while mixed, indicate that visualization of this sort may provide useful support for judging the relevance of documents, in particular by enabling users to make more accurate decisions about which documents to inspect in detail. Problems in the evaluation of such tools in interactive environments are discussed

Velez, MC, D Silver, and M Tremaine. 2005, "Understanding Visualization through Spatial Ability Differences." In IEEE Conference on Visualization, pp. 511-18.

Little is known about the cognitive abilities which influence the comprehension of scientific and information visualizations and what properties of the visualization affect comprehension. Our goal in this paper is to understand what makes visualizations difficult. We address this goal by examining the spatial ability differences in a diverse population selected for spatial ability variance. For example, how is, spatial ability related to visualization comprehension? What makes a particular visualization difficult or time intensive for specific groups of subjects? In this paper, we present the results of an experiment designed to answer these questions. Fifty-six subjects were tested on a basic visualization task and given standard paper tests of spatial abilities. An equal number of males and females were recruited in this study in order to increase spatial ability variance. Our results show that high spatial ability is correlated with accuracy on our three-dimensional visualization test, but not with time. High spatial ability subjects also had less difficulty with object complexity and the hidden properties of an object. 
Vernik, R, GS von Itzstein, and A Bouchard. 2007, "Imago: An Integrated Prototyping, Evaluation and Transitioning Environment for Information Visualisation." In 11th International Conference on Information Visualization, pp. 17-22.

This paper introduces Imago, an environment that supports the prototyping, evaluation and transitioning of information visualisation approaches into practice. The approach is based on the use of an underlying semantic model of contextual and visualisation knowledge and integrated evaluation capabilities to aid the transitioning process. We discuss the use of Imago in relation to our experiences in researching and providing information visualisation approaches for command and control activities.

Vervoort, JM, et al. 2010, "Stepping into Futures: Exploring the Potential of Interactive Media for Participatory Scenarios on Social-Ecological Systems." Futures 42(6):604-16.

In this paper, we present a strategy for the development of interactive media scenarios to help communicate uncertainties and complexities in coupled human and natural systems. Insights arising from Complex Adaptive Systems theory advocate the need for more adaptive perspectives on natural resources management. For the collaborative exploration of future complexities and uncertainties, participatory scenario development has proven to be a powerful approach. A range of communication strategies with benefits for conveying complexity, however, has not yet been adopted by scenario developers. We present a framework of criteria with which we structurally analyze the benefits of interactive media communication. First, we consider requirements of feasibility, flexibility and stakeholder contributions. Then, we synthesize criteria for the communication of Complex Adaptive Systems. Finally, we set criteria for communicatory clarity and engagement. Using this framework, we review several science communication fields, including landscape visualization, serious gaming and visual analytics. We then develop a strategy for interactive media communication in participatory scenario development, including two work-in-progress examples. This strategy employs mixed media, micro-games and accessible stakeholder contributions in a geo-web context, and is suitable for participatory work in live settings as well as on-line, from a local to a global scale.

Viau, C, et al. 2010, "The Flowvizmenu and Parallel Scatterplot Matrix: Hybrid Multidimensional Visualizations for Network Exploration." IEEE Transactions on Visualization and Computer Graphics 16(6):1100-8.

A standard approach for visualizing multivariate networks is to use one or more multidimensional views (for example, scatterplots) for selecting nodes by various metrics, possibly coordinated with a node-link view of the network. In this paper, we present three novel approaches for achieving a tighter integration of these views through hybrid techniques for multidimensional visualization, graph selection and layout. First, we present the FlowVizMenu, a radial menu containing a scatterplot that can be popped up transiently and manipulated with rapid, fluid gestures to select and modify the axes of its scatterplot. Second, the FlowVizMenu can be used to steer an attribute-driven layout of the network, causing certain nodes of a node-link diagram to move toward their corresponding positions in a scatterplot while others can be positioned manually or by force-directed layout. Third, we describe a novel hybrid approach that combines a scatterplot matrix (SPLOM) and parallel coordinates called the Parallel Scatterplot Matrix (PSPLOM), which can be used to visualize and select features within the network. We also describe a novel arrangement of scatterplots called the Scatterplot Staircase (SPLOS) that requires less space than a traditional scatterplot matrix. Initial user feedback is reported. 
Viegas, FB, and M Wattenberg. 2007, "Artistic Data Visualization: Beyond Visual Analytics." In 2nd International Conference on Online Communities and Social Computing held at the HCI International 2007, pp. 182-91.

Information visualization is traditionally viewed as a tool for data exploration and hypothesis formation. Because of its roots in scientific reasoning, visualization has traditionally been viewed as an analytical tool for sensemaking. In recent years, however, both the mainstrearning of computer graphics and the democratization of data sources on the Internet have had important repercussions in the field of information visualization. With the ability to create visual representations of data on home computers, artists and designers have taken matters into their own hands and expanded the conceptual horizon of infovis as artistic practice. This paper presents a brief survey of projects in the field of artistic information visualization and a preliminary examination of how artists appropriate and repurpose "scientific" techniques to create pieces that actively guide analytical reasoning and encourage a contextualized reading of their subject matter.

Viegas, FB, and M Wattenberg. 2006, "Technical Forum - Communication-Minded Visualization: A Call to Action." IBM Systems Journal 45(4):801-12.

In this paper we introduce the concept of communication-minded visualization (CMV), a visualization designed to support communication and collaborative analysis. Our emphasis is on the design of the user experience rather than the technical implementation challenges. We believe that designing for communication is essential because users do not interact with visualizations solely to gain personal insights. An insight that matters usually has to be communicated to others. To harness the power of visualization as a working tool for multidisciplinary teams, designers need to pay close attention to how visualization affects and enables the communication of discoveries and the discussion of ideas within multiple contexts

Vilanova, A, A Telea, and G Scheuermann. 2008, "Visual Analysis and Semantic Exploration of Error Aware Urban Change Detection." Symposium A Quarterly Journal In Modern Foreign Literatures 27(3).

Viola, I, H Hauser, and D Ebert. 2010, "Editorial Note for Special Section on Illustrative Visualization." Computers \& Graphics 34(4):335-36.

Voinea, L, J Lukkien, and A Telea. 2007, "Visual Assessment of Software Evolution." Science of Computer Programming 65(3):222-48.

Configuration management tools have become well and widely accepted by the software industry. Software Configuration Management (SCM) systems hold minute information about the entire evolution of complex software systems and thus represent a good source for process accounting and auditing. However, it is still difficult to use the entire spectrum of information such tools maintain. Currently, significant effort is being done in the direction of mining this kind of software repositories for extracting data to support relevant assessments. In this article we propose a concerted set of visualization tools and techniques for the assessment of software evolution based on the information stored into SCM systems. Firstly, we introduce a generic way to obtain models of source code at different levels of detail and from different perspectives. Secondly, we propose a set of visual representations and techniques to efficiently and effectively depict the evolution of these code models. These techniques target specific questions and assessments, from the detailed code developer perspective to the overview required by system architects 
and project managers. We detail the concrete implementation of two such code models and corresponding visual representations. The file view describes code change at line level across multiple versions of a single file, or small number of files. The project view shows changes at file level across complete software projects. All our views share the same visual and interactive techniques, enabling users to easily switch among and correlate between them. We implement our visual techniques to quickly and compactly display and navigate the evolution of tens of thousands of artifacts on a single screen. We demonstrate our techniques with several use cases performed on real world, industry-size code bases and outline the concrete findings and ways our visualizations helped in understanding various types of code changes.

Voinea, L, and A Telea. 2007, "Visual Data Mining and Analysis of Software Repositories." Computers \& Graphics 31(3):410-28.

In this article we describe an ongoing effort to integrate information visualization techniques into the process of configuration management for software systems. Our focus is to help software engineers manage the evolution of large and complex software systems by offering them effective and efficient ways to query and assess system properties using visual techniques. To this end, we combine several techniques from different domains, as follows. First, we construct an infrastructure that allows generic querying and data mining of different types of software repositories such as CVS and Subversion. Using this infrastructure, we construct several models of the software source code evolution at different levels of detail, ranging from project and package up to function and code line. Second, we describe a set of views that allow examining the code evolution models at different levels of detail and from different perspectives. We detail three views: the file view shows changes at line level across many versions of a single, or a few, files. The project view shows changes at file level across entire software projects. The decomposition view shows changes at subsystem level across entire projects. We illustrate how the proposed techniques, which we implemented in a fully operational toolset, have been used to answer nontrivial questions on several real-world, industry-size software projects. Our work is at the crossroads of applied software engineering (SE) and information visualization, as our toolset aims to tightly integrate the methods promoted by the InfoVis field into the SE practice.

von Pilgrim, J, K Duske, and P McIntosh. 2009, "Eclipse Gef3d: Bringing 3d to Existing 2d Editors." Information Visualization 8(2):107-19.

In this paper we present the Eclipse project GEF3D. It is a framework for three-dimensional (3D) editors and editors, based on the widely used two-dimensional (2D) graphical editing framework Eclipse Graphical Editing Framework (GEF). It enhances this framework, enabling programmers to easily implement 3D editors. As an Eclipse plugin GEF3D is seamlessly integrated into the Eclipse integrated development environment, allowing developers to work with one tool for developing and visualizing their software in $3 \mathrm{D}$. The third dimension enables the visualization of more complex relationships than provided by existing two-dimensional representations. In this paper we explain the architecture and certain design patterns of GEF3D in order to give researchers and developers interested in 3D software visualization an overview of how to use GEF3D and the features provided by the framework. We present the results of a usability evaluation, show how GEF3D is applied to embed an existing 2D editor into a 3D editor, and discuss performance issues. Information Visualization (2009) 8, 107-119. doi: 10.1057/ivs.2009.9 
Vora, PR, and MG Helander. 1997, "Chapter 38 - Hypertext and Its Implications for the Internet." in Handbook of Human-Computer Interaction, eds. GH Marting, KL Thomas and VP Prasad, pp. 877-914. North-Holland, Amsterdam.

Summary Vannevar Bush (1945) pointed out the inefficiency and artificiality of the index-based methods of storing and retrieving information, which required that "having found one item... [one must] emerge from the system and re-enter a new path" (p. 106). Bush claimed that "[t]he human mind does not work that way. It operates by association. With one item in tis grasp, it snaps instantly to the next that is suggested by the association of thoughts..." (Bush, 1945, p. 106).

Vrotsou, K, K Ellegard, and M Cooper. 2007, "Everyday Life Discoveries: Mining and Visualizing Activity Patterns in Social Science Diary Data." In 11th International Conference on Information Visualization, pp. 130-38.

The ability to identify and examine patterns of activities is a key tool for social and behavioural science. In the past this has been done by statistical or purely visual methods but automated sequential pattern analysis through sophisticated data mining and visualization tools for pattern location and evaluation can open up new possibilities for interactive exploration of the data. This paper describes the addition of a sequential pattern identification method to the visual activity-analysis tool, VISUALTimePAcTS, and its effectiveness in the process of pattern analysis in social science diary data. The results have shown that the method correctly identifies patterns and conveys them effectively to the social scientist in a manner that allows them quick and easy understanding of the significance of the patterns.

Wachter, SB, et al. 2003, "The Employment of an Iterative Design Process to Develop a Pulmonary Graphical Display." Journal of the American Medical Informatics Association 10(4):363-72.

Objective: Data representations on today's medical monitors need to be improved to advance clinical awareness and prevent data vigilance errors. Simply building graphical displays does not ensure an improvement in clinical performance because displays have to be consistent with the user's clinical processes and mental models. In this report, the development of an original pulmonary graphical display for anesthesia is used as an example to show an iterative design process with built-in usability testing. Design: The process reported here is rapid, inexpensive, and requires a minimal number of subjects per development cycle. Three paper-based tests evaluated the anatomic, variable mapping, and graphical diagnostic meaning of the pulmonary display. Measurements: A confusion matrix compared the designer's intended answer with the subject's chosen answer. Considering deviations off the diagonal of the confusion matrix as design weaknesses, the pulmonary display was modified and retested. The iterative cycle continued until the anatomic and variable mapping cumulative test scores for a chosen design scored above $90 \%$ and the graphical diagnostic meaning test scored above $75 \%$. Results: The iterative development test resulted in five design iterations. The final graphical pulmonary display improved the overall intuitiveness by $18 \%$. The display was tested in three categories: anatomic features, variable mapping, and diagnostic accuracy. The anatomic intuitiveness increased by $25 \%$, variable mapping intuitiveness increased by $34 \%$, and diagnostic accuracy decreased slightly by $4 \%$. Conclusion: With this rapid iterative development process, an intuitive graphical display can be developed inexpensively prior to formal testing in an experimental setting. 
Wade, A, and R Nicholson. 2010, "Improving Airplane Safety : Tableau and Bird Strikes." In IEEE Conference on Information Visualization, pp. 1-3.

Wagener, T, and J Kollat. 2007, "Numerical and Visual Evaluation of Hydrological and Environmental Models Using the Monte Carlo Analysis Toolbox." Environmental Modelling \& Software 22(7):1021-33.

The detailed evaluation of mathematical models and the consideration of uncertainty in the modeling of hydrological and environmental systems are of increasing importance, and are sometimes even demanded by decision makers. At the same time, the growing complexity of models to represent realworld systems makes it more and more difficult to understand model behavior, sensitivities and uncertainties. The Monte Carlo Analysis Toolbox (MCAT) is a Matlab library of visual and numerical analysis tools for the evaluation of hydrological and environmental models. Input to the MCAT is the result of a Monte Carlo or population evolution based sampling of the parameter space of the model structure under investigation. The MCAT can be used off-line, i.e., it does not have to be connected to the evaluated model, and can thus be used for any model for which an appropriate sampling can be performed. The MCAT contains tools for the evaluation of performance, identifiability, sensitivity, predictive uncertainty and also allows for the testing of hypotheses with respect to the model structure used. In addition to research applications, the MCAT can be used as a teaching tool in courses that include the use of mathematical models.

Wagman, M. 1995. The Sciences of Cognition: Theory and Research in Psychology and Artificial Intelligence. Praeger Publishers, Westport.

Wagman presents a general, unified theory of artificial and human intelligence under which the nature of human reasoning, problem solving, analogical thinking, and scientific discovery is examined from theoretical, research and computational perspectives. The work analyzes foundational issues regarding the nature of intelligent systems and intelligence, and significant and current research in the area is discussed. This book will be of interest to scholars dealing with psychology, artificial intelligence and cognitive science.

Walker, R. 2010, "Vastvis - Visual Analytics with Multiple Coordinated Views ( a ) ( B ) ( C ) (H ) ( D ) ( G ) ( F ) ( E )." In IEEE Symposium on Visual Analytics Science \& Technology, pp. 279-80.

Wall, J. 2010, "Prodv V - a Cas Se Study in Delive Ering Visu Ual Analy Ytics." In IEEE Symposium on Visual Analytics Science \& Technology, pp. 247-48.

WanAdnan, W, et al. 2007, "An Experimental Evaluation of Information Visualization Techniques and Decision Style on Decision Performance." In 11th Pacific Asia Conference on Information Systems, pp. U1214-U20.

The importance of individual characteristics has been emphasized in the human computer interaction and information visualization (IV) literature. However decision style, which is recognized as one of the key individual cognitive differences that affects system success, has received little attention in these areas. This study aims to examine how individual differences, IV techniques and task complexity influence decision performance and user preferences in a business decision support environment. The study adopted an experimental method, based on within-subject design approach. The results showed that there were significant differences in decision performance between IV techniques, individual differences and task. 
The results suggest that decision style is a significant moderator on the relationship between IV and performance. The findings have important implications for the DSS designers, and provide important research issues for future work.

Wang Baldonado, MQ, A Woodruff, and A Kuchinsky. 2000, "Guidelines for Using Multiple Views in Information Visualization." In Working Conference on Advanced Visual Interfaces (AVI '00), pp. 110-19.

Wang, C. 2010, "Poster : Dynamic Network Visualization in 1 . 5d." In IEEE Conference on Information Visualization, pp. 5-6.

Wang, G, et al. 2010, "Visualizing Differences of Dti Fiber Models Using 2d Normalized Embeddings." In IEEE International Conference on Information Visualization.

Wang, H, G Cai, and AM MacEachren. 2008, "Geodialogue: A Software Agent Enabling Collaborative Dialogues between a User and a Conversational Gis." In 20th IEEE International Conference on Tools with Artificial Intelligence, pp. 357-60.

Wang, L, D Tjondrongoro, and Y Liu. 2007, "Clustering and Visualizing Audiovisual Dataset on Mobile Devices in a Topic-Oriented Manner." in Advances in Visual Information Systems, eds. GP Qiu, et al., Vol 4781, pp. 310-21.

With the significant enhancement of telecom bandwidth and multi media-supported mobile devices occupying the market, consuming audiovisual contents on the move is no longer a hype. A lot of telecom operators are now porting traditional TV service to PDAs, $3 \mathrm{G}$ cell phones. However, several surveys suggest that direct migration of service from large screen to small screen may not comply with mobile users' consuming behaviors. In this paper, we first elaborate existing surveys together with our survey result to understand consumer interests in terms of consuming audiovisual contents on mobile devices. Based on the findings, we propose a novel solution to help user locate, gather, and cross-relate topics scattered in an array of contents across various domains. A web-based demo application has been implemented for PDA. The system evaluation indicates that this paradigm of organizing and presenting multimedia archives is very welcomed.

Wang, TD, et al. 2008, "Aligning Temporal Data by Sentinel Events: Discovering Patterns in Electronic Health Records." In 26th Annual CHI Conference on Human Factors in Computing Systems, pp. 457-66.

Electronic Health Records (EHRs) and other temporal databases contain hidden patterns that reveal important cause-and-effect phenomena. Finding these patterns is a challenge when using traditional query languages and tabular displays. We present an interactive visual tool that complements query formulation by providing operations to align, rank and filter the results, and to visualize estimates of the intervals of validity of the data. Display of patient histories aligned on sentinel events (such as a first heart attack) enables users to spot precursor, co-occurring, and aftereffect events. A controlled study demonstrates the benefits of providing alignment (with a $61 \%$ speed improvement for complex tasks). A qualitative study and interviews with medical professionals demonstrates that the interface can be learned quickly and seems to address their needs. 
Wang, TD, C Plaisant, and B Shneiderman. 2010, "Temporal Pattern Discovery Using Lifelines2." In IEEE International Conference on Information Visuazliation, pp. 4-6.

Wang, TD, et al. 2009, "Temporal Summaries: Supporting Temporal Categorical Searching, Aggregation and Comparison." Visualization and Computer Graphics, IEEE Transactions on 15(6):1049-56.

When analyzing thousands of event histories, analysts often want to see the events as an aggregate to detect insights and generate new hypotheses about the data. An analysis tool must emphasize both the prevalence and the temporal ordering of these events. Additionally, the analysis tool must also support flexible comparisons to allow analysts to gather visual evidence. In a previous work, we introduced align, rank, and filter (ARF) to accentuate temporal ordering. In this paper, we present temporal summaries, an interactive visualization technique that highlights the prevalence of event occurrences. Temporal summaries dynamically aggregate events in multiple granularities (year, month, week, day, hour, etc.) for the purpose of spotting trends over time and comparing several groups of records. They provide affordances for analysts to perform temporal range filters. We demonstrate the applicability of this approach in two extensive case studies with analysts who applied temporal summaries to search, filter, and look for patterns in electronic health records and academic records.

Wang, W, and A Lu. 2006, "Interactive Wormhole Detection in Large Scale Wireless Networks." In IEEE Symposium on Visual Analytics Science \& Technology, pp. 99-106.

Wang, X, et al. 2009, "Defining and Applying Knowledge Conversion Processes to a Visual Analytics System." Computers \& Graphics 33(5):616-23.

Knowledge-assisted visualization has been a fast growing field because it directly integrates and utilizes domain knowledge to produce effective data visualization. However, most existing knowledgeassisted visualization applications focus on integrating domain knowledge that is tailored only for specific analytical tasks. This reflects not only the different understandings of what "knowledge" is in visualization, but also the difficulties in generalizing and reapplying knowledge to new problems or domains. In this paper, we differentiate knowledge into two types, tacit and explicit, and suggest four conversion processes between them (internalization, externalization, collaboration, and combination) that could be included in knowledge-assisted visualizations. We demonstrate the applications of these four processes in a bridge visual analytical system for the US Department of Transportation and discuss their roles and utilities in real-life scenarios.

Wang, X, and S-w Lee. 2008, Integrating Visual Analysis with Ontological Knowledge Structure. Technical Rpt. CVC-UNCC-08-30, University of North Carolina.

Wang, X, E Miller, and K Smarick. 2008, "Investigative Visual Analysis of Global Terrorism." Computer Graphics 27(3):919-26.

Recent increases in terrorist activity around the world have made analyzing and understanding such activities more critical than ever. With the help of organizations such as the National Center for the Study of Terrorism and Responses to Terrorism (START), we now have detailed historical information on each terrorist event around the world since 1970. However, due to the size and complexity of the data, identifying terrorists' patterns and trends has been difficult. To better enable investigators in understanding terrorist activities, we propose a visual analytical system that focuses on depicting one of 
the most fundamental concepts in investigative analysis, the five W's (who, what, where, when, and why). Views in our system are highly correlated, and each represents one of the W's. With this approach, an investigator can interactively explore terrorist activities efficiently and discover reasons of attacks (why) by identifying patterns temporally (when), geo-spatially (where), between multiple terrorist groups (who), and across different methods or modes of attacks (what). By coupling a global perspective with the details gleaned from asking these five questions, the system allows analysts to think both tactically and strategically.

Wang, Y, J Shearer, and KL Ma. 2007, "Vica: A Voronoi Interface for Visualizing Collaborative Annotations." in Cooperative Design, Visualization, and Engineering, ed. YH Luo, Vol 4674, pp. 21-32.

Large-scale scientific investigation often includes collaborative data exploration among geographically distributed researchers. The tools used for this exploration typically include some communicative component, and this component often forms the basis for insight and idea sharing among collaborators. Minimizing the tool interaction required to locate "interesting" communications is therefore of paramount importance. We present the design of a novel visualization interface for representing the communications among multiple collaborating authors, and detail the benefits of our approach versus traditional methods. Our visualization integrates directly with the existing data exploration interface. We present our system in the context of an international research effort conducting collaborative analysis of accelerator simulations. 
Ware, C. 2004, "Appendix C - the Perceptual Evaluation of Visualization Techniques and Systems." in Information Visualization, pp. 393-404. Academic Press, San Diego.

Ware, C. 2004, "Chapter 1 - Foundation for a Science of Data Visualization." in Information Visualization, pp. 1-27. Academic Press, San Diego.

Ware, C. 2003, "Chapter 2 - Design as Applied Perception." in Hci Models, Theories, and Frameworks, ed. MC John, pp. 11-26. Morgan Kaufmann, San Francisco.

Ware, C. 2004, "Chapter 5 - Visual Attention and Information That Pops Out." in Information Visualization, pp. 145-86. Academic Press, San Diego.

Ware, C. 2004, "Chapter 7 - Visual Objects and Data Objects." in Information Visualization, pp. 227-58. Academic Press, San Diego.

Ware, C. 2004, "Chapter 8 - Space Perception and the Display of Data in Space." in Information Visualization, pp. 259-95. Academic Press, San Diego.

Ware, C. 2004, "Chapter 9 - Images, Words, and Gestures." in Information Visualization, pp. 297-316. Academic Press, San Diego.

Ware, C. 2004, "Chapter 10 - Interacting with Visualizations." in Information Visualization, pp. 317-50. Academic Press, San Diego.

Ware, C. 2004, "Chapter 11 - Thinking with Visualizations." in Information Visualization, pp. 351-86. Academic Press, San Diego.

Ware, C. 2000. Information Visualization. Morgan Kaufmann, San Francisco.

Ware, C. 2004. Information Visualization: Perception for Design. Morgan Kaufmann, San Francisco.

Ware, C, and M Plumlee. 2005, "Chapter 29 - 3d Geovisualization and the Structure of Visual Space." in Exploring Geovisualization, eds. D Jason, MM Alan and K Menno-Jan, pp. 567-76. Elsevier, Oxford.

Summary The problems of creating geovisualization interfaces are analyzed by characterizing space in three distinct ways. The first considers the nature of the perceptual structure of space. Here the primary reference system is the egocentric coordinate reference frame, centered on the user and oriented with respect to the user's view direction. The second characterization is in terms of the cost of gaining extra information by navigation. This navigation is fastest when it consists of simple eye movements, and is much slower with virtual flying interfaces. Zooming interfaces lie somewhere between. The third characterization is in terms of the cognitive mechanism we use to make visual queries on displays containing geographic information. Limits of visual working memory impose severe restrictions on our ability to inter-relate information from different parts of large geographic data spaces. We show how understanding these three aspects of space can be used to make specific predictions about which types of navigation interface will be most suitable for specified tasks. 
Ware, C, et al. 2002, "Cognitive Measurements of Graph Aesthetics." Information Visualization 1(2):10310.

Waser, J, et al. 2010, "World Lines." IEEE Transactions on Visualization and Computer Graphics 16(6):1458-67.

In this paper we present World Lines as a novel interactive visualization that provides complete control over multiple heterogeneous simulation runs. In many application areas, decisions can only be made by exploring alternative scenarios. The goal of the suggested approach is to support users in this decision making process. In this setting, the data domain is extended to a set of alternative worlds where only one outcome will actually happen. World Lines integrate simulation, visualization and computational steering into a single unified system that is capable of dealing with the extended solution space. World Lines represent simulation runs as causally connected tracks that share a common time axis. This setup enables users to interfere and add new information quickly. A World Line is introduced as a visual combination of user events and their effects in order to present a possible future. To quickly find the most attractive outcome, we suggest World Lines as the governing component in a system of multiple linked views and a simulation component. World Lines employ linking and brushing to enable comparative visual analysis of multiple simulations in linked views. Analysis results can be mapped to various visual variables that World Lines provide in order to highlight the most compelling solutions. To demonstrate this technique we present a flooding scenario and show the usefulness of the integrated approach to support informed decision making.

Waterworth, JA. 1997, "Chapter 39 - Multimedia Interaction." in Handbook of Human-Computer Interaction, eds. GH Marting, KL Thomas and VP Prasad, pp. 915-46. North-Holland, Amsterdam.

Summary Increasingly, sensory information is being provided through computers and digital media. The boundary between the real and the artificial is blurring as recordings of real events are merged with computer-generated sounds and images to create dynamic composites and interactive virtual worlds. The computer is becoming an interactive device for generating perceptual experience. This is a natural evolution of technology in response to the impressive array of senses that humans have, and their extraordinary ability to integrate sensory information into powerful perceptual models of their environment.

Wattenberg, M, and D Fisher. 2003, "A Model of Multi-Scale Perceptual Organization in Information Graphics." In IEEE Symposium on Information Visualization, pp. 23-30.

We propose a new method for assessing the perceptual organization of information graphics, based on the premise that the visual structure of an image should match the structure of the data it is intended to convey. The core of our method is a new formal model of one type of perceptual structure, based on classical machine vision techniques for analyzing an image at multiple resolutions. The model takes as input an arbitrary grayscale image and returns a lattice structure describing the visual organization of the image. We show how this model captures several aspects of traditional design aesthetics, and we describe a software tool that implements the model to help designers analyze and refine visual displays. Our emphasis here is on demonstrating the model's potential as a design aid rather than as a description of human perception, but given its initial promise we propose a variety of ways in which the model could be extended and validated. 
Weaver, C. 2000, "Improvisational Geovisualization of the 2000 United States Census." In AutoCarto, pp. 1-10.

Weaver, C. 2010, "Multidimensional Data Dissection Using Attribute Relationship Graphs." In IEEE Symposium on Visual Analytics Science \& Technology, pp. 75-82.

Weaver, C. 2008, "Multidimensional Visual Analysis Using Cross-Filtered Views." In IEEE Symposium on Visual Analytics Science and Technology, pp. 163-70.

Weaver, C. 2007, "Patterns of Coordination in Improvise Visualizations." In IS\&T/SPIE Conference on Visualization and Data Analysis, pp. 64950K-50K-12.

Weaver, C, et al. 2007, "Visual Exploration and Analysis of Historic Hotel Visits." Information Visualization 6(1):89-103.

Weaver, C, et al. 2006, "Visual Analysis of Historic Hotel Visitation Patterns." In IEEE Symposium on Visual Analytics Science and Technology, pp. 35-42.

Weghorst, S, et al. 2008, "Medical Interface Research at the Hit Lab." Virtual Reality 12(4):201-14.

Wehrend, S, and C Lewis. 1990, "A Problem-Oriented Classification of Visualization Techniques." In 1st IEEE Conference on Visualization, pp. 139-43.

Progress in scientific visualization could be accelerated if workers could more readily find visualization techniques relevant to a given problem. This paper describes an approach to this problem based on a classification of visualization techniques that is independent of particular application domains. A user breaks up a problem into subproblems, describes these subproblems in terms of the objects to be represented and the operations to be supported by a representation, locates applicable visualization techniques in a catalog, and combines these representation into a composite representation for the original problem. The catalog and its underlying classification provide a way for workers in different application disciplines to share methods.

Weick, KE. 1995. Sensemaking in Organizations. Sage, Thousand Oaks, CA.

Weinkauf, T, and H Theisel. 2010, "Streak Lines as Tangent Curves of a Derived Vector Field." IEEE Transactions on Visualization and Computer Graphics 16(6):1225-34.

Characteristic curves of vector fields include stream, path, and streak lines. Stream and path lines can be obtained by a simple vector field integration of an autonomous ODE system, i.e., they can be described as tangent curves of a vector field. This facilitates their mathematical analysis including the extraction of core lines around which stream or path lines exhibit swirling motion, or the computation of their curvature for every point in the domain without actually integrating them. Such a description of streak lines is not yet available, which excludes them from most of the feature extraction and analysis tools that have been developed in our community. In this paper, we develop the first description of streak lines as tangent curves of a derived vector field - the streak line vector field - and show how it can be computed from the spatial and temporal gradients of the flow map, i.e., a dense path line integration is required. We demonstrate the high accuracy of our approach by comparing it to solutions where the ground truth is analytically known and to solutions where the ground truth has been obtained using the classic streak line 
computation. Furthermore, we apply a number of feature extraction and analysis tools to the new streak line vector field including the extraction of cores of swirling streak lines and the computation of streak line curvature fields. These first applications foreshadow the large variety of possible future research directions based on our new mathematical description of streak lines.

Weisberg, RW. 1996, "Prolegomena to Theories of Insight in Problem Solving: A Taxonomy of Problems." in The Nature of Insight, eds. RJ Sternberg and JE Davidson, pp. 157-96. The MIT Press.

Weiss, K. 2010, "Nested Refinement Domains for Tetrahedral and Diamond Hierarchies." In IEEE Conference on Information Visualization, pp. 6-7.

Wen, Z, and MX Zhou. 2008, "Evaluating the Use of Data Transformation for Information Visualization." IEEE Transactions on Visualization and Computer Graphics 14(6):1309-16.

Data transformation, the process of preparing raw data for effective visualization, is one of the key challenges in information visualization. Although researchers have developed many data transformation techniques, there is little empirical study of the general Impact of data transformation on visualization. Without such study, It is difficult to systematically decide when and which data transformation techniques are needed. We thus have designed and conducted a two-part empirical study that examines how the use of common data transformation techniques impacts visualization quality, which in turn affects user task performance. Our first experiment studies the impact of data transformation on user performance in single-step, typical visual analytic tasks. The second experiment assesses the impact of data transformation in multi-step analytic tasks. Our results quantify the benefits of data transformation In both experiments. More Importantly, our analyses reveal that (1) the benefits of data transformation vary significantly by task and by visualization, and (2) the use of data transformation depends on a user's interaction context. Based on our findings, we present a set of design recommendations that help guide the development and use of data transformation techniques. (C) 2008 IEEE.

Westerberg, AW, et al. 1989, "A Future Computer Environment for Preliminary Design." in Foundations of Computer-Aided Process Design, eds. JJ In Siirola, IE Grossman and G Stephanopoulos, pp. 507-28. Elsevier, Amsterdam, Netherlands.

Consideration is given to the creation of future computer-based design environments to support a team-based preliminary design process. Adopting the view of L.L. Bucciarelli (1988), one can see design as a social process among the team members. Each team member has his (or her) own view of the artifact to be designed, and each operates in his own 'object world'. The authors list a set of attributes they see as needed for a computer-based environment to support this process. After suggesting that the activity of design is a form of continual evolutionary model development by the participants, they argue that it can be captured by providing a support system to record and organize models as they are developed and shared. Based on work with creating the modeling language ASCEND, the authors propose a number of specific concepts on which the design of such a support system can be based

Westerdahl, B, et al. 2006, "Users' Evaluation of a Virtual Reality Architectural Model Compared with the Experience of the Completed Building." Automation in Construction 15(2):150-65.

It is important that the properties of a Virtual Reality (VR) model support its purpose in the specific context in which it is used. This study investigated how employees of a company experienced a VR 
model of their yet-to-be-built office building. We also compared the VR experience with the employees' experience of the completed building. The results showed that the employees felt that the VR model was a useful aid in the decision-making process concerning their future workplace. In addition, The Semantic Environment Description Scale was used to compare the experience of the VR model with the experience of the real building. The results suggest that the VR model gave a fairly accurate representation of the real building.

Westerman, SJ, J Collins, and T Cribbin. 2005, "Browsing a Document Collection Represented in Twoand Three-Dimensional Virtual Information Space." International Journal of Human-Computer Studies 62(6):713-36.

This paper reports a study of information retrieval performance using an interface in which documents were represented by objects in a virtual environment. Spatial location was determined by semantic content, with inter-object distance representing semantic similarity of documents. The quality of spatial-semantic mapping was manipulated as was the number of dimensions (two versus three) in which document nodes were arranged. Participants were required to browse the information space and identify all documents relevant to a specified topic. Results indicated that participants were able to use threedimensional spatial mapping of semantic information to facilitate task performance, with performance being better when the quality of the mapping was higher. Strategy differences were identified, with participants adopting a more ['] exhaustive' approach when searching two-dimensional node arrangements, and a more ['] focused' approach for three-dimensional arrangements. Cognitive ability was not strongly associated with task performance, but participants of relatively lower cognitive ability tended to out-perform those of higher cognitive ability in three-dimensional conditions. Possible reasons for these findings are discussed.

Westerman, SJ, and T Cribbin. 2000, "Mapping Semantic Information in Virtual Space: Dimensions, Variance and Individual Differences." International Journal of Human-Computer Studies 53(5):765-87.

This paper reports two studies investigating the computer-based representation of the semantic information content of databases using object location in two- and three-dimensional virtual space. In the first study, the cognitive demands associated with performing an information search task were examined under conditions where the "goodness of fit" of the spatial-semantic "mapping" was manipulated. The effects of individual differences in spatial ability and associative memory ability also were considered. Results indicated that performance equivalence, between two- and three-dimensional interfaces, could be achieved when the two-dimensional interface accounted for between 50 and $70 \%$ of the semantic variance accounted for by the three-dimensional solution. A second study, in which automatic text analysis was used to generate two- and three-dimensional solutions for document sets of varying sizes and types, supported the conclusion that, for the purpose of information search, the amount of additional semantic information that can be conveyed by a three-dimensional solution does not outweigh the associated additional cognitive demands. 
Wetheimer, M. 1959. Productive Thinking. Harper, New York.

White, RW, G Marchionini, and G Muresan. 2008, "Evaluating Exploratory Search Systems: Introduction to Special Topic Issue of Information Processing and Management." Information Processing \& Management 44(2):433-36.

Whiting, MA, et al. 2006, "Threat Stream Data Generator : Creating the Known Unknowns for Test and Evaluation of Visual Analytics Tools." In AVI workshop on BEyond time and errors: novel evaluation methods for information visualization (BELIV'06).

Whiting, MA, J Haack, and C Varley. 2008, "Creating Realistic , Scenario-Based Synthetic Data for Test and Evaluation of Information Analytics Software." In Conference on BEyond time and errors: novel evaLuation methods for Information Visualization (BELIV'08).

Whitney, P, C Posse, and X Lei. 2006, "Towards an Extendable Software System for Information Integration." In WORLDCOMP'06.

Whitney, PD, C Posse, and X Lei. 2005, Towards a Unified Approach to Information Integration - a Review Paper on Data / Information Fusion. Technical Rpt. PNNL-15428, Pacific Northwest National Laboratory, Richland, WA.

Whyte, J, et al. 2008, "Visualizing Knowledge in Project-Based Work." Long Range Planning 41(1):7492.

This article considers how visual practices are used to manage knowledge in project-based work. It compares project-based work in a capital goods manufacturer and an architectural firm. Visual representations are used extensively in both cases, but the nature of visual practice differs significantly between the two. The research explores the kinds of knowledge that are (and aren't) developed and made visible in strategizing and planning activities. For example, whereas the emphasis of project-based work in the former firm is on exploitation of knowledge and it visualizes its project context largely in commercial and processual terms, the emphasis in the latter is on exploration and it uses a wide range of visual materials to understand physical interdependencies across the project boundary. We contend particular kinds of visual tools can help project teams step between exploration and exploitation within a project, and articulate the types of representations, foci of attention and patterns of interaction involved. The findings suggest that business managers can make more deliberate choices about how knowledge is made visible, and can change visual practice to align the project with exploring and exploiting opportunities. It raises the question: What don't you see within your organization? The work contributes to academic debates about managing through projects, strategising and organizing, while the focus on visual representation disrupts the tacit-codified dichotomy in the broad debate on knowledge and learning, and highlights the craft skills central to strategizing and organizing.

Wickham, H, et al. 2010, "Graphical Inference for Infovis." IEEE Transactions on Visualization and Computer Graphics 16(6):973-9.

How do we know if what we see is really there? When visualizing data, how do we avoid falling into the trap of apophenia where we see patterns in random noise? Traditionally, infovis has been concerned with discovering new relationships, and statistics with preventing spurious relationships from being 
reported. We pull these opposing poles closer with two new techniques for rigorous statistical inference of visual discoveries. The "Rorschach" helps the analyst calibrate their understanding of uncertainty and "line-up" provides a protocol for assessing the significance of visual discoveries, protecting against the discovery of spurious structure.

Wingyan, C, and A Leung. 2007, "Supporting Web Searching of Business Intelligence with Information Visualization." In IEEE/WIC/ACM International Conference on Web Intelligence, pp. 807-11.

In this research, we proposed and validated an approach to using information visualization to augment search engines in supporting the analysis of business stakeholder information on the Web. We report in this paper findings from a preliminary evaluation comparing a visualization prototype with a traditional method of stakeholder analysis (Web browsing and searching). We found that the prototype achieved a higher perceived usefulness and perceived analysis effectiveness and was perceived favorably in expediting the subjects' decision making and in helping them understand the analysis results. Overall, the proposed approach was found to augment traditional methods of analyzing business stakeholders. We discuss implications to researchers and practitioners and future directions.

Wing-Yi, C, Q Huamin, and M Wai-Ho. 2010, "Visualizing the Semantic Structure in Classical Music Works." Visualization and Computer Graphics, IEEE Transactions on 16(1):161-73.

A major obstacle in the appreciation of classical music is that extensive training is required to understand musical structure and compositional techniques toward comprehending the thoughts behind the musical work. In this paper, we propose an innovative visualization solution to reveal the semantic structure in classical orchestral works such that users can gain insights into musical structure and appreciate the beauty of music. We formulate the semantic structure into macrolevel layer interactions, microlevel theme variations, and macro-micro relationships between themes and layers to abstract the complicated construction of a musical composition. The visualization has been applied with success in understanding some classical music works as supported by highly promising user study results with the general audience and very positive feedback from music students and experts, demonstrating its effectiveness in conveying the sophistication and beauty of classical music to novice users with informative and intuitive displays.

Wipfli, S, and C Schneider. 2009, "The Sensitive Tapestry Built Architecture as a Platform for Information Visualization and Interaction." In 4th Information Visualization Conference, pp. 486-89.

This paper presents 'The Sensitive Tapesny', an interactive installation using body-input as a nontraditional user interface. The technical basis for this kind of media-enabled environment is a thermal imager that captures the activities in public areas at a large scale. The installation has been developed as a prototypical example in architectural education at the ETH Zurich, Science City. The aim of the project is to generate a novel experience, which shows the potential Of merging physical architecture and digital information. The article newly develops architecture as an interface that reveals information about the building itself its occupants, and/or its environment. It describes the research that employs design experimentation and information visualization with the use of computer-supported, interactive, visual representations of abstract data to amplify cognition. This questions the way in which one perceives the own body spatiality and motility in physical and augmented environments and how the particular experience created by this juxtaposition evokes one's awareness of the motility in the public. The paper 
suggests that introducing this kind of display in a social scenario can enrich the casual interaction Of people nearby and this might enhance social awareness and engagement.

Wise, JA, et al. 1995, "Visualizing the Non-Visual: Spatial Analysis and Interaction with Information from Text Documents." In IEEE Conference on Information Visualization, pp. 51-58.

Wiss, U, and D Carr. 1998, A Cognitive Classification Framework for 3-Dimensional Information Visualization. Technical Rpt. LTU-TR-9804-SE, Lulea University of Technology.

Wiss, U, D Carr, and H Jonsson. 1998, "Evaluating Three-Dimensional Information Visualization Designs: A Case Study of Three Designs." In IEEE Conference on Information Visualization, pp. 137-44.

A number of three-dimensional information visualization designs have been invented during the last years. However, comparisons of such designs have been scarce, making it difficult for application developers to select a suitable design. This paper reports on a case study where three existing visualization designs have been implemented and evaluated. We found that the three information visualization designs have inherent problems when used for visualizing different data sets, and that certain tasks can not be supported by the designs. A general methodology for evaluation is presented, which comprises evaluation of suitability for different data sets as well as evaluation of support for user tasks.

Wittenberg, C. 2004, "A Pictorial Human-Computer Interface Concept for Supervisory Control." Control Engineering Practice 12(7):865-78.

Increasing level of automation of production systems has lead to reduced numbers of operators and at the same time increase in the number of process information each operator has to supervise and control. This article presents a concept for the design of graphical user interfaces with pictorial elements in the domain of industrial automation. The concept uses techniques known from the three-dimensional computer graphics and information structure based on the operators task to present the production systems adapted to the human information processing. Experiments tested and evaluated the efficiency of this concept.

Wohlfart, E. 2007, "A Detailed Comparison of Information Visualization Tools Using a Reference Data Set." Vol Master's Thesis. Technical University of Vienna.

Wohlfart, E, et al. 2008, "Comparing Information Visualization Tools Focusing on the Temporal Dimensions." In 12th International Conference on Information Visualization, pp. 69-74.

Empirical comparisons and categorizations of information visualization tools lack important considerations: the former undervalue the need for a theoretical background, and the latter tend to have too much distance from the user because they do not consider definite user tasks. Therefore, our work combines these approaches and presents the results of both a qualitative evaluation and a recently published categorization. We focus on the visualization of temporal data and reveal that current tools realize only a small part of the visualization possibilities in this field. 
Wong, et al. 2009, "Designing a Collaborative Visual Analytics Tool for Social and Technological Change Prediction." Computer Graphics and Applications, IEEE 29(5):58-68.

This paper discussed about GreenOracle which an interdisciplinary team designed and developed. GreenOracle is a collaborative visual analytics tool for predicting global climate change's impact on US power grids and its implications for society and national security. These future scenarios provide critical assessments and information to help policymakers and stakeholders formulate a coherent and unified strategy toward shaping a safe and secure society.

Wong, PC. 2007, "Visual Analytics Science and Technology." Information Visualization:1-3.

Wong, PC, et al. 2006, "Have Green - a Visual Analytics Framework for Large Semantic Graphs." In IEEE Symposium on Visual Analytics Science and Technology, pp. 67-74.

A semantic graph is a network of heterogeneous nodes and links annotated with a domain ontology. In intelligence analysis, investigators use semantic graphs to organize concepts and relationships as graph nodes and links in hopes of discovering key trends, patterns, and insights. However, as new information continues to arrive from a multitude of sources, the size and complexity of the semantic graphs will soon overwhelm an investigations cognitive capacity to carry out significant analyses. We introduce a powerful visual analytics framework designed to enhance investigators natural analytical capabilities to comprehend and analyze large semantic graphs. The paper describes the overall framework design, presents major development accomplishments to date, and discusses future directions of a new visual analytics system known as Have Green.

Wong, PC, et al. 2006, "Graph Signatures for Visual Analytics." IEEE Transactions on Visualization and Computer Graphics 12(6):1399-413.

We present a visual analytics technique to explore graphs using the concept of a data signature. A data signature, in our context, is a multidimensional vector that captures the local topology information surrounding each graph node. Signature vectors extracted from a graph are projected onto a lowdimensional scatterplot through the use of scaling. The resultant scatterplot, which reflects the similarities of the vectors, allows analysts to examine the graph structures and their corresponding real-life interpretations through repeated use of brushing and linking between the two visualizations. The interpretation of the graph structures is based on the outcomes of multiple participatory analysis sessions with intelligence analysts conducted by the authors at the Pacific Northwest National Laboratory. The paper first uses three public domain data sets with either well-known or obvious features to explain the rationale of our design and illustrate its results. More advanced examples are then used in a customized usability study to evaluate the effectiveness and efficiency of our approach. The study results reveal not only the limitations and weaknesses of the traditional approach based solely on graph visualization, but also the advantages and strengths of our signature-guided approach presented in the paper. 
Wong, PC, et al. 2008, "A Dynamic Multiscale Magnifying Tool for Exploring Large Sparse Graphs." Information Visualization 7(2):105-17.

Wong, PC, et al. 2006, "Generating Graphs for Visual Analytics through Interactive Sketching." Visualization and Computer Graphics, IEEE Transactions on 12(6):1386-98.

We introduce an interactive graph generator, GreenSketch, designed to facilitate the creation of descriptive graphs required for different visual analytics tasks. The human-centric design approach of GreenSketch enables users to master the creation process without specific training or prior knowledge of graph model theory. The customized user interface encourages users to gain insight into the connection between the compact matrix representation and the topology of a graph layout when they sketch their graphs. Both the human-enforced and machine-generated randomnesses supported by GreenSketch provide the flexibility needed to address the uncertainty factor in many analytical tasks. This paper describes more than two dozen examples that cover a wide variety of graph creations from a single line of nodes to a real-life small-world network that describes a snapshot of telephone connections. While the discussion focuses mainly on the design of GreenSketch, we include a case study that applies the technology in a visual analytics environment and a usability study that evaluates the strengths and weaknesses of our design approach

Wong, PC, et al. 2004, "In-Spire Infovis 2004 Contest Entry." In IEEE Symposium on Information Visualization, pp. r2-r2.

This is the first part (summary) of a three-part contest entry submitted to IEEE InfoVis 2004. The contest topic is visualizing InfoVis symposium papers from 1995 to 2002 and their references. The paper introduces the visualization tool IN-SPIRE, the visualization process and results, and presents lessons learned.

Wong, PC, et al. 2006, "Have Green - a Visual Analytics Framework for Large Semantic Graphs." Journal of Graph Algorithms and Applications:67-74.

Wong, PC, et al. 2005, "Visual Analytics and Storytelling through Video." In IEEE Symposium on Information Visualization, pp. 6-7.

Wong, PC, et al. 2006, "Walking the Path : A New Journey to Explore and Discover through Visual Analytics." Information Visualization 5(4):237-49.

Wood, J. 2009, "Chapter 14 - Geomorphometry in Landserf." in Developments in Soil Science, eds. H Tomislav and IR Hannes, Vol 33, pp. 333-49. Elsevier.

Wood, J, et al. 2005, "Chapter 14 - Using 3d in Visualization." in Exploring Geovisualization, eds. D Jason, MM Alan and K Menno-Jan, pp. 293-312. Elsevier, Oxford.

Summary The notion of three-dimensionality is applied to five stages of the visualization pipeline. While 3D visualization is most often associated with the visual mapping and representation of data, this chapter also identifies its role in the management and assembly of data, and in the media used to display $3 \mathrm{D}$ imagery. The extra cartographic degree of freedom offered by using $3 \mathrm{D}$ is explored and offered as a motivation for employing 3D in visualization. The use of VR and the construction of virtual environments exploit navigational and behavioural realism, but become most useful when combined with abstracted 
representations embedded in a 3D space. The interactions between the development of geovisualization, the technology used to implement it and the theory surrounding cartographic representation are explored. The dominance of computing technologies, driven particularly by the gaming industry is discussed with particular reference to 3D geovisualization.

Woods, DD, and JC Watts. 1997, "How Not to Have to Navigate through Too Many Displays." in Handbook of Human-Computer Interaction, eds. GH Marting, KL Thomas and VP Prasad, pp. 617-50. North-Holland, Amsterdam.

Summary The first and most basic response of system developers to the expanding power of the computer and its increasing penetration into application areas has been to collect and make available more and more raw data. The modular nature of the computer as a medium for representation has allowed system developers to accommodate the expanded set of data easily (at least from a technology point of view) by adding more and more displays to the set of potentially observable frames hidden behind the narrow keyhole of the computer screen. The combination of a large field of raw data and a narrow keyhole have created a variety of problems for practitioners, people engaged in the practice of a profession or occupation.

Woolf, BP. 2009, "Chapter 4 - Teaching Knowledge." in Building Intelligent Interactive Tutors: StudentCentered Strategies for Revolutionizing E-Learning, pp. 95-135. Morgan Kaufmann, San Francisco.

Workman, M. 2010, "A Behaviorist Perspective on Corporate Harassment Online: Validation of a Theoretical Model of Psychological Motives." Computers \& Security 29(8):831-39.

Cyber harassment has been seen in the literature as a problem among school-aged children, or at the adult-level, as a legal problem involving attacks that have typically been associated with retaliation for some psychosocial or monetary gain. However, with the rise of the social networking phenomenon, cyber harassment has spread from school-aged children to a wider developmental and behavioral phenomenon. Companies and professionals are increasingly the targets of personal attacks on social network sites and in blog postings by "trolls" and "cyber bullies". Thus rather than gaining information, these kinds of attacks often disseminate misleading or false information to damage their targets, to interfere with them, or for the purposes of extortion. We conducted a randomized field study of a bounded population into motivations that may lead people to conduct these kinds of attacks, we validated the model empirically, and we drew some potential behavioral responses along with implications for further research.

Workman, M. 2007, "Cognitive Load Research and Semantic Apprehension of Graphical Linguistics." In 3rd Symposium of the Workgroup Human-Computer Interaction and Usability Engineering of the Austrian Computer Society, pp. 375-88.

In knowledge-work, there are increasing amounts of complex information rendered by information technology, which has led to the common term, information overload. Information visualization is one area where empirically tested semantic theory has not yet caught up with that of the underlying information storage and retrieval theory, contributing to information overload. In spite of a vast body of cognitive theory, much of the human factors research on information visualization has overlooked it. Specifically, information displays have facilitated the data gathering (ontological) aspects of human problem-solving and decision-making, but have exacerbated the meaning-making (epistemological) aspects of those activities by presenting information in linear rather than in graphical (holistic) forms. 
Drawing from extant empirical research, we present a thesis suggesting that cognitive load may be reduced when holistic information is imbued with transformational grammar to help alleviate the information overload problem, along with a methodological approach for investigation.

Workman, M. 2008, "An Experimental Assessment of Semantic Apprehension of Graphical Linguistics." Computers in Human Behavior 24(6):2578-96.

Visual displays of information (such as dashboards) have become very sophisticated in rendering world semantics but neglect display semantics, leading to what is commonly called information overload. Underlying storage and retrieval research has been utilizing semantic and cognitive theory to drive the current implementations of ontology markup using the resource description framework (RDF) and Web ontology language (OWL) for over a decade. Yet despite these semantically rich underlying description logics, and despite the very large and mature stream of cognitive and neuroscience theory literature on visual perception and attention, memory, and linguistics, this is one aspect within the area of information visualization and human factors research where empirically tested semantic theory has not yet caught up, and begs for theory-driven research into display semantics using what might be termed "graphical linguistics." We conducted an experiment to assess the cognitive effort of interpreting domain general knowledge using the same information represented in three forms, and found that graphical linguistics reduce cognitive effort for a specific type of task involving high-density time-sensitive information typically found in situation control rooms.

Wrede, S, et al. 2004, "Integration Frameworks for Large Scale Cognitive Vision Systems - an Evaluative Study." In 17th International Conference on Pattern Recognition, pp. 761-64.

Owing to the ever growing complexity of present day computer vision systems, system architecture has become an emerging topic in vision research. Systems that integrate numerous modules and algorithms of different $\mathrm{I} / \mathrm{O}$ and time scale behavior require sound and reliable concepts for interprocess communication. Consequently, topics and methods known from software and systems engineering are becoming increasingly important. Especially, framework technologies for system integration are required. This contribution results from a cooperation between two multinational projects on cognitive vision. It discusses functional and non-functional requirements in cognitive vision and compares and assesses existing solutions. 
Wright, W. 2009, "Research Report: Information Animation Applications in the Capital Markets." In IEEE Conference on Information Visualization, pp. 19-25.

Wu, AN, et al. 2009, "Civil: Support Geo-Collaboration with Information Visualization." In International ACM Conference on Supporting Group Work, pp. 272-75.

Teams of specialized experts, such as emergency management planning teams, while making decisions need to efficiently pool domain-specific knowledge, synthesize relevant information,,aid keep track of collaborators activities at a low interaction cost. This requires tools that allow monitoring both low-level information (e.g., individual actions and external events) and higher-order activities (e.g., how members contribute to groupwork). This paper presents design of CIVIL, a system prototype developed to support map-based decision-making. We report our empirical evaluation of the effects of visualizations oil the decision process and the final product.

Wu, L, D Lv, and W Cheng. 2010, "The Phased Evaluation and Information Visualization of City Building Fire Hazards." In 4th International Conference on Bioinformatics and Biomedical Engineering, pp. 1-4.

The fire developing process was divided into four partial periods, and the influential factors of every partial period were analyzed. Combining the workers' abilities and management conditions, the 3 evaluation model of building fire was made. Use the method of AHP to determine the degree of importance between factors of the peer grade, i.e., weight. The 3 level fuzzy comprehensive evaluation method was applied to evaluating the building fire hazard. Using modern computer technology VB6.0 and MapInfo, the visual information management system was constructed. The evaluation and classification of building fire hazards were made based on the basic data, and the result can be shown in the map at realtime. Taking the building A in Qingdao City as an example, the evaluation result was consistent with the practice. And the system can help to realize the visualization and dynamic management.

Wu, QS, et al. 2009, "Visualization of Security Events Using an Efficient Correlation Technique." In IEEE Symposium on Computational Intelligence in Cyber Security, pp. 61-68 ST - Visualization of Security Events Using.

Wu, Y, et al. 2010, "Visualizing Windows System Traces." In 5th international symposium on Software visualization pp. 123-32.

Wu, Y, et al. 2010, "Opinionseer: Interactive Visualization of Hotel Customer Feedback." IEEE Transactions on Visualization and Computer Graphics 16(6):1109-18.

The rapid development of Web technology has resulted in an increasing number of hotel customers sharing their opinionson the hotel services. Effective visual analysis of online customer opinions is needed, as it has a significant impact on buildinga successful business. In this paper, we present OpinionSeer, an interactive visualization system that could visually analyze alarge collection of online hotel customer reviews. The system is built on a new visualization-centric opinion mining technique thatconsiders uncertainty for faithfully modeling and analyzing customer opinions. A new visual representation is developed to conveycustomer opinions by augmenting well-established scatterplots and radial visualization. To provide multiple-level exploration, weintroduce subjective logic to handle and organize subjective opinions with degrees of uncertainty. Several case studies illustrate theeffectiveness 
and usefulness of OpinionSeer on analyzing relationships among multiple data dimensions and comparing opinionsof different groups. Aside from data on hotel customer feedback, OpinionSeer could also be applied to visually analyze customeropinions on other products or services.

Wyeld, T. 2009, "The Correlation between the Ability to Read and Manually Reproduce a 3d Image: Some Implications for 3d Information Visualisation." In 13th International Conference on Information Visualization, pp. 496-501.

Most of us can recognize common 3D objects depicted in drawings, photographs and computer graphics. But, few of us are able to manually reproduce them in a convincing manner. This paper discusses a psychology experiment that investigates the variability in individual drawing ability and the ability to read 3D images. Currently, Shepard and Metzlerpsilas Mental Rotation Test is the most popular test for spatial ability. This paper discusses the need to further investigate the correlation of 3D drawing ability and recognition and its potential effect on the legibility of the 3D information visualization application. This paper reports ongoing research in this field.

Xiang, Y, et al. 2005, "Visualizing Criminal Relationships: Comparison of a Hyperbolic Tree and a Hierarchical List." Decision Support Systems 41(1):69-83.

In crime analysis, law enforcement officials have to process a large amount of criminal data and figure out their relationships. It is important to identify different associations among criminal entities. In this paper, we propose the use of a hyperbolic tree view and a hierarchical list view to visualize criminal relationships. A prototype system called COPLINK Criminal Relationship Visualizer was developed. An experiment was conducted to test the effectiveness and the efficiency of the two views. The results show that the hyperbolic tree view is more effective for an "identify" task and more efficient for an "associate" task. The participants generally thought it was easier to use the hierarchical list, with which they were more familiar. When asked about the usefulness of the two views, about half of the participants thought that the hyperbolic tree was more useful, while the other half thought otherwise. Our results indicate that both views can help in criminal relationship visualization. While the hyperbolic tree view performs better in some tasks, the users' experiences and preferences will impact the decision on choosing the visualization technique.

Xiao, L, J Gerth, and P Hanrahan. 2006, "Enhancing Visual Analysis of Network Traffic Using a Knowledge Representation." In IEEE Symposium On Visual Analytics Science \& Technology, pp. 107-14.

Xiao, N, DA Bennett, and MP Armstrong. 2007, "Interactive Evolutionary Approaches to Multiobjective Spatial Decision Making: A Synthetic Review." Computers, Environment and Urban Systems 31(3):23252.

This paper reviews recent developments in evolutionary algorithms and visualization in the context of multiobjective spatial decision making. A synthetic perspective is employed to bridge these two areas and to create a unified conceptual framework that can be used to address a broad range of multiobjective spatial decision problems. In this framework, evolutionary algorithms are employed to generate optimal, or near-optimal, solutions to a problem being addressed. Alternatives created are then displayed in an interactive visual support system that can be used by decision makers to discover the competing nature of multiple objectives and to gain knowledge about the tradeoffs among alternatives. 
Xie, SH, et al. 2009, "Design and Evaluation of Extensions to Uml Sequence Diagrams for Modeling Multithreaded Interactions." Information Visualization 8(2):120-36.

Learning about concurrency and synchronization is difficult for novices. Our research seeks to support and improve the teaching and learning of concurrency concepts and to improve comprehension of the intricacies of multiple thread interactions. This paper describes a series of empirical studies in the first phase of our research. We began by conducting a comparative study to empirically evaluate the usability by novices of the existing variants of the UML sequence diagram notation in solving comprehension tasks involving multiple thread interactions. The results implied that a deliberately designed variant of this notation may provide better support for reasoning about concurrent behavior. We then investigated the factors that complicate learning, with the idea that the same complexities would also complicate comprehension tasks. In order to understand the practical difficulties novices encounter in learning about concurrency, we conducted an instructor interviewand an observational study. These investigations guided us in determining the desirable properties of a new notation. We then designed synchronizationadorned UML (saUML) sequence diagrams, which extend UML sequence diagrams with those properties. Finally, we performed four empirical studies to evaluate the usability and efficacy of saUML. Through these empirical studies, we were able to validate the benefits of saUML in enhancing novices' understanding of programs with different levels of synchronization complexity. Information Visualization (2009) 8, 120-136. doi: 10.1057/ivs.2009.6

$\mathrm{Xu}, \mathrm{K}$, et al. 2010, "Visual Analytics of Pandemic Spread." In IEEE Symposium on Visual Analytics Science \& Technology, pp. 2-3.

Xu, L, T-Y Lee, and H-W Shen. 2010, "An Information-Theoretic Framework for Flow Visualization." IEEE Transactions on Visualization and Computer Graphics 16(6):1216-24.

The process of visualization can be seen as a visual communication channel where the input to the channel is the raw data, and the output is the result of a visualization algorithm. From this point of view, we can evaluate the effectiveness of visualization by measuring how much information in the original data is being communicated through the visual communication channel. In this paper, we present an information-theoretic framework for flow visualization with a special focus on streamline generation. In our framework, a vector field is modeled as a distribution of directions from which Shannon's entropy is used to measure the information content in the field. The effectiveness of the streamlines displayed in visualization can be measured by first constructing a new distribution of vectors derived from the existing streamlines, and then comparing this distribution with that of the original data set using the conditional entropy. The conditional entropy between these two distributions indicates how much information in the original data remains hidden after the selected streamlines are displayed. The quality of the visualization can be improved by progressively introducing new streamlines until the conditional entropy converges to a small value. We describe the key components of our framework with detailed analysis, and show that the framework can effectively visualize 2D and 3D flow data.

$\mathrm{Xu}, \mathrm{S}, \mathrm{X}$ Chen, and D Liu. 2009, " Classifying Software Visualization Tools Using the Bloom's Taxonomy of Cognitive Domain." In 22nd Canadian Conference on Electrical and COmputer Engineering, pp. 13-18.

There are a lot of software visualization tools existing for various purposes. Therefore, how to choose the right visualization tool for a specific task becomes an important issue. Although there are a few 
taxonomies for classifying visualization tools, each has its own defects. This paper proposes a new classification schema based on the widely used Bloom's cognitive taxonomy. We summarize a set of questions for each level of the Bloom taxonomy using the well defined verbs, to identify if the visualization tool belongs to the individual cognitive level, which in turn can determine the purposes of the tool. We also conduct case studies on four tools to evaluate this new approach. The result demonstrates that the new classification schema can provide good guidance for people to choose a useful visualization tool.

Xueling, W, and R Fu. 2010, "Mechanism and Methods of Outdoor Ar Spatial Information Visualization Representation." In Second International Conference on Computer Modeling and Simulation, pp. 272-76.

Almost all human outdoor activities is closely related to geographical position, thus the development of GIS is more rapidly. From the perspective of spatial information visualization, GIS is a digital description of objective geographical world. As abstract models of geographical environment, analog maps and 2D electronic maps have respective limitations in vivid representing spatial information. Simultaneously, the vast majority of HCI is limited to plane display and is difficult to users to integrate into it. Relation between outdoor AR (augmented reality) technology and spatial information visualization representation embodies in the common basic cognition and mobility. Emergence of outdoor AR and mobile GIS provides the combination of outdoor AR technology and spatial information visualization representation with powerful theoretical foundation and technical channel, which offers new ideas and new interface to spatial information visualization representation and is committed to enhance and expand user's spatial cognition by overlaying virtual geographic objects on real scene. The paper firstly analyzes its research status, feasibility of the combination, basic characteristics and structure. Secondly, it discusses existing technologic prototypes from application-driven perspective and puts forward a research framework and discusses four key technologies: display device, tracking and positioning, virtual-real combination and HCI. Furthermore, it propose core algorithms including a hybrid registration method based on GPS and 3D electronic compass, registration methods using affine representation and geographical objects modeling etc. Lastly, it expatiate the applications and significance.

Yang, CC, H Chen, and K Hong. 2003, "Visualization of Large Category Map for Internet Browsing." Decision Support Systems 35(1):89-102.

Information overload is a critical problem in World Wide Web. Category map developed based on Kohonen's self-organizing map (SOM) has been proven to be a promising browsing tool for the Web. The SOM algorithm automatically categorizes a large Internet information space into manageable sub-spaces. It compresses and transforms a complex information space into a two-dimensional graphical representation. Such graphical representation provides a user-friendly interface for users to explore the automatically generated mental model. However, as the amount of information increases, it is expected to increase the size of the category map accordingly in order to accommodate the important concepts in the information space. It results in increasing of visual load of the category map. Large pool of information is packed closely together on a limited size of displaying window, where local details are difficult to be clearly seen. In this paper, we propose the fisheye views and fractal views to support the visualization of category map. Fisheye views are developed based on the distortion approach while fractal views are developed based on the information reduction approach. The purpose of fisheye views are to enlarge the regions of interest and diminish the regions that are further away while maintaining the global structure. On the other hand, fractal views are an approximation mechanism to abstract complex objects and control the amount of information to be displayed. We have developed a prototype system and conducted a user 
evaluation to investigate the performance of fisheye views and fractal views. The results show that both fisheye views and fractal views significantly increase the effectiveness of visualizing category map. In addition, fractal views are significantly better than fisheye views but the combination of fractal views and fisheye views do not increase the performance compared to each individual technique.

Yang, CC, and A Chung. 2004, "Intelligent Infomediary for Web Financial Information." Decision Support Systems 38(1):65-80.

The World Wide Web is the most popular information dissemination channel in the world. Such information space grows by an estimation of at least $10 \%$ every month. Many newspapers take the advantage to expand their services by providing real time electronic versions of news information on the Web in contrast to the traditional "ink-on-paper" newspapers. The timely financial news is very important for decision-making of investors. However, the tremendous amount of information releasing simultaneously creates the problem of information overloading, which reduces the decision-making capabilities significantly. As most of the investors are not experienced users of information retrieval systems, they spend extensive amount of time to identify the relevant information. Software agents act as intermediaries between the users and the information providers to notify users of recently published relevant information. In this paper, we present an intelligent agent that monitors the posting of the Web information providers and utilizes user profiles and user feedback to learn user preference and search for the Chinese Web financial information on behalf of users.

Yang, CC, and M Sageman. 2009, "Analysis of Terrorist Social Networks with Fractal Views." Journal of Information Science 35(3):299-320.

Social network visualization has drawn significant attention over recent years. It creates images of social networks that provide investigators with new insights about network structures and helps them to communicate those insights to others. Visualization facilitates the social network analysis. It supports the investigators to discover patterns of interactions among the social actors including detecting subgroups, identifying central actors and their roles, and discovering patterns of interactions among social actors. However, visualizing a large heterogeneous social network has several challenges. The large size of networks, complex relations among social actors and limited number of available pixels on a screen make it difficult to present important information clearly to investigators and hence reduce the capability of investigators to explore the networks. In this work, we propose the fractal views to construct a visual abstraction of a large and complex social network with users selected social actors as focuses. The fractal views are focus and context visualization techniques using an information reduction approach. It controls the amount of information displayed by focusing on the syntactic structure of information. It is useful in discovering knowledge from terrorist social networks for combating the war on terrorism. Such application has formed an important research topic, known as intelligence and security informatics, in recent years due to the terrorist attacks of September 112001 (9/11) and several other terror attacks that have occurred within the last decade. We present several case studies to demonstrate the capability of the proposed technique on analyzing the Global Salafi Jihad terrorist social network. It extracts the hidden relationships among terrorists through user interactions. In addition, we have conducted a user evaluation to assess the efficiency and effectiveness of fractal views. It shows that fractal views outperform fisheye views and zoom-in windows to support users in visualizing and analyzing terrorist social networks. 
Yang, J, et al. 2006, "Semantic Image Browser : Bridging Information Visualization with Automated Intelligent Image Analysis." In IEEE Symposium on Visual Analytics Science \& Technology.

Yang, J, et al. 2007, "Value and Relation Display: Interactive Visual Exploration of Large Data Sets with Hundreds of Dimensions." IEEE Transactions on Visualization and Computer Graphics 13(3):494-507.

Few existing visualization systems can handle large data sets with hundreds of dimensions, since high-dimensional data sets cause clutter on the display and large response time in interactive exploration. In this paper, we present a significantly improved multidimensional visualization approach named Value and Relation (VaR) display that allows users to effectively and efficiently explore large data sets with several hundred dimensions. In the VaR display, data values and dimension relationships are explicitly visualized in the same display by using dimension glyphs to explicitly represent values in dimensions and glyph layout to explicitly convey dimension relationships. In particular, pixel-oriented techniques and density-based scatterplots are used to create dimension glyphs to convey values. Multidimensional scaling, Jigsaw map hierarchy visualization techniques, and an animation metaphor named Rainfall are used to convey relationships among dimensions. A rich set of interaction tools has been provided to allow users to interactively detect patterns of interest in the VaR display. A prototype of the VaR display has been fully implemented. The case studies presented in this paper show how the prototype supports interactive exploration of data sets of several hundred dimensions. A user study evaluating the prototype is also reported in this paper.

Yang, J, MO Ward, and EA Rundensteiner. 2003, "Interactive Hierarchical Displays: A General Framework for Visualization and Exploration of Large Multivariate Data Sets." Computers \& Graphics$U k 27(2): 265-83$.

Numerous multivariate visualization techniques and systems have been developed in the past three decades to visually analyze and explore multivariate data being produced daily in application areas ranging from stock markets to the earth and space sciences. However, traditional multivariate visualization techniques typically do not scale well to large multivariate data sets, with the latter becoming more and more common nowadays. This paper proposes a general framework for interactive hierarchical displays (IHDs) to tackle the clutter problem faced by traditional multivariate visualization techniques when analyzing large data sets. The underlying principle of this framework is to develop a multi-resolution view of the data via hierarchical clustering, and to use hierarchical variations of traditional multivariate visualization techniques to convey aggregation information about the resulting clusters. Users can then explore their desired focus region at different levels of detail, using our suite of navigation and filtering tools. We describe this IHD framework and its full implementation on four traditional multivariate visualization techniques, namely, parallel coordinates (Inselberg and Dimsdale, Proceedings of Visualization (1990) 361; Wegman, J. Amer. Statist. Assoc. 411(85) (1990) 664), star glyphs (Siegel et al., Surgery 72 (1972) 126), scatterplot matrices (Cleveland and McGill, Dynamics Graphics for Statistics (1988)), and dimensional stacking (LeBlanc et al., Proceedings of Visualization 90 (1995) 271), as implemented in the XmdvTool system (Ward, Proceedings of Visualization 94 (1994) 326; Martin and Ward, Proceedings of Visualization 95 (1995) 271, Fua et al., Proceedings of Visualization 99 (1999) 43; Proceedings of Information Visualization 99 (1999) 58). We also describe an empirical evaluation that verified the effectiveness of the interactive hierarchical displays. (C) 2003 Elsevier Science Ltd. All rights reserved. 
Yang, JC, and SY Chen. 2010, "Effects of Gender Differences and Spatial Abilities within a Digital Pentominoes Game." Computers \& Education 55(3):1220-33.

Spatial ability is a critical skill in geometric learning. Several studies investigate how to use digital games to improve spatial abilities. However, not every learner favors this kind of support. To this end, there is a need to examine how human factors affect learners' reactions to the use of a digital game to support geometric learning. In this vein, this paper addresses this issue by developing a digital pentominoes game and examining the effects of two essential human factors, especially gender differences and spatial abilities, on students' performance. The results demonstrate that students' spatial abilities were significantly improved after they took the digital pentominoes game. The results also demonstrate that the digital game can reasonably reduce the differences between boys and girls. Moreover, the major gender differences lie within mental rotation among the three types of spatial ability and also mainly exist in the low spatial ability group. Finally, the findings are applied to develop a framework that can be used to enhance the understanding of gender differences and spatial abilities within the digital pentominoes game.

Yang, Y-c, and Y-y Jian. 2009, "Preliminary Studyon Electronic Map Based on Surface of Ellipsoid." Procedia Earth and Planetary Science 1(1):1158-64.

Map carrier not only decides the storage mode of map information, it also has important influence on mathematic foundation and expression mode of geographical information. Based on analysis of the limitations lying in the flat map, this paper introduces the concept of the digital map and electronic map on ellipsoid surface, elaborates on the superiority. The paper investigates the fundamental method of drawing, display and analysis of the digital map on ellipsoid surface farther. Compared with conventional map mode, the digital map on ellipsoid surface is a new concept, it fits the spatial data visual mode based on geographical features superlatively well and suits the people's cognitive and thought habit who regards earth surface as a ellipsoid; it carries out a reform of expression mode of the existing digital map, and will explore the analysis function and application scope of electronic map and digital map effectively.

Yannier, N, et al. 2008, "Using Haptics to Convey Cause-and-Effect Relations in Climate Visualization." Haptics, IEEE Transactions on 1(2):130-41.

We investigate the potential role of haptics in climate visualization. In existing approaches to climate visualization, different dimensions of climate data such as temperature, humidity, wind, precipitation, and cloud water are typically represented using different visual markers and dimensions such as color, size, intensity, and orientation. Since the number of dimensions in climate data is large and climate data needs to be represented in connection with the topography, purely visual representations overwhelm users. Rather than overloading the visual channel, we investigate an alternative approach in which some of the climate information is displayed through the haptic channel in order to alleviate the perceptual and cognitive load of the user. In this approach, haptic feedback is further used to provide guidance while exploring climate data in order to enable natural and intuitive learning of cause and effect relationships between climate variables. Our experiments with 33 human subjects show that haptic feedback significantly improves the understanding of climate data and the cause and effect relations between climate variables as well as the interpretation of the variations in climate due to changes in terrain. 
Yehezkel, C. 2002, "A Taxonomy of Computer Architecture Visualizations." ACM SIGCSE Bulletin 34(3):101-05.

Yen, BPC, and KYM Ng. 2003, "Development and Evaluation of Dynamic Virtual Object Catalogs." Information \& Management 40(4):337-49.

One of the most interesting commercial opportunities for Internet business is the ability to deploy catalogs on the Web. Existing electronic catalogs are able to provide comprehensive information, efficient data update, and economical development; however, the huge data volume due to high variety and fast turnover of customized products often causes their construction and maintenance to be unrealistic. This paper presents a Web-based dynamic virtual object catalog of customized products for Internet commerce and focuses on the comparison of system performance for various catalogs in different perspectives. The results show that the proposed catalog has tremendous advantages in development time, required cost, and storage space over existing catalogs under most conditions. (C) 2002 Elsevier Science B.V. All rights reserved.

Yener, Y, and S Özkadif. 2010, "The Suggested Metaphors Regarding on the Concept of "Cell" by Teacher Candidates of Biology, Science and Primary." Procedia - Social and Behavioral Sciences 2(2):1107-13.

The main purpose of this study is to define the perceptions of biology, science and primary teaching candidates studying at Selcuk University on the concept of "cell" using metaphors. The data of the study was obtained through the answers given to the complete-questions such as "cell is like...because,..." and "cell is similar to..., because..." The suggested metaphors were gathered under 6 groups: "administrative", "controller", "building stone", "information store", "cooperative", "connective". As a result, it was seen that almost two third of the participants defined cell as "building stone" and "cooperative" while the rest, one third, defined it as "administrative","controller", "information store" and "connective". The results of this study indicate important data on the fact that metaphors can be used as effective tools to reveal the students' personal perceptions on the concept of cell.

Yi, JS. 2010, "Implications of Individual Differences on Evaluating Information Visualization Techniques." In BELIV'10: Beyond time and errors: novel evaluation methods for Information Visualization, April 10-11, 2010.

As HCI researchers extensively investigated the individual differences after the seminal work of Egan [6], the individual differences should gain more attention from researchers in information visualization (InfoVis). Though more evaluation studies have taken individual differences into consideration, it is unclear that corresponding measures are comprehensively and sufficiently understood. Thus, in this posi- tion paper, the author attempt to compile evidence showing that some individual differences may avoid our prior investigations and to suggest other potential measures to increase our coverage. The author also hoped that this position paper works as a call for creating a standardized measurement tool for individual differences. 
Yi, JS, et al. 2007, "Toward a Deeper Understanding of the Role of Interaction in Information Visualization." Visualization and Computer Graphics, IEEE Transactions on 13(6):1224-31.

Even though interaction is an important part of information visualization (Infovis), it has garnered a relatively low level of attention from the Infovis community. A few frameworks and taxonomies of Infovis interaction techniques exist, but they typically focus on low-level operations and do not address the variety of benefits interaction provides. After conducting an extensive review of Infovis systems and their interactive capabilities, we propose seven general categories of interaction techniques widely used in Infovis: 1) Select, 2) Explore, 3) Reconfigure, 4) Encode, 5) Abstract/Elaborate, 6) Filter, and 7) Connect. These categories are organized around a user's intent while interacting with a system rather than the lowlevel interaction techniques provided by a system. The categories can act as a framework to help discuss and evaluate interaction techniques and hopefully lay an initial foundation toward a deeper understanding and a science of interaction.

Yi, JS, et al. 2008, "Understanding and Characterizing Insights: How Do People Gain Insights Using Information Visualization?" In Conference on BEyond time and errors: novel evaLuation methods for Information Visualization (BELIV'08), pp. 1-6.

Even though "providing insight" has been considered one of the main purposes of information visualization (InfoVis), we feel that insight is still a not-well-understood concept in this context. Inspired by research in sensemaking, we realized the importance of the procedural aspects in understanding insight. Thus, rather than asking "What is insight?" we instead focus on "How do people gain insights?" In an effort to better understand and characterize insight, we reviewed previous literature in InfoVis, seeking other researchers' comments and views on this concept. We found that: 1) Insights are often regarded as end results of using InfoVis and the procedures to gain insight have been largely veiled; 2) Four largely distinctive processes of gaining insight (Provide Overview, Adjust, Detect Pattern, and Match Mental Model) have been discussed in the InfoVis literature; and 3) These different processes provide some hints to understand the procedures in which insight can be gained from InfoVis. We hope that our findings help researchers and practitioners evaluate InfoVis systems and technologies in a more insight-oriented way.

Yin, M, et al. 1998, "Computer-Generated Time Lines for Visualizing and Editing Epidemiological and Biomedical Data." Computer Methods and Programs in Biomedicine 56(1):23-29.

Most biomedical data have a temporal dimension. Time-line displays spatialize this dimension and help the viewer comprehend large sets of complex data. If we add ways for users to selectively expand the details of data visible on a time line, even more information can be organized and accessed. Design issues for this kind of display include: how to display time scales that are often wider than the physical display space; how to display events with brief duration; how to display data for two or more events that overlap in time; how to manage the display of data details; how to allow database editing from a time line; and how to facilitate time-based analytical techniques. We describe a time-line display system that addresses each of these issues, and show how it can be used to organize data for an epidemiological study of parental radiation exposure and childhood leukemia. We also suggest further refinements of the time line technique for other biomedical applications. 
Yoon, B. 2008, "On the Development of a Technology Intelligence Tool for Identifying Technology Opportunity." Expert Systems with Applications 35(1-2):124-35.

Technology intelligence tools have come to be regarded as vital components in planning for technology development and formulating technology strategies. However, most such tools currently focus on providing graphical frameworks and databases to support the process of technology analysis. Techpioneer, the proposed tool in this paper, aims to offer decisive information in order to identify technology opportunities. To this end, the system uses textual information from technological document databases and applies morphology analysis to derive promising alternatives and conjoint analysis to evaluate their priority. In addition, the method used in developing a technology dictionary is presented, employing clustering and network analysis. This system also has the ability to communicate with experts in order to estimate the value of existing patents, which is inevitable for the priority-setting of alternatives, construct a morphological matrix and so on. This paper presents the system architecture and functions of this tool and moreover, illustrates the prototype implementation and case study of the same.

Yost, B, and C North. 2006, "The Perceptual Scalability of Visualization." Visualization and Computer Graphics, IEEE Transactions on 12(5):837-44.

Larger, higher resolution displays can be used to increase the scalability of information visualizations. But just how much can scalability increase using larger displays before hitting human perceptual or cognitive limits? Are the same visualization techniques that are good on a single monitor also the techniques that are best when they are scaled up using large, high-resolution displays? To answer these questions we performed a controlled experiment on user performance time, accuracy, and subjective workload when scaling up data quantity with different space-time-attribute visualizations using a large, tiled display. Twelve college students used small multiples, embedded bar matrices, and embedded timeseries graphs either on a 2 megapixel (Mp) display or with data scaled up using a $32 \mathrm{Mp}$ tiled display. Participants performed various overview and detail tasks on geospatially-referenced multidimensional time-series data. Results showed that current designs are perceptually scalable because they result in a decrease in task completion time when normalized per number of data attributes along with no decrease in accuracy. It appears that, for the visualizations selected for this study, the relative comparison between designs is generally consistent between display sizes. However, results also suggest that encoding is more important on a smaller display while spatial grouping is more important on a larger display. Some suggestions for designers are provided based on our experience designing visualizations for large displays.

You, Q, SF Fang, and P Ebright. 2007, "Visualizing Unstructured Text Sequences Using Iterative Visual Clustering." in Advances in Visual Information Systems, eds. GP Qiu, et al., Vol 4781, pp. 275-84.

This paper presents a keyword-based information visualization technique for unstructured text sequences. The text sequence data comes from nursing narratives records, which are mostly text fragments with incomplete and unreliable grammatical structures. Proper visualization of such text sequences can reveal patterns and trend information rooted in the text records, and has significant applications in many fields such as medical informatics and text mining. In this paper, an Iterative Visual Clustering (IVC) technique is developed to facilitate multi-scale visualization, and at the same time provide abstraction and knowledge discovery functionalities at the visualization level. Interactive visualization and user feedbacks are used to iteratively group keywords to form higher level concepts and keyword clusters, which are then feedback to the visualization process for evaluation and pattern 
discovery. Distribution curves of keywords and their clusters are visualized at various scales under Gaussian smoothing to search for meaningful patterns and concepts.

Yu, B, and G Cai. 2007, "A Query-Aware Document Ranking Method for Geographic Information Retrieval." In 4th ACM workshop on Geographical information retrieval - GIR '07, pp. 49-49.

Yu, C, et al. 2010, "A Data-Driven Paradigm to Understand Multimodal Communication in HumanHuman and Human-Robot Interaction." In 9th International Symposium on Intelligent Data Analysis, pp. 232-44.

Data-driven knowledge discovery is becoming a new trend in various scientific fields. In light of this, the goal of the present paper is to introduce a novel framework to study one interesting topic in cognitive and behavioral studies - multimodal communication between human-human and human-robot interaction. We present an overall solution from data capture, through data coding and validation, to data analysis and visualization. In data collection, we have developed a multimodal sensing system to gather fine-grained video, audio and human body movement data. In data analysis, we propose a hybrid solution based on visual data mining and information-theoretic measures. We suggest that this data-driven paradigm will lead not only to breakthroughs in understanding multimodal communication, but will also serve as a successful case study to demonstrate the promise of data-intensive discovery which can be applied in various research topics in cognitive and behavioral studies.

Yu, C, et al. 2009, "Visual Data Mining of Multimedia Data for Social and Behavioral Studies." Information Visualization 8(1):56-70.

With advances in computing techniques, a large amount of high-resolution high-quality multimedia data (video and audio, and so on) has been collected in research laboratories in various scientific disciplines, particularly in cognitive and behavioral studies. How to automatically and effectively discover new knowledge from rich multimedia data poses a compelling challenge because most state-ofthe-art data mining techniques can only search and extract pre-defined patterns or knowledge from complex heterogeneous data. In light of this challenge, we propose a hybrid approach that allows scientists to use data mining as a first pass, and then forms a closed loop of visual analysis of current results followed by more data mining work inspired by visualization, the results of which can be in turn visualized and lead to the next round of visual exploration and analysis. In this way, new insights and hypotheses gleaned from the raw data and the current level of analysis can contribute to further analysis. As a first step toward this goal, we implement a visualization system with three critical components: (1) a smooth interface between visualization and data mining; (2) a flexible tool to explore and query temporal data derived from raw multimedia data; and (3) a seamless interface between raw multimedia data and derived data. We have developed various ways to visualize both temporal correlations and statistics of multiple derived variables as well as conditional and high-order statistics. Our visualization tool allows users to explore, compare and analyze multi-stream derived variables and simultaneously switch to access raw multimedia data. Information Visualization (2009) 8, 56-70. doi: 10.1057/ivs.2008.32; published online 12 February 2009 
Yu, H, C Wang, and JH Chen. 2010, "Hierarchical Streamline Bundles for Visualizing 2d Flow Fields." In IEEE Conference on Information Visualization.

Yu, L, et al. 2010, "Fi3d: Direct-Touch Interaction for the Exploration of 3d Scientific Visualization Spaces." IEEE Transactions on Visualization and Computer Graphics 16(6):1613-22.

We present the design and evaluation of FI3D, a direct-touch data exploration technique for 3D visualization spaces. The exploration of three-dimensional data is core to many tasks and domains involving scientific visualizations. Thus, effective data navigation techniques are essential to enable comprehension, understanding, and analysis of the information space. While evidence exists that touch can provide higher-bandwidth input, somesthetic information that is valuable when interacting with virtual worlds, and awareness when working in collaboration, scientific data exploration in 3D poses unique challenges to the development of effective data manipulations. We present a technique that provides touch interaction with 3D scientific data spaces in 7 DOF. This interaction does not require the presence of dedicated objects to constrain the mapping, a design decision important for many scientific datasets such as particle simulations in astronomy or physics. We report on an evaluation that compares the technique to conventional mouse-based interaction. Our results show that touch interaction is competitive in interaction speed for translation and integrated interaction, is easy to learn and use, and is preferred for exploration and wayfinding tasks. To further explore the applicability of our basic technique for other types of scientific visualizations we present a second case study, adjusting the interaction to the illustrative visualization of fiber tracts of the brain and the manipulation of cutting planes in this context.

Yuan, X, et al. 2010, "Scalable Multi-Variate Analytics of Seismic and Satellite-Based Observational Data." IEEE Transactions on Visualization and Computer Graphics 16(6):1413-20.

Over the past few years, large human populations around the world have been affected by an increase in significant seismic activities. For both conducting basic scientific research and for setting critical government policies, it is crucial to be able to explore and understand seismic and geographical information obtained through all scientific instruments. In this work, we present a visual analytics system that enables explorative visualization of seismic data together with satellite-based observational data, and introduce a suite of visual analytical tools. Seismic and satellite data are integrated temporally and spatially. Users can select temporal ; and spatial ranges to zoom in on specific seismic events, as well as to inspect changes both during and after the events. Tools for designing high dimensional transfer functions have been developed to enable efficient and intuitive comprehension of the multi-modal data. Spreadsheet style comparisons are used for data drill-down as well as presentation. Comparisons between distinct seismic events are also provided for characterizing event-wise differences. Our system has been designed for scalability in terms of data size, complexity (i.e., number of modalities), and varying form factors of display environments.

Yue, J, et al. 2009, "A Blackboard-Based Approach Towards Predictive Analytics." In AAAI Spring Symposium.

Zachow, S, et al. 2009, "Visual Exploration of Nasal Airflow." Visualization and Computer Graphics, IEEE Transactions on 15(6):1407-14.

Rhinologists are often faced with the challenge of assessing nasal breathing from a functional point of view to derive effective therapeutic interventions. While the complex nasal anatomy can be revealed by 
visual inspection and medical imaging, only vague information is available regarding the nasal airflow itself: Rhinomanometry delivers rather unspecific integral information on the pressure gradient as well as on total flow and nasal flow resistance. In this article we demonstrate how the understanding of physiological nasal breathing can be improved by simulating and visually analyzing nasal airflow, based on an anatomically correct model of the upper human respiratory tract. In particular we demonstrate how various information visualization (InfoVis) techniques, such as a highly scalable implementation of parallel coordinates, time series visualizations, as well as unstructured grid multi-volume rendering, all integrated within a multiple linked views framework, can be utilized to gain a deeper understanding of nasal breathing. Evaluation is accomplished by visual exploration of spatio-temporal airflow characteristics that include not only information on flow features but also on accompanying quantities such as temperature and humidity. To our knowledge, this is the first in-depth visual exploration of the physiological function of the nose over several simulated breathing cycles under consideration of a complete model of the nasal airways, realistic boundary conditions, and all physically relevant timevarying quantities.

Zaientz, JD, SD Wood, and RL Hawkins. 2005, "Improving Information Assimilation by Modeling Warfighter Context." in Enabling Technologies for Simulation Science Ix, eds. DA Trevisani and AF Sisti, Vol 5805, pp. 153-64. SPIE.

In order to wage successful campaigns, the next generation of intelligence analysts and battle commanders will need to assimilate an enormous amount of information that will come from a wide range of heterogeneous data sources. Complicating this problem further is the fact that warfighters need to be able to manage information in an environment of rapidly changing events and priorities. The consequence of not addressing this problem, or not addressing it as effectively as hostile forces do, is a potential loss of assets, personnel, or tactical advantage. To design effective information displays there needs to be an extensible framework that models the warfighters context including characteristics of the information sources being displayed, the current Intelligence Surveillance Reconnaissance (ISR) picture or Common Operating Picture (COP), the warfighters current state and task, and the state of the information display. BINAH (Battlespace Information and Notification through Adaptive Heuristics) uses an agent-based modeling approach coupled with research into temporal and spatial reasoning, novel display management techniques, and development of a formal high-level language for describing model-based information configuration. The result is an information configuration pipeline designed to provide perceptual and cognitive analysis support to Air Force analysts engaged in Time-Critical Targeting target nomination. It has been integrated with the Air Force Research Laboratory's (AFRL) XML-based Joint Battlespace Infosphere (JBI) combat information management system and combines JBI delivered sensor data with a local user model and display strategies to configure a geospatial information display. The BINAH framework will provide a firm grounding for developing new (CISR)-I-4 displays that maximize the ability of warfighters to assimilate the information presented.

Zanbaka, CA, et al. 2005, "Comparison of Path Visualizations and Cognitive Measures Relative to Travel Technique in a Virtual Environment." Visualization and Computer Graphics, IEEE Transactions on 11(6):694-705.

We describe a between-subjects experiment that compared four different methods of travel and their effect on cognition and paths taken in an immersive virtual environment (IVE). Participants answered a set of questions based on Crook's condensation of Bloom's taxonomy that assessed their cognition of the IVE with respect to knowledge, understanding and application, and higher mental processes. Participants 
also drew a sketch map of the IVE and the objects within it. The users' sense of presence was measured using the Steed-Usoh-Slater presence questionnaire. The participants' position and head orientation were automatically logged during their exposure to the virtual environment. These logs were later used to create visualizations of the paths taken. Path analysis, such as exploring the overlaid path visualizations and dwell data information, revealed further differences among the travel techniques. Our results suggest that, for applications where problem solving and evaluation of information is important or where opportunity to train is minimal, then having a large tracked space so that the participant can walk around the virtual environment provides benefits over common virtual travel techniques.

Zavrel, J. 1996, "Neural Navigation Interfaces for Information Retrieval: Are They More Than an Appealing Idea?" Artificial Intelligence Review 10(5-6):477-504.

Neural networks have recently been proposed for the construction of navigation interfaces for Information Retrieval systems. In this paper, we give an overview of some current research in this area. Most of the cited approaches use (variants) of the well-known Kohonen network. The Kohonen network implements a topology-preserving dimensionality-reducing mapping, which can be applied for information visualization. We identify a number of problems in the application of Kohonen networks for Information Retrieval, most notably scalability, reliability and retrieval effectiveness. To solve these problems we propose to use the Growing Cell Structures network, a variant of the Kohonen network which shows a more flexible adaptation to the domain structure. This network was tested on two standard test-collections, using a combined recall and precision measure, and compared to traditional IR methods such as the Vector Space Model and various clustering algorithms. The network performs at a competitive level of effectiveness, and is suitable for visualization purposes. However, the incremental training procedures for the networks result in a reliability problem, and the approach is computationally intensive. Also, the utility of the resulting maps for navigation will need further improvement.

Zeckzer, D. 2010, "Visualizing Software Entities Using a Matrix Layout." In 5th international symposium on Software visualization pp. 207-08.

Zeiller, M. 2005, "A Case Study Based Approach to Knowledge Visualization." In Ninth International Conference on Information Visualisation, pp. 377-82.

Case studies are proposed as a research method on knowledge visualization that can deal with the multidisciplinarity, the large variety of research targets and the complex correlations of this type of information visualization utilized for supporting tasks of knowledge management. A suitable case structure is presented that documents the analyzed cases and allows for a comparative analysis of multiple cases. To be able to systematically evaluate and compare the applied visualization techniques a set of evaluation criteria is introduced.

Zellweger, P. 2005, "A Database Taxonomy Based on Data-Driven Knowledge Modeling." In International Conference on Integration of Knowledge Intensive Multi-Agent Systems, 2005., pp. 469-74.

Zenebe, A, L Zhou, and AF Norcio. 2010, "User Preferences Discovery Using Fuzzy Models." Fuzzy Sets and Systems 161(23):3044-63.

User preferences discovery aims to learn the patterns of user preferences for various services or items such as movies. Preferences discovery is essential to the development of intelligent personalization 
applications. Based on decision and utility theories, traditional approaches to preferences discovery explicitly query users about the behavior of value function, or utility of every outcome with respect to each decision criterion. Consequently, these approaches are generally error-prone and labor intensive. Although implicit elicitation approaches have been proposed to address the above limitations, extent approaches largely ignore multi-valued nature of item features and uncertainty associated with item features and user preferences. To address uncertainty due to vagueness and imprecision, this research proposed a general framework for preferences discovery based on fuzzy set theories. In addition, new fuzzy models were created for preferences discovery and representation. Further, an algorithm was developed to predict user preferences with uncertainty, and visualization of item features, user feedback, and the discovered preferences helped improve the interpretation of the discovered knowledge. The results of the simulation evaluation using a benchmark movie dataset revealed that the proposed preference discovery method: (1) doubled the accuracy of preference discovery as compared to random prediction; and (2) outperformed conventional techniques in making movie recommendation. These findings suggest that fuzzy models are effective for preferences patterns discovery, and personalized recommendation application.

Zeng, W, et al. 2010, "Supine and Prone Colon Registration Using Quasi-Conformal Mapping." IEEE Transactions on Visualization and Computer Graphics 16(6):1348-57.

In virtual colonoscopy, CT scans are typically acquired with the patient in both supine (facing up) and prone (facing down) positions. The registration of these two scans is desirable so that the user can clarify situations or confirm polyp findings at a location in one scan with the same location in the other, thereby improving polyp detection rates and reducing false positives. However, this supine-prone registration is challenging because of the substantial distortions in the colon shape due to the patient's change in position. We present an efficient algorithm and framework for performing this registration through the use of conformal geometry to guarantee that the registration is a diffeomorphism (a one-to-one and onto mapping). The taeniae coli and colon flexures are automatically extracted for each supine and prone surface, employing the colon geometry. The two colon surfaces are then divided into several segments using the flexures, and each segment is cut along a taenia coli and conformally flattened to the rectangular domain using holomorphic differentials. The mean curvature is color encoded as texture images, from which feature points are automatically detected using graph cut segmentation, mathematic morphological operations, and principal component analysis. Corresponding feature points are found between supine and prone and are used to adjust the conformal flattening to be quasi-conformal, such that the features become aligned. We present multiple methods of visualizing our results, including 2D flattened rendering, corresponding 3D endoluminal views, and rendering of distortion measurements. We demonstrate the efficiency and efficacy of our registration method by illustrating matched views on both the 2D flattened colon images and in the 3D volume rendered colon endoluminal view. We analytically evaluate the correctness of the results by measuring the distance between features on the registered colons.

Zeqian, S, and M Kwan-Liu. 2008, "Mobivis: A Visualization System for Exploring Mobile Data." In IEEE PacificVIS '08, pp. 175-82.

The widespread use of mobile devices brings opportunities to capture large-scale, continuous information about human behavior. Mobile data has tremendous value, leading to business opportunities, market strategies, security concerns, etc. Visual analytics systems that support interactive exploration and discovery are needed to extracting insight from the data. However, visual analysis of complex socialspatial-temporal mobile data presents several challenges. We have created MobiVis, a visual analytics 
tool, which incorporates the idea of presenting social and spatial information in one heterogeneous network. The system supports temporal and semantic filtering through an interactive time chart and ontology graph, respectively, such that data subsets of interest can be isolated for close-up investigation. "Behavior rings," a compact radial representation of individual and group behaviors, is introduced to allow easy comparison of behavior patterns. We demonstrate the capability of MobiVis with the results obtained from analyzing the MIT Reality Mining dataset.

Zhang, C, et al. 2007, "A New Virtual Dynamic Dentomaxillofacial System for Analyzing Mandibular Movement, Occlusal Contact, and Tmj Condition." in Digital Human Modeling, ed. VG Duffy, Vol 4561, pp. 747-56.

This paper describes a new virtual dynamic dentomaxillofacial system. Mechanical articulators have been used to reproduce mandibular movements and analyze occlusal contacts. With the help of VR and visualization technologies, virtual articulator systems can provide dynamic simulation and quantitative information visualization, enhance the functionality, and extend the system to additional application areas. We integrate mandibular movement simulation, occlusal analysis and TMJ analysis into our system, and design new algorithms to improve the results of analysis. This system is helpful to the education, the research, and the clinic in dentistry. An evaluation is conducted to prove the functionality and usability of the system.

Zhang, H, Y-F Li, and HBK Tan. 2010, "Measuring Design Complexity of Semantic Web Ontologies." Journal of Systems and Software 83(5):803-14.

Ontology languages such as OWL are being widely used as the Semantic Web movement gains momentum. With the proliferation of the Semantic Web, more and more large-scale ontologies are being developed in real-world applications to represent and integrate knowledge and data. There is an increasing need for measuring the complexity of these ontologies in order for people to better understand, maintain, reuse and integrate them. In this paper, inspired by the concept of software metrics, we propose a suite of ontology metrics, at both the ontology-level and class-level, to measure the design complexity of ontologies. The proposed metrics are analytically evaluated against Weyuker's criteria. We have also performed empirical analysis on public domain ontologies to show the characteristics and usefulness of the metrics. We point out possible applications of the proposed metrics to ontology quality control. We believe that the proposed metric suite is useful for managing ontology development projects.

Zhang, HC, M Zhao, and Publ. 2010, "E-Commerce Website Evaluation Studies Based on Information Visualization Technology." In International Conference on Engineering and Business Management, pp. 5540-43.

With the rapid development of the information techniques, internet and thousands of Web sites become the most significant channels of communication. Information technology has been introduced to commerce and trade field, evolving as the platform of information publicity and transaction. The emerge of Electronic Business brings us a convenient, efficient and saving business mode, realizes the possibility of trading and controlling the supply chain via visual business platform without traveling in the real world. It's a trend that the technique of Information Visualization will be applied in Electronic Business field as a popular and significant method of information management. Information Visualization illustrates huge amount of information and the complicate relation between them by employing graphics, images, and other visual forms. This paper discusses the using of Information Visualization techniques in 
Electronic Business field by using PersonalBrain software to analyze the popular Electronic Business platforms, and concentrates on the structure of the platform, the data reliability and the way of searches, etc.

Zhang, J, C Chen, and D Pellegrino. 2010, "Poster : A Taxonomy of Visual Representations and Analytic Tasks for Design of Text Visualization." In IEEE Conference on Information Visualization.

Zhang, J, et al. 2007, "Design and Development of Distributed Virtual Geographic Environment System Based on Web Services." Information Sciences 177(19):3968-80.

This paper aims to design and develop a Distributed Virtual Geographic Environment (DVGE) system. A DVGE system is an Internet-based virtual 2D and 3D environment that provides users with a shared space and a collaborative platform for publishing multidimensional geo-data, and for simulating and analyzing complex geo-phenomena. Users logging into the system from different clients can share distributed geo-information resources, including geo-data and geo-models, and can complete collaborative tasks. Web service technology provides effective solutions for constructing DVGE systems because of its ability to support multi-platform, multi-architecture, and multi-program-language interoperability on the Internet, but also because of its ability to share programs, data, and software. This paper analyzes the characteristics, relevant technologies, and specifications of web services, such as grid services, Open Geo-data Interoperability Specifications (OpenGIS), and Geography Markup Languages (GML). The architecture and working mechanisms of the DVGE system based on web services are then elaborated. To demonstrate DVGE systems based on web services, we examine a case study of water pollution in Yangzhou City, Jiangsu Province, China, using a prototype DVGE system that is developed with Jbuilder9.0 and Java3D 1.0 packages, and the Weblogic platform 8.1.

Zhang, J, et al. 2002, "Human-Centered Information Visualization." In International Workshop on Dynamic Visualization and Learning.

Zhang, L, et al. 2007, "Conservative Voxelization." The Visual Computer 23(9-11):783-92.

Zhang, N, et al. 2010, "Volumetric Modeling in Laser Bph Therapy Simulation." IEEE Transactions on Visualization and Computer Graphics 16(6):1405-12.

In this paper, we introduce a novel application of volume modeling techniques on laser Benign Prostatic Hyperplasia (BPH) therapy simulation. The core technique in our system is an algorithm for simulating the tissue vaporization process by laser heating. Different from classical volume CSG operations, our technique takes experimental data as the guidance to determine the vaporization amount so that only a specified amount of tissue is vaporized in each time. Our algorithm uses a predictorcorrector strategy. First, we apply the classical CSG algorithm on a tetrahedral grid based distance field to estimate the vaporized tissue amount. Then, a volume-correction phase is applied on the distance field. To improve the performance, we further propose optimization approaches for efficient implementation.

Zhang, P. 1998, "An Image Construction Method for Visualizing Managerial Data." Decision Support Systems 23(4):371-87.

High volume data with complicated relationships can render human decision-making a frustrating task. Computer-generated visualization is an approach that can assist decision-makers in gaining insight 
into the data so that eventually superior solutions can be developed. Current research in visualization has addressed how to deal with high volume data that have some inherent structures (such as hierarchy, network, or geographical relationships). Many management domains, however, have data that lack obvious structures to provide a base for computer-generated visualization. This paper reports a specially designed technique for visualizing such management data. Data objects involved in the decision-making tasks are assigned with geometry (called visual abstract) in Euclidean space. Then a set of image construction rules are applied to connect multiple visual abstracts into images that can be displayed on a computer screen. We use two business domains, manufacturing production planning and resource constrained project scheduling, to illustrate this visualization technique.

Zhang, S, and O Bodenreider. 2006, "Law and Order: Assessing and Enforcing Compliance with Ontological Modeling Principles in the Foundational Model of Anatomy." Computers in Biology and Medicine 36(7-8):674-93.

The objective of this study is to provide an operational definition of principles with which wellformed ontologies should comply. We define 15 such principles, related to classification (e.g., no hierarchical cycles are allowed; concepts have a reasonable number of children), incompatible relationships (e.g., two concepts cannot stand both in a taxonomic and partitive relation), dependence among concepts, and the co-dependence of equivalent sets of relations. Implicit relations--embedded in concept names or inferred from a combination of explicit relations--are used in this process in addition to the relations explicitly represented. As a case study, we investigate the degree to which the Foundational Model of Anatomy (FMA)--a large ontology of anatomy--complies with these 15 principles. The FMA succeeds in complying with all the principles: totally with one and mostly with the others. Reasons for non-compliance are analyzed and suggestions are made for implementing effective enforcement mechanisms in ontology development environments. The limitations of this study are also discussed.

Zhao, Q, and B Chen. 2006, "Temporal and Information Flow Based Event Detection from Social Text Streams." Social Text (Leuski 2004).

Zhen, W, and MX Zhou. 2008, "Evaluating the Use of Data Transformation for Information Visualization." Visualization and Computer Graphics, IEEE Transactions on 14(6):1309-16.

Data transformation, the process of preparing raw data for effective visualization, is one of the key challenges in information visualization. Although researchers have developed many data transformation techniques, there is little empirical study of the general impact of data transformation on visualization. Without such study, it is difficult to systematically decide when and which data transformation techniques are needed. We thus have designed and conducted a two-part empirical study that examines how the use of common data transformation techniques impacts visualization quality, which in turn affects user task performance. Our first experiment studies the impact of data transformation on user performance in single-step, typical visual analytic tasks. The second experiment assesses the impact of data transformation in multi-step analytic tasks. Our results quantify the benefits of data transformation in both experiments. More importantly, our analyses reveal that (1) the benefits of data transformation vary significantly by task and by visualization, and (2) the use of data transformation depends on a user's interaction context. Based on our findings, we present a set of design recommendations that help guide the development and use of data transformation techniques. 
Zhen, W, MX Zhou, and V Aggarwal. 2005, "An Optimization-Based Approach to Dynamic Visual Context Management." In IEEE Symposium on Information Visualization, pp. 187-94.

We are building an intelligent multimodal conversation system to aid users in exploring large and complex data sets. To tailor to diverse user queries introduced during a conversation, we automate the generation of system responses, including both spoken and visual outputs. In this paper, we focus on the problem of visual context management, a process that dynamically updates an existing visual display to effectively incorporate new information requested by subsequent user queries. Specifically, we develop an optimization based approach to visual context management. Compared to existing approaches, which normally handle predictable visual context updates, our work offers two unique contributions. First, we provide a general computational framework that can effectively manage a visual context for diverse, unanticipated situations encountered in a user system conversation. Moreover, we optimize the satisfaction of both semantic and visual constraints, which otherwise are difficult to balance using simple heuristics. Second, we present an extensible representation model that uses feature based metrics to uniformly define all constraints. We have applied our work to two different applications and our evaluation has shown the promise of this work.

Zheng, H-T, C Borchert, and Y Jiang. 2010, "A Knowledge-Driven Approach to Biomedical Document Conceptualization." Artificial Intelligence in Medicine 49(2):67-78.

Objective Biomedical document conceptualization is the process of clustering biomedical documents based on ontology-represented domain knowledge. The result of this process is the representation of the biomedical documents by a set of key concepts and their relationships. Most of clustering methods cluster documents based on invariant domain knowledge. The objective of this work is to develop an effective method to cluster biomedical documents based on various user-specified ontologies, so that users can exploit the concept structures of documents more effectively.Methods We develop a flexible framework to allow users to specify the knowledge bases, in the form of ontologies. Based on the user-specified ontologies, we develop a key concept induction algorithm, which uses latent semantic analysis to identify key concepts and cluster documents. A corpus-related ontology generation algorithm is developed to generate the concept structures of documents.Results Based on two biomedical datasets, we evaluate the proposed method and five other clustering algorithms. The clustering results of the proposed method outperform the five other algorithms, in terms of key concept identification. With respect to the first biomedical dataset, our method has the F-measure values 0.7294 and 0.5294 based on the MeSH ontology and gene ontology (GO), respectively. With respect to the second biomedical dataset, our method has the F-measure values 0.6751 and 0.6746 based on the MeSH ontology and GO, respectively. Both results outperforms the five other algorithms in terms of F-measure. Based on the MeSH ontology and GO, the generated corpus-related ontologies show informative conceptual structures.Conclusions The proposed method enables users to specify the domain knowledge to exploit the conceptual structures of biomedical document collections. In addition, the proposed method is able to extract the key concepts and cluster the documents with a relatively high precision. 
Zheng, Z, et al. 2010, "Vdvr : Verifiable Visualization of Projection-Based Data." IEEE Transactions on Visualization and Computer Graphics 16(6):1515-24.

Zhicheng, L, N Nersessian, and J Stasko. 2008, "Distributed Cognition as a Theoretical Framework for Information Visualization." Visualization and Computer Graphics, IEEE Transactions on 14(6):1173-80.

Even though information visualization (InfoVis) research has matured in recent years, it is generally acknowledged that the field still lacks supporting, encompassing theories. In this paper, we argue that the distributed cognition framework can be used to substantiate the theoretical foundation of InfoVis. We highlight fundamental assumptions and theoretical constructs of the distributed cognition approach, based on the cognitive science literature and a real life scenario. We then discuss how the distributed cognition framework can have an impact on the research directions and methodologies we take as InfoVis researchers. Our contributions are as follows. First, we highlight the view that cognition is more an emergent property of interaction than a property of the human mind. Second, we argue that a reductionist approach to study the abstract properties of isolated human minds may not be useful in informing InfoVis design. Finally we propose to make cognition an explicit research agenda, and discuss the implications on how we perform evaluation and theory building.

Zhong, Y. 2008, "Study on Cognitive Decision Support Based on Learning and Improvement of Mental Models." In 2008 ISECS International Colloquium on Computing, Communication, Control, and Management, pp. 490-94.

Zhou, H. 2009, "Visual Clustering in Parallel Coordinates and Graphs." in Computer Science, Vol Ph.D., pp. 125-25. Hong Kong University of Science and Technology (Hong Kong), Hong Kong.

Information visualization has emerged as a very active research field for multivariate and relational data analysis in recent years. It turns complex and abstract data such as demographic data, financial data, social networks, and paper citations into visual representations, and then users can exploit interactive computer graphics techniques and human visual capabilities to gain insight into the data. Parallel coordinates and graphs are two well-established methods in information visualization. However, when data become very large, the effectiveness of both methods is dramatically reduced as tens of thousands of lines can easily overwhelm the display and the resulting visual clutter will obscure any underlying patterns. Thus, clutter reduction for parallel coordinates and graphs is a very important research problem in information visualization. In this thesis, we introduce visual clustering as a new approach for clutter reduction and pattern detection. Compared with traditional clutter reduction methods such as filtering and brushing, visual clustering can enhance and reveal interesting patterns in the data while preserving the context. For parallel coordinates, we present a force-based optimization method to bundle polylines by adjusting their shapes, and a splatting framework to reveal features with animations. For graphs, a geometry-based edge grouping approach and an energy-based hierarchical visual clustering scheme are proposed. The effectiveness of these methods has been demonstrated through extensive experiments using both synthetic data and datasets from real applications. 
Zhou, H, and WJ Hou. 2009, "Multi-Hierarchy Information Visualization Research Based on ThreeDimensional Display of Products System." in Human Interface and the Management of Information: Information and Interaction, Pt Ii, eds. G Salvendy and MJ Smith, Vol 5618, pp. 287-94.

Currently, the information on the Web is countless, which is throughout tens of thousands of Web sites all over the world. And the Web site intertwined with each other through hyperlinks between documents. Regardless Of Such a big scale of the Web information, it will continue expanding. How to access to the information on the Web easily has become a problem needed to be solved urgently. However, the way of accessing to the information is far from satisfactory. Information visualization will play an increasingly important role in helping people understand the structure of the information space, finding information needed quickly and preventing the lost in the information ocean effectively. The paper used the Multi-hierarchy information visualization on a specific e-commerce web site, and established a three-dimensional products display system. According to the analysis of users on business web site, the establishment of a representative user model was established. In accordance with the user model, system function was analyzed and integrated, and task analysis was hierarchical. Based on the user's demand, the paper confirmed the content and the way of the showing. Finally the paper designed the system according to the information structure, interaction and information visualization.

Zhou, L, and G Hripcsak. 2007, "Temporal Reasoning with Medical Data--a Review with Emphasis on Medical Natural Language Processing." Journal of Biomedical Informatics 40(2):183-202.

Temporal information is crucial in electronic medical records and biomedical information systems. Processing temporal information in medical narrative data is a very challenging area. It lies at the intersection of temporal representation and reasoning (TRR) in artificial intelligence and medical natural language processing (MLP). Some fundamental concepts and important issues in relation to TRR have previously been discussed, mainly in the context of processing structured data in biomedical informatics; however, it is important that these concepts be re-examined in the context of processing narrative data using MLP. Theoretical and methodological TRR studies in biomedical informatics can be classified into three main categories: category 1 applies theories and models from temporal reasoning in AI; category 2 defines frameworks that meet needs from clinical applications; category 3 resolves issues such as temporal granularity and uncertainty. Currently, most MLP systems are not designed with a formal representation of time, and their ability to reason about temporal relations among medical events is limited. Previous work in processing time with clinical narrative data includes processing time in clinical reports, modeling textual temporal expressions in clinical databases, processing time in clinical guidelines, and building time standards for data exchange and integration. In addition to common problems in MLP, there are challenges specific to TRR in medical text, which occur at each level of linguistic structure and analysis. Despite advances in temporal reasoning in biomedical informatics, processing time in medical text deserves more attention. Besides the need for more research in temporal granularity, fuzzy time, temporal contradiction, intermittent events and uncertainty, broad areas for future research include enhancing functions of current MLP systems on processing temporal information, incorporating medical knowledge into temporal reasoning systems, resolving coreference, integrating narrative data with structured data and evaluating these systems. 
Zhou, MX, and SK Feiner. 1998, "Visual Task Characterization for Automated Visual Discourse Synthesis." In SIGCHI conference on Human factors in computing systems - CHI '98, pp. 392-99.

To develop a comprehensive and systematic approach to the automated design of visual discourse, we introduce a visual task taxonomy that interfaces high-level presentation intents with low-level visual techniques. In our approach, visual tasks describe presentation intents through their visual accomplishments, and suggest desired visual techniques through their visual implications. Therefore, we can charac- terize visual tasks by their visual accomplishments and implications. Through this characterization, visual tasks can guide the visual discourse synthesis process by specifying what presentation intents can be achieved and how to achieve them.

Zhou, Z, et al. 2004, "An Experimental Study on the Role of 3d Sound in Augmented Reality Environment." Interacting with Computers 16(6):1043-68.

Investigation of augmented reality (AR) environments has become a popular research topic for engineers, computer and cognitive scientists. Although application oriented studies focused on audio AR environments have been published, little work has been done to vigorously study and evaluate the important research questions of the effectiveness of three-dimensional (3D) sound in the AR context, and to what extent the addition of $3 \mathrm{D}$ sound would contribute to the AR experience. Thus, we have developed two AR environments and performed vigorous experiments with human subjects to study the effects of $3 \mathrm{D}$ sound in the AR context. The study concerns two scenarios. In the first scenario, one participant must use vision only and vision with 3D sound to judge the relative depth of augmented virtual objects. In the second scenario, two participants must cooperate to perform a joint task in a game-based AR environment. Hence, the goals of this study are (1) to access the impact of 3D sound on depth perception in a single-camera AR environment, (2) to study the impact of 3D sound on task performance and the feeling of ['] human presence and collaboration', (3) to better understand the role of 3D sound in humancomputer and human-human interactions, (4) to investigate if gender can affect the impact of 3D sound in AR environments. The outcomes of this research can have a useful impact on the development of audio AR systems, which provide more immersive, realistic and entertaining experiences by introducing 3D sound. Our results suggest that 3D sound in AR environment significantly improves the accuracy of depth judgment and improves task performance. Our results also suggest that 3D sound contributes significantly to the feeling of human presence and collaboration and helps the subjects to [']identify spatial objects'.

Zhu, B, and H Chen. 2005, "Information Visualization." Annual review of information science and technology 39(1):139-77.

Zhu, B, and H Chen. 2005, "Using 3d Interfaces to Facilitate the Spatial Knowledge Retrieval: A GeoReferenced Knowledge Repository System." Decision Support Systems 40(2):167-82.

Retrieving knowledge from a knowledge repository includes both the process of finding information of interest and the process of converting incoming information to a person's own knowledge. This paper explores the application of 3D interfaces in supporting the retrieval of spatial knowledge by presenting the development and the evaluation of a geo-referenced knowledge repository system. As computer screen is crowded with high volume of information available, 3D interface becomes a promising candidate to better use the screen space. A 3D interface is also more similar to the 3D terrain surface it represents than its 2D counterpart. However, almost all previous empirical studies did not find any supportive evidence for the application of 3D interface. Realizing that those studies required users to 
observe the 3D object from a given perspective by providing one static interface, we developed 3D interfaces with interactive animation, which allows users to control how a visual object should be displayed. The empirical study demonstrated that this is a promising approach to facilitate the spatial knowledge retrieval.

Zhu, B, and HC Chen. 2008, "Communication-Garden System: Visualizing a Computer-Mediated Communication Process." Decision Support Systems 45(4):778-94.

Archives of computer-mediated communication (CMC) Could be valuable organizational resources. Most CMC archive systems focus on presenting one of the three aspects of a CMC community: discussion content, participants' behavior, or social net-works among participants. Very few CMC archive systems support the easy integration of these three aspects. This paper thus describes two-phase research to propose an automatic approach that facilitates users' integrated understanding of discussion content and behavior of CIVIC participants. We validated the approach through the development and evaluation of a prototype system, the Communication-Garden system. (C) 2008 Elsevier B.V. All rights reserved.

Zhu, B, and HC Chen. 2005, "Information Visualization." Annual review of information science and technology 39:139-77.

Zhu, B, S Watts, and H Chen. 2010, "Visualizing Social Network Concepts." Decision Support Systems 49(2):151-61.

Social network concepts are invaluable for understanding the social network phenomena, but they are difficult to comprehend without computerized visualization. However, most existing network visualization techniques provide limited support for the comprehension of network concepts. This research proposes an approach called concept visualization to facilitate the understanding of social network concepts. The paper describes an implementation of the approach. Results from a controlled laboratory experiment indicate that, compared with the benchmark system, the NetVizer system facilitated better understanding of the concepts of betweenness centrality, gatekeepers of subgroups, and structural similarity. It also supported a faster comprehension of subgroup identification.

Zhu, W, and C Chen. 2007, "Storylines: Visual Exploration and Analysis in Latent Semantic Spaces." Computers \& Graphics 31(3):338-49.

Zhu, Y. 2007, "Measuring Effective Data Visualization." In 3rd International Symposium on Visual Computing, pp. 652-61.

In this paper, we systematically examine two fundamental questions in information visualization how to define effective visualization and how to measure it. Through a literature review, we point out that the existing definitions of effectiveness are incomplete and often inconsistent - a problem that has deeply affected the design and evaluation of visualization. There is also a lack of standards for measuring the effectiveness of visualization as well as a lack of standardized procedures. We have identified a set of basic research issues that must be addressed. Finally, we provide a more comprehensive definition of effective visualization and discuss a set of quantitative and qualitative measures. The work presented in this paper contributes to the foundational research of information visualization. (C) Springer-Verlag Berlin Heidelberg 2007. 
Zhu, Y, XY Suo, and GS Owen. 2007, "Complexity Analysis for Information Visualization Design and Evaluation." in Advances in Visual Computing, Pt I, eds. G Bebis, et al., Vol 4841 LNCS, pp. 576-85.

In this paper, we present a method for analyzing the complexity of information visualization. We have identified a number of factors that influence the efficiency of visual information read-off and integration. Here the complexity is measured in terms of visual integration, number of separable dimensions for each visual unit, the complexity of interpreting the visual attributes, and the efficiency of visual search. These measures are derived from well established psychological theories. Together they indicate the amount of cognitive load for comprehending a visualization design. This method is particularly useful for developers to quickly evaluate multiple design choices. Finally, we demonstrate our method through a complexity analysis on a computer security visualization program.

Zhu, Y, XY Suo, and GS Owen. 2009, "A Visual Data Exploration Framework for Complex Problem Solving Based on Extended Cognitive Fit Theory." in Advances in Visual Computing, Pt 2, Proceedings, eds. G Bebis, et al., Vol 5876 LNCS, pp. 869-78.

In this paper, we present a visual data exploration framework for complex problem solving. This framework consists of two major components: an enhanced task flow diagram and data visualization window. Users express their problem solving process and strategies using the enhanced task flow diagram, while multiple frames of visualizations are automatically constructed in the data visualization window and are organized as a tree map. This framework is based an extended Cognitive Fit Theory, which states that a data visualization should be constructed as a cognitive fit for specific tasks and a set of data variables. It also states that the structure of multiple data visualizations should match the structure of the corresponding tasks. Therefore, in our framework, data is presented in either visual or non-visual format based on the cognitive characteristic of the corresponding task. As users explore various problem solving strategies by editing the task flow diagram, the corresponding data visualizations are automatically updated for the best cognitive fit. This visual data exploration framework is particularly beneficial for users who need to conduct specific and complex tasks with large amount of data. As a case study, we present a computer security data visualization prototype.

Ziat, M, et al. 2007, "Haptic Recognition of Shapes at Different Scales: A Comparison of Two Methods of Interaction." Interacting with Computers 19(1):121-32.

In order to design a "haptic zoom", in this fundamental study, we compare two scaling methods by focusing on the strategies adopted by subjects who are using a sensory substitution device. Method 1 consists of a reduction of the sensor size and of its displacement speed. Speed reduction is obtained by a "human" movement adjustment (hand speed reduction). Method 2 consists of a straightforward increase in the dimensions of the image. The experimental device used couples a pen on a graphics tablet with tactile sensory stimulators. These are activated when the sensor impinges on the outline of the figure on the computer screen. This virtual sensor (a square matrix composed of 16 elementary fields) moves when the pen, guided by human hand movements, moves on the graphics tablet. The results show that the recognition rate is closely dependent on the size of the figure, and that the strategies used by the subjects are more suitable for method 2 than for method 1. In fact, half of the subjects found that method 1 inhibited their movements, and the majority of them did not feel the scaling effect, whereas this was clearly felt in method 2 . 
Ziefle, M. 2010, "Information Presentation in Small Screen Devices: The Trade-Off between Visual Density and Menu Foresight." Applied Ergonomics 41(6):719-30.

Small mobile devices are ubiquitous and must be designed with great care. One of the most serious challenges is how information on the small displays is presented optimally. This paper addresses the special problem of the increasing number of aged users. On the one hand, information displayed should be easily readable. This requires a low information density and a sufficiently large font size. On the other hand menu orientation is facilitated when the amount of information per screen is maximized and a large preview is allowed. This requires presenting as many functions as possible on the screen at a time. Thus, the tradeoff between readability and orientation demands is crucial. In the present study, this tradeoff was experimentally investigated. Two factors, font size ( $8 \mathrm{pt}, 12 \mathrm{pt}$ ) and the size of the preview (one or five functions per screen at a time) were varied and effects on navigation performance were observed. Forty older participants solved nine common phone navigation tasks twice consecutively on a simulated mobile phone. Both factors contributed to performance, but there was a significant interaction: navigation performance was optimal when font size and the size of the preview were large. The lowest performance was obtained when the preview was small and the font size large, showing that proper orientation is more important than visibility demands. The results can be used in ergonomic guidelines to optimized information presentation on small screens.

Ziegler, H, and DA Keim. 2010, "Visual Market Sector Analysis for Financial Time Series Data." In IEEE Symposium on Visual Analytics Science \& Technology, pp. 83-90.

Ziegler, H, T Nietzschmann, and DA Keim. 2007, "Visual Exploration and Discovery of Atypical Behavior in Financial Time Series Data Using Two-Dimensional Colormaps." In 11th International Conference Information Visualization (IV'07), pp. 308-15.

This work describes two pixel-based paradigms for visual financial time series data analysis that allow analyzing assets in overview and in detail, and offer improved insights into the characteristics of assets compared to traditional visualization methods. We contribute a two-dimensional color coding scheme for inter-Zintra-asset analysis that extends the two paradigms and supports the discovery of significant characteristics of assets, such as atypical " against-the-market" -behavior inform of exceptional stability in case of whole market losses, or the discovery of assets in a portfolio that - while still being profitable - notably underperform the market median. We apply our techniques on real world data sets, and focus on assets of the banks of Switzerland.

Ziemkiewicz, C, et al. 2010, "Visualization Theory : Putting the Pieces Together." In IEEE Symposium on Information Visualization.

Ziemkiewicz, C, and R Kosara. 2010, "Laws of Attraction: From Perceptual Forces to Conceptual Similarity." Visualization and Computer Graphics, IEEE Transactions on 16(6):1009-16.

Many of the pressing questions in information visualization deal with how exactly a user reads a collection of visual marks as information about relationships between entities. Previous research has suggested that people see parts of a visualization as objects, and may metaphorically interpret apparent physical relationships between these objects as suggestive of data relationships. We explored this hypothesis in detail in a series of user experiments. Inspired by the concept of implied dynamics in psychology, we first studied whether perceived gravity acting on a mark in a scatterplot can lead to errors 
in a participant's recall of the mark's position. The results of this study suggested that such position errors exist, but may be more strongly influenced by attraction between marks. We hypothesized that such apparent attraction may be influenced by elements used to suggest relationship between objects, such as connecting lines, grouping elements, and visual similarity. We further studied what visual elements are most likely to cause this attraction effect, and whether the elements that best predicted attraction errors were also those which suggested conceptual relationships most strongly. Our findings show a correlation between attraction errors and intuitions about relatedness, pointing towards a possible mechanism by which the perception of visual marks becomes an interpretation of data relationships.

Ziemkiewicz, C, and R Kosara. 2009, "Preconceptions and Individual Differences in Understanding Visual Metaphors." Computer Graphics Forum 28(3):911-18.

Understanding information visualization is more than a matter of reading a series of data values; it is also a matter of incorporating a visual structure into one's own thinking about a problem. We have proposed visual metaphors as a framework for understanding high-level visual structure and its effect on visualization use. Although there is some evidence that visual metaphors can affect visualization use, the nature of this effect is still ambiguous. We propose that a user's preconceived metaphors for data and other individual differences play an important role in her ability to think in a variety of visual metaphors, and subsequently in her ability to use a visualization. We test this hypothesis by conducting a study in which a participant's preconceptions and thinking style were compared with the degree to which she is affected by conflicting metaphors in a visualization and its task questions. The results show that metaphor compatibility has a significant effect on accuracy, but that factors such as spatial ability and personality can lessen this effect. We also find a complex influence of self-reported metaphor preference on performance. These findings shed light on how people use visual metaphors to understand a visualization. (C) 2009 The Eurographics Association and Blackwell Publishing Ltd.

Ziemkiewicz, C, and R Kosara. 2008, "The Shaping of Information by Visual Metaphors." Visualization and Computer Graphics, IEEE Transactions on 14(6):1269-76.

The nature of an information visualization can be considered to lie in the visual metaphors it uses to structure information. The process of understanding a visualization therefore involves an interaction between these external visual metaphors and the user's internal knowledge representations. To investigate this claim, we conducted an experiment to test the effects of visual metaphor and verbal metaphor on the understanding of tree visualizations. Participants answered simple data comprehension questions while viewing either a treemap or a node-link diagram. Questions were worded to reflect a verbal metaphor that was either compatible or incompatible with the visualization a participant was using. The results suggest that the visual metaphor indeed affects how a user derives information from a visualization. Additionally, we found that the degree to which a user is affected by the metaphor is strongly correlated with the user's ability to answer task questions correctly. These findings are a first step towards illuminating how visual metaphors shape user understanding, and have significant implications for the evaluation, application, and theory of visualization. 
Ziemkiewicz, C, and R Kosara. 2008, Understanding Information Visualization in the Context of Visual Communication. Technical Rpt. CVC-UNCC-07-08, Univeristy of North Carolina.

Zook, M, and M Dodge. 2009, "Mapping, Cyberspace." in International Encyclopedia of Human Geography, eds. K Rob and T Nigel, pp. 356-67. Elsevier, Oxford.

Cyberspace cartographies are a significant area of creativity in contemporary mapmaking, with a considerable amount of experimentation with visual metaphors, survey methods, data sources, and novel forms of user interaction with map representations. Mapping cyberspace can be usefully categorized into three distinct cartographic modes: (1) [']maps in cyberspace' which provide greater access and user interactivity to traditional geographic maps by placing them online; (2) [']maps of cyberspace' which represent the structures and operations of cyberspace itself; and (3) [']maps for cyberspace' which are designed for navigation through the virtual spaces of cyberspace. All three modes of cyberspace mapping create innovative forms of representation and have expanded the frontiers of cartography in terms of what is mappable and how users interact with maps. Alongside these achievements, however, remain age-old questions of the power and the politics of maps. While cyberspace is in some ways democratizing mapmaking, it has simultaneously provided governments and corporations with new opportunities for mapping and control.

Zuk, T, and S Carpendale. 2006, "Theoretical Analysis of Uncertainty Visualizations - Art. No. 606007." in Visualization and Data Analysis 2006 eds. RF Erbacher, et al., Vol 6060, pp. 6007-07. SPIE.

Although a number of theories and principles have been developed to guide the creation of visualizations, it is not always apparent how to apply the knowledge in these principles. We describe the application of perceptual and cognitive theories for the analysis of uncertainty visualizations. General principles from Bertin. Tufte, and Ware are outlined and then applied to the analysis of eight different uncertainty visualizations. The theories provided a useful framework for analysis of the methods, and provided insights into the strengths and weaknesses of various aspects of the visualizations.

Zuk, T, et al. 2006, "Heuristics for Information Visualization Evaluation." In AVI workshop on BEyond time and errors: novel evaluation methods for information visualization (BELIV'06), pp. 1-6.

Heuristic evaluation is a well known discount evaluation technique in human-computer interaction (HCI) but has not been utilized in information visualization (InfoVis) to the same extent. While several sets of heuristics have been used or proposed for InfoVis, it is not yet known what kind of heuristics are useful for finding general InfoVis problems. We performed a meta-analysis with the goal of exploring the issues of heuristic evaluation for InfoVis. This meta-analysis concentrates on issues pertaining to the selection and organization of heuristics, and the process itself. For this purpose, we used three sets of previously published heuristics to assess a visual decision support system that is used to examine simulation data. The meta-analysis shows that the evaluation process and results have a high dependency on the heuristics and the types of evaluators chosen. We describe issues related to interpretation, redundancy, and conflict in heuristics. We also provide a discussion of generalizability and categorization of these heuristics.

Zülch, G, and S Stowasser. 2003, "Chapter 25 - Eye Tracking for Evaluating Industrial Human-Computer Interfaces." in The Mind's Eye: Cognitive and Applied Aspects of Eye Movement Research, eds. J Hyönä, R Radach and H Deubel, pp. 531-53. North-Holland, Amsterdam. 
A.503 



\section{Distribution}

No. of

Copies

\# Name

Organization

Address

City, State and ZIP Code

\# Organization

Address

City, State and ZIP Code

Name

Name

Name

Name

Name (\#)

\# Name

Organization

Address

City, State and ZIP Code
No. of

\section{Copies}

\# Foreign Distribution

\# Name

Organization

Address

Address line 2

COUNTRY

\# Local Distribution

Pacific Northwest National Laboratory

Name

Name

Mailstop

Mailstop

Name

Mailstop

Name

Mailstop

Name 




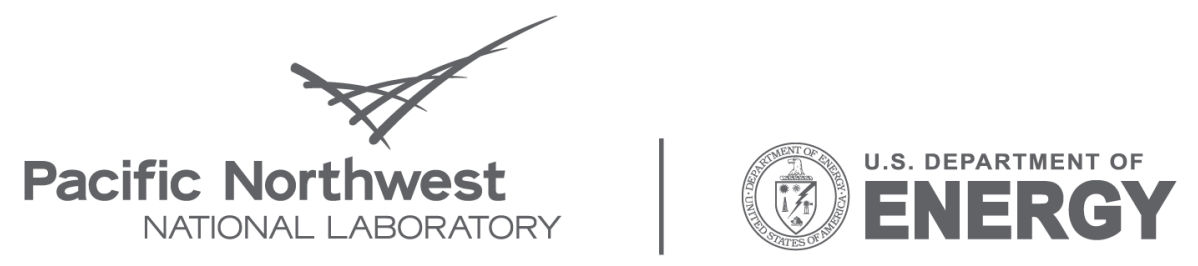

Proudly Operated by Battelle Since 1965

902 Battelle Boulevard

P.O. Box 999

Richland, WA 99352

1-888-375-PNNL (7665)

www.pnl.gov 